


\section{PRACTICAL APPLICATIONS IN BIOMEDICAL ENGINEERING}

Edited by Adriano O. Andrade, Adriano Alves Pereira, Eduardo L. M. Naves and Alcimar B. Soares 


\section{Practical Applications in Biomedical Engineering}

http://dx.doi.org/10.5772/3331

Edited by Adriano O. Andrade, Adriano Alves Pereira, Eduardo L. M. Naves and Alcimar B. Soares

\section{Contributors}

Suélia De Siqueira Rodrigues Fleury Rosa, Adson Rocha, José Conceição Carvalho, Christakis Constantinides, Adriano Andrade, Juraj Kronek, Ema Paulovicova, Zuzana Kronekova, Lucia Paulovicova, Jozef Luston, Thanh-Dinh Thanh Nguyen, Venketesh N. Dubey, Neil Vaughan, Michael Wee, Richard Isaacs, Ajay Sonar, James Carroll, Vasilios Papaioannou, loannis Pneumatikos, Geovani Rodrigo Scolaro, Fernando Mendes De Azevedo, Christine Boos, Roger Walz, Elena Chernolovskaya, Natalya Petrova, Marina Zenkova, Tatiana Vasilieva, Erika G. Meraz, Homer Nazeran, Lidia Rascon Madrigal, Nelly Gordillo Castillo, Carlos Ramos, Liza Rodriguez, Galba Maria Campos-Takaki, Walid A. Zgallai

\section{(c) The Editor(s) and the Author(s) 2013}

The moral rights of the and the author(s) have been asserted. All rights to the book as a whole are reserved by INTECH. The book as a whole (compilation) cannot be reproduced distributed or used for commercial or non-commercial purposes without INTECH's written permission. Enquiries concerning the use of the book should be directed to INTECH rights and permissions department (permissions@intechopen.com). Violations are liable to prosecution under the governing Copyright Law.

\section{(cc)BY}

Individual chapters of this publication are distributed under the terms of the Creative Commons Attribution 3.0 Unported License which permits commercial use, distribution and reproduction of the individual chapters, provided the original author(s) and source publication are appropriately acknowledged. If so indicated, certain images may not be included under the Creative Commons license. In such cases users will need to obtain permission from the license holder to reproduce the material. More details and guidelines concerning content reuse and adaptation can be foundat http://www.intechopen.com/copyright-policy.html.

\section{Notice}

Statements and opinions expressed in the chapters are these of the individual contributors and not necessarily those of the editors or publisher. No responsibility is accepted for the accuracy of information contained in the published chapters. The publisher assumes no responsibility for any damage or injury to persons or property arising out of the use of any materials, instructions, methods or ideas contained in the book.

First published in Croatia, 2013 by INTECH d.o.o.

eBook (PDF) Published by IN TECH d.o.o.

Place and year of publication of eBook (PDF): Rijeka, 2019.

IntechOpen is the global imprint of IN TECH d.o.o.

Printed in Croatia

Legal deposit, Croatia: National and University Library in Zagreb

Additional hard and PDF copies can be obtained from orders@intechopen.com

Practical Applications in Biomedical Engineering

Edited by Adriano O. Andrade, Adriano Alves Pereira, Eduardo L. M. Naves and Alcimar B. Soares

p. $\mathrm{cm}$.

ISBN 978-953-51-0924-2

eBook (PDF) ISBN 978-953-51-6287-2 


\section{We are IntechOpen, \\ the world's leading publisher of Open Access books}

Built by scientists, for scientists

\section{$4,000+$ \\ Open access books available \\ $116,000+$ \\ International authors and editors

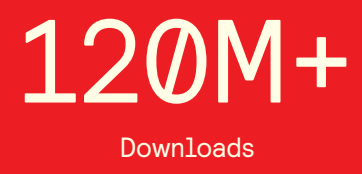

Our authors are among the

151

Countries delivered to

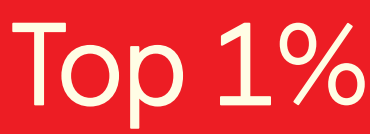

most cited scientists

Contributors from top 500 universities

$12.2 \%$

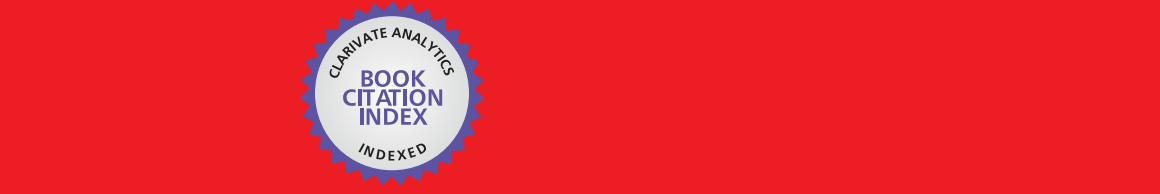

WEB OF SCIENCE ${ }^{\mathrm{M}}$

Selection of our books indexed in the Book Citation Index in Web of Science ${ }^{\mathrm{TM}}$ Core Collection (BKCI)

\section{Interested in publishing with us? \\ Contact book.department@intechopen.com}





\section{Meet the editor}

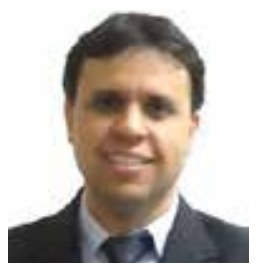

Dr. Adriano O. Andrade graduated in Electrical Engineering at the Federal University of Goiás (Brazil) in 1997. He received his MSc and PhD in Biomedical Engineering respectively from the Federal University of Uberlândia (Brazil) in 2000 and from the University of Reading (UK) in 2005. He completed a one year Post-Doctoral Fellowship at the Institute of Biomedical Engineering of the University of New Brunswick (Canada) in 2010. Currently, he is Professor in the Faculty of Electrical Engineering of the Federal University of Uberlândia. He has authored and co-authored more than 100 peer-reviewed publications in Biomedical Engineering. Dr. Andrade's primary area of research is focused towards getting information from the neuromuscular system in order to understand its strategies of organization, adaptation and controlling. His research interests include Biomedical Signal Processing and Modelling, Assistive Technology and Rehabilitation Engineering. 



\section{Contents}

\section{Preface XI}

Section 1 Biomedical Signal Processing and Modelling 1

Chapter 1 Human Tremor:

Origins, Detection and Quantification 3

Adriano O. Andrade, Adriano Alves Pereira,

Maria Fernanda Soares de Almeida,

Guilherme Lopes Cavalheiro, Ana Paula Souza Paixão,

Sheila Bernardino Fenelon and Valdeci Carlos Dionisio

Chapter 2 Second- and Third-Order Statistical Characterization of Non-Linearity and Non-Gaussianity of Adult and Fetal ECG Signals and Noise 25 Walid A. Zgallai

Chapter 3 Fractal Physiology, Breath-to-Breath Variability and Respiratory Diseases: An Introduction to Complex Systems Theory Application in Pulmonary and Critical Care Medicine 55 Vasilios Papaioannou and Ioannis Pneumatikos

Chapter 4 Wavelet Filter to Attenuate the Background Activity and High Frequencies in EEG Signals Applied in the Automatic Identification of Epileptiform Events 81 Geovani Rodrigo Scolaro, Fernando Mendes de Azevedo, Christine Fredel Boos and Roger Walz

Chapter 5 Impulse Oscillometric Features and Respiratory System Models Track Small Airway Function in Children 103 Erika G. Meraz, Homer Nazeran, Carlos Ramos, Liza Rodriguez, Lidia Rascón Madrigal and Nelly Gordillo Castillo

Chapter 6 Simulation of Subject Specific Bone Remodeling 141 Ajay Sonar and James Carroll 
Chapter 7 Prosthesis for Flow Control in the Esophagus as a New Technique for the Treatment of Obesity 169 Suélia de $S$. Rodrigues Fleury Rosa, Adson Ferreira da Rocha and José Conceição Carvalho

Chapter 8 Structure - Functions Relations in Small Interfering RNAs 187

Natalya S. Petrova, Marina A. Zenkova and Elena L. Chernolovskaya

Chapter 9 Microbiological Chitosan:

Potential Application as Anticariogenic Agent 229

Thayza Christina Montenegro Stamford, Thatiana Montenegro Stamford-Arnaud, Horacinna Maria de Medeiros Cavalcante, Rui Oliveira Macedo and Galba Maria de Campos-Takaki

Chapter 10 Antimicrobial and Anti-Adhesive Potential of a Biosurfactants Produced by Candida Species 245

Raquel Diniz Rufino, Juliana Moura de Luna, Leonie Asfora Sarubbo, Lígia Raquel Marona Rodrigues, José Antônio C. Teixeira and Galba Maria de Campos-Takaki

Chapter 11 Biocompatibility and Immunocompatibility Assessment of Poly(2-Oxazolines) 257 Juraj Kronek, Ema Paulovičová, Lucia Paulovičová, Zuzana Kroneková and Jozef Lustoň

Chapter 12 Bio-Medical Applications of the Electron-Beam Plasma 285

T. Vasilieva

Chapter 13 Functional Inorganic Nanohybrids for Biomedical Diagnosis 311

Thai-Hoa Tran and Thanh-Dinh Nguyen

Section 3 Biomedical Image Processing 341

Chapter 14 Study of the Murine Cardiac Mechanical Function Using Magnetic Resonance Imaging: The Current Status, Challenges, and Future Perspectives 343

Christakis Constantinides

Chapter 15 Biomedical Engineering in Epidural Anaesthesia Research 387

Venketesh N. Dubey, Neil Vaughan, Michael Y. K. Wee and Richard Isaacs 


\section{Preface}

Biomedical Engineering is an interdisciplinary and emergent area that combines engineering with life sciences. The idea of this book is to provide the reader with recent advances and applications in Biomedical Engineering in three particular fields of interest: Biomedical Signal Processing and Modelling, Biomaterials and Prosthetic Devices, and Biomedical Image Processing. The book is a collection of self-contained papers describing specific applications and reviews in these fields. Each paper was properly peer-reviewed by experts before final acceptance and inclusion in the book.

Biomedical Signal Processing and Modelling refers to the use of digital signal processing tools and mathematical models for the characterization and representation of biological systems. In many cases the information available from the system is a time-series related to its current state, hence the role of signal processing is to extract features from time-series that are able to quantify and characterize the state of the biological system. The use and implementation of models is of paramount relevance in life sciences and engineering, mainly when considering ethical and economic issues. However, there is a trade-off between model complexity and accuracy in the representation of the reality. Therefore, modelling biological systems is always a challenging task. The first six chapters of the book are dedicated to this exciting field. The study of these chapters can provide the reader with valuable practical examples of the use of signal processing and modelling. The ideas and methods employed can be easily extended to the reader's own research.

Biomaterials are substances that have been engineered to take a form which is used to direct, by control of interactions with components of living systems, the progress therapeutic and diagnostic procedures. In the past decade, there has been a great advance in this area allowing the improvement of medical treatment and diagnosis. Currently, nanostructures can be used as biomaterials capable of targeting specific living tissues, aiding the process of detection and monitoring of non-communicable diseases such as cancer that is one of the leading global killers. It is expected that in a near future biomaterials can be used in clinical routine as therapy to a number of diseases. Chapters from 7 to 13 illustrate the use of biomaterials in distinct contexts. In addition, some of these chapters also discuss implantable prosthetic devices, which can be seen as a type of biomaterial engineered for providing artificial extension of a 
body part. Prosthetic devices can be used for replacing missing body parts, and also to extend the body function.

Biomedical Image Processing concerns the use of image processing techniques for image analysis, compression and transmission. It is by itself an interdisciplinary research field attracting expertise from applied mathematics, computer science, engineering, statistics, physics, biology and medicine. Computer-assisted image analysis has already become part of clinical routine. However, the new development of high technologies and use of distinct types of imaging modalities have produced new challenges to the field. For instance, how to deal with the increasing volume of data without losing significant information that could be used for diagnosis and treatment. Furthermore, techniques of image analysis can be combined in virtual reality environments in a number of situations, e.g., surgery planning and execution, and also in training. The book brings two chapters (14 and 15) dedicated to this area. It is hoped that the information provided in these chapters can motivate the reader to further explore the field of Biomedical Image Processing.

Finally, I would like to thank my colleagues Dr. Adriano Alves Pereira, Dr. Eduardo Lázaro and Dr. Alcimar B. Soares, of the Postgraduate Program on Biomedical Engineering of the Federal University of Uberlândia in Brazil, for helping me with the task of reviewing the chapters of this book. I do hope the material we have organized can contribute to your knowledge and research.

Have a good reading!

Prof. Dr. Adriano O. Andrade

Biomedical Engineering Laboratory (BioLab), Faculty of Electrical Engineering, Federal University of Uberlandia, 


Section 1

Biomedical Signal Processing and Modelling 



\title{
Human Tremor: Origins, Detection and Quantification
}

\author{
Adriano O. Andrade, Adriano Alves Pereira, Maria Fernanda Soares \\ de Almeida, Guilherme Lopes Cavalheiro, Ana Paula Souza Paixão, \\ Sheila Bernardino Fenelon and Valdeci Carlos Dionisio
}

Additional information is available at the end of the chapter

http://dx.doi.org/10.5772/54524

\section{Introduction}

The human tremor is one of the most common movement disorders, which is characterized by repetitive and stereotyped movements. The origins of tremor are still not clear, however tremor can be associated with physiological phenomena, such as ageing, and with neurological disorders, for instance, Parkinson's disease. The first type of tremor is referred to as physiological tremor, whereas the latter as pathological tremor.

The clinical evaluation of tremor can be a valuable tool for the diagnosis of neuromuscular disorders and also for monitoring their progress. However, in a number of circumstances the discrimination between physiological and pathological tremor may not be clinically evident. In this context, the use of sensors for detecting tremor, and the data analysis tools employed in its quantification and classification are of paramount importance.

In this chapter, the origins, detection and quantification of tremor are discussed. The chapter begins with a review concerning the definition and classification of distinct types of tremor. A review of current theories that explain the origins of tremor are presented. The problem of detecting tremor by using electronic devices is addressed, and new advances in the area of tremor detection are introduced. A review and a critical discussion of the most common tools employed for tremor quantification and classification is provided. The chapter finishes by pointing out key unanswered questions in tremor research.

\section{Defining the human tremor}

Tremor is the most common movement disorder characterized by repetitive and stereotyped movements [1]. The human tremor is a clinical manifestation characterized by an 
involuntary, rhythmic, oscillatory movement of a body part that can be classified in many ways, depending on its etiology, phenomenology, frequency, location and pharmacological response [2; 3].

The rhythmic characteristic of tremor around a balanced position, as the regular rhythm, amplitude and frequency make easier the identification and also the differential diagnosis of tremor, distinguishing it from other involuntary movements.

The movement caused by tremor can be associated to many factors such as neurological disorders and natural processes. The latter is often referred to as physiological tremor and is present in greater or lesser degree, in all humans [2; 4]. The presence of severe tremor disorders causes many difficulties, and can also indicate the presence of diseases related to the central nervous system (CNS). However, the dividing landmark between physiological tremor and that resultant of dysfunctions is tenuous and has not been precisely established, since the changes in the CNS control that causes it can be associated to many factors. Some examples of pathological tremor, i.e. associated to neurological disorder factors, are the cerebellar, essential and parkinsonian tremor [5], and others, such as in psychogenic, orthostatic and neuropathic tremor, which are considered relatively rare in the medical literature [6].

According to phenomenology, or better, according to the circumstances in which tremor manifests it can be classified in two main types: resting tremor and action tremor [4]. The resting tremor can be observed when the body part in which it appears is not suffering the effects of gravity and the muscles are not contracted [4]. Usually, the resting tremor has the characteristic of adduction-abduction or flexion-extension. The main example of resting tremor is the parkinsonian tremor.

The action tremor appears during a voluntary muscle contraction [4]. The action tremor encompasses postural, kinetic, intentional, task-specific and isometric tremor.

The postural tremor can be observed when maintaining a voluntary position against the effect of gravity [4]. Some examples of postural tremor are the essential, cephalic, axial cerebellar and primary orthostatic tremor. The kinetic tremor can be observed while performing a voluntary movement [4], whereas the intentional tremor occurs during a movement, but specifically when there is the intention to hit a target, like in the test of finger-nose, while writing, speaking and handling objects. The cerebellar, essential and mesencephalic tremor are examples of intentional tremor.

The task-specific tremor is manifested almost exclusively during a specific motor movement, such as the activities of writing, drawing or playing a musical instrument [4]. Finally, the isometric tremor occurs when the affected segment is contracted without the occurrence of the displacement of the body segment [4]. Generally it can be observed in isometric muscle contraction, which occurs when force is exerted against a steady object, like in the act of pushing a wall or flexing the wrist on a surface.

Usually, the essential tremor and the enhanced physiological tremor are classified as postural tremor. The parkinsonian tremor is typically a resting tremor and decreases its 
amplitude with movement. Instead, the cerebellar tremor appears while performing a movement, therefore, is considered an action tremor, predominantly kinetic. In mesencephalic and Holmes' tremor it can be found a mixture of resting, postural and kinetic tremor with high amplitude and intensity.

Regarding the frequency of tremor, or rather, according to number of oscillations of the affected segment in a unit time, tremor can be classified into three main types: low frequency tremor (less than four cycles per second or Hertz); middle frequency tremor (between 4 and $7 \mathrm{~Hz}$ ) and high-frequency tremor (more than $7 \mathrm{~Hz}$ ) [4]. Physiological tremor usually presents high frequency $(8-12 \mathrm{~Hz}$ ) and low amplitude. Essential tremor reaches frequencies between 6-12 Hz while tremor from Parkinson's disease usually has frequencies between 4 and $6 \mathrm{~Hz}$. The cerebellar tremor is also a low frequency tremor (less than $5 \mathrm{~Hz}$ ), such as the mesencephalic tremor $(2-5 \mathrm{~Hz})$. However, the frequency range for tremor can vary depending on the patient condition and the type of treatment he is receiving.

Regarding to the location, it is possible to observe that tremor can occur in any part of the body; however the limb segments and the head are the most affected. There may be involvement of other body parts, such as the trunk, but this situation is not common [4].

According to drug response, beta-adrenergic blockers are commonly used in physiological tremor treatment. Alcohol, which can be used as therapeutic method, may be employed in patients with essential tremor. Tremor can also be reduced by relaxation, concentration, voluntary suppression and the increase of load on the affected extremity.

About two thirds of patients with essential tremor show considerable reduction of tremor for 45 to 60 minutes after the ingestion of alcohol; however alcohol cannot be used over a long period of time because in the course of time larger quantities will be required to produce similar effects, which can cause chronic alcoholism. Furthermore, when the effect of alcohol is over, tremor gets worse. The treatment of essential tremor is done with primidone or beta-blockers, another alternative is the use of the botulinum toxin direct in the affected muscles. Besides this, alprazolam has effect in the treatment of essential tremor. When this pathology is clinically intractable, a contralateral thalamotomy surgery is indicated.

The parkinsonian tremor can be reduced or controlled by the use of drugs with dopaminergic effects and with anticholinergics; in certain cases, a thalamotomy able to reach the thalamic nucleus, specially the lower ventral medial nucleus, is used in the case of unilateral Parkinson's disease. The constant thalamic high frequency stimulation is related with good results in essential and parkinsonian tremor. The deep thalamic cerebral stimulation is safer and more effective than thalamotomy, which requires the permanent placement of an electrode in the brain. Side effects of deep brain stimulation are reversible with the manipulation of the stimulation parameters.

The cerebellar tremor does not respond well to treatments. Usually substances that increase the gabaergic activity, like valproic acid, clonazepam and isoniazid, are used. The mesencephalic tremor does not respond well to some drugs either, especially its postural component. However, the resting component can improve with anticholinergics. The 
stereotactic surgery on the ventral medial nucleus of thalamus can control the postural component.

Thousands of people each year begin to present some type of motor dysfunction, which interferes in their daily activities and reduces significantly the quality of life of these individuals. A number of studies and governmental statistics have shown that the elderly population is the most affected by tremor and its consequences, which are responsible for physical limitations of these individuals [1].

The manifestation of the tremor can cause considerable functional incapacity leading to social isolation by interference in the activities of daily living (ADLs) and instrumental activities of daily living (IADL) such as eating, writing, dressing and maintaining some personal care [7].

Moreover, recent researches from the Brazilian Ministry of Health (available on www.saude.gov.br) suggest that signs like tremor and loss of balance do not always mean the presence of neurological diseases such as Parkinson's disease, which affects mainly the population with age over 50 years old. According to this Ministry about $25 \%$ of patients who exhibit signs of Parkinson do not have the disease. The imprecise diagnosis of these diseases and the consequent use of unnecessary or inappropriate drugs also results in waste of public resources.

Generally, current therapies are limited because they relieve symptoms more than cure. The most commonly used drugs are: propranolol, primidone, gabapentin, topiramate and others to be considered comprise in the second row like alprazolam, atenolol, sotalol and clonazepam and, in the third line: clozapine, nadolol, nimodipine, being the botulinum toxin the first line for hands, head and voice tremors, in cases of essential tremor.

Some studies have suggested that moderate tremor, which accompanies the natural aging process can be diagnosed as pathological tremor. It is also possible that the pathological tremor is wrongly diagnosed as physiological tremor [5].

The human tremor is a public health problem faced all over the world. Costs related to medical and social aspects, necessary for diagnosis and treatment of tremor, have grown constantly in past decades and currently reach billions of dollars in many countries. A treatment that seeks to mitigate the symptoms and create the possibility of a person with tremor accomplish everyday tasks constitute an important intervention. In this context, studies that contribute to the understanding of tremor are of paramount importance.

\section{Understanding the origins of human tremor}

The study of tremor is not new and it can be found in the biblical texts and documents of antiquity coming from India and Egypt [3]. The interest in the study of tremor increased over the past decades and, lately, many researches can be found in this area, especially related to the quantification of human tremor signals. Quantification of tremor allows studying it in an objective way, making it possible to establish relationships between the tremor activity and variables, such as age or the presence of neurological dysfunctions. 
Regarding to the study of tremor's origins only relatively recently such ideas have received great interest [8].

The movement caused by tremor can be associated to factors such as neurological disorders and natural processes. $[2 ; 11 ; 12]$ The former is called pathological tremor whereas the latter is often referred to as physiological tremor.

There are several hypotheses to explain the appearance of physiological tremor (PT). One explanation for the existence of physiological tremor is the effect of ballistocardiogram, i.e., the passive vibration of the body tissues produced by the mechanical activity of the heart [13], i.e., a result of mechanical reflexes of the heartbeat and also of neural reflexes [14]. Another hypothesis is that physiological tremor is induced by mechanical properties of limbs and motor neurons firings.

It is also believed that the physiological tremor is a peripheral manifestation of neural oscillatory activity in the central nervous system (CNS) and that some types of pathological tremors are resultant of distortions and amplifications of these central oscillations [8].

According to Hallett (1998), the sources of tremor can be summarized into three groups: mechanical, reflex and central oscillations [9].

The first source is the mechanical oscillations, in which joints and muscle movements satisfy the laws of physics and the complex joint-muscle-tendon system can be compared to masses and springs. Therefore, the oscillations can be interpreted as the movements of masses and springs [10].

The second source of tremor is the reflex oscillations that are reported in the central and peripheral circuits. On the peripheral circuit the path occurs from muscles to the spinal cord and from the spinal cord to the muscles. On the central circuit the path occurs from peripheral to the spinal cord and supraspinal segments including the brain, cerebellum, basal ganglia and cerebral cortex [10].

The third and last source of tremor is central oscillations that can be observed since the first recordings of the electroencephalography (EEG). The neural activity follows rhythmic behavior. Therefore, the cerebral cortex, the basal ganglia, the cerebellum and the brainstem nucleus are all involved in the genesis of tremor [10].

Detailed analysis of oscillations in the CNS are imprecise due to the difficulty in performing measurements directly in the human brain [8]. As these neural oscillations can directly influence motor control and indicate the status of the CNS, the interest in the study of various types of tremor, as peripheral manifestations of central oscillations, has grown in recent years.

We still do not have a precise definition of the origin of tremor in humans. It is believed that it is a product of several factors. Thus, the tremor is considered a peripheral oscillation that may also have, in addition to contributions of neural activities, activities originating from the motor units and from the resonances of reflex arcs [8].

Moreover, the pathological tremor can be associated with several factors, such as neurological disorders [2]. 


\section{How to detect and record tremor?}

There are several ways for measuring human tremor. However, even today, the most used methods are those that makes the use of severity scales [17; 18]. In these methods, the patients are asked to perform different drawing patterns such as spirals, circles and letters (Figures 1 to 9). These drawings are subsequently classified by neurologists according to a numeric scale, usually ranging from 0 (no visible tremor) to 5 (severe disabling tremor). The drawings made by patients are then compared with examples of previous researches, carried out taking into account others patients previously classified. Therefore, this type of classification consists in a visual comparison and contains the subjectivity of the expert responsible for the analysis. In addition, this analysis prevents the extraction of critical information from tremor activity, such as frequency, amplitude and speed.
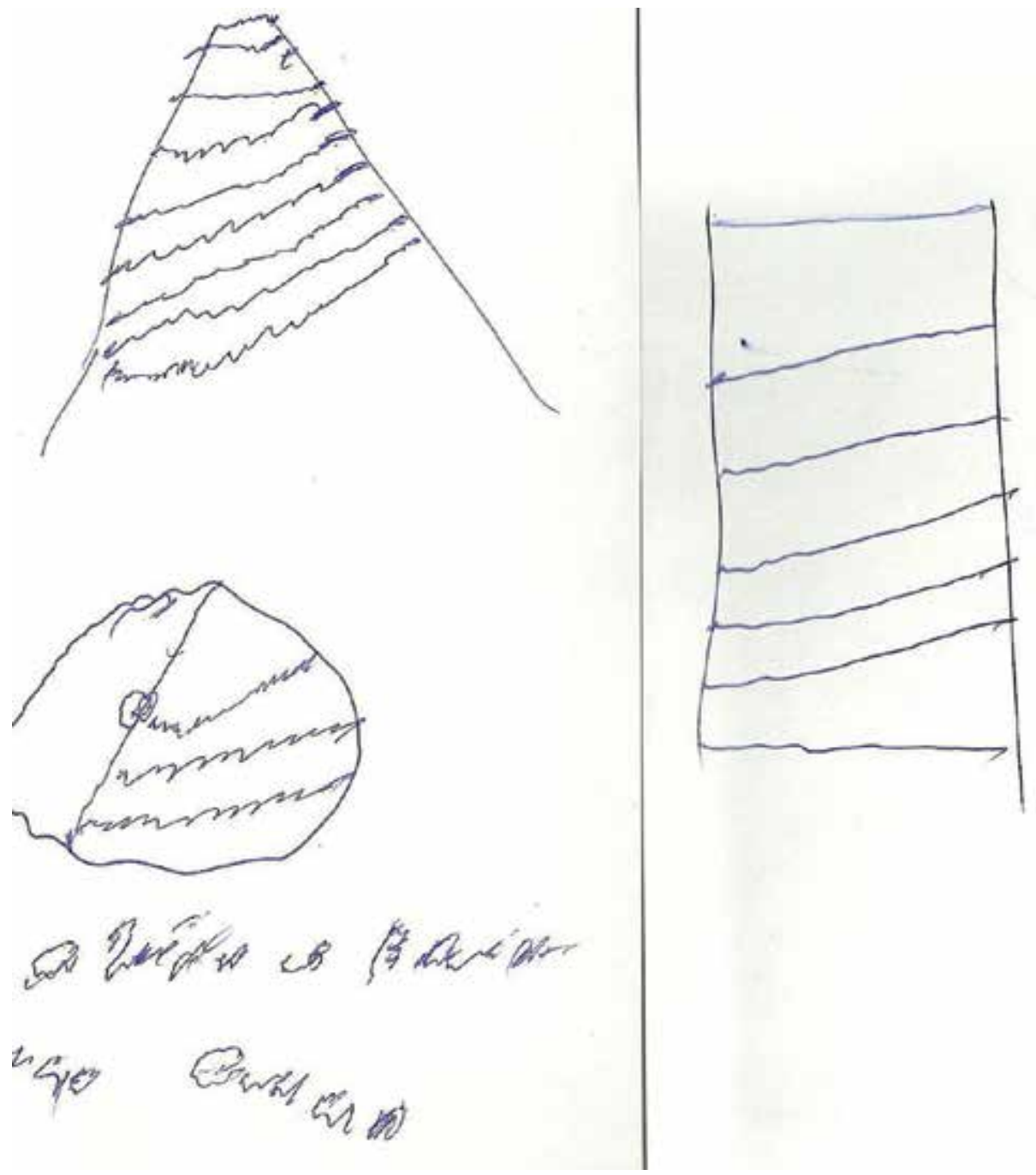

Figure 1. Action tremor. Male patient born in 1934. Date: 09/03/2009. 

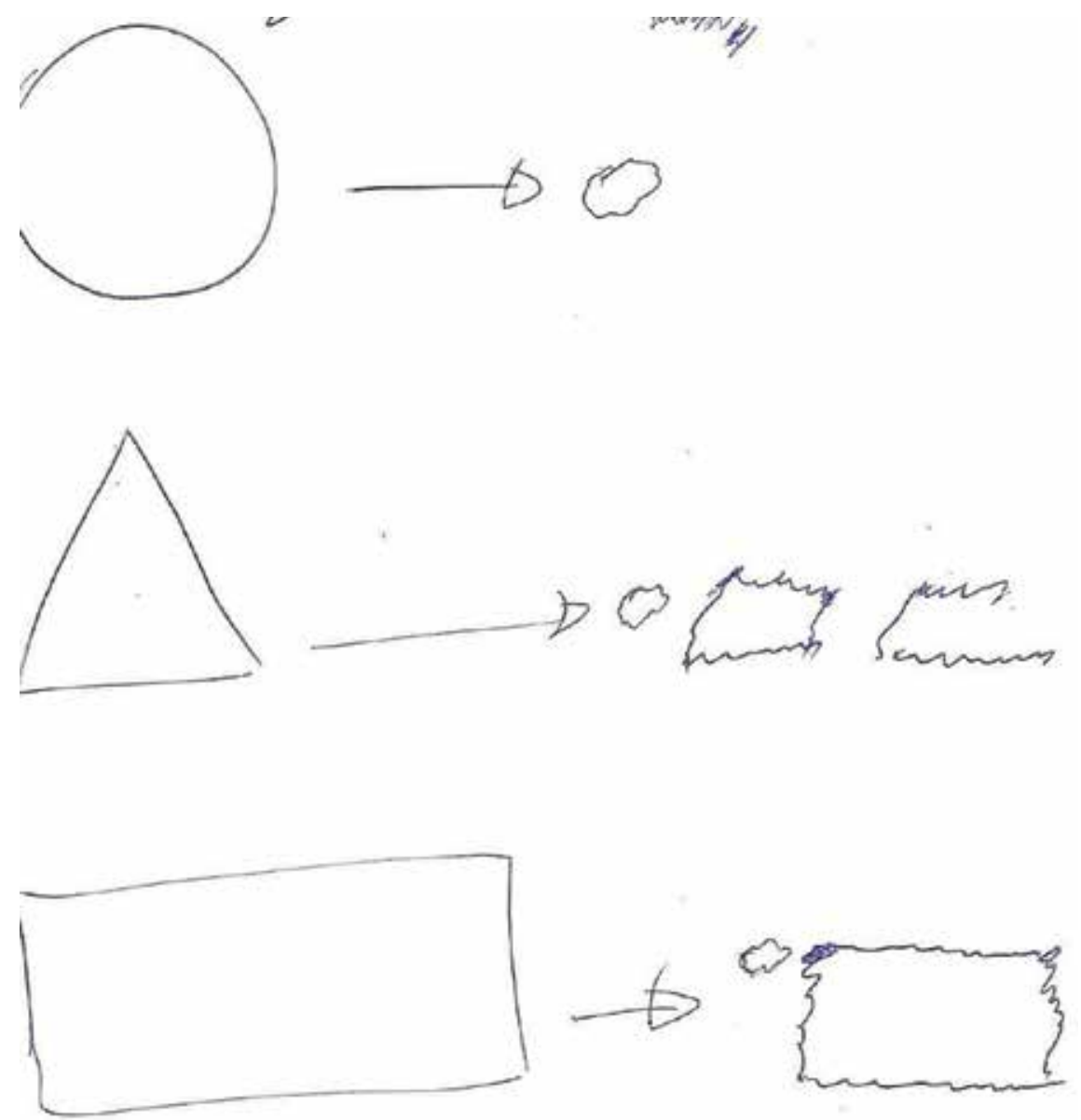

Figure 2. Action tremor. Male patient born in 1934. Date: 09/03/2009.

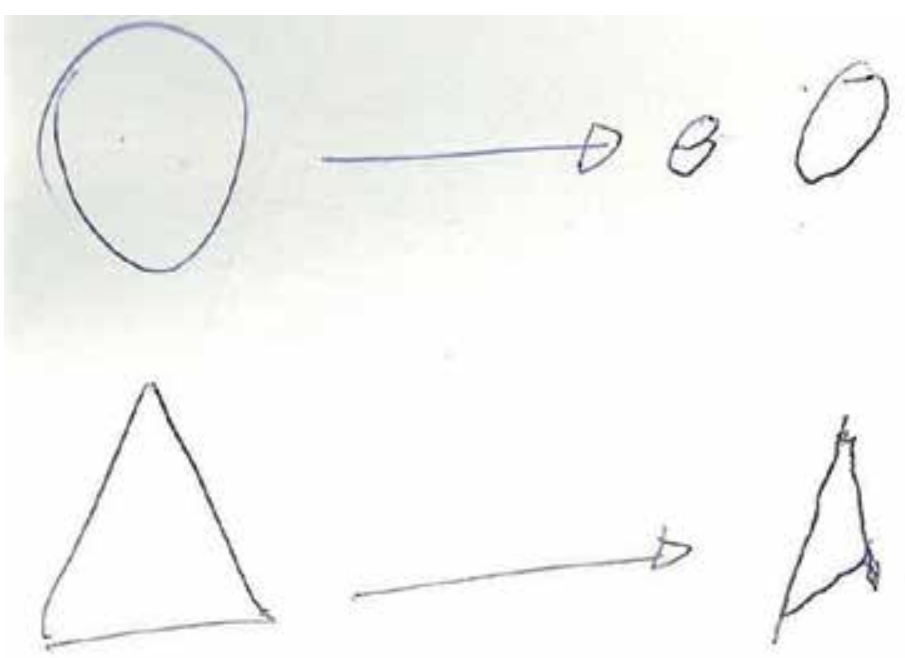

Figure 3. Action tremor. Male patient born in 1934 after treatment with levodopa. Date: 10/05/2010. 

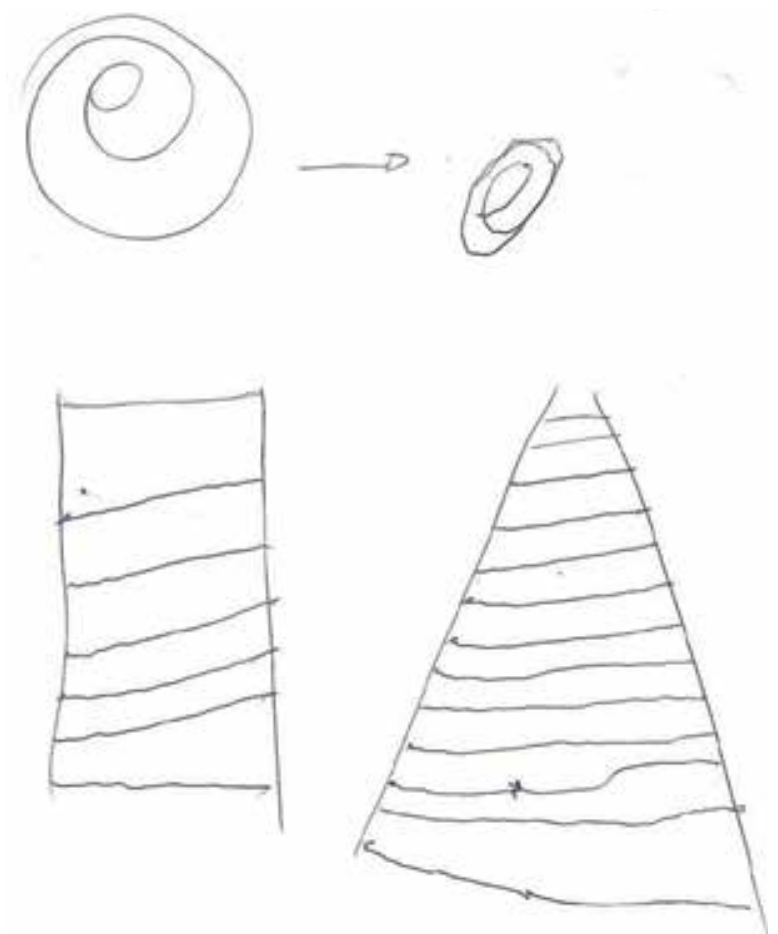

Figure 4. Action tremor. Male patient born in 1934 after treatment with levodopa. Date: 10/05/2010.
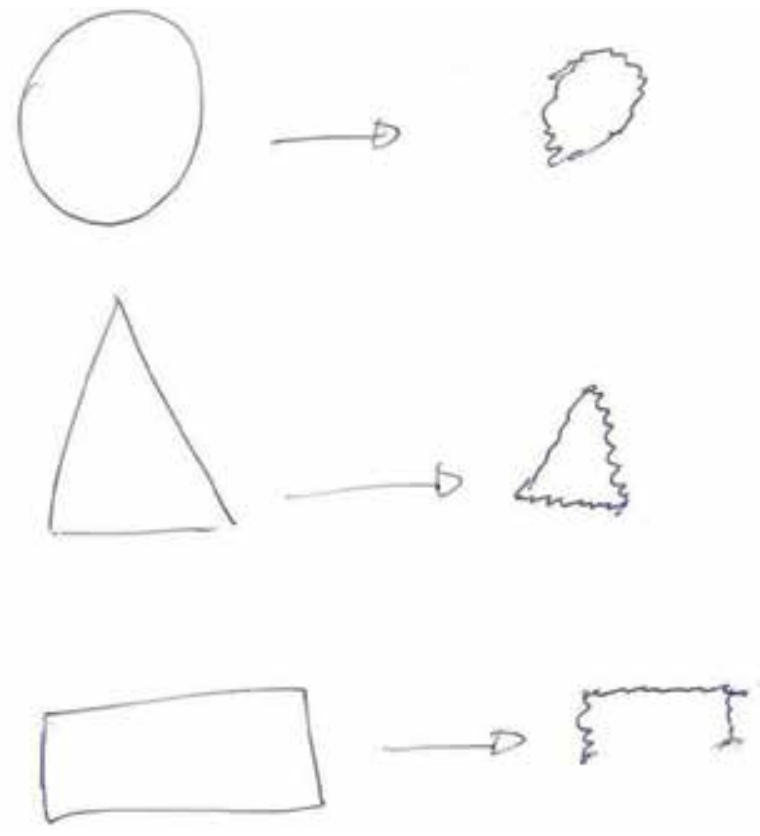

Figure 5. Male patient born in 1937. Vascular tremor: left hand tremor since 1989, dizziness, hypertension, patellar hyperreflexia, leucoaraiose, brain volume reduction, brain gap and microangiopathy. Date: 05/08/2010. 

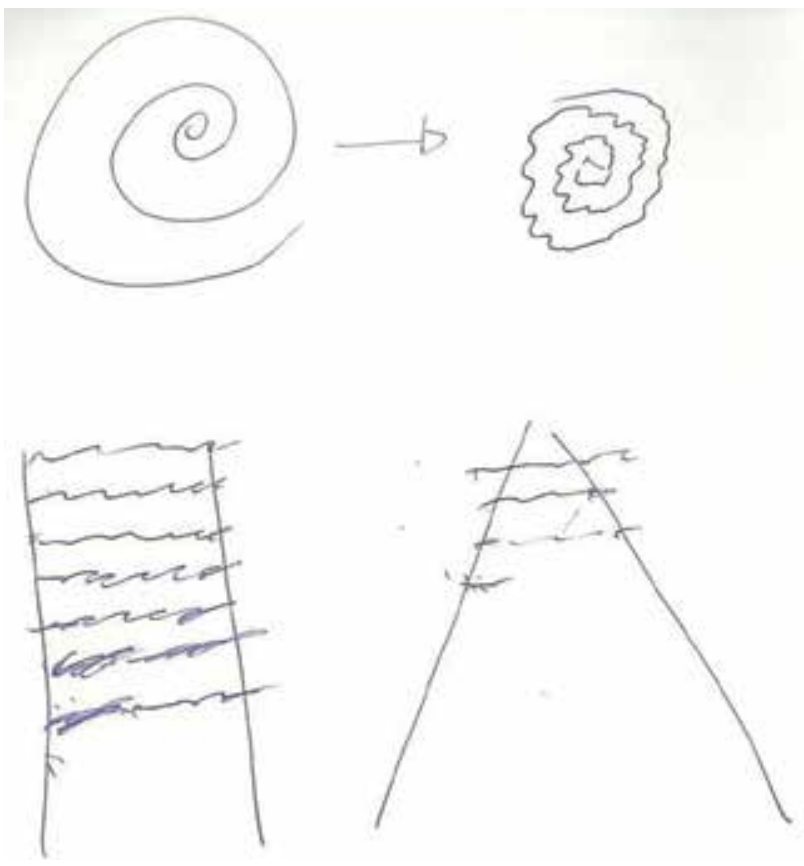

Figure 6. Male patient born in 1937. Vascular tremor: left hand tremor since 1989, dizziness, hypertension, patellar hyperreflexia, leucoaraiose, brain volume reduction, brain gap and microangiopathy. Date: 05/08/2010.
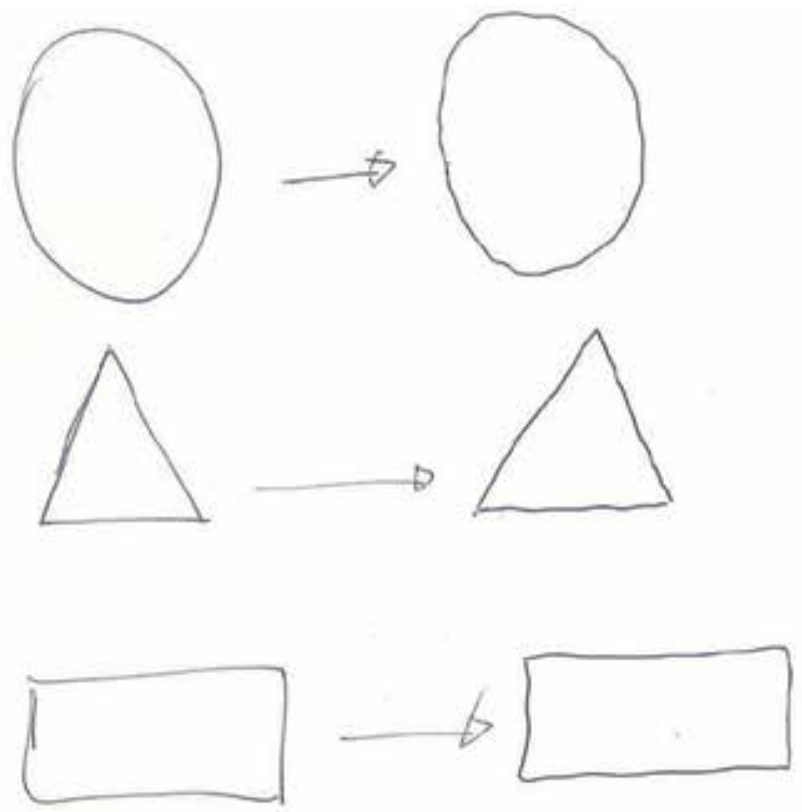

Figure 7. Female patient born in 1983. Hand tremor for four years that gets worse with anxiety and while handling objects. Absence of neurological signs. Normal dosage of calcium and parathyroid hormone. Absence of familiar cases. 


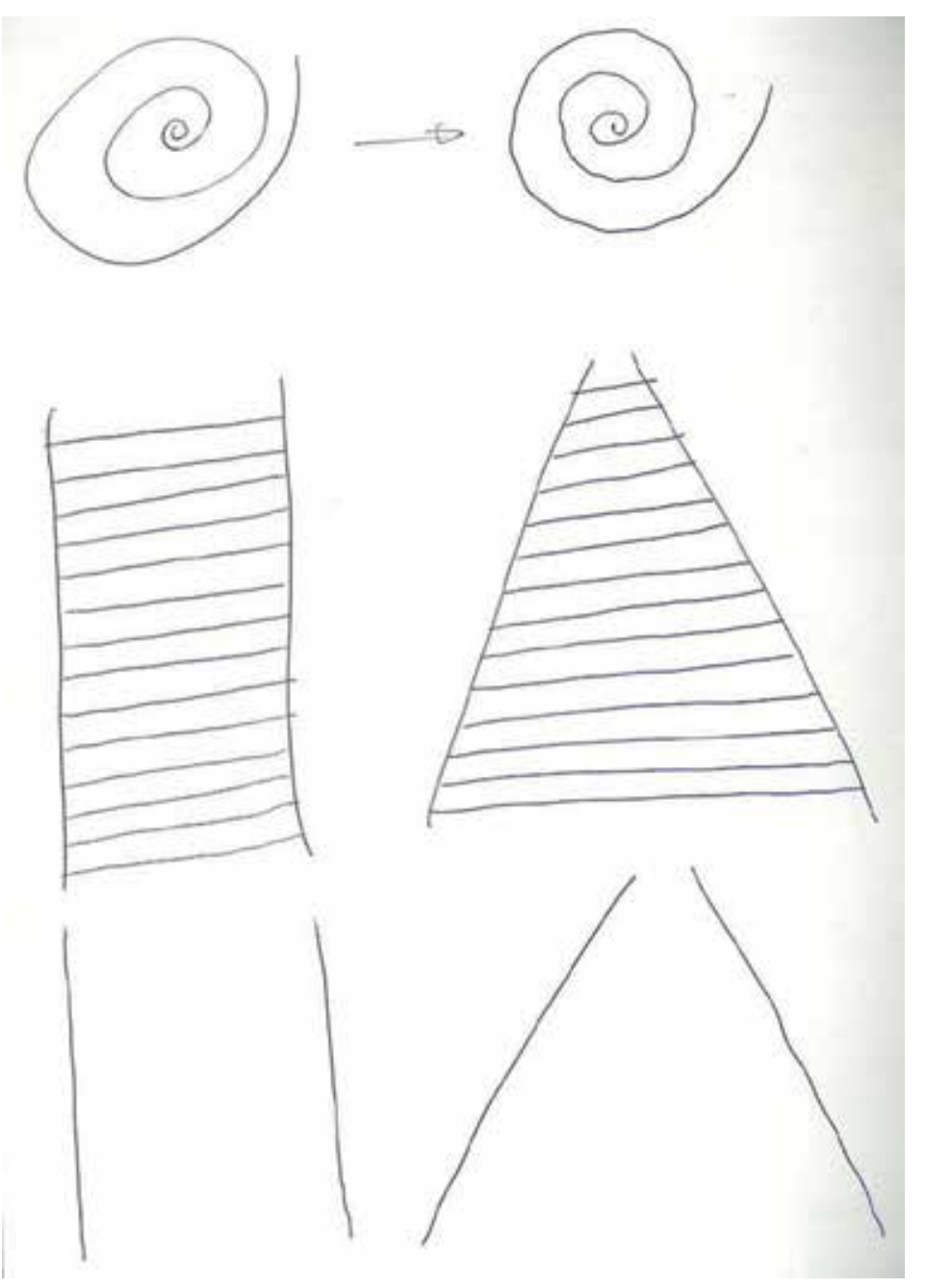

Figure 8. Female patient born in 1983. Hand tremor for four years that gets worse with anxiety and while handling objects. Absence of neurological signs. Normal dosage of calcium and parathyroid hormone. Absence of familiar cases. 

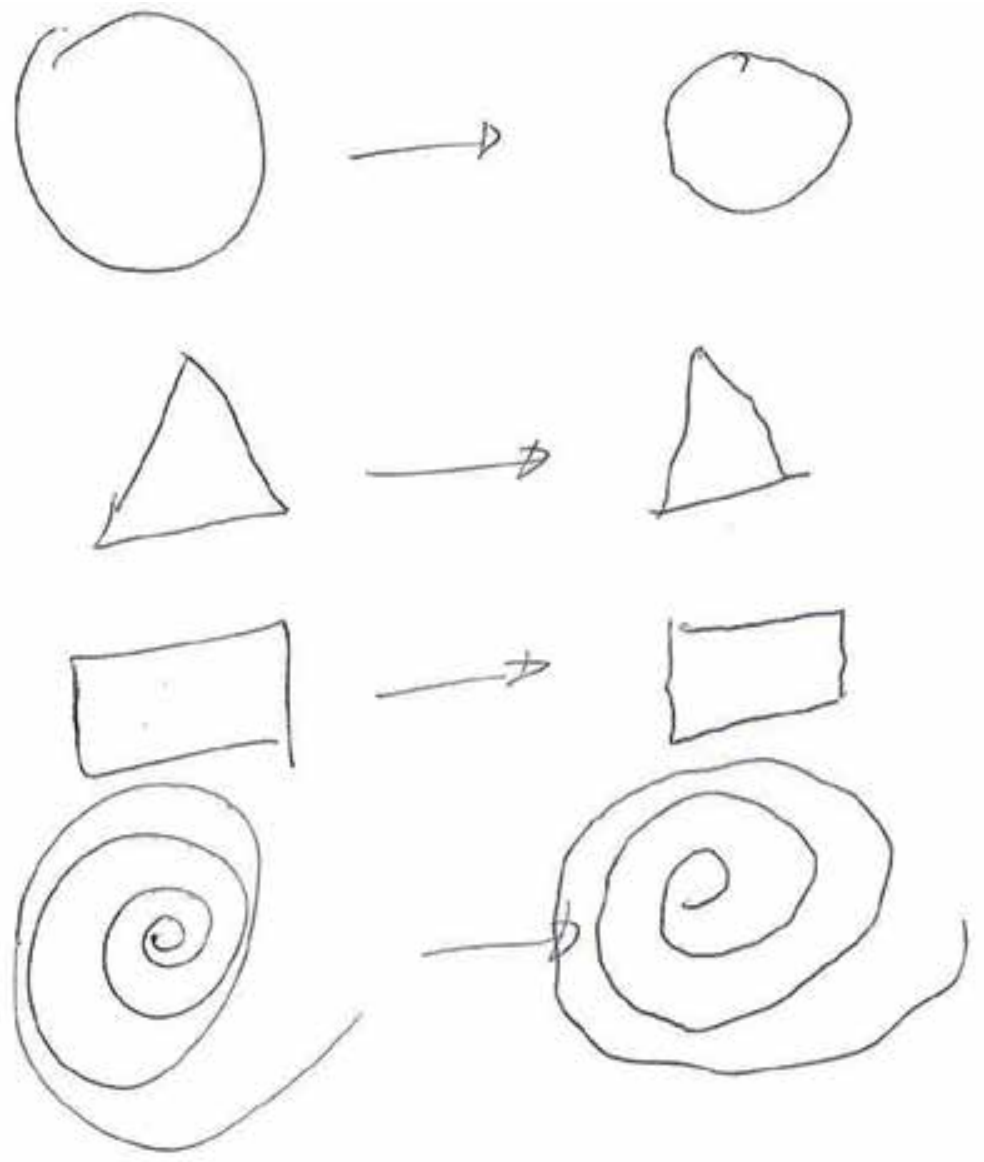

Figure 9. Female patient born in 1929. Left hand tremor, oblivion, normal neurological examination. Nuclear magnetic resonance of the brain: ischemic lacunar lesions, acute semioval center ischemia, slight brain reduction.

For the clinical diagnosis of tremor, it is necessary a complete and detailed medical history relative to factors, such as age of onset of tremor, family history, circumstances that modifies the tremor, use of drugs that can trigger the movement, existence of comorbidities, use of alcohol, smoking, anxiety, stress and depression. Besides this, it is important to do a clinical neurological exam analyzing the semiological aspects, with special focus on the type of tremor and how it presents, examining the patient standing, sitting, walking or performing movements of limbs (evidence of coordination of upper and lower limbs), supination and pronation movements, presence or absence of cog.

Regarding to physiological and essential tremor, there is no need for further investigation. Laboratory tests are important to rule out endocrine (thyroid) or other extrapyramidal 
diseases that manifest tremor, e.g. dosage of ceruloplasmin and copper, as well as eye examination (in Wilson's disease). Imaging and functional neuroimagins - PET and SPECTCT positron and photon emission tomography may be useful in differentiation between essential and parkinsonian tremors, using markers for dopamine transporter or striatal dopaminergic terminals.

The different forms of assessment of tremor can be divided into clinical and biomechanical evaluation that takes into account qualitative and quantitative analysis. Clinical evaluation is based on clinical studies dedicated to the understanding of the characteristics, evolution and treatment of diseases, which has tremor as one of its manifestations. Thus, these evaluations are basically composed of scales. Currently, rating scales such as Washington Heights-Inwood Genetic Study of Essential Tremor - WHIGET tremor rating scale - wTRS [19; 20], Fahn-Tolosa-Marin Tremor Rating Scale [21], and the Essential Tremor Rating Assessment Scale - TETRAS [22] are used to evaluate essential tremor during the clinical examination. Each tremor rating scale subjectively scale the intensity of tremor from 0 to 4 , generally corresponding (0) normal (1) slightly abnormal, (2) mildly abnormal (3), moderately abnormal, and (4) severely abnormal. Other scales are also used for evaluation of Parkinson's disease, such as UPDRS and Hoehn and Yahr. The UPDRS is a clinical tool for evaluating patients with this disorder. Recently, the Movement Disorder Society - MDS recommended a review, published in 2008, named MDS-UPDRS [16]. The MDS-UPDRS scale consists of a list of questions divided into four parts, in which the values 0-4 should be assigned depending on the severity of tremor: 0 - normal or smooth, 1 - minimum problems, 2 - mild impairment, 3 - moderate problems and 4 - serious problems. Another scale used to assess the level of Parkinson's diseases in patients is the Hoehn and Yahr. The scale is a simple staging that evaluates the overall severity of Parkinsonism based on bilateral motor dysfunction, involvement and the compromise of gait and balance. The original 5-point scale (Stage 1-5) was subsequently modified to a 7-point scale stages that included 1.5 and 2.5 in the 1990s [23].

The clinical evaluation of patients with pathological tremor is usually based on patterns that are obtained by observing groups of analysis. For each disease there is a standard for evaluation of patients. Some of these patterns are briefly described below.

MDS-sponsored UPDRS Revision (MDS-UPDRS) - Result of changes in the original pattern known as the Unified Parkinson's Disease Rating Scale (UPDRS), is the most used method for analyzing the development of Parkinson's disease. This tool is used for quantitative assessment and treatment of patients and consists in a list of questions divided into four parts, to which must be assigned values between 0 and 4 , depending on the severity of the problem: 0 - normal, 1 - light, 2-soft, 3 - moderate, and 4 - severe. The MDS-UPDRS maintains the structure of the UPDRS, i.e contains four parts. However, these component parts have been modified in order to promote integration with elements of non-motor Parkinson's disease: part I - non-motor experiences of daily living; Part II - motor daily experiences, part III - motor examination, part IV - motor complications. Parts I and II are evaluated according to the patient's own responses to a questionnaire. The tool analyzes the symptoms of Parkinson's disease through clinical evaluation and patient self-report [24]. 
Hoehn and Yahr Scale - This tool is used in the evaluation of patients with Parkinson's disease, classifying them into six stages, ranging between 0 and 5 [3]. In its original version, this scale comprises five stages of evaluating the severity of Parkinson's disease and covers comprehensive measures of signs and symptoms, including postural instability, rigidity, tremor and bradiscinesia [24]. Patients classified between stages I and III have light to moderate stage of the disease, as those who fall between stages IV and V have severe disabilities, and the stage $\mathrm{V}$ indicates an inability to move alone. A modified version of Hoehn and Yahr Scale also features two intermediate stages for evaluation of disease [24]. The protocol of this tool includes tests that evaluate the severity of resting, postural and kinetic tremors. Besides this, the test includes tasks such as extension of the arms, ingestion of liquids using spoons and cups, drawings of the spiral of Archimedes and exercises like touch the own nose with the finger. The protocol also includes specific instructions for scoring and that the expert can classify each task performed by the patient.

Washington Heights-Inwood Genetic Study of Essential Tremor (WHIGET) - It is the most used tool in clinical assessment of essential tremor. This tool has emerged from a study started in 1955, which aimed to investigate genetic aspects of essential tremor by using methods not yet implemented [3].

Bain - Clinical examination of Bain consists in performing a series of test that analyze the various components of tremor (resting tremor, postural tremor, kinetic tremor, intention tremor) [25]. The various components of the tremor are analyzed as follows: 1 - the resting component of the tremor of the head is measured with the patient lying on a couch with his head resting on cushions and postural component is collected with the patient sitting unsupported on the head and looking forward; 2 - component of the postural tremor of lower limbs is analyzed with the patient seated and with the extended leg, while the rest tremor is analyzed with the feet of the patient placed on the floor, the upper limbs are evaluated with the patient seated, three component of the tremor at rest is analyzed while the arms are relaxed and flat on the neck of the patient, while the postural component is analyzed with arms outstretched, hands pronated and fingers separated; 4 - the kinetic component is measured during the transitional phase of the test finger-nose and intentional component is measured while the finger of the subject gets closer to a target placed at reach. Vocal tremor is analyzed from the speech of each patient (patients should speak his own name, address and birthday) and, moreover, from the sound of singing from the patient, holding a musical note with the voice. All the tasks are scored from 0 to 10, as follows: 0-3 light, 4-6 - moderate, 7-9 - severe and 10 - very severe [25].

We can observe that clinical evaluation is not able to provide many answers regarding the evolution of the disease, since it does not consider the peculiarities of each patient and uses the subjectivity of experts during the evaluation and classification of the individuals.

Aiming to eliminate the subjectivity and limitations of the analysis methods based on scales some techniques to measure and analyse the tremor electronically have been developed. Thus, besides the methods employed in the clinical evaluation, many others are applied to evaluate the tremor in the laboratory. The most common methods are accelerometry, 
electromyography (EMG) and spirography [5]. The biomechanical analysis of the tremor involves qualitative and quantitative aspects, and its main methods of measuring are electromyography (EMG), magnetic tracker system, active optical markers, accelerometers, gyroscopes and spirography.

The most common method for eletronic evaluation of tremor is the accelerometry, which makes use of sensors to measure the acceleration of a body part [12;26; 27; 28]. Accelerometers are the main tool for the identification of tremor, easily observed by the large number of recent studies addressing the assessment of tremor $[29 ; 30 ; 31 ; 32 ; 33 ; 34$; 35]. The accelerometers measure linear acceleration forces in three orthogonal directions, being able to capture the movement of members produced by the action of gravity and muscle action, including tremors.

In accelerometry, data acquisition is performed by a sensor known as accelerometer that based on the Newton's second law, is capable of measuring the acceleration of a body. The accelerometer consists of an electromechanical device, usually based on the piezoelectric effect or the variation of capacitance which, when attached to any part of the body is capable of measuring acceleration forces or the movement caused by the tremor. This device generates a sequence of values (time-series) representing the instantaneous value of the acceleration as a function of time on the body part in which the sensor has been set. This series is stored and it can later be analyzed computationally.

Following this same logic (electronic evaluation, storage and computational analysis), other methods have been proposed, such as gyroscopes (evaluation of angular displacement) and speed / position transducers of many types [11;29].

Gyroscopes are devices used to measure angular velocity. It is a simple device to detect the rate of change in the orientation of each segment and are insensitive to gravitational force [36].

Another tool to detect tremor consists in the use of electromyography. The electromyographic signal (EMG) can be considered as the superposition of individual activity of several active motor units during muscle contraction and may be used to diagnose many types of neuromuscular disorders. The EMG signal may be picked up by electrodes placed on the skin surface or by means of needle / wire electrodes which are introduced into the muscle tissue $[26 ; 27 ; 28 ; 37]$.

Electromyography is an experimental technique concerned with the development, recording and analysis of myoelectric signals. The frequency $(\mathrm{Hz})$, mean amplitude $(\mathrm{mV})$ and pattern (synchronous or alternating) are used to evaluate the tremor [38].

In the acquisition of electromyographic signals for tremor analysis is common to use a specific task with the use of weights to reduce the influence of the heartbeat on the acquired electromyographic signal. For example, Elble (2003) compared the tremor in two groups of healthy individuals, a group of young (20-42 years old) and another group of elderly (70-92 years old). Elble analyzed the signals obtained in a state without load and with the addition of a weight of $300 \mathrm{~g}$. In this study, subjects were seated with the forearm supported and hands at rest or under load (palm and fingers extended in a straight line with the forearm). 
Currently, there are devices for movement capture with wireless technology, that are capable to integrate accelerometers and gyroscopes. These devices are light and easy to use, being commonly used for the study of tremor [39].

Furthermore, there are still new devices for tremor evaluation through videos [30] and tools that use accelerometers and transmit information through internet and Bluetooth technology [41]. The magnetic tracker system provides the movement displacement $(x, y$ and $\mathrm{z}$ ) and orientation (pitch, roll and yaw) of each body segment relative to a fixed transmitter [42]. From the active optical markers can be extracted the acceleration and the application of trigonometry makes possible the description of the vector orientation and the estimation of limb posture [43].

Tremor quantification can be used to control the administration of therapeutic drugs [39] and the optimization of deep brain stimulation [40].

The signal processing and analysis of tremor often involves the spectral analysis, based on Fast Fourier Transform - FFT [35; 39; 41], but this technique as modified weighted Fourier Linear Combiner -WFLC [44] is suitable for periodic or quasi-periodic estimation of motion with single dominant frequency, whereas Band-Limited Multiple Fourier Linear Combiner BMFLC [34] is suitable for estimation of band limited signals consisting of multiple frequency components. Other methods can also be found as Detrended Fluctuation Analysis (DFA) to analyze hand essential tremor time-series extracted from regions around the first three main frequency components of the tremor power spectra - PWS [45]. To increase the accuracy other modifications or algorithms are used [46; 47]. However, it is not possible to determine the best way to perform these analyses, since this depends on the objectives of the study. Therefore, it is not possible to reach any conclusion on the most appropriate methodologies for the detection and diagnosis of tremor [3].

Hand-drawing patterns are commonly assessed by means of visual rating scales.[48; 49] However, such scales provide only crude subjective estimates of tremor amplitude. In order to reduce the subjectivity and limitation of some methods based on visual scales, there have been developed a few strategies for electronically measuring tremor, such as accelerometry and digitizing tablets. The use of digitizing tablets is common and provides the possibility of tremor activity detection under kinetic conditions.

The usual function of a digitizing tablet is to enable the analysis of drawings directly on the computer. The measurement of tremor by using digitizing tablets is a non-invasive alternative for tremor detection that combines simplicity with the precision and versatility of computational methods. The digitizing tablet is able to inform the position of the tip of the pen on its surface. By using this property this device can detect the movement of a subject following standard drawing patterns placed on it $[27 ; 49 ; 50 ; 51 ; 52 ; 53 ; 54 ; 55 ; 56]$

Subjectively interpreted in previous decades, nowadays the digital spirography can provide quantitative data of the movement control [57]. The spirography has been considered valid and reliable to diagnose early Parkinson's disease [58], essential tremor [59] and to distinguish tremulous parkinsonian patients with normal presynaptic dopaminergic 
imaging from tremulous patients with Parkinson's disease [60]. In general, the signals are recorded and stored for posterior analysis. The analysis involves methods such as the radius-angle transformations of the two-dimensional spiral pictures that are captured from the original clinical information (shape, kinematics and dynamics) [57; 58]. In addition, more sophisticated tools and modern statistical algorithms can be used for data evaluation [59; 60].

Digitizing tablet was used by Almeida (2010) [5] with a technique known as spirography. This technique consists in the reproduction by the patient of the Archimedes' spiral according to an ideal model. Thus, a model of this spiral is displayed on the table surface and the patient should try to cover the route of the model as accurately as possible.

Several attributes of the spiral of Archimedes make its use attractive in tests for the detection of human tremor. First, it has a simple design and it is easily understood by subjects who can follow its trajectory. Secondly, the shape of the spiral is smooth with an increasing radius, reducing the occurrence of false-positive tremor caused by abrupt changes in the direction of motion

There are a number of research studies concerning the employment of digitizing tablets for both the quantification of pathological tremor and the detection of movement disorders [49; $50 ; 51 ; 52 ; 53 ; 54 ; 55 ; 56 ; 61]$. However, even with the advances in the technology of digitizing tablets, which allowed for more precision and accuracy in the measurement of movements, no study focusing upon the use of these devices, as a tool for investigating the relation between physiological tremor and ageing in kinetic conditions, was found in our literature survey. Although some authors, e.g., Wenzelburger et al.,[62] support the hypothesis that kinetic tremor is related to an enhancement of physiological tremor this assumption is not consensual $[26 ; 27 ; 63]$ and therefore additional studies in this area are required.

The different ways of evaluating the tremor, clinical and biomechanics, can be viewed as complementary and in general are used simultaneously, with the aim of compare the qualitative and quantitative data. Thus, it is expected that future improvement of existing tools, as well as the introduction of new tools better clarify this point.

\section{Author details}

Adriano O. Andrade*, Adriano Alves Pereira, Maria Fernanda Soares de Almeida, Guilherme Lopes Cavalheiro and Ana Paula Souza Paixão

Faculty of Electrical Engineering, Federal University of Uberlândia, Brazil

Sheila Bernardino Fenelon

Faculty of Medicine, Federal University of Uberlândia, Brazil

Valdeci Carlos Dionisio

Faculty of Physical Education, Federal University of Uberlândia, Brazil

" Corresponding Author 


\section{Acknowledgement}

The authors would like to express their gratitude to "Coordenação de Aperfeiçoamento de Pessoal de Nível Superior" (CAPES - Brazil), "Conselho Nacional de Desenvolvimento Científico e Tecnológico" (CNPq - Brazil) and "Fundação de Amparo à Pesquisa do Estado de Minas Gerais" (FAPEMIG - MG - Brazil) for the financial support.

\section{References}

[1] Bhagwath, G. Tremors in elderly persons: clinical features and management. Hospital Physician, v. 49, p. 31-49, 2001.

[2] Smaga, S. Tremor. American Family Physician, v. 68, n. 8, p. 1545-1553, 2003.

[3] Mansur, P. H. G. et al. A review on techniques for tremor recording and quantification. Critical Reviews in Biomedical Engineering, v. 35, n. 5, p. 343-362, 2007. ISSN 0278940X. Disponível em:

< http://www.begellhouse.com/journals/4b27cbfc562e21b8,4d8cbde20903daf0, 3889d79e52078054.html >.

[4] Borges, V.; Ferraz, H. B. Tremors. Revista Neurociências, v. 14, n. 1, p. 43-47, 2006.

[5] Almeida, M. F. S. et al. Investigation of Age-Related Changes in Physiological Kinetic Tremor. Annals of Biomedical Engineering, v. 38, n. 11, p. 3423-3439, 2010. ISSN 00906964. Disponível em: < http://dx.doi.org/10.1007/s10439-010-0098-z >.

[6] Wyne, K. T. A comprehensive review of tremor: an organized approach to the patient assessment is crucial to reaching an accurate diagnosis. Consider the constellation of signs and symptoms, and know the characteristics of each form of tremor. Journal of the American Academy of Physicians Assistants v. 18, n. 12, p. 43-50, 2005.

[7] Jankovic, J. Essential tremor: clinical characteristics. 2000. S21-5 ISBN 0028-3878. Disponível em:

< http://www.biomedsearch.com/nih/Essential-tremor-clinical-characteristics/ 10854348.html >.

[8] Mcauley, J. H.; Marsden, C. D. Physiological and pathological tremors and rhythmic central motor control. Brain, v. 123, n. 8, p. 1545-1567, August 1, 2000 2000. Disponível em: < http://brain.oxfordjournals.org/cgi/content/abstract/123/8/1545 >.

[9] Hallett, M. Overview of Human Tremor Physiology. Movement Disorders, v. 13, n. S3, p. 43-48, 1998. ISSN 1531-8257. Disponível em: < http://dx.doi.org/10.1002/mds.870131308 >.

[10] Grimaldi, G.; Manto, M.-U.; Manto, M. Tremor: From Pathogenesis to Treatment. San Rafael, California: Morgan \& Claypool Publishers 2008.

[11] De Lima, E. et al. Empirical mode decomposition: a novel technique for the study of tremor time series. Medical and Biological Engineering and Computing, v. 44, n. 7, p. 569-582, 2006. Disponível em: < http://dx.doi.org/10.1007/s11517-006-0065-x >.

[12] Deuschl, G.; Lauk, M.; Timmer, J. Tremor classification and tremor time series analysis. Chaos, v. 5, n. 1, p. 48-51, 1995. Disponível em: < http://link.aip.org/link/?CHA/5/48/1 >. 
[13] Bhidayasiri, R. Differential diagnosis of common tremor syndromes. Postgraduate Medical Journal, v. 81, n. 962, p. 756-762, December 1, 2005 2005. Disponível em: < http://pmj.bmj.com/content/81/962/756.abstract >.

[14] Young, R. R.; Hagbarth, K. E. Physiological tremor enhanced by manoeuvres affecting the segmental stretch reflex. Journal of Neurology, Neurosurgery \& Psychiatry, v. 43, n. 3, p. 248-256, March 1, 1980 1980. Disponível em:

< http://jnnp.bmj.com/content/43/3/248.abstract >.

[15] Mattos, J. P. D. Diagnóstico diferencial dos tremores. Arquivos de Neuro-Psiquiatria, v. 56, p. 320-323, 1998. ISSN 0004-282X. Disponível em:

< http://www.scielo.br/scielo.php?script=sci_arttext\&pid=S0004-282X1998000200027 $\&$ nrm $=$ iso $>$.

[16] Goetz, C. G. et al. Movement Disorder Society-sponsored revision of the Unified Parkinson's Disease Rating Scale (MDS-UPDRS): Process, format, and clinimetric testing plan. Movement Disorders, v. 22, n. 1, p. 41-47, 2007. ISSN 1531-8257. Disponível em: < http://dx.doi.org/10.1002/mds.21198>.

[17] Ramaker, C. et al. Systematic evaluation of rating scales for impairment and disability in Parkinson's disease. Movement Disorders, v. 17, n. 5, p. 867-876, 2002. ISSN 15318257. Disponível em: < http://dx.doi.org/10.1002/mds.10248>.

[18] Greffard, S. et al. Motor Score of the Unified Parkinson Disease Rating Scale as a Good Predictor of Lewy Body-Associated Neuronal Loss in the Substantia Nigra. Arch Neurol, v. 63, n. 4, p. 584-588, April 1, 2006 2006. Disponível em: < http://archneur.amaassn.org/cgi/content/abstract/63/4/584 >.

[19] Louis, E. D. et al. A teaching videotape for the assessment of essential tremor. Movement Disorders, v. 16, n. 1, p. 89-93, 2001. ISSN 1531-8257. Disponível em: < http://dx.doi.org/10.1002/1531-8257(200101)16:1<89::AID-MDS1001>3.0.CO;2-L >.

[20] Louis Ed, W. K. J. A. S. M. P. S. L. Y. Q. A. H. Validity of a performance-based test of function in essential tremor. Archives of Neurology, v. 56, n. 7, p. 841-846, 1999. ISSN 0003-9942. Disponível em: < http://dx.doi.org/10-1001/pubs.Arch Neurol.-ISSN-00039942-56-7-noc8137>.

[21] Stacy, M. A. et al. Assessment of interrater and intrarater reliability of the Fahn-TolosaMarin Tremor Rating Scale in essential tremor. Movement Disorders, v. 22, n. 6, p. 833838, 2007. ISSN 1531-8257. Disponível em: < http://dx.doi.org/10.1002/mds.21412 >.

[22] Mostile, G. et al. Correlation between Kinesia system assessments and clinical tremor scores in patients with essential tremor. Movement Disorders, v. 25, n. 12, p. 1938-1943, 2010. ISSN 1531-8257. Disponível em: < http://dx.doi.org/10.1002/mds.23201 >.

[23] Goetz, C. G. et al. Movement Disorder Society Task Force report on the Hoehn and Yahr staging scale: Status and recommendations The Movement Disorder Society Task Force on rating scales for Parkinson's disease. Movement Disorders, v. 19, n. 9, p. 1020-1028, 2004. ISSN 1531-8257. Disponível em: < http://dx.doi.org/10.1002/mds.20213>.

[24] Goulart, F.; Pereira, L. X. Main scales for Parkinson's disease assessment: use in physical therapy. Fisioterapia e Pesquisa, v. 2, n. 1, p. 49-56, 2004. 
[25] Bain, P. G. et al. Assessing tremor severity. Journal of Neurology, Neurosurgery \& Psychiatry, v. 56, n. 8, p. 868-873, August 1, 1993 1993. Disponível em: < http://jnnp.bmj.com/content/56/8/868.abstract $>$.

[26] Raethjen, J. et al. Determinants of physiologic tremor in a large normal population. Clinical Neurophysiology, v. 111, n. 10, p. 1825-1837, 2000. ISSN 1388-2457. Disponível em: < http://www.sciencedirect.com/science/article/B6VNP-419BFX0-J/2/ ee6af8163265c25d3ac795c893aa454e >.

[27] Elble, R. J. Characteristics of physiologic tremor in young and elderly adults. Clinical Neurophysiology, v. 114, n. 4, p. 624-635, 2003. ISSN 1388-2457. Disponível em: < http://www.sciencedirect.com/science/article/B6VNP-47XWVJ43/2/e2bb859ab434ea2b5c91345ae114f7d2 >.

[28] Morrison, S.; Mills, P.; Barrett, R. Differences in multiple segment tremor dynamics between young and elderly persons. The Journals of Gerontology Series A: Biological Sciences and Medical Sciences, v. 61, n. 9, p. 982-990, 2006. Disponível em: < http://biomed.gerontologyjournals.org/cgi/content/abstract/61/9/982 >.

[29] Salarian, A. et al. Quantification of tremor and bradykinesia in Parkinson's disease using a novel ambulatory monitoring system. IEEE Transactions on Biomedical Engineering, v. 54, n. 2, p. 313-322, 2007. ISSN 0018-9294.

[30] Uhríková, Z. et al. Validation of a new tool for automatic assessment of tremor frequency from video recordings. Journal of Neuroscience Methods, v. 198, n. 1, p. 110113, 2011. ISSN 0165-0270. Disponível em: < http://www.sciencedirect.com/science/article/pii/S0165027011001294 >.

[31] Kuncel, A. M. et al. Tremor reduction and modeled neural activity during cycling thalamic deep brain stimulation. Clinical Neurophysiology, v. 123, n. 5, p. 1044-1052, 2012. ISSN 1388-2457. Disponível em:

< http://www.sciencedirect.com/science/article/pii/S1388245711006250 >.

[32] Tsipouras, M. G. et al. An automated methodology for levodopa-induced dyskinesia: Assessment based on gyroscope and accelerometer signals. Artificial Intelligence in Medicine, v. 55, n. 2, p. 127-135, 2012. ISSN 0933-3657. Disponível em:

< http://www.sciencedirect.com/science/article/pii/S0933365712000322 >.

[33] Hilliard, J. D.; Frysinger, R. C.; Elias, W. J. Effective subthalamic nucleus deep brain stimulation sites may differ for tremor, bradykinesia and gait disturbances in Parkinson's disease. Stereotact Funct Neurosurg, v. 89, n. 6, p. 357-364, 2011. ISSN 10116125. Disponível em: < http://pubget.com/paper/22104373 http://gateway.proquest.com/ openurl?ctx_ver=Z39.88-

2004\&res_id=xri:pqd\&rft_val_fmt=info:ofi:fmt:kev:mtx:journal\&genre=article\&jtitle=Ste reotactic and Functional Neurosurgery\&issn=1011-61251423-0372\&atitle=Effective subthalamic nucleus deep brain stimulation sites may differ for tremor, bradykinesia and gait disturbances in Parkinson's disease.\&date=2011-01-01\&volume=89\&issue= 6\&spage=357 http://dx.doi.org/10.1159/000331269 >.

[34] Veluvolu, K. C.; Ang, W. T. Estimation of Physiological Tremor from Accelerometers for Real-Time Applications. Sensors, v. 11, n. 3, p. 3020-3036, 2011. ISSN 1424-8220. Disponível em: < http://www.mdpi.com/1424-8220/11/3/3020 >. 
[35] Sanchez-Ramos, J. et al. Quantitative Analysis of Tremors in Welders. International Journal of Environmental Research and Public Health, v. 8, n. 5, p. 1478-1490, 2011. ISSN 1660-4601. Disponível em: < http://www.mdpi.com/1660-4601/8/5/1478>.

[36] Tong, K.; Mak, A.; Ip, W. Command control for functional electrical stimulation hand grasp systems using miniature accelerometers and gyroscopes. Medical and Biological Engineering and Computing, v. 41, n. 6, p. 710-717, 2003. ISSN 0140-0118. Disponível em: < http://dx.doi.org/10.1007/BF02349979>.

[37] Timmer, J. et al. Cross-spectral analysis of physiological tremor and muscle activity. Biological Cybernetics, v. 78, n. 5, p. 359-368, 1998. Disponível em:

$<$ http://dx.doi.org/10.1007/s004220050440>.

[38] Milanov, I. Electromyographic differentiation of tremors. Clinical Neurophysiology, v. 112, n. 9, p. 1626-1632, 2001. ISSN 1388-2457. Disponível em:

< http://www.sciencedirect.com/science/article/B6VNP-43RJ9D3-7/2/ 4b5ec662a94e4eae13b0ef5cc9180537 >.

[39] Giuffrida, J. P. et al. Clinically deployable Kinesia ${ }^{\mathrm{TM}}$ technology for automated tremor assessment. Movement Disorders, v. 24, n. 5, p. 723-730, 2009. ISSN 1531-8257. Disponível em: < http://dx.doi.org/10.1002/mds.22445 >.

[40] Mera, T. et al. Kinematic optimization of deep brain stimulation across multiple motor symptoms in Parkinson's disease. J Neurosci Methods, v. 198, n. 2, p. 280-286, 2011. ISSN 0165-0270. Disponível em:

< http://pubget.com/paper/21459111

http://gateway.proquest.com/openurl?ctx_ver=Z39.88-

2004\&res_id=xri:pqd\&rft_val_fmt=info:ofi:fmt:kev:mtx:journal\&genre=article\&jtitle=Jou rnal of Neuroscience Methods\&issn=0165-02701872-678X\&atitle=Kinematic

optimization of deep brain stimulation across multiple motor symptoms in Parkinson's disease $\&$ date $=2011-03-31 \&$ volume $=198 \&$ issue $=2 \&$ spage $=280$

http://www.sciencedirect.com/science/article/pii/S0165-0270(11)00167-1 >.

[41] Barroso Júnior, M. C. et al. A telemedicine instrument for remote evaluation of tremor: design and initial applications in fatigue and patients with Parkinson's disease. Biomedical engineering online, v. 10, p. 14, 2011. Disponível em:

< http://ukpmc.ac.uk/abstract/MED/21306628 >.

[42] Ghassemi, M. et al. Bradykinesia in patients with Parkinson's disease having levodopainduced dyskinesias. Brain Research Bulletin, v. 69, n. 5, p. 512-518, 2006. ISSN 03619230. Disponível em:

< http://www.sciencedirect.com/science/article/pii/S036192300600061X >.

[43] Albert, M. V.; Kording, K. P. Determining posture from physiological tremor. Experimental brain research. Experimentelle Hirnforschung. Experimentation cerebrale, v. 215, n. 3-4, p. 247-255, 2011. Disponível em:

< http://ukpmc.ac.uk/abstract/MED/21997329>.

[44] Riviere, C. N.; Reich, S. G.; Thakor, N. V. Adaptive Fourier modeling for quantification of tremor. Journal of Neuroscience Methods, v. 74, n. 1, p. 77-87, 1997. ISSN 0165-0270. Disponível em: < http://www.sciencedirect.com/science/article/B6T04-3TCVRT9$\mathrm{T} / 2 / 6 f 12090 \mathrm{ac} 184 \mathrm{c} 878343120 \mathrm{~cd} 13881 \mathrm{~b} 90>$. 
[45] Blesic, S. et al. Scaling analysis of bilateral hand tremor movements in essential tremor patients. Journal of Neural Transmission, v. 118, n. 8, p. 1227-1234, 2011. ISSN 03009564. Disponível em: < http://dx.doi.org/10.1007/s00702-011-0581-1 >.

[46] Latt, W. T.; Veluvolu, K. C.; Ang, W. T. Drift-Free Position Estimation of Periodic or Quasi-Periodic Motion Using Inertial Sensors. Sensors, v. 11, n. 6, p. 5931-5951, 2011. ISSN 1424-8220. Disponível em: < http://www.mdpi.com/1424-8220/11/6/5931 >.

[47] Popovi $\backslash$, L. Z. et al. Adaptive band-pass filter (ABPF) for tremor extraction from inertial sensor data. Comput. Methods Prog. Biomed., v. 99, n. 3, p. 298-305, 2010. ISSN 01692607.

[48] Mergl, R. et al. Digitized analysis of handwriting and drawing movements in healthy subjects: methods, results and perspectives. Journal of Neuroscience Methods, v. 90, n. 2, p. 157-169, 1999. ISSN 0165-0270. Disponível em:

< http://www.sciencedirect.com/science/article/B6T04-3XBTV5W-7/2/ 9e2366c4d50d9aaadb2e8c877f27da34 >.

[49] Louis, E. D. et al. Is essential tremor symmetric?: observational data from a communitybased study of essential tremor. Archives of Neurology, v. 55, n. 12, p. 1553-1559, 1998. Disponível em: < http://archneur.ama-assn.org/cgi/content/abstract/55/12/1553 >.

[50] Feys, P. et al. Digitised spirography as an evaluation tool for intention tremor in multiple sclerosis. Journal of Neuroscience Methods, v. 160, n. 2, p. 309-316, 2007. ISSN 0165-0270. Disponível em: < http://www.sciencedirect.com/science/article/B6T044MC71C5-1/2/cc55be9051d4ab74e620ece8a142903e >.

[51] Elble, R. J. et al. Quantification of essential tremor in writing and drawing. Movement Disorders, v. 11, n. 1, p. 70-78, 1996. ISSN 1531-8257. Disponível em:

< http://dx.doi.org/10.1002/mds.870110113 >.

[52] Miralles, F.; Tarongí, S.; Espino, A. Quantification of the drawing of an Archimedes spiral through the analysis of its digitized picture. Journal of Neuroscience Methods, v. 152, n. 1-2, p. 18-31, 2006. ISSN 0165-0270. Disponível em:

< http://www.sciencedirect.com/science/article/B6T04-4H68NGJ-1/2/ 0934969b75770f9c6452893d336e093d >.

[53] Liu, X. et al. Quantifying drug-induced dyskinesias in the arms using digitised spiraldrawing tasks. Journal of Neuroscience Methods, v. 144, n. 1, p. 47-52, 2005. ISSN 01650270. Disponível em: < http://www.sciencedirect.com/science/article/B6T04-4DX27FD3/2/eacac6a2698f8a2b30d47c0d858d3356 >.

[54] Rudzińska M, I. A., Banaszkiewicz K, Bukowczan S, Marona M, Szczudlik A Quantitative tremor measurement with the computerized analysis of spiral drawing. Polish Journal Of Neurology And Neurosurgery, v. 41, n. 6, p. 510-516, 2007.

[55] Elble, R. J. et al. Tremor amplitude is logarithmically related to 4- and 5-point tremor rating scales. Brain, v. 129, n. 10, p. 2660-2666, 2006. Disponível em: $<$ http://brain.oxfordjournals.org/cgi/content/abstract/129/10/2660 >.

[56] Ulmanová, O. et al. Tremor magnitude: a single index to assess writing and drawing in essential tremor. Parkinsonism \& Related Disorders, v. 13, n. 4, p. 250-253, 2007. ISSN 1353-8020. Disponível em: < http://www.sciencedirect.com/science/article/B6TB94K7FJPP-4/2/e4e3eba11bec47a0d2e67de3a85c6ef4 >. 
[57] Pullman, S. L. Spiral analysis: a new technique for measuring tremor with a digitizing tablet. Movement Disorders, v. 13, n. 3, p. 85-89, 1998. ISSN 1531-8257. Disponível em: $<$ http://dx.doi.org/10.1002/mds.870131315>.

[58] Saunders-Pullman, R. et al. Validity of spiral analysis in early Parkinson's disease. Mov Disord, 2007. ISSN 08853185.

[59] Haubenberger, D. et al. Validation of digital spiral analysis as outcome parameter for clinical trials in essential tremor. Movement Disorders, v. 26, n. 11, p. 2073-2080, 2011. ISSN 1531-8257. Disponível em: < http://dx.doi.org/10.1002/mds.23808 >.

[60] Bajaj, N. P. S. et al. Can spiral analysis predict the FP-CIT SPECT scan result in tremulous patients? Movement Disorders, v. 26, n. 4, p. 699-704, 2011. ISSN 1531-8257. Disponível em: < http://dx.doi.org/10.1002/mds.23507>.

[61] Elble, R. J.; Sinha, R.; Higgins, C. Quantification of tremor with a digitizing tablet. Journal of Neuroscience Methods, v. 32, p. 193-198, 1990.

[62] Wenzelburger, R. et al. Kinetic tremor in a reach-to-grasp movement in Parkinson's disease. Movement Disorders, v. 15, n. 6, p. 1084-1094, 2000. ISSN 1531-8257. Disponível em:

< http://dx.doi.org/10.1002/1531-8257(200011)15:6<1084::AID-MDS1005>3.0.CO;2-Y>.

[63] Sturman, M. M.; Vaillancourt, D. E.; Corcos, D. M. Effects of aging on the regularity of physiological tremor. Journal of Neurophysiology, v. 93, p. 3064-3074, 2005. ISSN 00223077. Disponível em: < http://jn.physiology.org/cgi/content/full/93/6/3064 >. 


\title{
Second- and Third-Order Statistical \\ Characterization of Non-Linearity and \\ Non-Gaussianity of Adult and Fetal \\ ECG Signals and Noise
}

\author{
Walid A. Zgallai \\ Additional information is available at the end of the chapter
}

http://dx.doi.org/10.5772/52575

\section{Introduction}

This chapter investigates the application of digital signal processing techniques to ECG signals. The first few sections of this chapter are devoted to definitions and properties of cumulants, their spectra, and associated statistics. This is followed by describing the structural properties of the third-order cumulants of an adult male's chest ECG, maternal chest ECG, transabdominally-measured ECG, as well as fetal ECG signal using scalpelectrode. The non-linearity and non-stationarity of ECG signals are investigated using the bispectrum and bicoherence squared. The third-order cumulants, bispectra, and bicoherence squared of some noise components, namely, the baseline wander, electromyographic (EMG), and motion artefact noise isolated from the MIT/BIH databases are analysed. Finally, concluding remarks are discussed and summarised.

Adequate knowledge of the higher-order statistics (HOS) of both the maternal and fetal ECG signals must be acquired in order to pave the way for fetal QRS-complex identification and detection. There are several motivations behind using higher-order statistics in processing ECG signals. These motivations are:

i. ECG signals are predominantly non-Gaussian (Rizk et al., 1995; Rizk and Zgallai, 1999), and exhibit quadratic and higher-order non-linearities supported by third- and fourthorder statistics, respectively. It is worth mentioning that, in general, the third-order cumulants can support linear non-Gaussian, and non-linear signals.

ii. The maternal and fetal QRS-complex bispectral contours do not overlap with that of the baseline wander and that of the EMG above $-20 \mathrm{~dB}$ normalised to the peak of the 
maternal QRS-complex bispectrum (Zgallai, 2007). It is comparatively easy to detect and classify either using the bispectral contour template matching technique.

iii. In the HOS domain, the Gaussian noise diminishes if the data length is adequate (Nikias and Petropulu, 1993; Nam and Powers, 1994). This implies that it is possible, under certain conditions, to process the ECG signal in Gaussian noise-free domains. It was found (Rizk and Zgallai, 1999) that for ECG signals a minimum length of $1 \mathrm{sec}$ is adequately long to suppress Gaussian noise in the higher-order statistical domains, whilst not long enough to violate Hinich's criterion of local stationarity (Brockett et al. 1988). Hinich tests for Gaussianity and linearity were performed on ECG signals (Zgallai, 2007). ECG signals are non-stationary in the statistical sense, but relatively short data can be successfully treated with conventional signal processing tools primarily designed for stationary signals. For example, when dealing with individual cardiac cycles, non-stationarity is not an issue but when one takes on board the heart rate time series which is chaotic and multi-dimensional then it is not wise to assume stationarity for analysis purposes (Rizk et al. 2002).

iv. In the third-order domain all sources of noise with symmetric probability density functions (pdfs), e.g., Gaussian and uniform, will vanish. The ECG signals are retained because they have non-symmetric distributions (Zgallai, et al., 1997).

v. ECG signals contain quadratic and cubic non-linearities (Rizk et al., 1998). Such measurable quantities of non-linearity if not synthesised and removed before any further processing for the purpose of signal identification and classification could lead to poor performance with regard to fetal QRS-complex detection rates.

An adaptive third-order Volterra structure (Nam and Powers, 1994) has been used to synthesise the linear, quadratic non-linear, and cubic non-linear components of ECG signals. The removal of non-linearities in the transabdominal ECG signal yields an increase in the fetal heartbeat detection rates by up to $7 \%$ in the third-order cumulant matching technique (Zgallai, 2010), and 10\% in the bispectral contour template matching technique (Zgallai, 2012 a, Zgallai, 2012 b).

For noise identification and characterisation in the third-order statistical domain, use is made of the recorded normal ECG signals contained in the MIT/BIH databases (MIT/BIH, 1997). The third-order cumulants, bispectra, and bicoherence squared of some noise components, namely, the baseline wander, electromyographic (EMG) (Zgallai, 2009), and motion artefact noise isolated from the MIT/BIH databases are analysed. Knowing the statistics of those noise components, would facilitate the detection of ECG signals against a cocktail of background noise in either the cumulant or the bispectrum domain. Higher detection rate of fetal QRS-complex can be achieved in the enhanced fetal QRS-complex bispectrum domain against both maternal and motion artefact bispectral contribution (Zgallai, 2012 a). Bispectral enhancement has been carried out after removing the baseline wander, and in difficult cases, after linearisation (removing non-linearity from the noise contaminated maternal transabdominal signal). 


\section{Background and definitions}

\subsection{Cumulants}

Given a set of $n$ real variables $\left\{x_{1}, x_{2}, \ldots, x_{n}\right\}$, their joint moments of order, $r=k_{1}+k_{2}+\ldots+k_{n}$ are given by (Kravtchenko-Berejnoi, V. et al. 1995):

$$
\operatorname{Mom}\left[x_{1}^{k_{1}}, x_{2}^{k_{2}}, \ldots, x_{n}^{k_{n}}\right] \stackrel{\nabla}{=} E\left\{x_{1}^{k_{1}}, x_{2}^{k_{2}}, \ldots, x_{n}^{k_{n}}\right\}=\left.(-j)^{r} \frac{\partial^{r} \phi\left(\omega_{1}, \omega_{2}, \ldots, \omega_{n}\right)}{\partial \omega_{1}^{k_{1}} \partial \omega_{2}^{k_{2}} \ldots \partial \omega_{n}^{k_{n}}}\right|_{\omega_{1}=\omega_{2}=\ldots=\omega_{n}=0}
$$

where $\phi\left(\omega_{1}, \omega_{2}, \ldots, \omega_{n}\right) \underline{\nabla} E\left\{e^{j\left(\omega_{1} x_{1}, \omega_{2} x_{2}, \ldots, \omega_{n} x_{n}\right)}\right\}$ is their joint characteristic function. E\{. $\}$ denotes the expectation operator. Another form of the joint characteristic function is defined as the natural logarithm of $\phi\left(\omega_{1}, \omega_{2}, \ldots, \omega_{n}\right)$, i.e., (Nikias and Petropulu, 1993)

$$
\tilde{\Psi}\left(\omega_{1}, \omega_{2}, \ldots, \omega_{n}\right) \underline{=} \ln \left[\phi\left(\omega_{1}, \omega_{2}, \ldots, \omega_{n}\right)\right]
$$

For Gaussian processes, the logarithm of the characteristic function is a polynomial of degree two. Hence, all cumulants of order three and higher will be identically zero. The joint cumulants of order $r$ of the same set of random variables, are defined as the coefficients in the Taylor expansion of the second characteristic function about zero, i.e., (Nikias and Petropulu, 1993)

$$
\operatorname{Cum}\left[x_{1}^{k_{1}}, x_{2}^{k_{2}}, \ldots, x_{n}^{k_{n}}\right]=E\left\{x_{1}^{k_{1}}, x_{2}^{k_{2}}, \ldots, x_{n}^{k_{n}}\right\}=\left.(-j)^{r} \frac{\partial^{r} \tilde{\Psi}\left(\omega_{1}, \omega_{2}, \ldots, \omega_{n}\right)}{\partial \omega_{1}^{k_{1}} \partial \omega_{2}^{k_{2}} \ldots \partial \omega_{n}^{k_{n}}}\right|_{\omega_{1}=\omega_{2}=\ldots=\omega_{n}=0}
$$

Thus, the joint can be expressed in terms of the joint moments of a set of random variables. The moments of the random variable $\left\{x_{1}\right\}$ are defined as (Nikias and Petropulu, 1993)::

$$
\mathrm{m}_{1}=\mathrm{E}\left\{\mathrm{x}_{1}\right\} \cdot \mathrm{m}_{2}=\mathrm{E}\left\{\mathrm{x}_{1}^{2}\right\} \cdot \mathrm{m}_{3}=\mathrm{E}\left[\mathrm{x}_{1}^{3}\right]
$$

Cumulants are related to moments by (Nikias and Petropulu, 1993)

$$
\mathrm{c}_{1}=\mathrm{m}_{1} \cdot \mathrm{c}_{2}=\mathrm{m}_{2}-\mathrm{m}_{1}{ }^{2} \cdot \mathrm{c}_{3}=\mathrm{m}_{3}-3 \mathrm{~m}_{2} \mathrm{~m}_{1}+2 \mathrm{~m}_{1}^{3} .
$$

For three random variables $\mathrm{x}_{1}, \mathrm{x}_{2}$, and $\mathrm{x}_{3}$ the third-order cumulants are defined as (Kravtchenko-Berejnoi, V. et al. 1995):

$$
c_{3}^{x}\left(\tau_{1}, \tau_{2}\right)=m_{3}^{x}\left(\tau_{1}, \tau_{2}\right)-m_{1}^{x}\left[m_{2}^{x}\left(\tau_{1}\right)+m_{2}^{x}\left(\tau_{2}\right)+m_{2}^{x}\left(\tau_{2}-\tau_{1}\right)\right]+2 \cdot\left(m_{1}^{x}\right)^{3}
$$

\subsection{Properties of cumulants}

1. $c\left[a_{1} x_{1}, a_{2} x_{2}, \ldots, a_{n} X_{n}\right]=a_{1} a_{2} \ldots$ an $c\left[x_{1}, x_{2}, \ldots, x_{n}\right]$ (Nikias and Petropulu, 1993). 
2. Cumulants are symmetric functions in their arguments, e.g., $c\left[\mathrm{x}_{1}, \mathrm{x}_{2}, \mathrm{x}_{3}\right]=\mathrm{c}\left[\mathrm{x}_{2}, \mathrm{x}_{1}, \mathrm{x}_{3}\right]=$ $c\left[x_{3}, x_{2}, x_{1}\right]$, and so on (Nikias and Petropulu, 1993)..

3. If the random variables $\left\{\mathrm{x}_{1}, \mathrm{x}_{2}, \ldots, \mathrm{x}_{\mathrm{n}}\right\}$ can be divided into any two or more groups which are statistically independent, their nth-order cumulant is identical to zero; i.e. $c\left[\mathrm{x}_{1}, \mathrm{x}_{2}\right.$, $\left.\ldots, x_{n}\right]=0$, whereas in general $\operatorname{Mom}\left[x_{1}, x_{2}, \ldots, x_{n}\right] \neq 0$ (Nikias and Petropulu, 1993)..

4. If the sets of random variables $\left\{\mathrm{x}_{1}, \mathrm{x}_{2}, \ldots, \mathrm{x}_{\mathrm{n}}\right\}$ and $\left\{\mathrm{y}_{1}, \mathrm{y}_{2}, \ldots, \mathrm{y}_{\mathrm{n}}\right\}$ are independent, then $c\left[x_{1}+y_{1}, x_{2}+y_{2}, \ldots, x_{n}+y_{n}\right]=c\left[x_{1}, x_{2}, \ldots, x_{n}\right]+c\left[y_{1}, y_{2}, \ldots, y_{n}\right]$ (Nikias and Petropulu, 1993).

5. If the set of random variables $\left\{x_{1}, x_{2}, \ldots, x_{n}\right\}$ is jointly Gaussian, then all the information about their distribution is contained in the cumulants of order $n \leq 2$. Therefore, all cumulants of order greater than two $(n>2)$ have no new information to provide. This leads to the fact that all joint cumulants of order $n>2$ are identical to zero for Gaussian random vectors. Hence, the cumulants of order greater than two, in some sense, measure the non-Gaussian nature of a time series (Nikias and Petropulu, 1993).

\subsection{One-dimensional third-order cumulant slices}

Since higher-order cumulants and spectra are multi-dimensional functions, their computation may be impractical in some applications due to excessive crunching. This is caused by the large CPU time taken to calculate HOS functions, compared to SOS functions. It was suggested to use 1-d slices of multi-dimensional cumulants, and their 1-d Fourier transforms, as ways of extracting useful information from higher-order statistics of nonGaussian stationary processes (Nagata, 1970). The third-order cumulants of a non-Gaussian process, $\{x(k)\}$, is given by (Nikias and Petropulu, 1993):

$$
c_{3}^{x}\left(\tau_{1}, \tau_{2}\right)=c\left\{\mathrm{x}(k), \mathrm{x}\left(k+\tau_{1}\right), \mathrm{x}\left(k+\tau_{2}\right)\right\}
$$

One-dimensional slices of $\mathcal{C}_{3}^{x}\left(\tau_{1}, \tau_{2}\right)$ can be defined as (Nikias and Petropulu, 1993):

$$
r_{2,1}^{x}(\tau) \underline{=}\{x(k), \mathrm{x}(k), \mathrm{x}(k+\tau)\}=c_{3}^{x}(0, \tau)
$$

and

$$
r_{1,2}^{x}(\tau) \underset{=}{=}\{\mathrm{x}(k), \mathrm{x}(k+\tau), \mathrm{x}(k+\tau)\}={ }_{c_{3}}^{x}(\tau, \tau)
$$

Define the following even and odd functions (Nikias and Petropulu, 1993):

$$
S_{2,1}^{x}(\tau) \underline{=} \frac{1}{2}\left[r_{2,1}^{x}(\tau)+r_{1,2}^{x}(\tau)\right]
$$

and

$$
q_{2,1}^{x}(\tau) \underline{=} \frac{1}{2}\left[r_{2,1}^{x}(\tau)-r_{1,2}^{x}(\tau)\right]
$$

A 1-d spectrum could be defined as (Nikias and Petropulu, 1993): 


$$
R_{2,1}^{x}(\omega)=\sum_{\tau=-\infty}^{\infty} r_{2,1}^{x}(\tau) e^{-j \omega t}=\sum_{\tau=-\infty}^{\infty}\left\{s_{2,1}^{x}(\tau) \cdot \cos (\omega \tau)-j q_{2,1}^{x}(\tau) \cdot \sin (\omega \tau)\right\}
$$

\subsection{Cumulant spectra}

Higher order spectra are defined as the multi-dimensional Fourier transforms of the higher order statistics of the superimposed signals in the presence of noise (Nikias and Raghuveer, 1987; Rosenblatt, 1985; Brillinger, 1965). The nth-order cumulant spectrum of a process $\{x(k)\}$ is defined as the (n-1)-dimensional Fourier transform of the nth-order cumulant sequence. The nth-order cumulant spectrum is thus defined as (Nikias and Petropulu, 1993):

$$
C_{n}^{x}\left(\omega_{1}, \omega_{2}, \ldots, \omega_{n-1}\right)=\sum_{\tau_{1}=-\infty}^{+\infty} \cdots \sum_{\tau_{n-1}=-\infty}^{+\infty} c_{n}^{x}\left(\tau_{1}, \tau_{2}, \cdots, \tau_{n-1}\right) e^{-j\left(\omega_{1} \tau_{1}+\omega_{2} \tau_{2}+\ldots, \omega_{n} \tau_{n-1}\right)},
$$

where

$$
\left|\omega_{1}\right| \leq \pi \quad \text { for } \quad i=1,2, \ldots n-1 \text {, and } \quad\left|\omega_{1}+\omega_{2}+\ldots+\omega_{n-1}\right| \leq \pi
$$

\section{Special cases}

1. Power spectrum $(n=2)$ :

$$
C_{2}^{x}(\omega)=\sum_{\tau=-\infty}^{+\infty} c_{2}^{x}(\tau) e^{-j(\omega \tau)}
$$

where $c_{2}^{x}(\tau)$ is the covariance.

2. $\quad$ Bispectrum $(n=3)$ :

$$
C_{3}^{x}\left(\omega_{1}, \omega_{2}\right)=\sum_{\tau_{1}=-\infty}^{+\infty} \sum_{\tau_{2}=-\infty}^{+\infty} c_{3}^{x}\left(\tau_{1}, \tau_{2}\right) e^{-j\left(\omega_{1} \tau_{1}+\omega_{2} \tau_{2}\right)}
$$

where $c_{3}^{x}\left(\tau_{1}, \tau_{2}\right)$ is the third-order cumulant. Conventional higher-order statistics (HOS) estimates are asymptotically unbiased and consistent (Nagata, 1970), and easy to implement using FFT-based methods. The ability to resolve harmonic components is limited by the uncertainty principle of the Fourier transform. There are numerous methods for polyspectra estimation based on parametric methods. MA models have been treated in (Nikias, 1988; Friedlander and Porat, 1988). Spectral estimation methods based on non-causal AR models were developed in (Huzi, 1981). Methods based on ARMA models have been published (Lii, 1982). MA, AR and ARMA methods based on higher-order statistics were described (Mendel, 1988). A review of cumulant spectra and the asymptotic properties of their estimators were given in (Rosenblatt, 1983). Practical considerations for bispectral estimation were given (Subba Rao, 1983). The relationship between the bispectrum and conventional methods for their estimation was discussed (Zurbenko, 1982). 


\subsection{Non-stationarity and the OT region of the bispectrum}

The bispectrum of a stationary sampled process must be zero in the triangle region OT, i.e., the region defined by the triangle, OT $=\left\{\omega_{1}, \omega_{2}: \omega_{2} \leq \omega_{1}, \pi \leq \omega_{1}+\omega_{2} \leq 2 \pi-\omega_{1}\right\}$. The bispectrum in the OT region will be non-zero if the process is non-stationary. The bispectrum has 12 symmetric regions. The knowledge of the bispectrum in one triangular region is enough for a complete description of the bispectrum of a real process.

\subsection{Nth-order coherency function}

A normalised cumulant spectrum or the nth-order coherency index is a function that combines the cumulant spectrum of order $\mathrm{n}$ and the power spectrum. It is defined as (Nikias and Petropulu, 1993)

$$
P_{n}^{x}\left(\omega_{1}, \omega_{2}, \ldots, \omega_{n-1}\right) \stackrel{\nabla}{=} \frac{C_{n}^{x}\left(\omega_{1}, \omega_{2}, \ldots, \omega_{n-1}\right)}{\left[C_{2}^{x}\left(\omega_{1}\right) \cdot C_{2}^{x}\left(\omega_{2}\right) \cdots C_{2}^{x}\left(\omega_{n-1}\right) \cdot C_{2}^{x}\left(\omega_{1}+\omega_{2}+\ldots+\omega_{n-1}\right)\right]^{2}} .
$$

The third-order $(n=3)$ coherence index is also called bicoherence. The $n$th order coherence index is useful for the detection and characterisation of non-linearities in time series via phase relations of their harmonic components. The coherency index is used to differentiate between linear non-Gaussian processes and non-linear processes when both have non-zero cumulants. If the coherency index is zero, then the process is linear and Gaussian. If the $\mathrm{n}^{\text {th }}$ order coherency index is not frequency dependent, then the process is linear non-Gaussian. If the coherency index is frequency dependent, then the process is non-linear (Nikias and Petropulu, 1993).

\subsection{Statistical measures}

A statistical measure could be described as an unbiased estimate when the expected value of the estimated statistic is, asymptotically, equal to the true value. An estimate of the cumulant spectra is unbiased if

$$
E\left|\hat{C}_{3}^{x}\left(\omega_{1}, \omega_{2}\right)\right|=C_{3}^{x}\left(\omega_{1}, \omega_{2}\right)
$$

The bias is defined as the difference between the true value and the expected value.

\section{Second-order statistics of ECG signals}

\subsection{The probability density functions (pdfs) of ECG signals}

Three essential ECG signals are considered:

1. the maternal chest ECG signal. This is measured using one surface electrode positioned on the chest and one reference electrode on the thigh; 
2. The transabdominally-measured ECG signal which contains both maternal and fetal contributions amongst other deterministic and chaotic signals plus noise artefacts (Rizk et al., 2002). This is acquired using twin surface electrodes positioned near the mother's umbilicus and synchronised with the maternal chest signal;

3. the fetal scalp electrode ECG signal which will always be used as a reference signal in the assessment of any particular QRS detection technique based on non-invasive transabdominally-measured ECG signals. The non-symmetry of the probability density functions (pdfs) of the above mentioned signals is shown in the histograms of Fig. 1 and supports their third-order cumulants.

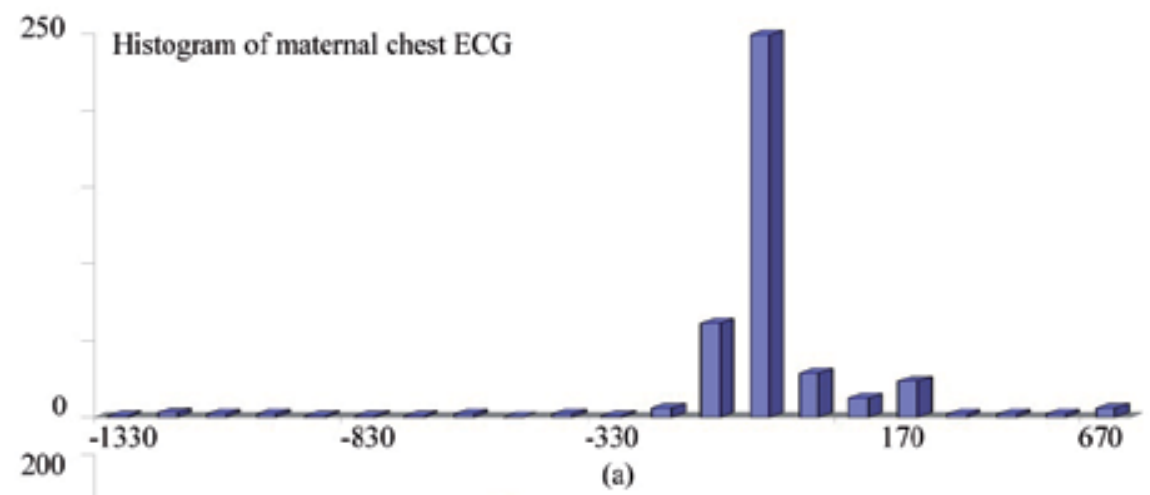

Histogram of fetal scalp electrode ECG
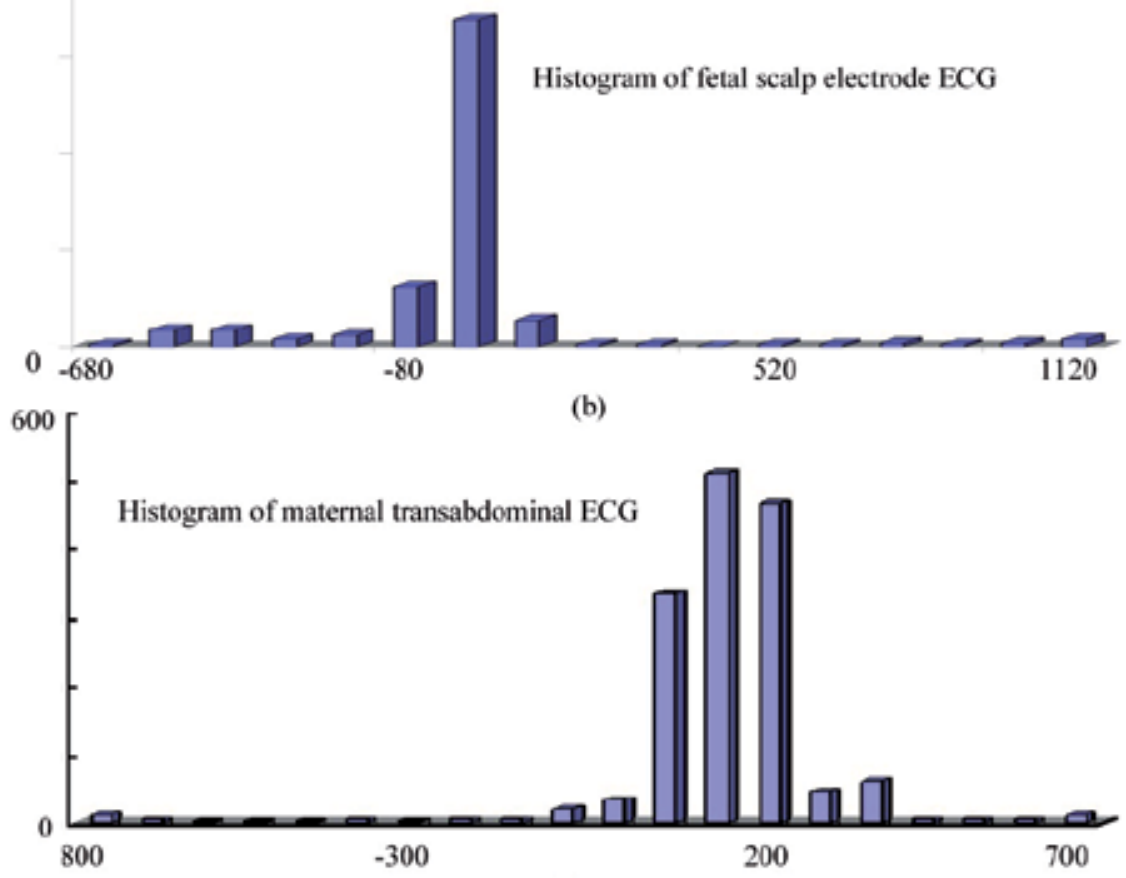

(c)

Figure 1. Histograms of typical templates of (a) a maternal chest ECG, (b) a fetal scalp electrode FECG, and (c) a maternal transabdominal ECG. They all show non-Gaussian distributions. 


\subsection{The second-order cumulants of ECG signals}

Fig. 2 (a) shows a full maternal transabdominal cardiac cycle (1000 ms) which has been divided into four segments, I, II, III, and IV. These segments represent (I) the predominantly maternal QRS-complex, (II) the first fetal heartbeat with maternal contribution, (III) QRSfree ECG, and (IV) the second fetal heartbeat with maternal contribution, respectively. Fig. 2 (b) shows a typical example of the second-order cumulants (auto-correlation functions) for the segments shown in Fig. 2 (a). Second-order statistics do not show any distinguishable features that could be used to differentiate between maternal QRS-complex, fetal heartbeat with maternal contribution, and QRS-free ECG contributions.

\subsection{The power spectrum of ECG signals}

Fig. 3 depicts the power spectrum using the FFT method for (a) fetal scalp electrode ECG signal (data length $500 \mathrm{~ms}$ ), (b) maternal transabdominal ECG (data length $1000 \mathrm{~ms}$ ). The maternal cardiac cycle begins $50 \mathrm{~ms}$ before the R-wave and ends $50 \mathrm{~ms}$ before the next Rwave. The subject is at the first stage of labour ( 40 weeks gestation). The FFT method reveals a fetal scalp electrode ECG principal spectral peak at $30 \mathrm{~Hz}$. The FFT method for the transabdominal cardiac cycle reveals the maternal principal spectral peak of $15 \mathrm{~Hz}$. However, the FFT does not show fetal spectral peak from the segmented transabdominal signal. There is a shallow peak at $28 \mathrm{~Hz}$ and a shifted peak at $42 \mathrm{~Hz}$ (Zgallai, 2007).

\section{Third-order statistics of ECG signals}

\subsection{Cumulants and their slices of cardiac cycles}

The maternal chest ECG is measured using the standard three-lead electrode system. The maternal transabdominally-measured signals are obtained using two surface electrodes. The electrode pair is set over the umbilicus, and lined up with the median vertical axis of the uterus. The ground electrode is located on the woman's hip. The fetal scalp electrode is used when deemed necessary. Multi-dimensional Third-order cumulants TOCs were computed for the above ECG signals as well as for the four segments of the maternal transabdominal cardiac cycles. The four segments were coded as I, II, III, and IV, each of length $250 \mathrm{~ms}$ which has been considered short enough as not to satisfy the assumption of non-stationarity, and long enough to meet the threshold of the higher-order statistical variances. The four coded segments ascribe to the following often occurring scenario; (I) Segment I, 0-250 ms; Predominantly maternal QRS-complex (no fetal QRS-complex present), (II) Segment II, $251-500 \mathrm{~ms}$; The first fetal heartbeat with maternal contribution, (III) Segment III, $501 \mathrm{~ms}-750 \mathrm{~ms}$; QRS-free ECG, and (IV) Segment IV, 751 - $1000 \mathrm{~ms}$; The second fetal heartbeat with maternal contribution.

Fig. 4 (a), (b), (c), and (d) each depicts ECG signals (upper panel) and their third-order cumulants (lower panel) for fetal scalp ECG (550 ms), maternal chest ECG (900 ms), and two different and randomly picked transabdominally-measured maternal ECGs (1000 ms each). The subject is at 40 weeks gestation after the water has been broken hence facilitating fetal scalp measurements. The maternal cardiac cycle begins $50 \mathrm{~ms}$ before the R-wave and ends $50 \mathrm{~ms}$ before the next R-wave. 

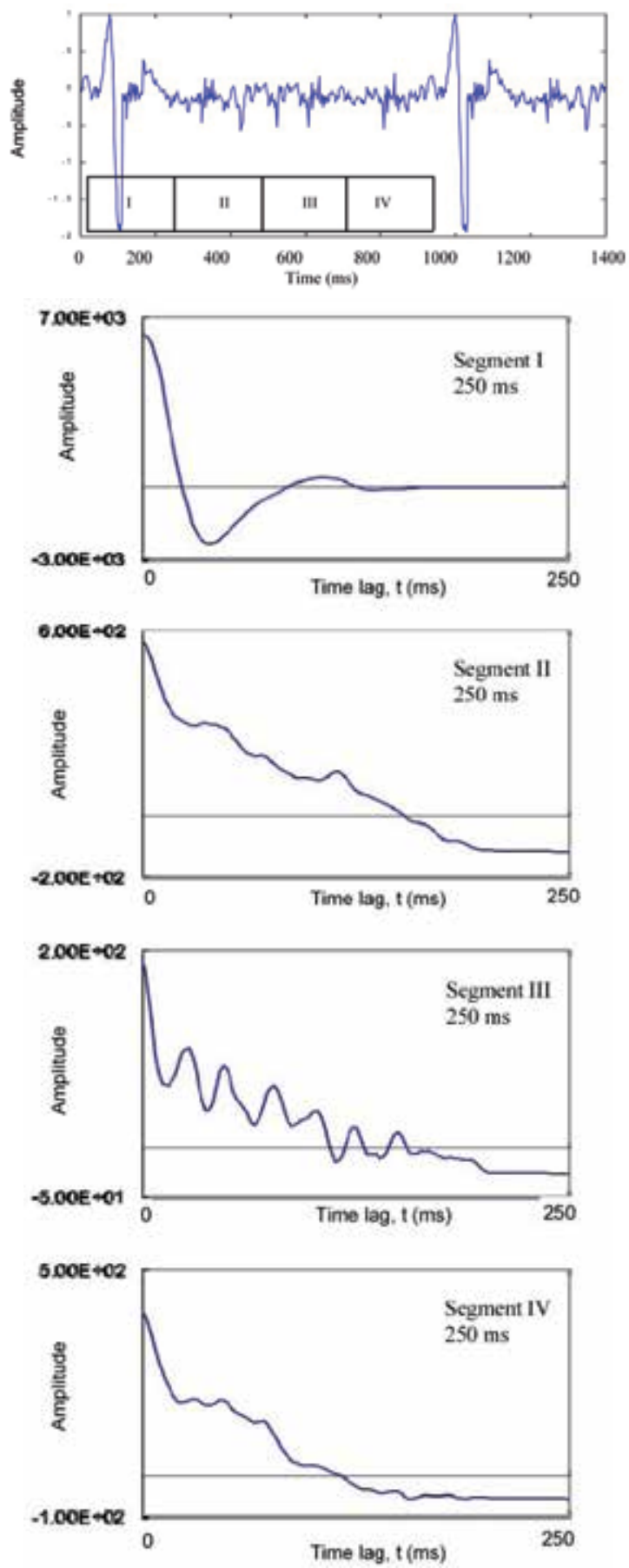

Figure 2. (a). Maternal transabdominal cardiac cycle $(1000 \mathrm{~ms})$ divided into four segments. The maternal cardiac cycle begins $50 \mathrm{~ms}$ before the R-wave and ends $50 \mathrm{~ms}$ before the next R-wave. The subject is at the first stage of labour (40 weeks gestation). (b). Typical examples of the second-order cumulants computed using the segments I, II, III, and IV shown in 2 (a) of maternal transabdominal ECG. 


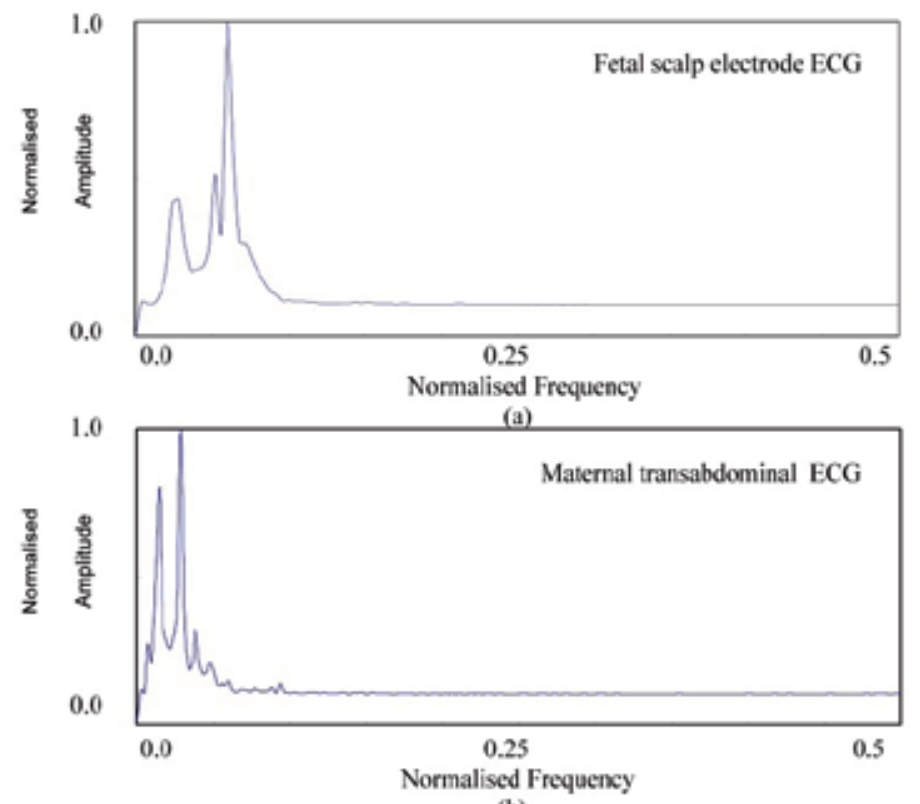

(b)

Figure 3. The power spectrum using the FFT method for (a) fetal scalp electrode ECG (data length 500 $\mathrm{ms}$ ), and (b) maternal transabdominal ECG signal (data length $1000 \mathrm{~ms}$ ). The maternal cardiac cycle begins $50 \mathrm{~ms}$ before the R-wave and ends $50 \mathrm{~ms}$ before the next $\mathrm{R}$-wave. The subject is at the first stage of labour (40 weeks gestation).

A quick glance at the similarities of the four cumulant patterns in Fig. 4 (a), (b), (c) and (d), gives a little hope of successful detection of the fetal presence in the maternal cardiac cycle. To complicate the matter further, the two transabdominal cumulants in Fig. 4 (c) and (d) look dissimilar even though both contain two fetal QRS-complexes. However, the best way to distinguish between those patterns is to slice them and look for discriminant features.

Fig. 5 shows the third-order cumulants and their diagonal (1.h.s.) and wall (r.h.s.) slices of one transabdominal cardiac cycle which is segmented into four segments of $250 \mathrm{~ms}$ each for (I) predominantly maternal QRS, (II) the first fetal heartbeat with maternal contribution, (III) QRS-free ECG, and (IV) the second fetal heartbeat with maternal contribution. The diagonal and wall TOC slices of the maternal segment (I) are easily distinguished from the diagonal and wall TOC slices of segments (II), (III), and (IV). Furthermore, the diagonal and wall TOC slices of fetal QRS segments (II) and (IV) are distinguishable from the diagonal and wall TOC slices of the QRS-free ECG segment (III) in that there is a distinguishable and wellformed peak at the origin in both diagonal and wall TOC slices. The peak of the QRS-free ECG segment is much narrower and more related to motion artefact than a signal.

Note that having computed the three-dimensional TOCs, either the diagonal or the wall slice could be used in the detection / classification process. Therefore, computing the full multi-dimensional TOC and then extracting individual slices is an unnecessary waste of the CPU time. So, why not compute any arbitrary 1-d slice directly without firstly having to 
compute the two-dimensional TOC and secondly extract the 1-d slice? The TOC-diagonal and the TOC-wall slices are straightforward to compute directly, by freezing one of the two cumulant lags and changing the other one. However, computing any other arbitrary slice requires the development of an auxiliary algorithm (Zgallai, 2007). It has been found that performing direct computations of the 1-d TOC slices instead of computing the 2-d TOC firstly and secondly extracting individual 1-d slices results in saving of more than $99 \%$ of the CPU time.

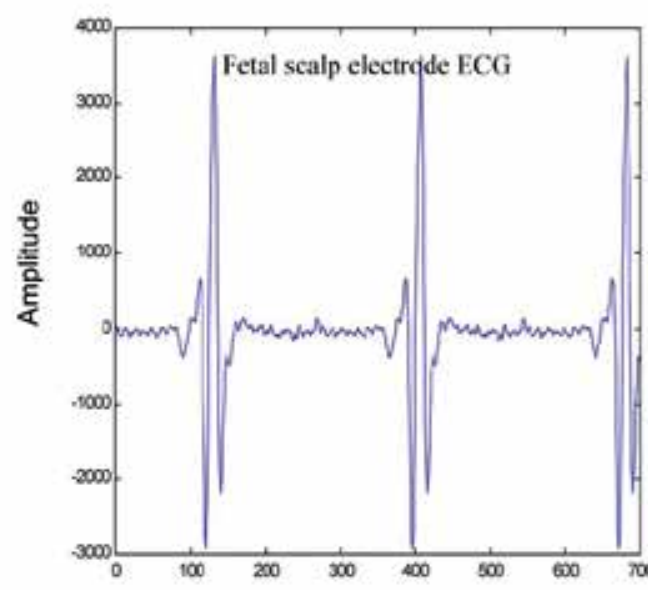

Sample

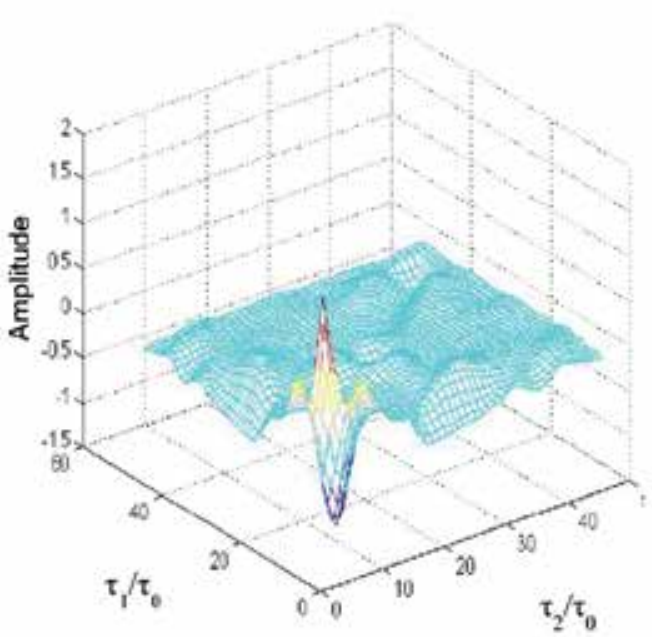

(a)

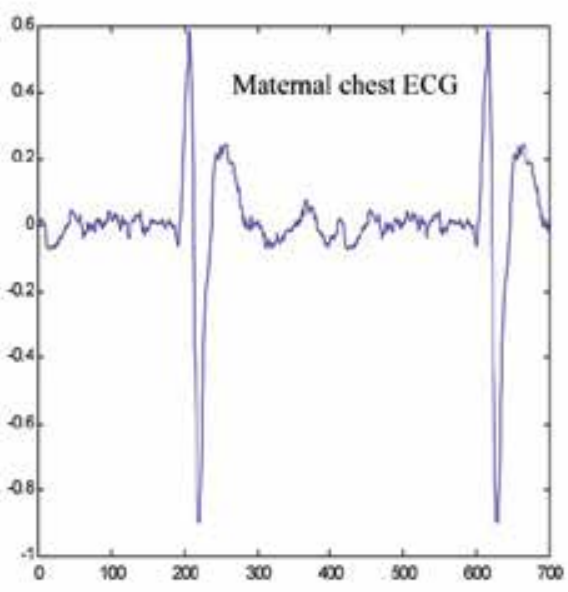

Sample

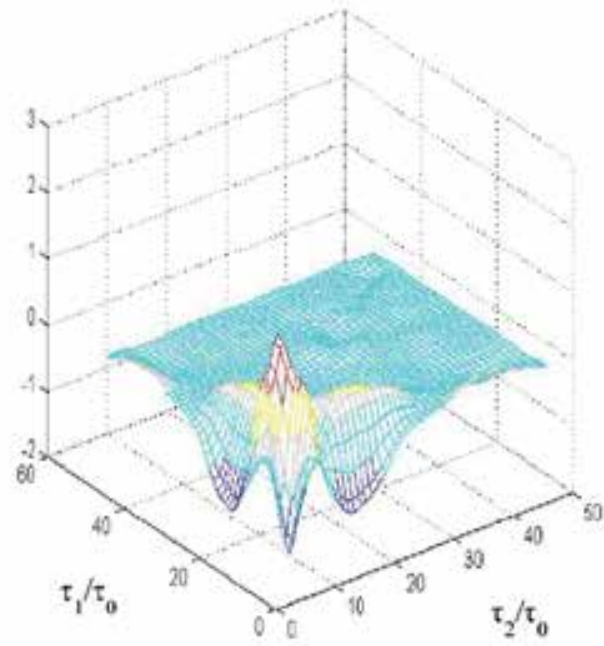

(b) 


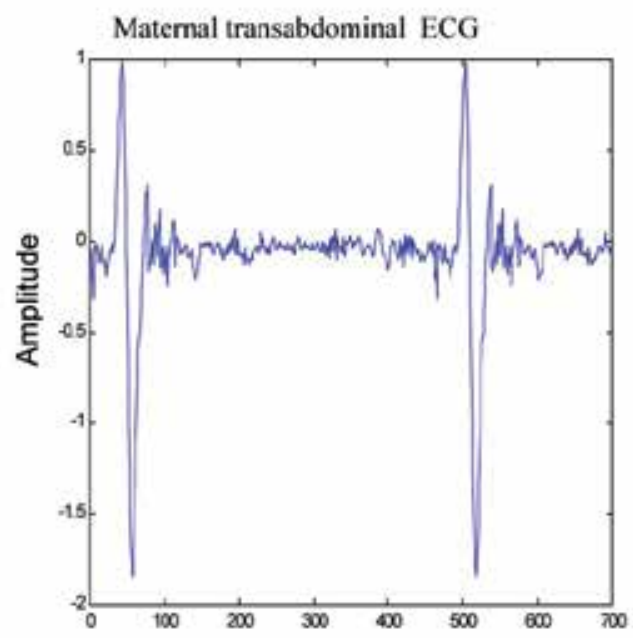

Sample

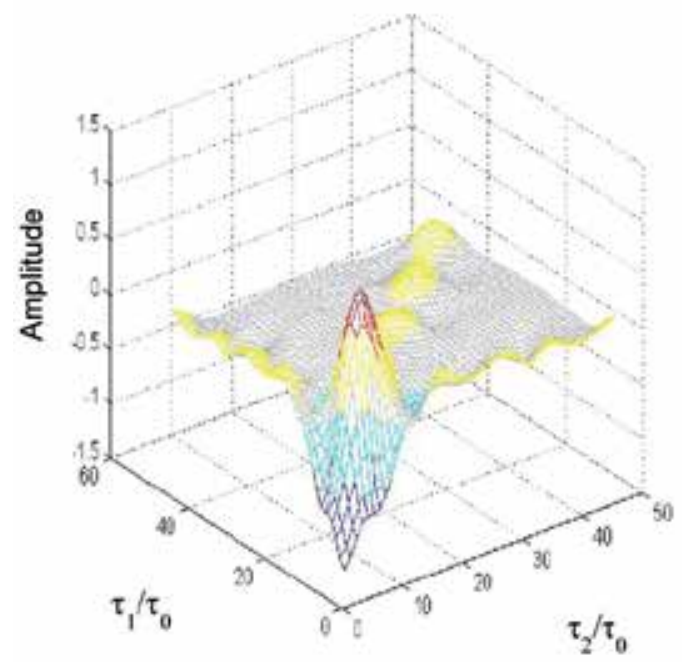

(c)

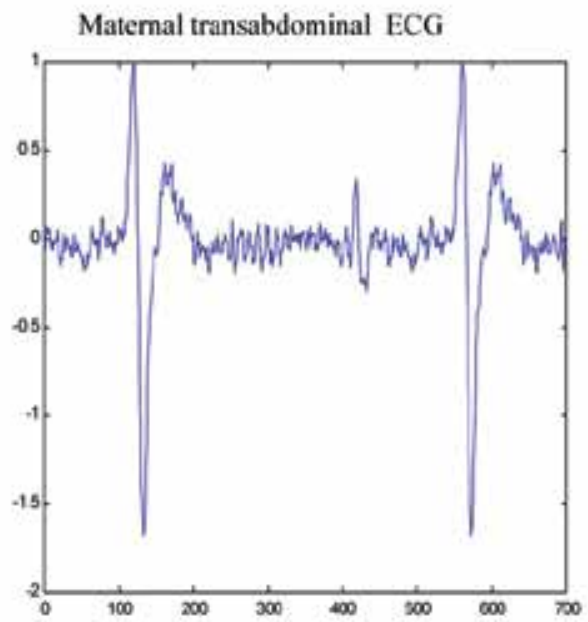

Sample

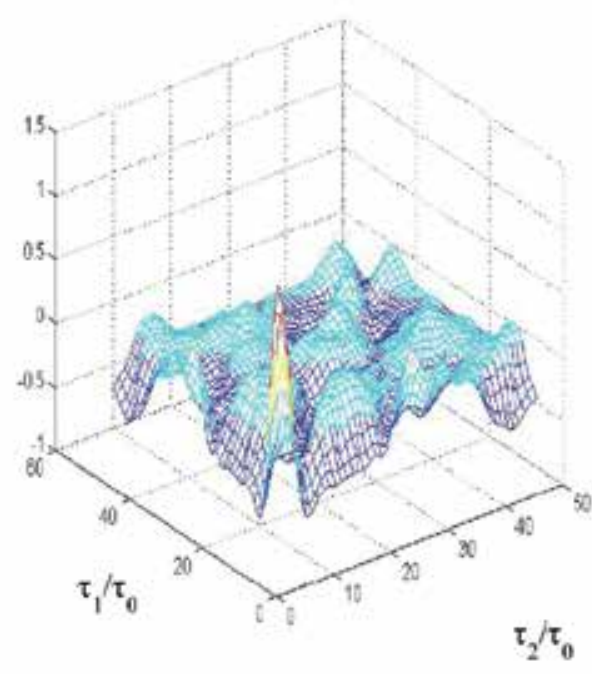

(d)

Figure 4. ECG signals (upper panel) and their third-order cumulants (lower panel) for (a) fetal cardiac cycle using fetal scalp electrode ( data length $550 \mathrm{~ms}$ ), (b) maternal chest cardiac cycle using one surface electrode and a reference electrode (data length $900 \mathrm{~ms}$ ), (c) and (d) are two maternal transabdominal cardiac cycles measured using twin surface electrodes (data length $1000 \mathrm{~ms}$ each). The maternal cardiac cycle begins $50 \mathrm{~ms}$ before the R-wave and ends $50 \mathrm{~ms}$ before the next $\mathrm{R}$-wave. The subject is at the first stage of labour, 40 weeks. 

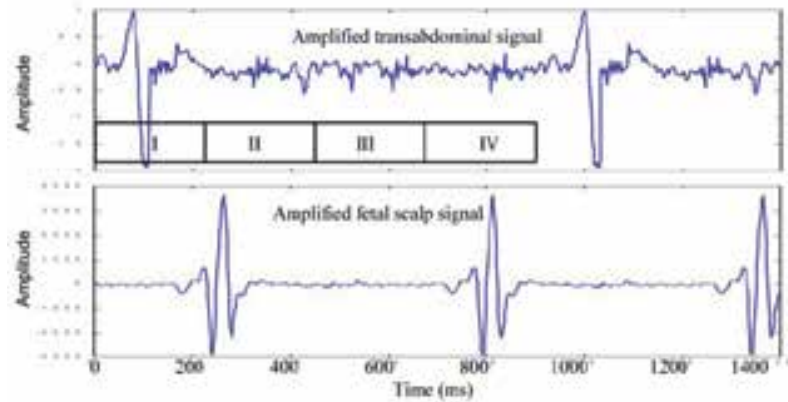

(a)

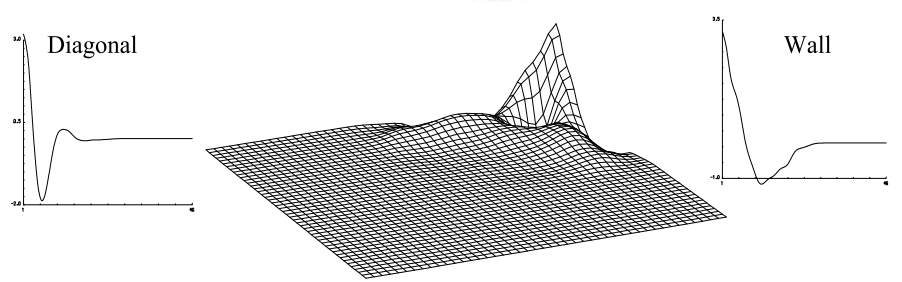

(b)
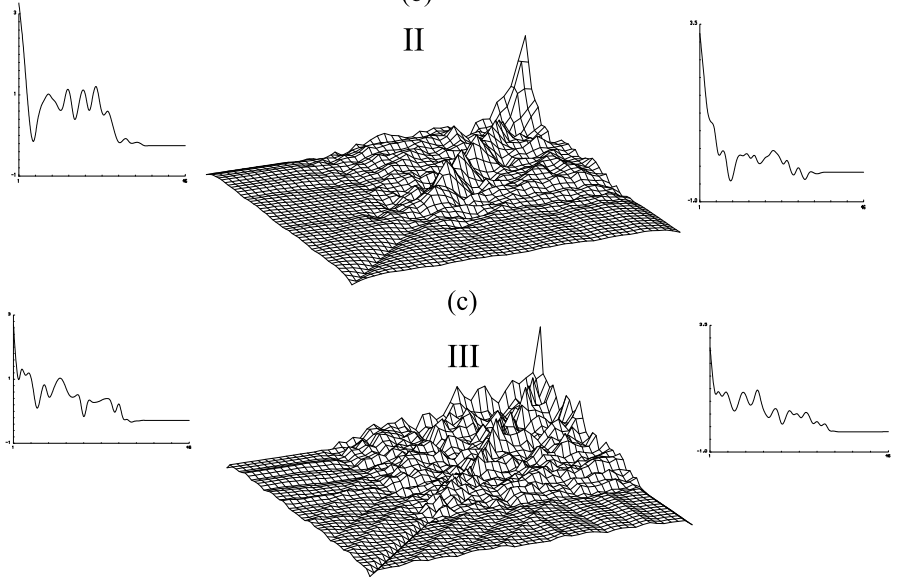

(d)
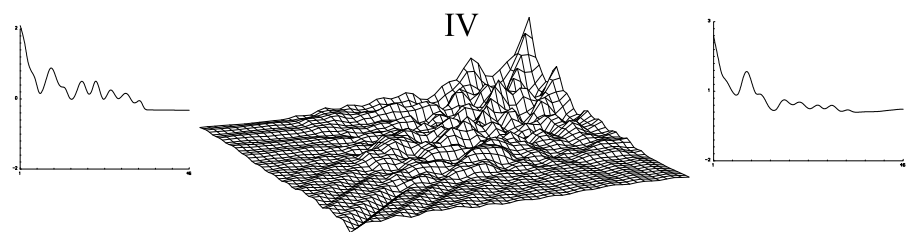

(e)

Figure 5. (a) Maternal transabdominal ECG signal (upper panel) and the synchronised fetal ECG signal measured using fetal scalp electrode (lower panel). (b), (c), (d) and (e) are the third-order cumulants and their diagonal (l.h.s.) and wall (r.h.s.) slices for segments I, II, III, and IV, respectively, each segment is 250 ms. Segment I: pre-dominantly maternal QRS-complex, segment II, the first fetal heartbeat with maternal contribution, segment III: QRS-free ECG, and segment IV: the second fetal heartbeat with maternal contribution. The maternal cardiac cycle begins $50 \mathrm{~ms}$ before the R-wave and ends $50 \mathrm{~ms}$ before the next Rwave. 
Fig. 6 shows four selected slices of the third-order cumulants computed using one cardiac cycle for each of the following; (a) and (b) an adult male and female chest, respectively, (c) maternal transabdominal, and (d) fetal scalp electrode ECG signal.

(a)

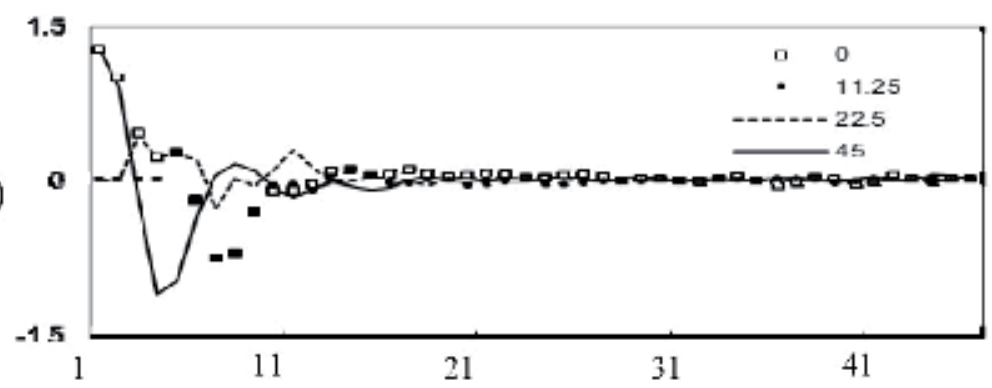

(b)

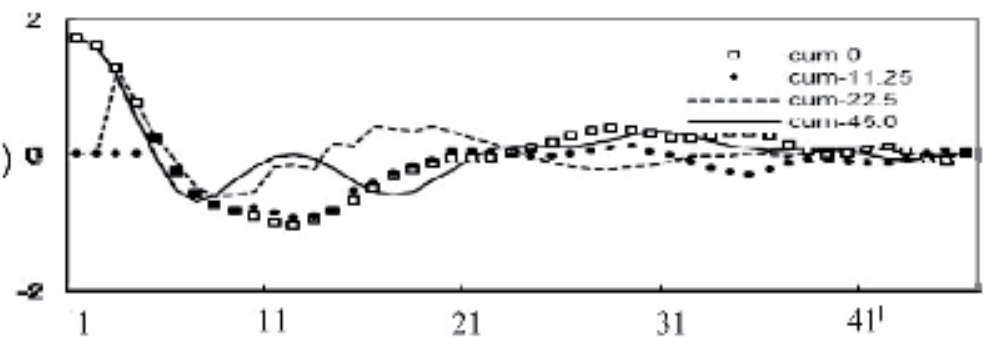

(c)
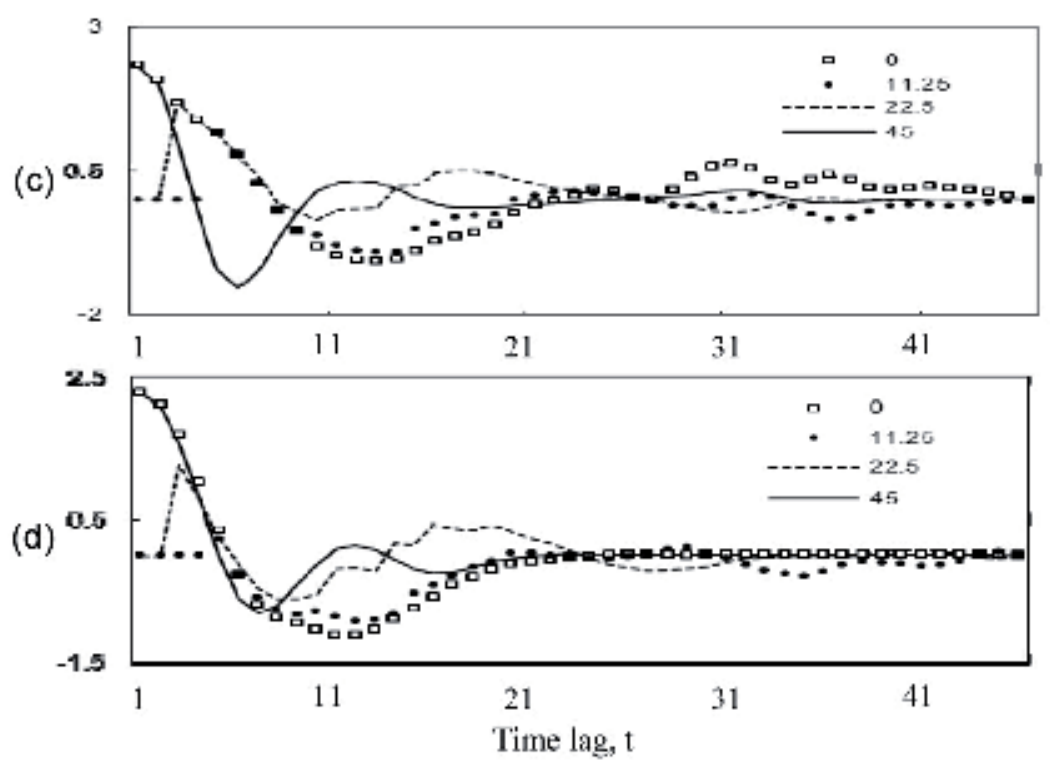

Figure 6. Third order cumulant slices at $0^{\circ}$ (wall), $11.25^{\circ}, 22.50^{\circ}$, and $45^{\circ}$ (diagonal) for (a) male chest cardiac cycle using one surface electrode (data length $1180 \mathrm{~ms}$ ), (b) maternal chest cardiac cycle using one surface electrode (data length $900 \mathrm{~ms}$ ), (c) maternal transabdominal cardiac cycle using twin surface electrodes (data length $1000 \mathrm{~ms}$ ), and (d) fetal cardiac cycle using fetal scalp electrode (data length 550 $\mathrm{ms}$ ). The maternal cardiac cycle begins $50 \mathrm{~ms}$ before the R-wave and ends $50 \mathrm{~ms}$ before the next R-wave. The female subject is at the first stage of labour, 40 weeks gestation. 


\subsection{The bispectrum, contour maps and slices for cardiac cycles}

Fig. 7 shows the bispectrum magnitudes (left panel) and the corresponding contours (right panel) using one cardiac cycle for; (a) fetal scalp electrode ECG, (b) maternal chest ECG, and (c) maternal transabdominal ECG signal. Before attempting to assess any advantages of the ECG bispectrum over and above the power spectrum one should regress,
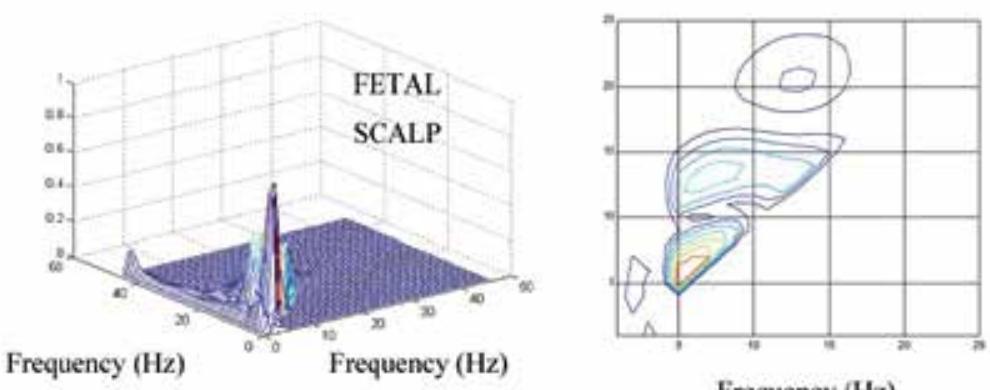

Frequency $(\mathrm{Hz})$

(a)
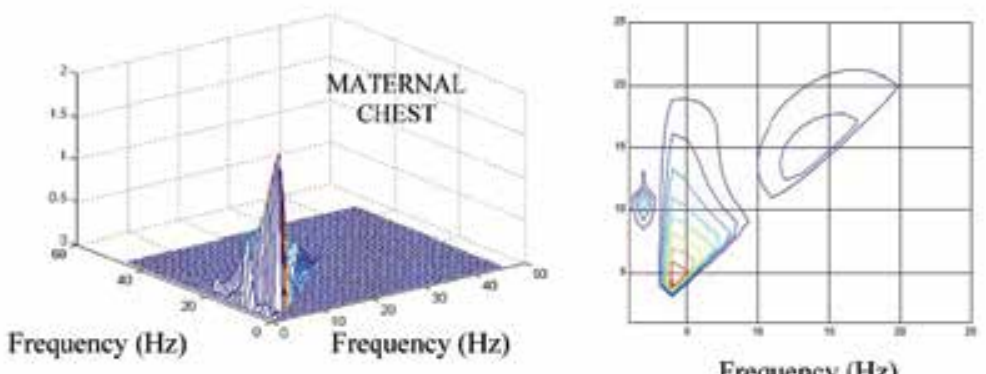

Frequency $(\mathbf{H z})$

(b)
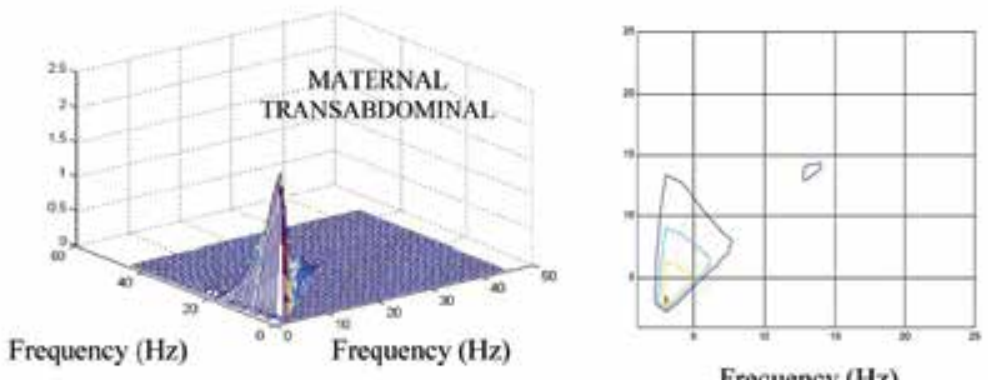

Frequency $(\mathrm{Hz})$

(c)

Figure 7. The bispectrum magnitude (left panel) and contour map (right panel) for (a) a fetal cardiac cycle using fetal scalp electrode (data length $550 \mathrm{~ms}$ ), (b) a maternal chest cardiac cycle (data length 1000 $\mathrm{ms}$ ), and (c) a maternal transabdominal cardiac cycle (data length $1000 \mathrm{~ms}$ ). The maternal cardiac cycle begins $50 \mathrm{~ms}$ before the $\mathrm{R}$-wave and ends $50 \mathrm{~ms}$ before the next $\mathrm{R}$-wave. The subject is at the first stage of labour, 40 weeks gestation. The direct method is used to calculate the bispectrum. 
for a moment, to view the power spectrum and locate the frequency ranges for adult and fetal QRS-complexes. The power spectrum of appropriately sampled ECG showed the QRScomplex principal peak in the frequency range from $15 \mathrm{~Hz}$ to $20 \mathrm{~Hz}$, and $25 \mathrm{~Hz}$ to $40 \mathrm{~Hz}$, for the maternal chest ECG and fetal scalp electrode ECG, respectively. The power spectrum has limitations as an estimator in terms of resolution, variance, and clarity of the spectrum to be able to produce clear and distinguishable peaks for the P-waves. An alternative spectrum estimator was used instead, namely, the multiple signal classification (MUSIC) pseudo-spectrum. The MUSIC-based pseudo-spectrum also showed that the principal peaks for the p-waves occupy a range from $5 \mathrm{~Hz}$ to $8 \mathrm{~Hz}$ for adults. The principal peaks for the Pwaves of the fetal scalp electrode ECG occupy a range from $8 \mathrm{~Hz}$ to $10 \mathrm{~Hz}$. The same MUSIC-based spectral estimators have revealed high local energy peaks around $5 \mathrm{~Hz}$ due to motion artefact (Zgallai, et al., 1997).

It is clearly seen in Fig. 7 that all significant twin-frequency peaks occur at frequencies lower than the p-wave and QRS-complex frequencies. It is very difficult to observe any p-wave or QRS-complex frequencies. The only thing that could be construed from these results is that the combined effect of the low temporal resolution resulting from using the whole cardiac cycle and the low spectral resolution inherent in the bispectrum formation, the QRScomplex twin peaks which should occur at frequency ranges from $(15 \mathrm{~Hz}, 15 \mathrm{~Hz})$ to $(20$ $\mathrm{Hz}, 20 \mathrm{~Hz})$ for adults and from $(25 \mathrm{~Hz}, 25 \mathrm{~Hz})$ to $(40 \mathrm{~Hz}, 40 \mathrm{~Hz})$ for fetal scalp electrode ECG are completely masked and cannot be found even at $-30 \mathrm{~dB}$ normalised to any significant low frequency peak. Instead, only low frequencies predominate (Zgallai, $2012 \mathrm{~b}$ ).

Fig. 8 shows the bispectra of fetal scalp electrode and maternal chest ECG signals (left panel) and the corresponding contour maps (right panel). The maternal cardiac cycle begins $50 \mathrm{~ms}$ before the R-wave and ends $50 \mathrm{~ms}$ before the next R-wave. The subject is at the first stage of labour, 40 weeks gestation. The bispectrum is calculated using the direct method which involves calculating a two-dimensional Fourier transform. Ten Hanning windows is used in calculating the bispectrum which are averaged for smoothing. The bispectral peaks of the fetal scalp electrode and maternal chest QRS-complexes exist at $(40 \mathrm{~Hz}, 40 \mathrm{~Hz})$ and $(13 \mathrm{~Hz}, 13$ $\mathrm{Hz})$, respectively. However, they are shifted, shallow and inconclusive even though they are centred near the right frequency pairs, $(30 \mathrm{~Hz}, 30 \mathrm{~Hz})$ for the fetal scalp electrode and (17 $\mathrm{Hz}, 17 \mathrm{~Hz}$ ) for the maternal chest ECG.

The temporal resolution could be improved by applying appropriate segmentations to the QRS-complexes. Instead of taking one cardiac cycle for an adult, which is on average 1000 $\mathrm{ms}$, the $250 \mathrm{~ms}$ QRS-complex segment which is centred on the R-wave and runs $125 \mathrm{~ms}$ in opposite directions is considered. This also applies to the fetal scalp electrode ECG signal but with a reduced QRS-complex length of typically $60 \mathrm{~ms}$.

Fig. 9 (top) depicts bispectral slices of the fetal QRS-complex which shows the correct position of a spectral peak at $30 \mathrm{~Hz}$ but only on the diagonal slice. Fig. 9 (middle and bottom) show maternal chest and transabdominal QRS-complex bispectrum slices. The maternal chest and abdomen both exhibit spectral frequencies of $17 \mathrm{~Hz}$ and $15 \mathrm{~Hz}$, respectively, but only on the diagonal slice. Considerable improvement has resulted due to 
improving the temporal resolution prior to the bispectral calculations for both fetal and maternal chest segmented QRS-complexes. However, looking at the maternal chest and transabdominal bispectral diagonal slices, lowering of the QRS peak frequency from $17 \mathrm{~Hz}$ to $15 \mathrm{~Hz}$ is observed.

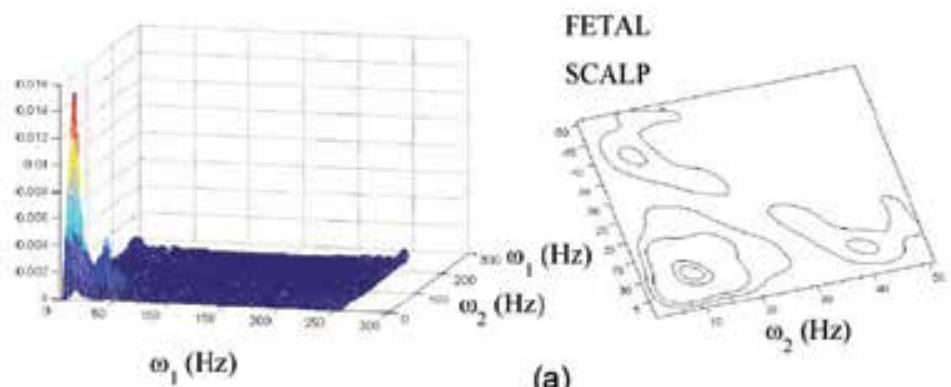

(a)

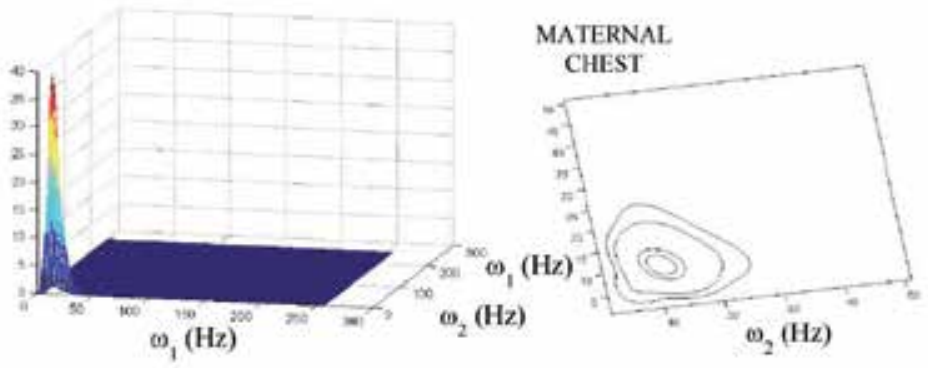

(b)

Figure 8. The bispectra of (a) a fetal scalp and (b) a maternal chest ECG signal (left panel) and the corresponding contour maps (right panel). The maternal cardiac cycle begins $50 \mathrm{~ms}$ before the R-wave and ends $50 \mathrm{~ms}$ before the next R-wave. The subject is at the first stage of labour, 40 weeks gestation. The bispectrum is calculated using the direct method. A Hanning window is used to calculate the bispectrum which is averaged for smoothing.

A possible cause of this shifting in the QRS-complex frequency peak is the susceptibility and lack of predictability of the bispectral representation of highly-complex multi-frequency signals. During labour contractions, the presence of very strong deterministic and chaotic signals emanating from the uterus, and the accompanying motion artefacts result in highly dimensional transabdominal signals (Rizk et al., 2000) is shown. Consequently it is very difficult to isolate with integrity the maternal and fetal QRS- complex spectral peaks without first resorting to super-resolution algorithms using eigenvector-based projections (Zgallai, 2007).

\subsection{Non-linearity of ECG signals}

The non-linearity in the ECG signal can be detected using the bicoherence squared. Fig. 10 depicts the bicoherence squared and their corresponding contour maps using one cardiac cycle for a fetal scalp electrode, maternal chest, and maternal transabdominal ECG. The bicoherence squared has peaks at the frequency pairs of $(6 \mathrm{~Hz}, 15 \mathrm{~Hz})$ and $(14 \mathrm{~Hz}, 14 \mathrm{~Hz})$ for the fetal scalp cardiac cycle, $(15 \mathrm{~Hz}, 15 \mathrm{~Hz})$ for the maternal chest cardiac cycle, and $(7.5$ 
$\mathrm{Hz}, 7.5 \mathrm{~Hz}$ ) for the maternal transabdominal cardiac cycle. These bicoherence peaks support non-linearity.

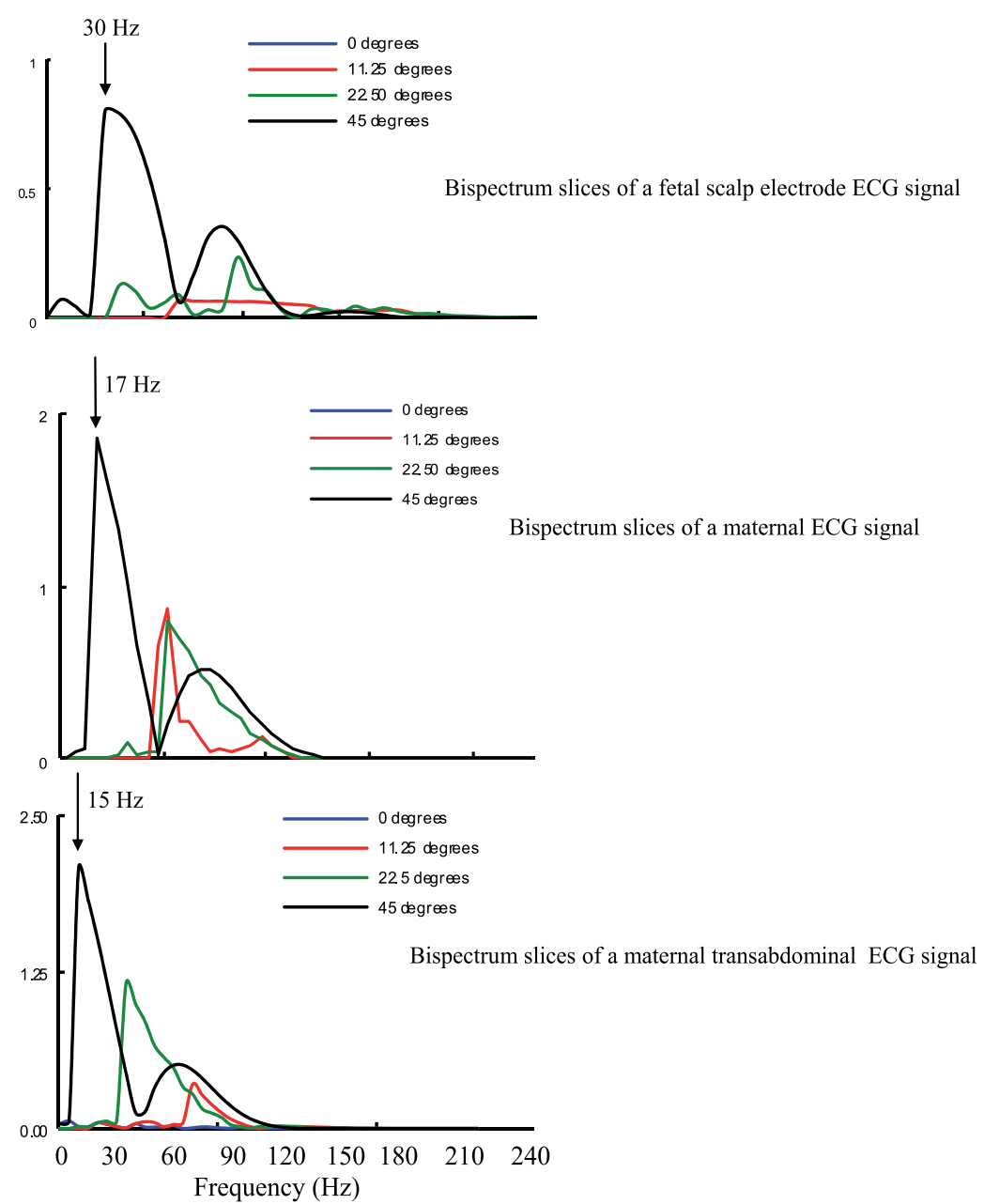

Figure 9. Bispectrum slices at $0^{\circ}$ (wall), $11.25^{\circ}, 22.50^{\circ}$, and $45^{\circ}$ (diagonal) for $250 \mathrm{~ms}$ segments of; fetal cardiac cycle using fetal scalp electrode (upper panel), maternal chest cardiac cycle (middle panel), and maternal transabdominal cardiac cycle (lower panel). The maternal cardiac cycle begins $50 \mathrm{~ms}$ before the R-wave and ends $50 \mathrm{~ms}$ before the next R-wave. The subject is at the first stage of labour, 40 weeks gestation.

\subsection{Proximity of the maternal and fetal QRS- complexes}

There is a general consensus that individual cardiac cycles are locally stationary. This was substantiated (Zgallai, 2007) by Hinich test (Hinich, 1982). However, when applying a highly dimensional signal such as the transabdominal ECG that have several individual non-linear and deterministic signals overlapping both in the time and frequency domains, all coexisting in a cocktail of noise and motion artefact, it is prudent to re-examine the 

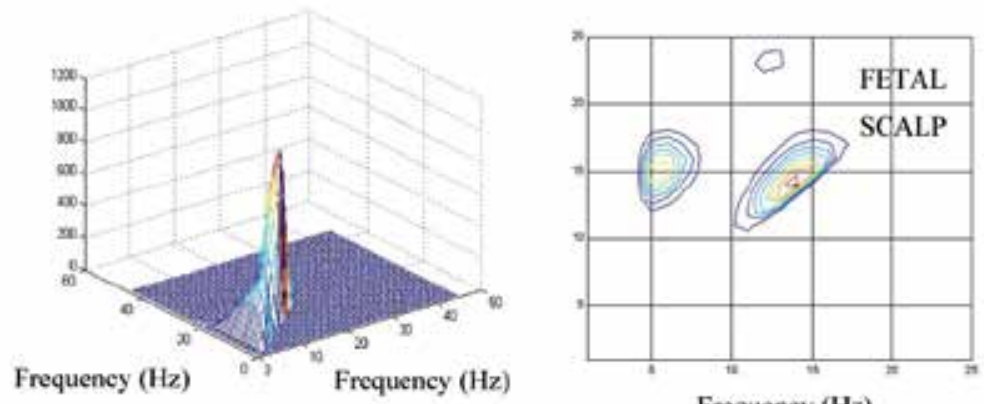

Frequency $(\mathrm{Hz})$

(a)
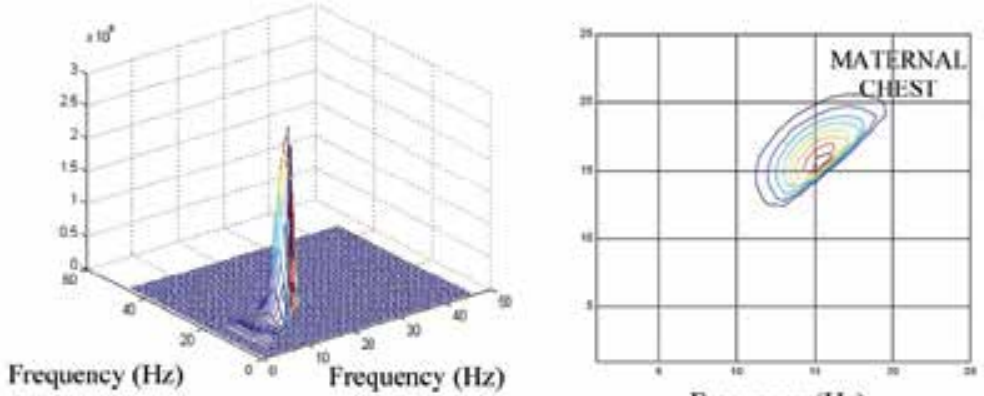

Frequency $(\mathrm{Hz})$

(b)
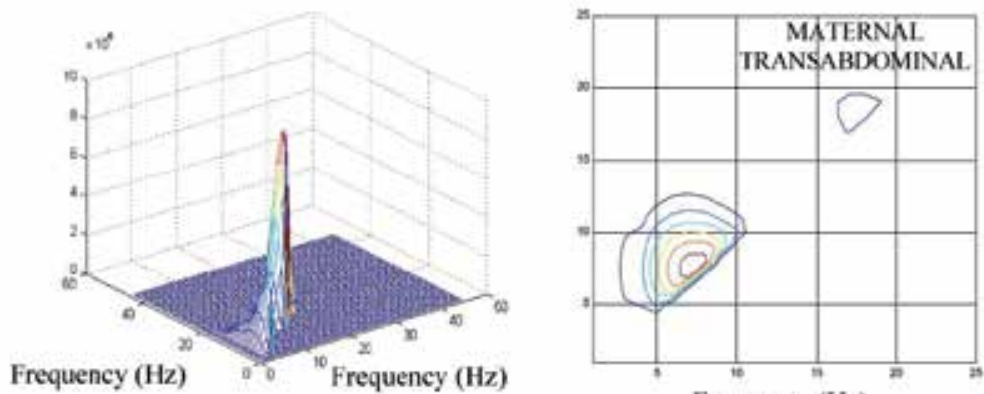

Frequency $(\mathrm{Hz})$

(c)

Figure 10. The bicoherence squared (left panel) and contour map (right panel) for (a) a fetal cardiac cycle using fetal scalp electrode (data length $550 \mathrm{~ms}$ ), (b) a maternal chest cardiac cycle (data length 1000 $\mathrm{ms}$ ), and (c) a maternal transabdominal cardiac cycle (data length $1000 \mathrm{~ms}$ ). The maternal cardiac cycle begins $50 \mathrm{~ms}$ before the R-wave and ends $50 \mathrm{~ms}$ before the next $\mathrm{R}$-wave. The subject is at the first stage of labour, 40 weeks gestation. The bispectrum is calculated using the direct method. A Hanning window is used to calculate the bispectrum which is averaged for smoothing.

validity of the stationarity assumption in relation to such signals. It is only natural to expect that the proximity of two non-linear signals such as the maternal and fetal QRS-complexes would result in non-linear (quadratic and higher-order) coupling and this in turn would invoke non-stationarity. The above is demonstrated to be true by inspecting the bispectral OT region shown in Fig. 11. The maternal cardiac cycle in Fig. 11 begins $50 \mathrm{~ms}$ 

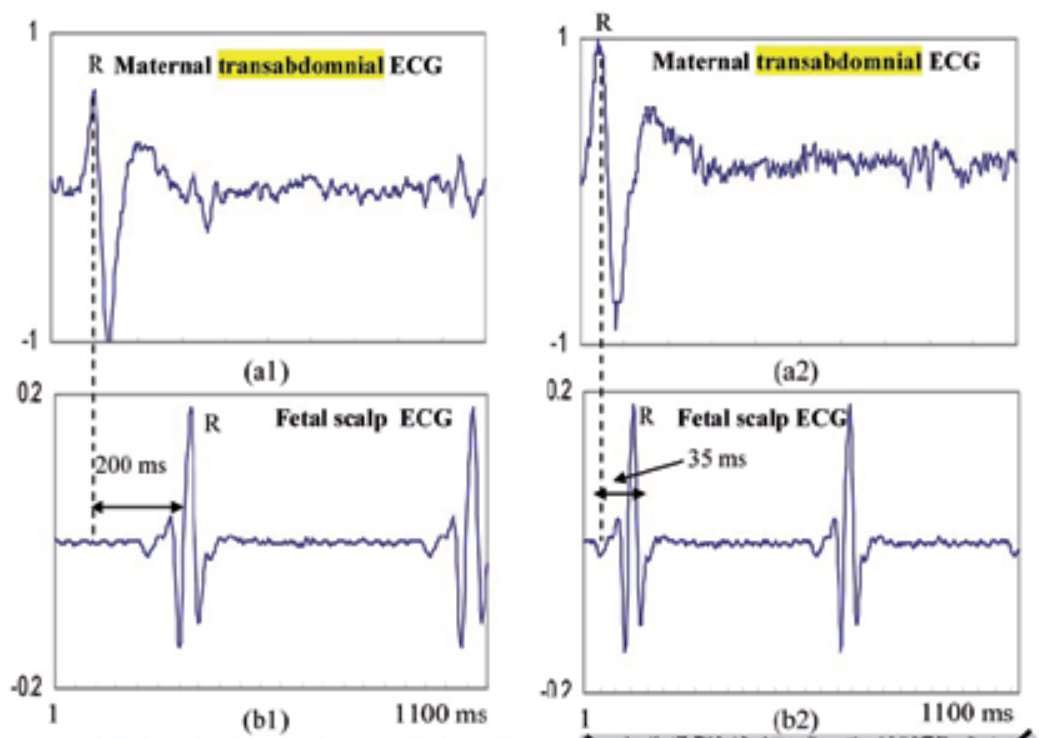

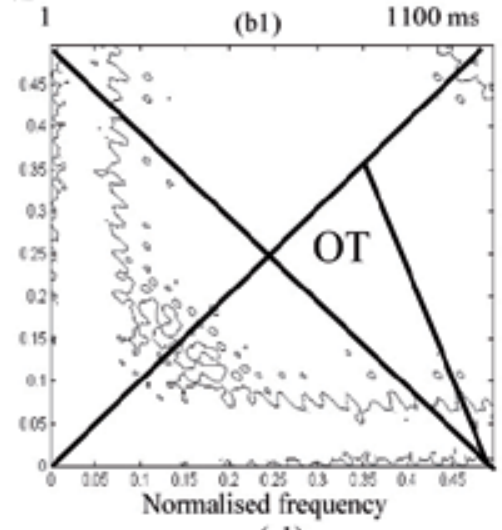

(cl)

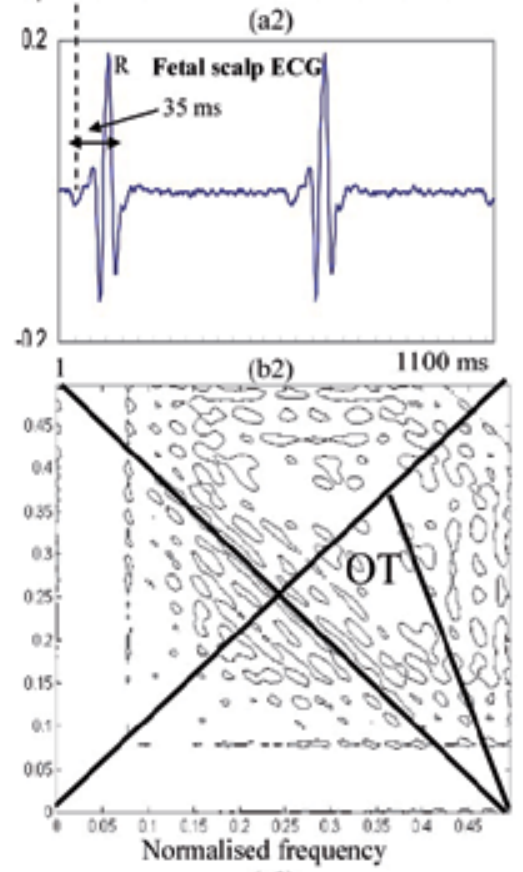

(c2)

Figure 11. (a1), (a2) Two typical examples of maternal transabdominal cardiac cycles, (b1) and (b2) are the corresponding fetal ECG signals using fetal scalp electrode. The first fetal QRS-complex in (b1) is separated from the maternal QRS-complex in (a1) by $200 \mathrm{~ms}$. The first fetal QRS-complex in (b2) is separated from the maternal QRS-complex in (a2) by $35 \mathrm{~ms}$. The corresponding bispectrum contour maps at a level of $-30 \mathrm{~dB}$ for the two cycles in (a1) and (a2) are shown in (c1) and (c2), respectively. The $\mathrm{R}$-wave of the first fetal QRS-complex in (b1) is separated from the R-wave of the maternal QRScomplex in (a1) by $200 \mathrm{~ms}$. The corresponding bispectrum in (c1) does not show extra activity in the OT region. The R-wave of the first fetal QRS-complex in (b2) is separated from the R-wave of the maternal QRS-complex in (a2) by $35 \mathrm{~ms}$. The corresponding bispectrum in (c2) shows extra activities in the OT region due to non-linear coupling between the mother and the baby.

before the R-wave and ends $50 \mathrm{~ms}$ before the next R-wave. The subject is at the first stage of labour, 40 weeks gestation. Fetal cardiac cycle data length is $550 \mathrm{~ms}$, and transabdominal ECG cardiac cycle data length is $1000 \mathrm{~ms}$. Two typical transabdominally measured maternal ECG cycles, ((a1), (a2)), and two synchronised fetal scalp ECG cycles ((b1), (b2)) are shown. The lower parts of the Figure, (c1) and (c2), consist of the corresponding maternal bispectral contour maps at a level of $-30 \mathrm{~dB}$. The two R-waves of the maternal and fetal QRS- 
complexes in (a1) and (b1), respectively, are separated by $200 \mathrm{~ms}$. The resultant bispectrum in (c1) does not support the OT region. However, the situation is totally different when the two R-waves are as close as $35 \mathrm{~ms}$ as shown in Fig. 11 (a2) and (b2). Now the OT region of the bispectrum in (c2) is fully occupied and non-stationary. This means conventional signal processing techniques cannot be used to separate the maternal and fetal QRS-complexes. This problem has been adequately solved by linearising (at least removing quadratic coupling) the transabdominal signal before attempting to separate individual QRScomplexes.

\subsection{Cumulants and bispectra of noise components}

The MIT/BIH databases (MIT/BIH, 1997) have recordings of the three main types of noise in ECG signals, namely, (a) baseline wander, (b) electro-myographic (EMG) noise, and (c) motion artefact. The following statistics help in the processing stages of the fetal heartbeat detection. When using super-resolution techniques requirement for Gaussian and nonGaussian extraction and suppression is eliminated except for the conventional removal of baseline wander which is embedded in all data acquisition systems (baby monitors).

\section{a. Baseline Wander noise}

Fig. 12 depicts second- and third-order statistics of a baseline wander noise segment of 10,000 samples (approximately $30 \mathrm{sec}$ ) extracted from the MIT/BIH databases. Both the bispectrum and the bicoherence squared show high peaks at low frequencies $(<5 \mathrm{~Hz})$. This means that the effect of the baseline wander noise on both maternal and fetal QRScomplexes at $15 \mathrm{~Hz}$ and $30 \mathrm{~Hz}$, respectively, is not significant. It is prudent to eliminate such noise in the pre-processing stage. One conventional method of eliminating baseline wander employs a high-pass filter such as Butterworth high-pass filter of order 5, cut-off frequency of $1 \mathrm{~Hz}$, a transition period of $1 \mathrm{~Hz}$, a minimum ripple of $-50 \mathrm{~dB}$ outside the main frequency lobe.

\section{b. Electromyographic noise}

Fig. 13 shows some statistics of an electromyographic (EMG) noise segment of 10,000 samples extracted from the MIT/BIH databases. The noticeable feature is that the bispectrum is confined to low frequencies less than $(10 \mathrm{~Hz}, 10 \mathrm{~Hz})$. This means that it will not interfere with the isolation of the adult QRS-complex bispectrum peak which occupies frequencies between $(15 \mathrm{~Hz}, 15 \mathrm{~Hz})$ and $(20 \mathrm{~Hz}, 20 \mathrm{~Hz})$, provided that an appropriate super-resolution technique is employed. But the bicoherence squared of the EMG noise is spread over a wide band of frequencies, up to $(120 \mathrm{~Hz}, 120 \mathrm{~Hz})$. The carpet effect of the non-linearity attributed to the EMG noise will be eliminated by linearising the transabdominal signal prior to fetal QRS-complex detection in the third-order statistical domain. Under broad signal and noise conditions, linearisation of the transabdominal ECG signal not only removes to a great extent the signal non-linearity, but also partially eliminates other types of non-linearity due to noise. 


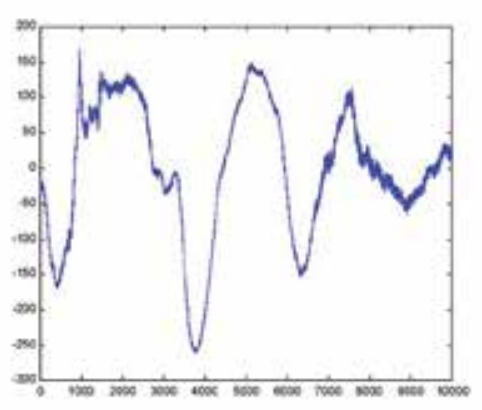

(a)

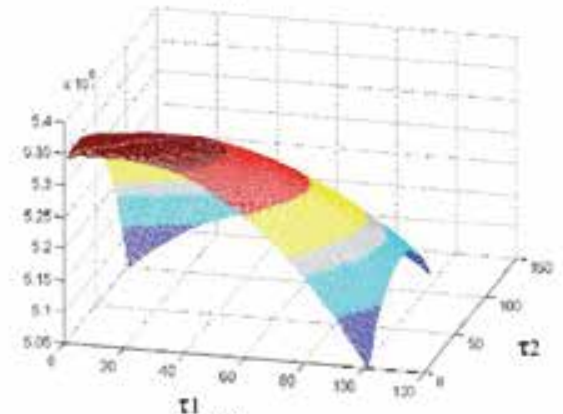

(c)
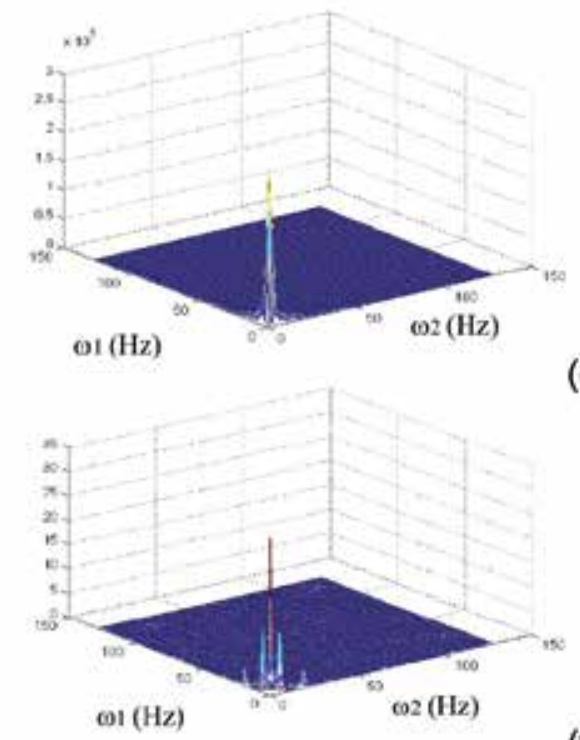

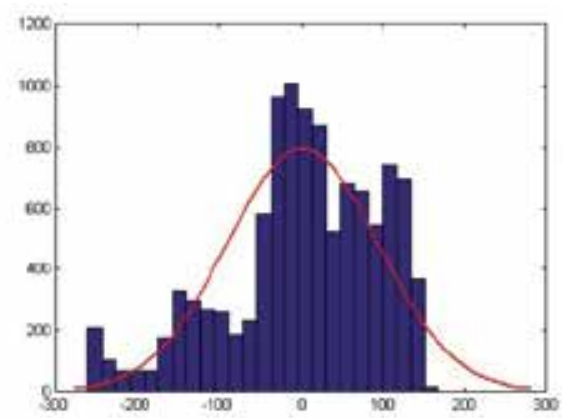

(b)

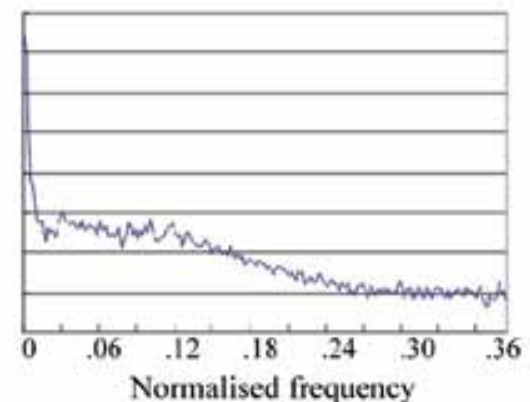

(d)

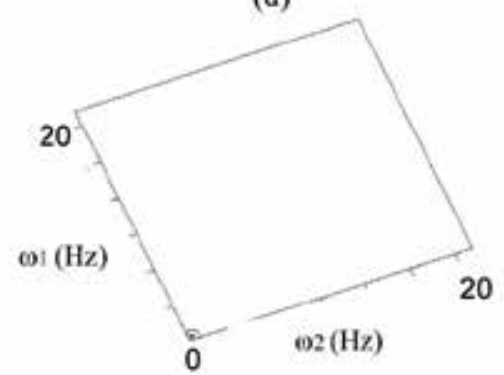

(e)

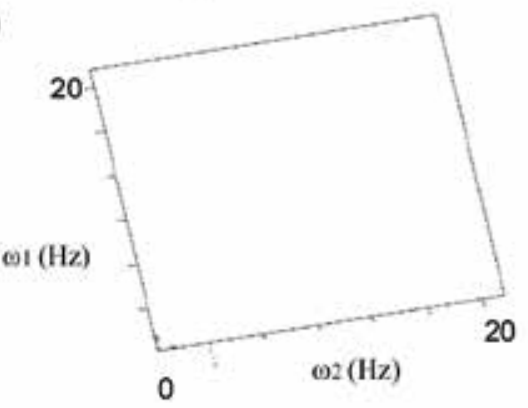

Figure 12. Characterisation of 10,000 samples of baseline wander noise extracted from the MIT/BIH database and sampled at 360 samples per second. (a) time series, (b) its histogram showing nonGaussian pdf, (c) third-order cumulants, (d) power spectrum using the averaged periodogram method, (e) the bispectrum (1.h.s.) calculated using the direct method with contour maps (r.h.s.) and (f) the bicoherence squared (l.h.s.) with contour maps (r.h.s.). 


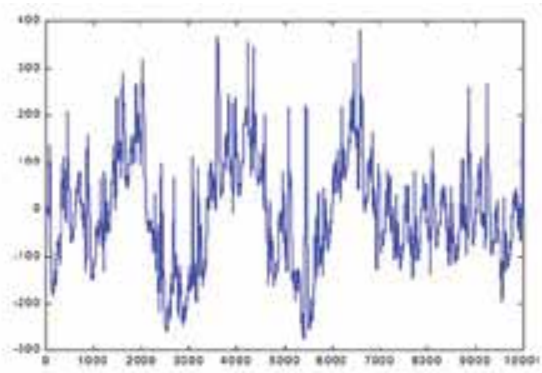

(a)

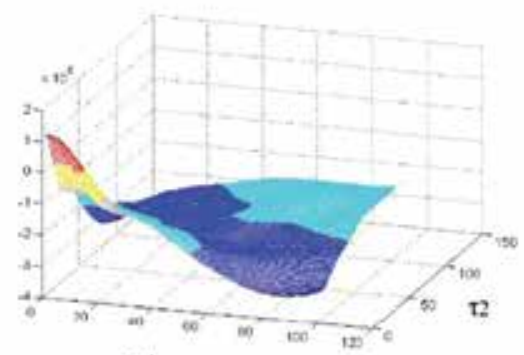

(c)
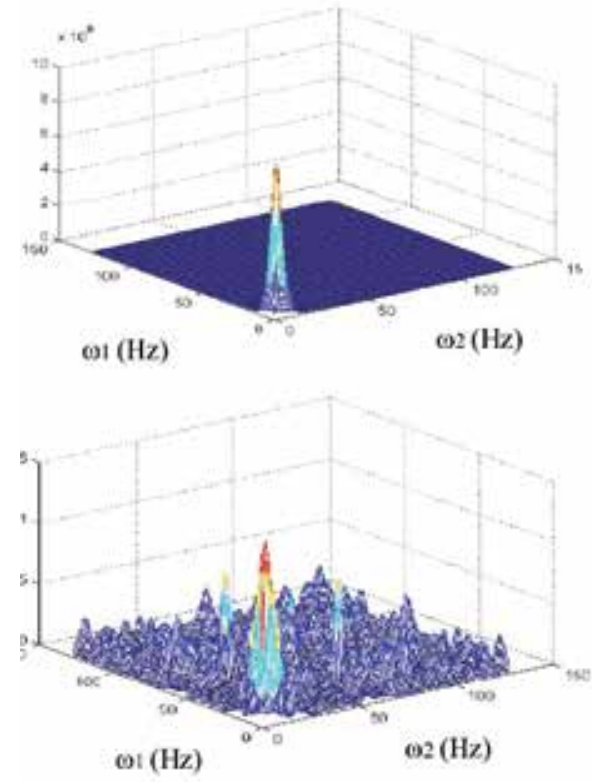

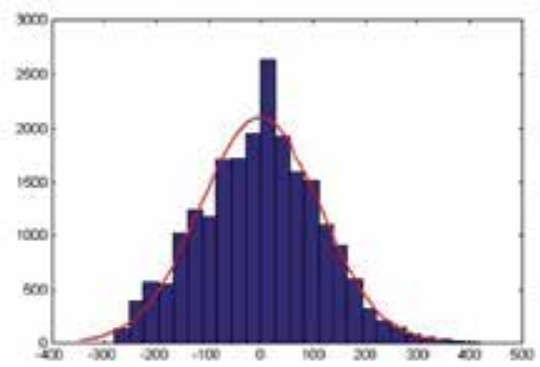

(b)

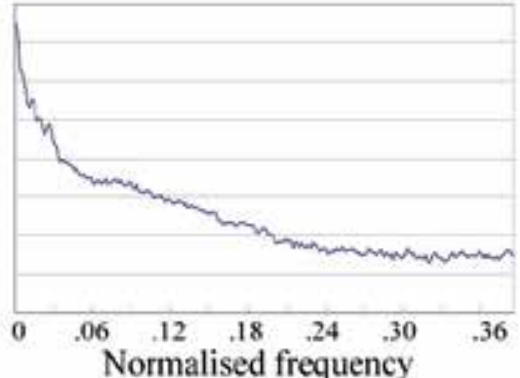

(d)

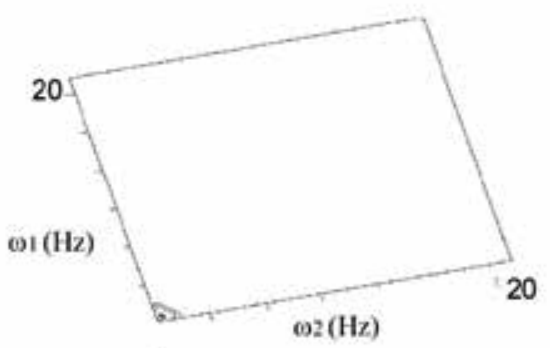

0

(e)

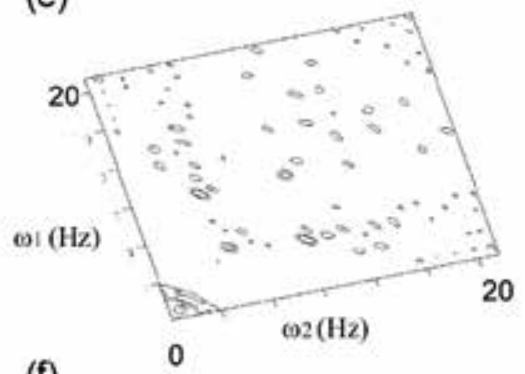

(f)

Figure 13. Characterisation of 10,000 samples of electromyographic noise extracted from the MIT/BIH database and sampled at 360 samples per second. (a) time series, (b) its histogram, (c) third-order cumulants, (d) power spectrum using the averaged periodogram method, (e) the bispectrum (1.h.s.) calculated using the direct method with contour maps (r.h.s.) and (f) the bicoherence squared (l.h.s.) with contour maps (r.h.s.). 


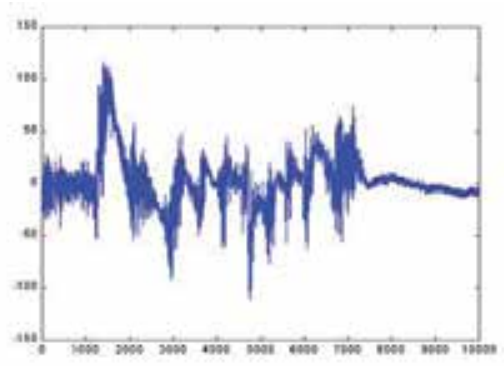

(a)

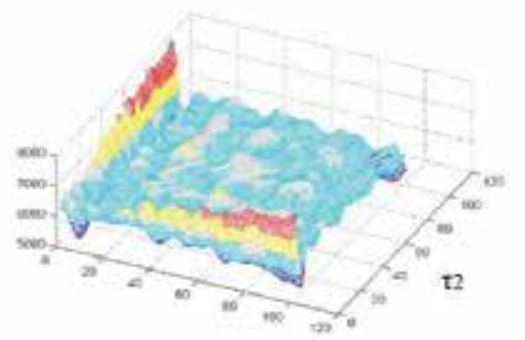

tI

(c)
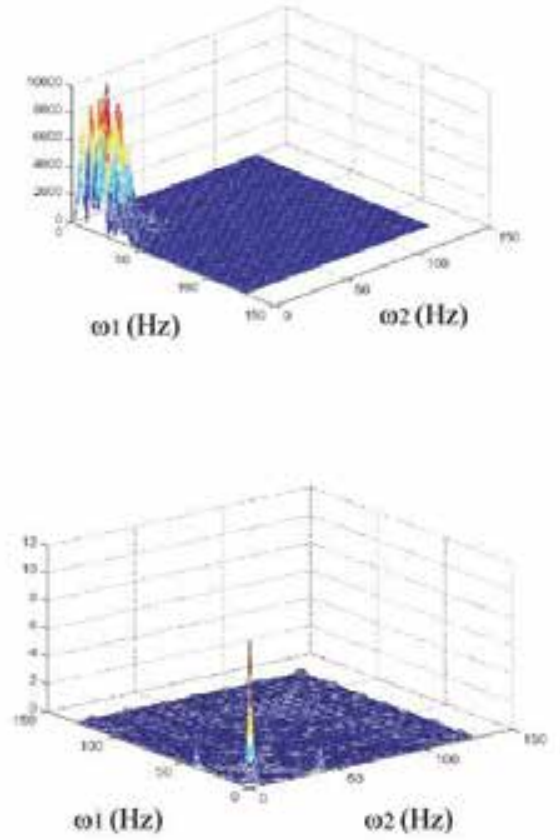

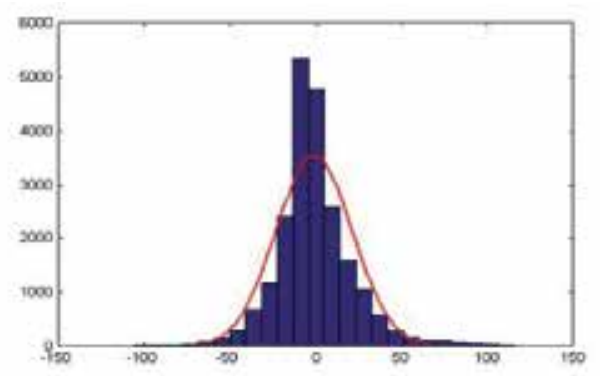

(b)

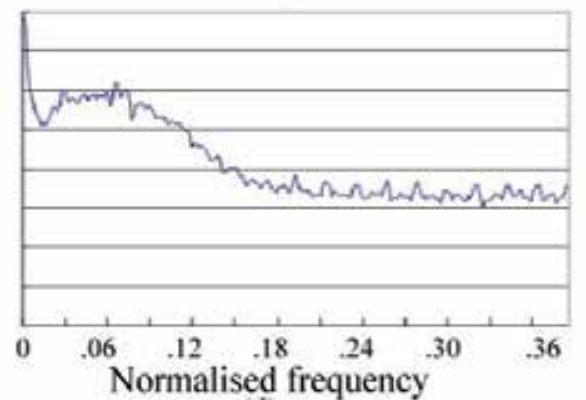

(d)

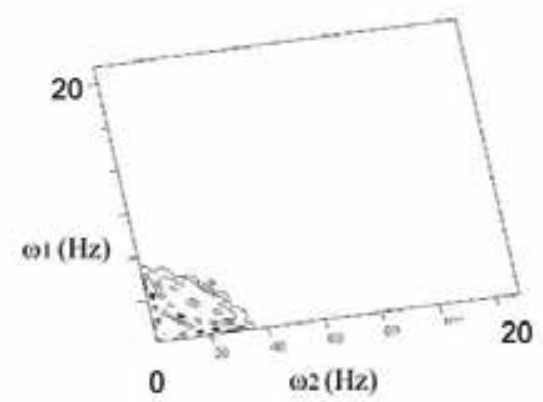

(e)

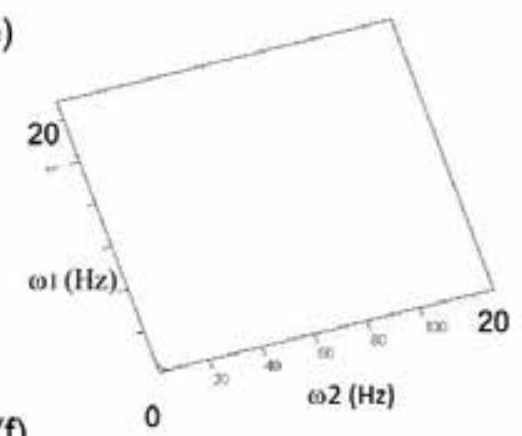

(f)

Figure 14. Characterisation of 10,000 samples of motion artefact noise extracted from the MIT/BIH database and sampled at 360 samples per second. (a) time series, (b) its histogram, (c) third-order cumulants, (d) power spectrum using the averaged periodogram method, (e) the bispectrum (l.h.s.) calculated using the direct method with contour maps (r.h.s.) and (f) the bicoherence squared (l.h.s.) with contour maps (r.h.s.). 


\section{c. Motion artefact noise}

Fig. 14 depicts second- and third-order statistics of a motion artefact noise segment of 10,000 samples extracted from the MIT/BIH databases. The bispectrum has many frequencies in the triangle region of $(0 \mathrm{~Hz}, 0 \mathrm{~Hz}),(0 \mathrm{~Hz}, 35 \mathrm{~Hz})$ and $(35 \mathrm{~Hz}, 0 \mathrm{~Hz})$. These bispectral frequencies of motion artefact would be overlapping with those of the maternal and fetal QRS-complexes, albeit at around $-20 \mathrm{~dB}$ level. However, the level of noise at the QRS-complex spectra is comparatively small and the effect of motion artefact on the detection of QRS-complexes is not noticeable. Fig. 14 (f) reveals that the bicoherence squared is rather confined to very low frequencies. As mentioned above, linearisation plays a definitive role.

\section{Discussion and conclusions}

The objective of this chapter is to introduce the subject of higher-order statistics (HOS) and its applications to the non-linear / non-Gaussian ECG signals to pave the way for employing HOS-based techniques as the solution to the formidable problem of transabdominal fetal heartbeat detection during labour. High detection rates can be accomplished by invoking the HOS-based techniques, namely, the third-order cumulant template matching and the bispectral contours template matching and which utilises a set of different levels of bispectral contours.

The key question that was attempted is why do HOS-based techniques yield the highest possible Fetal Heart Rate FHR? The reasons behind achieving high FHRs when using the HOS-based well-refined techniques are; (1) Under broad signal and noise conditions, higher-order cumulants and their spectra become high signal-to-noise ratio domains where detection, parametric estimation and signal classification can be performed. (2) The Gaussian noise diminishes in the HOS domains if the data length is adequate. For ECG signals, a minimum length of $1 \mathrm{sec}$ is sufficiently long to suppress Gaussian noise and maintain a low level of HOS variances in the HOS domains, whilst not sufficiently long to violate Hinich's criterion for local stationarity. (3) In the third-order domain all sources of noise with symmetric probability density functions (pdfs), e.g., Gaussian and uniform, will vanish. The ECG signals are retained because they have non-symmetric distributions. This implies that it is more than adequate to utilise only the TOCs and their bispectra. There is no need to seek higher-than-third-order statistics as implicated in all the Independent Component Analysis (ICA) applications to FHR detection. (4) The maternal and fetal QRSbispectral contours, which are used as the discriminant patterns in the identification and classification, only overlap with the bispectra of the baseline wander and that of the EMG at very low levels (around $-20 \mathrm{~dB}$ normalised to the peak of the maternal QRS-complex bispectrum). Therefore, it is comparatively easy to detect and classify QRS-complexes in ECG signals utilising either the TOC or the BIC template matching techniques.

\subsection{Direct computations of individual 1-d TOC slices}

It is also shown that, having computed the two-dimensional TOC, either the diagonal or the wall slice or a combination of the diagonal and wall slices is used in the detection / 
classification process. Therefore, computing the full multi-dimensional TOC and then extracting individual slices is an unnecessary waste of the CPU time. So, why not compute any arbitrary 1-d slice directly without firstly having to compute the two-dimensional TOC and secondly extract the 1-d slice? The TOC-diagonal and the TOC-wall slices are straightforward to compute directly, by freezing one of the two cumulant lags and changing the other one. However, to compute any other arbitrary slice requires the development of an auxiliary algorithm. It has been found that performing direct computations of the 1-d TOC slices instead of computing the 2-d TOC firstly, and secondly extracting individual 1-d slices results in saving of more than $99 \%$ of the CPU time. The same applies to the $2-\mathrm{d}$ bispectrum. However, it has to be borne in mind that it is the matching of the horizontal bispectral contours that is used in the Bispectral Contour (BIC) template matching technique instead of the 1-d polar bispectral slices. Because in order to use the 1-d polar bispectrum slices effectively one needs to use a minimum of 24 polar slices so as not to miss the capturing of rapid changes or null features in the bispectrum that could be used as discriminant patterns. Whereas for BIC contours the number of discriminant horizontal slices required for detection / classification does not exceed 10.

\subsection{Bispectral features of QRS-complexes}

The power spectrum of appropriately sampled ECG showed the QRS-complex principal peak in the frequency range from $15 \mathrm{~Hz}$ to $20 \mathrm{~Hz}$, and $25 \mathrm{~Hz}$ to $40 \mathrm{~Hz}$, for the maternal chest ECG and fetal scalp electrode ECG, respectively. Unfortunately, the power spectrum has limitations as an estimator in terms of resolution, variance, and clarity of the spectrum to be able to produce clear and distinguishable peaks for the P-waves. Therefore, an alternative spectrum estimator was used instead, namely, the multiple signal classification (MUSIC) pseudo-spectrum. The MUSIC-based pseudo-spectrum showed that the principal peaks for the p-waves occupy a range from $5 \mathrm{~Hz}$ to $8 \mathrm{~Hz}$ for adults. The principal peaks for the Pwaves of the fetal scalp electrode ECG occupy a range from $8 \mathrm{~Hz}$ to $10 \mathrm{~Hz}$. The same MUSIC-based spectral estimators have revealed high local energy peaks around $5 \mathrm{~Hz}$ due to motion artefact (Zgallai et al., 1997).

As with cumulants, their bispectra were computed for the above mentioned ECG data samples and segmentations using the direct method which involves calculating a twodimensional Fourier transform. The following bispectral peaks have been observed only on the bispectral diagonal slice at the following frequency pairs;

a. $(17 \mathrm{~Hz}, 17 \mathrm{~Hz})$ and $(15 \mathrm{~Hz}, 15 \mathrm{~Hz})$ for the maternal chest and the transabdominal ECGs, respectively. So, there is a shift in the bispectral peak from $17 \mathrm{~Hz}$ to $15 \mathrm{~Hz}$ in the transabdominal ECG.

b. $(30 \mathrm{~Hz}, 30 \mathrm{~Hz})$ and less prominently at $(20 \mathrm{~Hz}, 20 \mathrm{~Hz})$ for the fetal scalp electrode ECG.

\subsection{Quadratic coupling in transabdominally measured ECG signals}

It has been found in maternal transabdominal ECG signals that close proximity of the maternal and fetal QRS-complexes initiates additional quadratic and higher-order non- 
linearities that could be due to higher-order coupling of the maternal and fetal own harmonics and the concomitant mixing of the ECG signals and the non-linear uterine contraction interference signal. This so called coupling between maternal and fetal ECGs (Rizk et al., 2001) is manifested in a newly formed bicoherence squared peak(s) which did not exist in either of the isolated maternal bicoherence squared or the isolated fetal bicoherence squared computed from their respective ECG signals. The non-linear second- or third-order Volterra structure has been used (Rizk et al., 2001) to quantify the effect of this coupling, in part. The rest of the quantification process is carried out using the bicoherence squared.

It is worth mentioning that, depending on the bispectrum estimation method employed, the techniques for the detection and quantification of quadratic phase coupling are divided into two categories: the conventional and the parametric. The conventional techniques are based on the bicoherence spectrum and they are better qualifiers of the phase coupling (Kim and Powers, 1978; Kim and Powers, 1995). However, their resolution is limited by the uncertainty principle of the Fourier transform. On the other hand, the parametric techniques are based on the auto-regressive (AR) modelling of the third-order cumulants. Although the parametric AR methods are not good quantifiers, they possess a high resolution capability, much higher than the frequency resolution of the conventional methods (Nikias and Raghuveer, 1987; Raghuveer and Nikias, 1986). The so called coupling results in nonstationarity in the transabdominal ECG signal. This is evidenced by the filling of the bispectrum OT region which is used as a measure of non-stationarity in non-Gaussian signals.

\subsection{Noise identification in male and non-pregnant female adults}

For noise identification and characterisation in the third-order domain, the MIT/BIH databases were utilised (MIT/BIH 1997). Apart from Gaussian noise, there exist three types of non-Gaussian noise in ECG signals, namely, baseline wander, electromyographic (EMG), and motion artefact noise. 10,000 samples of each of these three types of noise are analysed. A brief summary of their third-order statistics is shown in Table 1.

\begin{tabular}{|l|l|l|l|}
\hline & Baseline wander & Electromyographic & Motion artifact \\
\hline $\begin{array}{l}\text { Third-order } \\
\text { cumulants }\end{array}$ & Support & Support & Support \\
\hline Bispectrum & Frequency $<5 \mathrm{~Hz}$ & Frequency $<10 \mathrm{~Hz}$ & Frequency $<35 \mathrm{~Hz}$ \\
\hline $\begin{array}{l}\text { Bicoherence } \\
\text { Squared }\end{array}$ & Frequency $<5 \mathrm{~Hz}$ & Frequency $<120 \mathrm{~Hz}$ & Frequency $<5 \mathrm{~Hz}$ \\
\hline
\end{tabular}

Table 1. Third-order statistics of three types of noise in ECG signals; baseline wander, electromyographic noise, and motion artefact.

The effect of the baseline wander noise on both the maternal and the fetal QRS-complexes at $15 \mathrm{~Hz}$ and $30 \mathrm{~Hz}$, respectively, is not significant. Table 1 shows that only the bispectrum of 
the motion artefact and the bicoherence squared of the EMG noise have frequencies that would potentially overlap with those of the QRS-complexes of the mother and the fetal, albeit at $-20 \mathrm{~dB}$ level. The bicoherence squared of the EMG noise is spread over a wide band of frequencies, up to $(120 \mathrm{~Hz}, 120 \mathrm{~Hz})$. The carpet effect of the non-linearity attributed to the EMG noise will be significantly reduced by linearising the transabdominal signal prior to fetal QRS detection in the third-order statistical domain. Under broad signal and noise conditions, linearisation of the transabdominal ECG signals not only removes to a great extent the signal non-linearity, but also partially eliminates other types of non-linearity due to noise or non-linearity due to strong uterine contractions.

It could be deduced from Table 1 that there would be overlapping between the bispectral frequencies of motion artefact and those of the maternal and the fetal QRS-complexes, albeit at around $-20 \mathrm{~dB}$ level. However, the level of noise at the QRS-complex spectra is comparatively small and by using QRS-complex tailor-made spectral windows, the effect of motion artefact on the detection of the QRS-complexes is not noticeable.

\section{Author details}

Walid A. Zgallai

Berkshire, UK

Dubai, UAE

\section{References}

Brillinger,D. R. (1965). “An Introduction to Polyspectra," Annals of Mathematical Statistics, vol. 36, pp. 1351-1374.

Brockett, P. L.; Hinich, M. J.; and Patterson, D. (1988). “Bispectral-Based Tests for the Detection of Gaussianity and Linearity in Time Series," Journal of the American Statistical Association, vol. 83, No. 403, pp. 657-664.

Friedlander, B. and Porat, P. (1988). "Performance analysis of MA parameter estimation algorithms based on higher-order moments," Proceedings of IEEE Int Conf Acoustics, Speech and Signal Processing (ICASSP), pp. 2412-2415, NY, April.

Hinich, M. J. (1982). “Test for Gaussianity and linearity of a stationary time series," Journal of time series analysis, vol. 3, No. 3, pp. 169-176.

Kim, Y. and Powers, E. J. (1978). “Digital Bispectral Analysis of Self-Excited Fluctuation Spectra," The Physics of Fluids, vol. 21, pp. 1452-1453.

Kim, S. B. and Powers, E. J. (1995). "Estimation of Volterra kernels via higher-order statistical signal processing," in Higher order statistical signal processing, (Boashash, Powers, and Zoubier, eds.), Ch. 7, pp. 213-267, 1995.

Kravtchenko-Berejnoi, V. et al. (1995). "On the use of tricoherent analysis to detect nonlinear wave-wave interactions," Signal Processing, vol. 42, pp. 291-309.

Lii, K. S. (1982). “Non-Gaussian ARMA model identification and estimation,” Proceedings of Business and Economics Statistics, ASA, pp. 135-141. 
Mendel, J. M. (1988). “Use of Higher Order Statistics in Signal Processing and System Theory: An Update," Proceedings of SPIE, Advanced Algorithms and Architectures for Signal Processing III, vol. 975, pp. 126-144.

MIT/BIH (1997) MIT/BIH Database: ECG Database Applications Guide, 10th Edition. HarvardMIT Division of Health Sciences and Technology, MIT Room 20A-113, Cambridge, MA 02139, USA.

Nagata, Y. (1970). "Lag joint probability, higher-order covariance function and higherOrder spectrum," Bulletin de la socie'te' Franco-Japonaise d'oceanographic, vol. 8, Part 2, pp. 78-94, May.

Nam, S. W.; Powers, E. J. (1994). "Application of Higher Order Spectral Analysis to Cubically Non-Linear System Identification," IEEE Transaction on Signal Processing, vol. 42, No. 7, pp. 1746-1765, July.

Nikias, C. L. (1988). "ARMA bispectrum approach to nonminimum phase system identification," IEEE Transaction on Acoustics, Speech, and Signal Processing, vol. ASSP-36, pp. 513-524, April.

Nikias, C. L.; Petropulu, A. P. (1993). Higher Order Spectra Analysis: A Nonlinear Signal Processing Framework, Prentice Hall.

Nikias, C. L. and Raghuveer, M. R. (1987). “Bispectrum estimation: A digital signal processing framework," IEEE Proceedings, vol. 75, No. 7, pp. 869-891, July.

Raghuveer, M. R. and Nikias, C. L. (1986). "Bispectrum estimation via parametric modeling," Signal Processing, Special issue on modern trends of spectral analysis, vol. 10, pp. 35-48, Jan.

Rizk, M., Zgallai, W. A., Carson, E., MacLean, A., \& Grattan, K. (2002). “Multi-fractility in Fetal Heart Beat Dynamics," The 2nd European Medical and Biological Engineering Conference, Austria, December.

Rizk M., Zgallai W. A., McLean, A., Carson, E., and Grattan, K. (2001) Virtues and Vices of Source Separation Using Linear Independent Component Analysis for Blind Source Separation of Non-linearly Coupled and Synchronised Fetal and Mother ECGs. IEEE Engineering in Medicine and Biology Conference, USA.

Rizk, M. et al. (2000) Novel decision strategy for P-wave detection utilising nonlinearly synthesised ECG components and their enhanced pseudospectral resonances. IEE Proceedings Science, Measurement \& Technology, Special section on Medical Signal Processing, vol. 147, No. 6, pp. 389-397, November

Rizk M. and Zgallai, W. (1999) "Higher Order Statistics Are Indispensable Tools in The Analysis of Electrocardiogram Signals," IEE Colloquium on Statistical Signal Processing, January.

Rizk, M. et al. (1998) "Higher-Order Ambulatory Electrocardiogram Identification and Motion Artefact Suppression With Adaptive Second- and Third-Order Volterra Filters," SPIE '98 Adv Sig Proc Algorithms, Arch, \& Implementations VIII Vol. 3461 pp. 417-431, USA, 19-24 July.

Rizk, M., Romare, D., Zgallai, W. A., Grattan, K., Hardiman, P., and O’Riordan, J. (1995) "Higher order statistics (HOS) in signal processing: Are they of any use?," IEE Colloquium, digest \#111, pp. 1/1-1/6, London, May. 
Rosenblatt, M. (1985). Statistical Sequences and Random Fields, Birkhauser, USA.

Rosenblatt, M. (1983). "Cumulants and Cumulant Spectra," in Handbook of Statistics, vol. 3, (D. Brillinger, and P. Krishnaiah, eds.), Amesterdam, Holland, pp. 369-387.

Subba Rao, T. (1983). "The Bispectral Analysis of Nonlinear Stationary Time Series with Reference to Bilinear Time Series Models," in Handbook of Statistics, vol. 3, (D. Brillinger, and P. Krishnaiah, eds.), Amesterdam, Holland, pp. 293-319.

Zgallai, W. A. (2012 a) Detection and Classification of Adult and Fetal ECG Using Recurrent Neural Networks, Embedded Volterra and Higher-Order Statistics, in Recurrent Neural Networks, El Hefnawi, M. and Mysara, M., Ed., InTech Open, ISBN 979-953-307-546-3.

Zgallai, W. A. (2012 b) Non-Invasive Fetal Heartbeat Detection Using Bispectral Contour Matching. The International Conference on Electronics and Biomedical Engineering Applications, Dubai, UAE, 7-8 January.

Zgallai, W. A. (2010). "Non-invasive fetal heartbeat detection using third-order cumulant slices matching and ANN classifiers," The $7^{\text {th }}$ International Association of Science and Technology for Development (IASTED), International Conference on Biomedical Engineering, IASTED, Austria, 17/02.

Zgallai, W. A. (2009) Embedded Volterra for Prediction of Electromyographic Signals During Labour. The $16^{\text {th }}$ IEEE International Conference on Digital Signal Processing (DSP), Greece, 05/07.

Zgallai, W. A. (2007) Advanced Robust Non-Invasive Fetal Heart Detection Techniques During Active Labour Using One Pair of Transabdominal Electrodes, PhD Thesis, City University London, UK.

Zgallai, W. A. Et al., (1997) MUSIC-Based Bispectrum Detector: A Novel Non-Invasive Detection Method For Overlapping Fetal and Maternal ECG Signals. Proceedings 19th IEEE International Conference Engineering in Medicine and Biology, EMBS, pp. 72-75, USA, October.

Zgallai W. A. et al., (1997) Third-order cumulant signature matching technique for noninvasive fetal heart beat identification. IEEE International Conference on Acoustics, Speech, and Signal Processing (ICASSP), vol. 5, pp 3781-3784, Germany.

Zurbenko, I. G. (1982). The spectral analysis of time series, Ch. 6, North-Holland Series in Statistics and Probability, vol. 3, pp. 169-176. 


\title{
Fractal Physiology, Breath-to-Breath Variability and Respiratory Diseases: An Introduction to Complex Systems Theory Application in Pulmonary and Critical Care Medicine
}

\author{
Vasilios Papaioannou and loannis Pneumatikos
}

Additional information is available at the end of the chapter

http://dx.doi.org/10.5772/3331

\section{Introduction}

Physiologic data measured at the bedside often display fluctuations at scales spanning several orders of magnitude. These fluctuations are extremely inhomogeneous and appear irregular and complex whereas in the medical literature, they are often regarded as noise and are neglected. However, they may carry information about the underlying structure or function of the heart and lungs. Examples include fluctuations in heart rate, respiratory rate, lung volume and blood flow [1]. The central task of statistical physics is to study macroscopic phenomena that result from continuous microscopic interactions among many different components. Particularly, physiologic systems such as the cardiovascular and respiratory systems, are good candidates for such an approach, since they include multiple components and are affected by varying neuro-autonomic inputs, continuously over time [1].

Healthy state exhibits some degree of stochastic variability in physiologic variables, such as heart and respiratory rate. This variability is a measure of complexity that accompanies healthy systems and is responsible, according to Buchman, for their greater adaptability and functionality related to pathologic systems [2]. Loss of this variability has been shown to precede the onset of sepsis and multiple organ dysfunction syndrome (MODS) [3-6]. Studying physiological signals of critically ill patients, such as heart and respiratory rate can easily identify 'hidden' information concerning inherent dynamics and overall variability within time series [4]. Recognition that physiologic time series contain such information, related to an extraordinary complexity that characterizes physiologic systems, defies traditional mechanistic approaches based on conventional biostatistical methodologies and 
has fueled growing interest in applying techniques from statistical physics, for the study of living organisms [6]. Through those techniques different 'physiomarkers' reflecting variability of various biosignals (e.g., heart rate variability that is the variability of R-R interval in the electrocardiogram) can be estimated. These indices of healthy complexity seem to fulfill the requirements of contemporary critical care medicine for better and more accurate early warning signs, since they are based on high-frequency measurements (sampling rate at least $250 \mathrm{~Hz}$ ). Different monitors sample original physiological signals at discrete sample intervals and the rate of sampling determines how well the signal is reconstructed. In this respect, and based on the Shannon-Nyquist theorem, accurate reproduction needs a sampling frequency at least two times the highest frequency component of a signal's frequency spectrum, otherwise the signal is undersampled. On the contrary, conventional biomarkers, such as different cytokines, are typically measured once per day, exhibit marked pleiotropy and poorly reflect inherent dynamics of the system under study, leading finally to loss of information regarding real time changes in patient's physiology. The combination of structural indices such as the left ventricular ejection fraction (LVEF) with autonomic function indices derived from heart rate variability analysis (HRV) has been recently proposed as the state-of-the-art method for risk assessment among patients with acute myocardial infarction or severe congestive heart failure [7].

In this respect, a few studies have explored indices derived from breathing pattern variability analysis in patients with pulmonary diseases or in critically ill patients during their stay in the Intensive Care Unit (ICU), for assessing readiness for liberation from mechanical ventilation, respectively. Reliable assessment of breathing variability involves a set of signal processing techniques that can be applied to various respiratory signals and can also extract different sets of information concerning intrinsic breathing dynamics.

\section{Signal processing techniques}

\subsection{Linear methods}

A considerable body of data suggests that healthy individuals exhibit breath-to-breath variability of breath components in a breath series [8,9]. Breath-to-breath variations have been traditionally treated as random uncorrelated white noise superimposed on the output of the respiratory controller $[9,10]$. According to Tobin, the random fraction aids respiratory system to perform tasks other than gas exchange, such as speaking $[8,11]$. Only simple statistics such as mean, variance and coefficient of variation ( $\mathrm{CV}=$ standard deviation/mean) can estimate random variational fraction after averaging over many breathing cycles. Since variability in complex living systems is not only an artefact of biological noise but also an intrinsic property of various control mechanisms, different types of deterministic (nonrandom) variability have been described in the pattern of breathing. These types include correlated and oscillatory fractions [12-14].

Autocorrelation analysis calculates coefficients that quantify the fraction of variational activity that is correlated on a breath-to-breath basis. Observations through time may be correlated with a lagged version of themselves. A set of time series values is taken as the first set and the 
same set is taken as the second, except lagged. If the autocorrelation coefficient retreats from 1.00 as the lag increases and then returns to nearly 1.00, the studied time series behaves in a periodic way. A plot of autocorrelation coefficients on the vertical axis with different lag on the horizontal axis is termed a correlogram; whereas time values at which autocorrelation coefficients reproaches 1.00 indicate periodicities within the time series [13].

Periodic signals can be decomposed into a frequency spectrum of oscillating signals. The different frequency components can be estimated through the Fast Fourier Transformation (FFT) of a time series. The method is called power spectrum density (PSD) and displays in a plot the relative contribution (amplitude) of each frequency $[15,16]$, whereas the area under the power spectral curve in a particular frequency band is considered to be a measure of variability (power) at that frequency. PSD of breathing signals can determine which fraction of variational activity is oscillatory at a particular frequency on a breath-to-breath basis. It has been proposed that the standard deviation (SD) for each breath component can be considered as a measure of gross breath-to-breath variability $[13,17]$.

\subsection{Non-linear methods}

The above methods have been described as linear, easy to interpret and accessible. However, their application supposes stationary time series behaviour, meaning stability of statistical properties of signals along time. In these cases, any variation in measurements is considered to be random sampling error around a 'true' mean [18]. Furthermore, they present insensitivity to the orderliness of data and lack the ability of describing systems' inherent dynamics. For instance, a time series can be variable but not complex. Conversely, a time series can be less variable but highly complex, therefore variability and complexity that better describes inherent dynamics of non-stationary signals, are two different and independent aspects of a time series [18]. For the above reasons, different nonlinear complexity assessment techniques have been studied as weaning descriptors in few human studies and for the estimation of breathing complexity in different experimental models. The methods mostly used include approximate entropy (ApEn), sample entropy (SampEn) and visual assessment through Poincaré plots [19-23].

ApEn was introduced by Pincus $[19,20]$ as a quantification of regularity in data and compares each group of consecutive measurements over a predefined time window to every other group of measurements of the same time length. ApEn is a measure of the likelihood that patterns recur over specified time intervals. Regular signals are expected to have low ApEn, while complex ones take on higher ApEn values. Due to ApEn's dependence on the record length an alternative statistic named sample entropy (SampEn) was introduced by Richmann and Moorman [21] with the benefit of reduced computational load.

Visual assessment methods include the application of Poincaré plots. For this plot analysis, each value of the original time series [e.g., respiratory rate (RR)] $R-R_{n}$ is plotted against the value of the immediately following $R-R_{n+1}$ for a predetermined segment. The plot can be quantified by the two values of standard deviations, SD1 and SD2 as indicators of the dispersion of RR points [22,23]. SD2 is defined as the dispersion of points along the line-of- 
identity whereas SD1 is defined as the dispersion of points perpendicular to the line-ofidentity through the centroid of the plot. Table 1 describes the mostly used methods for quantification of breathing variability and complexity.

Fractal analysis constitutes a subset of non-linear methods and will be discussed separately.

\begin{tabular}{|c|c|c|c|}
\hline Method & Type & Characteristics & Limitations \\
\hline $\begin{array}{l}\text { Fast Fourier } \\
\text { Transformation (FFT) } \\
{[15,16]}\end{array}$ & Linear & $\begin{array}{l}\text { Suitable for analysis } \\
\text { of stationary signals } \\
\text { of long duration }\end{array}$ & $\begin{array}{l}\text { Reduced capability of } \\
\text { detecting adjacent } \\
\text { peaks in signals with } \\
\text { short duration }\end{array}$ \\
\hline $\begin{array}{l}\text { Approximate entropy } \\
(\text { ApEn) }[19,20]\end{array}$ & Nonlinear & $\begin{array}{l}\text { Estimates regularity } \\
\text { within non stationary } \\
\text { signals. The higher its } \\
\text { values the more easily } \\
\text { predicted behavior of } \\
\text { the signal and vice } \\
\text { versa }\end{array}$ & $\begin{array}{l}\text { There are no } \\
\text { guidelines for } \\
\text { parameter selection } \\
\text { and dependence on } \mathrm{N} \\
\text { (length of data) when } \\
\text { short signals are } \\
\text { processed }\end{array}$ \\
\hline $\begin{array}{l}\text { Sample entropy } \\
\text { (SampEn) } \\
{[21]}\end{array}$ & Nonlinear & $\begin{array}{l}\text { Guidelines for } \\
\text { parameter selection } \\
\text { are provided. Low } \\
\text { computational load. } \\
\text { Better consistency } \\
\text { than ApEn }\end{array}$ & $\begin{array}{l}\text { Reduced accuracy for } \\
\text { short time series and } \\
\text { wide confidence } \\
\text { intervals for highly } \\
\text { correlated signals }\end{array}$ \\
\hline $\begin{array}{l}\text { Poincaré plots for } \\
\text { visual assessment } \\
{[22,23]}\end{array}$ & Nonlinear & $\begin{array}{l}\text { Easy and fast } \\
\text { estimation of signal } \\
\text { dynamics (periodic, } \\
\text { quasiperiodic, chaotic } \\
\text { pattern) }\end{array}$ & $\begin{array}{l}\text { No automation in } \\
\text { feature extraction }\end{array}$ \\
\hline
\end{tabular}

Table 1. Summary of the most frequently used linear and nonlinear methods for estimation of breathto-breath variability /complexity.

\subsection{Fractals and power law: Basic concepts}

Fluctuations of a variable can be characterised by its probability density distribution. A way of estimating its characteristics is the construction of a histogram after normalisation, so that the area under it will be equal to one. Often, this distribution $N(x)$ of a variable $x$ follows the so called power law form: $\mathrm{N}(\mathrm{x})=\mathrm{x}^{-\mathrm{d}}$ meaning that the relative frequency of a value $\mathrm{x}$ is proportional to $\mathrm{x}$ raised to the power of $-\mathrm{d}$. If we plot the logarithms of this relationship we have a linear equation: 


$$
\left[N(x)=x^{-d} \text { then, } \log (N)=-d^{*} \log (x)\right],
$$

whereas $d$ is the negative slope of a straight line fit to $N$. This slope is frequently called $\beta$ slope or exponent [24].

Power law distribution behaves differently than Gaussian distributions. Its tails are very long (long-tail distribution), representing the relative frequency of occurrence of large events. This means that the probability of large or rare events is much higher compared with a Gaussian. Power laws describe dynamics that have a similar pattern of change at different scales and they are called 'scale invariant'. On the contrary, Gaussians are characterised by typical values, such as those corresponding to their peaks [25]. Moreover, the power law describes a time series with many small variations and fewer and fewer large ones, whereas the pattern of variation is statistically similar regardless of its size. Magnifying or shrinking the scale of the signal reveals the same relationship, a property that has been called 'selfsimilarity' and is a fundamental characteristic of fractals [24,26]. Fractals are self similar objects because small parts of the structure at increasing magnification appear similar to the entire object. Akin to a coastline, fractals represent structures that have no fixed length, since it increases with increased magnification of measurement. This is why all fractals have noninteger dimensions, the so called fractal dimensions (FDs) [24,27].

The concept of fractals can be applied not only to structures that lack a characteristic length scale, but also to signals that lack a characteristic time scale. In this case, the relationship between the statistical properties of the fluctuations of the signal and the time window of observation follows the power law. The meaning of such behaviour is that future values in a time series are dependent on the past, displaying correlations over time, whereas the system that produces the signal exhibits a kind of memory [24,28].

In order to evaluate the power law of a signal it is necessary to compute the power spectrum. For that reason, a Fourier transformation is applied to the signal in order to decompose it to different frequency components that are included within the time series. Every time series can be considered as a sum of sinusoid oscillations with different frequencies. The fast Fourier transformation (FFT) that is a method for fast estimate of Fourier transform, transforms a signal to a sum of cosine and sine oscillations whose amplitudes determine their contribution to the whole signal. This frequency domain analysis displays the contribution of each sine wave as a function of its frequency, whereas its square is the power of that frequency in the whole spectrum of the signal [16]. The increased variability/complexity is a hallmark of health, whereas many large clinical studies in cardiovascular medicine have proven that loss of variability is associated with sudden cardiac death, post-myocardial infarction (MI) heart failure and ventricular fibrillation [29].

In the case of power law calculation, the plot of the log-log representation of the power spectrum (log power versus log frequency) gives rise to a straight line with a slope of approximately -1 . As the frequency increases the size of variation drops by the same factor (scale invariance). The values of the $\beta$ slope/exponent can reflect the inherent dynamics of a system. Values near 1 are supposed to reflect fractal-like behavior, whereas values lower 
than 0.5 represent a system without any correlations, lack of memory and finally chaotic-like and unpredictable evolution in time (white noise). On the contrary, values of $\beta$ slope higher than 1 or even near 1.5 characterize strong correlations within the signal and a highly predictable and almost periodic evolution in time (brown noise) [26,27]. Goldbereger [30] has studied cardiovascular dynamics in health and disease and has found that both unpredictable (random-walk) and periodic behaviors represent loss of physiologic function and correlate with lack of fractal properties of heart rate signals in patients with cardiovascular diseases. Similar results have been found also in critically ill patients with severe sepsis and septic shock [26].

\section{Breathing pattern dynamics: physiological and pathophysiological implications}

Neurons in the brainstem govern respiratory rhythm through a network of coupled oscillators. Critical components of this network are located in a specialised region of the brainstem called the pre-Botzinger complex (pre-BotC). This complex system can generate a wide variety of rhythms [31]. Focal activation of this region has been shown to increase the frequency of inspiratory motor bursts. Del Negro and colleagues [32] showed that progressively elevating neuronal excitability of the pre-BotC of neonatal rats in vitro by increasing artificial cerebrospinal fluid $\mathrm{K}^{+}$concentration causes periodic modulation of the inspiratory rhythm, reflected by cranial nerve XII motor discharge, in a well defined sequence of behavioural states, characterised by periodic oscillations, quasiperiodicity and ultimately disorganised aperiodic activity. In another experimental study with anesthetised adult cat models, Chen et al. [33] found that both focal hypoxia and chemical stimulation of pre-BotC can produce a marked excitation of phasic phrenic nerve discharge, characterized by high amplitude, rapid rate of rise, short duration bursts and reduced complexity, estimated with approximate entropy (low ApEn values).

The above studies support the hypothesis that central respiratory centers are responsible for different breathing patterns with various degrees of variability and complexity in different settings and levels of stimulation. In addition, they can also adapt ventilation to metabolic needs through integration of afferent information, since the respiratory pattern is created by integration of different inputs from chemoreceptors, chest wall and pulmonary receptors, the cerebrum, vagal afferents and non-respiratory central mechanisms [34,35].

Both hypercapnia and hypocapnia may alter ventilatory pattern. Jubran et al. [36] observed that hypercapnia in humans increases the gross variability of minute ventilation (MV) and $\mathrm{V}_{\mathrm{T}}$ but decreases that of inspiratory (Ti) and expiratory (Te) times. In addition, hypercapnia makes ventilation more monotonous with increased autocorrelation between adjacent values. Fiamma et al. [37] studied breath-by-breath variability and complexity of different ventilatory signals (instantaneous ventilation, tidal volume, mean inspiratory flow, inspiratory and expiratory times) in eight healthy subjects with different $\mathrm{CO}_{2}$ levels. Hypercapnia reduced breathing variability and increased all complexity indices whereas hypocapnia induced reciprocal changes. The authors concluded that chemoreceptors may exert a strong and inverse influence on ventilatory variability and complexity. 
Cortical and subcortical effects upon breathing patterns dynamics are also influenced by slow-wave sleep that seems to reduce respiratory complexity [38] whereas panic-anxiety disorders may increase it [39]. Furthermore, Samon and Bruce reported that breathing complexity decreases with anesthesia and vagotomy [40].

Apart from chemoreceptor signalling, chest wall and pulmonary receptors may continuously affect central neural output, especially during resistive breathing. Brack and Tobin [11] measured breathing variability over one hour in ten patients with restrictive lung disease and in seven healthy subjects. They found that variability of $\mathrm{Ti}$, Te and $\mathrm{V}_{\mathrm{T}}$, were significantly reduced in the patients group compared with the healthy group. Furthermore, autocorrelation coefficients were increased almost 3-fold in the patients group, indicating increased periodicity. According to the authors, the decreased breath-to-breath variability in restrictive lung disease patients is a compromise between increased effort and carbon dioxide clearance and arises from their voluntary control of ventilation, as they 'choose' to do it in order to reduce respiratory distress [11,41,42].

Of particular importance in the ICU setting is the potential impact of systemic inflammation on breath-to-breath dynamics as suggested by endotoxin response studies. In a clinical study of Preas and colleagues [43], 12 healthy subjects were randomized to receive endotoxin or saline. Administration of endotoxin after 3 to 4 hours increased RR, decreased $\mathrm{Ti}$, produced dyspnea, augmented autocorrelation coefficients within RR time series and decreased random fraction of variational activity of frequency. These changes were related to changes in arterial carbon dioxide tension. The authors concluded that endotoxin has a direct effect on respiratory controller function whose increased output causes dyspnea. They suggested that decrease in random fraction of breath variability, meaning reduced freedom to vary the respiratory cycle, was attributed to a decrease in circulation time between the lung and the chemoreceptors, secondary to an increase in cardiac output. Since ibuprofen, a cyclooxygenase inhibitor, did not abolish dyspnea, something that seems to happen in healthy exercising subjects, the authors proposed that endotoxin augments respiratory center output through other alternative pathways [44, 45].

\section{Fractals and power law in pulmonary physiology}

Many organs in different biological systems have fractal structure. Fractal branching reduces the distances over which materials are transported, providing rapid and efficient delivery of nutrients [46]. The lung offers many examples of self-similarity properties. Weibel and Gomez [47] first measured the morphology of human airways and found an exponential relationship between the diameter and the generation number of the conducting airways. Mandelbrot [48], who was the first who introduced the term fractals, discovered a unifying scaling pattern of the branching in the lung. Its higher fractal dimension corresponds to a more complex branching, whereas a lower one reflects a more homogeneous structure. Moreover, regional pulmonary blood flow has been shown by Glenny to exhibit spatial and temporal fractal patterns [49]. The structure of alveolar surface has been also found to be well described by power laws, reflecting scale invariance [50]. The 
probability distribution of airway opening during inspiration behaves also according to the power law [51].

Another property of fractals and power laws in pulmonary physiology is error tolerance during development. In simulations of airway morphogenesis during lung development, West [52] compared a power law branching rule with an exponential decaying one and found that in the first case, the system was less susceptible to errors introduced into the branching process. These same properties suggest that living systems are capable to operate similarly at different scales, meaning that whenever environmental conditions change they can adapt more easily to their surroundings.

Aging has been proven by Lipsitz and Goldbereger [30] to be significantly associated with loss of complexity of physiological signals, leading to decreased ability to adapt to different physiological insults. Using different algorithms for estimating fractal properties and power law behavior, these authors found that the $\beta$ slope of different signals in elderly was either reduced (decreased lower than 1) or augmented (increased higher than 1) compared to younger adults, indicating chaotic or periodic behavior, respectively. Peng and co-workers [53] showed that aging was associated with a breakdown of fractal dynamics of respiratory signals via a decrease in $\beta$ slope towards 0.5 (randomness). Concerning early stages of development in humans, one study [54] has found that ultrasonographic patterns for assessment lung maturity showed fractal properties with a power law behavior. In addition, the $\beta$ slope increased with gestational age from 28 to 38 weeks. Szeto and co-workers [55], calculated $\beta$ slopes of different respiratory signals in human fetus and showed its movement from randomness towards fractal behavior with gestational age. In conclusion, it seems that there is great variability in complexity with age in early life, after which complexity decreases with aging.

\section{Fractal properties of the lung in disease: data from clinical and experimental studies}

Alterations to fractal properties are related to different pathologies and could have clinical implications for diagnosis and treatment. Physiologic time series, such as heart and respiratory signals, show similar alterations in their power law behavior in different disease states. Mackey and Glass [56] have introduced the term 'dynamic diseases' to describe states with loss of fractal properties of organs and power law dynamics of signals that are produced form the above structures. For example, loss of heart rate variability that is the variability of the R-R in the electrocardiogram, has been found in patients with heart failure [57], atrial fibrillation, septic shock and multiple organ dysfunction syndrome [6,26]. In respiratory disorders, a classical example is the highly periodic variation in respiratory frequency, seen in Cheyne-Stokes respiration. Penzel [58] has observed loss of fractal properties of heart rate signals during episodes of obstructive sleep apnea.

Macklem [59] was the first who raised the question of whether airway function can be studied using tools from chaos theory and the paradigm of complex systems. Que and coworkers [60] studied the distribution of forced oscillatory resistance in asthmatics and 
demonstrated that lung function exhibit loss of fractal properties during severe asthma. Frey and colleagues [61] applied fractal methods to twice-daily peak expiratory flow (PEF) in asthmatic patients and showed that the $\beta$ slope was reduced, whereas it become more regular with standard long-acting $\beta 2$-agonist treatment and more random with short-acting $\beta 2$-agonist treatment, respectively. Moreover, the authors were able to demonstrate that the higher the $\beta$ exponent when a patient was not under any treatment, the larger the improvement of his condition upon administration of long-acting $\beta 2$-agonist therapy.

In another study, Suki and co-workers [51] studied the dynamics of airway opening and crackles, using a simple mathematic model of the periphery of airway tree. Suki found that the time series of crackles emitted during airway opening follows a power law distribution. Additionally, as the crackles propagate up the tree, the sound amplitude is attenuated at successive bifurcations, whereas its distribution follows the power law. The same has been found for the time intervals of the 'jumps' by which airway resistance decreases upon lung inflation by a constant flow. In a study of Boser and colleagues [62], the fractal dimension of airways was computed using autopsy material from three groups: fatal asthma, non-fatal asthma and non-asthma controls. The authors were able to show that the average FDs of both fatal (1.72) and non-fatal asthma groups (1.76) were significantly lower than that of the third control group (1.83, $\mathrm{p}<0.05)$, whereas the lower fractal dimension correlated with a decreased overall structural complexity and pathologic severity of disease.

Venegas and colleagues [63], using positron emission tomography (PET) imaging and computer modeling showed that in cases of bronchoconstriction and when smooth muscle activation reaches a critical level, localized clusters of poorly ventilated lung regions can develop abruptly in discrete steps. These steps are called avalanches and can lead to new stable conditions. Because of the fractal structure of the airways, small initial heterogeneities that are always present and particularly in the diseased lung, can be amplified, leading to sudden patches of poorly ventilated lung regions. Another implication is that since airways are organized into a fractal network embedded in the elastic parenchyma, the constriction of one airway can propagate and cause an avalanche-like constriction in large parts of the lung. The same holds true for the opposite process, where opening of airways during inhalation takes place in discrete steps [63-65].

Suki [51] has also demonstrated that airway opening upon inflation occur in avalanches with power law distribution of both the size and time intervals between them. The significance of these results is that the probability of finding a large avalanche is much higher than it would be if the distribution were Gaussian or exponential, so both the magnitude and timing of pressure excursions applied at the airways (i.e., using mechanical ventilation) may be critical in triggering the avalanche process of alveolar recruitment [24].

In conclusion, these studies in asthma show that when the airways are likely to approach their critical closing threshold pressure, a small stimulus can provoke a catastrophic cascade of airway closure and for that reason, there is such poor correlation between the trigger and the outcome in asthmatic patients. Moreover, the history of symptom fluctuations seems related to the structural changes of the airway tree (power law distribution of airway diameter) [65]. 
Airway recruitment may affect alveolar recruitment as well. Sujeer and co-workers [66] have found in mathematical models that the recruited volumes upon inflation with constant flow are distributed according to a power law with a $\beta$ slope equal to 2 . From the above findings it can be supposed that since alveolar recruitment is influenced by airway structure, then the pressure-volume curve may carry information about the airway tree [24]. Whether such models have any value in acute lung injury (ALI) is unclear. In this syndrome, it has been found that the opening pressure distribution does not seem to be always Gaussian, something that is assumed to be the case in the avalanche model [67]. More studies are needed to investigate the pattern of recruitment in ALI, particularly in the case of gravity effect upon ventilation-perfusion mismatch at the level of alveoli [68].

The application of fractal analysis has also shed light into the morphology of the lung in cases of emphysema. Computed tomography (CT) is a sensitive method for assessing lung structure in different pathologies. In general, low attenuation area (LAA) clusters are depicted in pixels with density less than 950 Hounsfield units. These areas incorporate mostly air and assigned a value of 1, whereas pixels with a density higher than 950 include tissue with a value 0 . Summing the number of pixels in a cluster gives the cluster size. In that way, a binary map of the lung can be constructed and a few studies have shown that in normal conditions, this map is highly heterogeneous [24]. Mishima and colleagues [69] have found that the probability distribution of LAA clusters follows a power law for both normal subjects and patients with chronic obstructive pulmonary disease (COPD). However, patients exhibited significantly smaller $\beta$ slopes or exponents, which did not correlate with pulmonary function tests except for diffusion capacity of the lung. The authors suggested that the neighboring smaller LAA clusters tend to coalesce and form larger clusters as the weak elastic fibers separating them break under tension. This process does not change the $\%$ LAA but decreases the number of small clusters in favor of larger ones, which result in a reduction of the $\beta$ slope. Another assumption derived from this study is that the likelihood of finding large LAA clusters is much higher in COPD patients than in normal controls [65].

Another possible application of fractals in pulmonary and critical care medicine seems to include the mechanical ventilation of critically ill patients. In an oleic acid injury animal model, Mutch and colleagues [70] introduced fluctuations according to an algorithm, to mechanical ventilation (biological variable tidal volume and respiratory frequency proportional to pre-defined minute ventilation values). Compared with conventional ventilation (with similar minute ventilation), this approach increased respiratory arrhythmia and oxygenation and decreased dead space. According to Suki [24,71], when fluctuations in the form of symmetrically distributed random noise is added to peak airway pressures (noisy ventilation), the mean does not change but isolated values can be augmented, leading to significant alveolar recruitment. In a mathematical model, the authors found that the recruited lung can be $200 \%$ larger in the case of biological variable ventilation than during conventional ventilation. Moreover, the standard deviation (SD) of the noise can be manipulated in order to achieve better oxygenation (system's output), a phenomenon called 'stochastic resonance', which has already been confirmed in animal models of ALI [71]. 


\section{Breathing variability and complexity indices as weaning predictors in mechanically ventilated patients}

Engoren and colleagues [72] studied 10 control patients who had undergone cardiac surgery within an interval of 12 hours prior to this experiment and 21 patients who required prolonged ( $>7$ days) ventilatory support. The control group was studied during a weaning trial of $5 \mathrm{~cm} \mathrm{H}_{2} \mathrm{O}$ continuous positive airway pressure (CPAP). The patient group was studied during 60-to-120 min trials of spontaneous ventilation with $5 \mathrm{~cm} \mathrm{H}_{2} \mathrm{O}$ positive endexpiratory pressure (PEEP) with a constant $\left(12.2+/-6.6 \mathrm{~cm} \mathrm{H}_{2} \mathrm{O}\right)$ level of pressure support (PS) for each trial. These patients passed 59 and failed 14 weaning trials. During spontaneous ventilation each breath's instantaneous respiratory rate and tidal volume were recorded and their ApEn values were calculated for the terminal 1000 breaths, in a series of 100, 300 and 1000 breaths. Receiver operating characteristic (ROC) curves identified cutpoint values of continuous variables that predicted a failed weaning outcome. While mean $\mathrm{V}_{\mathrm{T}}$ did not vary between groups, mean $\mathrm{RR}$ and frequency-tidal volume ratio increased progressively from the control group to both successful weaning (SW) and failure weaning (FW) groups. Conversely, ApEn of RR did not vary between every pair groups, but entropy of $\mathrm{V}_{\text {T }}$ increased significantly from the control to FW group and from SW to group FW, with no difference between control and SW groups. The ROC curves for ApEn- $V_{T}$ and frequencytidal volume ratio showed similar sensitivity and specificity for predicting weaning failure whereas ApEn-V $\mathrm{V}_{\mathrm{T}}$ proved to be successful in separating SW from FW, with similar area under the curve (AUC) values of $0.74,0.75$ and 0.73 for 100, 300 and 1000 breaths respectively. Since 100 breaths during a weaning trial may take only 3 to 4 minutes compared with 30 to 40 minutes required for a 1000 breaths trial, ApEn-Vт assessment can be of significant value, permitting a faster determination of weaning tolerance.

According to the authors [72], increased irregularity of the FW group indicated enhanced external inputs to the respiratory controller whereas increased regularity of the SW group suggested greater component autonomy. The first case implies that enhanced external inputs from peripheral receptors that measure $\mathrm{pH}, \mathrm{PcO} 2, \mathrm{Po} 2$ or from cortical areas that represent dyspnea or anxiety are responsible for increasing breathing complexity $[19,20]$. Conversely, many studies estimating heart rate variability have found inverse relations between mortality after myocardial infarction or endotoxemia and HRV [73,74]. It seems that patient's voluntary control over breathing (which does not exist for heart rate) is responsible for different effects of cardiac and pulmonary disease on regularity.

El-Khatib et al. [75] studied 52 patients diagnosed mostly with lung diseases and under mechanical ventilation in two phases: 1 . under synchronized intermittent mandatory ventilation (SIMV) with RR $\leq 4$ breaths/min and with no PS for 60 minutes and 2. during a CPAP trial of $5 \mathrm{~cm} \mathrm{H}_{2} \mathrm{O}$ with no PS ventilation for other 60 minutes. Thirty-nine patients (75\%) were successfully extubated and the remaining 13 patients failed weaning trial. In both patient groups, different breathing signals were collected (airway flow, volume, proximal airway pressure) and were divided into three intervals of 300 breaths. During SIMV trials, the spontaneous and mechanical peak flows (PF) versus tidal volume 
scattergrams were constructed and coefficients of variation for both variables were determined for each 300 breath interval separately and for the whole 900 breaths. During CPAP trials, the Kolmogorov entropy indicating unpredictability [76] within the flowvolume loops was estimated, along with the dimension of the respiratory breathing pattern, defined as the number of clusters or clouds of trajectories in the flow-volume loops space, for each data interval. The authors found that the CV of tidal volume and PF, the Kolmogorov entropy and the fractal dimension of the spontaneous breathing pattern were all significantly smaller in the successfully weaning group compared with the failure weaning group.

These results suggested that breath-to-breath variability during SIMV trials and complexity during CPAP trials were increased in patients who failed weaning trials. Patients from the two groups did not differ in terms of age, previous days on mechanical ventilation and blood gases at study entry, whereas the distribution of diagnosis was also similar between the two groups. Since the aim of this study was not to determine a cut-off threshold in terms of breathing pattern complexity for weaning prediction, no sensitivity or specificity were measured. Furthermore, the authors did not present any possible explanation of their results in terms of different pathophysiological mechanisms that may drive various levels of variability/ complexity.

In another study, Bien and colleagues [77] investigated breathing pattern variability in 78 mechanically ventilated patients with systemic inflammatory response syndrome (SIRS) who had undergone abdominal surgery. The patients were divided in the successfully weaning group $(n=57)$ and the failure weaning group $(n=21)$. Within 1 hour before the measurement of breathing variability and under ventilation with PS with a pressure level between 10 and $20 \mathrm{~cm} \mathrm{H} \mathrm{H}_{2} \mathrm{O}$, tidal volume, total breath duration (Ttot), Ti, Te and peak inspiratory flow were calculated. After obtaining these data, the ventilatory mode was switched to PS of $5 \mathrm{~cm} \mathrm{H}_{2} \mathrm{O}$ plus $5 \mathrm{~cm} \mathrm{H}_{2} \mathrm{O}$ PEEP for 30 minutes. The coefficients of variation and the SD1 and SD2 from the constructed Poincare plots for the above variables were determined for at least 300 breaths. Moreover, AUC were computed for ROC curves and compared with airway occlusion pressure, its ratio to the maximal inspiratory pressure (P0.1/Pimax), RR, RR/ $\mathrm{V}_{\mathrm{T}}$, and the product $\mathrm{P} 0.1^{*}\left(\mathrm{RR} / \mathrm{V}_{\mathrm{T}}\right)$. The authors found that average values and the CVs of the 5 measured parameters were significantly lower in the failure weaning group than in the successful one. In addition, Poincaré plot analysis showed that both SD1 and SD2 of the 5 breathing parameters were significantly reduced in the weaning failure group whereas the SD1-SD2 ratio showed no statistical significance. Finally, AUCs of the CVs of the 5 measured variables and both SD1 and SD2 did not differ significantly from those of the 5 clinically used weaning predictors. All the AUC values were within the range of 0.73 to 0.80 .

This was the first study reporting that WF patients exhibited a decreased breath-to-breath variability and complexity. According to the authors, the different results from the two previous studies may be due to different weaning protocols that were implemented and different patient characteristics. Their patients had a mean age of 68 years, no history of pulmonary disease and a short duration of ventilatory support (3 days) whereas patients in 
the El-Khatib study [75] had a mean age of 50 years with predominantly underlying lung diseases and a longer duration of mechanical ventilation (11 days). Finally, in the Engoren study [72] cardiac surgery patients were included and indices of cardiac function were not presented, especially when low cardiac output states are associated with a Cheyne-Stokes breathing pattern.

Recently, Wysocki and colleagues [78] examined breathing variability in 46 medical and surgical patients during 60 minutes spontaneous breathing trials (SBT) and during a 5month period in four French tertiary university hospitals' ICUs. There were 32 successful and 14 failure weaning cases. SBT was performed with complete disconnection from the ventilator, meaning none form of ventilatory support. The time series analysis was performed by two independent investigators, one of whom was blinded to clinical information. Furthermore, a signal processing technique for checking stationarity of breath time series was performed and nonstationary data were excluded from analysis. The authors reported significantly increased CVs of tidal volume, $\mathrm{Ti}$, Te, Ttot, Ti/Ttot and $\mathrm{V}_{\mathrm{T}} / \mathrm{Ti}$ in the SW group, whereas the duration of autocorrelation (number of lags) was significantly shorter in the same group compared with patients who failed weaning trials, meaning that low number of breaths were significantly autocorrelated in cases of weaning success.

The different results from Engoren's and El-Khatib studies [72,75] were attributed to the prevailing conditions during respiratory variability analysis. According to Caminal and Brochard, different levels of pressure support may alter breath-to-breath variability in an inverse way $[79,80]$, something that could have played a role in the two previous studies. Furthermore, it was postulated the low breathing variability could cause the deterioration of respiratory mechanics rather than result from it, by promoting microatelectasis. In conclusion, Wysocki's patients did not receive any ventilatory support during weaning trial, limiting accuracy of comparisons with populations from the first two studies.

In a recent study [81], we tried to investigate heart rate (HR) and respiratory rate complexity in patients with weaning failure or success, using both linear and nonlinear techniques. Forty-two surgical patients were enrolled in the study. There were 24 who passed and 18 who failed a weaning trial. Signals were analyzed for 10 minutes during two phases: 1. pressure support (PS) ventilation $\left(15-20 \mathrm{~cm} \mathrm{H}_{2} \mathrm{O}\right)$ and 2. weaning trials with PS: $5 \mathrm{~cm} \mathrm{H} \mathrm{H}_{2} \mathrm{O}$. Low and high frequency (LF, HF) components of HR signals, HR multiscale entropy (MSE), RR sample entropy, cross-sample entropy as an indication of coupling between cardiorespiratory signals and Poincaré plots were computed in all patients and during the two phases of PS. Multiscale entropy was recently introduced [82] for quantifying heart rate complexity. Briefly, for a given R-R time series, multiple 'coarsegraining' time series are constructed by averaging the data points within non-overlapping windows of increasing length $\tau$, where $\tau$ represents the scale factor. Subsequently, sample entropy (SampEn) that represents the negative natural logarithm of the conditional probability that two sequences similar for $\mathrm{m}$ points remain similar at the next point with a tolerance $r$, is calculated for each time series and then plotted against the scale factor, 
giving rise to the MSE curve. The sum of SampEn over all scaling factors represents the MSE of a signal. For our MSE analysis, the parameter $r$ was set at $15 \%$ of standard deviation (SD) of the time series, after normalization $(S D=1)$ and the parameter $\mathrm{m}$ (embedding dimension), that is the length of sequences to be compared was set at 2 (data length ranging from 100 to 5000 data points) [82]. Furthermore, we extracted two more features from MSE curves after log transformation of SampEn and scale factor, the fast slopes for small time scales, i.e., those defined by SampEn values between scale 1 and 5 and the long slopes for higher scale values (Figures $1 \& 2$ ).

We found that weaning failure patients exhibited significantly decreased RR sample entropy, LF and HF components, compared with weaning success subjects $(p<0.001)$. Their changes were opposite between the two phases, except for MSE that increased between and within groups $(\mathrm{p}<0.001)$. A new model including rapid shallow breathing index (RSBI), RR and cross sample entropies predicted better weaning outcome compared with RSBI, airway occlusion pressure at $0.1 \mathrm{sec}\left(\mathrm{P}_{0.1}\right)$ and $\mathrm{RSBI}^{*} \mathrm{P}_{0.1}$ (conventional model, $\mathrm{R}^{2}=0.887$ vs 0.463 , $\mathrm{p}<0.001)$. Areas under the curve were 0.92 vs 0.86 , respectively $(\mathrm{p}<0.005)$.

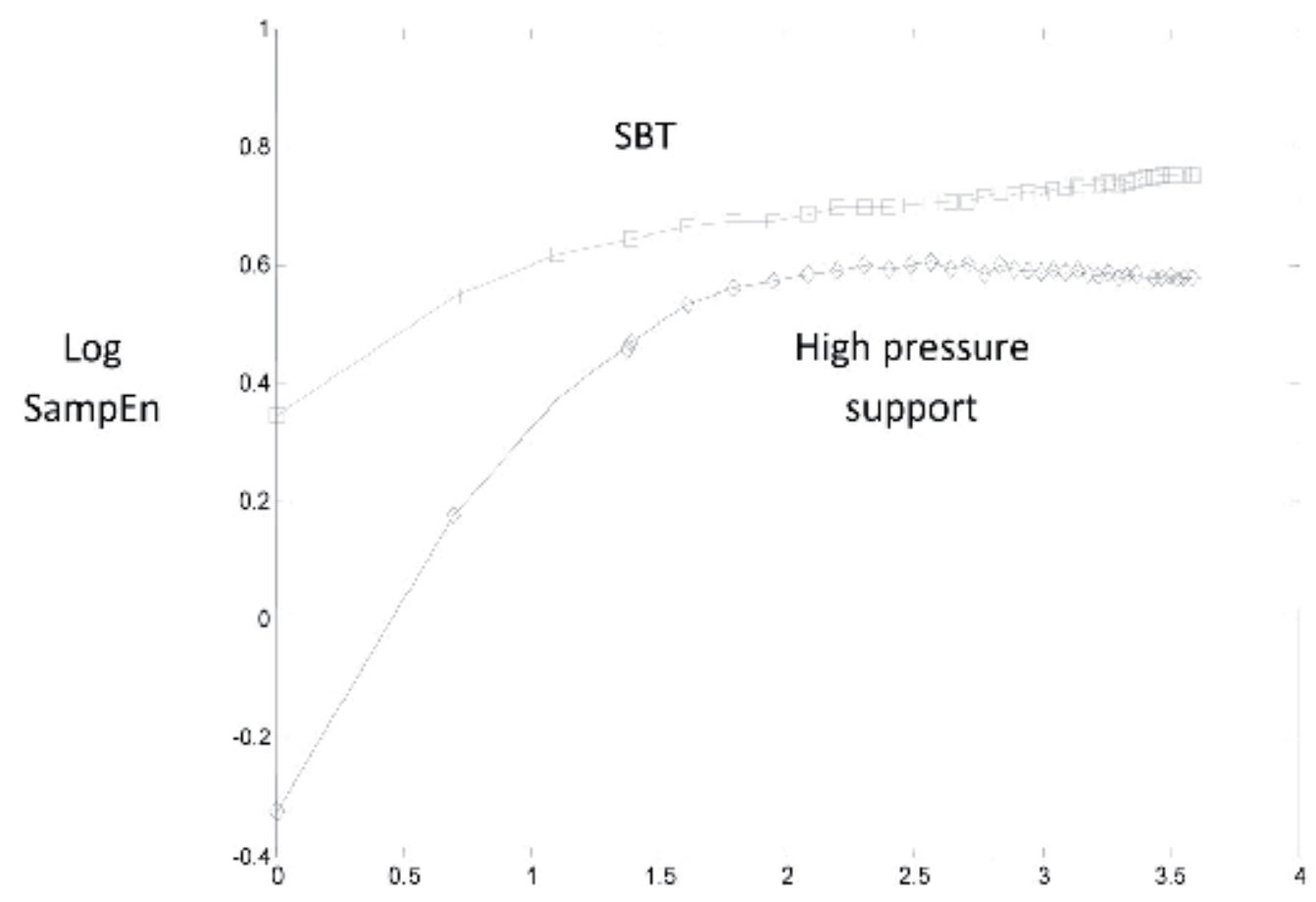

\section{Log scale factor}

Figure 1. Heart rate multiscale entropy (MSE) curves between phase H (high PSV) and spontaneous breathing trial (SBT) or phase L (low PSV) in a weaning failure patient. SampEn and scale factor have been logarithmically trans-formed. The MSE curve during the SBT has increased SampEn values; however, the slope during small scales (fast slope) is decreased compared with the high PSV phase. 


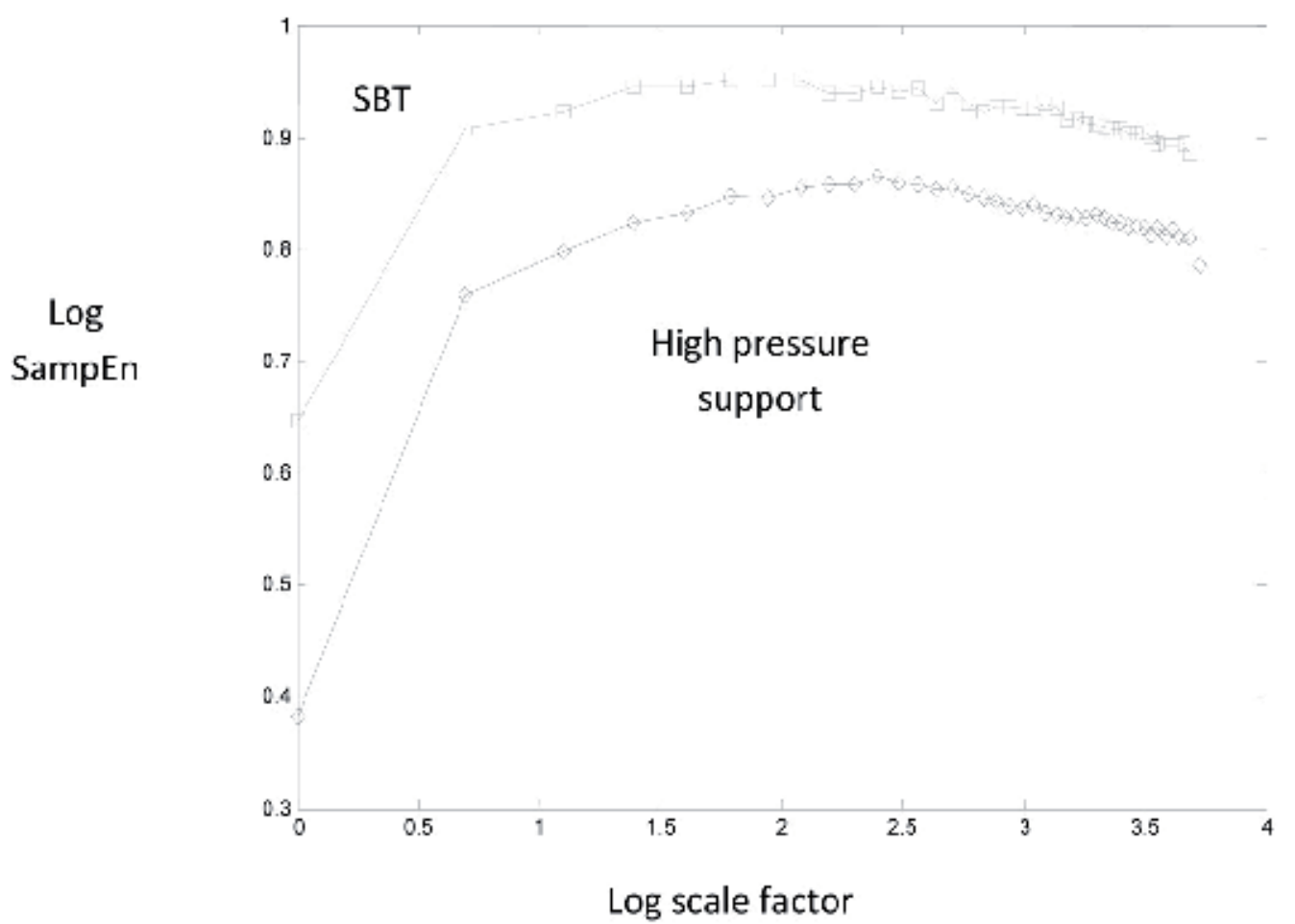

Figure 2. Heart rate multiscale entropy (MSE) curves between phase H (high PSV) and spontaneous breathing trial (SBT) or phase L (low PSV) in a weaning success patient. The MSE curve during the SBT has increased SampEn values; moreover, the slope during small scales (fast slope) looks similar with the slope during the high PSV phase.

Different profiles of MSE curves on small time scales between the two groups and according to fast slopes may be due to the impact of respiratory rhythm on heart rate dynamics (respiratory sinus arrhythmia). In general, the lower the amplitude of respiratory modulation, the higher the entropy values tend to be [82]. Thus, in both groups but especially in weaning failure patients, sum MSE was significantly increased between different PS levels, whereas reduced fast slopes could reflect different time constants of respiratory effect upon heart rate control mechanisms.

In another similar study [83], we enrolled thirty-two mechanically ventilated patients undertaking a weaning trial. There were 22 who passed and 10 who failed a weaning trial. Tidal volume and mean inspiratory flow were analyzed for 10 minutes during two phases: 1 . pressure support (PS) ventilation (15-20 $\mathrm{cm} \mathrm{H}_{2} \mathrm{O}$ ) and 2. weaning trials with PS: $5 \mathrm{~cm} \mathrm{H}_{2} \mathrm{O}$. Sample entropy (SampEn), fractal dimension (FD) and largest lyapunov exponents (LLE) of the two respiratory parameters were computed in all patients and during the two phases of PS. FD was computed according to the Higuchi method [84]. Briefly, FD was based on a measure of length $\mathrm{L}(\mathrm{k})$ of a time series, computed at different scales, by using a segment of $\mathrm{k}$ samples as a unit in each scale. The value of FD was calculated by a least-squares linear bestfitting procedure as the angular coefficient of the linear regression of the log-log graph of the 
mean of $\mathrm{k}$ values $\mathrm{Lm}(\mathrm{k})$ for $\mathrm{m}=1,2,3 \ldots \mathrm{k}$, with $\mathrm{k}$ being an interval time. The length $\mathrm{Lm}(\mathrm{k})$ originating from time $\mathrm{m}$ was calculated as the normalized sum of absolute differences between the values of point pairs that are ' $k$ samples distant' and the length of curve of the time interval $\mathrm{k}, \mathrm{L}(\mathrm{k})$ was calculated as the mean of the $\mathrm{k}$ values $\mathrm{Lm}(\mathrm{k})$. If the $\mathrm{L}(\mathrm{k})$ related to the scale used $(\mathrm{k})$ linearly in a log-log plot with slope FD, then the curve was said to show fractal dimension.

LLE were computed as follows: Complex systems are considered sensitive to initial conditions and exhibit an exponential divergence in the phase space, which describes in a 3dimensional axis their different states. Estimation of Lyapunov spectrum and largest Lyapunov exponents (LLE) can assess sensitivity to initial conditions. Briefly, if we consider two points in adjacent trajectories-states of the phase space with a distance between them $\mathrm{d}(0)$, after time $\mathrm{t}$ the average divergence (separation) will be:

$$
\left[d(t)=d(0) * e^{L L E *(i \Delta t)}\right]
$$

whereas LLE is the largest Lyapunov exponent. In this study, we computed LLE of mean inspiratory flow and tidal volume signals, using the algorithm proposed by Rosenstein [85], which seems to be useful, particularly in small data sets. Values higher than 0 reflect an unstable and unpredictable system, where nearby points will diverge to any arbitrary separation. Increased LLEs reflect increased sensitivity to initial conditions and characterize unpredictable variations, whereas low values indicate regularity [85].

Weaning failure patients exhibited significantly decreased respiratory pattern complexity, reflected in reduced sample entropy and lyapunov exponents of respiratory flow time series, compared to weaning success subjects $(p<0.001)$. In addition, their changes were opposite between the two phases of the weaning trials. A new model including rapid shallow breathing index (RSBI), its product with airway occlusion pressure at $0.1 \mathrm{sec}\left(\mathrm{P}_{0.1}\right)$, SampEn and LLE predicted better weaning outcome compared with RSBI, $\mathrm{P}_{0.1}$ and RSBI* $\mathrm{P}_{0.1}$ (conventional model, $\mathrm{R}^{2}=0.874$ vs $0.643, \mathrm{p}<0.001$ ). Areas under the curve were 0.916 vs 0.831 , respectively $(\mathrm{p}<0.05)$.

Vallverdu et al. [86] in another study with similar design compared with our two previous investigations examined heart rate and respiratory pattern complexity in 78 patients, during weaning trials and using information flow analysis, which describes the regularity of signals by estimating the auto- and mutual information functions. The authors were able to find reduced complexity and a more coupled nonlinear oscillator behavior in weaning failure subjects.

These results parallel those from Schmidt and colleagues [87] who reported increased LLE and Kolmogorov-Sinai entropy values of mean inspiratory flow signals in mechanically ventilated patients, after switching the ventilator from the pressure support mode to neurally adjusted ventilatory assist mode (NAVA). According to these authors, successful spontaneous breathing trials unmask underlying variability and complexity of central neural output, since inspiratory pressure inhibits the respiratory drive. This effect is nicely reflected through the increased complexity indices of flow and is responsible for better neuro-mechanical coupling. 


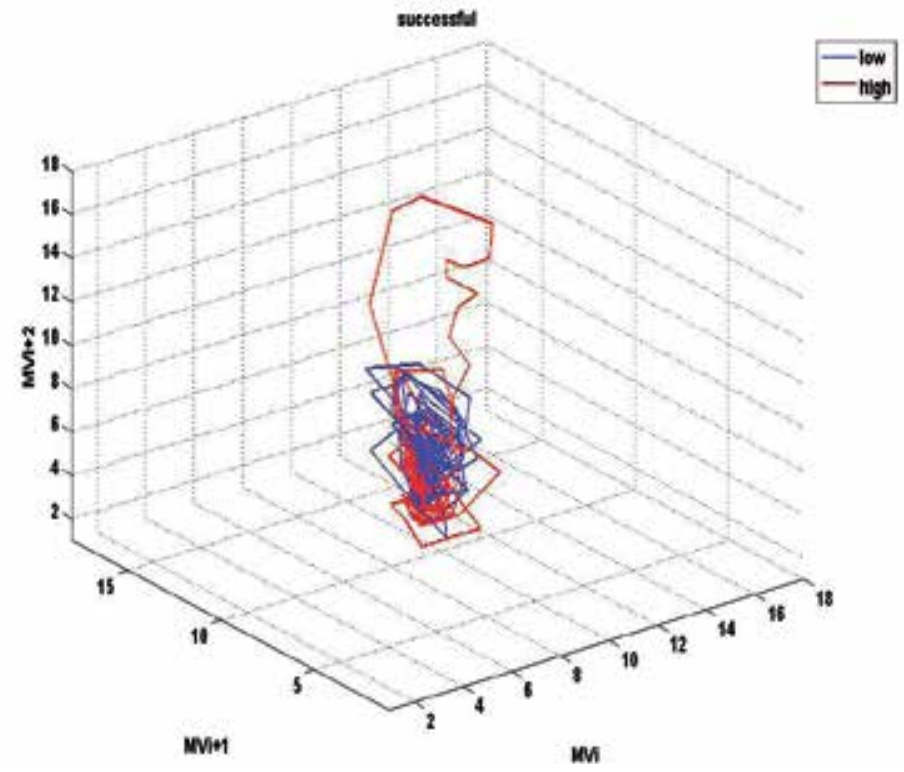

Figure 3. Phase space of minute ventilation (MV) in a weaning success patient. Different values $x(i)$ of minute ventilation were plotted against the following ones in tree-dimensional axes: $x, x+t$ and $x+2 t$, giving rise to the phase space. Red dots represent data during high PS ventilation, whereas blue dots represent data during the performance of a SBT.

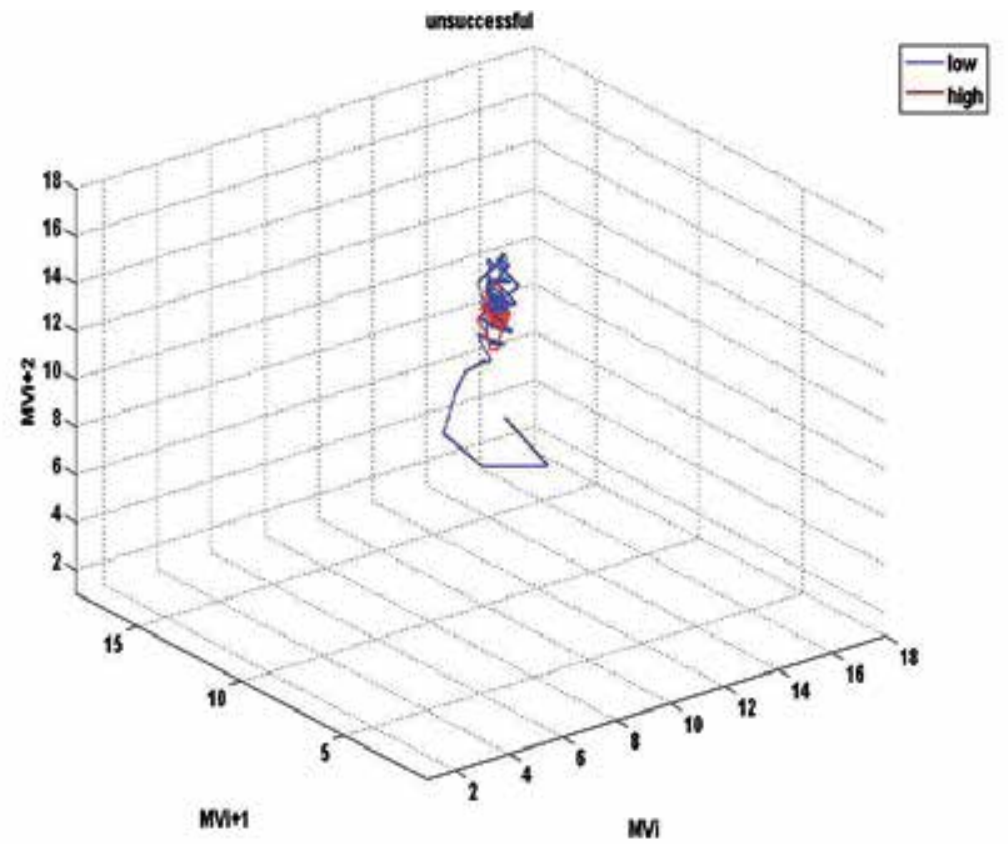

Figure 4. Phase space of minute ventilation in a weaning failure patient. Red dots represent data during high PS ventilation, whereas blue dots represent data during the performance of a SBT. Scattering of data seem highly reduced, compared with findings in Figure 3, whereas ventilation values are positioned in different parts of the space. 

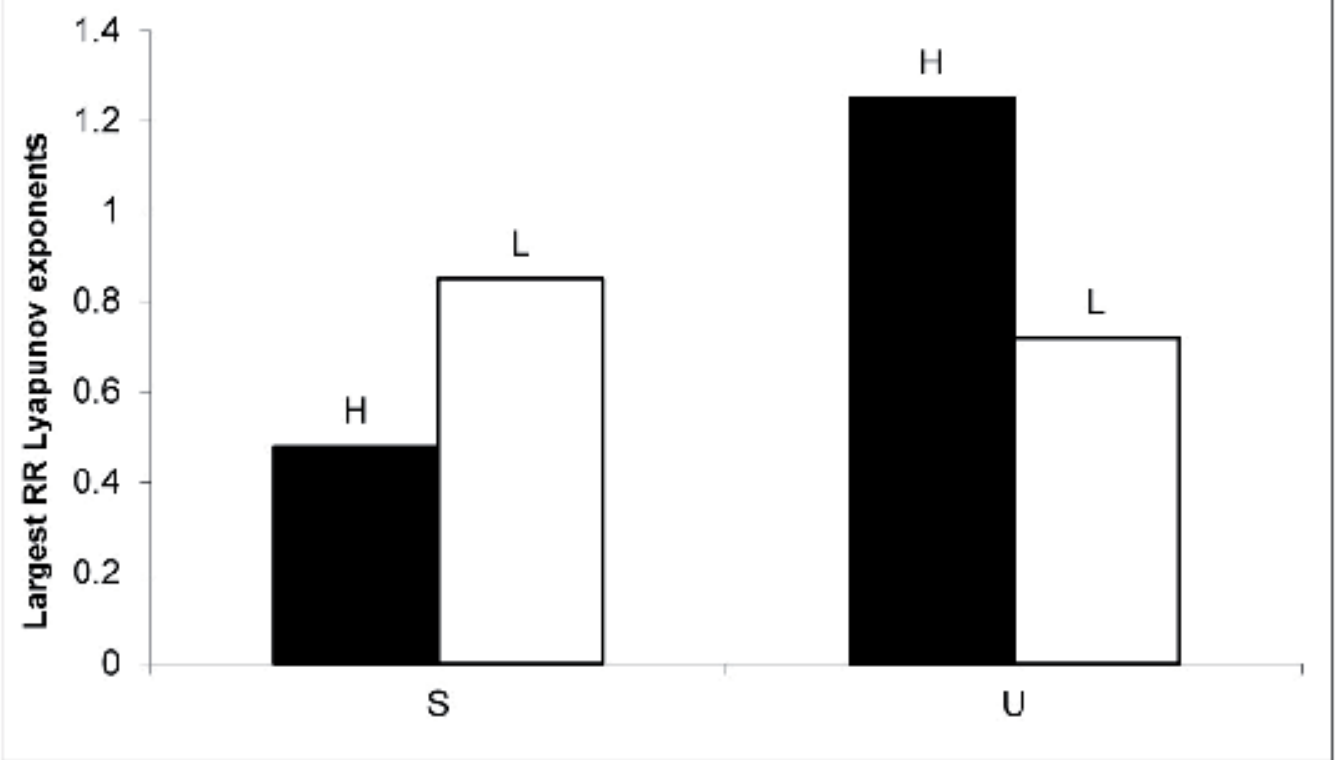

Figure 5. Distribution of LLE between the two groups of patients (S: successful weaning and U: unsuccessful), during high $(\mathrm{H})$ and low $(\mathrm{L})$ pressure support ventilation. It seems that SW group demonstrated an increase in LLE after shifting to spontaneous breathing, contrary to what have occurred to the FW group.

In another study, Mangin and colleagues [88] investigated ventilatory chaotic dynamics in 17 mechanically ventilated patients during switching the ventilator from the assist-control mode to pressure support mode. They were able to show that both fractal dimension and LLE were increased, particularly in 5 patients who were successfully extubated. Furthermore, the authors supposed that increased breathing complexity may also be attributed to higher vagal afferent feedback during unassisted breathing, as has already been shown by Sammon and Bruce [40].

These studies support our findings that transition between mechanical and spontaneous ventilation is associated with increased complexity of respiratory signals in weaning success patients, since duration of ventilation before the SBTs was similar between groups with different weaning outcome. Moreover, in a study of Burykin and Buchman [89] investigating cardiorespiratory dynamics and synchronization during controlled and unassisted breathing in 13 surgical patients, it was demonstrated that mechanical ventilation reduces significantly both heart and respiratory rate complexity whereas spontaneous respiration is more irregular with increased uncoupling of cardiorespiratory rhythms in weaning success patients.

\section{Conclusions}

According to Macklem [90], there is a continuum of thermodynamic systems that do not follow the $2^{\text {nd }}$ thermodynamic axiom (increase in entropy), since they exchange energy with 
their environment (open systems): from near to equilibrium, like crystals to far from equilibrium systems, like the weather. 'The amount of energy dissipated determines where the system is situated along the continuum. Between the crystals and weather a sudden phase transition occurs over a small range of energy consumption. It is only there where life can flourish'. From the above statement it is concluded that either a decrease in energy consumption, i.e., during myocardial ischemia, or an increase may both contribute to a shift away from stable state. It has been shown by Que [60] that during asthma, the local increase in metabolic rate is associated with increased variability of respiratory impedance to flow. The same author has proposed the term 'homeokinesis' instead of homeostasis [60], as a basic property of living systems that 'describes the ability of an organism to utilize external energy sources to maintain a highly organized internal environment fluctuating within acceptable limits in a far from equilibrium state'.

There is good basic science describing the physiological and fractal variability observed in respiratory dynamics and substantial evidence that conditions prevalent in the ICU impacts breath-to-breath variability. Different clinical studies have assessed the ability of markers of respiratory variability as predictors of successful separation from mechanical ventilation. However, these studies are not comparable since the authors implemented different weaning protocols in heterogeneous groups of patients and examined various indices of both breathing pattern variability (linear) and complexity (nonlinear) that by their nature capture different aspects of signal dynamics. It seems that the study of Wysocki [78] performed better, since it was conducted in 5 medical centres, stationarity tests were done and possible bias in the time series analysis was significantly controlled. Furthermore, lack of ventilatory support during weaning trial could have eliminated any possible impact of positive pressure ventilation upon breathing variability and could be responsible for the different findings between this and the first two studies. Unfortunately, the authors did not perform complexity nonlinear analysis. Since neither ApEn, nor any other complexity index was calculated, we cannot make assumptions regarding inherent breathing dynamics during discontinuation from mechanical ventilation, because variability and complexity may change in opposite directions.

We suggest that new studies are needed, for estimating both linear and non-linear indices of breathing variability and complexity for weaning outcome assessment, in different and homogeneous groups of patients, and comparing their diagnostic accuracy, along with other traditional and already used physiological predictors. Their association, in terms of 'coupling' or 'uncoupling' with other biosignals such as the electroencephalogram (EEG), with the aid of robust methods from signal processing theory (i.e., cross-ApEn, coherence etc) could uncover possible inter-relations between breathing and central nervous system dynamics during weaning trials.

However, we think that the development of a consensus between clinicians and modelers is urgently needed for the assessment of different methods used for breathing pattern variability prior to the performance of other studies. In the cardiology literature, the Task Force on HRV has standardized the use of a set of signal processing techniques, including target variables, frequency and duration of measurements and signal quality assessment, 
aiding in the development of better and more accurate diagnostic or prognostic indices in cardiovascular diseases [16]. Furthermore, many important questions remain unanswered such as the value of single or longitudinal variability measurements for clinically useful information or its prognostic capability for risk stratification between patients or within the same patient (trend analysis).

In conclusion, we believe that the most important aspect of research on breathing pattern dynamics remains the development of international databases of various respiratory signals, with free access from different investigators, such as the Web site Physionet (www.physionet.org), which has boosted research on heart rate dynamics in cardio-vascular medicine [91]. We also think that in addition, such efforts must also be undertaken by pulmonary physicians and those who treat patients with severe respiratory diseases. In conclusion, we suggest that implementation of fractal analysis and breathing pattern variability and complexity measurements in respiratory physiology will result in better understanding of different and dynamic changes during various pathologic states, whereas its impact upon prediction of severe complications will add value to such methods. Clinicians must begin to understand basic principles of complex systems theory and support an interdisciplinary approach for understanding pulmonary diseases, in order to treat their patients more effectively.

\section{Abbreviations}

ALI: acute lung injury

ApEn: approximate entropy

AUC: area under the curve

COPD: chronic obstructive pulmonary disease

CPAP: continuous positive airway pressure

$\mathrm{CV}$ : coefficient of variation

ECG: electrocardiogram

EEG: electroencephalogram

FD: fractal dimension

FFT: fast Fourier transformation

FW: failure weaning HRV: heart rate variability

ICU: intensive care unit

LAA : low attenuation area

LLE: largest lyapunov exponents

LVEF: left ventricular ejection fraction

MODS: multiple organ dysfunction syndrome

MSE : multiscale entropy

MV: minute ventilation

PEEP: positive end-expiratory pressure

PEF: peak expiratory flow

PET: positron emission tomography 
Pimax: maximal inspiratory pressure

P0.1: airway occlusion pressure at $0.1 \mathrm{sec}$

Pre-Botz: pre-Botzinger complex

PSD: power spectrum density

PSV: pressure support ventilation

ROC: receiver operating characteristic

RR: respiratory rate

RSBI: rapid shallow breathing index

SampEn: sample entropy

SBT: spontaneous breathing trial

SD: standard deviation

SIMV: synchronized intermittent mandatory ventilation

SIRS: systemic inflammatory response syndrome

SW: successful weaning

Te: expiratory time

Ti: inspiratory time

Ttot: total breath duration

Vт: tidal volume

\section{Author details}

Vasilios Papaioannou* and Ioannis Pneumatikos

Democritus University of Thrace, Alexandroupolis Hospital,

Intensive Care Unit, Alexandroupolis, Greece

\section{References}

[1] Suki B, Alencar AM. Fluctuations, noise and scaling in the cardio-pulmonary system. Fluctuations and Noise Letters. 2003; 3 (1): R1-R25.

[2] Buchman TG. The community of the self. Nature 2002; 420: 246-251.

[3] Godin PJ, Buchman TG. Uncoupling of biological oscillators: A complementary hypothesis concerning the pathogenesis of multiple organ dysfunction syndrome. Crit Care Med 1996; 24: 1107-1116.

[4] Kennedy H. Heart rate variability - a potential, noninvasive prognostic index in the critically ill patient. Crit Care Med 1998; 26: 213-214.

[5] Annane D, Trabold F, Sharshar T, et al. Inappropriate sympathetic activation at onset of septic shock. Am J Respir Crit Care Med 1999; 160: 458-65.

[6] Seely AJE, Christou NV. Multiple organ dysfunction syndrome: exploring the paradigm of complex nonlinear systems. Crit Care Med 2000; 28: 2193-2200.

[7] Priori SG, Aliot E, Blomstrom-Lundqvist C, et al. Task Force on Sudden Cardiac Death of the European Society of Cardiology. Eur Heart J 2001; 16: 1374-1450.

" Corresponding Author 
[8] Tobin MJ, Mador MJ, Guenther SM, et al. Variability of resting respiratory drive and timing in healthy subjects. J Appl Physiol 1988; 65: 309-317.

[9] Bruce EN. Temporal variations in the pattern of breathing. J Appl Physiol 1996; 84:10791087.

[10] Khoo MC, Kronauer RE, Strohl KP, et al. Factors inducing periodic breathing in humans. J Appl Physiol 1982; 53: 644-659.

[11] Brack T, Jubran A, Tobin MJ. Dyspnea and decreased variability of breathing in patients with restrictive lung disease. Am J Respir Crit Care Med 2002; 165:1260-1264.

[12] Tobin MJ, Yang KL, Jubran A, et al. Interrelationship of breath components in neighbouring breaths of normal eupneic subjects. Am J Respir Crit Care Med 1995; 152:1967-1976.

[13] Marchant BP. Time-frequency analysis for biosystems engineering. Biosyst Eng 2003; 85:261-281.

[14] Perez W, Tobin MJ. Separation of factors responsible for change in breathing pattern induced by instrumentation. J Appl Physiol 1985; 59:1515-1520.

[15] Lombardi F, Sandrone G, Pernptuner S et al. Heart rate variability as an index of sympathovagal interaction after myocardial infarction. Am J Cardiol 1987; 60:1239-1245.

[16] Task Force of the European Society of Cardiology and the North American Society of Pacing and Electrophysiology. Standards of measurement, physiological interpretation and clinical use. Circulation 1996; 93:1043-1065.

[17] Modarreszadeh M, Bruce EN, Gothe B. Nonrandom variability in respiratory cycle parameters of humans during stage 2 sleep. J Appl Physiol 1990; 69:630-639.

[18] Casaseca-de-la-Higuera P, Martin-Fernandez M, Alberola-Lopez C. Weaning from mechanical ventilation: a retro-spective analysis leading to a multimodal perspective. IEEE Trans Biomed Eng 2006; 53:1330-1345.

[19] Pincus SM, Gladstone IM, Ehrenkranz RA. A regularity statistic for medical data analysis. J Clin Monit 1991; 7:335-345.

[20] Pincus SM, Goldberger AL. Physiological time-series: what does regularity quantify. Am J Physiol Heart Circ Physiol 1994; 266:1643-1645.

[21] Richmann JS, Moorman JR. Physiological time-series analysis using approximate entropy and sample entropy. Am J Physiol Heart Circ Physiol 2000; 278:2039-2049.

[22] Webber CL, Zbilut JP. Dynamical assessment of physiological systems and status using recurrence plot strategies. J Appl Physiol 1994; 76:965-973.

[23] Kamen PW, Tonkin AM. Application of the Poincaré plot to heart rate variability: a new measure of functional status in heart failure. Aust NZ J Med 1995; 25:18-26.

[24] Suki B. Fluctuations and power laws in pulmonary physiology. Am J Respir Crit Care Med 2002; 166:133-137.

[25] West BJ, Goldberger AL. Physiology in fractal dimensions. Am Sci 1987; 75:354-365.

[26] Seeley A, Macklem P. Complex systems and the technology of variability analysis. Critical Care 2004; 8: R367-R384 (doi: 10.1186/cc2948).

[27] Goldberger AL. Non-linear dynamics for clinicians: chaos theory. Fractals and complexity at the bedside. Lancet 1996; 347:1312-1314. 
[28] Thamrin C, Stern G, Frey U. Fractals for physicians. Ped Respir Review 2010; 11:123131.

[29] Glenny RW, Robertson HT, Yamashiro S, Bassingthwaighte JB. Applications of fractal analysis to physiology. J Appl Physiol 1991; 70:2351-2367.

[30] Goldberger AL, Peng CK, Lipsitz LA. What is physiologic complexity and how does it change with aging and disease? Neurobiology of aging 2002; 23:23-26.

[31] Koshiya N, Smith JC. Neuronal pacemaker for breathing visualized in vitro. Nature 1999; 400:360-363.

[32] Del Negro CA, Wilson CG, Butera RJ, et al. Periodicity, mixed-mode oscillations and quasiperiodicity in a rhythm-generating neural network. Biophysical Journal 2002; 82:206-214.

[33] Chen X, Chon KH, Solomon IC. Chemical activation of pre-Botzinger complex in vivo reduces respiratory network complexity. Am J Physiol Regul Integr Comp Physiol 2005; 288:1237-1247.

[34] Mead M, Guyatt G, Cook D, et al. Predicting success in weaning from mechanical ventilation. Chest 2001; 120:400-424.

[35] Jubran A, Tobin MJ. Pathophysiologic basis of acute respiratory distress in patients who fail a trial of weaning from mechanical ventilation. Am J Respir Crit Care Med 1997; 155:906-915.

[36] Jubran A, Grant BJ, Tobin MJ. Effect of hyperoxic hypercapnia on variational activity of breathing. Am J Respir Crit Care Med 1997; 156:1129-1139.

[37] Fiamma MN, Straus C, Thibault S, et al. Effects of hypercapnia and hypocapnia on ventilatory variability and chaotic dynamics of ventilatory flow in humans. Am J Physiol Regul Integr Comp Physiol 2007; 292:1985-1993.

[38] Sako T, Burioka N, Suyama H, et al. Nonlinear behaviour of human respiratory movement during different sleep stages. Chronobiol Int 2001; 18:71-83.

[39] Yeragani VK, Radhakrishna RK, Tancer M, et al. Nonlinear measures of respiration: respiratory irregularity and increased chaos of respiration in patients with panic disorder. Neuropsychobiology 2002; 46:111-120.

[40] Sammon MP, Bruce EN. Vagal afferent activity increases dynamical dimension of respiration in rats. J Appl Physiol 1991; 70:1748-1762.

[41] Daubenspeck JA. Influence of small mechanical loads on variability of breathing pattern. J Appl Physiol 1981; 50: 299-306.

[42] Weinberger SE, Schwartzstein RM, Weiss JW. Hypercapnia. N Engl J Med 1989; 321:1223-1231.

[43] Preas HL, Jubran A, Vandivier RW, et al. Effect of endotoxin on ventilation and breath variability. Am J Respir Crit Care Med 2001; 164:620-626.

[44] Georgopoulos D, Bshouty Z, Younes M, et al. Hypoxic exposure and activation of the after-discharge mechanism in conscious humans. J Appl Physiol 1990; 69:1159-1164.

[45] DeLaCadena RA, Suffredini AF, Page JD, et al. Activation of the kallikrein-kinin system after endotoxin administration to normal human volunteers. Blood 1993; 81:3313-3317.

[46] West GB, Brown JH, Enquist BJ. The fourth dimension of life: fractal geometry and allometric scaling of organisms. Science 1999; 284:1677-1679. 
[47] Weibel ER, Gomez DM. Architecture of the human lung. Use of quantitative methods establishes fundamental relations between size and number of lung structures. Science 1962; 137:577-585.

[48] Mandelbrot BB. The fractal geometry of nature. New York: W.H. Freeman; 1977.

[49] Glenny RW, Polissar NL, Mckinney S, Robertson HT. Temporal heterogeneity of regional pulmonary perfusion is spatially clustered. J Appl Physiol 1995; 79:986-1001.

[50] Rigaut JP. An empirical formulation relating boundary lengths to resolution in specimens showing 'non-ideally fractal' dimensions. J Microsc 1984; 133:41-54.

[51] Suki B, Barabasi AL, Hantos Z, Petak F, Stanley HE. Avalanches and power-law behaviour in lung inflation. Nature 1994; 368:615-618.

[52] West BJ. Physiology in fractal dimensions: error tolerance. Ann Biomed Eng 1990; 18:135-149.

[53] Peng CK, Mietus JE, Liu Y, Lee C, Haudorff JM, Stanley HE, et al. Quantifying fractal dynamics of human respiration: age and gender effects. Ann Biomed Eng 2002; 30:683692.

[54] Prakash KN, Ramakrishnan AG, Suresh S, Chow TW. Fetal lung maturity analysis using ultrasound image features. IEEE Trans Inf Technol Biomed 2002; 6:38-45.

[55] Szeto HH, Cheng PY, Decena JA, Cheng Y, Wu DL, Dwyer G. Fractal properties in fetal breathing dynamics. Am J Physiol 1992; 263:141-147.

[56] Mackey MC, Glass L. Oscillation and chaos in physiological control systems. Science 1977; 197:287-289.

[57] Peng CK, Havlin S, Stanley HE, Goldberger AL. Quantification of scaling exponents and crossover phenomena in nonstationary heartbeat time series. Chaos 1995; 5:82-87.

[58] Penzel T, Kantelhardt JW, Grote L, Peter JH, Bunde A. Comparison of detrended fluctuation analysis and spectral analysis for heart rate variability in sleep and sleep apnea. IEEE Trans Biomed Eng 2003; 50:1143-1151.

[59] Macklem PT. Can airway function be predicted? Am J Respir Crit Care Med 1996; 153:19-20.

[60] Que CL, Kenyon CM, Olivenstein R, Macklem PT, Maksym GN. Homeokinesis and short-term variability of human airway caliber. J Appl Physiol 2001; 91:1131-1141.

[61] Frey U, Brodbeck T, Majumdar A, Taylor DR, Town GI, Silverman M, et al. Risk of severe asthma episodes pre-dicted from fluctuation analysis of airway function. Nature 2005; 438:667-670.

[62] Boser S, Park H, Perry S, Menache M, Green F. Fractal geometry of airway remodelling in human asthma. Am J Respir Crit Care Med 2005; 172:817-823.

[63] Venegas JG, Schroeder T, Harris S, Winkler RT, Melo MF. The distribution of ventilation during bronchoconstriction is patchy and bimodal: a PET imaging study. Respir Physiol Neurobiol 2005; 148:57-64.

[64] Venegas JG, Winkler T, Musch G. Self-organized patchiness in asthma as a prelude to catastrophic shifts. Nature 2005; 434:777-782.

[65] Frey U, Suki B. Complexity of chronic asthma and chronic obstructive pulmonary disease: implications for risk assessment, and disease progression and control. Lancet 2008; 372:1088-1099. 
[66] Sujeer MK, Buldyrev SV, Zapperi S, Andrade JS, Stanley HE, Suki B. Volume distributions of avalanches in lung inflation: a statistical mechanical approach. Phys Rev E 1997; 56:3385-3394.

[67] Martynowicz MA, Walters BJ, Hubmayr RD. Mechanisms of recruitment in oleic-acid injured lungs. J Appl Physiol 2001; 90:1744-1753.

[68] Gattinoni L, Dandrea L, Pelosi P, Vitale G, Pesenti A, Fumagalli R. Regional effects and mechanism of positive end-expiratory pressure in early adult respiratory distress syndrome. JAMA 1993; 269:2122-2127.

[69] Mishima M, Hirai T, Itoh H, Nakano Y, Sakai H, Muro S, et al. Complexity of terminal airspace geometry assessed by lung computed tomography in normal subjects and patients with chronic obstructive pulmonary disease. Proc Natl Acad USA 1999; 96:8829-8834.

[70] Mutch WA, Harms S, Ruth Graham M, Kowalski SE, Girling LG, Lefevre GR. Biologically variable or naturally noisy mechanical ventilation recruits atelectatic lung. Am J Respir Crit Care Med. 2000; 162:319-323.

[71] Suki B, Alencar AM, Sujeer MK, Lutchen KR, et al. Life-support system benefits from noise. Nature 1998; 393:127-128.

[72] Engoren M. Approximate entropy of respiratory rate and tidal volume during weaning from mechanical ventilation. Crit Care Med 1998; 26:1817-1823.

[73] Bigger JT, Fleiss JL, Steinman RC, et al. Frequency domain measures of heart period variability and mortality after myocardial infarction. Circulation 1992; 85:164-171.

[74] Goldstein B, Kepmpski MH, Stair D. Autonomic modulation of heart rate variability during endotoxin shock in rabbits. Critical Care Med 1995; 23:1694-1702.

[75] El Khatib M, Jamaleddine G, Soubra R, et al. Pattern of spontaneous breathing: potential marker for weaning outcome. Intensive Care Med 2001; 27:52-58.

[76] Grassberger P, Procaccia I. Characterization of strange attractors. Phys Rev Lett 1983; 50:346-349.

[77] Bien MY, Hseu SS, Yien HW, et al. Breathing pattern variability: a weaning predictor in postoperative patients re-covering from systemic inflammatory response syndrome. Intensive Care Med 2004; 30:241-247.

[78] Wysocki M, Cracco C, Teixeira A, et al. Reduced breathing variability as a predictor of unsuccessful patient separation from mechanical ventilation. Crit Care Med 2006; 34:2076-2083.

[79] Caminal P, Domingo L, Giraldo BF, et al. Variability analysis of the respiratory volume based on non-linear pre-diction methods. Med Biol Eng Comput 2004; 42:86-91.

[80] Brochard L. Breathing: does regular mean normal? Crit Care Med 1998; 26:1773-1774.

[81] Papaioannou V, Chouvarda I, Maglaveras N, Dragoumanis C, Pneumatikos I. Changes of heart and respiratory rate dynamics during weaning from mechanical ventilation: a study of physiologic complexity in surgical critically ill patients. J Crit Care 2011; 26(3):262-272.

[82] Costa M, Goldberger AL, Peng CK. Multiscale entropy analysis of biological signals. Phys Rev E Stat Nonlin Soft Matter Phys 2005; 71; 021906-1-18. 
[83] Papaioannou V, Chouvarda I, Maglaveras N, Pneumatikos I. Study of multiparameter respiratory pattern complexity in surgical critically ill patients during weaning trials. BMC Physiol 2011; 11 (1): 2, doi: 10.1186/1472-6793-11-2.

[84] Higuchi T. Approach to an irregular time series on the basis of the fractal theory. Physica D 1988; 31:277-283.

[85] Rosenstein M, Collins J, De Luca C. A practical method for calculating largest Lyapunov exponents for small data sets. Physica D 1993; 65:117-134.

[86] Vallverdu M, Tibaduisa O, Claria F, Hoyer D, Giraldo B, Benito S, Caminal P. Information flow to assess cardio-respiratory interactions in patients on weaning trials. Conf Proc IEEE Eng Med Biol Soc 2006; 1:1462-1465.

[87] Schmidt M, Demoule A, Cracco C, et al. Neurally adjusted ventilatory assist increases respiratory variability and complexity in acute respiratory failure. Anesthesiology 2010; 112:670-681.

[88] Mangin L, Fiamma MN, Straus C, et al. Source of human ventilatory chaos: lessons from switching controller mechanical ventilation to inspiratory pressure support in critically ill patients. Respiratory Physiology \& Neurobiology 2008; 161:189-196.

[89] Burykin A, Buchman TG. Cardiorespiratory dynamics during transition between mechanical and spontaneous ventilation in intensive care. Complexity 2008; 13 (6):4059.

[90] Macklem PT. Emergent phenomena and the secrets of life. J Appl Physiol 2008; 104:1844-1846.

[91] Goldberger AL, Amaral LAN, Glass L, et al. PhysioBank, PhysioToolkit, and PhysioNet: Components of a new research resource for complex physiologic signals. Circulation 2000; 101:215-220. 


\title{
Wavelet Filter to Attenuate the Background Activity and High Frequencies in EEG Signals Applied in the Automatic Identification of Epileptiform Events
}

\author{
Geovani Rodrigo Scolaro, Fernando Mendes de Azevedo, \\ Christine Fredel Boos and Roger Walz
}

Additional information is available at the end of the chapter

http://dx.doi.org/10.5772/53585

\section{Introduction}

Epilepsy is a chronic brain disease of unknown etiology, characterized by the occurrence of unprovoked epileptic seizures, causing brief disturbances in the normal activity of the brain [1]. In the epilepsy diagnosis process the visual analysis of Electroencephalographic signals (EEG) is still widely used by specialists. Its importance in diagnosis is due to the fact that you can get information about the patient's condition, recording the disturbance caused by epileptiform neuronal dysfunction, in the period that the patient is asymptomatic (without seizures) [2]. The EEG can also be used to help define the type of epileptic syndrome, provide information for planning of drug therapy and also to help in deciding the feasibility of surgery [3]. The most common elements in EEG signals of epileptic patients and important in the diagnosis of epilepsy are the spikes and sharp waves. The primary morphological difference between these two types of events is the duration that each presents. The spikes have durations between 40 and $80 \mathrm{~ms}$. The sharp waves have a duration between 80 and 200ms [3-4]. The process of reviewing the records of EEG is performed by trained and experienced specialists. However, this process is still a daunting task. The routine EEG records have durations between 20 and 40 minutes and are recorded from 21 to 32 channels, displayed in screen with 10 to 15 seconds each [3]. Few computer systems for automatic review of EEG have practical application. Many of these tend to identify a relatively large number of non-epileptiform events as positive, resulting in little or no effective economy of time [5]. Among the various non-epileptiform paroxysms, which generate more false positives in the automated detection of epileptiform events stand out 
high frequency noise, the alpha waves and especially the eyelid blinks (Figure 1). These patterns often occur in the EEG signals and they have characteristics similar to spikes and sharp waves being confused with epileptiform events.

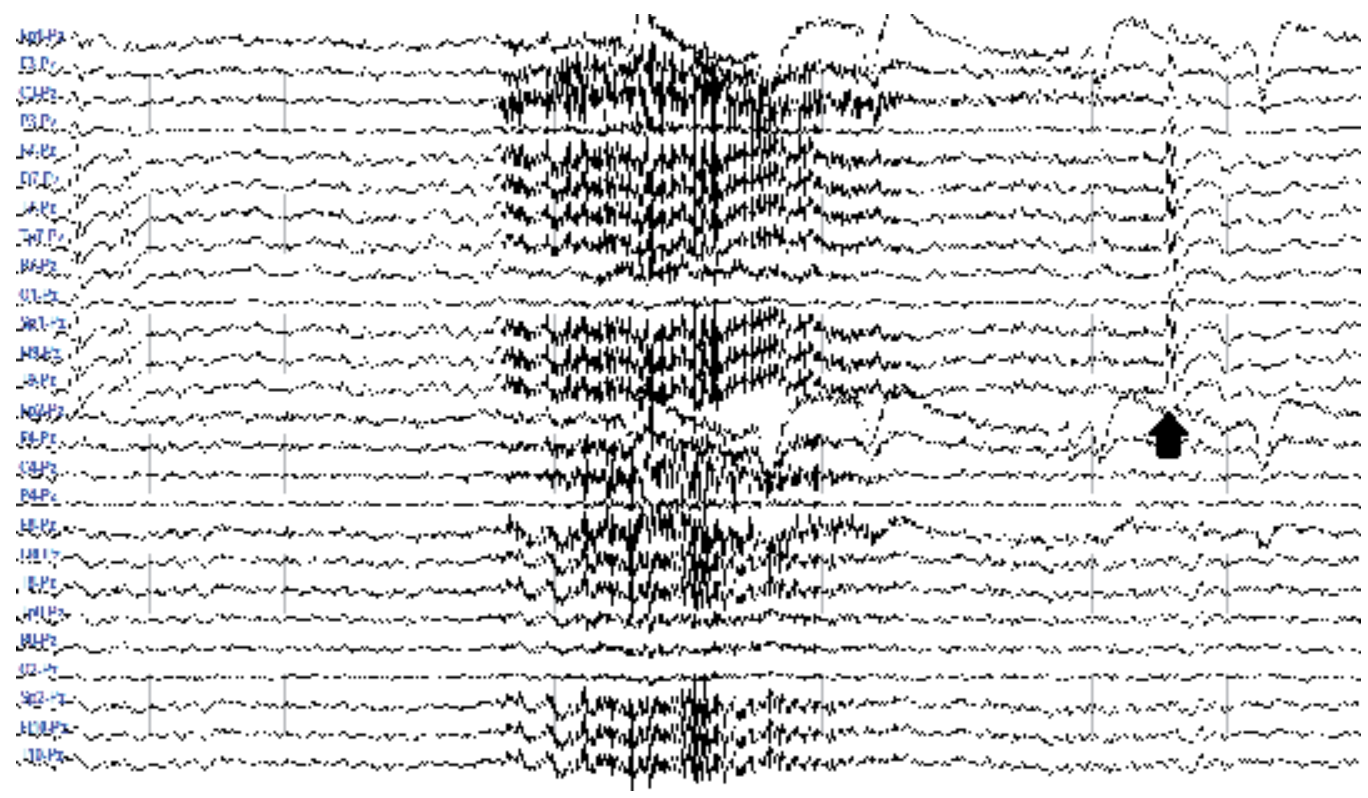

Figure 1. EEG screen with high frequency noise, eyelid blinks and epileptiform events (black arrow).

The low specificity of automated systems occurs due to variations in the EEG signals from patient to patient. This variation also occurs in the own patient due to different states of consciousness and behavior at the time of acquisition of these signals. Thus, it is difficult to establish a computational model of the epileptiform paroxysms, that can differentiate it from other activities present in the EEG.

The sensitivity of such systems can also be severely compromised by poor quality in the acquisition of an EEG signal. This is due to the large number of existing sources of artifacts. Despite this, most of the proposed automated systems fail to demonstrate they have achieved a rate of false positives per minute (FP / $\mathrm{min}$ ) acceptable [5].

This work will contribute to the automating process of the epilepsy diagnosis with a digital filter proposal based on Wavelet Transform, checking its feasibility of use to process the EEG signals.

\section{Material and methods}

\subsection{Bank of EEG signals}

The bank of EEG signals is composed by records of 11 patients truly epileptic. The used signals present the following settings: referential montage with $\mathrm{Pz}$ as the reference electrode, 32 channels, $512 \mathrm{~Hz}$ of sample rate, band limited $0.3-70 \mathrm{~Hz}$ and notch filter of $60 \mathrm{~Hz}$ to 
eliminate interferences caused by the power line. For the experiments were selected 600 events between spikes and sharp waves.

\subsection{Wavelet multiresolution analysis}

The analysis in time-frequency domain by Wavelet Transform is performed by taking a Wavelet prototype function called Mother-Wavelet. This Mother-Wavelet suffers dilations and translations, forming the Daughter-Wavelets (1) [6-7].

$$
\psi_{a, b}(t)=\frac{1}{\sqrt{a}} \cdot \psi\left(\frac{t-b}{a}\right)
$$

where $\psi(t)$ is the Mother-Wavelet and $\psi_{a, b}$ is the Daughter-Wavelet, $a^{-1 / 2}$ is the constant of energy normalization, $b$ is the translation factor and $a$ is the dilation factor.

The Continuous Wavelet Transform uses continuous parameters of time and scales [6]. Using discrete parameters to $a$ and $b(a \geq 1, b \geq 1)$ determines the Discrete Wavelet Transform (2).

$$
D W T(a, b)=\frac{1}{\sqrt{a_{0}^{i}}} \int_{-\infty}^{\infty} x(t) \cdot \psi^{*}\left(\frac{t-k b_{0} a_{0}^{i}}{a_{0}^{i}}\right) d t
$$

where $k$ and $i$ are integers, $b_{0}$ and $a_{0}$ are the parameters of translation and dilation, respectively.

The Wavelet Multiresolution Analysis is based in the computational implementation of the Discrete Wavelet Transform. The algorithm decomposes a discrete signal using filter banks, [6-8]. The set of filters $\mathrm{H}[\mathrm{n}]$ extract the average characteristics, defined as approximations of the signal $x$ and added to a set of filters G[n] extract the features of high-frequency defined as details of the signal $x[n]$ (Figure 2).

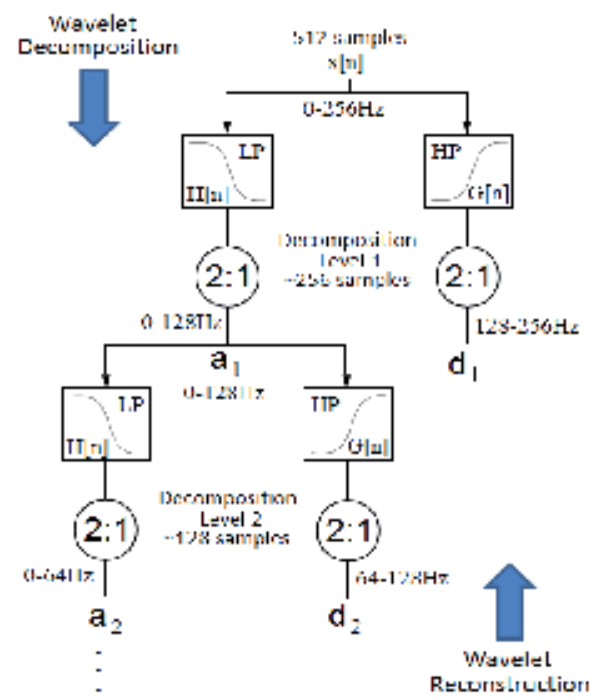

Figure 2. Representation of the Wavelet Multiresolution Analysis. 


\subsubsection{Filtering EEG signals using the denoising method}

The denoising method is a resource used in the decomposition process of the Wavelet Transform. This method is used to filter the signal through the manipulation of its coefficients in the Wavelet domain, before the reconstruction of the signal as shown in Figure 3.
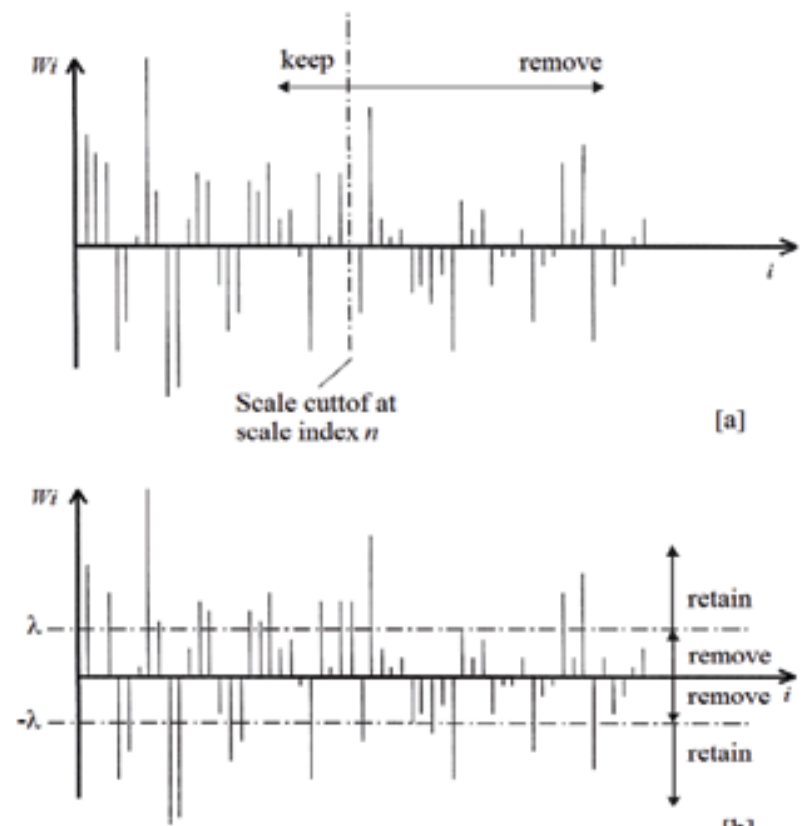

[b]

Figure 3. Representation of some ways to suppress coefficients in the Wavelet domain used to process a signal.

Once the full decomposition process was done from a particular Wavelet function it is possible to alter any of the coefficients in the transformed signal before performing the inverse transform (Figure 4).

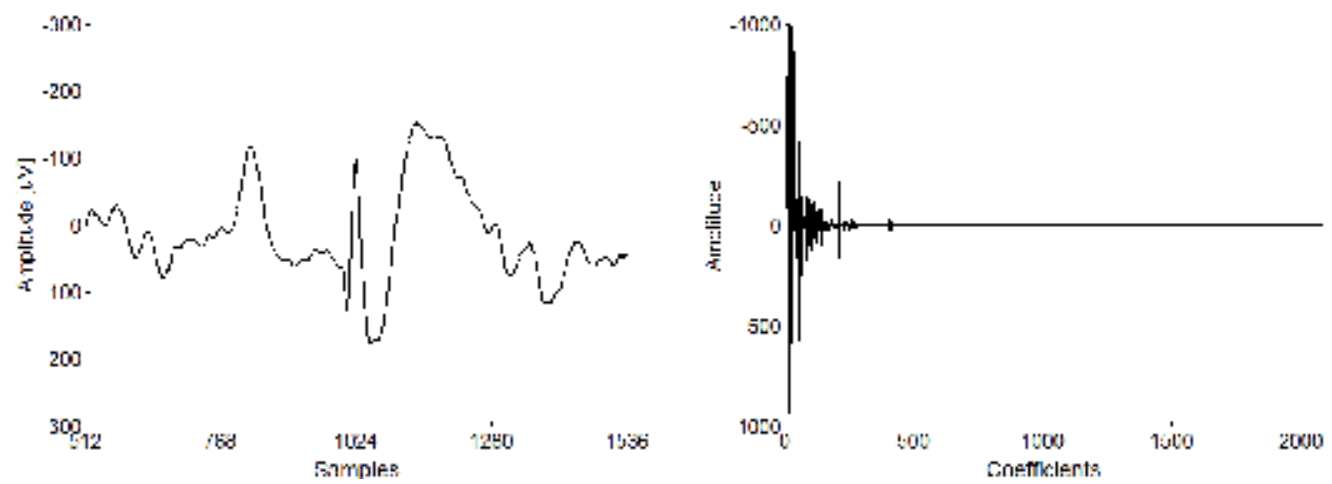

Figure 4. Original signal and the decomposed signal in the Wavelet domain. 
It can be possible to manipulate the coefficients in a variety of ways depending of the application [7]. For example, it is possible set groups or individual coefficients to zero from a specific scale (Figure 5[a]), increase or reduce the magnitude of them or even to choose some coefficients under or above a specific threshold $(\lambda)$ and set its values to zero or other value (Figure $5[b]$ ). Performing some of these ways it can be possible to use the Wavelet Transform with a digital filter. Further information see [7-10]. To demonstrate the behavior of a signal processed by denoising method was used an event epileptiform decomposed into six levels, generating the levels A6 and D6, D5, D4, D3, D2, D1 (Figure 5[a]). For better visualization the original signals (in black) and the processed signals (in blue) were superimposed, as a way to contrast the changes made between them. In Figure 5[a] is presented the first form of filtering selecting a given level of decomposition. The level A6 was removed from the decomposed signal, setting zero values to the corresponding coefficients (begining of signal to the red mark). Eliminating this level of approximation, the lower frequency of the signal will also be eliminated after its reconstruction. The signal resulting from this process (blue) compared with the original signal (black) shows a reduction of low frequency oscillations, highlighting the peak of the epileptiform event.In Figure $5[\mathrm{~b}]$ is presented the second form of filtering resulting from choice of a particular decision threshold (in this case four standard deviations). Removing the coefficients
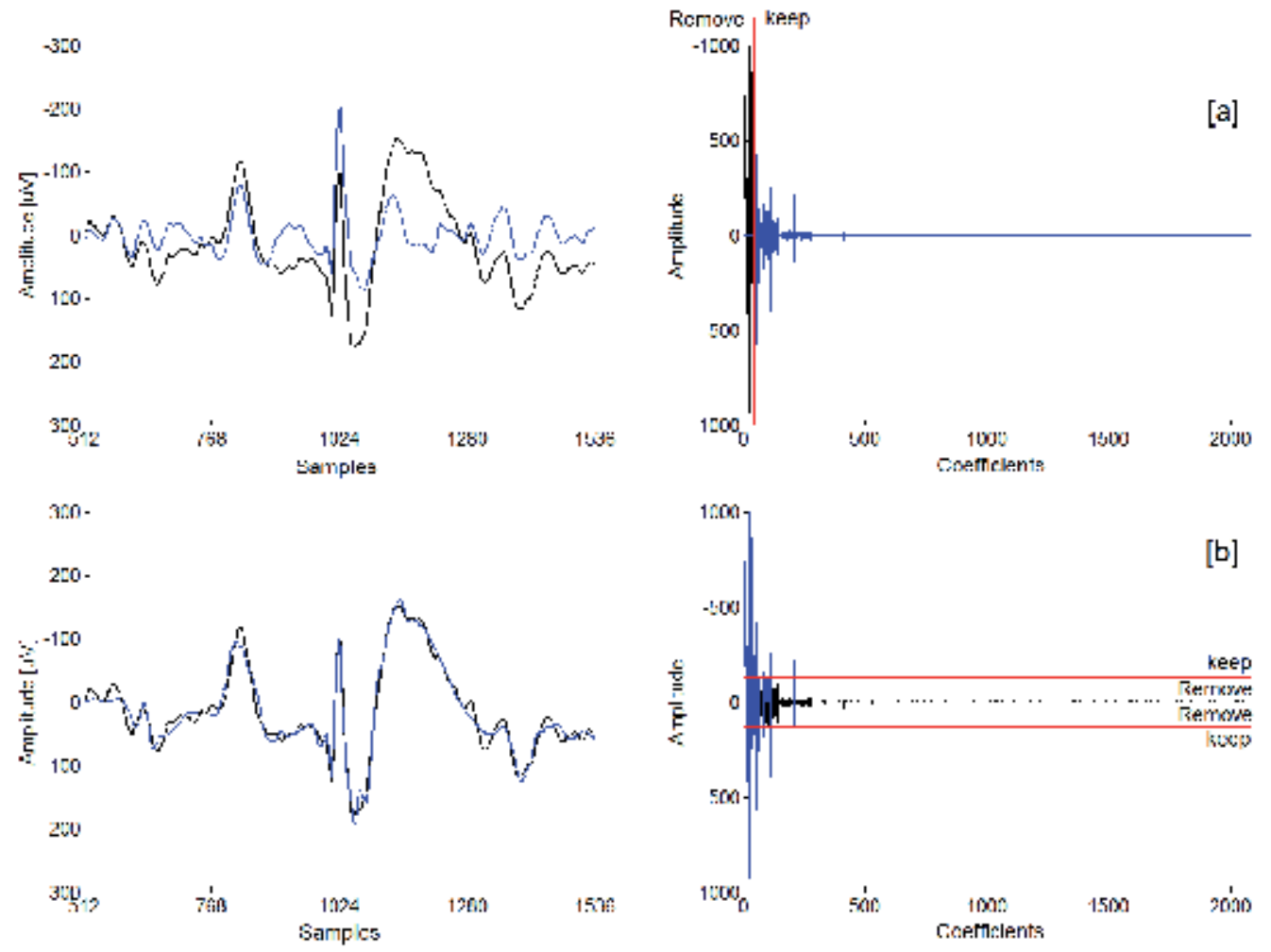

Figure 5. Denoising method to filtering EEG signals. In [a] decomposition using scales, [b] decomposition using a threshold. 
between the thresholds (marked in red) and replacing their values by zero, the high frequencies of the signal also change. High frequencies are increasingly attenuated as the threshold increases, making this a low-pass filter. Removing the coefficients that exceed the defined threshold, the low frequencies of the signal will be attenuated, making this process a high-pass filter. The filter proposed here is based on the first form of filtering, where the unnecessary frequencies of the EEG signals are eliminated, manipulating specific decomposition levels of the signal, which will be better explained in the next section.

\section{Methodology}

\subsection{Proposal for a filter to processing EEG signals using the wavelet transform}

An interesting feature of the Wavelet Transform, observed through the experiments, is that this tool when used properly has the capability to act as a digital filter. Through Figure 6 can be observed the stages of decomposition and reconstruction of a signal. In this example, the signal is decomposed and reconstructed into 6 levels, generating an approximation level A6 and six levels of detail, D6, D5, D4, D3, D2 and D1. The decomposed signal contains all frequency components of the original signal, grouped by level of decomposition.

When a signal is reconstructed from a specific level of approximation or detail, only the frequencies that covers this level in particular will be used to generate this signal. In other words, we have specific bands of the original signal, fragmented in secondary reconstructed signals (A6, D6, D5, D4, D3, D2 and D1). Performing the sum between the reconstructed signals, the original signal is obtained. On the other hand, performing the difference between them, a signal without especific frequencies is obtained. Based on these considerations a digital filter was designed using the Wavelet Transform for preprocessing the EEG signals, which may be used in the localization and identification process of epileptiform events.

In the initial experiments with the epileptiform events the signals were decomposed and reconstructed into six levels, generating the level of approximation A6 and levels of detail D1 to D6. Each of detail levels was analyzed individually. Some peculiarities were observed as the morphology of the generated signal, the amplitude and repeatability of the detail level that produces the largest number of reconstructed signals, morphologically similar to the original signal. We observed a repeating pattern in the level of detail D4, D5 and D6, which coincide with the frequency band defined for the epileptiform events. The experiments showed that the low frequencies of the original signal were retained in the approximation A6 (0-4 Hz). The frequency range of the original signal, which are not of interest including the frequency of $60 \mathrm{~Hz}$, were retained in detail D1 to D3 $(256-32 \mathrm{~Hz})$. These observations suggest that, when performing an addition operation between the reconstructed signals of the details D4, D5 and D6, we obtain a signal free of interference. This has been confirmed later with some experiments. The resulting signal from this process present only the frequencies between 4 and $32 \mathrm{~Hz}$. This band of frequencies include the frequencies of the epileptiform events (from 5 to $25 \mathrm{~Hz}$ ), so the signal generated has all the frequencies required for proper reconstruction of epileptiform events without the frequencies tha include the noises (Figure 7). 


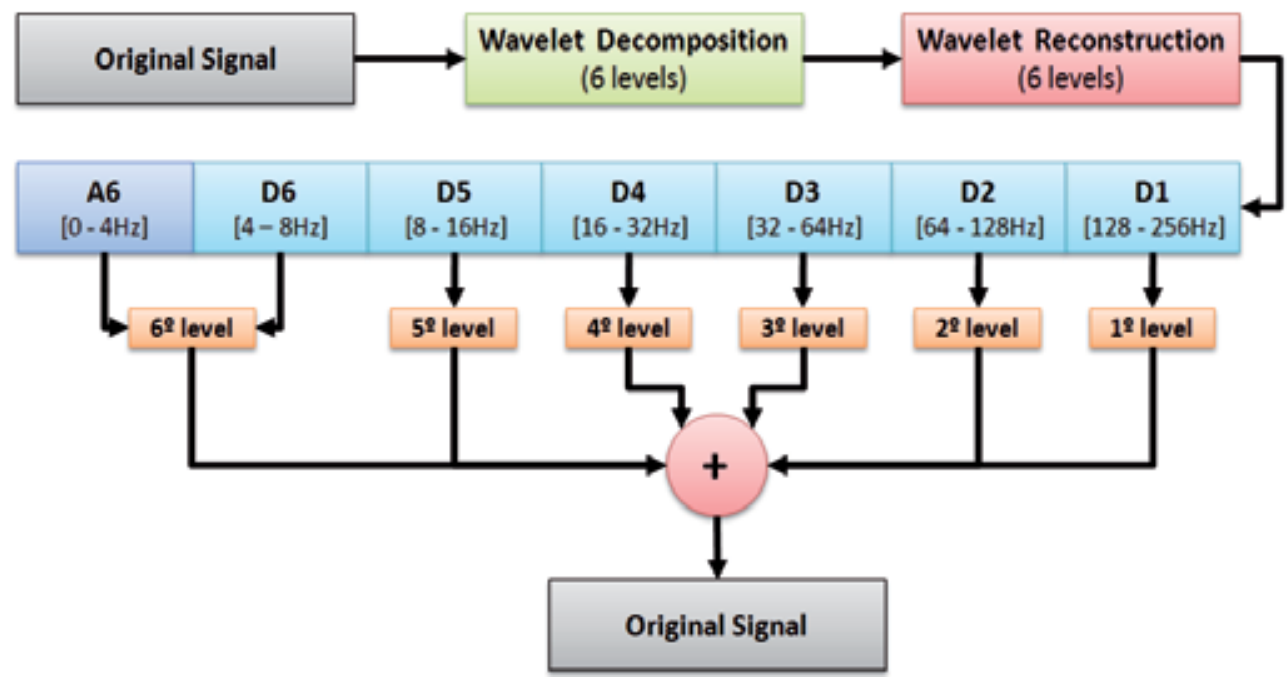

Figure 6. Process of decomposition and reconstruction of a signal.

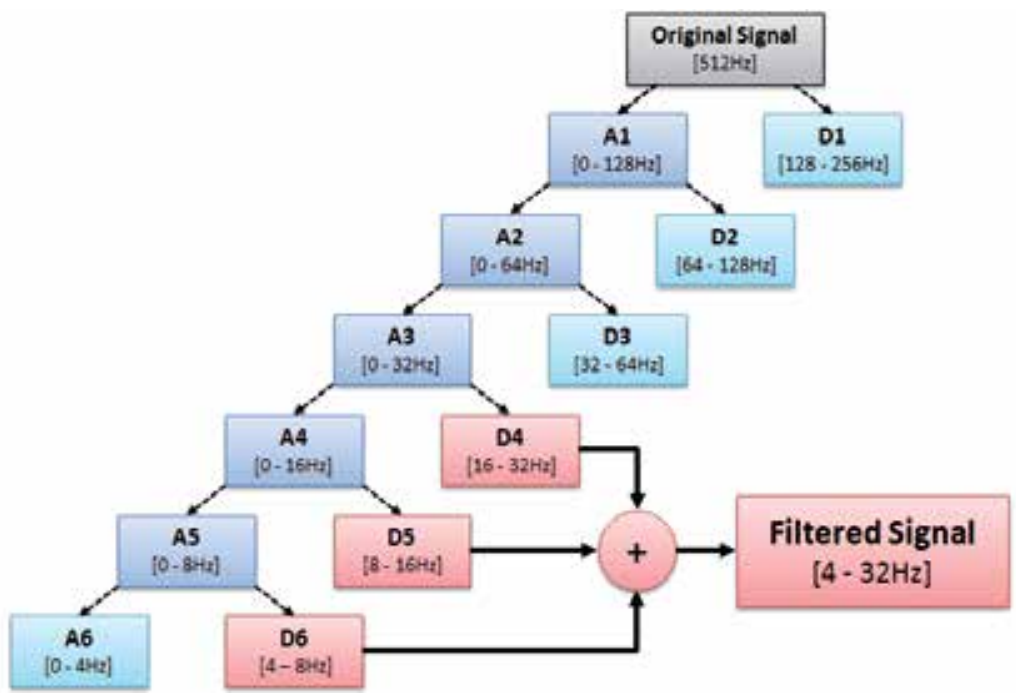

Figure 7. Proposal for a Wavelet filter using only the details of the decomposed signal. This filter is proposed performing an addition operation between levels of detail to obtain a signal free from interference, like the baseline and low frequency oscillations, as well as high frequency interference, including the $60 \mathrm{~Hz}$ noise

\subsection{Choice of the wavelet function to use with the proposed filter}

In this work the Wavelet Transform is employed as a digital filter. Thus, it was decided to make an inquiry to find the most appropriate Wavelet function for use with the filter, that contain few coefficients, and little distortion of the signal after filtering process. In initial experiments we used the energy of each Wavelet function as a criterion of choice. The function that had the highest average of accumulated energy and a reduced number of 
coefficients would be the chosen function. The experiment consisted of applying a set of epileptiform events in known Wavelet functions. We used the Wavelet function families: Daubechies, Symlets, Coiflets, Biortogonal and Reverse Biortogonal, totaling 65 functions. The set of signals contains 600 epileptiform events with different durations (Figure 8).

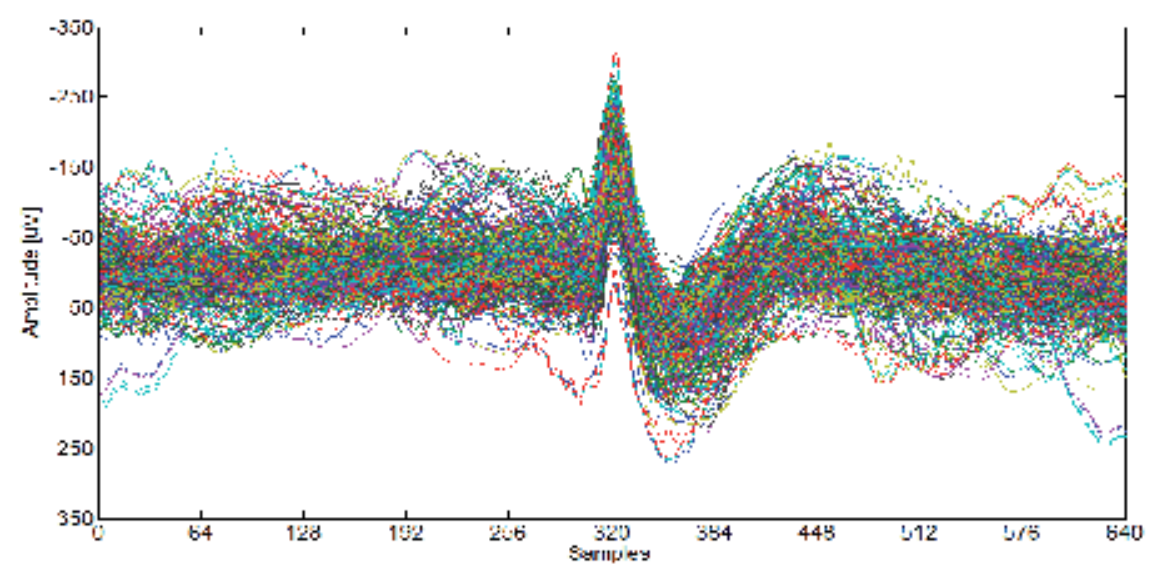

Figure 8. Representation of the selected events for the choice of the Wavelet function to be used in the filtering of signals.

All epileptiform events were applied to each of the Wavelet functions selected for this study. It was calculated energy of each decomposed epileptiform event, which were accumulated and used for obtaining the energy average of each Wavelet function used. For the tests with the proposed filter were chosen 10 functions with the highest cumulative average energy. The values obtained are shown in Table 1 .

\begin{tabular}{lll}
\hline Wavelet function & Coefficients & Energy [\%] \\
\hline bior3.1 & 4 & 89.57 \\
sym14 & 28 & 79.46 \\
sym15 & 30 & 79.40 \\
sym13 & 26 & 79.34 \\
sym12 & 24 & 77.45 \\
coif5 & 30 & 76.53 \\
rbio3.9 & 20 & 76.49 \\
rbio2.8 & 18 & 75.51 \\
sym10 & 20 & 75.18 \\
sym11 & 22 & 75.16 \\
\hline
\end{tabular}

Table 1. Visualization of 10 Wavelet functions with highest energy values and their respective number of coefficients for the epileptiform events used.

To test the obtained function was used a epoch of EEG signal, containing an epileptiform event, low frequency oscillations, high frequency and noise. This signal was applied in four functions from Table 5 and the filtered signals are presented in Figure 9. 


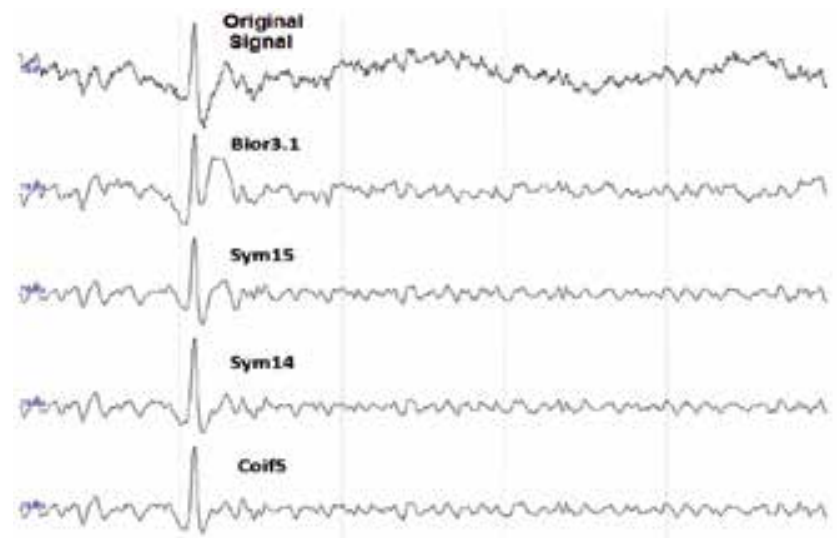

Figure 9. Examples of Wavelet filtering with some functions from table 5.

The results showed that the low frequency oscillations and high frequency noise present in the original signal was attenuated, highlighting only the epileptiform events. However, depending on the Wavelet function used the event presents distinct forms due to the characteristics of each Wavelet function. Other experiments were conducted to find the Wavelet function that most preserve the morphology of epileptiform events after processing, but also attenuate satisfactorily the low frequency oscillations and high frequency noise. In order to evaluate these changes between the original signals and the filtered signals were computed the associated error with the alteration in the morphology of the signals processed by the Wavelet filtering through the correlation index and the root mean square error (RMSE) between each original epileptiform event and the filtered ones.

\subsubsection{Calculation of correlation and root mean square errors}

For this experiment were analyzed the signal epoch of 1s, the epileptiform event (spike and slow-wave complex) and the isolated peak (spike only). These three types of signals are represented by Figure 10.

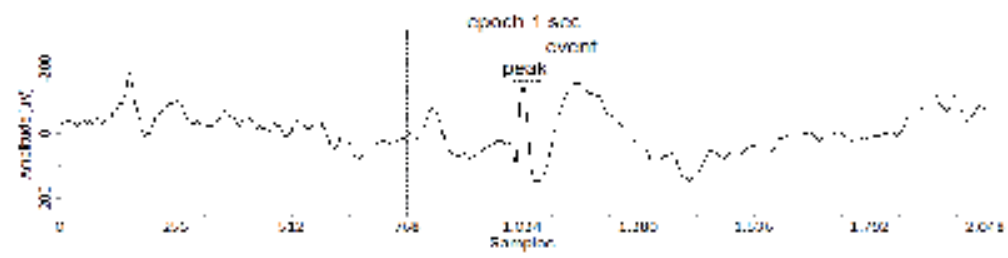

Figure 10. Representation of the categories defined for the analysis of the Wavelet functions.

All of the events selected were applied in each of the Wavelet functions, where were calculated the RMSE and the correlations for each of the categories defined above. Figure 11 presents the procedure performed with the Wavelet functions selected for calculating the RMSE and the correlation between the original signals and the filtered ones. Only for illustrative purposes were used the functions DB4 and RBio2.8, which were identified as the most appropriate functions for the filter proposed. 

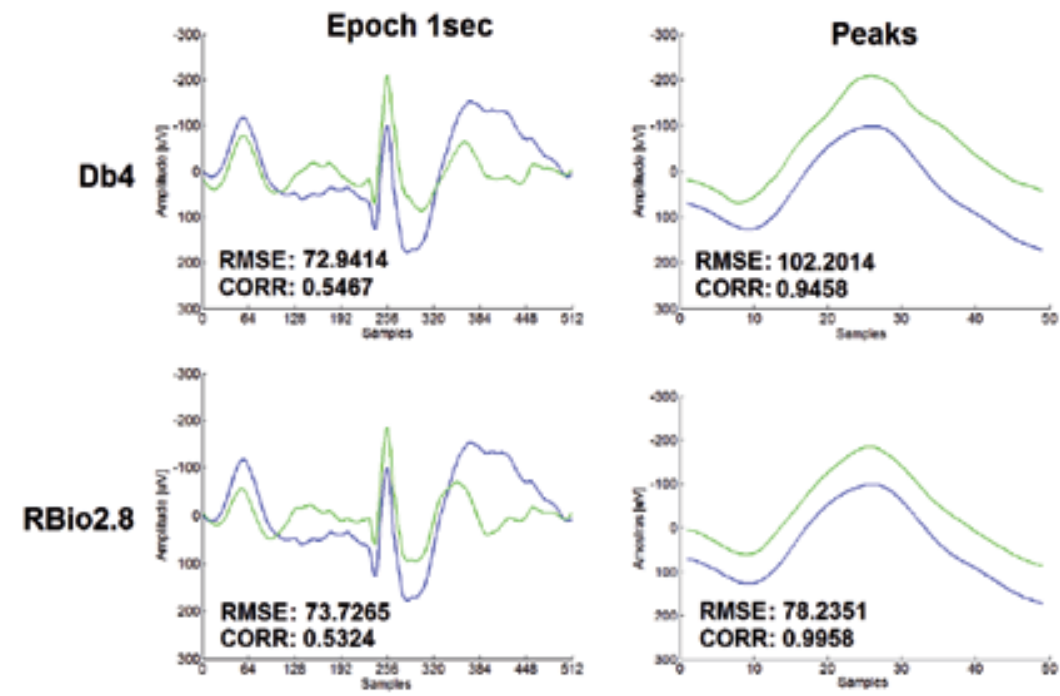

$\square$ Original Signal

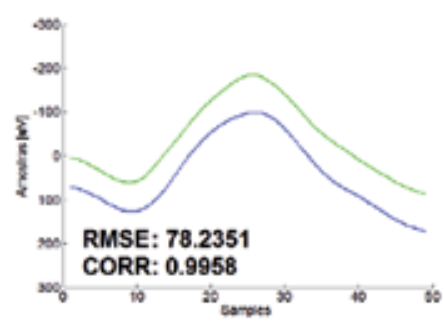

$\square$ Filtered Signal

Figure 11. Analysis representation of the epochs of $1 \mathrm{~s}$ signal and the event peak.
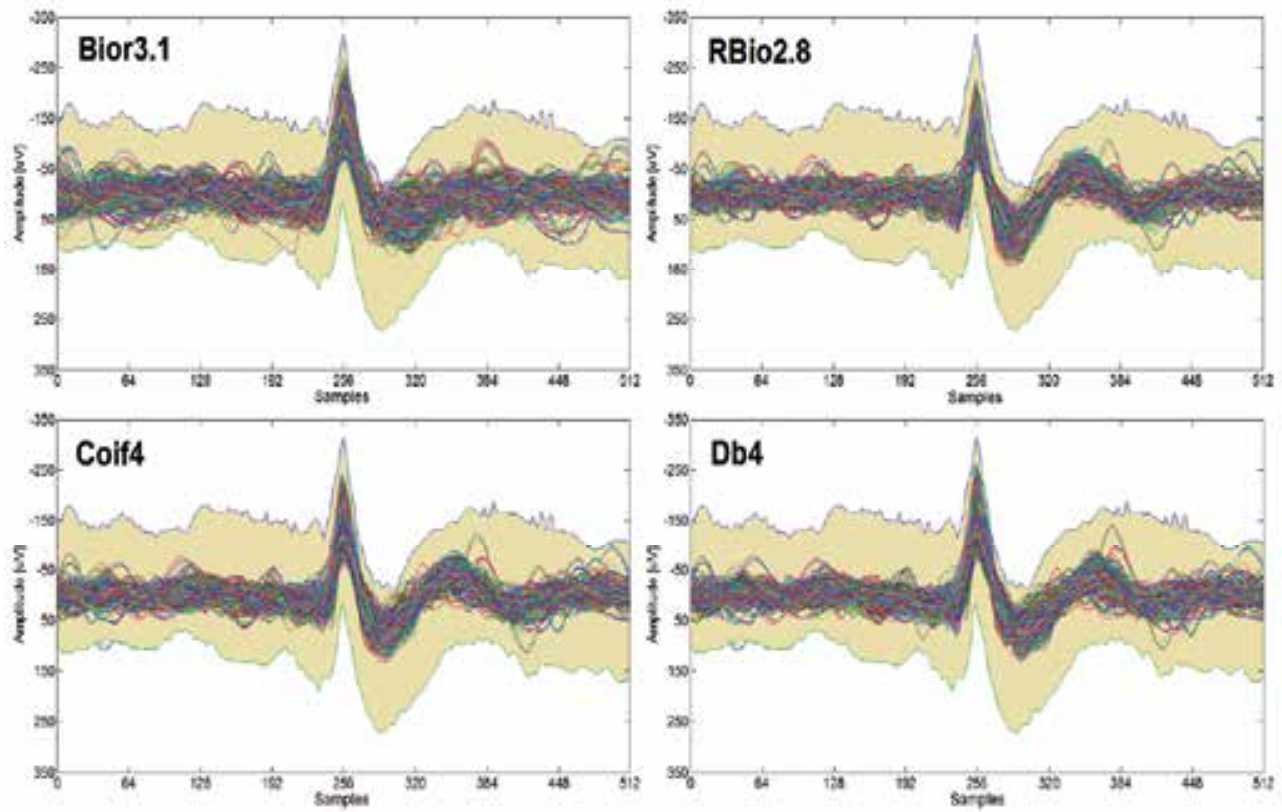

Figure 12. Demonstration of the Wavelet filter with the four obtained Wavelet functions, showing all epileptiform events after processing with the filter, contrasting with the original signals in the background. 
After calculation of the RMSE and correlations were obtained three other Wavelet functions, which were considered appropriate for use with the proposed filter. These functions are Rbio2.8, Coif4 and DB4. These functions were chosen because they presented the highest values of correlation and the lowest RMSE in all of the experiments carried out by category. Figure 12 presents a demonstration of before and after the filtering, performed for each one of the obtained Wavelet functions. It can be observed that in each graph there is an overlap of the original signals envelopes (backward) and the envelopes of the filtered signals (forward), showing the behavior of each selected Wavelet function for the filter proposed in this paper.

\section{Results}

\subsection{Evaluation of the selected wavelet functions}

This section presents the results obtained with the evaluation of the Wavelet functions best suited for use with the proposed filter. The experiments consisted of applying the set of epileptiform events and calculate the correlations and RMSE between the individual peaks of the original and filtered epileptiform events, between original events and the filtered events and between the original epochs and filtered epochs. The results were grouped into tables, which show the obtained values for each category analyzed.

\subsubsection{Results obtained through the calculation of the correlations}

Table 2 shows the results of the correlation between the peaks of the original events and the peaks of the filtered events, where the function RBio2.8 had the highest correlation value.

\begin{tabular}{llll}
\hline Wavelet function & Coefficients & Energy [\%] & Correlation between the peaks \\
\hline rbio2.8 & 18 & 75.51 & 0.985988 \\
coif2 & 12 & 63.90 & 0.985208 \\
rbio2.6 & 14 & 70.53 & 0.984620 \\
bior2.8 & 18 & 68.32 & 0.984608 \\
bior2.6 & 14 & 64.56 & 0.984165 \\
rbio6.8 & 18 & 73.29 & 0.984156 \\
bior6.8 & 18 & 73.47 & 0.983519 \\
sym8 & 16 & 71.83 & 0.982686 \\
sym6 & 12 & 67.04 & 0.982597 \\
sym4 & 8 & 59.07 & 0.982411 \\
\hline
\end{tabular}

Table 2. Correlation between the peaks of the original events and the filtered events.

Table 3 shows the obtained values through the calculation of the correlations between the original events and filtered epileptiform events. The function DB4 showed the highest value for average correlation between the original events and the filtered events. 


\begin{tabular}{llll}
\hline Wavelet function & Coefficients & Energy [\%] & Correlation between the events \\
\hline $\mathrm{db} 4$ & 8 & 48.30 & 0.833480 \\
$\mathrm{sym} 7$ & 14 & 67.69 & 0.824208 \\
$\mathrm{db} 8$ & 16 & 59.38 & 0.820907 \\
$\mathrm{db} 12$ & 24 & 65.23 & 0.812157 \\
coif5 & 30 & 76.53 & 0.810064 \\
rbio1.3 & 6 & 55.36 & 0.803467 \\
coif4 & 24 & 74.03 & 0.795365 \\
bior3.1 & 4 & 89.57 & 0.779477 \\
db15 & 30 & 67.40 & 0.775226 \\
coif3 & 18 & 70.16 & 0.771351 \\
\hline
\end{tabular}

Table 3. Correlation between the original events and the filtered events.

Table 4 presents the values obtained by calculating the average correlation between the epoch of the original signal and the epoch of the filtered signal. Again the function DB4 showed the highest correlation between the original signal and the filtered signal.

\begin{tabular}{llll}
\hline Wavelet function & Coefficients & Energy [\%] & Correlation between the epocs 1 sec \\
\hline db4 & 8 & 48.30 & 0.717658 \\
coif5 & 30 & 76.53 & 0.693210 \\
sym7 & 14 & 67.69 & 0.692088 \\
coif4 & 24 & 74.03 & 0.690788 \\
rbio1.3 & 6 & 55.36 & 0.690375 \\
db8 & 16 & 59.38 & 0.690320 \\
sym4 & 8 & 59.07 & 0.682411 \\
coif3 & 18 & 70.16 & 0.679416 \\
db12 & 24 & 65.23 & 0.675012 \\
db15 & 30 & 67.40 & 0.673080 \\
\hline
\end{tabular}

Table 4. Correlation between the original epochs and the filtered epochs.

Through use of the correlation index as a measure of the distortion of the signals processed morphologies, it was the two Wavelet functions. The function RBio2.8 stood out because it had the highest correlation value in the evaluation of the peaks (spikes) of epileptiform events. The function DB4 obtained the highest values of correlation in the evaluation of the events (spike-wave), and in the evaluation of the epochs.

\subsubsection{Results obtained from the RMSE}

Table 5 shows the RMSE values calculated between the peaks of the original epileptiform events and the peaks of the filtered epileptiform events. In this experiment the function that had the lowest error value was the function Coif4.

Table 6 presents the RMSE values obtained calculed between the original events (spike-wave) and the filtered events. The Wavelet function that had the lowest error value was the DB4. 


\begin{tabular}{llll}
\hline Wavelet function & Coefficients & Energy [\%] & RMSE between the peaks \\
\hline coif4 & 24 & 74.03 & 30.496005 \\
coif5 & 30 & 76.53 & 31.265593 \\
coif3 & 18 & 70.16 & 31.537512 \\
db4 & 8 & 48.30 & 31.725582 \\
db15 & 30 & 67.40 & 31.763431 \\
db11 & 22 & 64.09 & 31.833546 \\
sym12 & 24 & 77.45 & 31.937828 \\
sym4 & 8 & 59.07 & 32.571695 \\
sym10 & 20 & 75.18 & 32.621571 \\
db7 & 14 & 56.47 & 32.666603 \\
\hline
\end{tabular}

Table 5. RMSE values between the peaks of the original events and the peaks of the filtered events.

\begin{tabular}{llll}
\hline Wavelet function & Coefficients & Energy [\%] & RMSE between the events \\
\hline $\mathrm{db} 4$ & 8 & 48.30 & 37.659730 \\
sym7 & 14 & 67.69 & 38.648321 \\
db8 & 16 & 59.38 & 38.897637 \\
coif5 & 30 & 76.53 & 39.530448 \\
db12 & 24 & 65.23 & 39.739856 \\
rbio1.3 & 6 & 55.36 & 40.126242 \\
coif4 & 24 & 74.03 & 40.473673 \\
bior3.1 & 4 & 89.57 & 40.847805 \\
db15 & 30 & 67.40 & 41.935201 \\
coif3 & 18 & 70.16 & 42.023981 \\
\hline
\end{tabular}

Table 6. RMSE values between the original events (spike-wave) and the filtered events.

Table 7 presents the values obtained with the analysis of the epochs of $1 \mathrm{sec}$ of the original signals and the epochs of $1 \mathrm{sec}$ of the filtered signals. The function that showed the lowest error was the function DB4.

\begin{tabular}{llll}
\hline Wavelet function & Coefficients & Energy [\%] & RMSE between the epochs 1 sec \\
\hline db4 & 8 & 48.30 & 36.799603 \\
coif5 & 30 & 76.53 & 37.957277 \\
coif4 & 24 & 74.03 & 38.034667 \\
sym7 & 14 & 67.69 & 38.061023 \\
db8 & 16 & 59.38 & 38.129404 \\
rbio1.3 & 6 & 55.36 & 38.243990 \\
sym4 & 8 & 59.07 & 38.353757 \\
coif3 & 18 & 70.16 & 38.511218 \\
db15 & 30 & 67.40 & 38.828296 \\
db11 & 22 & 64.09 & 38.835923 \\
\hline
\end{tabular}

Table 7. RMSE values between the original epochs of $1 \mathrm{sec}$ and the filtered epochs of $1 \mathrm{sec}$. 
The experiments showed the function DB4 proved to be the best choice for use with the filter, according to the values of correlation and RMSE. This function is indicated when there is the need to maintain the morphology of the whole epoch of the filtered signal. When there is the need to preserve only the peaks of the filtered signal the RBio2.8 and Coif4 are the Wavelet functions more suitable for this task. If is only necessary to attenuate the normal background activity of the EEG, without concern for preserving the morphology of the epoch the Wavelet function Bior3.1 is the most appropriate to do this. Because this particular function have few coefficients, their use in filtering the EEG signals offers superior performance to other functions presented. However, this function shows greater distortion of the signal after filtering than the others. Table 8 shows the values of correlation and RMSE for a epoch of $1 \mathrm{sec}$.

\begin{tabular}{lllll}
\hline Wavelet function & Coeffs. & Energy [\%] & Correlation epochs 1 sec & RMSE epochs 1 sec \\
\hline $\mathrm{db} 4$ & 8 & 48.30 & 0.717658 & 36.799603 \\
coif4 & 24 & 74.03 & 0.690788 & 38.034667 \\
rbio2.8 & 18 & 75.51 & 0.666828 & 39.029143 \\
bior3.1 & 4 & 89.57 & 0.666274 & 39.463159 \\
\hline
\end{tabular}

Table 8. Wavelet functions obtained from the experiments performed with the calculation of the correlations and RMSE.

\subsection{Evaluation of the proposed filter}

In this section will be presented screen of the original EEG signals containing baseline fluctuations, high-frequency noise, low frequency oscillations and epileptiform events. All the signals were filtered using the function $\mathrm{Db} 4$.

\subsubsection{Experiments with the proposed filter applied in EEG Signals}

Figure 13 presents a segment of EEG signal contaminated by muscle artifacts. Can be observed the low frequency fluctuations present in the original signal were attenuated in the filtered signal. The high frequencies generated by the muscles suffer little attenuation in relation to the original signal leaving only frequencies below $32 \mathrm{~Hz}$.

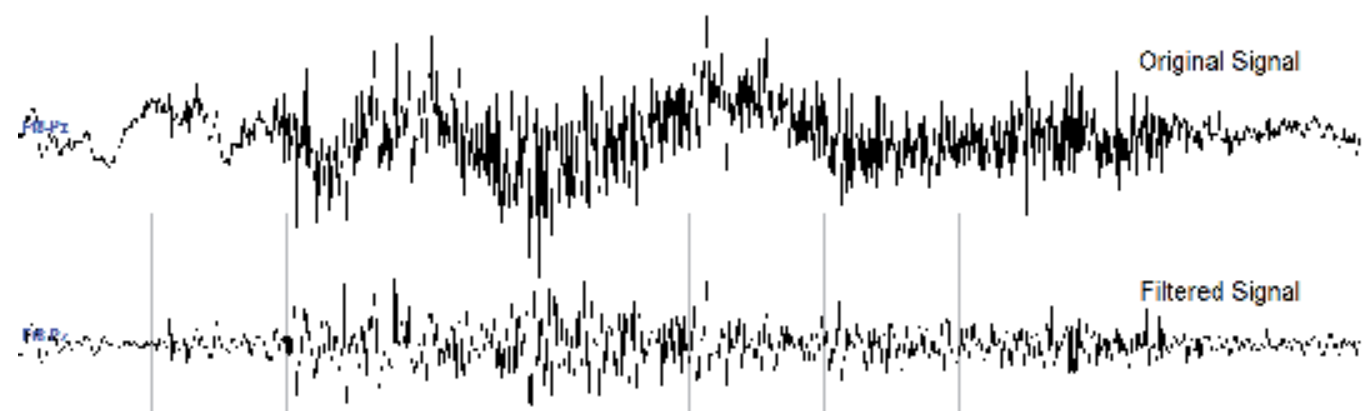

Figure 13. EEG signal containing muscle artefacts. 
Figure 14 shows a segment of the EEG signal which has large fluctuations of base line. It can be observed that the proposed filter attenuated the baseline oscillations and small oscillations of low frequencies apparent at the beginning of the original signal were also attenuated.

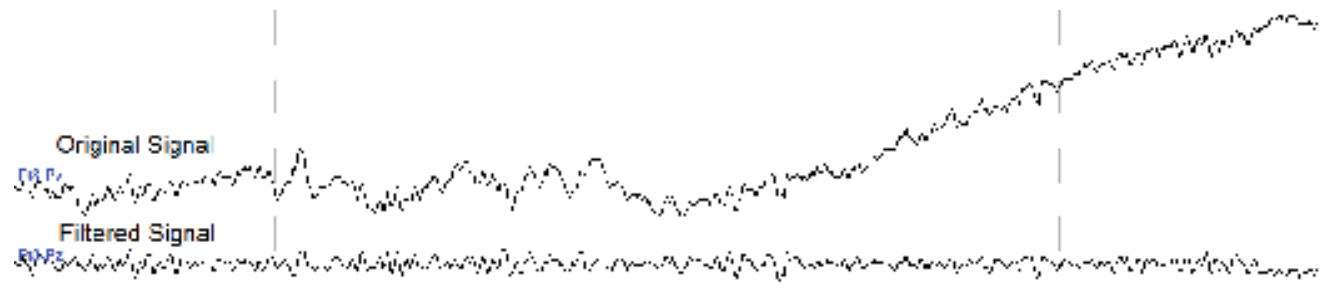

Figure 14. EEG signal containing base line oscillations.

Figure 15 presents a segment of the EEG signal containing high frequency noise and the filtered signal by Wavelet filter.

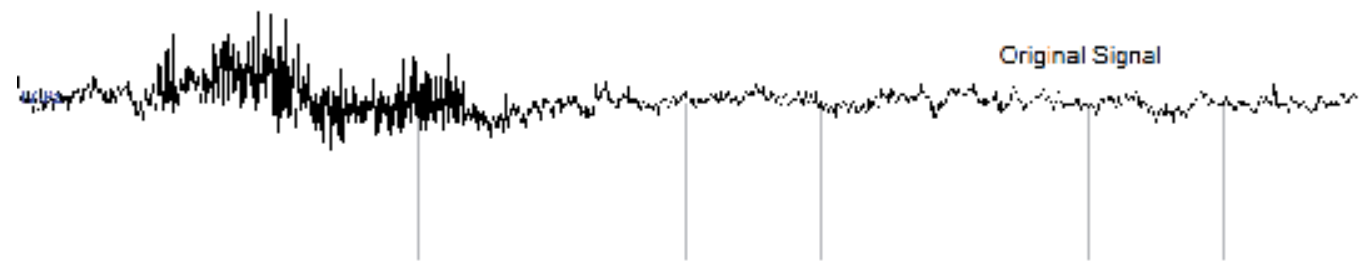

Filtered Signal

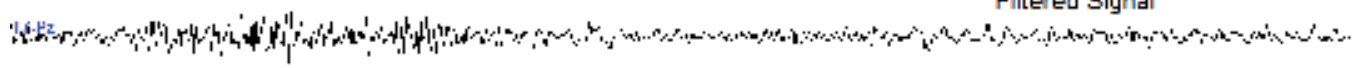

Figure 15. EEG signal containing noise of high frequency.

Figure 16 presents a segment of EEG containing epileptiform events and small lowfrequency oscillations. Can be observed that the low-frequency oscillations were attenuated highlighting the epileptiform events.

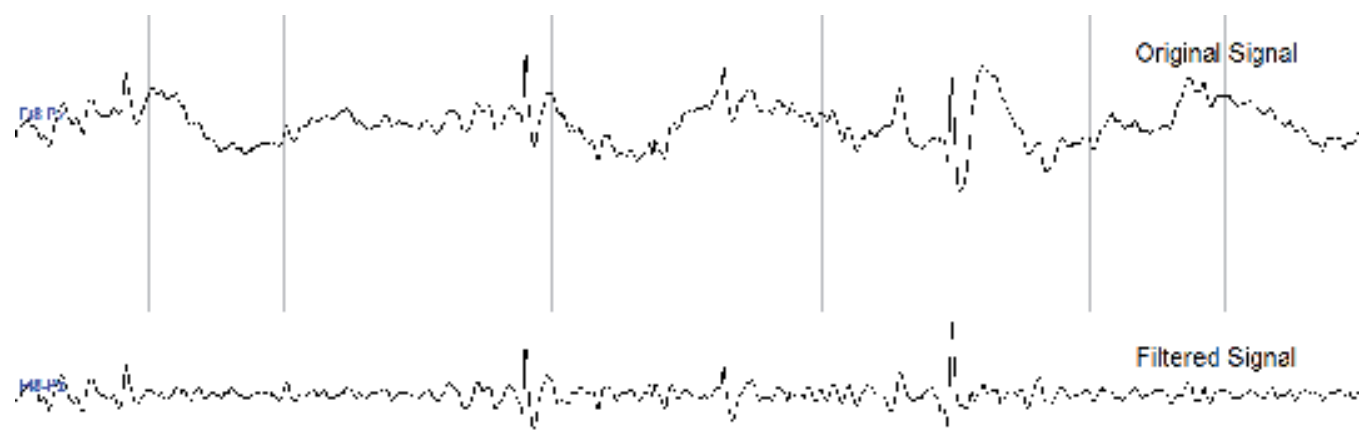

Figure 16. EEG signal containing some epileptiform events. 


\subsubsection{Experiments with the proposed filter applied in screens of EEG signals}

In the previous section have been shown figures containing segments of EEG signals, which were filtered by the proposed filter. This section presents individual screens of EEG signals containing the most different conditions found in the used records. These screens show noise of high and low frequencies, fluctuations of base line and some screens with epileptiform events. The screens were processed with the Wavelet function Db4, which was chosen for the proposed filter. Figure 17 presents a plot of EEG containing large fluctuations of base line. At the top right there is the presence of some epileptiform events, featuring a field of potential.

It can be observed that there was attenuation in the fluctuations of base line and other oscillations present in the original signal, highlighting the epileptiform events (Figure 18).

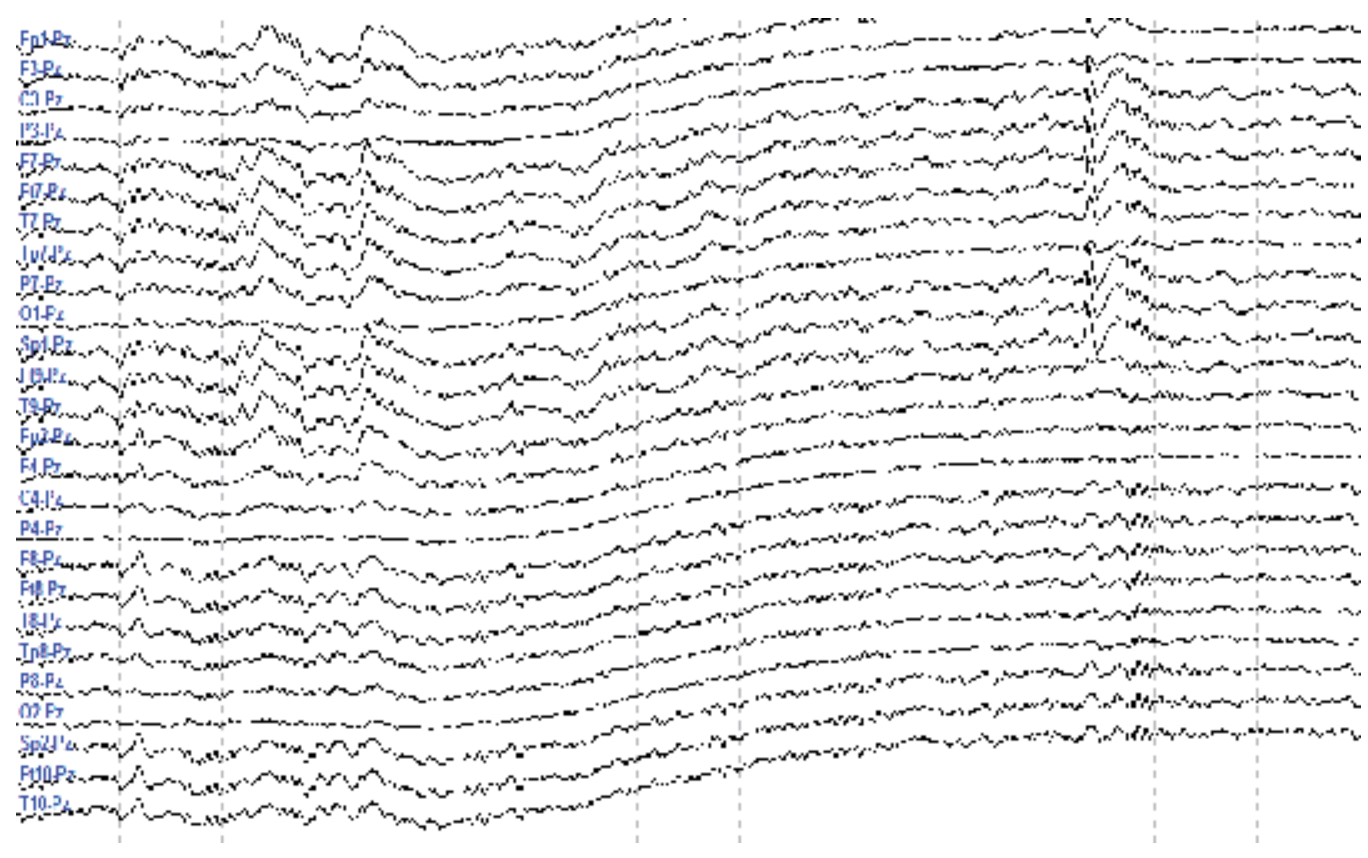

Figure 17. EEG screen containing large base line fluctuations and some epileptiform events. 


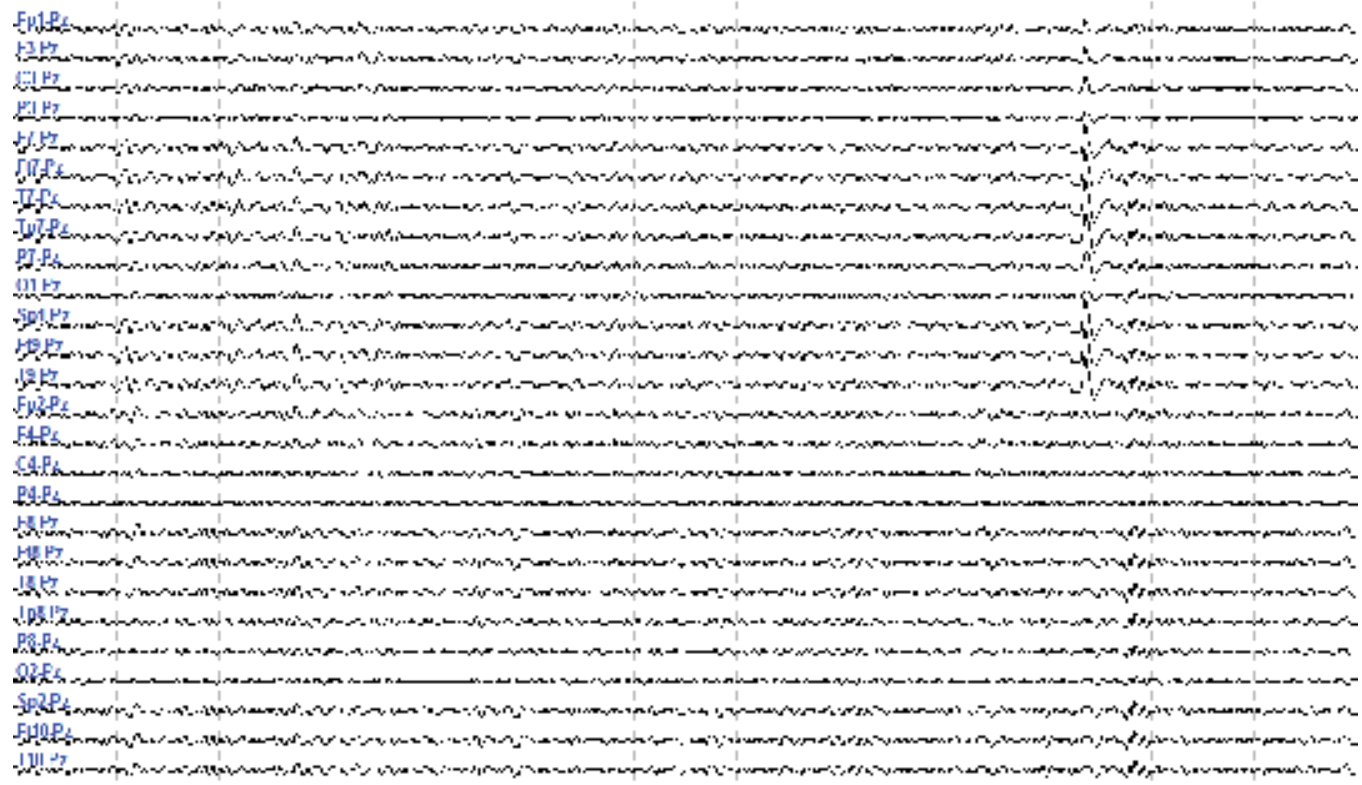

Figure 18. Screen of EEG signals filtered by proposed filter.

Figure 19 presents a plot of EEG containing many low-frequency oscillations, as well as high frequency noise.

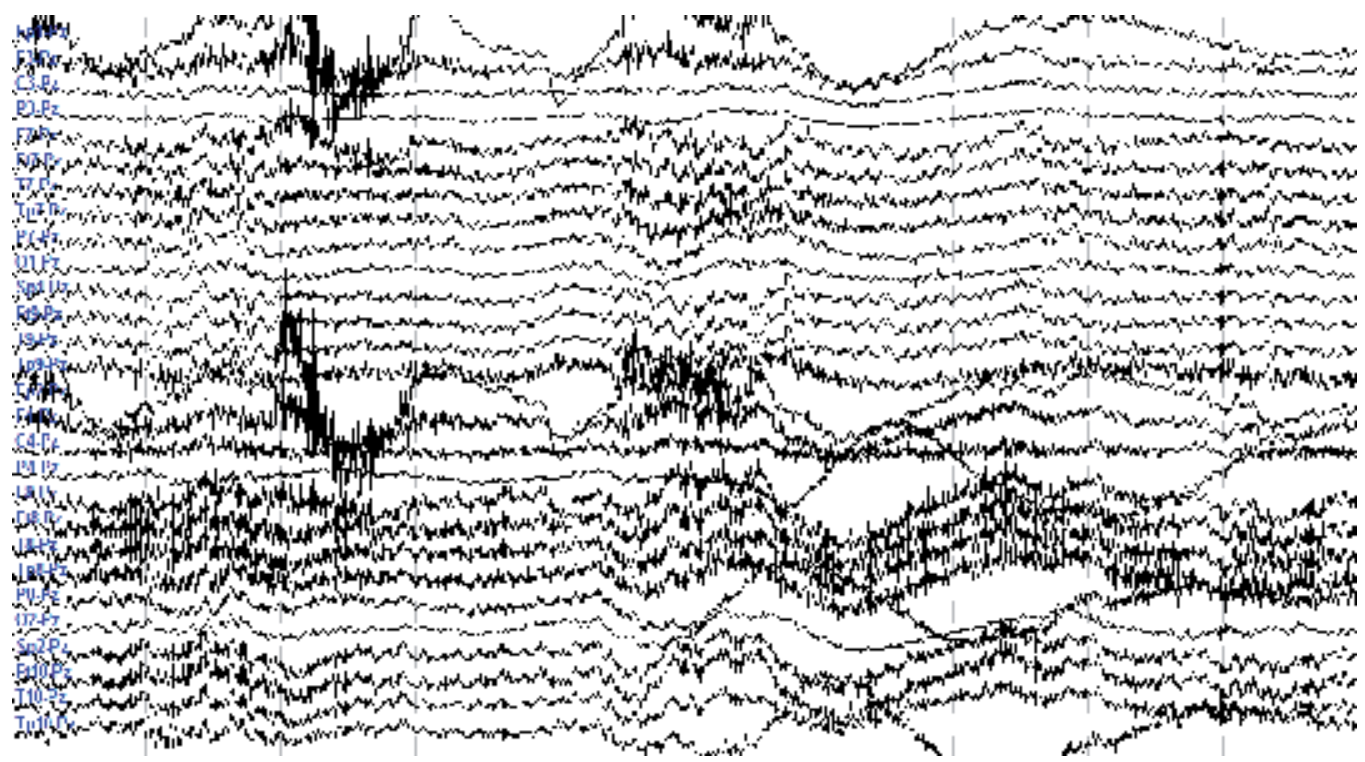

Figure 19. Screen of EEG signals containing noise of low and high frequencies.

Through the Figure 20 can be seen that the low frequency oscillations were attenuated, but some frequencies below $32 \mathrm{~Hz}$ remained in the processed signal. 


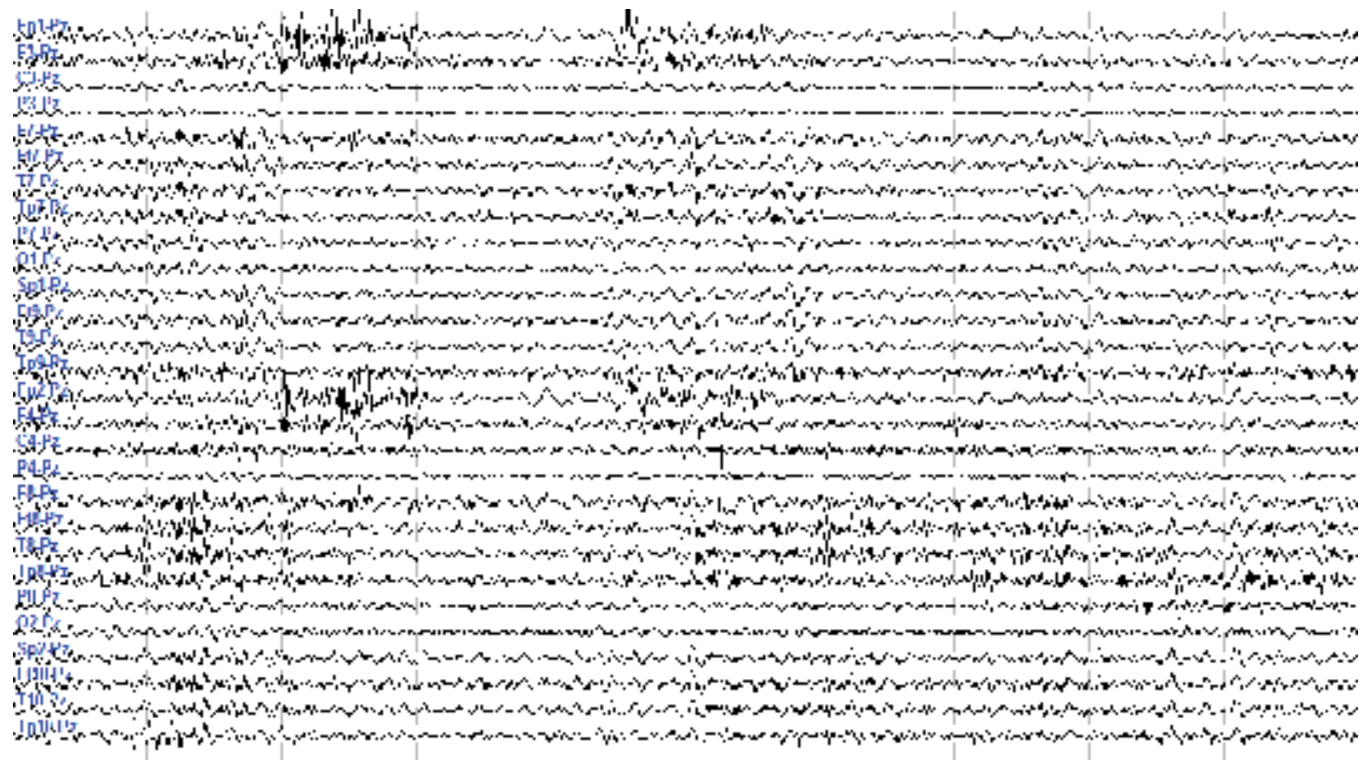

Figure 20. Screen of EEG signals filtered by proposed filter.

The Figure 21 presents a screen containing epileptiform events in two different periods of time. Can be observed small low frequency oscillations in the higher channels that were attenuated.

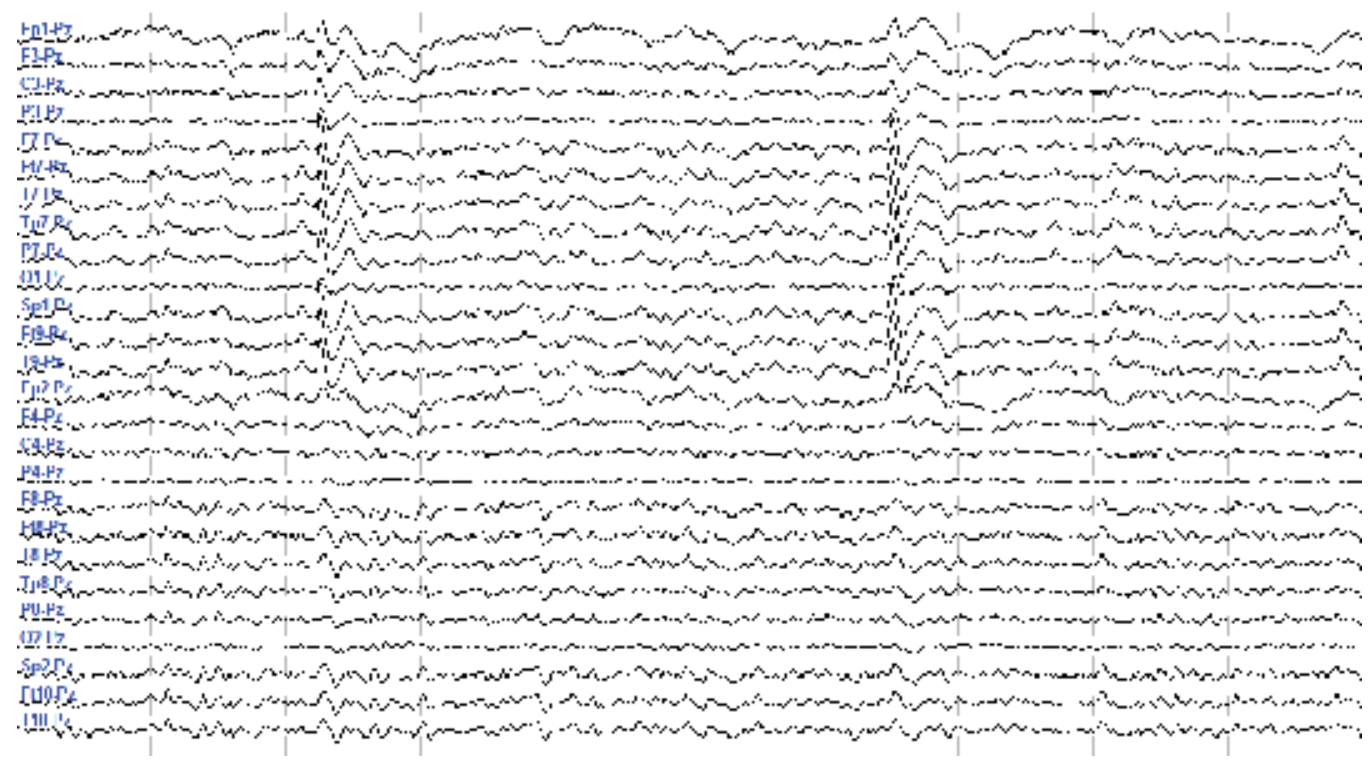

Figure 21. Screen of EEG signals containing epileptiform events in different periods of time.

In Figure 22 can be seen that the epileptiform events are more prominent in EEG signals, due to the fact the filter have attenuated all oscillations that were present in the signal. 


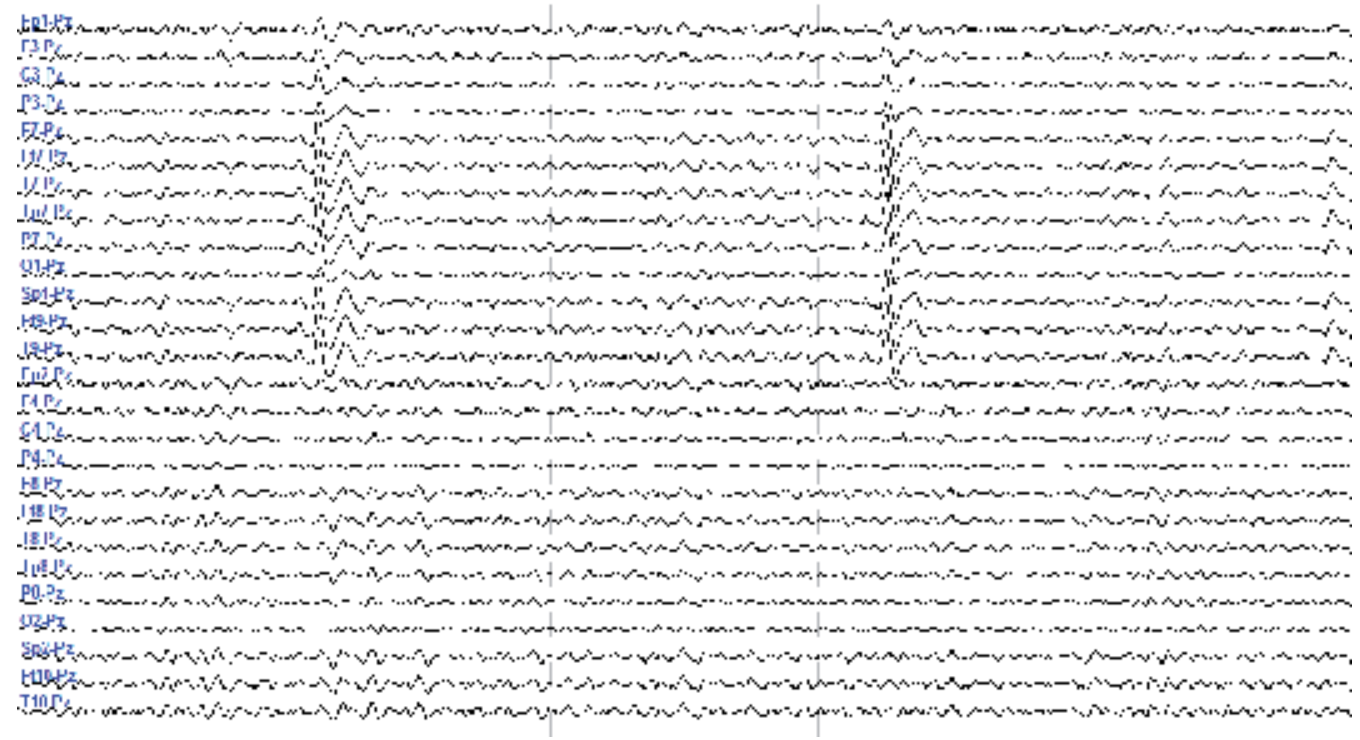

Figure 22. Screen of EEG signals filtered by proposed filter.

Figure 23 presents a screen containing blinks, muscle artifacts, noise and high frequencies, and at the center of the screen some epileptiform events.

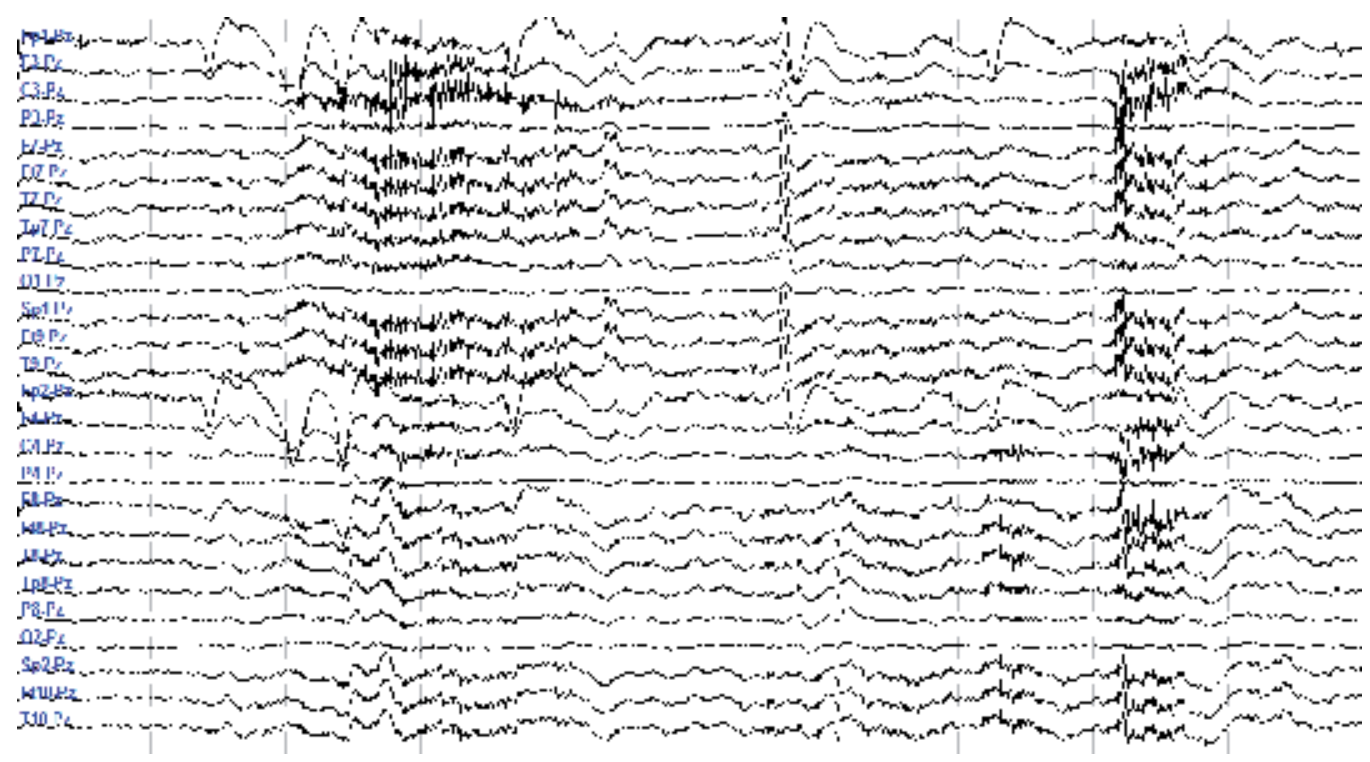

Figure 23. Screen containing blinks, muscle artefacts, high frequencies and at the centre of the screen epileptiform events.

In Figure 24 it can be seen that the predominant blinks in the front channels and the muscle artefacts were attenuated and the epileptiform events were highlighted. 


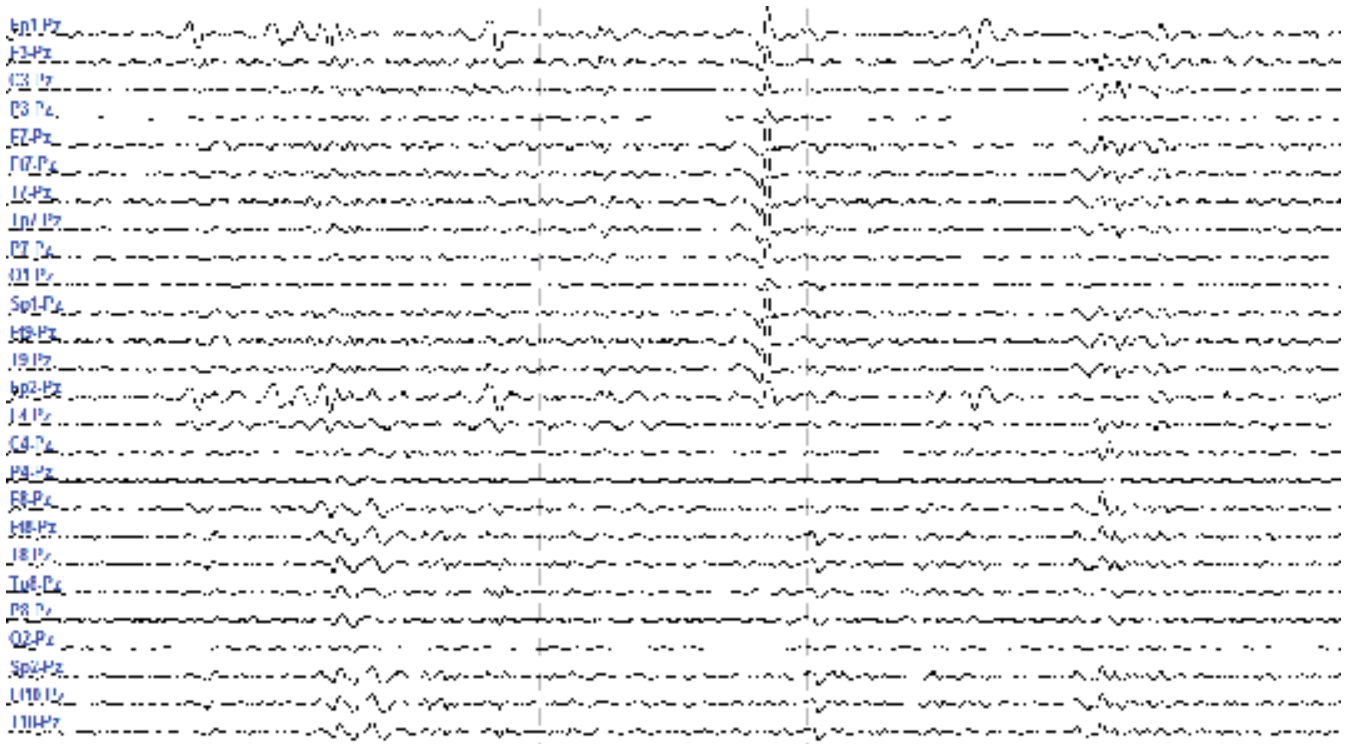

Figure 24. Screen of EEG signals filtered by proposed filter.

The Figure 25 presents a EEG screen contaminated by noise of $60 \mathrm{~Hz}$, eyelid blink, some oscillations and at the center of the screen epileptiform events (black arrow) obviously masked due to noise.

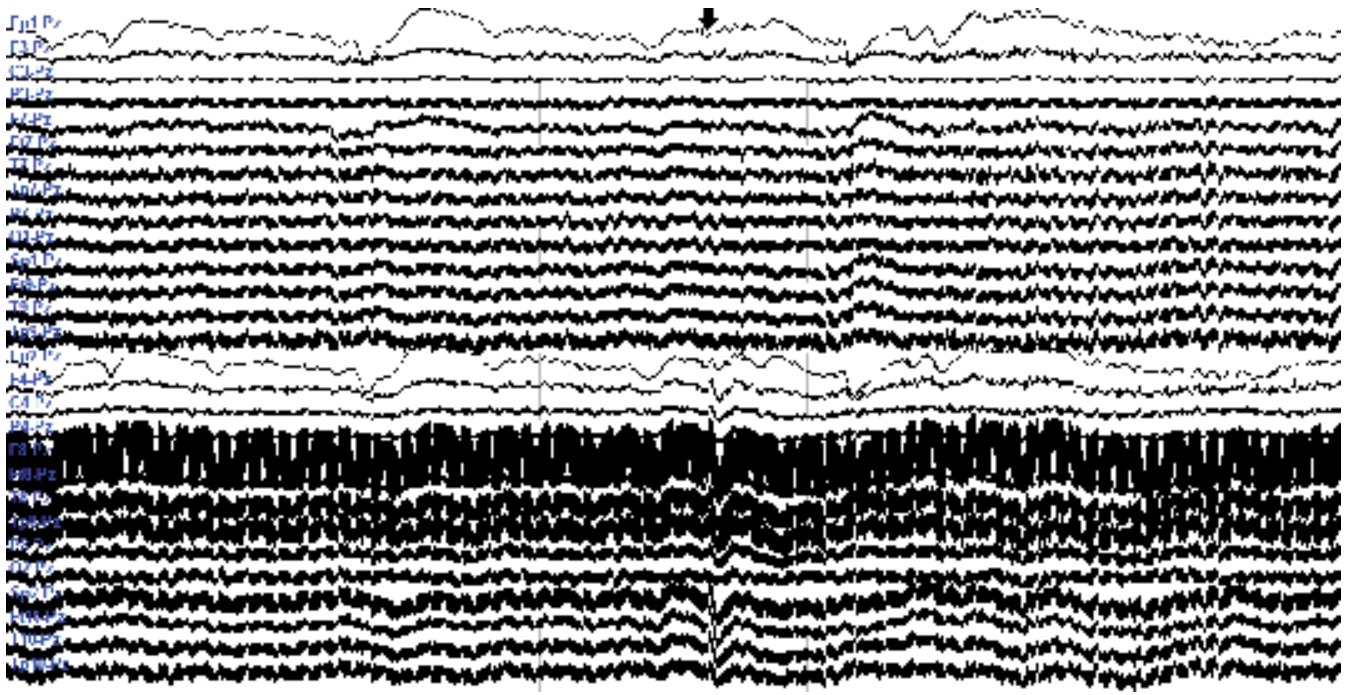

Figure 25. EEG screen containing noise of $60 \mathrm{~Hz}$ and epileptiform events masked by the noise.

In Figure 26 we can observe that the noise from the power supply has been attenuated, as well as the oscillations and eyelid blinks, showing the epileptiform events that previously did not appear. 


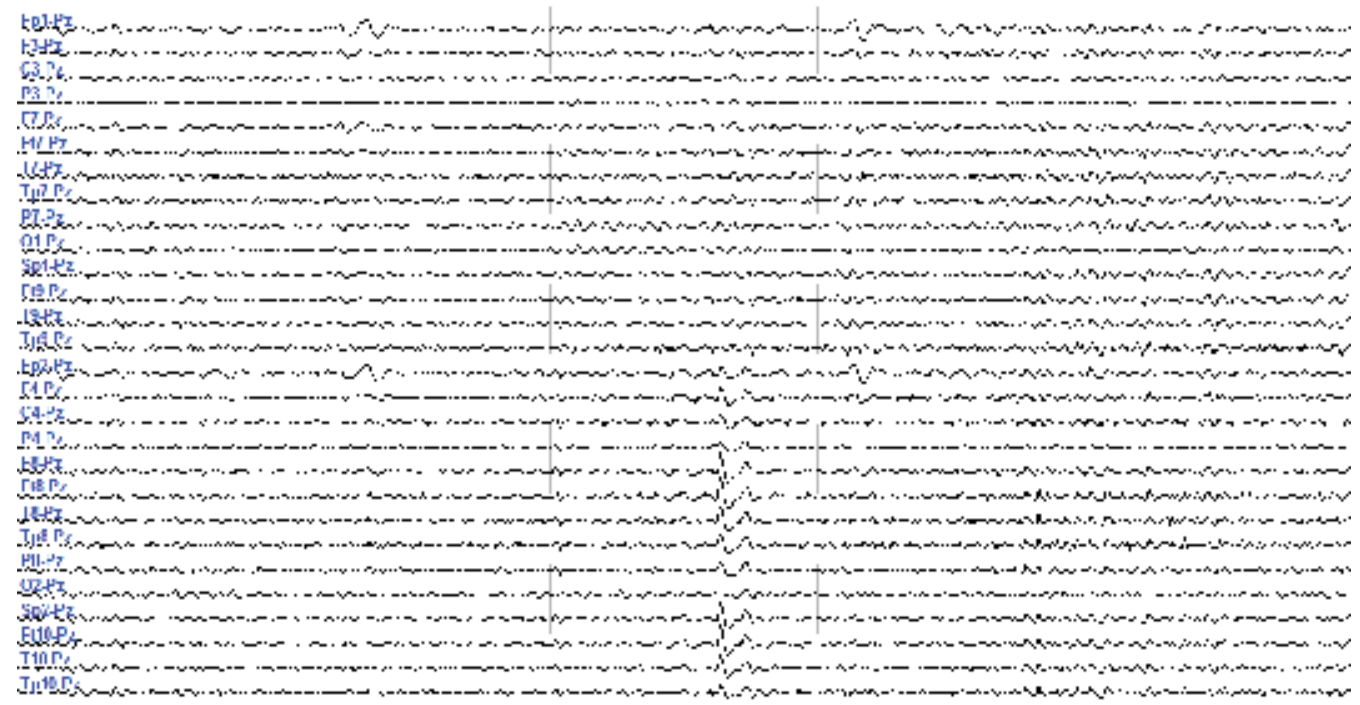

Figure 26. EEG screen processed by the proposed filter.

\section{Conclusion}

This work presented a study about the capability of the Wavelet Transform to be used to develop a digital filter to attenuate the background activity in the EEG signals. Four Wavelet functions were select from 65 evaluated. According to the experiments the Wavelet function $\mathrm{Db} 4$ proved to be the best function for the development of a digital filter in this application, according to researches performed by [9-12]. The function Db4 is indicated when there is the need to preserve the epoch of the filtered signal more like the original epoch. When there is the need to preserve the peaks of the epileptiform events the adequate function is the Rbio2.8. If the need is only to attenuate the background activity without the concern of preserves the morphology of the peaks in the epileptiform events the adequate function is the Bior3.1. Due to the fact of this function have reduced number of coefficients its application improves performance in filtering process.

\section{Author details}

Geovani Rodrigo Scolaro, Fernando Mendes de Azevedo, Christine Fredel Boos and Roger Walz

Institute of Biomedical Engineering, Federal University of Santa Catarina, Brazil

\section{References}

[1] Blum, A.S., Rutkove, S.B. The Clinical Neurophysiology Primer. Totowa, New Jersey: Humana Press Inc; 2007.

[2] Montenegro, M.A., Cendes, F., Guerreiro, M.M., Guerreiro, C.A. EEG in Clinical Practice. São Paulo: Lemos Editorial; 2001. 
[3] Pillai, J., Sperling, M.R. Interictal EEG and the Diagnosis of Epilepsy, Epilepsia (Vol. 47). Blackwell Pub./ ILAE; 2006.

[4] Lüders, H.O., Noachtar, S. Atlas and Classification of Electroencephalography: Introduction to the Evaluation of the Electroencephalogram. São Paulo: Lemos Editorial; 2000.

[5] Wilson, S. B., \& Emerson, R. Spike Detection: a review and comparison of algorithms. Clinical Neurophysiology 2002; 113, 1873-1881.

[6] Mallat, S. A Wavelet Tour of Signal Processing. USA: Academic Press; 1999

[7] Addison, P.S. The Illustrated Wavelet Transform Handbook: Introductory Theory and Applications in Science, Engineering, Medicine and Finance. New York: Taylor \& Francis Group; 2002.

[8] Indiradevi, K.P., Elias, E., Sathidevi, P.S., Dinesh Nayak, S., \& Radhakrishnan, K. A multi-level wavelet approach for automatic detection of epileptic spikes in the electroencephalogram. Computers in Biology and Medicine 2008; 38, 805-816.

[9] Wiltschko, A.B., Gage, G.J., Berke, J.D. Wavelet filtering before spike detection preserves waveform shape and enhances single-unit discrimination. Journal of Neuroscience Methods 2008; Elsevier.

[10] Adeli, H., Zhou, Z., \& Dadmehr, N. Analysis of EEG records in an epileptic patient using wavelet transform. Journal of Neuroscience Methods 2003; 123, 69-87.

[11] Subasi, A. EEG signal classification using wavelet feature extraction and a mixture of expert model. Expert Systems with Applications 2007; 32, 1084-1093.

[12] Scolaro, G.R., Azevedo, F.M., Boos, C.F. Evaluation of Different Wavelet Functions Applied in the Development of Digital Filters to Attenuate the Background Activity in EEG Signals. World Congress on Medical Physics and Biomedical Engineering 2012, Beijing, China. 


\title{
Impulse Oscillometric Features and Respiratory System Models Track Small Airway Function in Children
}

\author{
Erika G. Meraz, Homer Nazeran, Carlos Ramos, Liza Rodriguez, \\ Lidia Rascón Madrigal and Nelly Gordillo Castillo
}

Additional information is available at the end of the chapter

http://dx.doi.org/10.5772/52579

\section{Introduction}

Asthma is an inflammatory condition of the airways resulting in their hyper-reactivity, generating increased mucus, mucosal swelling and airway smooth muscle contraction all of which contribute to (partial) airway obstruction. The symptoms include chest tightness, coughing and wheezing, and in severe cases shortness of breath and low blood oxygen (1). According to Dorland's medical dictionary small airway impairment (SAI) is a chronic obstructive bronchitis with narrowing of the bronchioles and small bronchi. The term small airways refer to about $7^{\text {th }}$ to $19^{\text {th }}$ generation of airways with an inner diameter of about 2 to $0.5 \mathrm{~mm}$. These airways are considered to be an important site of inflammation in asthma and chronic obstructive pulmonary disease (COPD). The atopic manifestation just prior to asthma could be early small airway disease (SAD) and then if inflammation persists, asthma would appear. SAD includes a spectrum of inflammatory and fibrotic pulmonary diseases centered on the small conducting airways.

According to the American Academy of Allergy Asthma \& Immunology, Asthma and allergies stroke 1 out of 12 Americans and approximately 25 million Americans ( $8 \%$ of the U.S. population) had asthma in 2009. About 1 in 10 children (10\%) had asthma and 1 in 12 adults (8\%) had asthma in 2009. For the period 2008-2010, asthma prevalence was higher among children than adults. For the period 2008-2010, asthma prevalence was higher among multiplerace, black, and American Indian or Alaska Native persons than white persons. Asthma costs in the US grew from about $\$ 53$ billion in 2002 to about $\$ 56$ billion in 2007, about a 6\% increase. Compared with adults, children had higher rates for asthma primary care and emergency department visits, similar hospitalization rates, and lower death rates. Asthma was linked to 
3,447 deaths (about 9 per day) in 2007. Internationally asthma has a rising prevalence in low and middle income countries and it is reaching a steady level in high income countries. An estimated 300 million people worldwide suffer from asthma, with 250,000 annual deaths attributed to the disease; almost all of these deaths are avoidable (2).

In Mexico, 10\% (approximately 10 million people), of the population suffer from asthma. It is the most common cause of chronic illnesses and emergency hospitalizations in children according to the Mexican College of Allergy, Asthma and Pediatric Pulmonology (3).

Assessment of respiratory function is important in diagnosis and monitoring of asthma and other respiratory diseases in children (4). The pulmonary function test most commonly used to detect asthma is spirometry, which measures the volume of air that can be moved in or out of the lungs as a function of time with rapid and maximal inspiratory and expiratory efforts. This requires a considerable degree of cooperation from the subject, which is difficult to achieve in older children and almost impossible to achieve by younger children. This makes the diagnosis of asthma difficult owing to the lack of objective measurements for younger children (5). Furthermore, it has been reported that some asthmatic patients do not improve spirometrically, despite clinical improvement with treatment (6). This is of concern because if asthma is not appropriately controlled, it can lead to permanent airway damage.

In contrast to forced spirometry, the Forced Oscillation Technique (FOT) superimposes small air pressure perturbations on the natural breathing of a subject to measure lungs mechanics (respiratory impedance). The Impulse Oscillometry System (IOS) measures respiratory impedance by using short pulses (impulses) of air pressure. It has been developed as a patientfriendly lung function test that minimizes demands on the patient and requires only passive cooperation of the patient wearing a nose clip, keeping lips tightly closed about a mouthpiece and breathing normally through the mouth. IOS has been used with success to assess lung function in healthy and asthmatic children and adolescents (4-32). In infants and children, reversible airway obstruction and bronchial hyperresponsiveness (BHR) are significant components contributing to the diagnosis of bronchial asthma (27). All this evidence, which will be described in detail, confirms that lung function in children and adolescents is sensitively and accurately assessed by the IOS, before and after bronchodilation. To build upon this evidence and demonstrate the potential enhancements in the clinical utility of IOS, here we present respiratory system Model Parameters and selected IOS features derived from Pre- and Post-bronchodilation data acquired from Anglo and Hispanic children, that offer significant improvements in quantitative evaluation of small airway impairments and assessment of asthma in this population.

In 1991, the American Thoracic Society published guidelines focusing on spirometry as the most widely used lung function test, where they presented Reference Values for spirometric parameters for Caucasian and Black men and women. They also mentioned that it is common practice to interpret the results of lung function tests in relation to Reference Values and in terms of whether or not they are considered to be within the "normal" range (33).

The European Respiratory Society in 1995 published a workshop report (34) about “Reference Values for Residual Volume (RV), Functional Residual Capacity (FRC) and Total 
Lung Capacity (TLC)" in which it was mentioned that reference values play an important role in establishing whether the measured volumes fall within an expected range for healthy individuals of the same sex, similar stature, age, and other characteristics. They also point out that comparing reference with measured values is fraught with difficulties, as this may result in disease being undetected and as a consequence untreated. It is also mentioned that FRC is the only lung volume that can be measured routinely with accuracy and reliability, and in addition it is stated that attempts have been made to evaluate TLC and RV. In this report reference values (values for healthy subjects) and prediction equations for lung volumes for children and adults are obtained using different techniques like helium dilution and body plethysmography. These values are presented for different heights. However, this report does not include reference values and prediction equations based on the FOT or IOS.

Few studies have been developed to obtain Reference Values for healthy children using the FOT and IOS (35-42). It is essential to have IOS Reference Values for children, as this technique has been shown to be very effective in the detection of lung abnormalities. Therefore, here we intend to make an effort towards establishing normal IOS Reference Values in North American Anglo and Hispanic children 5 to 19 years old. We also aim to present baseline (pre-) and postbronchodilation IOS parameters for Anglo and Hispanic children with Probable Small Airway Impairment (PSAI), Small Airway Impairment (SAI) and Asthma.

IOS data generate frequency-dependent curves of respiratory impedance (resistance and reactance) that are visually analyzed to recognize changes in their shape and magnitude and distinguish healthy respiratory function from dysfunction. The IOS data can be deployed to develop mechanical and equivalent electrical circuit models of the respiratory impedance to evaluate and quantify lung mechanics. In these equivalent models, electrical components analogous to mechanical resistance, compliance, and inertance inherent in the respiratory system are used. Therefore, estimates for these Model Parameters based on IOS measurements could be used as baseline measures for better detection, diagnosis, and treatment of different respiratory diseases (43).

Previous work by our research group for more than a decade has focused on development and analysis of different equivalent electrical circuit models for human respiratory impedance. This effort to date has demonstrated that the performance of extended Resistance Inductance Capacitance (eRIC) model and the augmented RIC (aRIC) model (an improvement of the eRIC model) ranked in the middle of a series of conventional models developed over the past several decades in terms of total cumulative error. However, they provide parameter estimates that are physiologically more realistic and in line with expected values in normal subjects and those suffering from pulmonary diseases (43-51), than previous models.

The IOS data collected from children for this study were partially analyzed and presented in several publications (52-61), the latest results of this research are presented in this chapter.

Here we determine the eRIC and aRIC model parameter estimates in addition to sensitive IOS measures of lung function in Normal $(\mathrm{N})$ or Healthy $(\mathrm{H})$, PSAI, SAI and Asthmatic (A), Anglo and Hispanic children. We further evaluate the performance of these models in 
quantifying lung function in this population and analyze the correlation of these Model Parameters with sensitive IOS measures of lung function.

\section{Pulmonary function tests and previous studies}

Pulmonary function refers to how the lungs perform gas exchange. Pulmonary function testing is a practical application of Respiratory Physiology and is necessary for understanding abnormalities in lung function and the effects of treatments. Pulmonary function tests help to determine the severity of functional impairments or defects and the extent to which treatments restore normal function (62). In this section we first review two important pulmonary function tests: Spirometry and Impulse Oscillometry. We then perform a literature review of several studies that have been carried out in previous years to compare several Pulmonary Function Tests (PFTs) to assess the ability of FOT and IOS to measure pulmonary function and to discriminate between impaired and non-impaired conditions.

\subsection{Spirometry}

Spirometry is the most common PFT; it is a measurement of maximal airflow after deep inspiration to fill up the lungs. It can provide information about the size of the breathing tubes (mainly large airways) and about the presence of blockages to airflow (63). The measurements usually obtained from spirometry are (64):

- $\quad$ FVC (Forced vital capacity): Total volume of air that can be exhaled during a maximal forced expiration

- $\quad$ FEV 1 : Forced expiratory volume in seconds. It is the volume of air expired in the first second of maximal expiration

- $\quad$ FEV $_{1}$ / FVC: Percentage of the FVC expired in one second

- FEF $_{25 \%-75 \%}$ : Average expired flow over the middle half of FVC. It represents the average flow from the point at which $25 \%$ of the FVC has been exhaled to the point at which $75 \%$ of the FVC has been exhaled

- $\quad \mathbf{F E V}_{6:}$ Forced expiratory volume in six seconds

- $\quad$ PE : Peak expiratory flow represents the maximal expiratory flow rate achieved

The National Asthma Education and Prevention Program (NAEPP) Guidelines previously considered FEV 1 as the "gold standard" to assess asthma severity and control, but several studies have suggested that most children have normal or near normal FEV 1 even when they are symptomatic. Now the NAEPP has added $\mathrm{FEV}_{1} / \mathrm{FVC}$ ratio as an impairment criterion to classify asthma severity and control. The most important pulmonary function abnormalities seen in asthmatic children are decreases in the $\mathrm{FEV}_{1} / \mathrm{FVC}$ and the $\mathrm{FEF}_{25 \%}-75 \%$, while $\mathrm{FEV}_{1}$ remain in the normal range in spite of asthma severity (65).

\subsection{Forced Oscillation Technique (FOT)}

The fundamental principle of FOT is that respiratory mechanics can be measured from superimposition of external pressure oscillations on the respiratory system during resting 
breathing (66). Therefore, FOT superimposes small external pressure signals on the natural breathing to determine a subject's breathing mechanics. FOT measures respiratory impedance to this applied forced pressure oscillations produced by a loud speaker (67). FOT is indicated as a reliable diagnostic tool to obtain tidal breathing analysis. One of the great advantages of FOT over other pulmonary function tests is that the results measured are independent of the subject respiratory pattern, therefore it is effort independent; it requires only passive cooperation from the subject breathing normally through a mouth piece, keeping lips airtight closed around it, while wearing a nose clips occluding the nares (68). FOT has been used in humans for more than 50 years; it has been used in children with three major clinical aims (69):

1. To characterize the lung function abnormalities of chronic respiratory diseases in children

2. As a diagnostic tool, especially to recognize asthma and bronchial responsiveness

3. To study the physiological mechanisms and pathophysiology of diverse conditions involving and/or threatening the respiratory system

FOT applied at oscillation frequencies between 3 and $35 \mathrm{~Hz}$ can provide helpful information to help distinguish between large and small airways. The use of multiple oscillation frequencies in FOT allows a separation of large airways from small airways. Frequencies below $15 \mathrm{~Hz}$, low oscillation frequencies, have been shown to be transmitted more distally (peripherally) in the lungs, whilst frequencies higher than $20 \mathrm{~Hz}$, high oscillation frequencies, can reach only the intermediate size airways. As a result low oscillation frequencies reflect small and large airways, while high oscillation frequencies merely reflect large airways. Therefore, changes in large airway resistance cause uniform changes in resistance at all oscillation frequencies $(3-35 \mathrm{~Hz})$, whereas changes in small airway resistance result in noticeable changes in low frequency $(3-15 \mathrm{~Hz})$ resistance with small or no change in high frequency resistance. Peripheral airways include all airways with a diameter less than $2 \mathrm{~mm}$, and large airways are those with diameters greater than $4 \mathrm{~mm}(66)$.

One of the most remarkable features of FOT in relation to spirometry is that it has a relatively greater sensitivity to peripheral airways disease; due to the fact that spirometry does not provide a clear indication of peripheral airway obstruction regardless of the information contained in the flow-volume curve and the values of mid-flow rates $\left(\mathrm{FEF}_{25 \%-75 \%)}(68)\right.$.

\subsection{Impulse Oscillometry System (IOS)}

In 1956 Dubois presented the first study on FOT; in this study FOT was applied using sinusoidal oscillations with multiple single frequencies between 2 and $18 \mathrm{~Hz}$. After this study several modifications of FOT were developed, until 1993 when the pulse technique was improved and commercially produced by the German company Jaeger. It was named Impulse Oscillometry System (IOS), as an easier to use method to measure respiratory resistance $(\mathrm{R})$ and reactance $(\mathrm{X})$. The advantages of IOS include good time resolution. It measures 5 pulses per second, with continuous resolution in the frequency domain using a 
Fourier Integral (65-66) to calculate respiratory impedance. The IOS, as FOT, superimposes small air pressure perturbations on the natural breathing of a subject to measure the impedance of the respiratory system, offering an easy to use method as it does not require any effort from the subject being tested. An additional advantage is the simplicity of the hardware needed to generate the forced oscillations, allowing smaller, more efficient electronic and mechanical structures with minimal power loss (68).

Some disadvantages of the IOS have to be recognized. The fact that IOS measures spontaneous breathing from a subject allows biological variability, and to counteract this fact multiple tests are required to be performed in a subject in order to establish reliable mean values of IOS parameters. A special aspect of applying pulses of pressure is that they are applied within a very short time causing a higher impact on the respiratory system compared with other lung function tests, and this may be perceived as an unpleasant sensation during the measurements (68).

A Jaeger MasterScreen IOS (Viasys Healthcare, Inc. Yorba Linda, CA, USA) was used in this study. The system was calibrated every day using a 3-L syringe for volume calibrations and a reference resistance $(0.2 \mathrm{KPa} / \mathrm{L} / \mathrm{s})$ for pressure calibrations. Children were asked to wear a nose clip, while breathing normally through a mouthpiece and were instructed to tightly close their lips around it to avoid air leakage. Three to five IOS test replicates were performed on each subject to ensure reproducible tests without artifacts caused by air leaks, swallowing, breath holding or vocalization (9). In each IOS test impulses were applied for a period of 30 to 45 seconds. IOS data were carefully reviewed off line and quality-assured by our expert clinician to reject segments affected by airflow leak or swallowing artifacts. Coherence was also used as a 'quality assurance index'; it is an index of causality between the input and the output of a linear system, therefore if the system is nonlinear or if it is contaminated by extraneous noise then the coherence is lower than expected. Therefore, measurements with low coherence were excluded in this research to avoid problems with artifacts. Coherence is considered by researchers as a useful guide to quality assurance (67).

\subsubsection{IOS parameters}

IOS is a multifrequency oscillation method; it provides measures of respiratory mechanics in terms of respiratory impedance as a function of frequency $Z(f)$.

Respiratory Impedance is the transfer function of pressure $(\mathrm{P})$ and flow $(\mathrm{Q})$, derived from the superimposed forced oscillation, after being separated from the respiratory pressure and flow.

$$
Z(f)=P(f) / Q(f)
$$

The respiratory Impedance $(\mathrm{Z})$ measured by IOS is a complex quantity and consists of a real part called respiratory Resistance (R) and an imaginary part called respiratory Reactance (X).

$$
Z(f)=R(f)+j X(f)
$$


IOS also includes hallmarks such as Resonant Frequency (Fres) and Reactance Area (AX) also known as the "Goldman Triangle". IOS yields these indices over a selected frequency range of 3 to $35 \mathrm{~Hz}(68)$.

\section{A. Impedance Parameters: Respiratory Resistance (R) and Respiratory Reactance (X)}

\section{a. Respiratory Resistance (R)}

The real part of the Impedance $(Z)$ corresponds to the Resistance $(R)$, which includes the resistance of the proximal (central) and distal (peripheral) airways as well as lung tissue and chest wall while these latter resistances are usually negligible. In healthy adult subjects, $\mathrm{R}$ is nearly independent of oscillation frequency. When an airway obstruction occurs, either central or peripheral, R5 (Resistance at $5 \mathrm{~Hz}$ ) is increased above normal values. Central airway obstruction elevates $\mathrm{R}$ evenly independent of oscillation frequency. Peripheral airways obstruction is highest at low oscillation frequencies and falls with increasing frequency; this is called the negative frequency-dependence of Resistance (fdR). As peripheral resistance increases, $R$ becomes more frequency dependent. Small children normally present frequency-dependence of resistance, and this may be greater than in adults in the presence of peripheral airflow obstruction. Resistance is measured in $\mathrm{cmH}_{2} \mathrm{O} / \mathrm{L} / \mathrm{s}$ or $\mathrm{KPa} / \mathrm{L} / \mathrm{s}(68)$.

\section{b. Respiratory Reactance (X)}

The imaginary part of $Z$, the respiratory Reactance $(X)$, includes the mass-inertive forces of the moving air column expressed in terms of Inertance (I) and the Elastic properties (compliance) of lung periphery expressed as capacitance (C) in electrical terms (68).

$$
X(f)=\omega \cdot I-(1 / \omega . C)
$$

where

$$
\omega=2 \pi f\{0<f \leq f \max \}
$$

$\mathrm{C}$ represents the ability of the respiratory system to store energy, primarily located in the lung periphery. The component of $X$ associated with $C$ is defined to be negative in sign. It means $C$ is dominant at low oscillation frequencies, meanwhile the component of $X$ related to I is positive in sign, meaning that I's property is more prominent at high oscillation frequencies (see figure 1). Reactance is measured in $\mathrm{cmH}_{2} \mathrm{O} / \mathrm{L} / \mathrm{s}$ or $\mathrm{KPa} / \mathrm{L} / \mathrm{s}(68)$.

\section{B. Other IOS parameters: Resonant Frequency (Fres), Reactance Area (AX) and Frequency dependence of resistance $\left(\mathbf{R}_{5}-\mathbf{R}_{25}\right)$}

\section{a. Resonant Frequency}

The Resonant Frequency ( $\left.F_{r e s}\right)$ is the point at which $C$ and I are equal, therefore reactance is zero and is measured in Hertz (1/s) (68). 


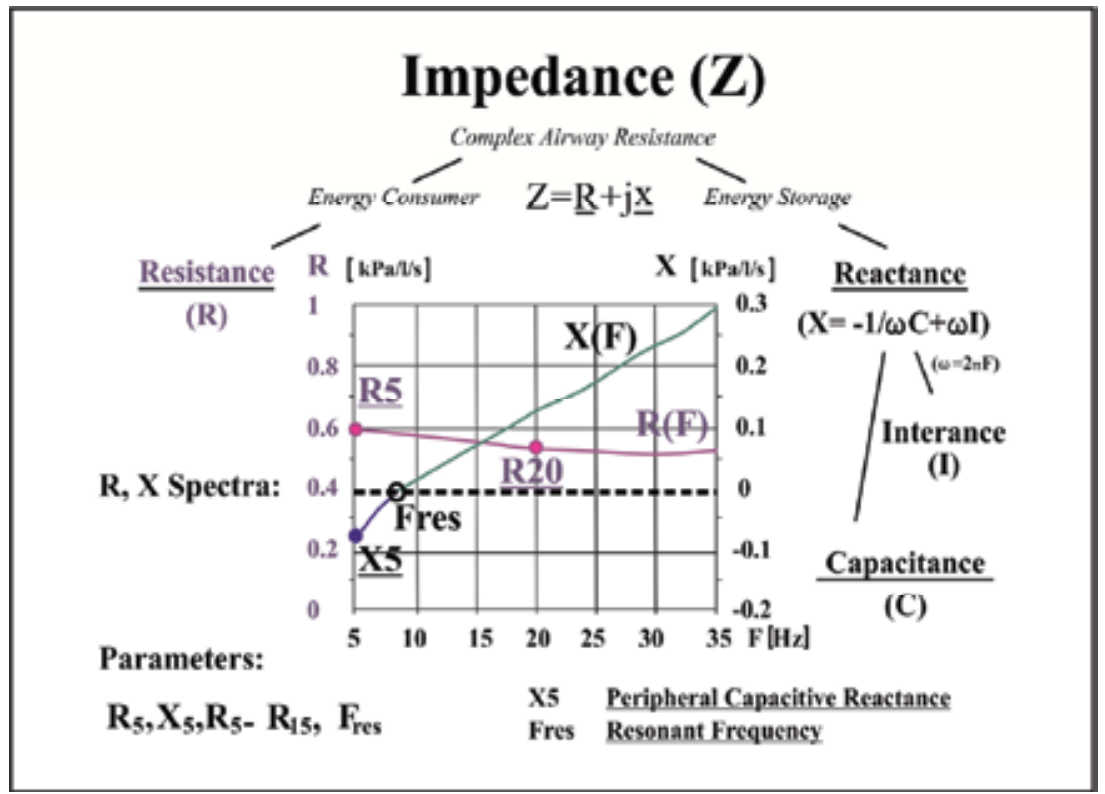

Figure 1. IOS parameters [76]

$$
\omega_{0} . I=\left(1 / \omega_{0} \cdot C\right)
$$

This parameter should not be interpreted as a particular respiratory system mechanical property; instead it can be used as a suitable marker to separate low frequency from high frequency impedance. Respiratory system abnormalities cause $F_{\text {res }}$ value to be increased [74].

\section{b. Reactance Area (AX)}

The Reactance Area (AX), - the "Goldman Triangle" - was introduced by Michael Goldman in his study on "Clinical applications of forced oscillations" (67); AX is the integrated low frequency respiratory reactance magnitude between $5 \mathrm{~Hz}$ and $\mathrm{F}_{\text {res, }}$ and is measured in $\mathrm{cmH}_{2} \mathrm{O} / \mathrm{L}$ or $\mathrm{KPa} / \mathrm{L}$. $\mathrm{AX}$ is a practical $\mathrm{FO}$ index related to respiratory compliance. $\mathrm{AX}$ is a single quantity that reflects changes in the degree of peripheral airway obstruction and closely correlates with $\mathrm{fdR}(68) . A X$ is a useful and sensitive index of peripheral airway function (66).

Figure 2 shows data collected from a Normal (N) child and a child with Small Airway Impairment (SAI) for this research as an example. In this figure it can be observed that the AX (the Goldman's Triangle) area is bigger for the child with SAI than for the normal child. It is interesting to notice that the values of Fres are very close for both children. 


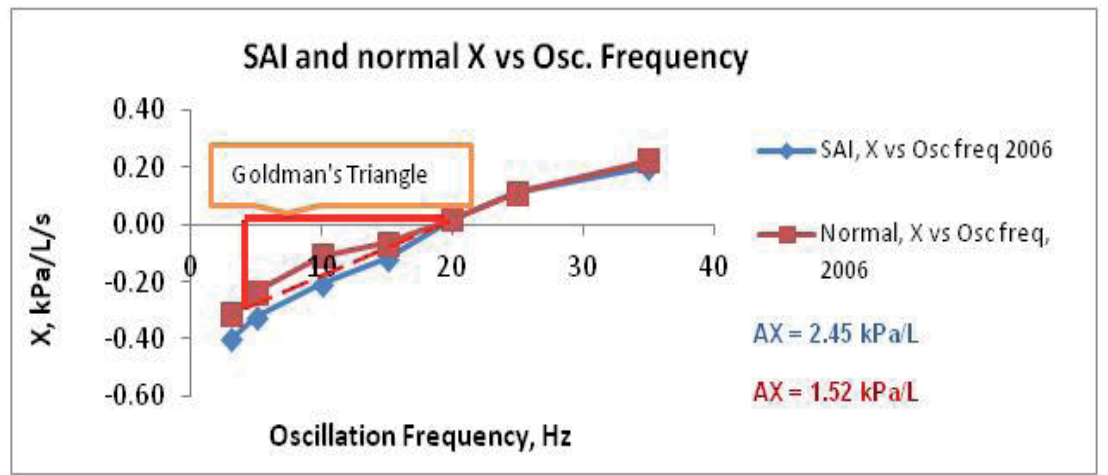

Figure 2. Reactance measurements in a Normal and a SAI child as a function of oscillation frequency.

\section{a) Frequency-dependence of resistance ( $f d R$ or R5-R20)}

It is simply the subtraction of the measured resistance at $20 \mathrm{~Hz}$ from the resistance at 5 $\mathrm{Hz}$ or $3 \mathrm{~Hz}$. Frequency-dependence of resistance is a characteristic of peripheral airway dysfunction (66).

$$
R 5-R 20=R 5-R 20
$$

Changes in AX with treatment interventions parallel changes in frequency-dependence of R. It has been suggested by Goldman et al. (66) that the magnitudes of frequency dependence of $\mathrm{R}$ and $\mathrm{AX}$ appear to reflect a similarly predominant influence of peripheral airway mechanical function.

Frequency-dependence of resistance occurs in healthy children, and to a better extent in children with respiratory system distresses (67). There is now plenty of evidence that peripheral airway inflammation is present in asthma patients, and frequencydependence of resistance occurs significantly in asthma (68).

\subsection{Bronchodilation phenotype}

The bronchodilation response as a physiological response to short-acting beta agonist medicines has been recommended to demonstrate reversibility of airflow obstruction consistent with the definition of asthma (65).

Bronchoconstriction is defined as increased tone of airway smooth muscles due to inflammation; and bronchodilation is defined as decrease in smooth muscle tone, and as a result a decrease in inflammation. When an increase of airways smooth muscle tone occurs, $\mathrm{R}$ increases due to a corresponding decrease in airway lumen. $\mathrm{R}$ also increases due to inflammation or edema. In asthmatics, high and low frequency $\mathrm{R}$ decreases after bronchodilation, showing a larger decrease in low-frequency $\mathrm{R}$ and a resultant decrease in frequency-dependence of resistance. In addition FOT has been reported to demonstrate larger sensitivity to inhaled corticosteroid or to $\beta$-agonist inhalation than spirometry (68). 
According to a study on PFT in preschool children in 2007, FOT has been successfully performed in different settings, and a number of studies have demonstrated that FOT was capable of identifying airway obstruction and reactions to bronchodilators and bronchoconstrictors (71). Several studies have been developed to assess bronchodilator responses using FOT. Marotta et al. (7) performed a study in 4-year old children concluding that IOS bronchodilator responses are remarkably abnormal in this population (children presented a significant bronchodilator response), and that IOS is a useful diagnostic tool in detection of early asthma development. Oostveen et al. (72) performed a comprehensive review on methodology, recommendations and future developments of FOT in clinical practice stating that FOT is a reliable method to assess bronchial hyperresponsiveness in adults and children. Ortiz et al. (8) performed an IOS study in children 2 to 5 years old in El Paso, Texas, finding that IOS is an acceptable method of assessing airway responses to bronchoactive drugs in this age group. In a more recent study related to the use of FOT to detect bronchodilation in children, Bar-Yishay et al. (73) concluded that FOT could reliably measure response to bronchodilator therapy. Recently Song et al (13) researched the utility of impulse oscillometry in young children with asthma finding that asthmatic children differed from control subjects in IOS-assessed bronchodilator response and that there were some significant correlations between bronchodilator responses of spirometric and IOS parameters. Galant et al. (65) stated that bronchodilator response (BDR) would appear to give important additional information about airway inflammation and found that IOS is a promising test to identify asthmatic preschoolers.

All this evidence confirms that lung function in children and adolescents is sensitively and accurately assessed by IOS, before and after bronchodilation. Nevertheless few longitudinal Forced Oscillation (FO) data exist in healthy subjects or in those with airflow obstruction. Oostveen et al. (72) noted the need for a practical FO index to define airway obstruction.

\subsection{IOS studies}

\subsubsection{IOS vs. Spirometry}

Table 1 provides a summary of several IOS vs Spirometry studies, with their descriptions, different IOS parameters analyzed and conclusions.

Table 1 summarizes the utility of different IOS indexes of lung function in relation to spirometric parameters in different subpopulations studied between 2002 and 2009. The conclusions emphasize the necessity to analyze these parameters to determine their efficacy to differentiate between Healthy and Impaired respiratory systems. In our study we focused on the most significant IOS indexes of lung function and pushed the boundary by further analyzing the respiratory impedance Model Parameters to differentiate between Healthy and Impaired respiratory conditions in children. 


\begin{tabular}{|c|c|c|c|}
\hline Researchers & $\begin{array}{l}\text { Evaluated } \\
\text { Population }\end{array}$ & $\begin{array}{l}\text { Evaluated } \\
\text { Parameters }\end{array}$ & Conclusions \\
\hline Marota et al. [7] & $\begin{array}{l}\text { asthmatic and non- } \\
\text { asthmatic }\end{array}$ & R5, X5, Fres & IOS bronchodilator responses are remarkably \\
\hline \multirow[t]{2}{*}{2003} & 4 years & & abnormal in this population. \\
\hline & & & IOS is a useful tool for asthma assessment \\
\hline \multirow{4}{*}{$\begin{array}{l}\text { Goldman et al. } \\
\text { [9] } \\
2002\end{array}$} & asthmatics & R5, R5-R15, AX & $\begin{array}{l}\text { Significant differences between R5, R5-R15 and } \\
\text { AX were seen. }\end{array}$ \\
\hline & 10-17 years & & Spirometric indices showed no change. \\
\hline & & & IOS parameters are sensitive measures \\
\hline & & & $\begin{array}{c}\text { of bronchomotor tone changes in these } \\
\text { adolescents. }\end{array}$ \\
\hline \multirow[t]{3}{*}{ Saadeh et al. [11] } & asthma symptoms & R5, R5-R15, AX & $\begin{array}{l}\text { Some asthmatic patients manifest normal } \\
\text { spirometry }\end{array}$ \\
\hline & $4-62$ years & & despite continuing symptoms, these patients \\
\hline & & & may be more sensitively managed using IOS \\
\hline \multirow[t]{4}{*}{ Gaylor et al. [6] } & asthmatics & R5, R5-R15, AX & $\begin{array}{c}\text { IOS shows systematic improvements after } \\
\text { inhaled }\end{array}$ \\
\hline & $5-80$ years & & $\begin{array}{c}\text { levalbuterol, FO is more sensitive than } \\
\text { spirometry }\end{array}$ \\
\hline & & & $\begin{array}{c}\text { and IOS should be considered before changing } \\
\text { therapy in }\end{array}$ \\
\hline & & & asthmatic patients whose $\mathrm{FEV}_{1}$ fails to improve \\
\hline \multirow[t]{4}{*}{ Vink et al. [12] } & asthmatics & $\begin{array}{c}\mathrm{R} \text { and } \mathrm{X} \text { at } 5-35 \\
\mathrm{~Hz}\end{array}$ & $\begin{array}{c}\text { Resistance values measured at } 5 \mathrm{~Hz} \text { showed to } \\
\text { be }\end{array}$ \\
\hline & $5-17$ years & & superior to PEF measurements, \\
\hline & & & $\begin{array}{l}\text { IOS parameters can be easily used as an } \\
\text { indirect }\end{array}$ \\
\hline & & & measure of airflow obstruction. \\
\hline \multirow[t]{6}{*}{ Song et al. [13] } & $\begin{array}{l}\text { asthmatics and } \\
\text { controls }\end{array}$ & $\begin{array}{l}\text { R5, R10, R20, } \\
\text { R35 }\end{array}$ & $\begin{array}{l}\text { Spirometry did not present statistically } \\
\text { significant }\end{array}$ \\
\hline & 3-6 years & & differences between groups. \\
\hline & & & $\begin{array}{c}\text { There were some significant correlations } \\
\text { between }\end{array}$ \\
\hline & & & $\begin{array}{l}\text { bronchodilator responses of spirometry and } \\
\text { IOS parameters. }\end{array}$ \\
\hline & & & IOS is a useful diagnostic tool and \\
\hline & & & $\begin{array}{l}\text { might be a helpful objective outcome } \\
\text { measure. }\end{array}$ \\
\hline \multirow[t]{2}{*}{ Song et al. [14] } & $\begin{array}{l}\text { asthmatics and } \\
\text { controls }\end{array}$ & $\begin{array}{c}\mathrm{R} \text { and } \mathrm{X} \text { at } 5-35 \\
\mathrm{~Hz}\end{array}$ & \multirow{2}{*}{$\begin{array}{c}\text { FEV } 1 \text { and PEFR showed significant correlation } \\
\text { with } \\
\text { impedance and resistance at 5,10,20 and } 35 \mathrm{~Hz} \\
\text { in both groups, }\end{array}$} \\
\hline & $7-15$ years & R5-R20 & \\
\hline
\end{tabular}




\begin{tabular}{|c|c|c|c|}
\hline Researchers & $\begin{array}{l}\text { Evaluated } \\
\text { Population }\end{array}$ & $\begin{array}{c}\text { Evaluated } \\
\text { Parameters }\end{array}$ & Conclusions \\
\hline & & & $\begin{array}{c}\text { FVC correlated with } \mathrm{Z} \text { and } \mathrm{R} \text { at } 10,20 \text { and } 35 \\
\mathrm{~Hz} \text { in both groups. } \\
\text { IOS is an appropriate measure of lung } \\
\text { function } \\
\text { when spirometry and PEF can not be } \\
\text { performed. }\end{array}$ \\
\hline $\begin{array}{c}\text { Antonova et al. } \\
{[15]}\end{array}$ & $\begin{array}{l}\text { mild and moderate } \\
\text { asthmatics } \\
6 \text { years }\end{array}$ & $\mathrm{Z}, \mathrm{R} 5, \mathrm{X} 5, \mathrm{~F}_{\text {res }}$ & $\begin{array}{l}\text { No significant differences for spirometry were } \\
\text { found, } \\
\text { IOS presented significant increase in total } \\
\text { impedance }(\mathrm{Z}), \mathrm{R} 5 \\
\text { and Fres, and significant decrease in X5. } \\
\text { IOS may be more sensitive than spirometry } \\
\text { for assessment of } \\
\text { inflammatory process and degree of asthma } \\
\text { severity. }\end{array}$ \\
\hline $\begin{array}{l}\text { Linares et al. } \\
\text { [16] } \\
2002\end{array}$ & $\begin{array}{l}\text { asthmatics } \\
6-15 \text { years }\end{array}$ & R5, X5, Fres & $\begin{array}{l}\text { There were good correlation between } \\
\text { spirometry and IOS, } \\
\text { best correlation: R5 and } \mathrm{FEV}_{1} \text {, and } \mathrm{R} 5 \text { and } \\
\text { FEF } 25 \text {. }\end{array}$ \\
\hline $\begin{array}{l}\text { Lewis-Brown et } \\
\text { al. [17] } \\
2005\end{array}$ & $\begin{array}{l}\text { asthmatics } \\
\text { 5-18 years }\end{array}$ & R5 & $\begin{array}{l}\text { Spirometry and IOS should be used together in } \\
\text { asthma } \\
\text { evaluation. }\end{array}$ \\
\hline $\begin{array}{l}\text { Nieto et al. [24] } \\
\qquad 2006\end{array}$ & mild asthma & $\begin{array}{c}\text { Z5, R5, } \\
\text { R20,X5,Fres }\end{array}$ & $\begin{array}{l}\text { Z5, R5, R20, X5, and Fres showed improvements, } \\
\text { no changes were found in the control group. } \\
\text { IOS is more sensitive than conventional } \\
\text { spirometry. }\end{array}$ \\
\hline Hur et al. [25] & children & $\mathrm{X} 5$ and $\mathrm{R} 5$ & $\begin{array}{c}\text { IOS parameters were more discriminative } \\
\text { than FEV for } \\
\text { detecting decreased lung function and showed } \\
\text { a good } \\
\text { correlation with } \mathrm{FEV}_{1} \text {. }\end{array}$ \\
\hline Larsen et al. [26] & $\begin{array}{l}\text { mild to moderate } \\
\text { asthma } \\
\text { 6-14 years }\end{array}$ & $\mathrm{AX}$ & $\begin{array}{l}\text { AX was unique in reflecting ongoing } \\
\text { improvement } \\
\text { in contrast to spirometric values, AX might } \\
\text { detect } \\
\text { alterations in airway mechanics not reflected by } \\
\text { spirometry. }\end{array}$ \\
\hline $\begin{array}{l}\text { Hellinckx et al. } \\
\text { [28] }\end{array}$ & $\begin{array}{l}\text { healthy and asthmatic } \\
\text { kindergarten children } \\
2.7 \text { - } 6.6 \text { years old }\end{array}$ & $\begin{array}{c}\mathrm{R} \text { and } \mathrm{X} \text { at 5-35 } \\
\mathrm{Hz}, \mathrm{F}_{\mathrm{res}}\end{array}$ & $\begin{array}{l}\text { No significant differences between groups for } \\
\text { IOS parameters } \\
\text { A change in R5 of } 40 \% \text { is to be considered as the } \\
\text { cut-off } \\
\text { for a "positive" bronchodilator response. }\end{array}$ \\
\hline
\end{tabular}




\begin{tabular}{|c|c|c|c|}
\hline Researchers & $\begin{array}{l}\text { Evaluated } \\
\text { Population }\end{array}$ & $\begin{array}{l}\text { Evaluated } \\
\text { Parameters }\end{array}$ & Conclusions \\
\hline $\begin{array}{l}\text { Graw-Panzer et } \\
\text { al. [29] }\end{array}$ & 46 inner-city children & R5 and R20 & $\begin{array}{c}\text { There is increased airway resistance as } \\
\text { measured by }\end{array}$ \\
\hline \multirow[t]{2}{*}{2009} & with asthma & & $\begin{array}{l}\text { IOS when there is airway obstruction measured } \\
\text { by }\end{array}$ \\
\hline & $4-20$ years & & $\begin{array}{l}\text { spirometry. A mean drop of R5 by }-24 \text { was } \\
\text { found to be significant }\end{array}$ \\
\hline
\end{tabular}

Table 1. IOS vs Spirometry Studies

\subsubsection{IOS vs Other techniques}

Other researchers have compared IOS with spirometry and other techniques. Table 2 presents a summary of several such studies, with their descriptions, different IOS parameters analyzed and conclusions.

\begin{tabular}{|c|c|c|c|}
\hline \multicolumn{4}{|c|}{ IOS, Spirometry and Whole body Plethysmography } \\
\hline Researchers & $\begin{array}{l}\text { Evaluated } \\
\text { Population }\end{array}$ & $\begin{array}{l}\text { Evaluated } \\
\text { Parameters }\end{array}$ & General Conclusions \\
\hline $\begin{array}{l}\text { Olaguibel } \\
\text { et al. [18] }\end{array}$ & asthmatics & R5, R20, X5 & $\begin{array}{l}\text { IOS was well accepted for young asthmatic children } \\
\text { and }\end{array}$ \\
\hline \multirow[t]{2}{*}{2005} & 3-6 year old & & $\begin{array}{l}\text { produced reproducible and sensitive indices of lung } \\
\text { function, }\end{array}$ \\
\hline & & & $\begin{array}{c}\text { R5 correlated with spirometry and plethysmographic } \\
\text { values. }\end{array}$ \\
\hline \multicolumn{4}{|c|}{ IOS and Whole body Plethysmography } \\
\hline $\begin{array}{l}\text { Tomalak et } \\
\text { al. [19] }\end{array}$ & $\begin{array}{l}\text { chronic respiratory } \\
\text { diseases }\end{array}$ & R5, R20, R35 & All three resistances correlated significantly with \\
\hline 2006 & $\begin{array}{l}\text { (asthma, allergic } \\
\text { diseases, cystic }\end{array}$ & & $\begin{array}{c}\text { plethysmographic Raw and the strongest correlation } \\
\text { was observed for R5. }\end{array}$ \\
\hline & $\begin{array}{c}\text { fibrosis, } \\
\text { bronchiectasis and } \\
\text { lung }\end{array}$ & & $\begin{array}{l}\text { IOS may be useful in diagnosing children with } \\
\text { obstructive respiratory diseases. }\end{array}$ \\
\hline & fibrosis) & & \\
\hline & 5-18 years & & \\
\hline
\end{tabular}




\begin{tabular}{|c|c|c|c|}
\hline \multicolumn{4}{|c|}{ O2) and Whole Body Plethysmography } \\
\hline $\begin{array}{l}\text { Bisgaard et } \\
\text { al. [20] }\end{array}$ & suspected asthma & $\begin{array}{l}\mathrm{R} \text { and } \mathrm{X} \text { at } 5-35 \\
\mathrm{~Hz}\end{array}$ & $\begin{array}{c}\text { IOS total respiratory impedance }(\mathrm{Z}), \mathrm{Rint} \text {, and Ptc,O2 } \\
\text { changed }\end{array}$ \\
\hline \multirow[t]{2}{*}{1995} & \multirow[t]{2}{*}{ 4-6 years } & & $\begin{array}{l}\text { in parallel with sRaw and FEV1, these three } \\
\text { parameters }\end{array}$ \\
\hline & & & $\begin{array}{l}\text { provide convenient indices of changes in lung } \\
\text { function. }\end{array}$ \\
\hline \multicolumn{2}{|l|}{$\begin{array}{l}\text { Klug et al. } \\
\qquad[10]\end{array}$} & \multirow[t]{2}{*}{$\begin{array}{l}\mathrm{R} \text { and } \mathrm{X} \text { at } 5-35 \\
\mathrm{~Hz}\end{array}$} & $\begin{array}{l}\text { All the evaluated techniques reliably reflect short- } \\
\text { term }\end{array}$ \\
\hline 1996 & $2-4$ years & & changes in lung function. \\
\hline \multicolumn{4}{|c|}{ IOS, Interrupter Technique Resistance (Rint) and Whole Body Plethysmography } \\
\hline $\begin{array}{l}\text { Klug et al. } \\
\text { [4] }\end{array}$ & \multirow[t]{2}{*}{$\begin{array}{l}\text { Caucasian, no } \\
\text { chronic diseases }\end{array}$} & \multirow[t]{2}{*}{$\begin{array}{l}\text { R5,R10,X5,X10, } \\
\text { Z5,Fres }\end{array}$} & $\begin{array}{l}\text { Techniques require minimal cooperation and } \\
\text { allowed }\end{array}$ \\
\hline 1998 & & & $\begin{array}{l}\text { measurement of lung function in } \mathbf{8 0} \% \text { of the tested } \\
\text { children. }\end{array}$ \\
\hline $\begin{array}{l}\text { Nielsen et } \\
\text { al. [21] }\end{array}$ & $\begin{array}{l}\text { asthmatics and } \\
\text { controls }\end{array}$ & \multirow[t]{2}{*}{$\mathrm{R} 5, \mathrm{X} 5$} & Whole body plethysmography (sRaw) was superior \\
\hline 2000 & $2-5$ years & & in separating both groups. \\
\hline $\begin{array}{l}\text { Nielsen et } \\
\text { al. [23] }\end{array}$ & $\begin{array}{l}\text { asthmatics and } \\
\text { controls }\end{array}$ & \multirow[t]{2}{*}{$\mathrm{R} 5, \mathrm{X} 5$} & Whole body plethysmography (sRaw) was superior \\
\hline 2001 & $2-5$ years & & in separating both groups. \\
\hline \multicolumn{4}{|c|}{ IOS, Spirometry and exhaled nitric oxide (eNO) } \\
\hline $\begin{array}{l}\text { Todaki et al } \\
\qquad[30]\end{array}$ & $\begin{array}{c}62 \text { asthmatics, } 13 \\
\text { wheezy and }\end{array}$ & $\begin{array}{l}\text { R20 and R5- } \\
\text { R20 }\end{array}$ & A significant decrease occurred in the eNO level \\
\hline 2009 & 17 healthy children & & and it correlated with maximal expiratory flow at $50 \%$ \\
\hline
\end{tabular}

Table 2. IOS vs other Techniques Studies

Table 2 summarizes the utility of different IOS indexes of lung function in relation to parameters acquired from a variety of other pulmonary function test in different 
subpopulations studied between 1995 and 2009. The conclusions emphasize the necessity to analyze these IOS parameters to determine their efficacy to differentiate between Healthy and Impaired respiratory systems.

\subsubsection{IOS studies}

Table 3 presents a summary of several IOS studies, with their descriptions, different IOS parameters analyzed and conclusions.

\begin{tabular}{|c|c|c|c|}
\hline Researchers & Evaluated Population & $\begin{array}{c}\text { Evaluated } \\
\text { Parameters }\end{array}$ & General Conclusions \\
\hline Ortiz et al. [8] & asthmatics & X5 & $\begin{array}{l}\text { IOS is an acceptable method to assess airway } \\
\text { response }\end{array}$ \\
\hline 2002 & 2-5 years & & to bronchoactive drugs in this age group. \\
\hline $\begin{array}{l}\text { Goldman et al. } \\
\qquad[22]\end{array}$ & asthmatics & R5, R5-R20, AX & $\begin{array}{l}\text { IOS indices are sensitive measures of lung } \\
\text { mechanical }\end{array}$ \\
\hline 2008 & $2-5$ years & & $\begin{array}{c}\text { responses to bronchodilators in this group of } \\
\text { children. }\end{array}$ \\
\hline $\begin{array}{c}\text { Menendez et al. } \\
\text { [31] }\end{array}$ & asthmatics & R5,R5-R20, AX & IOS R5, R5-R20, AX are sensitive measures of \\
\hline \multirow[t]{2}{*}{2008} & $2-5$ years & & \multirow{2}{*}{$\begin{array}{l}\text { lung mechanics responses to SABA and LABA } \\
\text { in } \\
\text { pre-school children with asthma }\end{array}$} \\
\hline & & & \\
\hline Jee et al. [32] & asthmatics and & $R, X$, Fres and $A X$ & IOS parameters were significantly different \\
\hline \multirow[t]{2}{*}{2010} & $\begin{array}{l}\text { chronic couugh } \\
\text { (controls) }\end{array}$ & & between groups in the methacholine challenge \\
\hline & children & & \\
\hline
\end{tabular}

Table 3. IOS studies

The outcomes of most recent studies carried out between 2002 and 2010 presented in table 3 show the utility of different IOS parameters including the AX, "the Godlman's Triangle", as sensitive measures to assess lung function.

As mentioned before, a number of previous IOS studies, summarized in tables 1, 2 and 3, demonstrate that IOS is able to sensitively and accurately evaluate lung function in children and adolescents, using Pre- and Post-bronchodilation conditions and bronchial challenge. These studies further show that that different IOS parameters at different frequencies serve as quantitative indicators to evaluate the Pre- and Post-bronchodilation response and bronchial challenge results. It is also remarkable that very few studies, only the most recent ones, reported the analysis of the AX parameter, which could offer critical information about lung function in children and adolescents as stated by Goldman et al. (9, 22), Nieto et al. (24), Larsen et al. (26) and Menendez et al. (31). Therefore, here we aim to statistically evaluate the performance of all IOS measured and calculated parameters (Resistances and Reactances, Fres, AX, frequency-dependence of resistance R3-R20 and R5-R20) over 3-35 Hz 
during pre-post- bronchodilation conditions. We place special emphasis on the previously observed most significant IOS and Model Parameters and will determine which one of these parameters is more effective in differentiating between Pre- and Post-bronchodilation conditions.

\subsubsection{IOS reference values - Previous studies}

Table 4 below presents a summary of several studies performed to determine Reference FOT and IOS values in children.

\begin{tabular}{|c|c|c|c|}
\hline Researchers & $\begin{array}{c}\text { Method } \\
\text { Used }\end{array}$ & Evaluated Population & Conclusions \\
\hline $\begin{array}{l}\text { Clement et al. } \\
\text { [35] } \\
1987\end{array}$ & FOT & $\begin{array}{l}\text { Belgian } \\
403 \text { healthy children } \\
4 \text { to } 20 \text { years }\end{array}$ & $\begin{array}{l}\mathrm{R} \text { and } \mathrm{X} \text { vs frequency data depended on } \\
\text { age or height, on sex, and slightly on weight. } \\
\text { With growth } \mathrm{R} \text { and the frequency-dependence } \\
\text { of } \mathrm{R} \text { decrease while } \mathrm{X} \text { increases. } \\
\text { Adult values of } \mathrm{R} \text { and } \mathrm{X} \text { can be observed at } \\
15 \text { years of age in girls and at } 18 \text { years in boys. }\end{array}$ \\
\hline $\begin{array}{l}\text { Ducharme et } \\
\text { al. [36] }\end{array}$ & FOT & $\begin{array}{c}\text { North American } \\
\text { children } \\
\text { (white,black,asian,oth } \\
\text { ers) } \\
206 \text { healthy children } \\
3 \text { to } 17 \text { years } \\
\end{array}$ & $\begin{array}{l}\text { Height is the best predictor for total respiratory } \\
\text { resistance at } 8,12 \text { and } 16 \mathrm{~Hz} \text { in children. }\end{array}$ \\
\hline $\begin{array}{l}\text { Frei et al. [37] } \\
\quad 2005\end{array}$ & IOS & $\begin{array}{c}\text { North American } \\
222 \text { white children } \\
3 \text { to } 10 \text { years old } \\
100 \text { to } 150 \mathrm{~cm} \text { in } \\
\text { height }\end{array}$ & $\begin{array}{l}\text { Standing height was the only significant } \\
\text { predictor for all variables } \\
\text { Resistance and Fres decreased by height, } \\
\text { but also by age, and reactance increased. }\end{array}$ \\
\hline $\begin{array}{l}\text { Dencker et al. } \\
\text { [38] } \\
2006\end{array}$ & IOS & $\begin{array}{l}\text { Swedish } \\
2 \text { to } 10 \text { years } \\
360 \text { children } \\
90-160 \mathrm{~cm}\end{array}$ & $\begin{array}{l}\text { All variables were related to body height, } \\
\text { and most of them were weakly related to weight. }\end{array}$ \\
\hline $\begin{array}{l}\text { Nowowiejska } \\
\text { et al. [39] } \\
2008\end{array}$ & IOS & $\begin{array}{l}\text { Polish } \\
626 \text { healthy children } \\
\text { aged 3-18 }\end{array}$ & $\begin{array}{c}\text { Body height was the best predictor and resistances } \\
\text { were best predicted with exponential } \\
\text { models while reactances with linear ones. } \\
\text { R decreased with height while } \mathrm{X} \text { increased. }\end{array}$ \\
\hline $\begin{array}{l}\text { Amra et al. [40] } \\
2008\end{array}$ & IOS & $\begin{array}{c}\text { Iranian } \\
509 \text { healthy children }\end{array}$ & $\begin{array}{c}\text { These measurements can be used clinically } \\
\text { to help diagnose and monitor respiratory } \\
\text { disorders, independent of effort. }\end{array}$ \\
\hline $\begin{array}{l}\text { Wee et al. [41] } \\
\qquad 2007\end{array}$ & IOS & $\begin{array}{l}\text { Korean } \\
92 \text { children }\end{array}$ & $\begin{array}{l}\text { IOS is a feasible method to measure the respiratory } \\
\text { resistance in children. The reference values using IOS }\end{array}$ \\
\hline
\end{tabular}




\begin{tabular}{|c|c|c|c|}
\hline Researchers & $\begin{array}{c}\text { Method } \\
\text { Used }\end{array}$ & Evaluated Population & Conclusions \\
\hline & & 7 to 12 years old & $\begin{array}{r}\text { seems to be useful to diagnose a variety } \\
\text { of respiratory diseases. }\end{array}$ \\
\hline Jee et al. [42] & IOS & Korean children & $\begin{array}{r}\text { Healthy young children had better results in IOS } \\
\text { parameters than main reference values reported } \\
\text { before. }\end{array}$ \\
& 143 & 3-6 years
\end{tabular}

Table 4. IOS Reference Values

Table 4 shows that only few studies have been developed in order to determine Reference IOS Values for children.

In 1991, the American Thoracic Society published guidelines focusing on spirometry as the most widely used lung function test. This document states that it is common practice to interpret the results of lung function tests in relation to Reference Values and in terms of whether or not they are considered to be within the "normal" range (33).

The European Respiratory Society published a workshop report in 1995 (34) about some commonly measured spirometry parameters such as RV, FRC and TLC. In this document it is stated that reference values play an important role in establishing whether the measured respiratory volumes fall within an expected range for healthy individuals of the same sex, similar stature, age, and other characteristics. In this report reference values (values for healthy subjects) and prediction equations for lung volumes for children and adults are obtained using different techniques like helium dilution and body plethysmography. These values are presented for different heights. However, this report does not include reference values and prediction equations based on the FOT or IOS.

From table 4 we observe that it is crucial to have IOS Reference Values for children. These have been very effective in the detection of lung abnormalities, as demonstrated in table 1, 2, and 3. Therefore, in our research here we attempt to take the first steps towards establishing IOS Reference Values in Healthy North American Anglo and Hispanic children 5 to 19 years old. We also aim to present baseline (pre-) and post-bronchodilation IOS parameter values for this subpopulation of children with Probable Small Airway Impairment (PSAI), Small Airway Impairment (SAI) and Asthma (A).

\section{Respiratory system models}

The IOS and FOT impedance curves can be represented by equivalent electrical circuit models of the human respiratory system with components analogous to the resistances, compliances and inertances inherent in the characterization of this system. Respiratory system component values could be estimated using well-established parameter estimation methods. These could then be used to assist physicians in the diagnosis and treatment of different respiratory diseases (43). 
In our research we have special interest in the small airways disease (SAD) or small airway impairment (SAI) and asthma. An effective means to characterize small airway dysfunction is by integrating realistic models of lung mechanics based on physiological measurements made by FOT or IOS and other techniques (74).

Different equivalent electrical circuit models with lumped parameter components representing the resistances, inertances, and compliances of the respiratory system have been developed and analyzed over the years by different research groups (44).

Previous work by our research team has focused on development and analysis of six different equivalent electrical circuit models of human respiratory impedance. Our efforts to date, have demonstrated that the performance of the extended RIC (eRIC) and augmented RIC (aRIC) models rank in the middle of a series of conventional models developed over the past several decades in terms of total cumulative error. However, they provide parameter estimates that are physiologically more realistic and in line with expected values in healthy subjects and those suffering from pulmonary diseases than previous models (43-51).

In the following paragraphs we will introduce the two respiratory system models analyzed in this study: the Extended Resistance-Inertance-Compliance (eRIC) and the Augmented Resistance-Inertance-Compliance (aRIC) models.

\section{a. Extended RIC (eRIC) Model}

This model is proposed as an improvement of the RIC model, with an additional Peripheral resistance $\left(R_{p}\right)$ connected in parallel with the capacitance. Therefore, the eRIC model is composed of central (large airway) Resistance $\left(R_{c}\right)$, large airway Inertance (I), peripheral (small airway) Compliance $\left(C_{p}\right)$ and peripheral (small airway) Resistance $\left(R_{p}\right)$. This added $R_{p}$ allows for the frequency-dependence of resistance observed in impedance data, which is not possible for the RIC model. Rp models the small airways resistance. On the other hand the eRIC model can be also considered as a simplification of either DuBois' (with $I_{t}$ equal to zero and $C_{t}$ equal to infinity) or the Mead's model (with $C_{l}, C_{w}$ equal to infinity and $C_{e}$ equal to zero) (43). The electrical equivalent circuit for the eRIC model is shown in Figure 3:

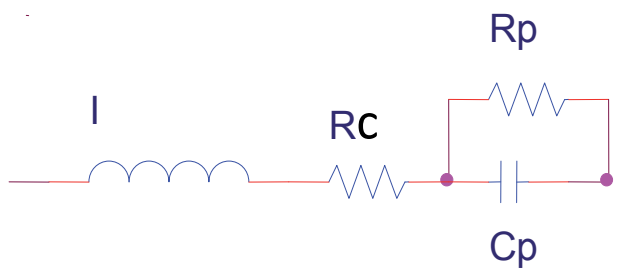

Figure 3. eRIC model (47).

The eRIC model impedance is calculated as follows: 


$$
Z=R c+\frac{R_{p}}{1+\left(\omega R_{p} C p\right)^{2}}+j\left(\omega I-\frac{\omega R_{p}^{2} C_{p}}{1+\left(\omega R_{p} C p\right)^{2}}\right)
$$

\section{b. Augmented RIC Model}

This model was proposed as an improvement to the eRIC model and it can be considered as a simplification of the Mead's model. aRIC is composed of central (large airway) Resistance (Rc), large airway Inertance (I), peripheral (small airway) Compliance $(\mathrm{Cp})$, peripheral (small airway) Resistance (Rp) and an additional compliance Ce (see figure 4), representing extrathoracic compliance. Its additional capacitance $\mathrm{Ce}$, representing extrathoracic compliance, is thought to increase the real part of the respiratory system's impedance at the higher frequencies due to upper airways shunt effects as observed in IOS data (44).

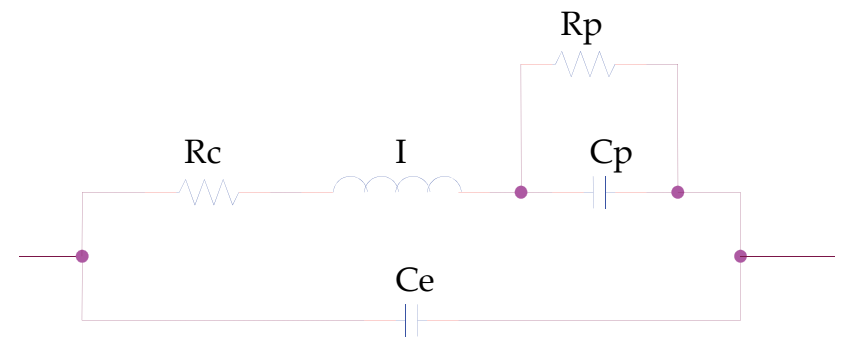

Figure 4. aRIC Model (47).

The aRIC impedance is given by:

$$
\begin{gathered}
Z=\frac{A\left(R c A+R_{p}\right)}{\left[A\left(1-\omega^{2} I C_{e}\right)+\left(\omega^{2} R_{p}^{2} C p C_{e}\right)\right]^{2}+\left[\omega C_{e}\left(R c A+R_{p}\right)\right]^{2}}+ \\
j \frac{\omega\left(I A-R_{p}^{2} C p\right)\left[A-\omega^{2} C_{e}\left(I A-R_{p}^{2} C p\right)\right]-\omega C_{e}\left(R c A+R_{p}\right)^{2}}{\left[A\left(1-\omega^{2} I C_{e}\right)+\left(\omega^{2} R_{p}^{2} C p C_{e}\right)\right]^{2}+\left[\omega C_{e}\left(R c A+R_{p}\right)\right]^{2}}
\end{gathered}
$$

\subsection{Parameter estimation technique}

Lung properties of a subject can be characterized by determination of the parameters of a respiratory system model that best fits its behavior. This information can then be used, with comparison to Reference Values, to determine underdeveloped features or existence of pathological conditions (55).

For this research the eRIC (Rc, I, Rp and Cp) and aRIC (Rc, I, Rp, Cp and Ce) model parameters were estimated using the average values of Resistances ( $R$ values) and Reactances (X values) from 5 to $25 \mathrm{~Hz}$ (R5, R10, R15, R20, R25, X5, X10, X15, X20 and X25) for each child tested. 
In Table 5 an example of respiratory system's IOS-based Resistance and Reactance values for a healthy male child (15 years old, $181.6 \mathrm{~cm}$ height and $84.1 \mathrm{~kg}$ weight) are presented. These IOS data were recorded before and after the use of a bronchodilator (Pre-B and post-B). In Table 6 an example of the Model Parameters calculated for the same child is given.

\begin{tabular}{|c|c|c|c|c|c|c|c|c|c|c|c|}
\hline Pre-B & R5 & R10 & R15 & R20 & R25 & X3 & X5 & X10 & X15 & X20 & X25 \\
\hline & 0.32 & 0.27 & 0.27 & 0.28 & 0.3 & -0.11 & -0.08 & -0.01 & 0.03 & 0.06 & 0.08 \\
\hline Post-B & R5 & R10 & R15 & R20 & R25 & X3 & X5 & X10 & X15 & X20 & X25 \\
\hline & 0.3 & 0.26 & 0.26 & 0.28 & 0.3 & -0.12 & -0.08 & 0 & 0.04 & 0.08 & 0.11 \\
\hline
\end{tabular}

Table 5. Respiratory system's Resistance $(\mathrm{kPa} / \mathrm{l} / \mathrm{s})$ and Reactance $(\mathrm{kPa} / \mathrm{l} / \mathrm{s})$ values for a healthy male child.

\begin{tabular}{|c|c|c|c|c|c|c|c|c|c|c|c|}
\hline pre-B & aRIC & $\begin{array}{c}\mathbf{R c} \\
(\mathrm{kPa} / \mathrm{l} / \mathrm{s}) \\
\end{array}$ & $\begin{array}{c}\mathbf{R p} \\
\mathrm{kPa} / \mathrm{l} / \mathrm{s})\end{array}$ & $\begin{array}{c}\mathbf{I} \\
\left(\mathrm{kPa} / \mathrm{l} / \mathrm{s}^{2}\right)\end{array}$ & $\begin{array}{c}\mathrm{Cp} \\
(1 / \mathrm{kPa}) \\
\end{array}$ & $\begin{array}{c}\mathrm{Ce} \\
(1 / \mathrm{kPa})\end{array}$ & eRIC & $\begin{array}{c}\mathbf{R c} \\
(\mathrm{kPa} / \mathrm{l} / \mathrm{s}) \\
\end{array}$ & $\begin{array}{c}\mathbf{R p} \\
\mathrm{kPa} / \mathrm{l} / \mathrm{s})\end{array}$ & $\begin{array}{c}\mathrm{I} \\
\left(\mathrm{kPa} / 1 / \mathrm{s}^{2}\right) \\
\end{array}$ & $\begin{array}{c}\mathrm{Cp} \\
(1 / \mathrm{kPa}) \\
\end{array}$ \\
\hline & & 0.256 & 0.209 & 0.001 & 0.233 & 0.004 & & 0.277 & 0.314 & 0.0007 & 0.282 \\
\hline post-B & aRIC & $\begin{array}{c}\text { Rc } \\
(\mathrm{kPa} / \mathrm{l} / \mathrm{s}) \\
\end{array}$ & $\begin{array}{c}\mathbf{R p} \\
(\mathrm{kPa} / \mathrm{l} / \mathrm{s})\end{array}$ & $\begin{array}{c}\mathbf{I} \\
\left(\mathrm{kPa} / \mathrm{l} / \mathrm{s}^{2}\right)\end{array}$ & $\begin{array}{c}\mathrm{Cp} \\
(1 / \mathrm{kPa})\end{array}$ & $\begin{array}{c}\mathrm{Ce} \\
(1 / \mathrm{kPa})\end{array}$ & eRIC & $\begin{array}{c}\mathbf{R c} \\
(\mathrm{kPa} / \mathrm{l} / \mathrm{s})\end{array}$ & $\begin{array}{c}\mathbf{R p} \\
(\mathrm{kPa} / \mathrm{l} / \mathrm{s})\end{array}$ & $\begin{array}{c}\mathbf{I} \\
\left(\mathrm{kPa} / \mathrm{l} / \mathrm{s}^{2}\right) \\
\end{array}$ & $\begin{array}{c}\mathrm{Cp} \\
(1 / \mathrm{kPa})\end{array}$ \\
\hline & & 0.245 & 0.239 & 0.001 & 0.241 & 0.004 & & 0.274 & 0.58 & 0.0008 & 0.289 \\
\hline
\end{tabular}

Table 6. Model Parameters calculated for a healthy male child.

Estimating Model Parameters is comparable to curve-fitting. Consequently a suitable error criterion $\mathrm{E}$ has to be selected and minimized. For this research the least square (LS) criterion was selected as follows:

$$
E=\Sigma[Z R(f)-Z R, e s t(f)]^{2}+[Z x(f)-Z x, e s t(f)]^{2}
$$

where

$$
f=5,10,15,20,25 \mathrm{~Hz}
$$

This LS criterion was used to minimize the sum of the squared errors between the measured IOS ZR and Zx and the estimated resistive ZR,est and the estimated reactance Zx,est at frequencies between 5 to $25 \mathrm{~Hz}$ (at $5 \mathrm{~Hz}$ intervals). The LS criterion was selected due to its commonplace use, its relation with other system identification algorithms and its availability in different software packages (44).

Due to the nonlinear dependence of the aRIC and eRIC impedance functions on the model parameters, the Matlab lsq-nonlin (nonlinear LS) was used in both algorithms, which are based on Newton's Method. Each estimation run began with an initial random guess, a parameter estimate vector produced by a random number generator appropriately weighted. Random initial guesses ranging consistently from 0 to 5,0 to 0.5 , and 0 to 0.05 were used to estimate the values of resistances, capacitances and inductances, respectively. 
For each child's averaged IOS data a total of 50 iterations were used to find parameter estimates minimizing the error function, with the Matlab program stopping each time when E (error value) changed by less than a factor of $10^{-9}$ from one iteration to the next one. Therefore, the LS error value at the end provided a measure of the goodness of fit to the given test data for each model (44).

\section{Research method design and statistical analysis}

This study was developed as part of a NIH-funded research to perform IOS evaluation of lung function in Anglo and Hispanic children 5-19 years old living in the El Paso, Texas area. The data were collected at Western Sky Medical Research clinic and in a Health Fair held in a Socorro District school over a 3 years period (2006-2008). The IOS data collected for this research were quality-assured by our expert clinician and pulmonologist, the late Professor Michael Goldman and were then classified by him into four categories: Normal, Probable Small Airway Impairment (PSAI), Small Airway Impairment (SAI) and Asthma (A).

The data presented in this study were collected in 2008. The data collected were Pre- and Post-bronchodilation data. The date collected analyzed in this study were acquired from 47 children in 2008. Three to five IOS test replicates were performed on each subject to ensure reproducible tests without artifacts caused by air leaks, swallowing, breath holding or vocalization. IOS data were carefully reviewed off line and quality-assured to reject segments affected by airflow leak or swallowing artifacts. A Jaeger MasterScreen IOS (Viasys Healthcare, Inc. Yorba Linda, CA, and USA) was used in this study. The system was calibrated every day before data collection using a 3-L syringe for volume calibrations and a reference resistance $(0.2 \mathrm{KPa} / \mathrm{L} / \mathrm{s})$ for pressure calibrations. Children were asked to wear a nose clip, while breathing normally through a mouthpiece and were instructed to tightly close their lips around it to avoid air leakage. Children tested using a bronchodilation medicine, called Levalbuterol (Xopenex), were tested (pre-bronchodilation), performing 3 to 5 IOS tests. Then the medicine (Xopenex) was given to the children using a nebulizer for 6 minutes and after that the children were asked to rest for 10 minutes; and finally after this waiting period the children were again tested recording 3 to 5 IOS tests (postbronchodilation).

This research study was supported in part by NIH grant \#1 S11 ES013339-01 A1: UTEPUNM HSC ARCH Program on Border Asthma. The research protocol was approved by the Institutional Review Board of the University of Texas at El Paso. Informed consents were obtained from children over 18 years and the parents or care givers of children under 18 years. A questionnaire on asthma or allergy symptoms provided by the ARCH Program was then completed by the participants or their parents.

Statistical analyses of IOS measured and calculated parameters as well as the eRIC and aRIC model parameters between Pre- and Post-bronchodilation data were made using tdistribution (differences of the means) test and statistical significance was established at a $\mathrm{p}$ $<0.05$ level. 


\section{Results and discussions}

The IOS data collected from 47 children in 2008 and Model Parameters calculated from them, under pre-bronchodilation and post-bronchodilation conditions were analyzed. The eRIC and aRIC Model Parameters were calculated over the frequency range of 5 to $25 \mathrm{~Hz}$.

The IOS and Model Parameters selected for analysis were selected from our previous publications as they produced significant results to differentiate between Healthy and Impaired as well as, between Pre- and Post- bronchodilation conditions. These parameters were chosen due to their ability to evaluate respiratory system's properties and impairments. The IOS, eRIC and aRIC Model Parameters analyzed were: R3, R5, R3-R20, R5R20, X3, X5, AX, Fres, eRIC Cp, eRIC Rp, aRIC Cp and aRIC Rp. In addition to our previous published results, we included IOS parameters at $3 \mathrm{~Hz}$ (R3, X3, R3-R20) to our analysis here to evaluate their performance.

After quality assurance by our expert clinician, the collected IOS data from all 47 children were classified into four groups: 6 Healthy (or Normal), 4 PSAI, 11 definite SAI and 24 Asthmatics. Children in these four groups were either Anglo or Hispanic based upon their parent's declarations in the questionnaires.

Mean \pm standard deviation and range values (Pre- and Post-B data collected in 2008) for each IOS and Model Parameters analyzed are presented in Table 7 for Healthy children, in Table 8 for PSAI children, in Table 9 for children with SAI, and in Table 10 for those with Asthma.

\begin{tabular}{|c|c|c|c|c|c|}
\hline \multicolumn{6}{|c|}{ IOS Measurements and Model Parameters } \\
\hline \multicolumn{6}{|c|}{ Healthy Group $N=6$} \\
\hline \multicolumn{3}{|c|}{ Pre-Bronchodilation } & \multicolumn{3}{|c|}{ Post-Bronchodilation } \\
\hline & Range & Mean \pm SD & & Range & Mean \pm SD \\
\hline $\mathbf{R} 3(\mathrm{kPa} / \mathrm{l} / \mathrm{s})$ & $0.34-0.59$ & $0.41 \pm 0.09$ & $\mathbf{R 3}(\mathrm{kPa} / \mathrm{l} / \mathrm{s})$ & $0.30-0.54$ & $0.38 \pm 0.08$ \\
\hline $\mathbf{R 5}(\mathrm{kPa} / \mathrm{l} / \mathrm{s})$ & $0.29-0.51$ & $0.35 \pm 0.08$ & $\mathbf{R 5}(\mathrm{kPa} / \mathrm{l} / \mathrm{s})$ & $0.25-0.48$ & $0.33 \pm 0.08$ \\
\hline $\mathbf{R 3}-\mathbf{R 2 0}(\mathrm{kPa} / \mathrm{l} / \mathrm{s})$ & $0.05-0.12$ & $0.09 \pm 0.02$ & $\mathbf{R 3}-\mathbf{R 2 0}(\mathrm{kPa} / \mathrm{l} / \mathrm{s})$ & $0.03-0.13$ & $0.08 \pm 0.04$ \\
\hline $\mathbf{R 5}-\mathbf{R 2 0}(\mathrm{kPa} / \mathrm{l} / \mathrm{s})$ & -0.08 & $0.03 \pm 0.03$ & $\mathbf{R 5}-\mathbf{R 2 0}(\mathrm{kPa} / \mathrm{l} / \mathrm{s})$ & -0.1 & $0.03 \pm 0.04$ \\
\hline $\mathbf{X} 3(\mathrm{kPa} / \mathrm{l} / \mathrm{s})$ & -0.12 & $-0.16 \pm 0.05$ & $\mathbf{X} 3(\mathrm{kPa} / \mathrm{l} / \mathrm{s})$ & -0.13 & $-0.15 \pm 0.05$ \\
\hline $\mathbf{X 5}(\mathrm{kPa} / \mathrm{l} / \mathrm{s})$ & -0.06 & $-0.10 \pm 0.02$ & $\mathbf{X 5}(\mathrm{kPa} / \mathrm{l} / \mathrm{s})$ & -0.05 & $-0.10 \pm 0.02$ \\
\hline $\mathbf{A X}(\mathrm{kPa} / \mathrm{l})$ & $0.18-0.36$ & $0.26 \pm 0.06$ & $\mathbf{A X}(\mathrm{kPa} / \mathrm{l})$ & $0.16-0.36$ & $0.24 \pm 0.07$ \\
\hline Fres (1/s) & $10.26-13.46$ & $11.18 \pm 1.15$ & Fres $(1 / \mathrm{s})$ & $9.6-13.74$ & $10.99 \pm 1.76$ \\
\hline eRIC Cp $(1 / \mathrm{kPa})$ & $0.2073-0.2816$ & $0.2346 \pm 0.0284$ & eRIC Cp (1/kPa) & $0.1894-0.2892$ & $0.2297 \pm 0.0362$ \\
\hline $\mathbf{R p}(\mathrm{kPa} / 1 / \mathrm{s})$ & $0.2163-0.8016$ & $0.5665 \pm 0.2788$ & $\mathbf{R p}(\mathrm{kPa} / \mathrm{l} / \mathrm{s})$ & $0.2227-0.5801$ & $0.3501 \pm 0.1630$ \\
\hline aRIC Cp $(1 / \mathrm{kPa})$ & $0.1851-0.2331$ & $0.2091 \pm 0.0169$ & aRIC Cp $(1 / \mathrm{kPa})$ & $0.1436-0.2411$ & $0.2087 \pm 0.0366$ \\
\hline $\mathbf{R p}(\mathrm{kPa} / 1 / \mathrm{s})$ & $0.1891-0.7206$ & $0.3922 \pm 0.2057$ & $\mathbf{R p}(\mathrm{kPa} / \mathrm{l} / \mathrm{s})$ & $0.1992-0.6173$ & $0.3151 \pm 0.1710$ \\
\hline
\end{tabular}

Table 7. Healthy children IOS and Model Parameters Pre- and Post-B (N=6) 
Impulse Oscillometric Features and Respiratory System Models Track Small Airway Function in Children 125

\begin{tabular}{|c|c|c|c|c|c|}
\hline \multicolumn{6}{|c|}{ IOS Measurements and Model Parameters } \\
\hline \multicolumn{6}{|c|}{ PSAI Group $N=4$} \\
\hline \multicolumn{3}{|c|}{ Pre-Bronchodilation } & \multicolumn{3}{|c|}{ Post-Bronchodilation } \\
\hline & Range & Mean \pm SD & & Range & Mean \pm SD \\
\hline $\mathbf{R 3}(\mathrm{kPa} / \mathrm{l} / \mathrm{s})$ & $0.47-0.99$ & $0.67 \pm 0.25$ & $\mathbf{R} 3(\mathrm{kPa} / \mathrm{l} / \mathrm{s})$ & $0.37-0.96$ & $0.60 \pm 0.27$ \\
\hline $\mathbf{R 5}(\mathrm{kPa} / \mathrm{l} / \mathrm{s})$ & $0.39-0.84$ & $0.57 \pm 0.21$ & $\mathbf{R 5}(\mathrm{kPa} / \mathrm{l} / \mathrm{s})$ & $0.30-0.87$ & $0.50 \pm 0.26$ \\
\hline $\mathbf{R 3}-\mathbf{R 2 0}(\mathrm{kPa} / \mathrm{l} / \mathrm{s})$ & $0.14-0.44$ & $0.30 \pm 0.15$ & $\mathbf{R 3}-\mathbf{R 2 0}(\mathrm{kPa} / \mathrm{l} / \mathrm{s})$ & $0.14-0.39$ & $0.26-0.12$ \\
\hline $\mathbf{R 5}-\mathbf{R 2 0}(\mathrm{kPa} / \mathrm{l} / \mathrm{s})$ & $0.07-0.29$ & $0.19 \pm 0.11$ & $\mathbf{R 5}-\mathbf{R 2 0}(\mathrm{kPa} / \mathrm{l} / \mathrm{s})$ & $0.07-0.30$ & $0.17 \pm 0.10$ \\
\hline $\mathbf{X} 3(\mathrm{kPa} / \mathrm{l} / \mathrm{s})$ & -0.43 & $-0.28 \pm 0.11$ & $\mathbf{X} 3(\mathrm{kPa} / \mathrm{l} / \mathrm{s})$ & -0.12 & $-0.24 \pm 0.06$ \\
\hline $\mathbf{X 5}(\mathrm{kPa} / \mathrm{l} / \mathrm{s})$ & -0.18 & $-0.22 \pm 0.09$ & $\mathbf{X 5}(\mathrm{kPa} / \mathrm{l} / \mathrm{s})$ & -0.11 & $-0.17 \pm 0.05$ \\
\hline $\mathbf{A X}(\mathrm{kPa} / \mathrm{l})$ & $0.57-2.57$ & $1.31 \pm 0.95$ & $\mathbf{A X}(\mathrm{kPa} / \mathrm{l})$ & $0.43-2.20$ & $1.11 \pm 0.82$ \\
\hline $\operatorname{Fres}(1 / s)$ & $15.53-17.36$ & $16.44 \pm 0.92$ & Fres $(1 / s)$ & $12.47-17.19$ & $15.37 \pm 2.54$ \\
\hline eRIC Cp (1/kPa) & $0.0445-0.1616$ & $0.0963 \pm 0.0556$ & eRIC Cp $(1 / \mathrm{kPa})$ & $0.0243-0.1636$ & $0.0978 \pm 0.0621$ \\
\hline $\mathbf{R p}(\mathrm{kPa} / \mathrm{l} / \mathrm{s})$ & $0.4398-0.6730$ & $0.5443 \pm 0.1211$ & $\boldsymbol{R p}(\mathrm{kPa} / \mathrm{l} / \mathrm{s})$ & $0.3539-0.6174$ & $0.4886 \pm 0.1180$ \\
\hline aRIC Cp $(1 / k P a)$ & $0.0389-0.1616$ & $0.0858 \pm 0.0572$ & aRIC Cp $(1 / \mathrm{kPa})$ & $0.0077-0.1636$ & $0.0861 \pm 0.0690$ \\
\hline $\mathbf{R p}(\mathrm{kPa} / \mathrm{l} / \mathrm{s})$ & $0.3561-0.6730$ & $0.5116 \pm 0.1403$ & $\mathbf{R p}(\mathrm{kPa} / \mathrm{l} / \mathrm{s})$ & $0.3539-0.8784$ & $0.5339 \pm 0.2423$ \\
\hline
\end{tabular}

Table 8. PSAI children IOS and Model Parameters Pre- and Post-B (N=4)

\begin{tabular}{|c|c|c|c|c|c|}
\hline \multicolumn{6}{|c|}{ IOS Measurements and Model Parameters } \\
\hline \multicolumn{6}{|c|}{ SAI Group N=11 } \\
\hline \multicolumn{3}{|c|}{ Pre-Bronchodilation } & \multicolumn{3}{|c|}{ Post-Bronchodilation } \\
\hline & Range & Mean \pm SD & & Range & Mean \pm SD \\
\hline $\mathbf{R 3}(\mathrm{kPa} / \mathrm{l} / \mathrm{s})$ & $0.55-0.91$ & $0.73 \pm 0.12$ & $\mathbf{R 3}(\mathrm{kPa} / \mathrm{l} / \mathrm{s})$ & $0.50-0.92$ & $0.67 \pm 0.14$ \\
\hline $\mathbf{R 5}(\mathrm{kPa} / \mathrm{l} / \mathrm{s})$ & $0.46-0.72$ & $0.61 \pm 0.09$ & $\mathbf{R 5}(\mathrm{kPa} / \mathrm{l} / \mathrm{s})$ & $0.43-0.78$ & $0.57 \pm 0.12$ \\
\hline $\mathbf{R 3}-\mathbf{R 2 0}(\mathrm{kPa} / \mathrm{l} / \mathrm{s})$ & $0.25-0.57$ & $0.38 \pm 0.10$ & R3-R20(kPa/l/s) & $0.16-0.51$ & $0.30 \pm 0.10$ \\
\hline $\mathbf{R 5}-\mathbf{R 2 0}(\mathrm{kPa} / \mathrm{l} / \mathrm{s})$ & $0.17-0.38$ & $0.26 \pm 0.06$ & R5-R20(kPa/l/s) & $0.07-0.37$ & $0.20 \pm 0.08$ \\
\hline $\mathbf{X} 3(\mathrm{kPa} / \mathrm{l} / \mathrm{s})$ & -0.32 & $-0.32 \pm 0.11$ & $\mathbf{X} 3(\mathrm{kPa} / \mathrm{l} / \mathrm{s})$ & -0.27 & $-0.26 \pm 0.08$ \\
\hline $\mathbf{X 5}(\mathrm{kPa} / \mathrm{l} / \mathrm{s})$ & -0.27 & $-0.25 \pm 0.09$ & $\mathbf{X 5}(\mathrm{kPa} / \mathrm{l} / \mathrm{s})$ & -0.2 & $-0.20 \pm 0.07$ \\
\hline $\mathbf{A X}(\mathrm{kPa} / \mathrm{l})$ & $1.10-2.73$ & $1.87 \pm 0.52$ & $\mathbf{A X}(\mathrm{kPa} / \mathrm{l})$ & $0.54-2.61$ & $1.36 \pm 0.59$ \\
\hline Fres $(1 / s)$ & $17.37-23.15$ & $19.47 \pm 1.95$ & Fres $(1 / s)$ & $16.8-23.41$ & $19.44 \pm 2.26$ \\
\hline eRIC Cp $(\mathrm{l} / \mathrm{kPa})$ & $0.0356-0.0710$ & $0.0533 \pm 0.0105$ & eRIC Cp (1/kPa) & $0.0358-0.1485$ & $0.0733 \pm 0.0291$ \\
\hline $\mathbf{R p}(\mathrm{kPa} / \mathrm{l} / \mathrm{s})$ & $0.3126-0.9167$ & $0.5885 \pm 0.1990$ & $\mathbf{R p}(\mathrm{kPa} / \mathrm{l} / \mathrm{s})$ & $0.3082-0.8075$ & $0.5023 \pm 0.1651$ \\
\hline aRIC Cp $(1 / k P a)$ & $0.0150-0.1007$ & $0.0409 \pm 0.0230$ & aRIC Cp (1/kPa) & $0.0185-0.1485$ & $0.0485 \pm 0.0404$ \\
\hline $\mathrm{Rp}(\mathrm{kPa} / \mathrm{l} / \mathrm{s})$ & $0.3486-0.7291$ & $0.4992 \pm 0.1100$ & $\mathrm{Rp}(\mathrm{kPa} / \mathrm{l} / \mathrm{s})$ & $0.3243-0.5810$ & $0.4359 \pm 0.0982$ \\
\hline
\end{tabular}

Table 9. SAI children IOS and Models Parameter Pre- and Post-B (N=11) 


\begin{tabular}{|c|c|c|c|c|c|}
\hline \multicolumn{6}{|c|}{ IOS Measurements and Model Parameters } \\
\hline \multicolumn{6}{|c|}{ Asthma Group N=24 } \\
\hline \multicolumn{3}{|c|}{ Pre-Bronchodilation } & \multicolumn{3}{|c|}{ Post-Bronchodilation } \\
\hline & Range & Mean \pm SD & & Range & Mean \pm SD \\
\hline $\mathbf{R 3}(\mathrm{kPa} / \mathrm{l} / \mathrm{s})$ & $0.45-1.77$ & $0.82 \pm 0.26$ & $\mathbf{R 3}(\mathrm{kPa} / \mathrm{l} / \mathrm{s})$ & $0.37-1.31$ & $0.62 \pm 0.20$ \\
\hline $\mathbf{R 5}(\mathrm{kPa} / \mathrm{l} / \mathrm{s})$ & $0.39-1.28$ & $0.68 \pm 0.19$ & $\mathbf{R 5}(\mathrm{kPa} / \mathrm{l} / \mathrm{s})$ & $0.32-1.04$ & $0.52 \pm 0.16$ \\
\hline $\mathbf{R 3}-\mathbf{R 2 0}(\mathrm{kPa} / \mathrm{l} / \mathrm{s})$ & $0.14-1.24$ & $0.44 \pm 0.22$ & $\mathbf{R 3}-\mathbf{R 2 0}(\mathrm{kPa} / \mathrm{l} / \mathrm{s})$ & $0.10-0.78$ & $0.30 \pm 0.14$ \\
\hline $\mathbf{R 5 - R 2 0}(\mathrm{kPa} / \mathrm{l} / \mathrm{s})$ & $0.07-0.75$ & $0.30 \pm 0.14$ & R5-R20 $(\mathrm{kPa} / \mathrm{l} / \mathrm{s})$ & $0.05-0.52$ & $0.20 \pm 0.10$ \\
\hline $\mathbf{X} 3(\mathrm{kPa} / \mathrm{l} / \mathrm{s})$ & -0.74 & $-0.34 \pm 0.15$ & $\mathbf{X} 3(\mathrm{kPa} / \mathrm{l} / \mathrm{s})$ & -0.55 & $-0.27 \pm 0.13$ \\
\hline $\mathbf{X 5}(\mathrm{kPa} / \mathrm{l} / \mathrm{s})$ & -0.69 & $-0.29 \pm 0.14$ & $\mathbf{X 5}(\mathrm{kPa} / \mathrm{l} / \mathrm{s})$ & -0.47 & $-0.21 \pm 0.11$ \\
\hline $\mathbf{A X}(\mathrm{kPa} / \mathrm{l})$ & $0.45-6.61$ & $2.24 \pm 1.23$ & $\mathbf{A X}(\mathrm{kPa} / \mathrm{l})$ & $0.28-4.35$ & $1.38 \pm 0.90$ \\
\hline Fres $(1 / \mathrm{s})$ & $14.44-24.19$ & $19.48 \pm 2.64$ & $\operatorname{Fres}(1 / \mathrm{s})$ & $12.76-20.86$ & $17.68 \pm 2.35$ \\
\hline eRIC Cp (1/kPa) & $0.0200-0.1782$ & $0.0593 \pm 0.0347$ & eRIC Cp $(1 / \mathrm{kPa})$ & $0.0047-0.2466$ & $0.0848 \pm 0.0527$ \\
\hline $\mathbf{R p}(\mathrm{kPa} / \mathrm{l} / \mathrm{s})$ & $0.2925-1.8113$ & $0.6749 \pm 0.2949$ & $\mathbf{R p}(\mathrm{kPa} / \mathrm{l} / \mathrm{s})$ & $0.2318-1.2561$ & $0.5050 \pm 0.2148$ \\
\hline aRIC Cp $(1 / \mathrm{kPa})$ & $0.0148-0.1522$ & $0.0570 \pm 0.0390$ & aRIC Cp $(1 / \mathrm{kPa})$ & $0.0047-0.2087$ & $0.0776 \pm 0.0519$ \\
\hline $\mathbf{R p}(\mathrm{kPa} / \mathrm{l} / \mathrm{s})$ & $0.2250-1.6536$ & $0.5840 \pm 0.2848$ & $\mathbf{R p}(\mathrm{kPa} / \mathrm{l} / \mathrm{s})$ & $0.1604-1.1443$ & $0.4288 \pm 0.2033$ \\
\hline
\end{tabular}

Table 10. Asthmatic children IOS and Model Parameters Pre- and Post-B (N=24)

With the tabulation of IOS and Model Parameters we achieved one of the goals of this research, to present baseline (Pre-) and Post-bronchodilation IOS parameters in Anglo and Hispanic children, classified in four groups: Healthy (table 7), Probable Small Airway Impairment (PSAI) (table 8), Small Airway Impairment (SAI) (table 9) and Asthma (table 10).

As expected, in the Healthy group (table 7), comparing Pre- and Post-bronchodilation IOS and Model Parameters mean values, there are almost no differences between Pre- and Postbronchodilation measurements. In table 8 , for the PSAI group we observe a modest difference between the mean IOS and Model Parameters during the Pre and Postbronchodilation conditions. In contrast, in tables 9 and 10 we observe a noticeably bigger difference between the IOS and Model Parameters mean values, comparing Pre and Postbronchodilation conditions. Also as expected, the biggest difference between Pre- and Postbronchodilation conditions exists (table 10), in the Asthmatic children group, for all the IOS and Model Parameters mean values.

Our expert clinician confirmed that the range of values of all analyzed features: R3, R5, R3R20, R5-R20, X3, X5, AX, Fres, eRIC Cp, eRIC Rp, aRIC Cp and aRIC Rp obtained for the $\mathrm{SAD}$ and Asthmatic groups were comparable to those values he has observed over many years in other asthmatic children of the same age range. Going to increasingly abnormal levels of "diagnostic classification," R3, R5, R5-R20, AX, Fres and Rp continue to increase from Normal to PSAI to SAI to Asthma, while X3, X5 and Cp decreased in this progression. 
Figures 5, 6, 7, 8, 9, 10 and 11 show in a more graphical fashion the changes in the most significant IOS and Model Parameters mean values for each of the four groups.

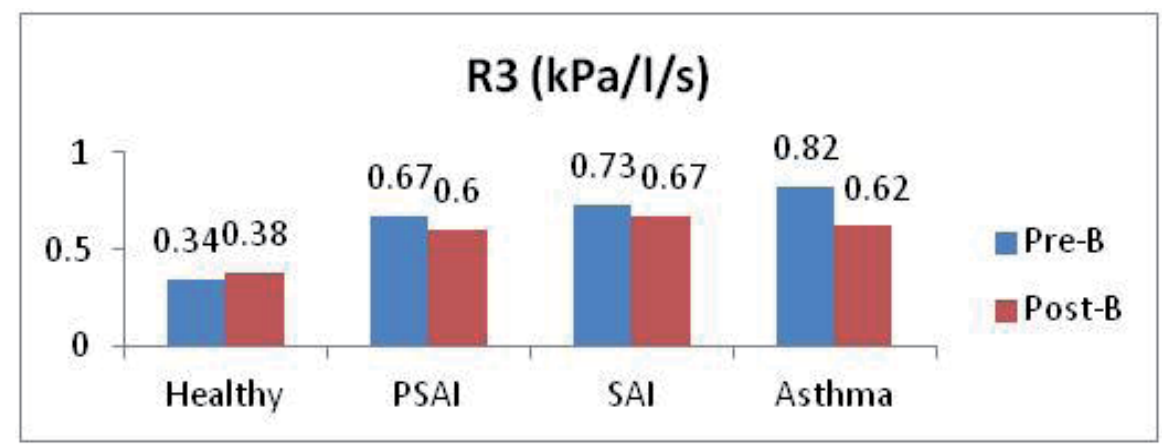

Figure 5. R3 mean values for the H, PSAI, SAI and Asthmatic children under Pre- and Post-B conditions.

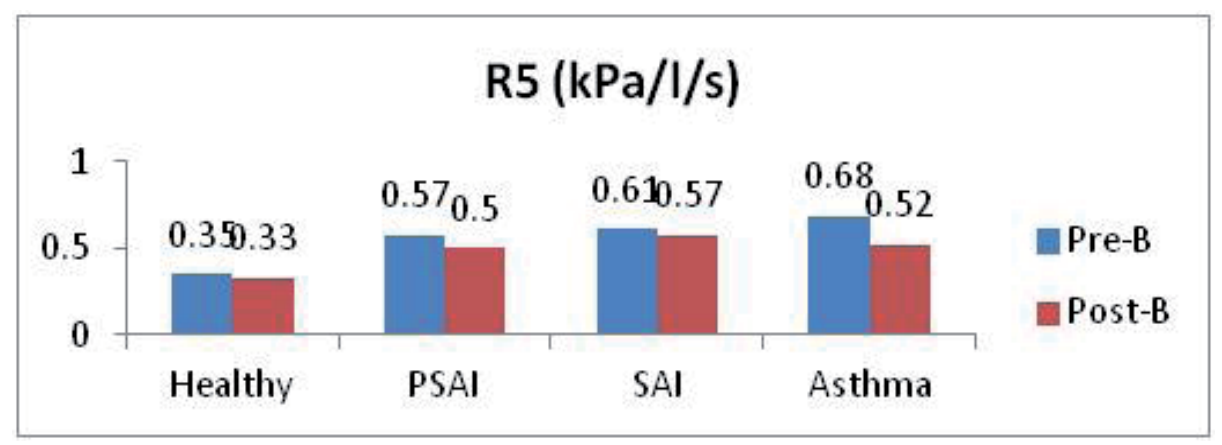

Figure 6. R5 mean values for the H, PSAI, SAI and Asthmatic children under Pre- and Post-B conditions.

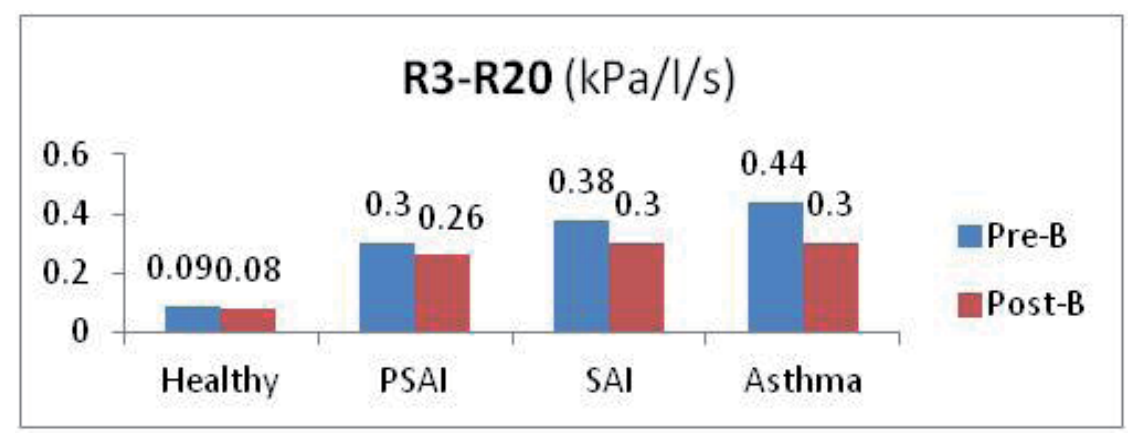

Figure 7. R3-R20 mean values for the H, PSAI, SAI and Asthmatic children under Pre- and Post-B conditions. 


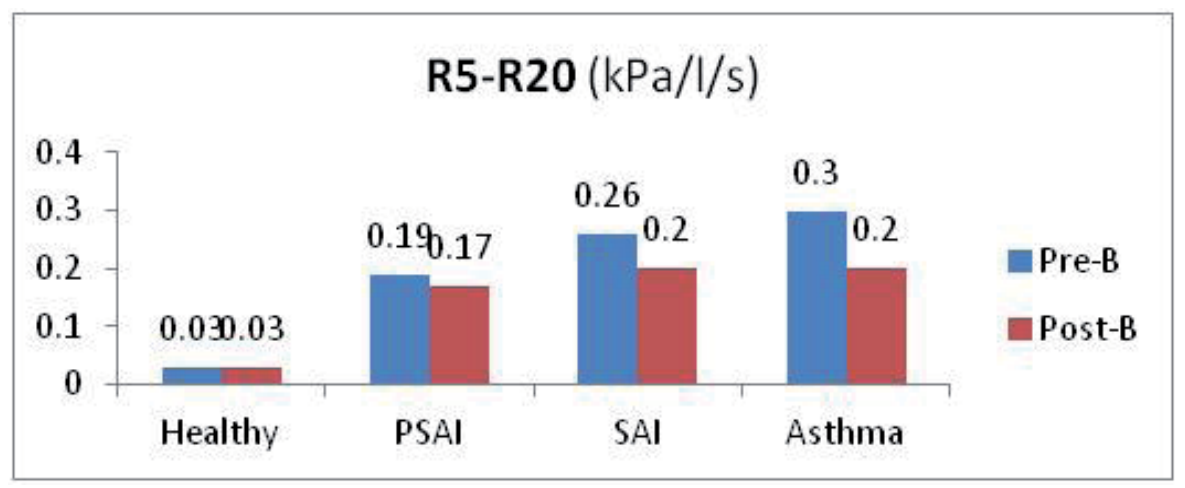

Figure 8. R5-R20 mean values for the H, PSAI, SAI and Asthmatic children under Pre- and Post-B conditions.

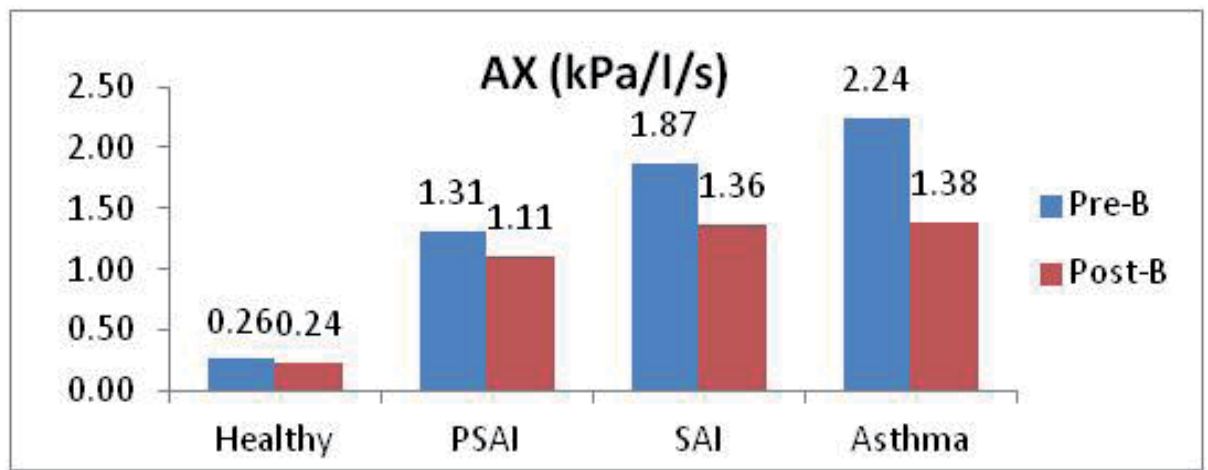

Figure 9. AX mean values for the H, PSAI, SAI and Asthmatic children under Pre- and Post-B conditions.

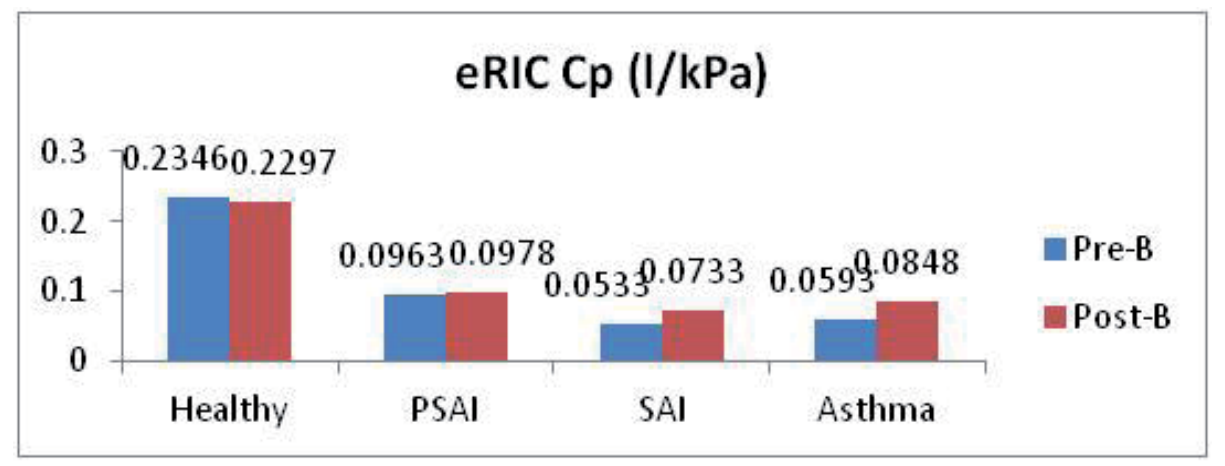

Figure 10. eRIC Cp mean values for the H, PSAI, SAI and Asthmatic children under Pre- and Post-B conditions. 


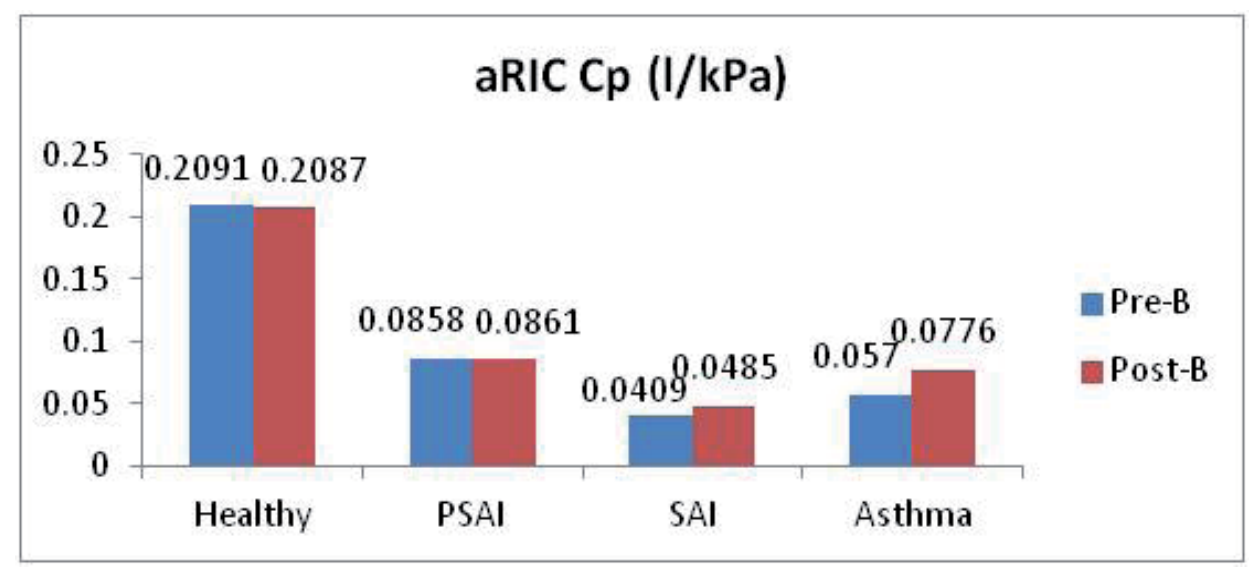

Figure 11. aRIC Cp mean values for the H, PSAI, SAI and Asthmatic children under Pre- and Post-B conditions.

Similarities between Normal and PSAI groups and between SAI and Asthmatic groups were also observed.

From tables 7,8,9, and10 and figures 7,8,9, 10 and 11 we can observe that the most significant IOS and Model Parameters for children classified as Healthy or PSAI were comparable, as these parameters showed a modest difference between these groups. However, these same parameters exhibited clear increases in R3, R5, R3-R20, R5-R20, AX, Fres, and Rp, and a clear decrease in $\mathrm{X} 3, \mathrm{X} 5$ and $\mathrm{Cp}$ going from Normal to PSAI. Going to increasingly abnormal levels of "diagnostic classification," R5, R5-R20, AX, Fres and Rp continue to increase from Healthy to PSAI to SAI to Asthmatic conditions, while X5 and Cp decrease in this progression. Differences between SAI and Asthmatic children were also modest.

In the statistical analysis performed for this research comparing Pre- and Post-B IOS and eRIC and aRIC Model Parameters in the Normal or Healthy group, no significant differences were observed (see Table 11).

\begin{tabular}{|c|c|}
\hline \multicolumn{2}{|c|}{ Healthy (Normal) Group } \\
\hline & $\begin{array}{c}\text { Pre-B vs Post-B } \\
\text { p values }\end{array}$ \\
\hline All IOS and Model Parameters & $>0.05$ \\
\hline
\end{tabular}

Table 11. Healthy group $\mathrm{p}$ values.

Comparing Pre- and Post-B IOS and eRIC and aRIC Model Parameters for the PSAI group only R3, R35 and eRIC Rc showed significant differences (see Table 12.)

Comparing Pre- and Post-B IOS and eRIC and aRIC Model Parameters for the SAI group the following parameters showed significant differences: R3,R5,X3,X5,X10,X15,R3-R20,R5R20,AX,aRIC Rp, eRIC Rp, eRIC I and eRIC Cp (see Table 13). 


\begin{tabular}{|c|c|}
\hline \multicolumn{2}{|c|}{ PSAI Group } \\
\hline & $\begin{array}{c}\text { Pre-B vs Post-B } \\
\text { p values } \\
\end{array}$ \\
\cline { 1 - 1 } $\mathbf{R 3}(\mathrm{kPa} / \mathrm{l} / \mathrm{s})$ & $<0.05$ \\
\hline $\mathbf{R} 35(\mathrm{kPa} / \mathrm{l} / \mathrm{s})$ & $<0.03$ \\
\hline eRIC $\mathbf{R c}(\mathrm{kPa} / \mathrm{l} / \mathrm{s})$ & $<0.04$ \\
\hline
\end{tabular}

Table 12. PSAI group $\mathrm{p}$ values.

\begin{tabular}{|c|c|}
\hline \multicolumn{2}{|c|}{ SAI Group } \\
\hline & $\begin{array}{c}\text { Pre-B vs Post-B } \\
\text { p value }\end{array}$ \\
\hline R3 (kPa/l/s) & $<0.02$ \\
\hline R5 (kPa/l/s) & $<0.05$ \\
\hline X3 (kPa/l/s) & $<0.02$ \\
\hline X5 (kPa/l/s) & $<0.005$ \\
\hline $\mathbf{X 1 0}(\mathrm{kPa} / \mathrm{l} / \mathrm{s})$ & $<0.001$ \\
\hline X15 (kPa/1/s) & $<0.005$ \\
\hline R3-R20 (kPa/l/s) & $<0.001$ \\
\hline R5-R20 (kPa/l/s) & $<0.005$ \\
\hline $\mathrm{AX}(\mathrm{kPa} / \mathrm{l} / \mathrm{s})$ & $<0.0002$ \\
\hline aRIC Rp (kPa/l/s) & $<0.01$ \\
\hline eRIC Rp (kPa/l/s) & $<0.02$ \\
\hline $\mathrm{I}\left(\mathrm{kPa} / \mathrm{l} / \mathrm{s}^{2}\right)$ & $<0.05$ \\
\hline $\mathrm{Cp}(\mathrm{l} / \mathrm{kPa})$ & $<0.03$ \\
\hline
\end{tabular}

Table 13. SAI group $p$ values

Comparing Pre- and Post-B IOS, eRIC and aRIC Model Parameters for the Asthmatic group all of the parameters showed significant differences with the exception of aRIC Ce and eRIC I (see Table 14).

Significant differences between these groups in the aforementioned IOS and Model Parameters were found. Comparing Normal Pre-B to PSAI Pre-B, all of the IOS and Model Parameters showed significant differences, with the exception of aRIC Rp and eRIC Rp. Comparing Normal (Healthy) Post-B and PSAI Post-B, the majority of the parameters showed significant differences with the exception of R3, R5, eRIC Rp and aRIC Rp. Comparing PSAI Pre-B and SAI Pre-B data, only three parameters showed significant differences between these groups: Fres, eRIC Cp and aRIC Cp. For PSAI Post-B and SAI 
Post-B, only Fres showed a significant difference. Finally comparing SAI Pre-B vs Asthma Pre-B, and SAI Post-B vs Asthma Post-B, no significant differences were found.

\begin{tabular}{|c|c|}
\hline \multicolumn{2}{|c|}{ Asthmatic Group } \\
\hline & $\begin{array}{c}\text { Pre-B vs Post-B } \\
\text { p value }\end{array}$ \\
\hline R3 $(\mathrm{kPa} / \mathrm{l} / \mathrm{s})$ & $<1.5 \mathrm{E}-9$ \\
\hline $\mathbf{R} 5(\mathrm{kPa} / \mathrm{l} / \mathrm{s})$ & $<1.6 \mathrm{E}-10$ \\
\hline $\mathrm{R} 10(\mathrm{kPa} / \mathrm{l} / \mathrm{s})$ & $<9.7 \mathrm{E}-08$ \\
\hline $\mathrm{R} 15(\mathrm{kPa} / \mathrm{l} / \mathrm{s})$ & $<0.0002$ \\
\hline $\mathbf{R} 20(\mathrm{kPa} / \mathrm{l} / \mathrm{s})$ & $<0.0002$ \\
\hline $\mathbf{R} 25(\mathrm{kPa} / \mathrm{l} / \mathrm{s})$ & $<3.9 \mathrm{E}-07$ \\
\hline R35 (kPa/l/s) & $<1.7 \mathrm{E}-06$ \\
\hline X3 $(\mathrm{kPa} / \mathrm{l} / \mathrm{s})$ & $<0.005$ \\
\hline X5 (kPa/l/s) & $<0.002$ \\
\hline $\mathrm{X} 10(\mathrm{kPa} / \mathrm{l} / \mathrm{s})$ & $<0.0003$ \\
\hline X15 (kPa/l/s) & $<1.4 \mathrm{E}-6$ \\
\hline $\mathrm{X} 20(\mathrm{kPa} / \mathrm{l} / \mathrm{s})$ & $<0.0002$ \\
\hline $\mathrm{X} 25(\mathrm{kPa} / \mathrm{l} / \mathrm{s})$ & $<0.01$ \\
\hline X35 (kPa/l/s) & $<0.003$ \\
\hline R3-R20 (kPa/l/s) & $<5.6 \mathrm{E}-06$ \\
\hline R5-R20 (kPa/l/s) & $<2.5 \mathrm{E}-06$ \\
\hline $\mathbf{A X}(\mathrm{kPa} / \mathrm{l} / \mathrm{s})$ & $<3.1 \mathrm{E}-08$ \\
\hline Fres $(1 / \mathrm{s})$ & $<0.0002$ \\
\hline aRIC $\quad \operatorname{Rc}(\mathrm{kPa} / \mathrm{l} / \mathrm{s})$ & $<0.006$ \\
\hline $\mathbf{R p}(\mathrm{kPa} / \mathrm{l} / \mathrm{s})$ & $<4.5 \mathrm{E}-07$ \\
\hline $\mathbf{I}\left(\mathrm{kPa} / \mathrm{l} / \mathrm{s}^{2}\right)$ & $<0.002$ \\
\hline $\mathrm{Cp}(1 / \mathrm{kPa})$ & $<0.0003$ \\
\hline eRIC Rc $(\mathrm{kPa} / \mathrm{l} / \mathrm{s})$ & $<0.002$ \\
\hline $\mathbf{R p}(\mathrm{kPa} / \mathrm{l} / \mathrm{s})$ & $<4.5 \mathrm{E}-06$ \\
\hline $\mathrm{Cp}(\mathrm{l} / \mathrm{kPa})$ & $<0.0003$ \\
\hline
\end{tabular}

Table 14. Asthmatic group $\mathrm{p}$ values 
These selected parameters seem to be sensitive and reliable indices of lung function for respiratory disease classification using Impulse Oscillometric data, eRIC and aRIC Model Parameters.

The correlation coefficient ( $r$ ) between children Heights and these IOS and Model Parameters was also analyzed. The parameters showed a good correlation, the best correlation was observed for $\mathrm{R} 5$ with $\mathrm{R}=0.764$, and the lowest $r$ value $(0.542)$ was observed for X5. In Table 15 the values for these correlations are presented.

\begin{tabular}{|c|c|}
\hline \multicolumn{2}{|c|}{ Correlation with Height } \\
\hline Parameters & $\mathbf{r}$ \\
\hline $\mathrm{R} 3(\mathrm{kPa} / \mathrm{l} / \mathrm{s})$ & 0.762 \\
\hline $\mathrm{R} 5(\mathrm{kPa} / \mathrm{l} / \mathrm{s})$ & 0.764 \\
\hline $\mathrm{X} 3(\mathrm{kPa} / \mathrm{l} / \mathrm{s})$ & 0.557 \\
\hline $\mathrm{X} 5(\mathrm{kPa} / \mathrm{l} / \mathrm{s})$ & 0.542 \\
\hline $\mathrm{R} 3-\mathrm{R} 20(\mathrm{kPa} / \mathrm{l} / \mathrm{s})$ & 0.688 \\
\hline $\mathrm{R} 5-\mathrm{R} 20(\mathrm{kPa} / \mathrm{l} / \mathrm{s})$ & 0.62 \\
\hline $\mathrm{AX}(\mathrm{kPa} / \mathrm{l} / \mathrm{s})$ & 0.741 \\
\hline $\mathrm{Fres}(1 / \mathrm{s})$ & 0.666 \\
\hline $\mathrm{eRIC} \mathrm{Cp}(\mathrm{l} / \mathrm{kPa})$ & 0.762 \\
\hline $\mathrm{Rp}(\mathrm{kPa} / \mathrm{l} / \mathrm{s})$ & 0.513 \\
\hline $\mathrm{aRIC} \mathrm{Cp}(\mathrm{l} / \mathrm{kPa})$ & 0.717 \\
\hline $\mathrm{Rp}(\mathrm{kPa} / \mathrm{l} / \mathrm{s})$ & 0.643 \\
\hline
\end{tabular}

Table 15. Correlation coefficients (r) between Height and IOS and respiratory system Model Parameters for 2008 data.

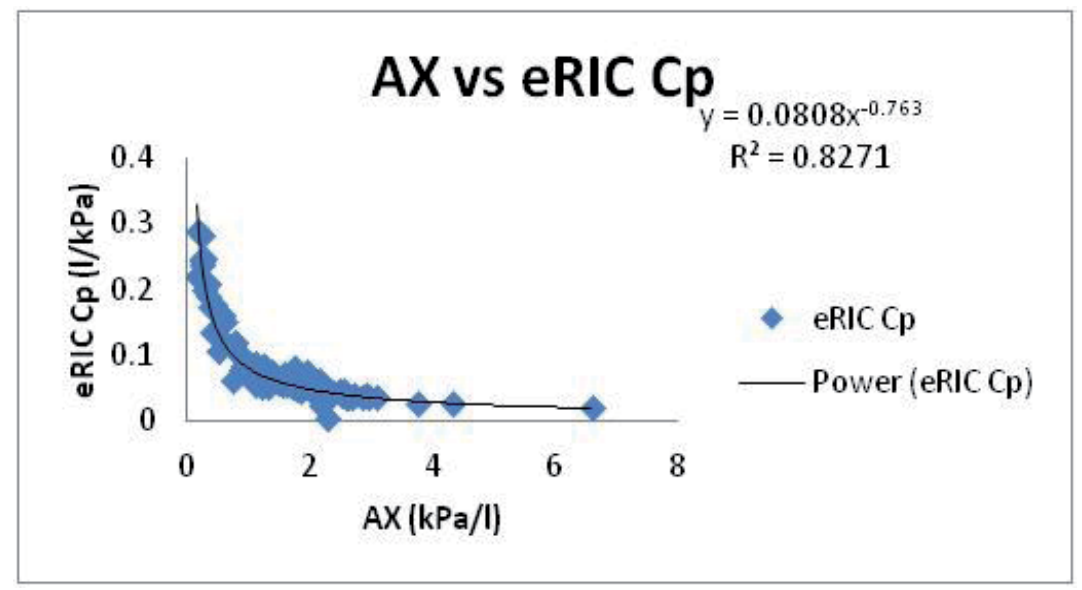

Figure 12. Correlation between AX and eRIC Cp for Pre- and Post-B data. 
The statistical correlation between AX and both models' Cps, and R3-R20 and R5-R20 with both models' Rps were also evaluated. The best correlation observed was between AX and eRIC C $p$ with a value of $r=0.909$. The correlation between AX and aRIC Cp had a lower $r$ value equal to 0.760 . For the correlation between R3-R20 and eRIC Rp, $r$ was equal to 0.589 . The correlation between R5-R20 and eRIC Rp was $r=0.516$. For R3-R20 and aRIC $R p, r=0.787$. And finally the correlation between R5-R20 and aRIC Rp was $r=0.731$. Figure 12 illustrates the best correlation observed. This was between AX and eRIC Cp.

\section{Conclusions}

While expert clinician diagnostic classification distinguished between children based on 4 levels of perceived normality or absence thereof of the visual patterns of IOS data, with the essential features characterizing the differences being associated with abnormalities group mean IOS and the aRIC model data appear to fall into two distinctly different groups: either Normal or Asthmatic, with the essential features characterizing the differences being associated with abnormalities of peripheral airways.

Our present and previous studies (52-61, 75), have demonstrated that equivalent electrical circuit Model Parameters of the human respiratory system are able to track changes in the respiratory system function after bronchodilation. Both the eRIC and aRIC models clearly distinguished between children who were Normal (Healthy) or possibly had mild SAI, who showed no significant changes with bronchodilation-BD), and those who were Asthmatic with SAI, both at baseline and at Pre- to Post-BD conditions. The eRIC model showed an apparently larger peripheral airway compliance $(\mathrm{Cp})$ than the aRIC model, probably because it might include some of the "extrathoracic airway compliance" (Ce). On the other hand, the eRIC model is more parsimonious, and the parameter, $\mathrm{Ce}$, that may be difficult for physicians to understand, appears to show no significant change Post-BD in the Asthmatic group.

It can be observed that children classified as Normal (Healthy) or possible SAI were relatively similar in both IOS and the aRIC model parameters. However, they showed clear increases in R3, R5, R3-R20, R5-R20, AX, Fres, and Rp, and a clear decrease in X3, X5 and Cp going from Normal to PSAI.

Going to increasingly abnormal levels of "diagnostic classification," R3, R5, R5-R20, AX, Fres and Rp continue to increase from Healthy to PSAI to SAI to Asthma, while X3, X5 and $\mathrm{Cp}$ decrease in this progression. Differences between SAI and Asthmatic children were modest.

The features used in this work seem to be sensitive and reliable indices for automatic respiratory disease classification using Impulse Oscillometry data. The correlation between AX and the eRIC Cp was the best correlation in both Pre-B and Post-B work.

Our expert clinician confirmed that the range of values of all analyzed features: R3, R5, R3R20, R5-R20, X3, X5, AX, Fres, eRIC Cp, eRIC Rp, aRIC Cp and aRIC Rp obtained for the 
SAD and Asthmatic groups were comparable to those values he has observed over many years in other asthmatic children of the same age range.

Comparing all the IOS parameters for Pre- and Post-B data and the eRIC and aRIC Model Parameters in the Normal group, no significant differences were observed. Also comparing Pre- and Post-B parameters for the PSAI group only R3, R5 and the eRIC Rc showed significant differences. Similarly evaluating Pre- and Post-B values for the SAI group, the following parameters showed significant differences: R3, R5, X3, X5, X10, X15, R3-R20, R5R20, AX, the aRIC Rp, eRIC Rp, eRIC I and eRIC Cp. Finally evaluating Pre- and Post-B values for the Asthmatic group all of the parameters showed significant differences with the exception of the aRIC Ce and eRIC I.

The selected IOS and model derived parameters (R3, R5, R3-R20, R5-R20, X3, X5, AX, Fres, the eRIC Cp, eRIC Rp, aRIC Cp and aRIC Rp) showed a good correlation with children Heights.

In this research study focusing on children with and without SAI (Healthy), the eRIC Model Parameters showed to be consistent and to some extent more closely correlated with IOS measures compared to the aRIC model parameters. As eRIC is more intuitive, less complex and a more parsimonious model (75), it may be considered a more suitable diagnostic tool for clinical applications than the aRIC model.

IOS lung function data are similarly well-modeled by the eRIC (without upper airway shunt compliance) and aRIC models (with upper airway shunt compliance), which are reduced versions of the popular Mead's model developed at Harvard several decades ago, based on the close correlations of their corresponding parameters excluding Ce. The eRIC model is a more parsimonious and equally powerful model in capturing the differences between SAI and $\mathrm{H}$ children, therefore it is recommended as a clinically-preferred model of lung function based on IOS data.

In summary, we conclude that the IOS parameters AX and the eRIC model-derived parameter $\mathrm{Cp}$ are the most reliable parameters to track small airway function in children before and after bronchodilation. AX (the "Goldman Triangle"), representing the integrated low frequency respiratory reactance magnitude between $5 \mathrm{~Hz}$ and Fres, and the eRIC Cp corresponding to the peripheral (small airway) compliance demonstrated superior diagnostic discrimination compared to all other parameters analyzed and emerged as useful and reliable indices of small airway function in children.

Further work in a larger number of $\mathrm{H}$ and SAI children is required to establish normal values for these sensitive indices and enable researchers in this field to perform more effective and timely evaluation, detection, diagnosis, and treatment of different respiratory diseases. Also future work should be performed in order to collect data from a larger sample of children and perform a statistical analysis in order to evaluate IOS parameters and both models (eRIC and aRIC) performances to evaluate these changes in lung function. A definitive choice between the eRIC and aRIC models will require further assessments in a larger sample of children. 


\section{Author details}

Erika G. Meraz

Universidad Autónoma de Ciudad Juárez, Mexico

University of Texas at El Paso, USA

Lidia Rascón Madrigal and Nelly Gordillo Castillo

Universidad Autónoma de Ciudad Juárez, Mexico

Homer Nazeran, Carlos Ramos and Liza Rodriguez

University of Texas at El Paso, USA

\section{References}

[1] American Lung Association Asthma and Allergy, Asthma \&Children. http://www.lung.org/lung-disease/asthma/learning-more-about-asthma/symptomsdiagnosis-and-treatment.html (accessed June 18 2012).

[2] AAAAI. American Academy of Allergy Asthma and Inmunology. http://www.aaaai.org/about-the-aaaai/newsroom/asthma-statistics.aspx (accessed June 18 2012).

[3] COMPEDIA. Colegio Mexicano de Pediatras Especialistas en Inmunología Clínica y Alergias. http://www.compedia.org.mx/archivos/docs/6_asma_bron.pdf. (accessed June 18 2012).

[4] Klug, B and Bisgaard, H. Specific Airway Resistance, Interrupter Resistance, and Respiratory Impedance in Healthy Children, and Respiratory Impedance in Healthy Children Aged 2-7 Years. Pediatric Pulmonology 1998; 25, 322-331.

[5] Nielsen, KG and Bisgaard, H. The Effect of Inhaled Budesonide on Symptoms, Lung Function, and Cold Air and Methacholine Responsiveness in 2- to 5-year-old Asthmatic Children. Am J Respir Crit Care Med 2000; 162, 1500-1506.

[6] Gaylor, P B, et al. Forced Oscillation using Impulse Oscillometry (IOS) Provides Objective Responses to Inhaled Corticosteroids (ICS) in Asthmatic Patients when FEV1 Fails to Improve. J Allergy and Clin Immun 2003; 111, S135.

[7] Marotta, A, et al. Impulse Oscillometry provides an effective measure of lung dysfunction in 4-year-old children at risk for persistent asthma. J Allergy Clin Immunol 2003; 112, 317-321.

[8] Ortiz, G and Mendez, R. The Effects of Inhaled Albuterol and Salmeterol in 2- to 5-YearOld Asthmatic Children as Measured by Impulse Oscillometry. Journal of Asthma 2002; 39(6), 531-536.

[9] Goldman, M D, et al. Within- and Between-Day Variability of Respiratory Impedance, Using Impulse Oscillometry in Adolescent Asthmatics. Pediatric Pulmonology 2002; 34, 312-319.

[10] Klug, B and Bisgaard, H. Measurement of Lung Function in awake 2-4 Year-Old Asthmatic Children During Methacholine Challenge and Acute Asthma: A Comparison of the Impulse $\mathrm{O}$ Technique, the Interrupter Technique, and Transcutaneous 
Measurments of Oxygen vs W-B Plethysmography. Pediatric Pulmonology 1996; 21, 290-300.

[11] Saadeh, C K, et al. Forced Oscillation Using Impulse Oscillometry (IOS) Detects False Negative Spirometery in Symptomatic Patients with Reactive Airways. Journal of Allergy and Clinical Immunology 2003; 3, S136.

[12] Vink, G R, et al. Impulse Oscillometry: A measure for Airway Obstruction. Pediatric Pulmonology 2003; 35, 214-219.

[13] Song, T W, et al. Utlility of impulse oscillometry in young children with asthma. Pediatr Allergy Immunol 2008; 19, 763-768.

[14] Song, T W, et al. Correlation between spirometry and impulse oscillometry in children with asthma. Acta Pediátrica 2008; Vol. 97, pp. 51-54.

[15] Antonova E.A., et al. Sensitivity impulse oscillometry and spirometry for assessment degree of severity of bronchial asthma in preschool children. ERS Annual Congress 2006: September 2-6, 2006, Munich, Germany.

[16] https://www.ersnetsecure.org/public/prg_congres.detail?ww_i_session=13872\&ww_c_ mode=TEXTE-SPECIAL (accessed June 2012)

[17] Linares, M, Concha, I and Meter, R. Correlation between spirometry and respiratory resistance and reactance measured by impulse oscillometry in asthmatic children. Revista Chilena de enfermedades respiratorias 2002, 18 (2) 90-98.

[18] Lewis-Brown, K, Hildreth, G and Maupin, K d. The association of spirometry with impulse oscillometry in pediatric patients with asthma. Chest 2005; 188s.

[19] Olaguibel, J M, et al. Comparative analysis of the bronchodilator response measured by impulse oscillometry (IOS), spirometry and body plethysmography in asthmatic children. J Invest Allergol Clin Immunol 2005; 15 (2), 102-106.

[20] Tomalak, W, et al. Impulse Oscillometry vs. Body Plethysmography in Assessing Respiratory Resistance in Children. Pediatric Pulmonology 2006; 41, 50-54.

[21] Bisgaard, $\mathrm{H}$ and Klug, B. Lung function measurement in awake young children. Eur Resp J 1995; 8, 2067-2075.

[22] Nielsen, K G and Bisgaard, H. Lung Function Response to Cold Air Challenge in Asthmatic and Healthy Children of 2-5 years of age. Am J Respir Crit Care Med 2000; 161, 1805-1809.

[23] Goldman, M D, et al. Impulse oscillometry sensitively measures long and short acting bronchodilator in asthmatic children. In Biomedical Engineering Recent Developments: Proceedings of the Twenty Fourth SBEC 2008, April 18-20, 73-76, El Paso, TX, USA.

[24] Nielsen, K G and Bisgaard, H. Discriminative Capacity of Bronchodilator Response Measured with Three Different Lung Function Techniques in Asthmatic and Healthy Children Aged 2 to 5 Years. Am J Respir Crit Care Med 2001; 164, 554-559.

[25] Nieto, A, et al. Montelukast improves pulmonary function measured by impulse oscillometry in children with asthma (Mio study). Respiratory Medicine 2006; 100 (7), 1180-1185.

[26] Hur, H Y, et al. A comparison between impulse oscillometry system and spirometry for detecting airway obstruction in children. Korean Journal of Pediatrics 2008; 51 (8), 842847. 
[27] Larsen, G L, et al. Impulse oscillometry versus spirometry in a long-term study of controller therapy for pediatric asthma. J Allergy Clin Immunol 2009; 123 (4), 861-867.

[28] Frey, U. Forced oscillation technique in infants and young children. Paedriatric Respiratory Reviews 2005; 6, 246-254.

[29] Hellinckx, J, et al. Bronchodilator response in 3-6 years old healthy and stable asthmatic children. Eur Respir J 1998; 12, 438-443.

[30] Graw-Panzer, K D, et al. Comparing Bronchodilator Response Measured by Impulse Oscillometry to Spirometry in Children with Asthma. Am J Respir Crit Care Med 2009; $179, \mathrm{~A} 4858$.

[31] Todaki, H, et al. Effect of bronchoconstriction on exhaled nitric oxide levels in healthy and asthmatic children. Ann Allergy Asthma Immunol 2009; 102 (6), 469-74.

[32] Menendez, R, et al. Impulse Oscillometry (IOS) measures effects of short and long acting Beta-Agonists (SABA, LABA) on Lung Mechanics in 2-5 year old children with asthma. J Allergy Clin Immunol 2008; 121 (2), S211.

[33] Jee, H M, et al. Usefulness of bronchial hyperresponsiveness measured with impulse oscillation technique in preschool children. J Asthma 2010, 47 (3), 227-232.

[34] Becklake, M and Crapo, R O. Lung Function Testing: Selection of Reference values and interpretative strategies. Am Rev Respir Dis 1991, 144, 1202-1218.

[35] Stocks, J and Quanjer, H. Reference values for residual volume, functional residual capacity and total lung capacity. Eur Respir J 1995, 8, 492-506.

[36] Clement, J, et al. Reference values of total respiratory resistance and reactance between 4 and $26 \mathrm{~Hz}$ in children and adolescents aged 4-20 years. Bull Eur Physiopathol Respir $1987 ; 23,441-448$.

[37] Ducharme, F M, Davis, G M and Ducharme, G R. Pediatric Reference Values for Respiratory Resistance Measured by Forced Oscillation. CHEST 1998; 113, 1322-1328.

[38] Frei, J, et al. Reference values in children 100 to $150 \mathrm{~cm}$ in height and 3 to 10 years of age. CHEST 2005; 128, 1266-1273.

[39] Dencker, $M$, et al. Reference values for respiratory system impedance by using impulse oscillometry in children aged 2-11 years. Clin Physiol Funct Imaging 2006; 26, 247-250.

[40] Nowowiejska, B, et al. Transient Reference values for Impulse Oscillometry for children aged 3-18 years. Pediatric Pulmonology 2008; 43, 1193-1197.

[41] Amra, B, Soltaninejad, F and Golshan, M. Respiratory Resistance by Impulse Oscillometry in Healthy Iranian Children aged 5-19 years. Iran J Allergy Asthma Immunol 2008; 7 (1), 25-29.

[42] Wee, Y S, et al. Reference Values of respiratory system impedance using impulse oscillometry in school-aged children in Korea. Korean J Pediatr 2007; 50 (9), 862-867.

[43] Jee, H M, et al. Reference Values for lung function testing using spirometry and impulse oscillometry in healthy preschool-aged children in Korea. ERS Annual Congress 2010: September 18-22 2010, Barcelona, España.

http://www.ers-education.org/pages/default.aspx?id=2320\&idBrowse=85383\&det=1 (accessed: March 15, 2011). 
[44] Diong, B, et al. Modeling Human Respiratory Impedance: Comparing the Best Method with the Least Estimation Errors. IEEE Engineering in Medicine and Biology Magazine 2007; 48-55.

[45] Diong, B, et al. The augmented RIC model of the human respiratory system. Med Biol Eng Comput 2009; 47 (4), 395-404.

[46] Rajagiri, A, et al. Can Asthma in Children be Detected by the Estimated Parameter Values of the Augmented RIC Model?. IEEE-Engineering in Medicine and Biology Society, 28th Annual International Conference 2006: Conference Proceedings, August 30 - September 3, 2006, 5595-5598.

[47] Rajagiri, A, et al. Evaluation of Augmented RIC Model of Adult Respiratory Impedance Based on Impulse Oscillometry Data. Int. Conf. Biomedical Engineering 2005: Conference Proceedings, December 2005.

[48] Rajagiri, A and Diong, B. Evaluation of Augmented RIC Model of Child Respiratory Impedance Based on Impulse Oscillometry Data. Proc. IEEE Region 5 Technical Conference 2006: Conference Proceedings, April 7-9, 2006, 291-295.

[49] Baswa, S, et al. Evaluation of Respiratory System Models Based on Parameter Estimates from Impulse Oscillometry Data. IEEE Engineering in Medicine and Biology 27th Annual Conference 2005: Conference Proceedings, September 1-4, 2005, 3, 2958-2961.

[50] Nguyen, T-U, et al. A study of IOS Data Using Two Mead-related Models of Respiratory Impedance". 29th Annual International Conference of the IEEE EMBS 2007: Conference, August 23-26, 2007, 1078-1081.

[51] Nguyen, T-U and Diong, B. Evaluation of Two Mead-related Models of Adult Respiratory Impedance Based on IOS Data. IEEE Region 5 Technical Conference 2007: Conferene Proceedings, April 20-21, 2007, 303-308.

[52] Woo, T, et al. A Comparison of Various Respiratory Models Based on Parameter Estimates From Impulse Oscillometry Data. 26th Annual International Conference of the IEEE EMBS 2004: Conference Proceedings, September 1-5, 2004, 5, 3828-3831.

[53] Meraz, E, et al. Modeling Human Respiratory Impedance in Anglo Asthmatic Children." In Biomedical Engineering Recent Developments 2007: Proceedings of the Twenty Third Southern Biomedical Engineering Conference 2007, April 13-15, 108-113.

[54] Meraz, E, et al. Modeling Human Respiratory Impedance in Hispanic asthmatic children 5 to 17 years old. In Biomedical Engineering Recent Developments 2008: Proceedings of the Twenty Fourth Southern Biomedical Engineering Conference 2008, April 18-20, 87-90.

[55] Meraz, E, et al. Modeling Human Respiratory Impedance in Normal children 5 to 17 years old. In Biomedical Engineering Recent Developments 2008: Proceedings of the Twenty Fourth Southern Biomedical Engineering Conference 2008, April 18-20, 85-86.

[56] Meraz, E, et al. Impulse Oscillometric Features of Lung Function: Towards ComputerAided Classficiation of Respiratory Diseases in Children. 30 th Annual Internatinal Conference of the IEEE EMBS 2008: Conference Proceedings, Vancouver, Canada, August 20-24, 2008, 2443-2446. 
[57] Meraz, E, et al. Normal Impulse Oscillometry (IOS) Lung Function Parameters in Adolescents Residing in el Paso, TX. In Annals of Epidemiology 2008: Proceedings of the Annual Meeting of the American College of Epidemiology 2008, 18 (9), 72.

[58] Goldman, M. et al. Abnormality of Oscillometric Resistances and Reactances is Greater in Hispanic than Anglo Children in El Paso, TX. CHEST 2008, Pediatric Pulmonary, Sleep, and Critical Care. October 2008, 134, 4.

[59] Meraz, E, et al. Modeling Human Respiratory Impdance in Hispanic Asthmatic Children. 29th Annual International Conference of the IEEE EMBS 2007: Conference Proceedings, Lyon, France, August 23-26, 2007, 4251-4254.

[60] Meraz, E, et al. Respiratory System Model Parameters Track Changes in Lung Function After Bronchodilation. In Biomedical Engineering Recent Developments 2009: Proceedings of the 25th SouthernBiomedical Engineering Conference 2009, May 15-17, 319-322.

[61] Meraz, E, et al. Forced Oscillation (IOS) measures in airway-impaired and normal children over 2 years. 19th European Respiratory Society Annual Congress 2009: Conference Abstracts, Austria, Vienna, September 12-16, 2009, 34 (53), 681s.

[62] Meraz, E G, et al. Analysis of impulse oscillometric measures of lung function and respiratoryModel Parameters in small airway-impaired and healthy children over a 2year period. BioMedical Engineering OnLine. 2011; 10, 21. http://www.biomedicalengineering-online.com/content/10/1/21 (Accessed June 2012)

[63] Beachey, W. Respiratory Care Anatomy and Physiology: Foundations for Clinical Practice. Second edition. St Louis, Missouri, USA. Mosby: Elsevier, 2007.

[64] Children's Hospital of Pitsburg. Routing Testing Spirometry. http://www.chp.edu/CHP/Routine+Testing. (Accessed March 2010)

[65] Barreiro, T J and Perrillo, I. An approach to interpreting spirometry. 2004, 69 (5), 11071115.

[66] Galant, S. Lung Function Measurement in the Assessment of Childhood Asthma: Recent Important Developments. Curr Opin Allergy Clin Immunol 2010; 10 (2), 149-154.

[67] Goldman, M D, Saadeh, C and Ross, D. Clinical applications of forced oscillation to assess peripheral airway function. Respiratory Physiology \& Neurobiology 2005; 148, 179-194.

[68] Goldman, M D. Clinical Application of Forced Oscillation. Pulmonary Pharmacology \& Therapeutics 2001; 14, 341-350.

[69] Smith, H J, Reinhold, P and Goldman, M D. Forced Oscillation technique and impulse oscillometry. Eur Respir Mon 2005; 31, 72-105.

[70] Marchal, F and Hall, G L. Forced Oscillation Technique. Eur Respir Mon 2010; 47, 121136.

[71] Carefusion. http://www.carefusion.com/images/IOS_Special_Edition.pdf. (Accessed June 2011)

[72] Beydon, $\mathrm{N}$ et al. on Behalf of the American Thoracic Society/ European Respiratory Society Working Group on Infant and Young Children Pulmonary Function Testing. An Official American Thoracic Society/European Respiratory Society Statement: 
Pulmonary Function Testing in Preschool Children. Am J Respir Crit Care Med. 2007; 175, 1304-1345.

[73] Oostveen, E, et al. The forced oscillation technique in clinical practice: methodology, recommendations and future developments. Eur Respir J. 2003; 22, 1026-1041.

[74] Bar-Yishay, E, et al. Use of the Forced Oscillation Technique to Detect Bronchodilation in children: Experience from the Schneider Children's Medical Center of Israel. Isr Med Assoc J 2009; 11 (4), 198-200.

[75] Lahzami, S and King, G G. Targeting small airways in asthma: the new challenge of inhaled corticosteroid treatment. Eur Respir J 2008; 31, 1145-1147.

[76] Goldman MD, Nazeran H, Ramos C, Toon E, Oates K, Bilton D, Meraz E, Hafezi N, Diong B. Electrical Circuit Models of the Human Respiratory System Reflect Small Airway Impairment Measured by Impulse Oscillation (IOS). IEEE EMBS 32nd Annual International Conference 2010: Conference Proceedings, Buenos Aires, Argentina, 2010, 2467-2472. 


\title{
Simulation of Subject Specific Bone Remodeling
}

\author{
Ajay Sonar and James Carroll \\ Additional information is available at the end of the chapter
}

http://dx.doi.org/10.5772/51822

\section{Introduction}

Vertebral compression fractures are very common low-bone-density related conditions among the elderly and have a significant adverse health effect [1]. According to a survey by the National Osteoporosis Foundation, osteoporosis related fractures cost an estimated \$19 billion in 2005 and the cost is predicted to go up to $\$ 25.3$ billion by the year of 2025 [2]. The fractures affects up to $25 \%$ of postmenopausal women [3]. Men are equally affected by this, but because of greater peak bone mass, it occurs about 10 years later than women [4]. The thoraco-lumbar region in the vertebra is the most common region where fractures occur, with T8, T12, L1 and L4 being the most commonly fractured segments [5]. The vertebral compression fracture can be avoided by preventative medication and physical therapy if the trend of the bone structure change is known. However, directly monitoring the density and structural change of patient's bones is practically impossible based on current technology. Examination of the patients' bones at regular intervals is costly, and not very helpful: the patients have to wait for months or years to know the effect of their lifestyles on their vertebra, while at this point any bone changes might already have occurred and damages made. Instead, simulation, as an cost-effective and non-intrusive method, is a better way to "know" the bone change in advance based on realistic input of loads and patients conditions, and thus prevent bone fractures occurring.

Changes in the cancellous bone structure have been studied since Julius Wolff first discovered the adaptive nature of the bone [6]. Different simulation techniques and models have been proposed $[7,8]$. However, in the previous studies standard load which is within the nominal range experienced by the human vertebra were used. In reality this load varies for different human subjects for various activities performed. This was a limitation in terms of the ability to measure accurate loads. With technologies like the state-of-the-art motion capture systems combined with musculoskeletal modeling and simulation softwares, accurate loads on different segments of the body can be calculated. This helps in more accurate simulation results.

This study aims at developing a simulation tool to predict the long term effects on the micro-architecture of the vetebral cancellous bone, given the person's present daily lifestyle, 


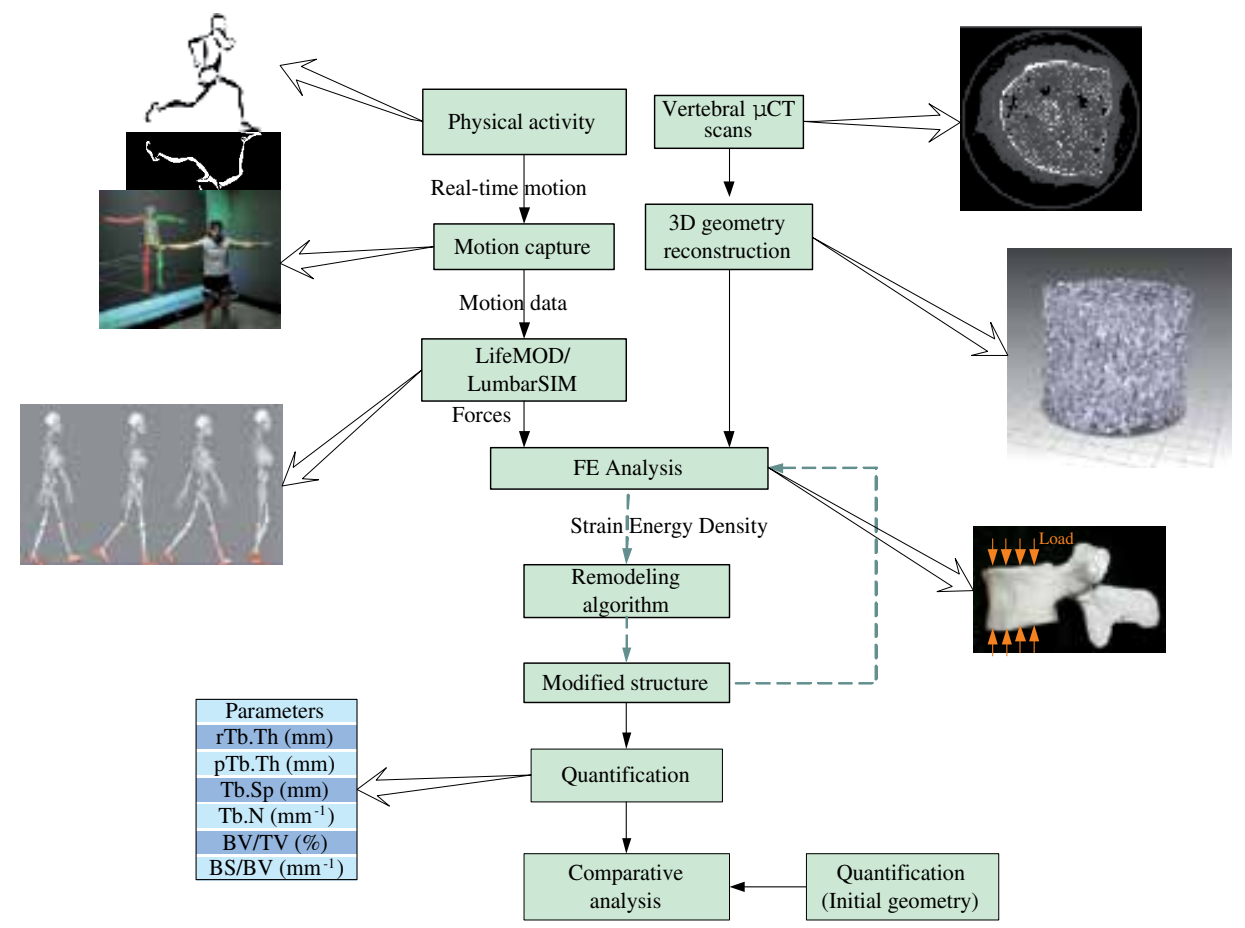

Figure 1. Schematic of proposed remodeling cycle

within a reasonable time frame (hours or days). Modules are created based on different purposes of simulation, and these modules are integrated into one efficient simulation method (Figure 1) that covers from the acquisition of individual motion data to the prediction of the changes under various physical loads. The process starts with scanning of the vertebral bodies to obtain $\mu \mathrm{CT}$ images. The $\mu \mathrm{CT}$ images are micro-computed tomography $(\mu \mathrm{CT})$ images generated using $\mathrm{x}$-rays. The result is a $2 \mathrm{D}$ cross section of a 3D object which shows the internal structure of the 3D object. In this particular case, pre-scanned $\mu \mathrm{CT}$ images were obtained. These images are processed to separate the bone and the marrow. A 3D model of the bone is constructed from the processed images. Quantifying parameters are calculated on the 3D model of the bone. After calculating the loads on the vertebrae caused by different activities, finite element methods are used to calculate the stresses within the structure caused by those loads. This triggers the remodeling process which continues until a steady state is reached and there are no more significant changes in the structure. If the method can differentiate and quantify the vertebra bone structure changes under different types of physical loads, then the method can be used to produce desirable physical therapy against the bone fractures and density loss. The details of the method are discussed in the following sections.

\section{Material and methods}

Bone by its nature is dynamic. It undergoes constant structural changes as a result of adaptation and remodeling. The purpose of remodeling is to prevent the accumulation of damage, adapt the internal architecture to external loads [9]. To effectively study the 


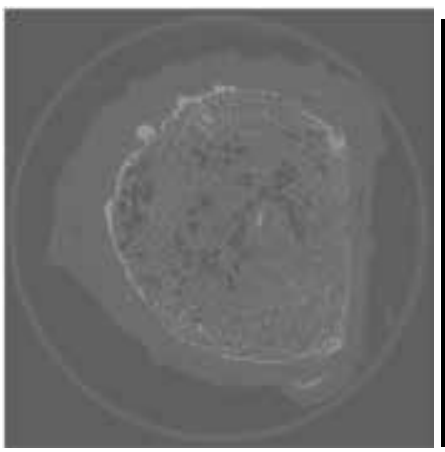

(a)

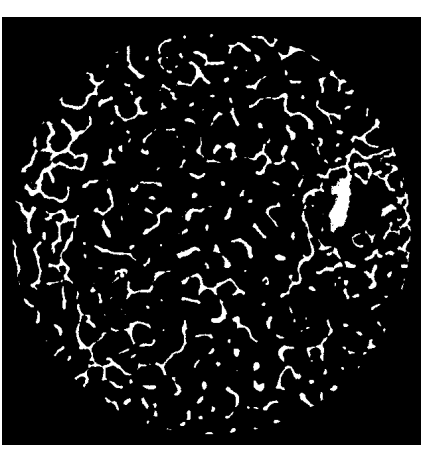

(b)

Figure 2. (a)Original $\mu \mathrm{CT}$ scan (b)Binary image after thresholding the ROI

remodeling phenomenon, it is important to know the components of the bone and the remodeling process $[10,11]$.

\subsection{Image processing}

Tomography is widely used in medical radiology to produce noninvasive, diagnostic, cross-sectional images of bone and tissue structure in human patients. As the input to our modeling, we use a series of $2 \mathrm{D} \mu \mathrm{CT}$ images, i.e., tomographic images whose resolution is in the micrometer range [12]. Figure 2(a) shows a sample image which is 16 bit gray-scale image, equivalent to histological sections used for microscopic slides. In order to effectively reconstruct a 3D geometry of the bone, it is important to identify the various regions in the gray scale image. The image shows bone, marrow and any artifact that might have been introduced due to the instrument and the imaging technique or post-processing technique used by the image reconstruction software. The goal of this section is to segment the bone region in the $\mu \mathrm{CT}$ images and generate a $3 \mathrm{D}$ geometry from these images.

An important step in the analysis of $\mu \mathrm{CT}$ images is segmentation. This process separates the bone from the surrounding marrow substance. Because of the difference in the density between the bone and the marrow, the region occupied by the calcified bone is lighter compared to the region occupied by the the marrow. An automated global thresholding method proposed by Otsu [13], which chooses the threshold to minimize the interclass variance of the black and white pixels in a grayscale image, was used to separate the bone from marrow. Since the focus of this project is on the remodeling and adaptation of the cancellous bone, a region of interest was chosen from the center of the vertebra. This removes the cortical shell and any artifacts present at the edge during scanning. The diameter of the ROI was arbitrarily chosen to be 480 pixels. To maintain the aspect ratio (diameter/height) of that of a single vertebral body (1.33), a total of 360 images $(480 / 360=1.33)$ were chosen from the entire set. These cored images were then thresholded to separate the bone matrix form the surrounding marrow (Figure 2(b)).

Once all the images were thresholded and segmented, the next step is to construct the 3D geometry from them. 3D geometry can be represented in many ways. One way is to store a list of vertices on the surface and a list of indices that form connected triangular facets from those 
vertices. This is an efficient and the most commonly used file format to store 3D geometries. An isosurface algorithm is used to extract connected triangular facet information. The result of this is a 3D surface geometry representing the cored section of the cancellous bone (Figure 3).

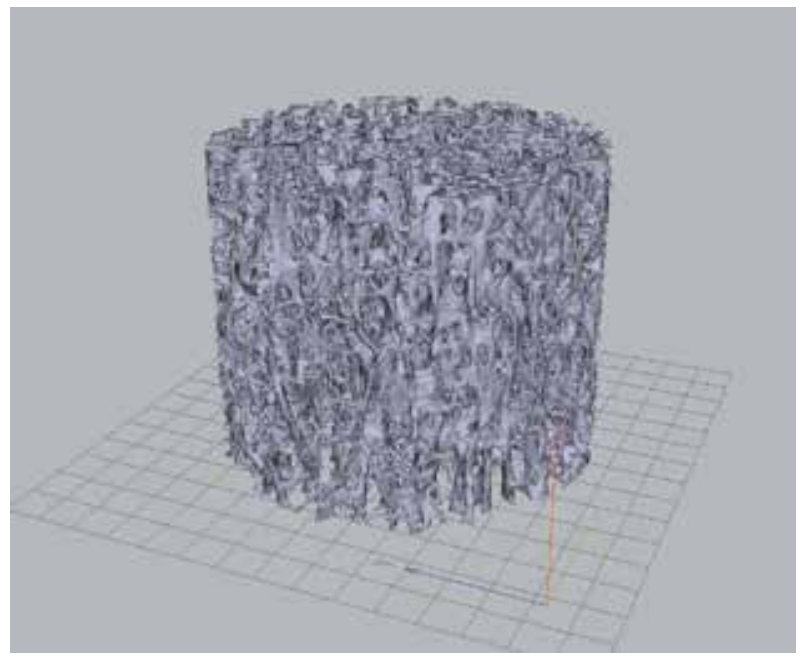

Figure 3. Surface model of the segmented core

Another useful way to represent the 3D geometry is using voxels. A voxel is a 3D pixel element, i.e., a cube of user specified dimensions. The resolution of the voxel depends on the resolution and the slice thickness of the $\mu \mathrm{CT}$ image. If the $\mu \mathrm{CT}$ images are stacked on top of each other in a sequence, the slice number in the stack defines the $Z$ coordinate. A right hand coordinate system is used throughout the document unless otherwise mentioned. The $X$ and $Y$ value of each pixel representing the bone in an image slice along with the slice number of that particular image in the stack, defines the $X, Y$ and $Z$ coordinate of each voxel. An example of the voxel model is illustrated in Figure 4. Although the surface model is a concise and efficient way of representing the 3D geometries, the voxel model is useful in calculating the various parameters that quantify the structure as explained in the following section.

\section{2. $3 \mathrm{D}$ thinning algorithm}

Thinning can be considered as a type of data compression where the important geometric features of a structure are retained without having to store a large amount of data to represent the actual geometry. Because of this, thinning is used in 3D object and feature recognition methods. Recognizing the features of a 3D geometry helps in accurately measuring the change in those features. Thus thinning serves as an intermediate step in calculating the direct 3D quantifying parameters of the bone. Thinning generates a mid-axis for a rod-like structure and a mid-plane for a plate-like structure. The process involves systematically shaving off the surface voxels without changing the topology of the structure until the skeleton of the structure is reached, at which point no more surface voxel can be removed. The skeletons computed by various thinning methods produce spurious curves and surface branches $[15,16]$. These short branches do not add any useful information. Pruning the unwanted short branches from both the curves and the surfaces with user controllable 


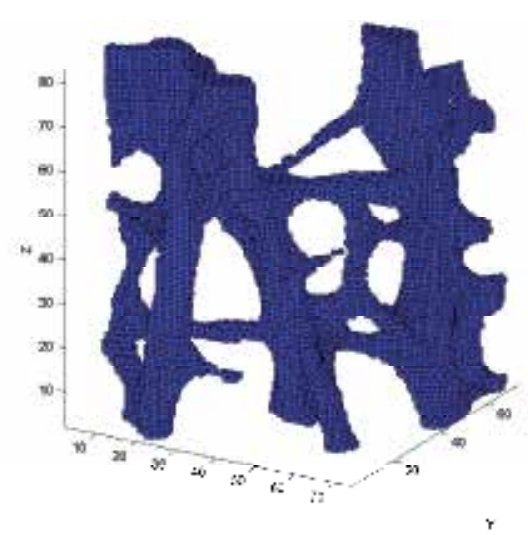

Figure 4. Voxel model of the segmented core

parameters is an useful feature, especially for complex structures like cancellous bone. Ju et al. [17] proposed an efficient pruning algorithm, which handles both the curve and the surface branches so that the final skeleton is both topology preserving and shape depicting. A slight variation of the method proposed by Ju et al. has been successfully implemented in MATLAB. The overview of the process in given below,

1. Find the bounday voxel

2. Find the critical points in the set of boundary voxels

- A point is a critical point if its removal changes the topology of the structure or if it is a curve or a surface end point

3. Remove the non-critical points from the set of bondary points

4. Repeate steps 1-3 untill there are no more boundary points to remove

The next step in thinning is pruning. Noisy and redundant surface and curve edges are removed in this step. This works on the same principal as morphological 2D image operators, erosion and dilation. These two operations are performed on the set of object points or voxels. Similar to the morphological operation on 2D images, opening has the effect of removing small irregular protrusions and produces a smooth edge. In the complete algorithm to calculate the skeleton of an 3D object, the thinning and pruning processes are run alternatively on the object points $[11,17]$.

\subsubsection{Validation of thinning algorithm}

The performance of thinning algorithm was tested on shapes with varying structural complexity in structure. It was first tested on a simple CAD geometry of regular grid of rods of known dimension and thickness. As expected the algorithm generated a clean one voxel thick mid-axis. This is shown in Figure 5, where the gray region represents the CAD structure and the blue line is the calculated mid-axis. This structure was rotated by 5 and 30 around the 
$\mathrm{X}$-axis, and the skeleton was calculated on both the geometries. The result on these structures is discussed in [11].

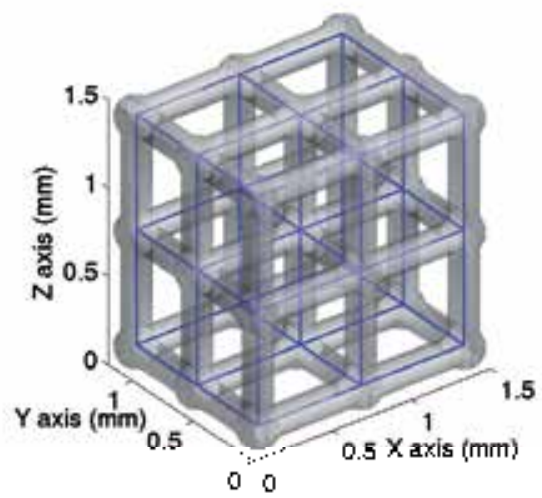

Figure 5. CAD model and its mid-axis overlapped on it.

Next, the algorithm was tested on a CAD model with plates in it and $\mu \mathrm{CT}$ scans of Duocel open cell aluminum foam with 20 pores per inch. In terms of the structure metal foam falls between the regular CAD file and the cancellous bone. The results and explanation in given in [11]. Finally a cancellous bone sample was used to calculate mid-axes; the results of which are shown in Figure 6. This information will be used in the next section (2.3) to calculate parameters quantifying the structure.
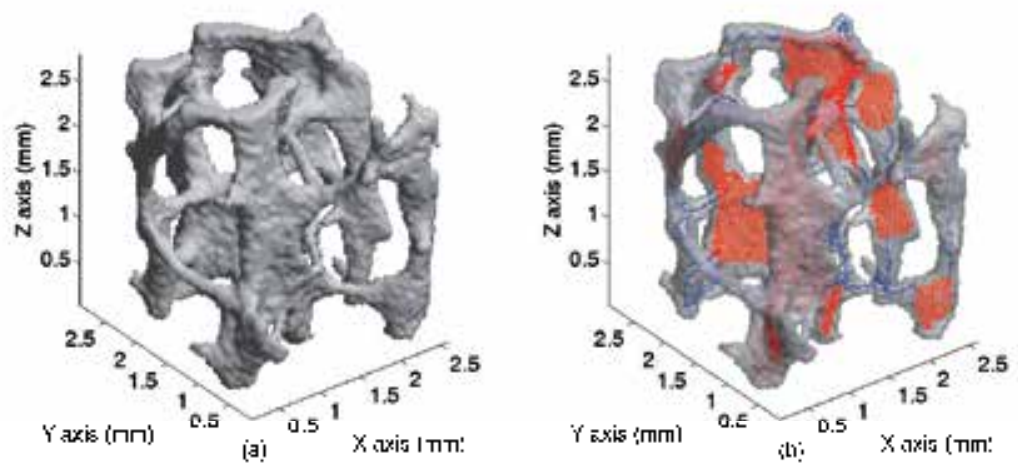

Figure 6. (a) Bone sample and (b) its skeleton overlapped on it.

\subsubsection{Parallelization of thinning algorithm}

The thinning algorithms are iterative in nature, i.e., voxels are traversed and removed either layer by layer or sequentially. As the size or the resolution of the geometry increases, the time taken to calculate the skeleton increases. For a complex structure like the cancellous bone within a vertebra, it becomes practically impossible to calculate the skeleton on a single 
machine within a reasonable time frame (minutes or even hours). To get an approximation of the computational time required to do such a calculation, Figure 7 shows a plot of time required to do such a calculation for different size geometries. If the calculations were being done on an entire vertebra, the time taken would be too long to be of any practical use.

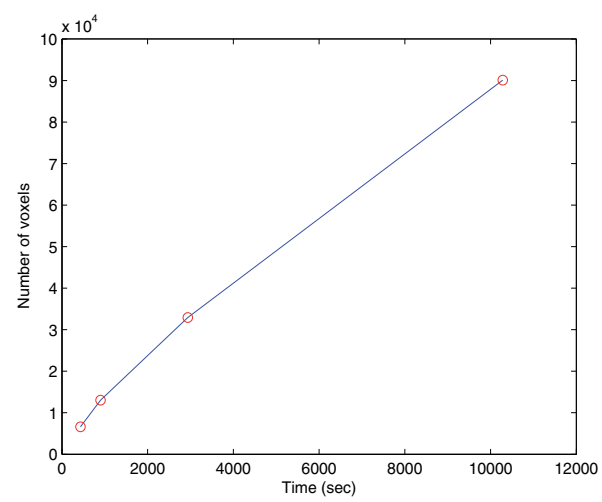

Figure 7. Time taken to calculate the skeleton.

One solution to this problem is to share the data across multiple processors and do the calculations in a distributed way. The concept of parallel computation is not new [18, 19]. Various techniques exists to perform parallel computations on a given data set. However, these algorithms are specific to an application and the literature on parallel computation of medial axes for the purpose of quantification is scarce. A simple and efficient method was developed and implemented using MATLAB's Parallel Computing Toolbox (PCT) and Distributed Computing Server toolboxes [14]. The concept is to split the data into smaller fragments which is customizable in terms of size and dimensions, send it to different processor cores, perform 3D thinning on the individual segments with the knowledge of how the segments are shared, collect the resulting data and combine the fragments to generate the final skeleton of the original structure. The details of the implementation are discussed in [11].

\subsection{Quantification}

Cancellous bone undergoes constant change in its micro-architecture. This change is more prominent in bone disorders like osteoporosis. As a result, the bone mass changes. Bone mass alone is not sufficient to quantify these changes. Clinical studies have shown that the cancellous structure experiences significant changes which affect the mechanical properties of the bone changes [20,21]. This change in the micro-architecture of cancellous bone leads to decrease in bone strength and eventually lead to vertebral compression fractures [22]. A prominent change of plate-like structures to rod-like structures was shown by Ding et al. [21] after the age of 60 years. Loss of entire struts or conversion of plates to struts due to perforation was shown by Parfitt et al. [23]. These previous data show that apart from the overall bone mass, cancellous bone goes through important structural changes.

Apart from the visual assessment of the structural changes, different structural parameters are used to characterize these changes $[24,25]$. In the past, these parameters were studied by 
the examination of the 2D cross-sections of cancellous bone biopsies. The 3D morphometric parameters are then derived from 2D images using stereological methods assuming a fixed structual model [24]. Recent advances in $\mu \mathrm{CT}$ have made it possible to acquire these parameters directly from $3 \mathrm{D} \mu \mathrm{CT}$ image $[26,27]$ without any underlying model assumptions. The calculated midaxes can also be used to decompose the bone into rods and plates [17]. Further, the orientation of the rods can also be calculated. By decomposing the cancellous bone into rods and plates, the parameters can be calculated for the rods and plates seperately. A comparison between the indirect calculated values assuming fixed model structure and the model independent direct calculation is made in [27]. These comparisons show that making assumptions on the type of cancellous bone structure leads to errors in the calculated values. Hence, a model-independent calculation of the parameters was used.

\subsubsection{Parameter calculations}

Based on the literature search the following set of parameters were chosen to characterize the bone sample [24, 27]. They are: bone volume fraction, which is a ratio of bone volume to the total volume (BV/TV); surface density, which is the ratio of bone surface area to the bone volume (BS/BV); trabecular thickness (Tb.Th); trabecular separation (Tb.Sp); and the trabecular number (Tb.N). The cancellous thickness, separation and number are measured directly from the 3D geometry with the method proposed by Hildebrand et al. [26] as described later in the section.

The bone volume (BV) is the volume enclosed by the bone surface. This does not include the marrow space. Total volume (TV) is the volume of the bone including the marrow space. Since the bone geometry is irregular, it is difficult to calculate the volume. To overcome this problem, the bone geometry is split into smaller known geometries (a tetrahedron in this case) and add the volume of each element [11]. Tetrahedral mesh generation algorithm [28] was used to divide the bone in to smaller elements. The bone surface area is calculated by adding the area of all the triangular facets generated by the isosurface function [10,11].

Before calculating the other three parameters, the skeleton of the structure is calculated as described in section 2.2. The thickness calculation uses a volume-based approach rather than the conventional surface based approach [26]. According to the volume-based approach, the local thickness $(\tau)$ is defined as below. If $V$ is the set of object points, then the local thickness measured at point $p \in V$ is the diameter of the largest sphere centered at point $q$ containing the point $p$ and completely inside the structure at the same time (Figure 8). The mean thickness of the structure is defined as the arithmetic mean of all the the local thicknesses. To implement this on a discrete 3D volume, the Euclidean distance from a point $(q)$ to the nearest background point is assigned to that point. This is calculated using a fast distance map algorithm proposed by Maurer et al. [29]. This distance is equivalent to the radius $r$ of the sphere centered at that point $q$. The local thickness measured at point $q$ is given by Equation 1 where, $\operatorname{sph}(q, r)$ is the set of points within the sphere centered at $q$ with a radius $r$.

$$
\tau(q)=2 * \max (r \mid \operatorname{sph}(q, r) \subseteq V)
$$

Figure 8 illustrates this concept. The blue line is the object boundary, red line is the mid-axis of the object. If $p$ is a point within the structure and $p \in \operatorname{sph}(q, r)$ then the local thickness of point 
$p$ is given by $2 * r$, the diameter of the largest sphere which encloses point $p$. Since the points on the mid-axes are the center of largest diameter sphere that can be fit within the structure, the local thickness of all the points on the mix-axes are calculated. The mean thickness of the structure is the average of all the local thicknesses calculated for that structure.

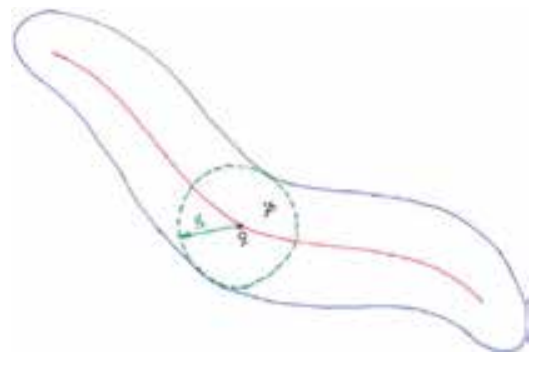

where,

$r=$ Distance map at point $q$

$p=A$ point within the object

Figure 8. Thickness calculation of a structure.

Calculating the trabecular separation (Tb.Sp) is similar except the maximum diameter sphere is fit within the marrow space. For trabecular number (Tb.N), the sphere fit within the marrow space is extended all the way to the mid-axes of the adjacent trabeculae.

\subsubsection{Algorithm verification}

The quantifying algorithm explained and implemented in the previous sections was verified on three different types of structures, a 3D CAD model of regular grid and known dimension with only rods, a similar CAD model with both rods and plates, and finally on $\mu \mathrm{CT}$ images of open cell metal foam structure with 20 pores per inch. The 3D CAD models have an aspect ratio of 1.3 which is the same as vertebral bone [30]. Since the parameters are measured on digitized images, the resolution of the $\mu \mathrm{CT}$ images have a significant effect on the calculated values. The $3 \mathrm{D}$ CAD models were imported into MATLAB with varying resolutions, and the set of parameters were calculated for each resolution. The error vs. resolution graph is plotted for each of the parameters for the 3D CAD model (Figure 9). As can be seen from these graphs, as the resolution increases (in other words, the size of voxels decreases) the error becomes small. The other cause of error due to digitization is how well the voxels are aligned with the surface boundary of the 3D geometry.

The quantifying parameters were measured on this CAD after rotating it around the $x$-axis by $5^{\circ}$ and then by $30^{\circ}$. The same procedure was repeated on a CAD model with both rods and plates. The results of this is discussed in [11].

The third structure used for verification was a metal foam sample scanned at a resolution of $28.7 \mu \mathrm{m} /$ pixel. The average calculated values of the parameters for this structure are shown in Table 1.

To find the accurateness of the results, struts were randomly picked from the metal foam sample. The thickness of these struts were measured by hand by fitting spheres within the 


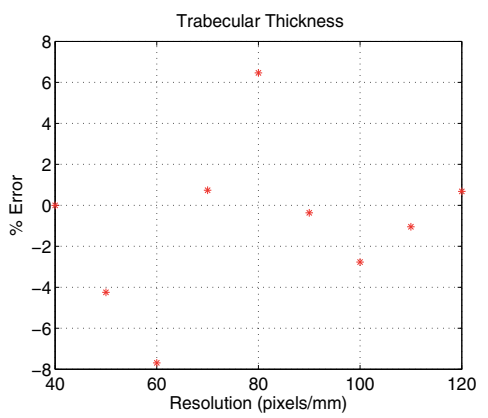

(a)

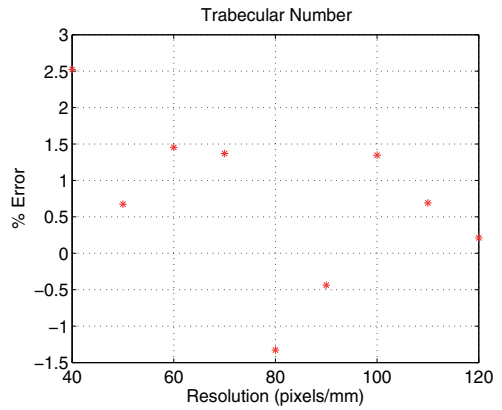

(c)

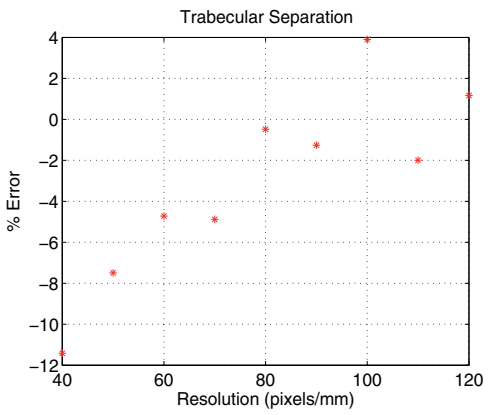

(b)

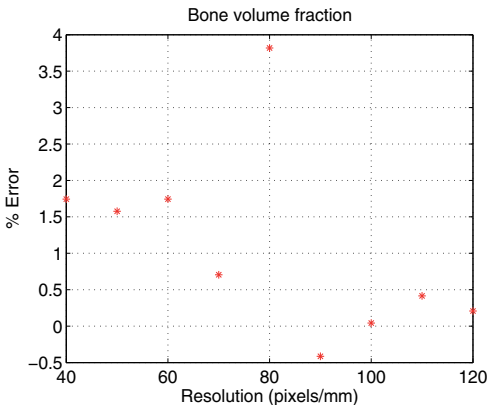

(d)

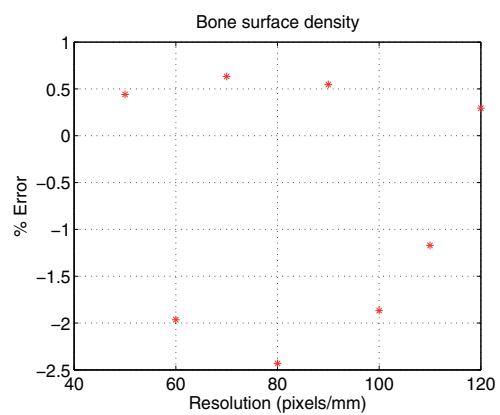

(e)

Figure 9. Parameters of CAD model in its original orientation (a) Trabecular thickness (b) Trabecular separation (c) Trabecular number (d) Bone volume fraction (e) Bone surface density.

\begin{tabular}{|l|l|l|l|}
\hline Parameters & Calculated values & Measured values & $\%$ Error \\
\hline rTb.Th $(\mathrm{mm})$ & 0.3276 & 0.3198 & $-2.4405 \%$ \\
\hline pTb.Th $(\mathrm{mm})$ & 0.4618 & 0.4558 & $-1.3163 \%$ \\
\hline Tb.Sp $(\mathrm{mm})$ & 0.7118 & 0.7312 & $2.6532 \%$ \\
\hline Tb.N $\left(\mathrm{mm}^{-1}\right)$ & 1.0999 & 0.9991 & $-10.08 \%$ \\
\hline BV $/ \mathrm{TV}(\%)$ & 0.1301 & 0.1319 & $1.3646 \%$ \\
\hline BS/BV $\left(\mathrm{mm}^{-1}\right)$ & 8.3297 & 8.4213 & $1.0877 \%$ \\
\hline
\end{tabular}

Table 1. Calculated parameters of the metal foam sample. 
struts. To measure the angle, cylinders were fit to the strut and the angle of the axis of the cylinder was taken as the true angle of the strut. These values were then compared to the calculated values from the quantifying algorithm. The metal foam sample has 300 struts in it. To assess the results with a $95 \%$ confidence level and within a 5\% confidence interval, the sample size was determined to be 169 [42]. Lillefors test for normality was performed on the data sets using the MATLAB's Statistical toolbox which proved that the both of the data sets come from data which is normally distributed. Thus the verification process shows that the calculated values are within an acceptable range of $\pm 5 \%$ of error. It was also concluded that the resolution and the orientation of the scans of the object have significant effect on the calculated values. As the resolution increases the error in the calculated values are generally reduced.

\subsection{Lumbar load calculation}

The skeleton is a load bearing structure. Skeletal segments are connected to each other by joints of various degrees of freedom. Each joint in the body experiences different amounts of load depending on the type of activity done by the human subject. This subject specific loading data can be used to predict the state of the bone structure with techniques that have been studied, tested and verified to comply to the rules of bone remodeling (2.5). The realistic loading data can be collected by surgically placing the load sensors into the subject's body at a desired location [31,32]. But this is not a practical solution to be used as a clinical tool. An alternative solution is to use motion data of the subject to drive a human body model in a musculoskeletal modeling and simulation software like lifemod [43].

\subsubsection{Motion capture (MOCAP)}

The first step is to capture the motion of a subject. The Vicon motion capture system was used for this purpose. The setup consists of 10 ViconMX cameras. A full body Plug-in-Gait marker set with 39 markers were used. $9 \mathrm{~mm}$ retro-reflective markers were fixed on to the black jumpsuit, worn by the user, with velcro. The details of the marker set are given in [11]. For the preliminary study, to show the proof of concept, two different types of motion were chosen: walking, and rope jumping exercise. Basic subject specific data was recorded for each subject. Two subjects were used in this test. Once the subject were markered up, s/he was asked to perform the two tasks and the data was collected using Vicon Nexus software. Figure 10 shows a screen shot of the Vicon Nexus software illustrating the camera setup and the subject within the capture volume. A custom MATLAB script was written to convert the motion capture data from Vicon system into LifeMOD compatible format.

\subsubsection{Detailed lumbar model in LifeMOD}

LifeMOD is a virtual human modeling and simulation software. It is built on MD ADAMS software which is a standard for mechanical system simulation. Given the basic information such as age, height, weight and gender, LifeMOD can generate a full body model with the basic bones, muscles and joints based on its anthropometric database. This basic model can be refined according to the users needs by adding more muscles and/or bone segments. It gives the user the ability to develop a full-body or a body segment with all the bones, joints and simulate muscles with varying levels of detail. With the ability to import the motion 


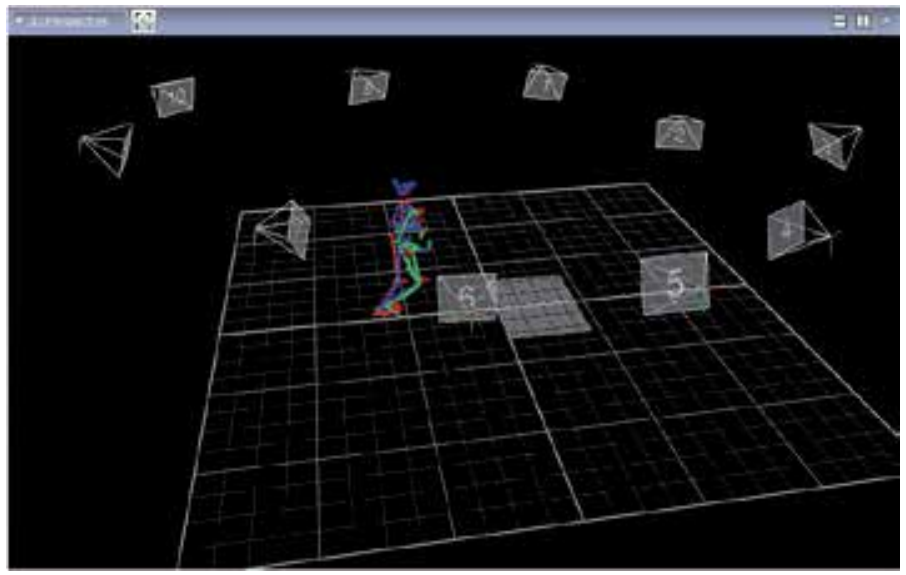

Figure 10. Camera setup for motion capture.

capture data and to perform forward and inverse dynamic simulations, it can calculate the force, displacement and torques on each of the body segments. This provides an important tool to calculate the forces acting on the L4 vertebrae without having to insert a pressure sensor into the subject. Because of this ability, the remodeling techniques can eventually be put to practical use.

The full body model generated by LifeMOD, along with the anthropometric data, treats the central torso containing the lumbar vertebrae as one segment (Figure 11). In order to calculate the loads on L4 vertebrae this model needed to be refined by adding individual vertebral bodies and intervertebral discs in the lumbar section. The components involved in creating a detailed lumbar section is shown in Figure 12. The adjacent segments, vertebral bodies, are connected to each other via spinal or the intervertebral discs. The intervertebral discs are kinematic constraint connecting adjacent vertebral bodies. These are implemented as a 6 degree of freedom force between any two given segments which include all the connective tissue between them. The disc is formed as a spring force. The values for stiffness and damping coefficient are used from the anthrompmetric data base in LifeMOD.

The spinal discs are connected to the vertebral segments at user specified landmark locations. A landmark is any significant location on a segments or bones in the model. It is an anatomical marker which represents position and orientation and moves with the segment to which it is attached to. The landmarks on the vertebral bodies were placed according to the locations specified in the literature [33]. The spinal discs connect to the inferior landmark on the vertebral body above them and superior landmark on the vertebral body below them (Figure 12). A detailed lumbar model was created in LifeMOD and incorporated in the full body model replacing the existing central torso section (Figure 13).

\subsubsection{Load calculation with MOCAP data}

With the full body model refined to include the detailed lumbar section, Motion capture (MOCAP) data is imported in to LifeMOD. Along with the $x, y$ and $z$ coordinates of the marker for each time-step the MOCAP data file contains the subject specific data (age, height, weight and lengths of body segments). This information is used by LifeMOD to scale the 


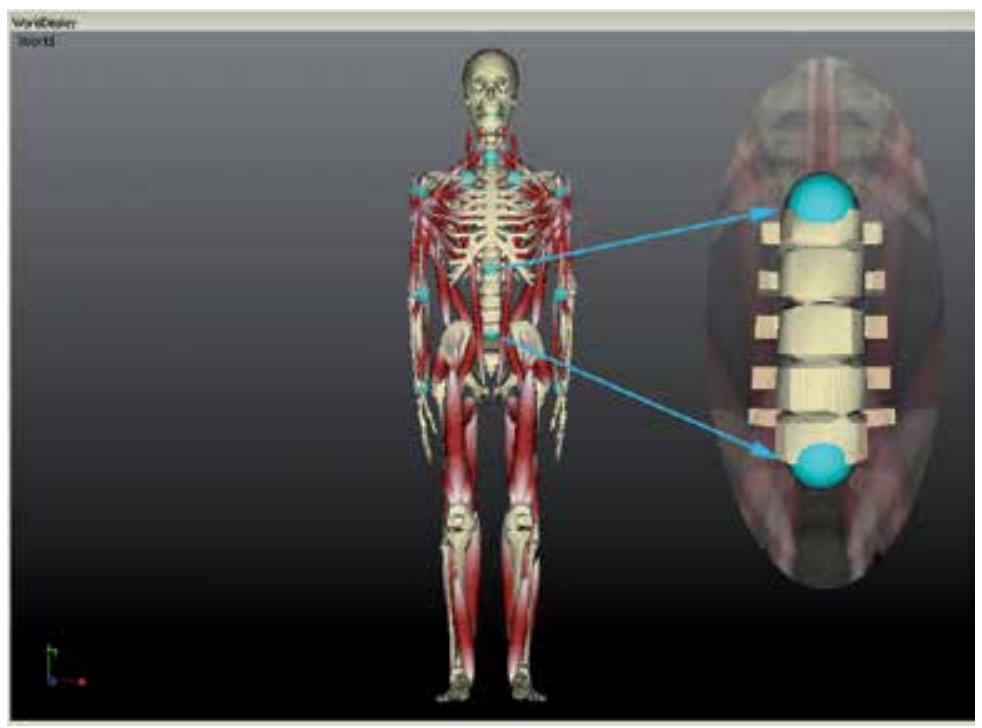

Figure 11. Fullbody model with the central torso enlarged.

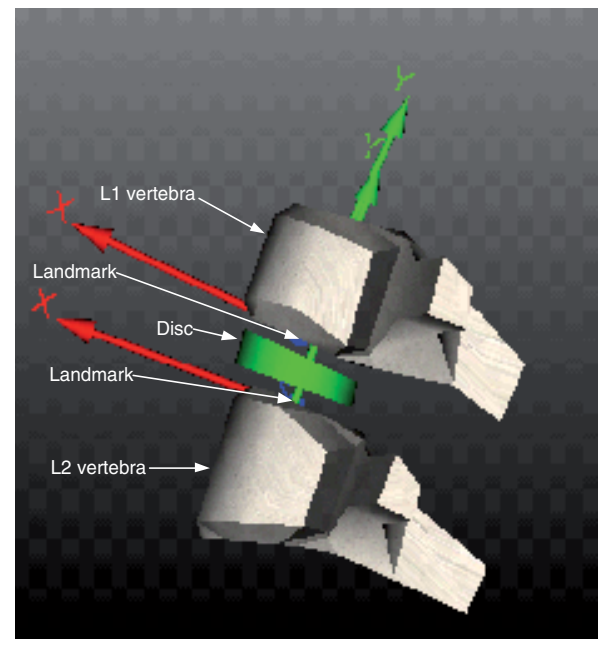

Figure 12. Exploded view of the lumbar vertebrae with spinal disc

musculoskeletal model and all its segments to match the subject data. Apart from the automatic scaling and positioning, manual tweaking to position the segments in the correct position by visual inspection of the landmark locations was necessary. The final body model along with the motion capture data is shown in Figure 14. The yellow spheres represent the marker agents imported through the motion capture data and the red spheres represent the locations on the body segments to which the marker agents are attached. Within LifeMOD the motion data (yellow spheres) and the marker locations on the body segment (red spheres) are linked via spring elements. The use of spring elements becomes clear in the next step. This helps to make adjustments for small differences in the human body model and the actual human subject. 


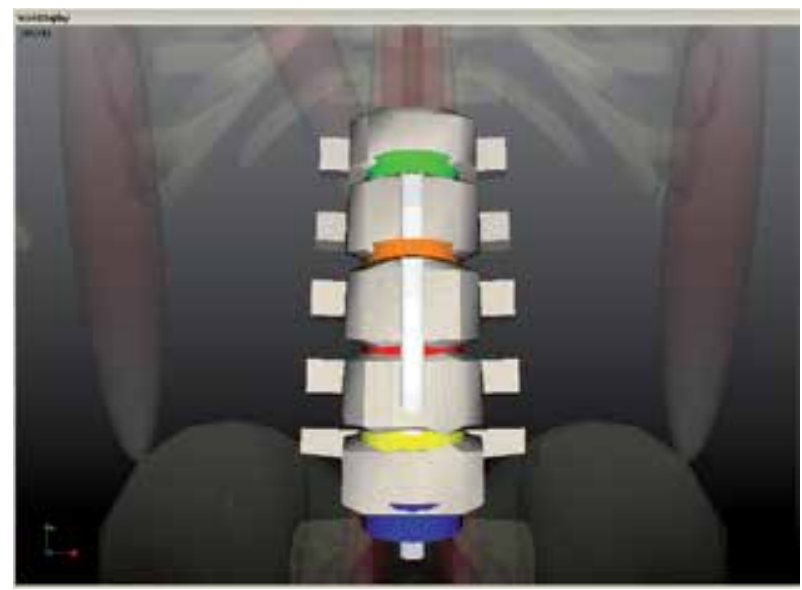

Figure 13. Closeup of the detailed lumbar model created in LifeMOD.

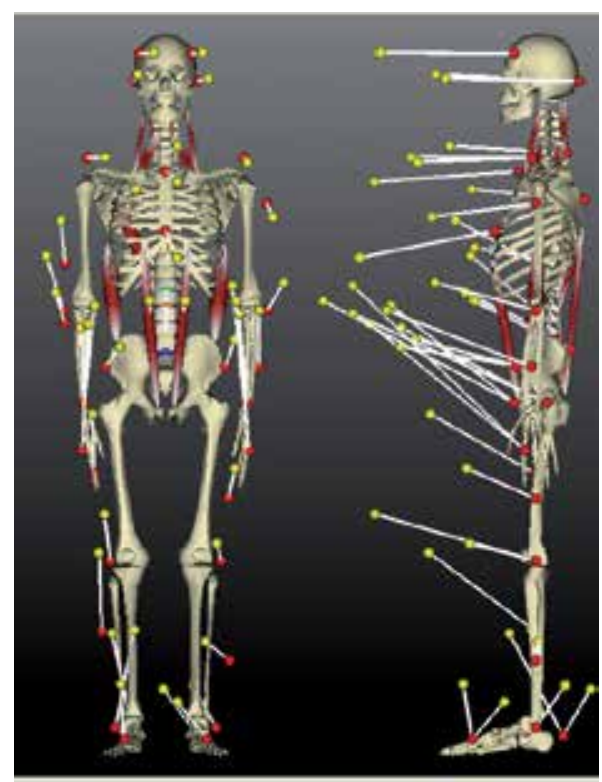

Figure 14. Body model along with the motion capture data.

With the motion capture data imported into LifeMOD, the next step is to perform an equilibrium analysis (or Settling run). In this process the model is fit to the data positions. The body segments are repositioned, while still maintaining the joint definitions, to match the position represented by the motion capture data. It is a dynamic analysis in which the data driven marker agents (yellow spheres) are fixed and the body segments are moved to find minimum energy configuration in the springs connecting the marker agents (red spheres). While recording the motion data, the markers placed on the body segment might not be exactly on the specified location. This means that, during equilibrium analysis the markers on the body segments will never overlap with the motion capture data points. The equilibrium analysis helps to reposition the model to align with the initial position of motion agent data. 
Having the motion agents and the body segment markers agents connect via spring elements allows for minor differences between the locations of these two marker sets. The final body position after equilibrium analysis is shown in Figure 15.

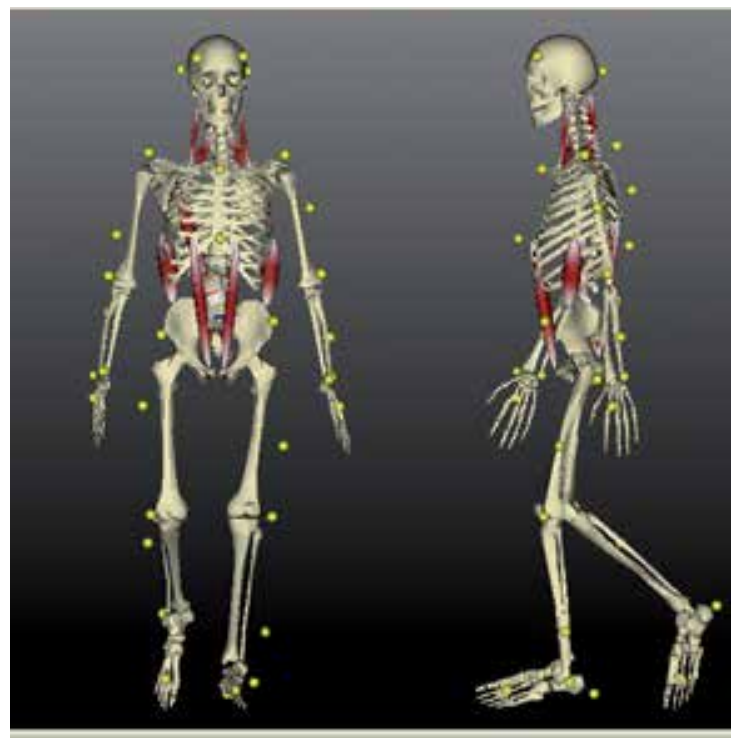

Figure 15. Body model after equilibrium analysis.

The next step is to perform the inverse dynamic simulation. During the inverse dynamic simulation, the joints and muscles are set as recording elements and the human body model is manipulated by the motion agents. The recording elements collect the angular motion and muscle lengthening and shortening data. After the inverse dynamic simulation, the motion agents are disabled and the recorded angular movements and the lengthening and shortening patterns are used in torque and force functions in joints and muscles. The joints and muscles are set to active from recording state. These active elements then drive the model along the curves recorded by the inverse-dynamics simulation as the PD-servo drivers replicate the joint and muscle data as closely as possible. At the end of the forward dynamic simulation the forces of the discs in all three directions ( $x, y$ and $\mathrm{z}$ axis) are calculated by LifeMOD.

The computed loads acting vertically down on L4 vertebra for two different activities (walking and rope jumping exercise) are shown in Figures 16 and 19. These values were comparable with load values for different activities in the literature $[31,32,34]$. The load values calculated from LifeMOD are used in the remodeling algorithm explained in the next section (2.5).

\subsection{Adaptive bone remodeling}

Many theoretical and computational models have been proposed to investigate and simulate the dynamic behavior of the bone $[9,35]$. With high resolution imaging methodologies such as $\mu \mathrm{CT}$ becoming more accessible, the study of cancellous remodeling began to take in to account the influence of cellular activity in 2 and 3 dimensions $[9,25]$. Simulation involving effect of metabolic bone formation deficit and the micro structural bone formation deficit were also developed $[9,36]$. Effect of age-related changes on cancellous plate thickness and the number 
of plates in the cancellous bone showed that the number of plates per unit volume is a more significant predictor of bone loss than a decrease in the plate thickness [46].

\subsubsection{Remodeling algorithm overview}

Different mathematical control models of mechanical bone mass regulation have been proposed [37, 38]. These models assumed a continuous feedback loop between the maintenance of bone mass and local strain values in the tissue. The idea of functional adaptation of the cancellous structure regulated by the local activity of cells was proposed by Roux [39]. The local activity of the cells is governed by the mechanical stimuli. Mullender et al. [37] proposed that the osteocytes act as sensors of mechanical signals or mechanoreceptors because of their favorable architecture and distribution. The mathematical model uses the amplitude of strain energy density as the mechanical signal that the osteocyte senses. The osteocytes in turn send this signal to the bone surface. The signal intensity decreases exponentially with distance (Equation 2).

$$
\begin{aligned}
& f_{i}(x)=e^{-\left(d_{i}(x) / D\right)} \\
& \text { where, } \\
& \qquad \begin{array}{l}
d_{i}=\text { distance between osteocyte } i \text { and surface location } x \\
D=\text { Distance form osteocyte } i \text { where its effect } \\
\text { has reduced to } e^{-1} \text { or } 36.8 \%
\end{array}
\end{aligned}
$$

The effective stimulus $P(x, t)$ at any given surface location $x$ is the sum of all the osteocyte signals within the regions of influence of nearby osteocytes (Equation 3) $([7,37])$.

$$
\begin{aligned}
& P(x, t)=\sum_{i=1}^{N} f_{i}(x) \mu_{i} R_{i}(t) \\
& \text { where, } \\
& \quad \mu_{i}=\text { Mechanosensitivity of the osteocyte } i \\
& R_{i}(t)=\text { Strain energy density rate } \\
& \quad \text { at osteocyte location } i
\end{aligned}
$$

The local change in the relative density $m$ on the surface of cancellous bone tissue is expressed as [7],

$$
\frac{d m}{d t}=\left\{\begin{array}{l}
\tau\left\{P(x, t)-k_{t h}\right\}-r_{o c} \text { if } P(x, t)>k_{t h} \\
0 \text { if } P(x, t) \leq k_{t h}
\end{array}\right.
$$

where $k_{t h}$ is the threshold of bone formation, $r_{o c}$ is the relative amount of bone resorbed by osteoblasts and $\tau$ is the time constant regulating the rate of the process. This was chosen to be 1 making the simulation process measured in simulation time. The relative density at any location within the bone varies between 0 (for no bone) to 1 (fully mineralized bone). When the relative density value reaches zero, the osteocyte at that location disappears. 
The resorption is regulated by the presence of micro cracks or by disuse [7, 8]. Resorption due to the presence of micro cracks is assumed to be constant,

$$
p(x, t)=d \quad(\text { spatially random })
$$

Resorption due to disuse is dependent on the strain and is given by the following equation, where $a=1.6$ and $c=12.5$ are constants.

$$
p(x, t)= \begin{cases}c[a-P(x, t)] & \text { if } P<a, \\ 0 & \text { if } P \geq a\end{cases}
$$

Osteocytes respond to dynamic loads [40,41]. The response of the osteocytes is measured in strain energy density (SED) rate. This SED rate can be determined by static finite element analysis using an equivalent static load of the dynamic loading signal. Consider a periodic loading signal $\sigma_{e}(t)$, oscillating between 0 and a maximum amplitude of $\sigma$, given by Equation 7 , which has a frequency of $f \mathrm{~Hz}(\omega=2 \pi f)$.

$$
\sigma_{e}(t)=\frac{1}{2} \sigma(1+\cos \omega t)
$$

Then the final form of equivalent static load (ESL) is given by Equation 8,

$$
\sigma^{\prime}=2 \sigma \sqrt{f} \Rightarrow E S L
$$

The previous studies have provided proof of the regulatory mechanism of the Wolff's law. The idea proposed in this research is the use of the realistic mechanical loading data on the L4 vertebra (Section 2.4) to run the remodeling algorithm. The load data is then used in the remodeling algorithm to predict the modified cancellous structure. The schematic of the proposed method is shown in the Figure 1. The advantage of this is that the effect of different activities on the strength and structure of cancellous bone can be studied. Another advantage is by using the motion data of different everyday activities, a daily effective load value can be calculated and this in turn is used to drive the remodeling process to simulate the changes in the cancellous bone structure.

\subsubsection{Equivalent static load calculation (ESL)}

The effective static load calculation of two different activities, walking and rope jumping exercise, are compared here. The load calculated from LifeMOD for walking motion of the subject is shown in Figure 16. The amplitude of the dynamic load oscillates between a minimum of $242.79 \mathrm{~N}$ and a maximum of $866.04 \mathrm{~N}$, with a peak to peak amplitude of $623.25 \mathrm{~N}$. The first few data points were discarded as the model goes through a settling run in which the motion agent markers are repositioned to align with the motion agents. The last few data points were discarded to make sure none of the reflective markers on the subject were missing as a result of being close to or going out of the capture volume. The data retained is between the two vertical red lines at time 0.8 seconds and time 3.98 seconds.

The frequency of the load cycle is equal to the frequency of the subject walking at a self selected pace. Within the Vicon Nexus software two events of gait cycle, toe off and heel strike, are 


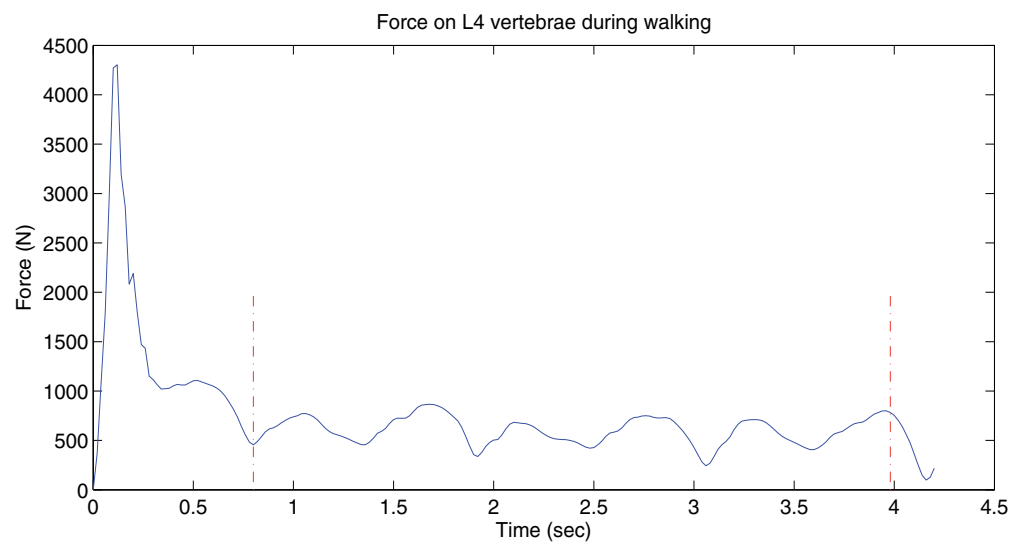

Figure 16. Load acting in superior-inferior direction (vertically down) on L4 vertebra during walking.

detected. A heel strike is defined as the instance the heel of the foot touches the ground and the toe off is defined as the instance when only the big toe of the reference foot is in contact with the ground. These two events, for both the feet, are shown in Figure 17. The toe offs are indicated by the red upward pointing arrows and the heel strikes are indicated by the black diamonds. Knowing the total duration of the capture data and the number of toe offs, the frequency of walking is determined. In this case, the first left foot toe off occurs at frame 331 and the last toe off occurs at frame 651 with the total number of frames in between being 338 . With a total toe offs of 3 and at a capture rate of 100 frames/second, the time between toe-offs is 1.1267 seconds and the frequency equals to $0.8875 \mathrm{~Hz}$. With this information the effective static load can be calculated to be $1174.29 \mathrm{~N}$. This calculated load is used on the segment of cancellous bone in the finite element algorithm.

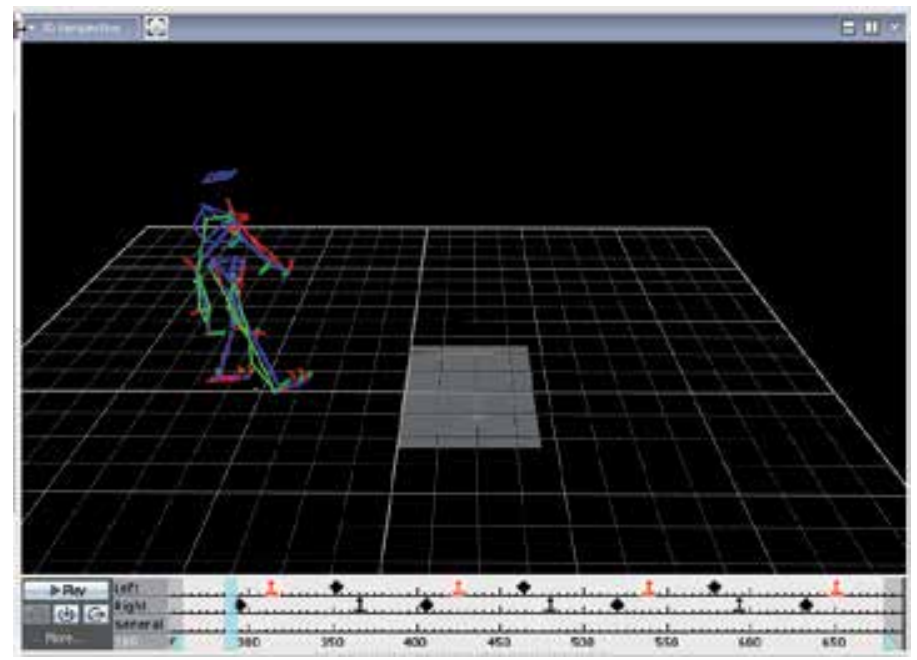

Figure 17. Walking gait cycle showing heel strike and toe off.

The second activity was rope jumping exercise. The calculated load signal on L4 is shown in Figure 19. Once again the first and last few data points are discarded and signal from 1.732 
seconds to 7.721 seconds is retained. The signal oscillates between a maximum amplitude of $1247 \mathrm{~N}$ and a minimum amplitude of $145.9 \mathrm{~N}$. This has a peak to peak amplitude of $1101.1 \mathrm{~N}$ for the dynamic signal. The frequency is determined similar to the method in walking gait. Between frames 110 and 804 for the first and last toe-off, there are 14 toe-offs (Figure 17). This gives 49.57 frames or 0.4957 seconds between toe-offs with a 100 frames per second capture rate. Thus the frequency of the load signal is $2.0173 \mathrm{~Hz}$. With this, the ESL is calculated to be $3127.82 \mathrm{~N}$. Once again, this calculated load is used on the segment of cancellous bone in finite element algorithm to calculate the stresses and strains within the bone segment.

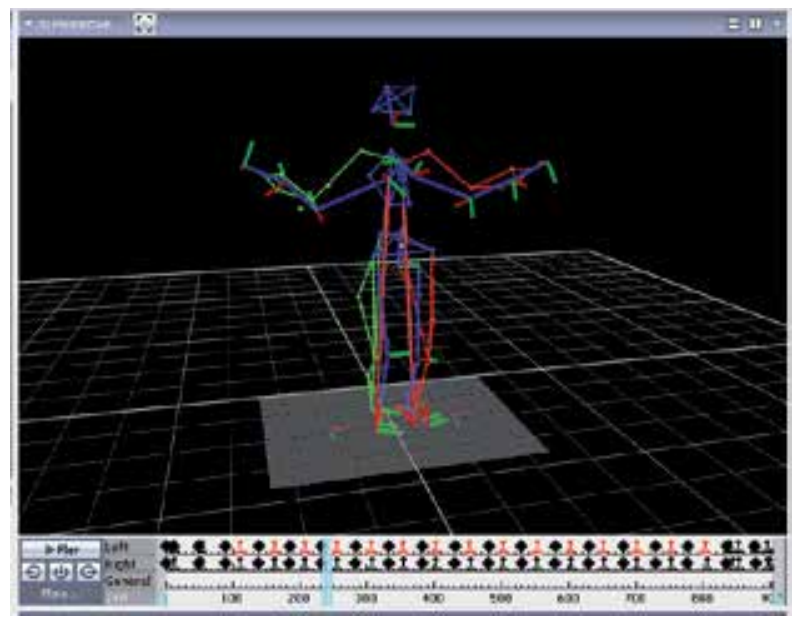

Figure 18. Rope jumping exercise showing heel strike and toe off.

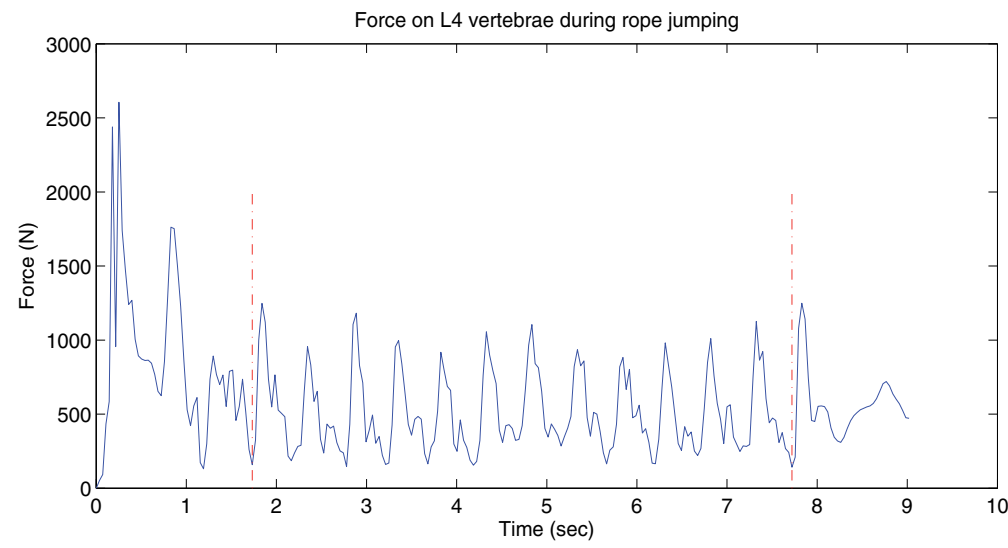

Figure 19. Load acting superior-inferior direction (vertically down) on L4 vertebra during rope jumping exercise.

\subsubsection{Remodeling results}

To calculate the stress and strain values within the bone material, a finite element solver specifically designed for biomechanical applications is used [44]. This is a three step process, 
sample preparation, finite element analysis and post processing of the data. These three steps are explained below.

A small volume of cancellous bone is extracted from the stack of $\mu \mathrm{CT}$ images. A 3D geometry of the sample is constructed. At this stage a tetrahedral mesh is created using a 3D tetrahedral finite element mesh generation algorithm [45]. A custom MATLAB code was written to assign material properties to the tetrahedral mesh and add a top and bottom rigid body plates with compressive loads on them [11]. Vertebral cancellous bone has an elastic modulus of 5,000 $\mathrm{MPa}$ and Poison's ratio of $0.3[8,37]$. The top and bottom of the bone segment are attached to rigid bodies whose translations are constrained along $X$ and $Y$ axes and rotation is constrained about $\mathrm{X}, \mathrm{Y}$ and $\mathrm{Z}$ axes. A positive force (compressive) is applied on the rigid bodies equivalent to the calculated ESL from the previous section. The solver is set to perform a quasi-static analysis ignoring the inertial effects. After the solver completes the analysis, the stress and strain values at each element location are known. The strain energy density value is calculated first at the element location and then interpolated to find the value at each osteocyte locations.

The calculated strain energy density values mapped on the bone sample are shown in Figure 20. With the SED values calculated, along with the other parameters required for the remodeling algorithm explained in section 2.5.1, the bone goes through the process of increasing/decreasing the bone density values until the density value either goes above 1 or below 0.01 . At that point, the voxel whose density goes below 0.01 is removed and new 6-neighbor voxels are added around the voxel whose density value goes above 1 . Each time there is a change in the structure, new stress, strain and SED values are calculated.

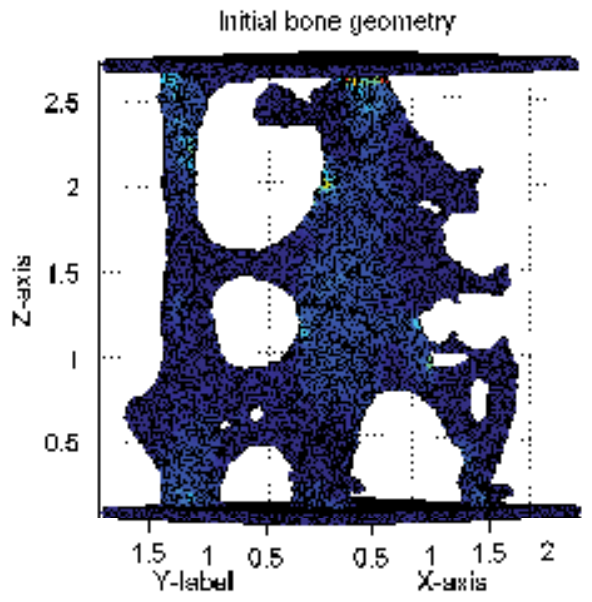

Figure 20. Calculated SED values mapped on the initial geometry of the bone sample.

This process is repeated until there is no further significant change in the structure between iterations. This is decided by comparing the quantifying parameters calculated at every 5 th iteration (Figure 22, 24). The final structure of a bone sample for the corresponding action of walking is shown in Figure 21 overlapped with the SED values. Figure 23 shows the final geometry of the same bone sample for rope jumping exercise. The results show that the bone microarchitecture responds differently to different loads. In this initial test, only the vertical component (Z-axis) of the load is applied. Hence, the structure seems to evolve into vertical 
struts. This can be further refined by adding the horizontal components ( $X$ and Y-axes) of the load. In which case, the horizontal struts might be retained and/or modified to adapt to the loads in the horizontal directions.

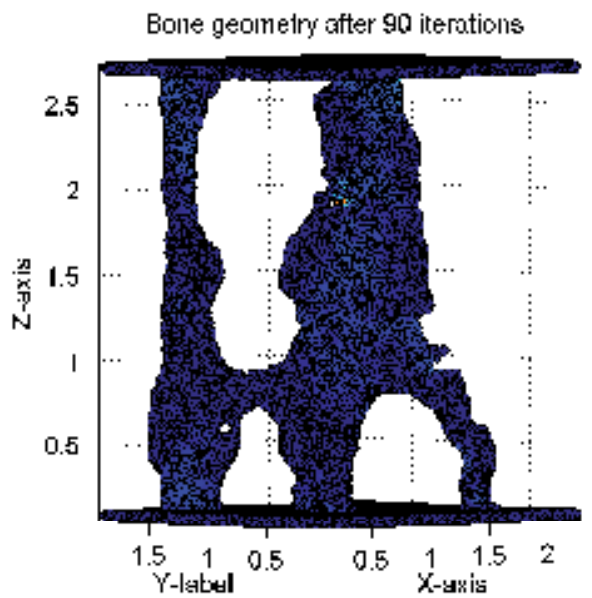

Figure 21. Final geometry of the bone structure (Applied load corresponds to walking).

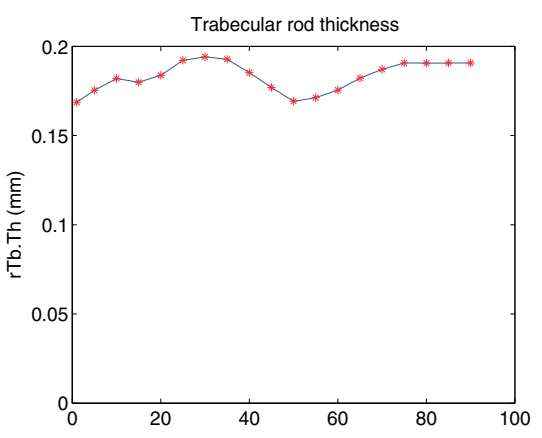

(a)

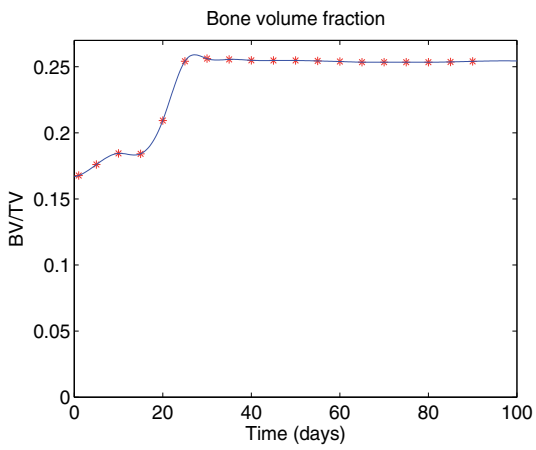

(c)

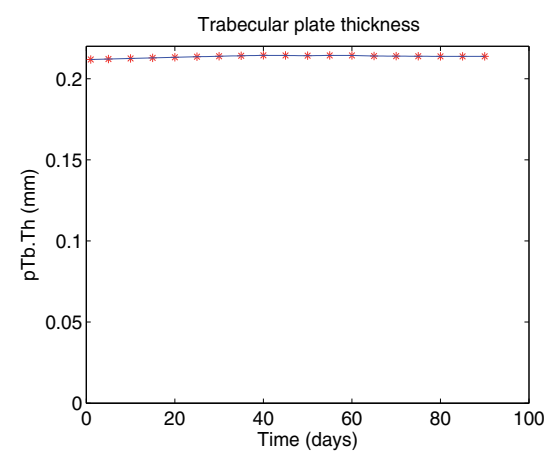

(b)

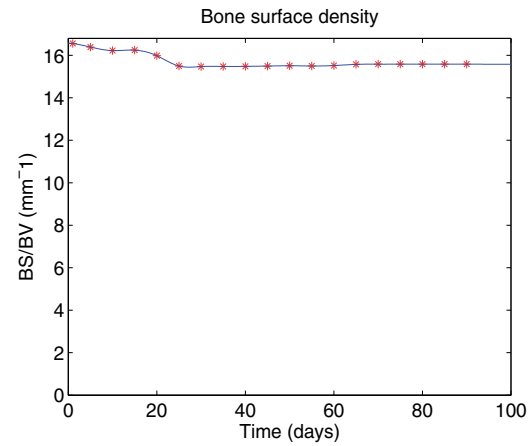

(d)

Figure 22. Variation in the quantifying parameters during remodeling as a result of walking exercise. 


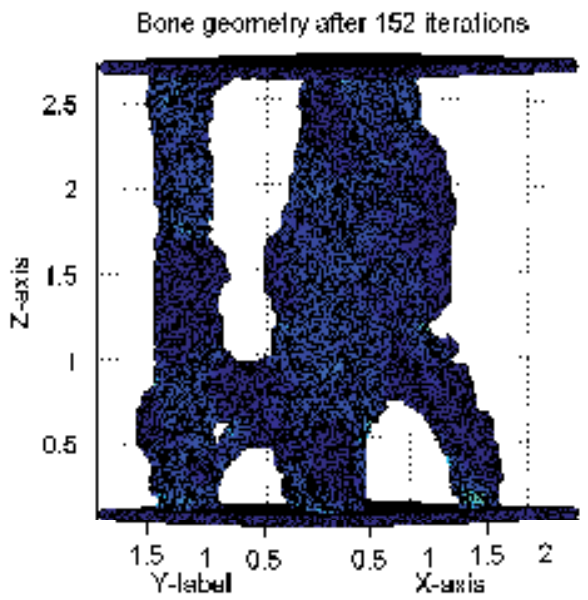

Figure 23. Final geometry of the bone structure (Applied load corresponds to rope jumping exercise).

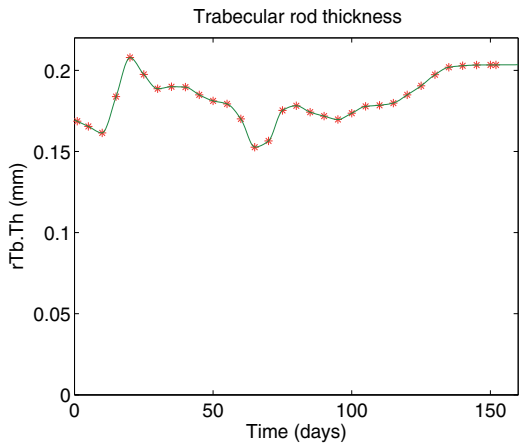

(a)

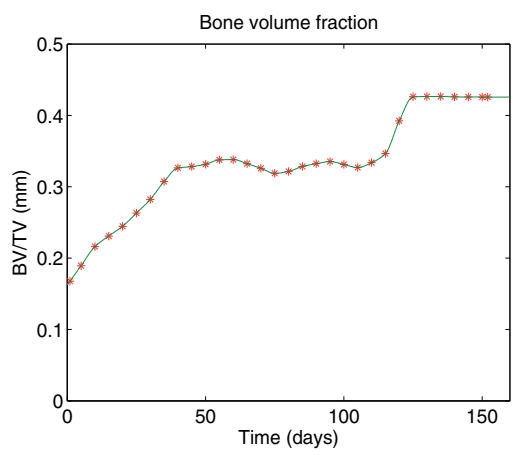

(c)

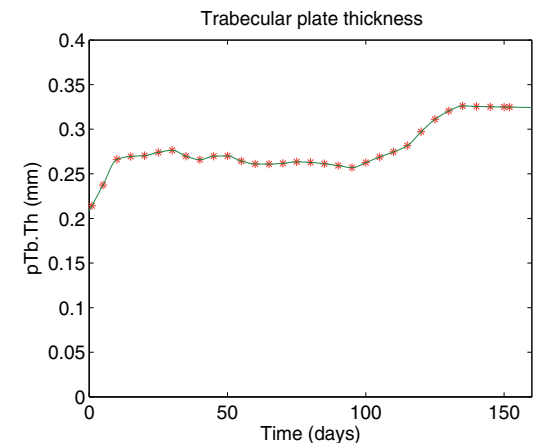

(b)

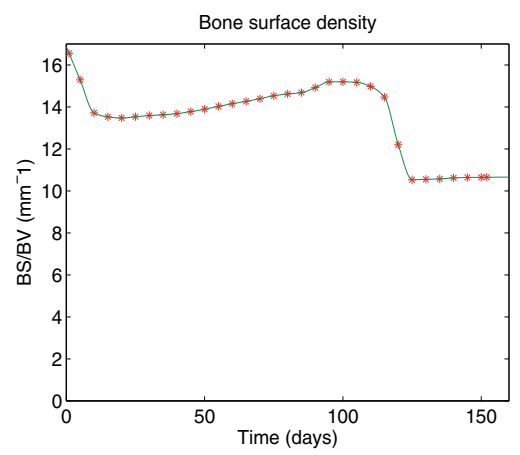

(d)

Figure 24. Variation in the quantifying parameters during remodeling as a result of rope jumping exercise.

\section{Conclusion and future work}

Successful integration of the proposed method is demonstrated in this study. Effects of different physical activities on the adaptation of the cancellous bone structure and 
their quantifying parameters are shown. The results suggest that the different loadings corresponding to different activities have varying effect on the microarchitecture of the bone. Thus, the loading conditions on the bone can be changed to produce desired results, i.e., stronger or at least not a weak structure. In general, this method has applications in physiotherapy, assessing/avoiding injuries in various sports, and studying the effects of different types of bone implants over a period of time.

Although the proposed remodeling cycle is successfully integrated and implemented in this study, there are still rooms for improvement. Some of the limitations of this study which would be the focus of the future work are, 1) the $\mu \mathrm{CT}$ scans of the vertebra are not in vivo, 2) effect of only one motion is included in the simulation which can be refined by including the daily loading activity, 3) Loading on the only the vertical direction is considered which may be extended to include all the three forces. Some of the other possibilities for improving the performance would be to look into the use of Graphical Processing Units (GPU) for computation. Use of GPU may be more cost efficient in a clinical environment. Based on the results, this study suggests the above mentioned to be addressed in the future work.

\section{Acknowledgements}

The authors would like to thank Dr. Kathleen Issen and Dr. Laurel Kuxhaus for their valuable advice and constructional critics and Nicole Corbiere for helping with $\mu \mathrm{CT}$ scanning validation.

\section{Author details}

Ajay V. Sonar

Graduate student, Department of Electrical and Computer Engineering, Clarkson University, Potsdam, USA

James J. Carroll

Associate Professor, Department of Electrical and Computer Engineering, Clarkson University, Potsdam, USA

\section{References}

[1] Old J.L, Calvert M (2004) Vertebral compression fractures in the elderly. American family physician. 69: 111-116.

[2] About Osteoporosis (2001). Available: http:/ / www.nof.org/node/40/. Accessed on: Dec 2011.

[3] Melton III L.J (1997) Epidemiology of spinal osteoporosis. Spine. 22:2s:11s.

[4] Campion J.M, Maricic M.J (2003) Osteoporosis in men. American family physician. 67:1521-1526.

[5] Patel U, Skingle S, Campbell G.A, Crisp A.J, Boyle I.T (1991) Clinical profile of acute vertebral compression fractures in osteoporosis. Theumatology. 30:418-421.

[6] Wolff J (1892) Das Gesetz der Transformation der Knochen. Berlin, Hirschwald.

[7] Huiskes R, Ruimerman R, van Lenthe G.H, Janssen J.D (2000) Effects of mechanical forces on maintenance and adaptation of form in trabecular bone. Nature. 405:704-706. 
[8] Ruimerman R, Huiskes R, van Lenthe G.H, Janssen J.D (2001) A computer-simulation model relating bone-cell metabolism to mechanical adaptation of trabecular architecture. Computer Methods in Biomechanics and Biomedical Engineering. 4:433-448.

[9] Van Der Linden J.C, Verhaar J, Weinans H (2001) A Three-Dimensional Simulation of Age-Related Remodeling in Trabecular Bone. J Bone Miner Res. 16:688-696.

[10] Sonar A, Kuxhaus L, Carroll J (2010) Simulation of subject specific bone remodeling and virtual reality visualization, Virtual Rality. InTech. pp. 273Ü290.

[11] Sonar A (2011) Simulation of subject specific bone remodeling. Thesis, (PhD) Clarkson University.

[12] Flannery B.P, Deckman H.W, Roberge W.G, D'amico K.L (1987) Three-dimensional X-ray microtomography. Science. 237:1439.

[13] Otsu, N. and others (1979) A threshold selection method from gray-level histograms. IEEE Transactions on systems, Man, and Cybernetics. 9:62-66.

[14] Parallel Computing Toolbox (2011). Available:

http://www.mathworks.com/products/parallel-computing/?s_cid=HP_FP_ML_parallel. Accessed on: Dec 2011.

[15] Palágyi K, Balogh E, Kuba A, Halmai C, Erdőhelyi B, Sorantin E, Hausegger K (2001) A sequential 3D thinning algorithm and its medical applications. Information Processing in Medical Imaging. 2082:409-415.

[16] Saha P.K, Chaudhuri B.B, Dutta Majumder D (1997) A new shape preserving parallel thinning algorithm for 3D digital images. Pattern Recognition. 30(12):1939-1955.

[17] Ju T, Baker M.L, Chiu W (2007) Computing a family of skeletons of volumetric models for shape description. Computer-Aided Design. 39(5):352-360.

[18] Garland M, Le Grand S, Nickolls J, Anderson J, Hardwick J, Morton S, Phillips E, Zhang Y, Volkov V (2008) Parallel computing experiences with CUDA. Micro, IEEE. 28(4):13-27.

[19] Jeyarajan T, Daniel G, Julia A. S, Anne T, Vicente G (2011) On the Usage of GPUs for Efficient Motion Estimation in Medical Image Sequences. International Journal of Biomedical Imaging. 2011.

[20] Mosekilde L (1988) Age-related changes in vertebral trabecular bone architecture-assessed by a new method. Bone. 9(4):247-250.

[21] Ding M, Hvid I (2000) Quantification of age-related changes in the structure model type and trabecular thickness of human tibial cancellous bone. Bone. 26(3):291-295.

[22] Kleerekoper M, Villanueva A.R, Stanciu J, Rao D.S, Parfitt A.M (1985) The role of three-dimensional trabecular microstructure in the pathogenesis of vertebral compression fractures. Calcified tissue international. 37(6):594-597.

[23] Parfitt A.M, Mathews C.H, Villanueva A.R, Kleerekoper M, Frame B, Rao D.S (1983) Relationships between surface, volume, and thickness of iliac trabecular bone in aging and in osteoporosis. Implications for the microanatomic and cellular mechanisms of bone loss. Journal of clinical investigation. 72(4):1396.

[24] Parfitt, A.M. and Drezner, M.K. and Glorieux, F.H. and Kanis, J.A. and Malluche, H. and Meunier, P.J. and Ott, S.M. and Recker, R.R. (1987) Bone histomorphometry: Standardization of nomenclature, symbols, and units. Journal of Bone Mineral Research. 2(6):595-610. 
[25] Müller R, Hayes W.C (1997) Biomechanical competence of microstructural bone in the progress of adaptive bone remodeling. Proceedings of SPIE. 3149:69.

[26] Hildebrand T, Ruegsegger P (1997) A new method for the model-independent assessment of thickness in three-dimensional images. Journal of Microscopy. 185(1):67-75.

[27] Hildebrand T, Laib A, Müller R, Dequeker J, Rüegsegger P (1999) Direct three-dimensional morphometric analysis of human cancellous bone: microstructural data from spine, femur, iliac crest, and calcaneus. Journal of Bone and Mineral Research. 14:1167-1174.

[28] iso2mesh: a 3D surface and volumetric mesh generator for MATLAB/Octave (2011) Available: http://iso2mesh.sourceforge.net/cgi-bin/index.cgi. Accessed on: Dec 2011.

[29] Maurer Jr C.R, Qi R, Raghavan V (2003) A linear time algorithm for computing exact euclidean distance transforms of binary images in arbitrary dimensions. IEEE Transactions on Pattern Analysis and Machine Intelligence. 25(2):265-270.

[30] Gilad I, Nissan M (1985) Sagittal evaluation of elemental geometrical dimensions of human vertebrae. Journal of anatomy. 143:115.

[31] Schultz A.B, Andersson G.B.J, Haderspeck K, Ortengren R, Nordin M, Bjork R (1982) Analysis and measurement of lumbar trunk loads in tasks involving bends and twists. Journal of Biomechanics. 15(9):669-675.

[32] Wilke H.J, Neef P, Caimi M, Hoogland T, Claes L.E (1999) New in vivo measurements of pressures in the intervertebral disc in daily life. Spine. 24(8):755.

[33] VAN Sint J.S (2007) Color atlas of skeletal landmark definitions: guidelines for reproducible manual and virtual palpation.

[34] Schultz A, Andersson G, Ortengren R, Haderspeck K, Nachemson A (1982) Loads on the lumbar spine. Validation of a biomechanical analysis by measurements of intradiscal pressures and myoelectric signals. The Journal of bone and joint surgery. American volume. 64(5):713.

[35] Thomsen J.S, Mosekilde L, Boyce R.W, Mosekilde E (1994) Stochastic simulation of vertebral trabecular bone remodeling. Bone. 15(6):655.

[36] Linden J.C, Verhaar J, Pols H, Weinans H (2003) A simulation model at trabecular level to predict effects of antiresorptive treatment after menopause. Calcified tissue international. 73(6):537-544.

[37] Mullender M.G, Huiskes R (1995) Proposal for the regulatory mechanism of Wolff's law. Journal of orthopaedic research. 13(4):503-512.

[38] Huiskes R, Weinans H, Grootenboer H.J, Dalstra M, Fudala B, Slooff T.J, (1987) Adaptive bone-remodeling theory applied to prosthetic-design analysis. J Biomech. 20(11-12):1135-1150.

[39] Roux W (1881) Der zuchtende Kampf der Teile, oder die "'Teilauslese"' im Organismus (Theorie der "'funktionellen Anpassung"'. Leipzig: Wilhelm Engelmann.

[40] Turner C.H, Owan I, Takano Y (1995) Mechanotransduction in bone: Role of strain rate. American Journal of Physiology- Endocrinology And Metabolism. 269(3):E438.

[41] Lanyon L.E, and Rubin C.T (1984) Static vs dynamic loads as an influence on bone remodelling. Journal of biomechanics. 17(12):897-905.

[42] Bennett, J.O. and Briggs, W.L. and Triola, M.F. () Statistical reasoning for everyday life. 
[43] LifeModeler, Inc.

Available: http://www.lifemodeler.com. Accessed on: Dec 2011

[44] Mass S.A, Rawlins D, Weiss J.A, Ateshian G (2011) FEBio 1.4 Uesr's Manual.

[45] Fang Q, Boas D.A (2009) Tetrahedral mesh generation from volumetric binary and grayscale images. Biomedical Imaging: From Nano to Macro, 2009. ISBI'09. IEEE International Symposium on. 1142-1145.

[46] Parfitt A.M, Mathews C.H, Villanueva A.R, Kleerekoper M, Frame B, Rao D.S (1983) Relationships between surface, volume, and thickness of iliac trabecular bone in aging and in osteoporosis. Implications for the microanatomic and cellular mechanisms of bone loss. Journal of Clinical Investigation. 72(4):1396. 
Section 2

Biomaterials and Prosthetic Devices 



\title{
Prosthesis for Flow Control in the Esophagus as a New Technique for the Treatment of Obesity
}

\author{
Suélia de S. Rodrigues Fleury Rosa, \\ Adson Ferreira da Rocha and José Conceição Carvalho
}

Additional information is available at the end of the chapter

http://dx.doi.org/10.5772/50992

\section{Introduction}

Obesity is becoming increasingly common, with at least 400 million obese adults worldwide and the World Health Organization (WHO) projecting that this statistic will reach 700 million in another five years [1]. The body, which is altered by excessive weight, has its biological process modified. This results in several diseases. Different diseases such as heart disease and strokes, high blood pressure, type 2 diabetes, cancer, asthma, sleep apnea, osteoarthritis are all related to obesity [2].

According to a recent survey by the Brazilian Society of Endocrinology and Metabolism (SBEM) obesity in Brazil has increased, on average, $240 \%$ over the past 20 years. Today, methods of treatment for obesity are: a) diets; b) non-pharmacological treatments; c) pharmacological treatments; d) surgeries. All the aforementioned methods have the same principle -an energy deficit is necessary to cause weight loss.

Among the techniques available for treating obesity, surgical techniques are more efficient. It is known that surgical methods offer effective results, maintaining $50 \%$ weight loss for 10 years. Two types of surgical treatments are practiced: a reduction of the stomach volume to diminish food ingestion and the use of a stomach-intestine bypass to produce practically no absorption of nutrients [3].

In type 2 diabetes mellitus (T2DM), treatment with pharmacologic therapies in order to control blood sugar only slows progression of the disease. Bariatric surgery has been implicated in the complete resolution of type 2 diabetes mellitus in several clinical studies [4].

Multidisciplinary studies for treating obesity and type 2 diabetes mellitus have been developed. Doctors and Engineers are working together to deal with the problem and find 
solutions. This is being carried out especially with biomaterials and in biomedical engineering where new devices are being developed.

GI Dynamics ${ }^{\circledR}$ is developing a novel endoscopic treatment for obesity and T2DM. The EndoBarrier $^{\mathrm{TM}}$ gastrointestinal liner is a removable implant that resides in the proximal intestine [3]. Toga, from Satiety, Inc., still in its trial stages, inserts flexible stapling devices through the mouth and into the stomach, and then uses suction to gather stomach tissue and staple it together, creating a small stomach pouch near the esophageal junction and thereby increasing satiety [1]. Furthermore, the methods used neuroregulation activation and gastric implants for activation of the vagus nerve as a way to control hunger [1,4].Thus, it is worth noting that there are difficulties in proposing an efficient obesity treatment. In other words, the control and cure of the multiple causes of obesity associated with eating habits is a difficult problem.

In this study, we designed an esophageal flow control module (CFE) made from a biosynthetic material based on natural latex (extracted from the rubber tree Hevea brasiliens). The intended purpose of the CFE is to help to control the speed and the volume of food ingested, and, as a result, to help in treatments to reduce weight.

The CFE proposed is classified as a restrictive surgical procedure. It is inserted into the esophagus ( $3 \mathrm{~cm}$ after the passage of the upper sphincter) and filled with gas. Experimental animal research has shown satisfactory results. The device has the mechanical characteristics necessary for use inside the esophagus. Furthermore, the animals did not have problems with gastric and behavioral changes, nutrition, blood disorders or irritation because of the CFE. Therefore, this unprecedented technique, which can solve the obesity problem, allows a decreased food intake by decreasing the flow through the esophagus.

Therefore, even though the device needs to be tested on humans, the efficiency of this procedure has been ratified, especially given the success of the evaluation in dogs. Weight loss was confirmed (and validated) between two groups of animals tested. One group had the module and the other did not (however both maintained the same dietary and environmental routine). A biomaterial, latex, is used in its development, given its prominence (in Brazil). There are non-gummy, rubber, hydrocarbon particles in the composition of natural latex. The particles are suspended in an aqueous solution phase in which there is on average $36 \%$ hydrocarbons, $1,4 \%$ proteins, $1,6 \%$ carbohydrates, $1 \%$ neutral lipids, $0,6 \%$ glycolipids plus phospholipids, $0,5 \%$ inorganic components, $58,5 \%$ water and $0,4 \%$ of other substances. For this reason, Hevea brasiliensis latex is a complex cytoplasmic system in which the rubber particles and the nonrubbery particles (also called non-gummy) are dispersed in an aqueous cytosolic phase. It is a national low-cost product, which is also easy to handle. Latex, plus other substances, was initially used as a material that induces healing in damaged esophageal walls. Results showed that a natural latex biomembrane with polylysine has biochemical characteristics that make it capable of interfering in the process of tissue repair thus promoting the rapid and regular formation of new tissue. It is also easy to handle, dispensing with complex techniques for manufacturing and use $[5,6,7]$. Based on these data, other studies utilizing latex have been done, such as using latex for myringoplasty in humans [8] and applying latex biomembranes 
for treating ischemic leg ulcers. Here, the goal is for the biomembrane to act as an inducing agent for healing tissues [9]. In [10], this membrane was employed in recurrent umbilical hernias and its effectiveness was established in twelve dairy cows.

In orthopaedic research aimed at more effective bone regeneration, latex was efficient in repairing tibial fractures in a rabbit, demonstrating great potential for this kind of application. In skull fractures in rats, the results were similar and the researchers believe that new investigations indicate its use in osteoporosis, odontology and facial bone reconstruction [11]. In [12], they developed a new microperforated vascular prosthesis model, made of tissue covered with a compound derived from rubber tree natural latex (Heveabrasiliensis) and used an expanded a polytetrafluoroethylene prosthesis as control in the contralateral pelvic limb in the same animal. The study was done applying two prostheses in 15 dogs. The microperforated latex and fabric graft showed satisfactory structural qualities (adaptability, elasticity, impermeability and possibility of suture) as a vascular substitute. It stimulated endothelial growth, beyond contact regions with the artery in anastomoses and was bicompatible with the dog's arterial system, showing adequate tissue integration. In [13], they evaluated using a natural latex mould in the postoperative surgical preparation of the neovagina with the objective of inducing healing and to keep the cavity functional in nine patients with Mayer-Rokitansky-Küster-Hauser (MRKH) syndrome. The results confirmed the properties of tissue replacement and regeneration of natural latex derived from Hevea brasiliensis and acceleration of the healing process with no rejection. In [14], they developed a topical latex membrane for controlled drug release. In other medical areas, latex has been studied and successfully utilized in the healing process of ulcers, and in burned areas on the body's surface and in conjunctiva reconstruction [8]. Due to these promising results, using latex was the basis for making the new device proposed here for treating and controlling diabetes and obesity.

A study was conducted concerning esophageal diseases in order to propose a device and its placement technique. It was found that patients with obstructive diseases of the esophagus (caustic stenosis, chagasic megaesophagus and esophageal cancer) have high weight loss as a result of reducing the flow of food, which can be seen in $[15,16,17,18]$. Because these esophageal diseases cause a reduction of the esophageal lumen or obstruct the emptying of the organ, they lead to the patient losing weight. In this context, the CFE® module was developed for helping in the treatment of obesity and it also can be used for diabetes treatment due to the significant weight loss achieved with its use. This chapter describes the engineering and design used in the development of the device and the tests already carried on it aiming at its efficacy and security evaluation in order to offer to the global community a new option for obesity and diabetes treatments.

\section{Materials and methods}

Whereas a decrease in food consumption may be secondary to esophageal obstruction and considering that there is the possibility for inserting devices that may alter its diameter (which takes place in the treatment of bleeding due to esophageal varices), it was plausible 
to suppose that developing the $\mathrm{CFE}{ }^{\circledR}$ module may lead to reducing food intake.The objective is to evaluate the possibility of CFE® insertion into the esophagus which causes, during its use, resistance to the passage of food bolus. This makes food ingestion slower due to a reduction in the lumen of the esophagus, resulting in the need for prolonged chewing.

\subsection{Latex preparation}

The latex used here had already been through the centrifuging process, with sulfur and resin suspensions, having the objective of providing elasticity and the necessary strength for the final compound. From this preparation process, the latex is configured as a compound which upon contact with the skin, vulcanizes, making it adhesive. To be removed, however, just pure water is used. On glass surfaces, its removal is facilitated due to the low friction that glass provides.

When handling the latex, glass rods were used for mixing, as well as a glass container for storage, plastic wrap to protect it from contact with the air and cotton flannel for cleaning. An important point for any application that uses raw latex is the uniformity of its properties, since this is an essential characteristic. To obtain this characteristic, the latex source should be the same, i.e. the latex should come from trees of the same clone.

\subsection{Manufacturing the CFE® module}

The CFE® module was manufactured aiming to develop a mechanical device, with biocompatible and flexible material, in the shape of an $8 \mathrm{~cm}$ long cylindrical balloon, inflatable with gas by a valve system with an approximately $1 \mathrm{~cm}$ diameter conduit. The dimension established for length refers, approximately, to the size of the upper third of the esophagus. Incorporated into the module were radiography visualizations and fixing devices, containing within them a barium contrast indicator for controlling the position for $\mathrm{x}$-rays. At one of the ends, there is a thread for fixing the module to a dental crown, which should be attached to the superior molar with the aim of preventing the module from migrating to the stomach in the event of accidental disinflation.

Manufacturing the CFE® module begins with preparing the environment, the raw material and the moulds and then follows a series of steps, such as: immersion of the glass moulds into a container containing liquid latex, drying the moulds in an oven, analyzing the thickness, removing the solidified latex from the moulds, assembly of module and final inspection.

\subsection{Cadaver test}

An experimental feasibility study was conducted, testing the placement and removal of the CFE® device in the esophagus of a cadaver at the Institute of Forensic Medicine (IFM). After being applied, the module was inflated with the aid of a manometer adapted to a pressure of $120 \mathrm{~mm} \mathrm{Hg}$. Its placement was made with delicate maneuvers aimed at preventing alterations to the module to be examined. 


\subsection{In-vitro study}

With the objective of estimating the minimal time to carry out an intervention, in the event that it descends to the stomach and in order that it not cause intestinal obstruction, an experiment was conducted in the Laboratory of Engineering and Innovation - LEI at the Gama Campus of the University of Brasília. This experiment compared the volume of BIB $(400 \mathrm{ml}$ to $700 \mathrm{ml})$ and (CFE $146 \mathrm{ml}$ ) with the goal of comparing its volumes and its emptying capacity in the case it is punctured. The calculation employed for obtaining the CFE volume used a technique based on solids of revolution and the time was obtained from simple arithmetic means. There were four CFE modules used as samples and these were immersed into an environment similar to the stomach. The method utilized was to inflate the CFEs with the same pressure to be applied in humans (60 $\mathrm{mmHg}-$ minimum), withdrawal of the scalp ${ }^{1}$ number 27 , immersion of the CFE in the environment with the hole made in the sample by the scalp on the upper part of the module thereby forcing partial emptying, since if it were not punctured, it would not empty as in the test done in [7].

\subsection{Preparation for clinical trial}

The choice for using a dog - as an animal for testing and for weight loss and/or as a validator of the CFE module - was made in light of findings raised with medical veterinarians. It was verified that the animal would be an excellent subject for analysis in this study keeping in mind the anatomical-physiological similarity between human and canine esophagi. Allied with this, above all, factors that denote affability with such animals and also, the facility of controlling their eating routine, as well as the food ingestion speed of the animal, associated with little chewing and the absence of psychological pressure (as opposed to what a human develops when the goal is losing weight). To conduct the experimental procedure, eight adult dogs, of no specific breed, males and females, with body weights varying between 9.2 and 17.8 $\mathrm{kg}$ were selected. Before the experiment, the animals were kept at the Veterinarian Hospital kennel at the Federal University of Goiás to be submitted to quarantine procedures that include: vaccination (Duramune Max, single dosage), application of ecto and endoparasiticides (administered through $100 \mathrm{mg}$ of mebendazole, twice a day for three days), in addition to laboratory exams like blood count, urinalysis, hormonal and blood biochemical evaluations. Furthermore, improvements in the nutritional conditions of the animals was sought, through the implementation of a daily diet of dry dog food, supplemented with semi-moist dog food rich in nutrients (Dudog()). The animals were vaccinated against rabies and DHLPPC (distemper, hepatitis and adenovirus, leptospirosis, parainfluenza, parvovirus, and coronavirus) and dewormed with a broad spectrum dewormer. Ectoparasites were eliminated with a two-dose application of pour-on parasiticide. Blood was taken from all the animals and when hemoparasitoses was present, doxycycline was applied for 21 days, and diminazene in two applications with an interval of 15 days.

\footnotetext{
${ }^{1}$ The scalps peripheral intravenous comprise: siliconized needle stainless steel bezel with thin walls and biangular and three faceted; wings malleable and flexible, adjustable anatomically as handling (known as "butterfly"); transparent vinyl tube.
} 


\subsection{Experimental model for analyzing weight loss}

It has been suggested that application is the placement and utilization of the CFE® module in the upper third of the esophagus ( $3 \mathrm{~cm}$ after the passage of the upper sphincter) of the dogs through video-endoscopy over a period of seven days as a weight loss method. The research protocol was previously submitted and approved by the Ethics Committee for Human and Animal Medical Research at the Federal University of Goiás (UFG) (process number 060/2008, registered on SAPP-WEB number 33256). To conduct the experimental procedure, eight adult dogs, of no specific breed, males and females, with body weights varying between 9.2 and $17.8 \mathrm{~kg}$ were selected. Before the experiment, the animals were kept at the Veterinarian Hospital kennel at the Federal University of Goiás to be submitted to quarantine procedures in addition to laboratory exams like blood count, urinalysis, hormonal and blood biochemical evaluations.

\subsubsection{Plan for placement}

The technique proposed for this new method is based on the mechanical decrease of the esophageal lumen, which directly influences a decrease in the speed and volume of food consumption, without altering the digestive tract or bringing about physiological and nutritional alterations: the outcome of the technique is weight loss. To achieve this, the CFE module was tested experimentally on animals, with the objective of verifying its capacity for accomplishing weight loss in the animal.

\subsubsection{Definition of the groups}

For this experiment, six dogs of no special breed, males and females, with body weight varying between 9.780 to $18.100 \mathrm{~kg}$ were selected. The process for recruiting and handling the animals followed the same norms described in the previous experiment. At this point, note that the animals were divided into two groups - the weight control-group (GCp) and the test-group (GT). In the weight control-group, the animals received the same dietary treatment and the same routine as the test-group; however, the module was not placed in the subjects in the first group. In the test-group, the module was placed following the same steps as the previous experiment, as shown in Table 1.

\begin{tabular}{|c|c|c|c|c|}
\hline \multirow{2}{*}{$\begin{array}{c}\text { Groups } \\
(\mathrm{n}=2)\end{array}$} & \multirow{2}{*}{$\begin{array}{c}\text { Weight }(\mathrm{kg}) \\
\bar{x}\end{array}$} & $\mathrm{~F}$ & $\mathrm{M}$ & \multirow{2}{|c|}{$\begin{array}{c}\text { Sex } \\
(\mathrm{mmHg})\end{array}$} \\
\cline { 3 - 4 } & 12.84 & 1 & 1 & 60 \\
\hline GC & 11.56 & 1 & 1 & 100 \\
\hline GT1 & 12.10 & 0 & 2 & 120 \\
\hline GT2 & 14.63 & 0 & 2 & 140 \\
\hline GT3 & & & \\
\hline
\end{tabular}

Table 1. Group Distribution. 


\subsubsection{Video-endoscopy placement}

The video-endoscopy procedure was conducted according to the same steps as described in the previous experiment.

\subsubsection{Post-placement care}

The post-placement period and the routine of the animals were carried out similar to the previous experiment.

\subsubsection{Evaluation parameters}

The parameters used in the evaluation were volume, speed, ingestion time, nutritional, hormonal and complete blood count, in addition to weight loss.

\subsubsection{Volume, speed and ingestion time}

The same diet (nutritional, quantity, and schedule characteristics) was chosen for evaluating the behavior of the dogs while eating, as well as for measuring the food left over (which was weighed and recorded). Another point evaluated was the time spent eating in both groups which is approximately proportional to the volume ingested - through clinical observation, a timer and a precision scale.

\subsubsection{Nutritional, hormonal and complete blood count}

The nutritional, hormonal and complete blood count was investigated by blood tests done before and after the placement. The tests done were classified as nutritional and blood count tests.

\subsubsection{Weight loss}

Weighing of the animals was done before the placement and after every two days until the module was withdrawn. Weights in the beginning and in the end of the experiment were compared, and comparisons were also made between the control and test groups.

\subsection{Evaluation instruments}

\subsubsection{Clinical observation}

Clinical observation was one of the evaluation instruments used, in which a daily chart was filled out with data referring to the following data bases: 1) reaction of dog before and after meals; 2) reaction of dog during ingestion of water; 3) stool consistency; 4) amount voided; 5) behavior (irritated, agitated, normal); 6) gagging, cough, vomit, choking; 7) signs of dysphagia; 8) behavior in the presence of other dogs; 9) blood tests; 10) weight at the end of the day. 


\subsubsection{X-rays}

Radiographic follow-up of the animals was done of the cervical region on the lateral-right (in decubito) side, immediately after placement of the module, and every two days until its removal. The test was done in the radiology laboratory at the Veterinary School at UFG, according to the following protocol: $55 \mathrm{kV}, 300 \mathrm{~mA}, 0.04$ second of exposure in a right-lateral ducubitus position. A normal esophagus is not visible radiographically, because it is a close to collapsing organ and the module behaves like a foreign body (filled with gas), making it possible to visualize it radiographically. Thus, due to the radiopacity of the gas, it is possible to determine location by means of image.

\subsubsection{Video-endoscopy}

A video-endoscopy was performed on the 15th day, after withdrawal of the module. The procedure was analogous to that used during placement, utilizing the same endoscopic material.

\subsection{Exclusions}

There were no exclusions of the dogs, including those that expelled the modules. All of them, at the end of the experiment were evaluated.

\subsection{Statistical study}

The statistical hypothesis t-student test was used here to make a comparison between the groups (small samples). All discussions of the present study were carried out with a $99 \%$ confidence interval.

\section{Results}

In Figure 1, the CFE module developed with its morphological macroscopic appearance can be observed.

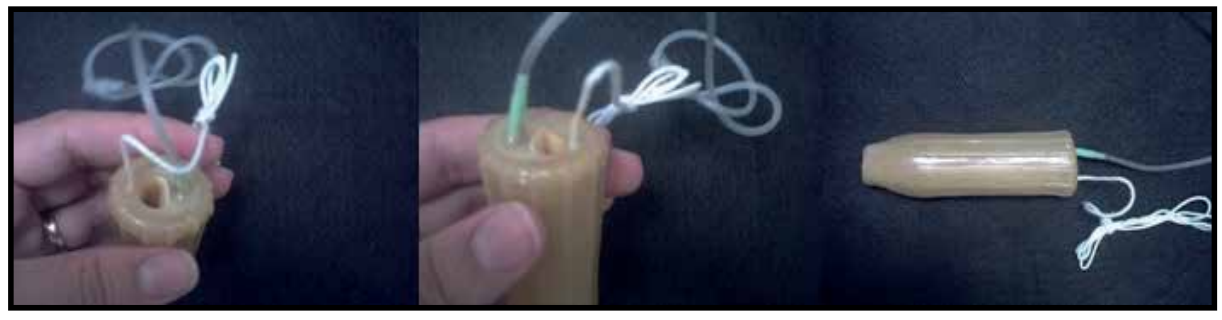

Figure 1. Picture of the esophageal flow control module - CFE; from left to right: upper and side views

The materials used in the manufacturing and packaging of the module that did withstand high temperatures, were sterilized with ethylene oxide. The materials (moulds) that withstand high temperatures were sterilized by steam autoclave. 


\subsection{Cadaver test}

In this study, a guide needed for pulling the device through to the inside of the esophagus was developed. During the removal procedure, the external wall of the module was perforated for deflation and it was removed with endoscopic forceps.

\subsection{In-vitro study}

It was found that if the module is inflated with the volume and pressure as described above,upon reaching the stomach it would take, via simple arithmetic mean, 49 hours to partially empty. With this partial emptying, it could pass through the pylorus and into the intestine, a situation similar to what occurred in the experiment presented in [7]. On that, note that among the restrictive techniques already in practice, endoscopic intervention is done to remove the object from the stomach before it descends into the intestine. In the case of the CFE - in the event that it becomes lodged in the stomach between one radiography and another, meaning during a 24-hour period, we would have ample time for removing it endoscopically without causing intestinal obstruction. It is worth mentioning in a detailed analysis carried out, it was noted that studies apply synthetic prosthesis in patients as temporary substitutes for the esophagus, and they are made of diverse materials such as: silicone, Marlex $®$, Teflon ${ }^{\circledR}$, collagen, and a mixture of collagen and silicone. The amount of time the prosthesis remained inside the body was on average from four to five weeks, from its placement until its exit which corresponds to the end of this treatment, when expelled in the feces after this period without causing intestinal obstruction. In [6], which is used as a basis for this work, among the groups evaluated, cases occurred where the latex prosthesis was expelled by the animal, without causing intestinal obstruction. Thus, the scope of radiographic evaluations adopted (every 24 hours) was done to prevent the possibility of it passing from the stomach to the intestine.

\subsection{Weight loss analysis experiment}

This analysis sought to verify if the diet offered was bringing about weight loss in the animals. Considering this, the food provided was initiated ten days before placing the module: the animals accepted the food well given that the ingredients used were appealing. With this procedure, weight loss did not take place in any dog. On the contrary, some animals experienced weight gain.

\subsubsection{Volume, speed and ingestion time}

The food provided was semi-moist, made for each dog individually with measurements calculated according to the animal's weight, as indicated by the manufacturer. When preparing the food, solid dog food was used along with meat pâté for dogs and a dietary supplement called Dudog ${ }^{\circledR}$. With the assistance of a veterinary doctor plus the manufacturer's instructions, the dietary composition was established for each dog which is presented in Table 2. 


\begin{tabular}{|c|c|c|c|c|}
\hline & Dog weight $(\mathrm{kg})$ & Solid dog food (g) & Meat (spoonful) & Dudog $^{\circledR}$ (slices) \\
\hline \multirow{4}{*}{ GCp } & 13,100 & 331 & 2 & 4 \\
\cline { 2 - 5 } & 13,400 & 339 & 2 & 4 \\
\hline \multirow{3}{*}{ GT1 } & 15,780 & 410 & 3 & 6 \\
\cline { 2 - 5 } & 9,780 & 247 & 1 & 2 \\
\cline { 2 - 5 } & 10,120 & 256 & 1 & 6 \\
\hline
\end{tabular}

Table 2. Amount of food offered to each dog, mixed with 1 liter of water.

The containers used were identified with name of each dog, being "deducted" from their weight at each meal offered. All meals provided were weighed after having been mixed with water in a blender. During feeding, the dog was monitored. When done eating, the container was removed and weighed again, so that the exact amount of the volume ingested, per meal per dog, was obtained.

However, it was observed that the volume of food ingestion in group GT1 was less than the volume in group GCp. The accumulation of leftovers was observed in the feeding of all subjects in group GT1, having at times volumes up to $1 \mathrm{~kg}$. This fact indicated that the method restricted the volume of food consumed by the dogs, but without causing apparent discomfort. Another point that should be put forth is that in the observation, there was great care taken to verify if there was evidence of satiety after eating in the dogs with the module. To this end, the container was left longer to confirm the dog's level of satiety. Faced with this situation, the animals did not go back and enjoy the rest of the contents, even when it was offered directly to them. This fact is a strong indicator that the dogs felt satiated. The speed and the time for ingesting the food were two other items observed, given that in the first two days, it was clearly noted that in group GT1, both (time and ingestion) were greater than those in group GCp.

During the eating process, the dogs in group GT1 made pauses in their food ingestion. This decreased and later it normalized over the subsequent days. It is believed that this situation occurred due to the esophagus module accommodating itself. After, it was observed that there was an inversion, since the food ingestion time of group GT1 was reduced and the amount of food consumed became gradually less. It must also be put into evidence that each dog was offered two meals a day - one at 09:00 and another at 18:00. 


\subsubsection{Nutritional, hormonal and complete blood count}

The complete blood count was collected through the Vacutainer ${ }^{\circledR}$ system, taken from the arteriovenous group in a tube with four drops of anti-coagulant (10\% EDTA solution), then performing the homogenization of the samples with slow and regular movements. From the samples, hemoglobin, hematocrit (the percentage occupied by red blood cells or erythrocytes in total blood volume, because a decrease in it may indicate anaemia), and total plasma protein (TPP) were evaluated. The data collected was analyzed statistically following the Tukey test $(\mathrm{p}<0,05)$, in the ANOVA module of STATISTICA software, with repeated measurements and a non-parametric test, for qualitative variables. The data obtained from the blood count are described in Table 3 below, thus allowing evaluation of the effects of the esophageal module, as well as evaluating how it affects the blood tissue.

\begin{tabular}{|l|c|c|c|c|c|c|}
\hline \multirow{2}{*}{} & \multicolumn{2}{|c|}{ Hemoglobin $(\mathrm{g} / \%)$} & \multicolumn{2}{|c|}{ Hematocrit $(\%)$} & \multicolumn{2}{|c|}{$\operatorname{TPP}^{*}(\mathrm{~g} / \%)$} \\
\cline { 2 - 7 } & Average & $\mathrm{DP}( \pm)$ & Average & $\mathrm{DP}( \pm)$ & Average & $\mathrm{DP}( \pm)$ \\
\hline GCp & 8,16 & 0,83 & 30,19 & 3,02 & 7,21 & 0,37 \\
\hline GT1 & 8,08 & 0,95 & 30,25 & 3,36 & 7,33 & 0,54 \\
\hline
\end{tabular}

Table 3. Evaluation of the hemoglobin, hematocrit and total plasma protein. Values do not differ ( $p>$ $0,05) .{ }^{*} \mathrm{TPP}=$ Total plasma protein.

It was also verified that the average values obtained from the evaluation do not differ. Therefore, it may be suggested that the method does not promote alterations in the blood tissue, since when the hemoglobin concentration was evaluated, there was no difference between the groups $(p>0,05)$, considering that the same result was obtained in the hematocrit and TPP evaluations.

However, it is known that blood count evaluations may suffer variations according to the nutritional condition of the animals, climatic factors, infections and release of blood components. Nevertheless, in this study the parameters were maintained within the physiological values.

\subsubsection{Weight loss}

Specifically in relation to weight loss, in all of the experiments conducted in this study which involved dogs, the animals' weights were monitored. Weight loss took place to a slight extent $(\approx 1,3 \%)$, as well as to a great extent $(\approx 6,3 \%)$, for the weight of the dogs in the analysis presented in item 7.3 of this study, which used the module. Lower indices were observed in recently arrived dogs from the Center for Zoonoses, and the higher indices were exhibited in the dogs that had already been at the Veterinary Hospital for a longer period of 
time. It is believed that this occurred because the dogs that had recently arrived were under their ideal weight.

In this analysis in which the two groups GCp (without module) and GT1 (with module) were compared, it was observed that the group in which the module was applied (for seven days), weight loss took place. This fact is attributed to a decrease in the volume of food ingested, brought about by the module placed in the esophagus. In Table 4, data related to the dogs submitted to the test are presented. For ten days, semi-moist food, before the module's placement, was provided to the two groups and after the module's placement, the dogs remained for seven more days on the same dietary routine.

The data in Table 4 are described next. In the Start column, the original weight of the dog is presented. The GCp lines correspond to the weights of the dogs in the control group that did not use the module and the GT1 lines are of the dogs in which the module was placed. The dogs were fully fed over a period of 10 days without the module. The weight of the dogs at the end of 10 days is shown in the "10 days (final)" column. Then, the modules were installed only in group GT1, and remained in the dogs for a period of 7 days, and the final weights, after 7 days, are presented in the "7 days (final)" column. The column furthest to the right indicates the weight loss percentage in relation to the weight of the dogs in both groups on the date of the module's placement.

\begin{tabular}{|l|c|c|c|c|}
\hline & $\begin{array}{c}\text { Dog weight } \\
(\mathrm{kg}) \\
\text { Start }\end{array}$ & $\begin{array}{c}\text { Dog weight } \\
(\mathrm{kg}) \\
10 \text { days (final) }\end{array}$ & $\begin{array}{c}\text { Dog weight (kg) } \\
7 \text { days (final) }\end{array}$ & Weight change (\%) \\
\hline \multirow{4}{*}{ GCp } & 13,100 & 13,480 & 14,000 & $(+) 3,71$ \\
\cline { 2 - 5 } & 13,400 & 13,400 & 13,590 & $(+) 1,39$ \\
\cline { 2 - 5 } & 15,780 & 16,200 & 16,630 & $(+) 2,58$ \\
\hline \multirow{3}{*}{ GT1 } & 9,780 & 9,900 & 9,540 & $(-) 3,63$ \\
\cline { 2 - 5 } & 10,120 & 12,180 & 11,140 & $(-) 8,54$ \\
\cline { 2 - 5 } & 18,100 & 19,800 & 17,950 & $(-) 9,35$ \\
\hline
\end{tabular}

Table 4. Animal weight variation².

\footnotetext{
${ }^{2}$ The values shown in the table are an average of the weights.
} 


\subsection{Clinical trial}

After placing the module, the animals were evaluated over a period of 15 days. No dog died and there was no exclusion. At the end of the procedures associated with the experiment, the dogs received hygiene care, food and the necessary clinical-laboratorial evaluations, in order that they be donated to owners registered in the Veterinary Hospital data bank (which in fact did take place).

\subsubsection{Lack of appetite}

No grave alteration in appetite was observed, such as anorexia. However, all of the groups ate less than what was observed before placing the module, thus characterizing a state of hyporexia. The semi-moist food provided was of the same consistency and quantity, and accepted by all of the animals, who also did not show any clinical manifestation suggesting dysphagia. None of them had clinical alterations during the period following.

\subsubsection{Absence of voiding and/or defecation}

In all groups, voidance was normal, present and without difficulty. But defecation took place with less frequency, was not daily, and had a soft consistency.

\subsubsection{Range of pressure values}

Concerning the range of pressure values, based on the results observed here and in the pilot study in dogs, a pressure range from $60 \mathrm{mmHg}$ up to $120 \mathrm{mmHg}$ is suggested for modules with construction characteristics similar to those applied in this experiment. As such, an exact definition of applied pressure should be made based on the diameter of the dog's esophagus, since the greater the diameter, the greater the pressure must be. This fact must be taken into consideration and evaluated prior to the endoscopy because there is a reasonable dimensional variation, given the vast number of breeds that are found in this species, each having distinct anatomical features.

Clinical observation shows that the expected outcome was achieved with the placement and fixedness of the module for GC and GT1, while preserving the dog's welfare and only minimally altering its physical condition. In groups GT2 and GT3, which used greater pressure, the dogs' state remained normal after a long period of adaptation, which altered the condition of the animal for a longer period.

\subsection{Damage to the esophageal wall}

The esophagus was analyzed in all of the animals, including the dog belonging to group GT3 that expelled the module 20 hours after placement, and one dog in group GT2 in which the module descended to the region caudal region of the stomach 9 days after placement. It must be emphasized that in all groups, there was no visible alteration to the esophageal wall. This finding was made after endoscopic macroscopic evaluation of the dogs' 
esophageal wall, after removal of the module, which was compared with the endoscopy that had been previously done. Nonetheless, after the endoscopic exam, it was observed that the entire esophageal wall was intact, i.e. a normal appearance of the esophageal wall was preserved having a bright and rosy coloring, without food residuals or ulcerations. The folds were normal as was the frequency of the peristaltic waves.

\section{Discussion}

At this point, it needs to be restated that obesity is a disease that affects an individual as a whole. It interferes with mental, physical and social aspects, and is not a direct result of psychological disorders, but it is a target for prejudice and discrimination [19 ]. Hence, when obese people seek out a doctor for treatment, they are in search of not only a healthy body, but also to "discover themselves". However, there are many problems that these individuals face. Standing out from these issues are marital problems, mental disorders, anxiety, depression, excessive eating, low self-esteem, guilt complex and no self-acceptance [20].

There are many factors that cause obesity as already described in this study. But in general, the main cause of obesity remains to be an altered or inadequate eating behavior. From this point of view, it is of fundamental importance that those who are obese are not only seeking solutions in order to control their weight: it is essential to provide conditions for reeducating such people.

When researching currently available treatments for all ranges of obesity, one point in common was observed - decreasing food intake - because depending on how the treatments are carried out each technique in its own way, seeks to achieve this goal. However, the failures, having unfavorable consequences to the patient, amongst other drawbacks, point to the need for finding new techniques.

In light of such a necessity, a new device was designed in this study for treating such cases. The device presented in this study is called an esophageal flow control module (CFE). It must be emphasized that this is a totally original method using a natural raw material extracted from the Heveabrasiliensis. An extremely simple, handmade method is used for manufacturing it, which is based on observations and products that already exist in this surgical field.

In this sense, it is important to consider that in terms of the surgical procedures utilized here, the method (proposed here) qualifies as a restrictive technique. This is particularly because the objective of the procedures is to reduce the volume of food ingested. Acknowledging that the esophagus is the organ for placement, it should be pointed out that this technique has disadvantages and restrictions for placement, which have also been described in this study.

The basis for this proposal then is founded upon presenting a proposal for reducing food intake, however, with some significant differences that are listed here: 1) the organ for 
placement is the esophagus - in its upper third; 2) reduction of the lumen in the esophagus works mechanically, which blocks the quantity of food going from the mouth to the stomach, limiting the ingestion speed of solid foods; 3) placement takes place by means of endoscopy; 4) no alteration to the digestive tract is made, which neither brings about dysfunction nor compromises the absorption of nutrients; the procedure does not cause esophageal lesions, compared to the "gastric band" method which causes lesions in $10 \%$ of cases; 5) similar to the other methods, there is the necessity for chewing correctly, however, upon ingesting food of improper consistency, the food comes back up (before reaching the stomach), a positive point in this treatment, since in the other methods, vomiting crises triggered by incorrect mastication may develop into anorexia and bulimia; 6) similarly to the other methods, multi-professional treatment, dietary planning and exercise are also necessary.

In this study, the results show that placing a module inflated with gas in the esophagus, does not lead to behavior alterations in the animals. In humans, this feature had already been observed in studies that use esophageal balloon applications. In [21], short esophageal balloons are used $(3 \mathrm{~cm}$ length and $1 \mathrm{~cm}$ in diameter) and long balloons $(16 \mathrm{~cm}$ in length and $0,8 \mathrm{~cm}$ in diameter) for measuring the pressure of the esophageal wall. In [22], notations on the pressure inside the esophagus with a long balloon led to reports that the best results are reached when measurements are made at the middle third, showing that in this region the shape of the pressure versus volume curve is not affected by changes in body posture. Another application to be shown is aimed at tubular prostheses used to maintain patent malignant strictures of the esophagus and seal tracheo-esophageal fistulae, when they are fully expanded, reaching $18 \mathrm{~mm}$ to $25 \mathrm{~mm}$ in diameter and $8 \mathrm{~cm}$ to $14 \mathrm{~cm}$ length [23]. In the procedures that are involved in this study, no macroscopic damage was observed in the esophageal wall where the module was placed, or in the entire length of the esophagus. A great advantage of this module in relation to the other $\mathrm{BIB}^{\circledR}$ methods and the gastric band is how simple it is to manufacture, with the raw material "latex" estimated to be low-cost, easy to handle and highly biocompatible.

As for flow control in the esophagus, there are no reports in literature of studies with this objective. This may be because no one correlated a common denominator between esophageal illnesses that reduce the diameter of the esophagus and marked weight loss. To this end, the model presented in this study seeks to promote a reduction in the esophageal lumen to achieve weight loss.

So, mainly based on the results obtained and in the experiments conducted in dogs, a new procedure is revealed here for treating cases of obesity, derived from a natural source, which imbues it with a low-cost. But, it is probable that alterations and improvements will take place in the module aiming at obtaining better results for applying it in humans.

Thus, the results reached suggest the possibility of controlling the volume of food intake by means of a mechanical system placed in the esophagus. In obtaining results associated with weight loss - without causing significant harm or nutritional changes or alterations 
in the blood, and furthermore without altering the digestive tract -, through a minimally invasive procedure, is a fact that is appealing within the current context of society. But, in validation of the conclusions reached in the experiments that support this study, it was revealed that a desire such as this has not yet been achieved from the scientifically proven premises.

\section{Conclusion}

The effects of mastication exert an influence on the mechanisms that trigger satiety. It implies nutritional re-education in individuals with obesity and also weight loss, as observed in previous studies. One can read in studies by many authors that new studies regarding procedures for treating obesity are necessary, particularly with the goal of improving the results and decreasing the costs and morbidity of obese patients. But, the basic, necessary characteristics for comprising a new treatment should be founded. On a minimally invasive procedure, without side effects, that would not interfere or modify gastro-intestinal anatomy.

\section{Author details}

Suélia de S. Rodrigues Fleury Rosa*, Adson Ferreira da Rocha and José Conceição Carvalho Engineering \& Innovation Laboratory,

University de Brasilia, Gama Campus, Setor Central, Gama-DF, Brazil

\section{References}

[1] Rutherford JJ.Biomedical engineering and the obesity epidemic.Engineering in Medicine and Biology Magazine, 2010, Jan-Fev, p.24-30.

[2] Dib N, Elhajj I, Antoun S, Al-Hajj G. Gastrectomy Surgical Assistive Instrument for Accurate Remnant Stomach Volume. Proceedings of the IEEE International Conference on Robotics and Biomimetics Bangkok, Thailand, February 21 - 26, 2009, p. 518-523.

[3] Partida, M.A.; Cardiel, E.; Rojano, M.E.; Urrutia, R.; Hernandez, P.R..Experimental Gastric Stimulator for Refractory Obesity Treatment.Electrical and Electronics Engineering, 2006 3rd International Conference on Veracruz, Sept. 6-8, 2006 p. 01-04.

[4] Fishman E, Melanson D, Lamport R, Levine A, GI Dynamics, Inc., Lexington, MA. A Novel Endoscopic Delivery System for Placement of a Duodenal-Jejunal Implant for the Treatment of Obesity and Type 2 Diabetes. 30th Annual International IEEE EMBS Conference Vancouver, British Columbia, Canada, August 20-24, 2008 p. 2501-2503.

[5] Mrué F. (1996). Substituição do Esôfago Cervical por Prótese Biossintética de látex: estudo experimental em cães, Dissertação de Mestrado, Faculdade de Medicina da Universidade de São Paulo, Ribeirão Preto, 114p.

" Corresponding Author 
[6] Mrué F. (2000). Reparo de lesões parciais do esôfago cervical utilizando biomembrana de látex natural com polilisina, Monografia do exame de qualificação, Faculdade de Medicina da Universidade de São Paulo, Ribeirão Preto, 33p.

[7] Rodrigues S. S. (2008). Desenvolvimento de um sistema para controle de fluxo esofagiano para tratamento da obesidade, Tese de Doutorado, Faculdade de Tecnologia, Departamento de Engenharia Elétrica da Universidade de Brasília, Brasília, DF, 106p.

[8] Oliveira J.A.A, Hyppolito M. A., Coutinho-Netto J., Mrué F. (2003). “Miringoplastia com a utilização de um novo material biossintético". In: Revista Brasileira de Otorrinolarigol., v. 69, 649-55.

[9] Frade M.A., Cursi I.B., Andrade F.F. (2004). "Management of diabetic skin wounds with a natural latex biomembrane". In: Medicina Cutánea Ibero-Latino-Americana; 32,157-62.

[10] Rabelo E.R. et al., (2005). “Emprego do compósito látex, poliamida e polilisina a 01\% na correção cirúrgica de hérnias umbilicais recidivantes em bovinos leiteiros". In: Acta ScientiaeVeterinariae., v.33, n. 2, 169-175.

[11] Zimmermann M. (2007). A membrana de látex como implante para correção de defeitos musculares em cães e coelhos, Dissertação de mestrado, Faculdade de Medicina Veterinária, Universidade Federal de Santa Maria, Santa Maria, 52p.

[12] Brandão M.L., Coutinho Netto J.,Thomazini J. A., Lachat J. J.,Muglia V. F, Piccinato C. E. (2007). "Prótese vascular derivada do látex". In: Jornal Vascular Brasileiro, 6(2), 130-141.

[13] Carvalho B.R. et al., (2008a). "Molde de látex natural (Hevea brasiliensis) para neovaginoplastia". In: Rev. Bras. Ginecol. Obstet., Rio de Janeiro, v. 30, n. 1, 31-35.

[14] Herculano R.D. et al., (2010). "Metronidazole release using natural rubber latex as matrix". In: Mat. Res., São Carlos, v. 13, n. 1, 57-61.

[15] Barbosa H., Barichello A.W., Vianna A.L., Mendelssonh P., Souza J.A.G. (1981). "Megaesôfago chagásico: tratamento pela cardioplastia à Thal". In: Revista do Colégio Brasileiro de Cirurgiões, 7(1), 16-29.

[16] Ellenbogen G., Ishioka S., Pinotti H.W., Raia A. (1982). "Resultado do tratamento cirúrgico do megaesôfago não avançado. Aspectos morfológicos do esôfago operado". In: Revista Paulista de Medicina, v. 99(2), 12-6.

[17] Cecconello I. (1984). "Tratamento do megaesôfago chagásico". In: Revista Ass. Med.Brasil, 30, 1-12.

[18] Carvalho J.C.; Silva Neto W.B.; Bretones Filho W. (1988b). "Transdiaphragmatic esophago-gastrostomy for dolichomegaesophasus". In: ABCD Arquivos Brasileiros de Cirurgia Digestiva, 3(3), 65-68.

[19] Franques, A.R.M (2002). Participação da psiquiatria e do psicólogo na fase perioperatória - participação do psicólogo. In: Garrido Júnior, A. B.; Ferraz, F. L.; Marshesini, J. B.; Szego, T. Cirurgia da obesidade. São Paulo: Atheneu, 75-79.

[20] Marchesini, S.D. (2002). Distúrbios psíquicos e obesidade. In: Garrido Júnior A. B.; Ferraz, F. L.; Marshesini, J. B.; Szego, T. In: Cirurgia da obesidade. São Paulo:Atheneu, 2533.

[21] Maynar M. et al. (1988). “Esophageal Structures: Balloon Dilation”. In: Radiology, 167, 703-706. 
[22] Karason S., Karlsen K.L., Lundin S. et al. (1999). “A simplified method for separate measurements of lung and chest wall mechanics in ventilator-treated patients". In: ActaAnaesthesiolScand, 43, 308-315.

[23] Kwan K.Y., Kaler K.V.I.S., Mintchev M. P. (2002). “High-pressure balloon catheter for real-time pressure monitoring in the esophagus". In: Institute of Electrical and Electronic Engineers Sensors Journal, 1211-1214. 


\title{
Structure - Functions Relations in Small Interfering RNAs
}

\author{
Natalya S. Petrova, Marina A. Zenkova and Elena L. Chernolovskaya
}

Additional information is available at the end of the chapter

http://dx.doi.org/10.5772/53945

\section{Introduction}

RNA interference is an evolutionary conserved mechanism of specific gene silencing induced by double stranded RNA homologous to the target mRNA. Small interfering RNAs (siRNAs) are widely used for the control of gene expression in molecular biology and experimental pharmacology. Currently, siRNAs are successfully used for the validation of potent drug targets for anti-cancer therapy. However, application of siRNAs as therapeutics is limited by their sensitivity to ribonucleases, poor cellular uptake and rapid size-mediated renal clearance. These challenges must be overcome to develop a successful siRNA-based drug. Many of these limitations could be resolved with the use of chemical modifications improving the siRNA properties.

This review examines recent data regarding principals of the design of siRNA for the silencing of therapeutically relevant genes. A particular focus will be made on chemical modifications and their impact on siRNA potency, nuclease resistance and duration of the silencing effect. The types of chemical modifications, their location in siRNA structure influence siRNA properties in different modes: modulation of the interaction with RNAi proteins, the thermal stability and thermoasymmetry of the duplex and the sensitivity to the degradation by ribonucleases.

Special attention will be paid to the design of siRNA for the silencing of thermodynamically unfavorable targets: mutant and chimerical genes. In this case the utilization of computer algorithms for the selection of active siRNA cannot be applied. Modification of siRNA structure aimed at the correction of the thermoasymmetry by incorporation of nucleotide substitutions, blocking incorporation of the sense strand in the RISC complex by truncation of one overhang or inactivation of the sense strand can be successfully used. Mismatches in the central part of the duplex can facilitate the cleavage and dissociation of the "passenger" (sense) strand, whereas selective chemical modification protects the non-perfect duplex from accelerated degradation. 
Peptides, steroids and other hydrophobic lipid groups can be attached to siRNA, extending the siRNA circulation time and enhancing direct cellular uptake. The potential of bioconjugation of siRNA with different biogenic molecules in altering the bioavailability and distribution of siRNAs following in vivo delivery will be discussed. The combination of these approaches can lead to the development of siRNAs with therapeutic value.

\section{Mechanism of RNAi}

RNAi phenomenon was found during transfection of dsRNAs in C.elegans [1] and is inherent in different organisms (flies, vertebrates, higher plants [2, 3, 4]). RNAi mechanism was initially examined in details in Drosophila melanogaster, but later it was found that the mechanism is highly conservative between the organisms. There are two stages of RNAi (fig. 1): at the first stage (phase of initiation) specific ribonuclease Dicer binds to and cleaves long dsRNAs yielding short (21-23 nt) duplexes with 2-overhanged nucleotides at the 3'ends (or siRNAs); at the second stage (effector phase) siRNAs molecules incorporate into multiprotein complex (RISC - RNA-induced silencing complex). One of siRNA strands ("passanger") undergo cleavage and dissociation from the complex upon RISC activation, the other strand ("guide") remains in the complex. Activated complex RISC ${ }^{*}$ specifically binds to RNA target and cleaves it (fig. 1$)[5,6,7,8,9,10]$. Long dsRNAs (> $30 \mathrm{bp}$ ) activate the innate immune response in the mammalian cells (except for non-differentiated or low differentiated cells) resulting in non-specific RNA degradation by RNase L, activation of

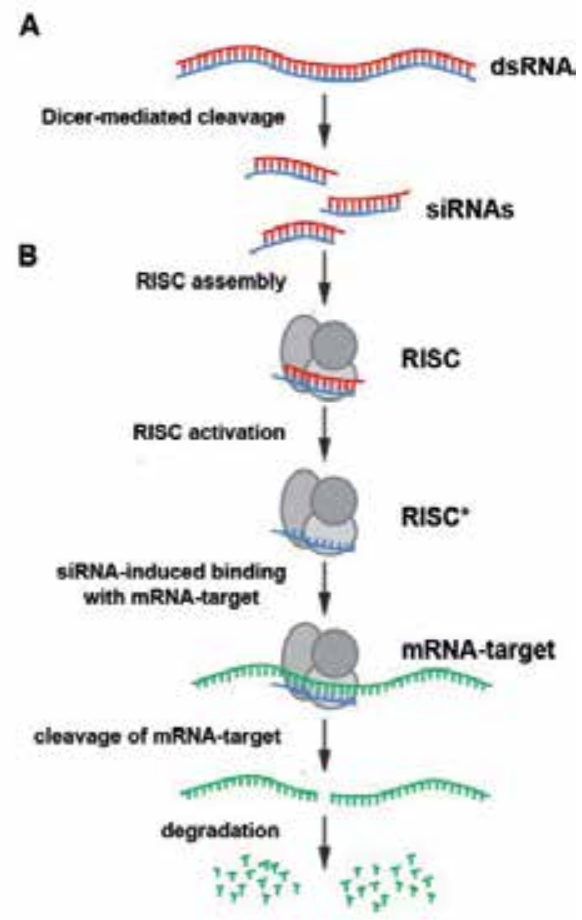

Figure 1. Scheme of RNAi. A. Initiation phase B. Effector phase. 
protein kinase PKR that inhibits translation. The expression of numerous genes, including genes encoding interferons and cytokines, alters [11, 12]. Thus, sequence-specific decrease of target mRNA level is not observed. It was found that RNAi in mammalian cells can be induced by chemically or enzymatically (in vitro) synthesized siRNAs or endogenously expressed siRNAs 19-21 bp in length. These siRNAs mimic the products of long dsRNAs processing by Dicer and can be involved directly into the effector phase of RNAi mechanism passing the phase of initiation (fig. 1) [13, 14, 15, 16, 17].

The selection of the "guide" strand is a key step determining the efficacy of RNAi induced by synthetic siRNAs. At the first step of RISC* assembly the intermediate complex RLC (RISC Loading Complex) consisting of Dicer, siRNA and R2D2 (D.melanogaster) or TRBP [18, 19, 20] (Homo sapiens) forms (fig. 2 A). R2D2 protein along with its human analog contains two dsRNA-binding domains and a Dicer binding domain.

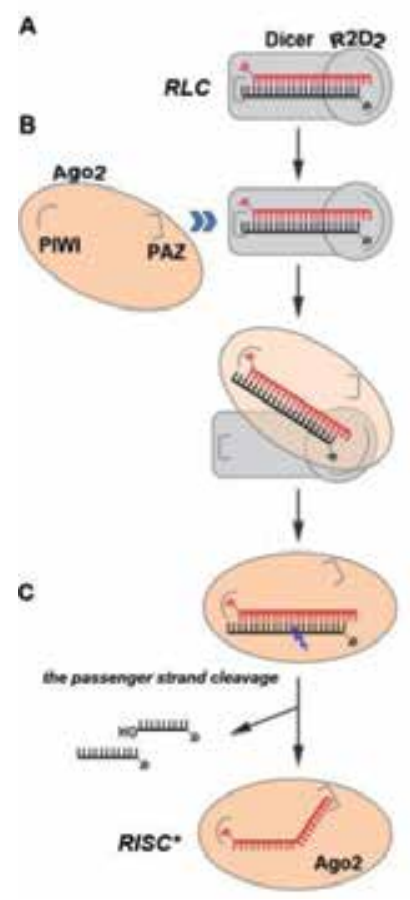

Figure 2. RISC* assembly. A. RLC-complex, consisting of Dicer, dsRNA-binding protein, R2D2 and siRNA. B. Interaction of Ago2 with RLC. C. RISC* formation.

Dicer contains dsRNA-binding domain and PAZ-domain (PIWI/Argonaute/Zwille), that has an affinity to $3^{\prime}$-overhangs of siRNA [21, 22]. It was detected that R2D2 preferably binds with more thermodynamic stable flank of the siRNA duplex, whereas Dicer interacts with less stable siRNA flank [20, 23, 24, 25]. The orientation of siRNA relative to the protein complex Dicer-R2D2 determines the positioning of siRNA in the complex with Ago2 (a catalytic part of RISC $^{*}$ ) (fig. 2 B). Ago2 is a protein of Argonaute family, containing PAZ and PIWI domains. The structure of PIWI domain similar to that of RNase H determines endoribonuclease activity 
of PIWI [26, 27]. Ago2 cleaves the one of the siRNA strand ("passenger" strand), another strand ("guide" strand) remains into the RISC* and guides the target RNA recognition and cleavage (fig. $2 \mathrm{C}$ ). The structure of the complex containing siRNA and Ago2 effects the selection of the strand (sense or antisense) that incorporates into RISC ${ }^{*}$, hence, determines the efficacy of mRNA-target cleavage. Evidently, the antisense strand that is homologous to the sequence of mRNA-target has to be "guide" strand in order to cleave mRNA. Ago2 replaces the Dicer-R2D2 dimer (fig. 2 B), interacting with Dicer via PAZ domain [28] whereas the phosphate group at the 5 '-end of the strand from the Dicer side ("guide" strand) interacts with PIWI domain that contains $\mathrm{Mg} 2+$ ion and basic amino acids.

The nucleotides located at 2 - 6 positions of the "guide" strand stabilizes the interaction between siRNA and Ago2 [29, 30,31]. The presence of the phosphate at the 5 -end of the "guide" strand is essential for RISC* assembly [32, 33]. This orientation of siRNA with respect to catalytic PIWI-domain provides the cleavage of the complementary strand ("passenger" strand) between 9th and 10th nucleotides that facilitates the strand dissociation (fig. 2 C) [34, 35]. Ago2 and the "guide" strand are the main components of

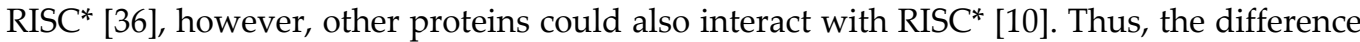
between thermodynamic stability of the duplex termini (thermodynamic asymmetry) determines the orientation of Dicer-R2D2 dimer and as a result, the structure of Ago2siRNA complex. Therefore, thermodynamic properties of siRNA play a key role in the strand selection [20,23, 24, 25] providing the preferential selection of the strand with low thermodynamic stability at the 5 '-end of the duplex as the "guide" strand [37, 38].

It was shown, that the perfect match between 2 - 12 nt of the "guide" strand (corresponding to one turn of dsRNA helix) and the target mRNA is essential for effective recognition and binding of RISC* with mRNA target [39]. The mechanism of RNA-target cleavage (occurs between $10^{\text {th }}$ and $11^{\text {th }}$ nt relative to the $5^{\prime}$-end of the "guide" strand [40]) and the following dissociation are similar to that of the "passenger" strand (described above). Cleaved target RNA and the "passenger" strand are degraded by ribonucleases after dissociation, whereas RISC $^{*}$ becomes available for acting in catalytic mode [7, 9, 17, 41]. Unlike antisense oligodeoxyribonucleotides (ODN), whose efficacy of action is determined by the efficacy of ODN hybridization with mRNA-target (fig. 3) [42], the formation of the duplex between the "guide" strand of siRNA and mRNA- target occurs due to the helicase activity of RISC* and virtually independent from the hybridization properties of the oligoribonucleotide [43]. Silencing of gene expression by siRNAs is observed at the much lower ON concentrations than in the case of antisense ODN (IC50 for siRNAs is $100-1000$ times lower, than IC50 for antisense ODNs) [43, 44].

Another mechanism of RISC activation - "bypass" route - employs the dissociation of the siRNA strands without preliminary cleavage of the sense strand by Ago2 [35]. This type of RISC activation is inherent in miRNAs, containing unpaired bases in the central part, critical for Ago2 endoribonuclease activity (fig. 4). Moreover, the "bypass" rout was observed for siRNAs with chemically modified nucleotides surrounding the cleavage site (between 9th and 10th nt of the "passenger" strand [45]) impeding Ago2 action [34, 35, 46]. Silencing of gene expression proceeds via the arrest of mRNA translation, since RISC* binds to mRNA- 
target together with translation suppressor proteins (for example, helicase RCK/p54 from DEAD box family) [47]. Complex of mRNA and proteins is deposited in "P-bodies" (processing bodies - distinct foci in the cytoplasm involved in mRNA turnover) [48]. In "Pbodies" mRNA decapping followed by ribonuclease cleavage occurs. In the other cases, mRNA complexed with proteins can be deposited in "P-bodies" for a long time without degradation (fig. 4). Later, mRNAs can escape from "P-bodies" and be involved in translation machinery or can be degraded in a described above manner [10].

A

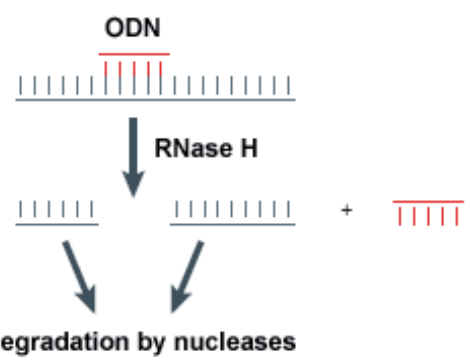

B

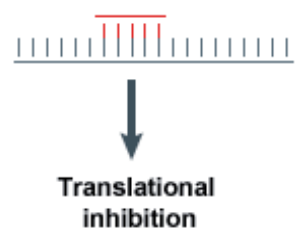

Figure 3. Silencing of gene expression by antisense ODNs: A. The degradation of the duplexes: ODN / mRNA or ODN / pre-mRNA by RNase H. B. Inhibition of mRNA translation or pre-mRNA splicing by ODNs those are not RNase H substrates [43].

The "bypass" mechanism of RISC activation is less efficient than the "classical" one, since in this case RISC ${ }^{*}$ does not act in a catalytic mode. The efficiency of RISC* assembly also is limited by the low rate of ATP-dependent dissociation of intact siRNA strands [35, 33, 39].

Thermodynamic stability siRNA duplex is an additional parameter determining the efficiency of RISC* assembly, hence, the efficiency of RNAi. Earlier developed algorithms for the selection of active siRNA sequences suggest selection of mRNA targets with approximately $50 \%$ GC-content. In the later studies, the percentage of $\mathrm{G}+\mathrm{C}$ nucleotides in the structure of effective siRNAs varied from 30 to $50 \%$ [49, 50]. It was shown that low thermodynamic stability of the central part of the duplex (from 9th to 14th nt counting from 5 '-end of the antisense strand) arising from AU-rich sequences or the presence of mismatches is a hallmark of active siRNAs [37]. The average difference between Gibbs energy of central parts of active and inactive siRNAs was found to be about $1.6 \mathrm{kcal} / \mathrm{mol}$. Unlike thermodynamic asymmetry, unstable center of duplex is not a sufficient criteria for selection of active siRNA [37].

Structural features of mRNA-target also effects the RNAi efficacy [51, 52, 53, 54]. The availability of the mRNA sequence for binding with siRNA (the absence of the hairpins in the secondary structure or overlapping with the binding regions of regulatory factors) as well as in the case of the ribozymes and the antisense ODN [55] can influence the silencing efficacy. In order to prove this, siRNAs targeted to mRNA sequences with different binding availability were synthesized [53]. It was shown that siRNAs targeted to the region of the initiation of translation or the $3^{\prime}$-end of mRNA-target were inactive. Whereas siRNAs targeted to the regions forming the hairpins displayed low and average silencing activity. As expected highperformance siRNAs were those targeted to unstructured regions of mRNA [51, 52, 54]. 
Thus, the analysis of the secondary structure of mRNA-target and thermodynamic properties of siRNA duplex play important role in the selection of active inhibitors of gene expression.

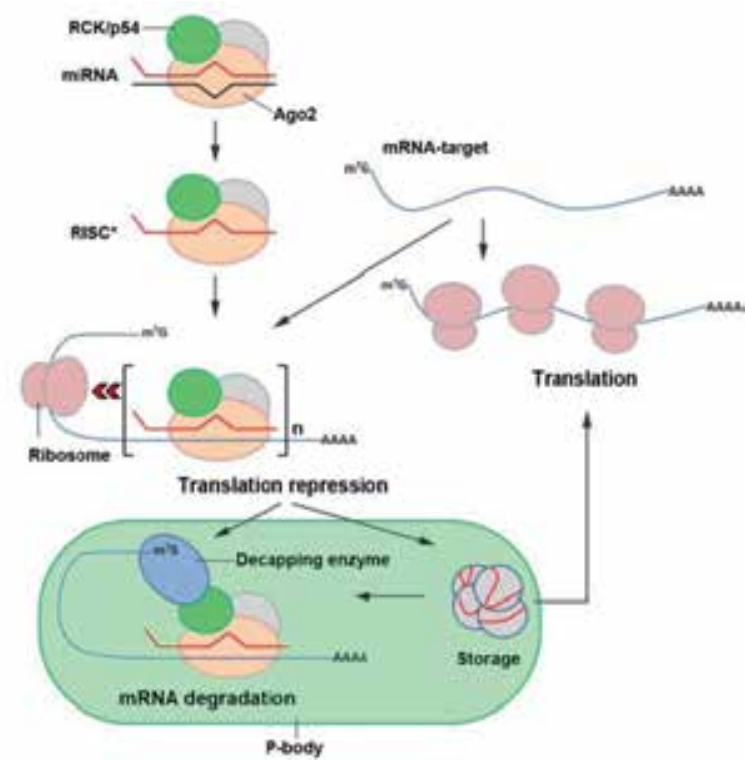

Figure 4. Scheme of RNAi with the "bypass" rout of RISC* assembly (miRNA-like action). RISC* binds with mRNA-target ( $\mathrm{n}$ is equal 1 , if the antisense strand is fully complementary to mRNA) [10].

\section{Chemical modifications: The influence on RNAi efficacy}

Currently, the development of RNAi-based drugs is an important aim in pharmacology. The optimization of siRNAs structure is required for biomedical applications; chemical modifications could be successfully used for this purpose. Different types of chemical modifications were widely used in antisense ODNs before the discovery of RNAi [42]. The data collected in these experiments can be applied for optimization of the properties of siRNAs. There are two types of factors affecting both antisense ODNs and siRNAs efficacy: systemic factors that act on the level of the organism and intracellular factors defining the activity of the therapeutics in the cells. The first type of factors includes: i) fast (approx. 5 min) siRNA elimination from organism resulted from its hydrophobicity and size of the molecule (approx. $14 \mathrm{kDa}$ ) [56, 57], ii) high nuclease sensitivity of siRNA [58, 59, 60, 61, 62] and iii) inefficient or/and non-specific delivery into target cells [63]. Intracellular factors are related to RISC assembly and the efficiency of binding to and cleaving the target.

\subsection{Chemical modifications of the nucleotides}

\subsubsection{Modifications of ribose (furanose) ring}

All ribose modifications can be divided in two main groups: the modifications related to the replacement of hydrogen atoms in furanose by different groups and structural modifications of the furanose cycle. 


\subsubsection{Substituents in furanose}

The $2^{\prime}-\mathrm{OH}$ group of the ribose is the main target for modification, since this group is involved in the phosphodiester bonds cleavage by endoribonucleases via trans-etherification mechanism [64]. Thus, the modification of 2'-OH defends siRNA against ribonucleases [62]. The size of the substituent is an important characteristic determining its tolerance by RNAi machinery. The small groups (2'-O-methyl (2'-O-Me), 2'-fluoro (2'-F) etc.) (fig. $5 \mathrm{~A})$ virtually do not disturb the conformation of siRNA duplex and are better tolerated than bulky groups: 2'-O-methoxyethyl (2'-O-MOE), 2'-O-allyl (2'-O-allyl) etc. The replacement of 2'-OH by electrophilic groups also stabilizes C3'-endo conformation of the ribose (fig. 6) which corresponds to A-helix geometry of the duplex obligatory for effective RNAi [58]. This sugar conformation arrange for the axial location of the substituents reducing total energy of the system and increasing the affinity to the complementary RNA target. As a result the duplex melting temperature $(\Delta \mathrm{Tm})$ increases approximately $1^{\circ} \mathrm{C}$ per modification [65].

2'-O-methyl modification is one of the widely used 2'- modifications for the enhancement of nuclease resistance of siRNA $[59,62]$ and impeding the induction of the interferon response in eukaryotic cells $[64,66,67,68,69,70]$. Obviously, the number and location of the modified nucleotides in the duplex are crucial for the silencing. The increase of the number of modifications was shown to decrease the silencing activity of siRNAs; totally modified siRNAs frequently had no activity $[58,59,71]$. However, in a number of experiments the activity of siRNA with the totally modified sense strand was compatible with that of unmodified analog [72, 73]. This can be related to functional unequivalence of siRNA strands [74, 75]. The introduction of 2 - 4 2'-O-Me modifications in the both strands of siRNA is well tolerated. Moreover, in the case of selective modification of the nucleasesensitive sites within siRNA the increase of the duplex nuclease resistance and prolonged silencing were detected [59].

The C3'-endo conformation could be stabilized by introduction of 2'-F-modified nucleotides in siRNA (fig. $5 \mathrm{~A}$ ). Since fluorine atom is more electronegative than oxygen atom, the increase of the binding affinity between siRNA strands and target RNA $\left(\Delta \operatorname{Tm} 2-4{ }^{\circ} \mathrm{C}\right.$ per modification) is observed [65]. Remarkably, the silencing activity of 2'-F-modified siRNAs virtually does not depend on the number of modifications in contrast to 2'-O-Me-modified siRNAs. The silencing activity of siRNAs with 2 '-F-modifications in the sense and the antisense strands was compatible with that of unmodified analogs [76]. The replacement of pyrimidine nucleotides in the duplex with 2'-F-analogs was well tolerated by RNAi machinery both in vitro [46, 77, 73] and in vivo [78]. The silencing activity of siRNA containing 2'-F modifications located in the site of Ago2 cleavage and unmodified analog was similar [79], as well as long-term silencing effects in vivo of modified siRNAs and unmodified analogs [78]. It should be noted that epimers of $2^{\prime}$-F-nucleotides ( $2^{\prime}$-fluoro- $\beta$-Darabinonucleotides, FANA (fig. 5 A)) with DNA-like C2'-endo-conformation of furanose cycle [80] (fig. 7) introduced in siRNA structure also improve the nuclease resistance of the duplex. It was found that partial modification of both strands with FANA or total modification with FANA of the sense strand of siRNAs ensure the A-geometry helix [81] and effective RNAi. These modifications were shown to alter the duplex thermodynamic stability insignificantly [76]. 


\section{A}

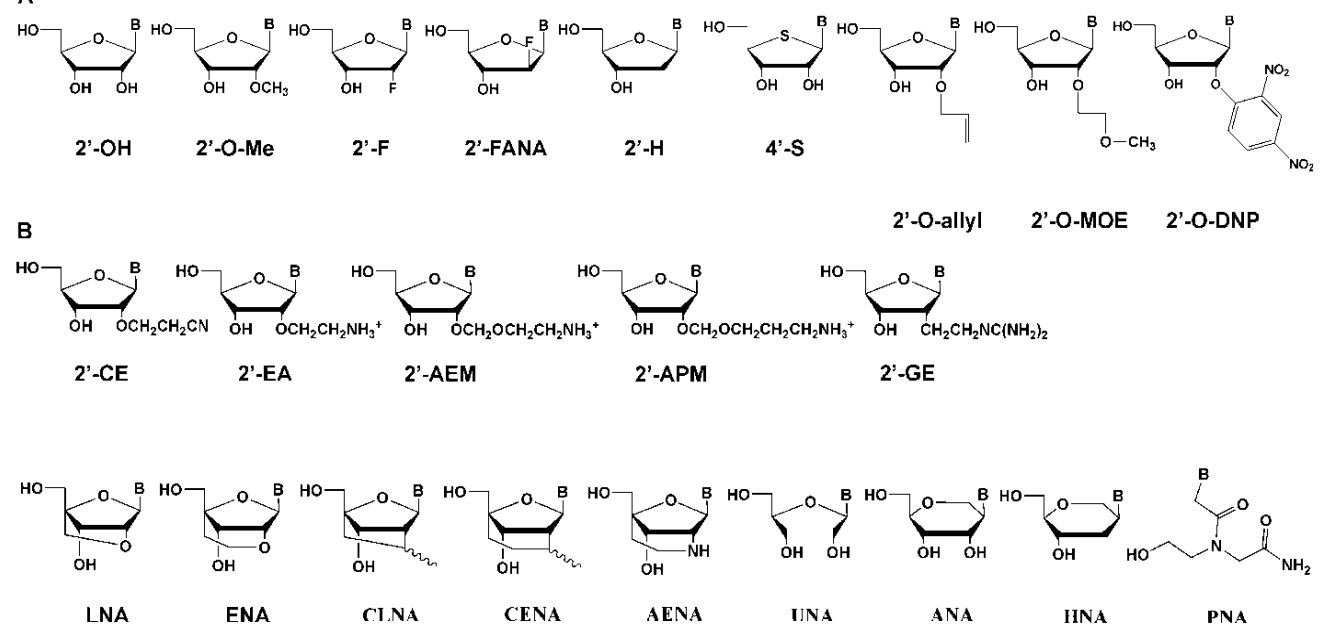

C

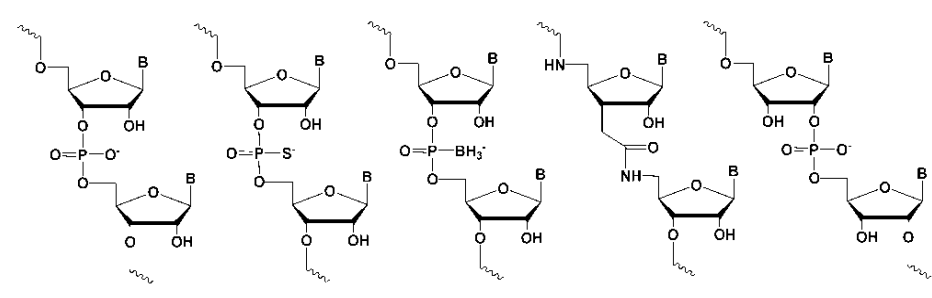

PO

PS

PB

3',5'-amide

2',5'-PO

D<smiles>[R]n1ccc(=O)[nH]c1=O</smiles><smiles>[R]c1c[nH]c(=O)[nH]c1=O</smiles><smiles>[R]N1CCC(=O)NC1=S</smiles>

uridine (U) pseudouridine ( $\Psi$ ) 2-thiouridine (s2U)

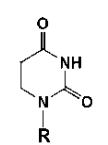

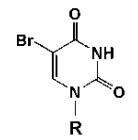

$\mathbf{R}$

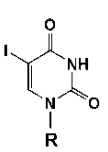

5-iodo-U

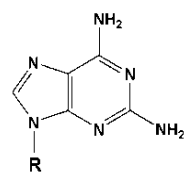

2,6-diaminopurine

Figure 5. Analogs of nucleotides and nucleosides used for siRNA modification. A. Substituents in furanose. B. Structural modifications of furanose cycle. C. Backbone modifications. "B" - base (A-C). D. Nucleobase modifications. " $R$ " - ribose residue.

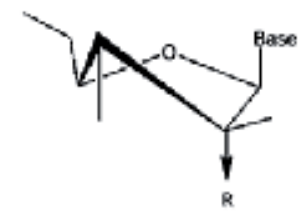

Figure 6. C3'-endo conformation of the ribose with the 2'-substituent. $\mathrm{R}$ - electron-acceptor group.

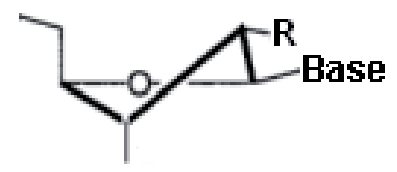

Figure 7. C2'-endo (DNA-like) conformation of ribose with the substituent (R) in 2'-position. 
On the contrary, the replacement of ribonucleotides of siRNAs with deoxyribonucleotides characterized by $\mathrm{C2}^{\prime}$-endo-conformation of the furanose cycle (fig. $5 \mathrm{~A}$ ) reduces their affinity to mRNA-target $\left(\Delta \mathrm{Tm}-0.5^{\circ} \mathrm{C}\right.$ per modification) (fig. 7) [65]. Mainly, deoxyribonucleotides are used for the defense of siRNA overhangs from exonucleases [13, 59]. However, the modification of the other duplex regions does not impede effective RNAi [40,82]. The introduction of deoxyribonucleotides in the duplex region responsible for the recognition of the target mRNA, so called "seed" region (about $8 \mathrm{nt}$. from the $5^{\prime}$ end of the antisense strand [29]) results in the increase of siRNA specificity since lesser stability of DNA/RNA hybrids as compared with RNA duplex [82]. siRNAs with the totally modified antisense or sense strands had no activity regardless of preferable for RNAi A-helix geometry of the duplexes [32].

Introduction of the bulky groups in the 2'-position of the furanose causes the conformational disturbance of the siRNA duplexes resulting in the decrease of siRNA silencing activity [76]. However, the modification of duplex termini and overhangs with 2'-O-methoxyethyl (2'-O-MOE) or 2'-O-allyl groups (fig. $5 \mathrm{~A}$ ) was tolerant $[46,83,84]$. The random replacement of $2^{\prime}-\mathrm{OH}$ in the both strands of the siRNA (up to $70 \%$ ) by 2,4dinitrophenyl ethers (2'-O-DNP) (fig. $5 \mathrm{~A}$ ) causes the increase of the silencing activity. Since the thermodynamic stability of the modified duplex was comparable with that of unmodified duplex [85] (that indicates the similar hybridization properties [86]), the observed effect may be related to the increase of the nuclease resistance and cellular uptake of the modified siRNA $[85,86,87]$. In order to increase the nuclease resistance of the siRNA duplexes, other chemical modifications - 2'-aminoethoxymethyl (2'-AEM), 2'aminopropoxymethyl (2'-APM), 2'-aminoethyl (2'-EA), 2'-cyanoethyl (2'-CE), 2'guanidinoethyl ( $2^{\prime}$-GE) etc.- may be used (fig. 5 A). It was shown that totally modified siRNAs were inactive, whereas the silencing activity of the partially modified siRNAs depends on the location of the modifications in the duplex structure [88].

Introduction of the modifications at the $4^{\prime}$-position of the furanose results in the slight increase of the RNA-duplex termostability ( $\triangle \operatorname{Tm} 1{ }^{\circ} \mathrm{C}$ per modification) $[89,90]$. Usually the $4^{\prime}$-thio (4'-S) ribonucleosides (fig. $5 \mathrm{~A}$ ) are used for modification of siRNA, especially, at the termini [91, 92, 93]. The replacement of the several ribonucleotides at the $5^{\prime}$-end of the antisense strand [91] or four ribonucleotides at the sense strand termini and at the 3 '-end of the antisense strand [93] by 4'-S- analogs only slightly decrease the silencing activity of siRNAs, whereas the modification of the central part of the duplex significant inhibits RNAi [91, 92]. The introduction of the 4'-S-modifications in siRNA structure increases nuclease resistance of the duplex and improves the pharmacokinetics of siRNA. Unfortunately, this modification simultaneously increases the binding of siRNAs with blood serum components and increases their cytotoxicity [65].

\subsubsection{Structural modifications of the furanose ring}

This type of modifications includes bicyclic derivatives of the nucleotides (LNA, ENA, CLNA, CENA, AENA et al.), acyclic nucleotides (UNA, PNA) or nucleotides containing pyranose ring (ANA, HNA) instead of ribose (fig. 5 B). LNA and ENA (2'-O,4'-C-methylene- 
and 2'-O,4'-C-ethylene bicyclic nucleotide analogs are the frequently used bicyclic derivatives which provide the significant increase of the duplex thermostability ( $\triangle \mathrm{Tm}$ up to $10{ }^{\circ} \mathrm{C}$ per modification) [94, 95]. The ribose in LNA and ENA is fixed in C3'-endoconformation (fig. 6) due to the methylene or ethylene "bridge" between 2'- and 4'positions, respectively.

Experimental data on the activity of LNA- and ENA-modified siRNAs are contradictory. In some cases modifications of the $5^{\prime}$ - and/or $3^{\prime}$-ends of the both siRNA strands were well tolerant $[65,96,97,98]$, whereas in the other studies ENA-analogs at the 3 '-end of the sense strand of siRNAs reduced or abolished the silencing activity [74]. The difference in activity may be connected with the difference in the thermodynamic asymmetry of the modified siRNA [37, 38]. The replacement of the several ribonucleotides with LNA in the antisense strand was shown to be well tolerant $[71,96]$, whereas extensive modification was tolerant only in the case of sisiRNA with segmented sense strand [99] (see section 1.3.5).

The thermodynamic stability and nuclease resistance of the siRNA could be increased by introduction of HNA and ANA nucleotides with pyranose ring (hexitol nucleic acid and altritol nucleic acid, respectively (fig. 5 B)) instead of ribose [100, 101]. The modified duplex contaning these analogs adopts the A-helix geometry [100], however, the location of the ANA- or HNA-analogs in siRNA structure is critical for silencing activity. Remarkably, the introduction of the ANA- or HNA-analogs at the 3'-termini of one or both strands maintain or increase the silencing activity $[102,103]$. The presence of ANA-analogs at the $5^{\prime}$-end of the antisense strand substantially decreased the siRNA activity. It was suggested that modifications at the $5^{\prime}$-end of the antisense strand impede the action of cellular kinases due to steric obstacles [103]. The decrease of the phosphorylation efficacy of the 5 'terminal nucleotide of the antisense strand is critical for efficient interaction of siRNA with Ago2 PIWI domain followed by mRNA-target cleavage [32,33]. siRNAs with HNA or ANAanalogs of nucleotides in the central part of the sense strand display the effective gene silencing, whereas, similar modifications of the antisense strand substantialy decreased the siRNA activity [88, 103].

In contrast to siRNA duplex stabilizing modifications, the presence of acyclic nucleotide analogs (UNA) (fig. 5 B) in the siRNA increase the conformational flexibility of the duplex and decrease its thermodynamic stability $\left(\Delta \mathrm{Tm}-5\right.$ to $-8{ }^{\circ} \mathrm{C}$ per modification). The location of the UNA-analogs in siRNA was shown to play a key role, since one modification can result in the significant reduction or augmentation of the silencing activity of siRNA [99, 104]. It was suggested that the replacement of ribonucleotides at the 3 '-end of the sense strand increases the duplex thermodynamic asymmetry and acquires antisense strand incorporation into RISC ${ }^{*}[104,105]$. It was found experimentally that the introduction of 1 3 UNA nucleotides at the 3 '-end of the sense strand, including the 3 '-overhangs, increase to some extent the siRNA activity [106, 107]. Quite the opposite, the modification of the first and the second positions of the "guide" strand abolishes the 5'-end phosphorylation and reduce siRNA silencing activity.

Peptide nucleic acids analogs (PNA) containing N-(2-aminoethyl)-glycin polyamide backbone could be used for modification of siRNA. It was shown that in the antiparallel 
PNA/RNA duplex RNA adopts the right-handed helix characterized by anticonformation at the N-glycosidic bond and C3'-endo-conformation of the ribose [108], with the similar to the RNA-like A-helix geometry [58]. PNAs are stable in the human blood serum as well as in the cellular extract; they are resistant to protein kinase A and several types of peptidases [109]. It was found that the introduction of PNA-analogs in the sense and / or in the antisense strands of the duplex increase nuclease resistance of siRNA. The increase of the siRNA silencing activity was observed when modifications were introduced in the sense strand. In the other cases the activity of PNA-containing siRNA was comparable with activity of the unmodified siRNA [110]. Due to the functional inequivalence of siRNA strands the modifications in the sense strand a better tolerated than modifications in the antisense strand.

Thus, virtually all ribose modifications provide the increase of the nuclease resistance of siRNA. However, the optimization of their number and location in the duplex structure (depending on the type of modification) is required to make them tolerable in the process of RNAi.

\subsubsection{Backbone modifications}

This type of modifications includes the replacement of phosphate group (PO) with phosphorothioate (PS) or boranophosphonate (PB) groups, the replacement of the $3^{\prime}, 5^{\prime}$ phosphodiester bond with 2',5'-bond or the amide bond instead of the ester bond (fig. $5 \mathrm{C}$ ). All of these modifications increase the nuclease resistance of siRNA, however their impact on the efficacy of RNAi is varied [76]. PS-modification is known to reduce the melting temperature of the duplex and oligoribonucleotide binding affinity [71]. However, the experimental data related to the activity of PS-modified siRNAs are discrepant. The comparable silencing activity of the PS-modified siRNAs and their unmodified analogs was shown in the studies [83,111], whereas in the other studies the silencing activity of the PSmodified siRNAs was lower, than that of their unmodified analogs [58, 73, 112]. The modification of the central part of the duplex decreases siRNA activity [113]. Remarkably, siRNAs with blunt 3 '-ends containing totally PS-modified one or both strands were active [73]. However, high binding affinity of the phosphorothioate analogs to serum albumin, IgG, IgM, lactoferrin and the cellular membrane receptors is disadvantageous [114]. It results in the development of toxic effects both in vitro and in vivo, even siRNAs containing alternate PS-analogs were toxic in cell cultures [83, 111]. Introduction of boranophosphonate analogs (fig. 5C) increases nuclease resistance of the duplex as compared with PScontaining and unmodified siRNA [65] and increases RNAi efficacy. It was found that an optimal position for PB-modifications is the terminal region of the sense strand of siRNA, whereas the modification of the central part of the duplex resulted in the significant decrease of silencing activity [65]. This effect is likely connected with the inhibition of the sense strand cleavage by Ago2 during RISC ${ }^{*}$ formation. The replacement of the $3^{\prime}, 5^{\prime}$ - by $2^{\prime}, 5^{\prime}$-phosphodiester bond or 3',5'-amide bond (fig. 5 C) increases the nuclease resistance of the duplex and is tolerant when located in the sense strand [115] or the $3^{\prime}$-overhangs of siRNA [116]. 
Thus, the most promising modification of the siRNA backbone is the boranophosphonate modification, since it provides the increase of the nuclease resistance of the duplex and its silencing activity and is not accompanied by the development of toxic effects. However, the price of PB-analogs limits their usage.

\subsubsection{The chemical modifications of the nucleobases}

In contrast to the other types of modifications, the modifications of heterocyclic bases (nucleobases) have no influence on the nuclease resistance of RNAi. Earlier it was found that the presence of modified nucleobases in the antisense ODN altered their hybridization properties [42]. It is known that the replacement of the uridine by 5-bromo- or 5-iodouridine (fig. $5 \mathrm{D}$ ) improves their ability to interact with adenine owing to the increase of the acidity of the imine and results in the stabilization of the base pair [117]. The replacement of the adenine by 2,6-diaminopurine results in the formation of the additional $\mathrm{H}$-bond, hence, in the stabilization of the nucleotide base pair [58]. At the start, it was suggested that the introduction of these analogs in the antisense strand of siRNA will enhances its binding affinity to mRNA and increases the RNAi efficacy. Unexpectedly, the decrease of silencing activity of modified siRNA in comparison with unmodified ones was observed. This effect is possibly related with the decrease of the dissociation rate of siRNA duplex on the step of RISC* formation [58]. The replacement of uridine by 2-thiouridine $\left(\mathrm{s}^{2} \mathrm{U}\right)$ or pseudouridine $(\psi)$ (fig. $5 \mathrm{D}$ ) provides the increase of the duplex thermodynamic stability due to the stabilization of the ribose in the C3'endo-conformation (fig. 6) [118, 119]. Dihydrouridine (D) contains non-aromatic ring that does not participate in staking interactions (fig. 7) which resulted in the destabilization of siRNA [120], but one substitution with D at the $3^{\prime}$-end of the sense or the antisense strand does not change siRNA silencing activity. The replacement of $U$ by $\Psi$ and $s^{2} U$ at the $3^{\prime}$-end of the sense strand reduces the silencing activity, whereas introduction of the same modification at the $3^{\prime}$ end of the antisense strand creates favorable thermodynamic asymmetry and provides siRNAs with higher activities than the parent siRNA. Thus, these modifications could be applied for the design of thermoasymmetric siRNAs [120] (see section 3.3).

\subsection{Chemical modification of siRNA termini}

This type of modifications includes the modification of the nucleotides at the 3 '-overhangs and the $5^{\prime}$-ends of the duplex. These modifications could be used for the solution of different problems: i) to increase the nuclease resistance of siRNA towards exoribonucleases (inverted 3'-3' or 5'-5' deoxyriboses [61, 121] (fig. 8), dideoxycytosine [122], ENA-analog of the thymidine [74] etc.), ii) to facilitate asymmetric RISC* assembly (the replacement of the $5^{\prime}-\mathrm{OH}$ by $5^{\prime}$-OMe group in the terminal nucleoside [123]) and ii) to provide the efficient accumulation of siRNA in cells (attachment of lipophilic molecules [57, 124, 125], folic acid [126], peptides [14, 127, 128], aptamers [129]). The fluorescent residues [111] and biotin [130] are widely used as a termini modification for siRNA detection.

The type of the applicable terminal modification has to be selected experimentally. In many cases the modification of the 5'- and 3 '-ends of the sense ("passenger") strand is well 
tolerated [59, 131, 111, 130], however the data on modification of the antisense ("guide") strand are discrepant. It is known that the phosphorylation of 5 '-OH groups of the antisense strands of synthetic siRNAs by the kinases [33, 122] is essential for interaction with PIWIdomain of Ago2 and for correct selection of the "guide" strand [29, 30, 31]. Therefore the replacement of the $5^{\prime}-\mathrm{OH}$ group by the $5^{\prime}-\mathrm{OMe}$ in the sense strand blocks its phosphorylation and the incorporation into RISC* as a "guide" strand [123]. On the other hand, the same modification of the antisense strand resulted in the decrease of siRNA silencing activity $[59,131,122]$. However, the attachment of the fluorescein residue to the 5 'phosphate of the antisense strand via hexamethylene linker is well tolerated [111], suggesting that this modification does not prevent the interactions of the 5 -terminal phosphate of siRNA with PIWI-domain. The $3^{\prime}$-end of the antisense strand recognized by PAZ-domains of Dicer and Ago2 [21] is less sensitive to modification [14, 33, 59]. It was shown that the attachment of puromycin or biotin to the 3 '-end of the antisense strand [130] or the replacement of the 3 -terminal ribonucleotide by $\mathrm{ddC}$, or the attachment of the aminopropyl linker via phosphodiester bond [122] virtually does not change the silencing activity. However, the introduction of the 2-hydroxyethylphosphate, ENA-analog of thymidine [74] or fluorescent dyes [111] at the 3 '-end of the antisense strand abolished the silencing. The replacement of the terminal nucleotides of siRNA in both strands by $5^{\prime}-5^{\prime}-$ or $3^{\prime}-3^{\prime}$-dioxyribose (fig. 8) results in the increase of the nuclease resistance and does not reduce the silencing activity $[59,61]$.

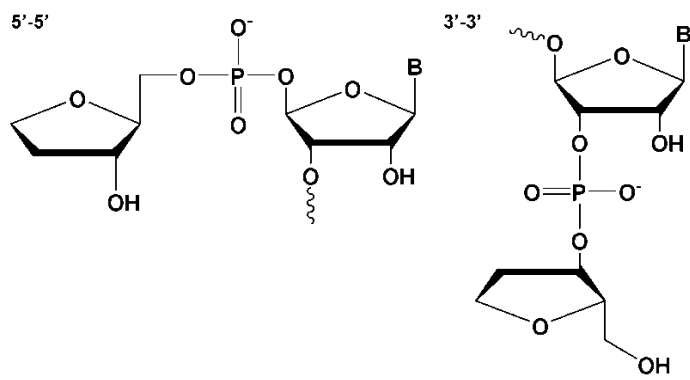

Figure 8. Inverted deoxyribose applied for the protection of $5^{\prime}$ - or $3^{\prime}$-termini of siRNA.

\subsubsection{Bioconjugates}

Anionic siRNA cannot effectively pass through the electrostatic and hydrophobic barriers of the cellular membrane to enter the cytoplasm and to induce RNAi. Conjugation of siRNAs with the lipophilic molecules (cholesterol, derivatives of the oleic, lithocholic and lauric acids), peptides, antibodies, aptamers and other compounds is an effective tool to overcome this problem [132, 133]. The cholesterol was suggested as the first candidate for conjugation, since the natural mechanisms of cholesterol transport exist in mammalian organisms. Apolipoprotein B (ApoB) expressed in the liver and intestine cells is involved in the assembly and secretion of the lipid-protein particles known as very-low-density lipoproteins (VLDL) and low-density lipoproteins (LDL) and in the transport and metabolism of the cholesterol. Since ApoB located on the cellular surface is specific to LDL-receptors, responsible for the delivery of the lipoproteins into cell [132, 134], it was suggested that 
LDL-receptors could deliver the cholesterol-conjugated siRNAs inside the cells via receptormediated endocytosis. It was proved experimentally that LDL-receptors bind with the LDLparticles preliminary associated with cholesterol-conjugated siRNA or the conjugate of siRNA and the oleic or lithocholic acids, however the mechanism of their penetration into cell is not well-defined [125] (Table 1). Moreover, the involvement of the transmembrane

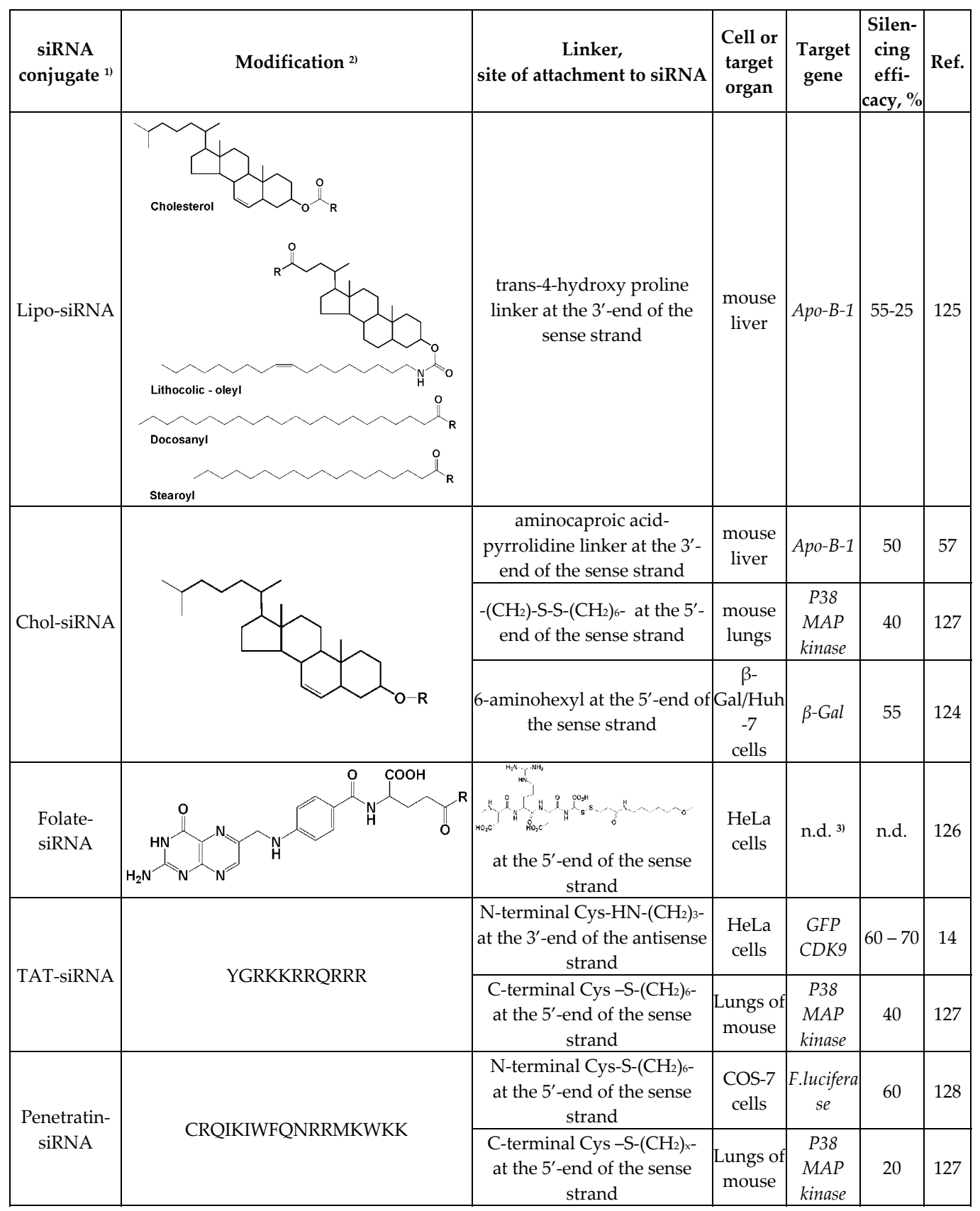




\begin{tabular}{|c|c|c|c|c|c|c|}
\hline $\begin{array}{c}\text { Transportan- } \\
\text { siRNA }\end{array}$ & CLIKKALAALAKLNIKLLYGASNLTWG & $\begin{array}{l}\text { N-terminal Cys-S- }\left(\mathrm{CH}_{2}\right)_{6-} \\
\text { at the } 5^{\prime} \text {-end of the sense } \\
\text { strand } \\
\end{array}$ & $\begin{array}{c}\text { COS-7 } \\
\text { cells }\end{array}$ & $\begin{array}{c}\text { F.lucifera } \\
\text { se }\end{array}$ & 50 & 128 \\
\hline $\begin{array}{l}\text { Aptamer- } \\
\text { siRNA }\end{array}$ & & $\begin{array}{l}\text { at the } 5^{\prime} \text {-end of the sense } \\
\text { strand }{ }^{4)}\end{array}$ & $\begin{array}{c}\text { LNCaP } \\
\text { and PC- } \\
3 \text { cells }\end{array}$ & $\begin{array}{l}\mathrm{Bcl}-2 \\
\text { PLK1 }\end{array}$ & 80 & 129 \\
\hline \multirow{2}{*}{$\begin{array}{l}\text { Antibody- } \\
\text { siRNA }\end{array}$} & Antibodies to insulin receptor & $\begin{array}{l}\text { biotin-tetraethylene glycol } \\
\text { at the } 3 \text { '-end of the sense } \\
\text { strand }\end{array}$ & $\begin{array}{c}\text { HEK } 293 \\
\text { cells }\end{array}$ & \multirow{2}{*}{$\begin{array}{c}\text { F.lucife- } \\
\text { rase }\end{array}$} & $>90$ & 141 \\
\hline & Antibodies to transferrin receptor & $\begin{array}{l}\text { biotin-tetraethylene glycol } \\
\text { at the } 3^{\prime} \text { - or 5'-end of the } \\
\text { sense strand }\end{array}$ & $\begin{array}{c}\text { Brain of } \\
\text { mouse }\end{array}$ & & $69-81$ & 142 \\
\hline
\end{tabular}

1) Lipo - lipophilic residues, $\mathrm{Chol}$ - cholesterol residue.

2) siRNA with linker are denoted «R».

${ }^{3}$ In this investigation the biological activity of the conjugate was not measured (n.d.).

4) The conjugate of siRNA and aptamer synthesized by in vivo transcription (without linker).

Table 1. Bioconjugates of siRNAs

protein SID-1 in the transport of the lipophilic conjugates was also shown [135]. It was suggested that SID-1 facilitates the dsRNA penetration into cells forming the channels for diffusion or mediating the interactions with other proteins [125, 135]. The successful use of the cholesterol and the derivatives of lithocholic, oleic and lauric acids for siRNA modification was demonstrated in vitro [124] and in vivo [57] (Table 1). The conjugate of siRNA and cholesterol, attached to the $5^{\prime}$-end of the sense strand, is able to penetrate into the human liver cells in the absence of the transfection agents. siRNA bearing cholesterol in the sense strand inhibits $\beta$-Gal gene expression more effectively than siRNAs with the cholesterol in the antisense strand or in the both strands. In the presence of the transfection agent the activity of the unmodified siRNA was comparable with that of the conjugates [124]. The accumulation of siRNA-Chol was detected in liver, heart, kidneys, fatty and pulmonary tissues after intravenous injection of the radioactively labeled conjugates (containing cholesterol at the $3^{\prime}$-end of the sense strand) in mice. It should be noted that cells of these organs express LDL-receptors at high level. Other examples of cholesterol-modified siRNAs with high penetration ability are presented in the Table 1.

The conjugation of siRNAs with the peptides could also improve their cellular accumulation (Table 1). 11 amino acid cationic peptide derived from cell-permeable Tat protein and responsible for its nuclear localization was used in a number of studies [14, 127]. It was found that the attachment of this peptide (TAT-peptide) with additional cysteine to the $3^{\prime}$ end of the antisense strand of siRNA results in its effective accumulation in HeLa cells and effective silencing of the target genes EGFP and CDK9 [14]. The endocytosis was suggested to be the mechanism of the conjugates penetration into cells [136, 137].

The addition of the cell penetrating peptides (transportan and penetrantin) to the 5 '-end of the sense strand of siRNA improves the penetration ability and the silencing activity of antiEGFP and anti-GL2 siRNAs in COS-7, C166-GFP, EOMA-GFP and CHO-AA8-Luc Tet-Off 
cells [128] (Table 1). It was suggested that penetration of the conjugates does not occur via pino- or endocytosis, but is mediated by diffusion through plasma membrane [128]. Besides the increase of transfection efficacy, the attachment of the peptides to siRNAs may enhance their specificity. Bioconjugates of siRNAs with peptides inhibiting the RISC assembly and containing specific sequence cleavable by cell-specific peptidases are promising agents for cell-specific gene silencing [138]. The efficient inhibition of the exogenous GFP expression was observed in choriocarcinoma Jeg-3 cells after electroporation of the conjugate of siRNA and a peptide with the "LEVD" sequence recognized by caspase-4, whereas in caspase- 4 deficient HEK 293 cells the conjugate was inactive [138].

Another approach to the enhancement of siRNA cellular accumulation is the delivery of siRNA conjugated with antibodies or aptamers [132]. The aptamers are structured synthetic nucleic acids with size less than $15 \mathrm{kDa}$ (Table 1), they can be chemically modified for the defense from the nucleases [139]. The penetration of these conjugates occurs via specific interactions with receptors on the surface of the target cells, providing the effective cellular accumulation of siRNAs [132]. The conjugates of anti-BCL2 or anti-PLK1 (polo-like kinase 1) siRNA and PSMA-receptor specific aptamer, effectively penetrate into prostate cancer LNCaP cells and induce effective gene silencing [129]. Injection of these conjugates into tumor expressing PSMA-receptors results in tumor regression [140]. It was shown also that the conjugates of siRNA and anti-transferrin or anti-insulin monoclonal antibodies attached to siRNA via streptavidin-biotin linker effectively reduces the exogenous F.luciferase expression in HEK 293 cells and rat glial cells implanted in the brain [141, 142] (Table 1).

The attachment of the folic acid to siRNA is a promising approach to increasing cellular uptake of siRNA. These conjugates can penetrate into virtually all human cancer cells, since expression of the expression of the folate receptors on the surface of the cancer cells is substantially higher than in normal cells [143]. The efficient accumulation of fluoresceinlabeled siRNA with folate attached to the $5^{\prime}$ - or $3^{\prime}$-end of the sense strand via disulfide bond was observed in solid tumor in mice [126] (Table 1). In the other studies [144, 145] the delivery of siRNA into cells was performed with folate-containing structures. These experiments (in vivo and in vitro) verified the advantage of siRNA modification by folate: specific delivery into cancer cells and high silencing effect of these siRNAs was observed.

The introduction of biomolecules in siRNA is one of the promising approaches of non-viral delivery. In contrast to other non-viral methods (cationic lipids and polymers, high-pressure injections) the advantages of conjugation include the cell-specificity and the absence of toxic effect [63, 146, 147, 148, 149]. The employment of cholesterol- and folate-contaning siRNAs in vivo is less specific, since LDL- and folate-receptors are expressed by different cells [125, 150], however, this approach is could be usefully applied when strict cell or tissue selectivity is not required.

\subsection{Combination of chemical modifications}

The application of different modifications simultaneously is a widely-used approach for the optimization of siRNA properties. Two main strategies are considered in order to increase 
nuclease resistance of siRNA. The first one is based on the extensive modification of siRNA. In this case optimal design of the duplex (i.e. selection of modifications) could be created experimentally by the analysis of the data on nuclease resistance and biological activity. For example, different types of modifications were used in totally modified siRNA, displaying high activity in mice infected with Hepatitis B virus. The sense strand of this siRNA contains deoxyribonucleotides instead of the purine ribonucleotides, 2'-F-modifications and $5^{\prime}-5^{\prime}$ - and $3^{\prime}-3^{\prime}$-terminal deoxyribonucleotides instead of the pyrimidine bases; the antisense strand contains alternating 2'-F / 2'-O-Me groups; one PO-bond between 3'-terminal nucleotides was replaced by PS-bond [61] (Table 2). Other examples of siRNA containing different combinations of chemical modifications are presented in the table 2. However, the extensive modification of siRNA often is not well-tolerated by RNAi machinery and rather often leads to the reduction or blocking of the silencing activity [40, 59, 99]. Therefore, minimization of the number of modifications in siRNA structure is required. It was shown, that duplexes with limited number of LNA-modifications could be active and as resistant to nucleases as the totally modified 2'-O-Me / 2'-F-siRNAs, which were inactive [40, 59, 99, 99] (Table 2). Since siRNAs are degraded mainly by endoribonucleases [59], it was suggested that the increase of the thermostability will increase the nuclease resistance [99]. On the other hand, it is known that the pattern of siRNA degradation in the presence of serum is similar to the cleavage by RNase A [62]. Therefore, the selective modification of the nuclease sensitive sites of siRNAs, mapped in the presence of serum, represents an alternative approach to the extensive modification of siRNA. This rational approach of siRNA modification allows to minimize the number of modified nucleotides in the duplex and as a consequence, to maintain its silencing activity [76]. The optimization of siRNA design also includes the defense of the 3 '-terminal nucleotides against exonuclease cleavage (see section 1.2.2).

The combinations of chemical modifications are used for optimization of other siRNA properties. In order to achieve in vivo both the efficient cellular uptake and their high nuclease resistance, the 2'-O-Me-modified or/and PS-modified siRNAs conjugated with cholesterol were used $[57,151]$. In order to increase the thermoasymmetry of the duplex, the nucleotide analogs with opposite effect on the siRNA thermostability can be used simultaneously. For instance, LNA- and UNA-analogs introduced in siRNA simultaneously was shown to enhance the silencing activity of the siRNA due to the increase of thermoasymmetry of the duplex: duplex stabilization of the duplex by LNA-analogs at the 5 '-end and destabilization by UNA-analogs at the 3'-end of the sense strand [99].

The increase of the RNAi efficacy was also observed after stabilization of the 3 '-end of the antisense strand of siRNA by $s^{2} U$ or $\psi$ analogs and destabilization of the $5^{\prime}$-end by introduction of $\mathbf{D}$ at the $3^{\prime}$-end of the sense strand [120]. It should be noted that the decrease of the thermostability of the duplex at the $3^{\prime}$-end of the sense strand is more preferable in comparison with the stabilization of the duplex at the $5^{\prime}$-end of the sense strand if the duplex stabilization is not needed [99].

Thus, in order to optimize siRNA design by chemical modifications the following principles should be considered: modifications should provide 1) the RNA-like A-helix geometry of the duplex; 2) the access of the terminal 5'-OH group of the antisense strand for 


\begin{tabular}{|c|c|c|c|c|}
\hline $\begin{array}{l}\text { Structure of the modified siRNA analog: } \\
5,- \text { sense strund }-3 \text {, } \\
3^{,}-\text {antisense strand }-5,1\end{array}$ & $\tau_{1, t}, \mathrm{~h}$ & Turset & $\begin{array}{l}\text { Silencing } \\
\text { efflency, } \%\end{array}$ & Ref. \\
\hline $\begin{array}{l}\text { B_GGACUUCUCUCAAUUUUCUTT_B } \\
\text { TsTTCCUGAAGAGAGUUAAAAGA }\end{array}$ & $48-72$ & $\begin{array}{l}\text { RNA of hepatile } \\
\text { B virus }\end{array}$ & 70 & 61 \\
\hline 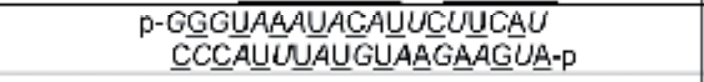 & $\therefore 7$ & $\begin{array}{l}\text { Endogenous } \\
\text { gene } P T E N\end{array}$ & 90 & 77 \\
\hline TTUsUsCsCsUsCsUsAsGsUsUsGsUsAsAsAsAsGsU & 48 & $\begin{array}{l}\text { Endogenous } \\
\text { geac Survivin }\end{array}$ & 75 & 72 \\
\hline $\begin{array}{l}\text { GCGGAUCUUGAAGUUCACCUU } \\
\text { UUCGCCUAGAACUUCAAGUGG }\end{array}$ & $\therefore 18$ & $\begin{array}{c}\text { Endogenous } \\
\text { gene GFP }\end{array}$ & 90 & 152 \\
\hline $\begin{array}{l}\text { GACGUAAACGGCCACAAGUTCU } \\
\text { UCGCUGCAUUUGCCGGUGUUCA }\end{array}$ & $-i$ & $\begin{array}{l}\text { Cndugerikus } \\
\text { gene } \theta G F P\end{array}$ & 87 & 99 \\
\hline $\begin{array}{c}\text { GACGUAAACGGCCACAAGUUC } \\
\text { CGCUGCAUUUGCCGGUGUUCA }\end{array}$ & - & $\begin{array}{l}\text { Endogenous } \\
\text { gene ELt? }\end{array}$ & 26 & 99 \\
\hline
\end{tabular}

1) Bold letters - deoxyribonucleotides, underlined letters - 2'-O-Me, italics - 2'-F-analogs, B_- 5'-5'-, _B - 3'-3'-inverted deoxyribose, underlined bold letters - LNA-analogs, $\mathrm{s}$ - phosphorothioate bond between nucleotides.

2) "_" data is not available.

Table 2. Extensive and selective modification of siRNA

\begin{tabular}{|c|c|c|}
\hline siRNA property & The role of chemical modification & Examples of modifications \\
\hline Thermostability & $\begin{array}{c}\text { To increase the thermostability of siRNA by } \\
\text { providing the optimal conformation of nucleotides } \\
\text { for base pairing } 65,99,120 \text {. }\end{array}$ & $\begin{array}{l}\text { 2'-O-Me, 2'-F, 2'-F-ANA, 2'- } \\
\text { O-MOE, 2'-O-DNP, 4'-S, } \\
\text { LNA, ENA et al. }\end{array}$ \\
\hline Thermoasymmetry & $\begin{array}{l}\text { To increase the thermoasymmetry of the duplex by } \\
\text { the decrease of thermostability at the } 3^{\prime} \text {-flank of the } \\
\text { sense strand or/and the increase of the } \\
\text { thermostability at the } 5^{\prime} \text {-flank of the sense strand of } \\
\text { the duplex } 99,105,107,120 \text {. }\end{array}$ & $\begin{array}{l}\text { UNA, LNA, nucleobase } \\
\text { modifications }\end{array}$ \\
\hline Nuclease resistance & $\begin{array}{c}\text { To increase the duplex nuclease resistance by } \\
\text { introduction of the chemically modified analogs of } \\
\text { nucleotides in its structure }{ }^{153,76} \text {. }\end{array}$ & Virtually all modifications \\
\hline $\begin{array}{l}\text { Duration of circulation in } \\
\text { the bloodstream }\end{array}$ & $\begin{array}{l}\text { To increase siRNA affinity to serum proteins and the } \\
\text { time of circulation } 57,65 \text {. }\end{array}$ & PS, 4'-S, Chol \\
\hline Cellular uptake & $\begin{array}{l}\text { To provide the effective mechanisms of siRNA } \\
\text { penetration: receptor-mediating endocytosis, } \\
\text { diffusion via cellular membrane or other ways (if the } \\
\text { mechanism is unknown) }{ }^{153,86,126} \text {. }\end{array}$ & $\begin{array}{l}\text { Chol, } 2^{\prime}-\mathrm{O}-\mathrm{DNP}, \\
\text { folate }\end{array}$ \\
\hline Target cellular uptake & $\begin{array}{l}\text { To increase the specificity of uptake by the } \\
\text { attachment of the molecules with high binding } \\
\text { affinity to surface of the cells of particular organs and } \\
\text { tissues }^{154} \text {. }\end{array}$ & $\begin{array}{l}\text { Peptides, } \\
\text { antibodies, } \\
\text { aptamers }\end{array}$ \\
\hline Specificity of action & $\begin{array}{l}\text { To increase the specificity of the silencing by: } 1 \text { ) the } \\
\text { increase of duplex thermoasymmetry }{ }^{99,105,107,120 ; 2)} \\
\text { alteration of binding affinity of the antisense strand } \\
\text { of siRNA to mRNA-target }{ }^{155,99,82} ; 3 \text { ) the absence of } \\
\text { receptors providing the activation of immune } \\
\text { response }{ }^{64} ; 4 \text { ) blocking the phosphorylation of the } 5^{\prime} \text { - } \\
\text { OH group of the sense strand of siRNA }{ }^{123} \text {. }\end{array}$ & $\begin{array}{l}\text { 1) see above; } \\
\text { 2) UNA, 2'-O-Me, } \\
\text { deoxyribonucleotides } \\
\text { 3) 2'-O-Me } \\
\text { 4) 2'-O-Me }\end{array}$ \\
\hline
\end{tabular}

Table 3. Influence of the chemical modifications on siRNA properties 
phosphorylation; 3) low thermostability of the 5 '-end of the antisense strand, hence, the modification has to increase the favorable thermoasymmetry or has no effect on the duplex thermostability.

The information on the influence of chemical modifications on siRNA properties is summarized in the table 3 .

\section{The impact of the siRNA structure on the efficiency of RNAi}

The investigation of the structures of natural siRNAs formed by dsRNA processing with Dicer and endogenous miRNA revealed the common siRNA structures. "Classical" small interfering RNA resembles duplexes 18 - 23 bp in length [18, 22, 156, 157] with $2 \mathrm{nt}$ overhangs at 3'-ends [40, 45] (fig. 9).

However, it was clearly demonstrated, that the silencing activity of structurally similar duplexes with different sequences varies significantly. As it was mentioned above thermodynamic asymmetry of the duplex and to a lesser extend the structure of mRNA target determines the siRNA activity [44, 45, 57, 58,60]. Possibility to optimize the duplex structure was demonstrated in a number of publications. Introduction of nucleotide substitutions in siRNA, resulting in a formation of non-canonical base pairs or mismatches $[38,158,159]$ is a perspective approach for the optimization of the thermodynamic properties of the duplex. The other approach utilize the inactivation of the sense strand of siRNA (for example, segmentation) to guarantee the incorporation of the antisense strand into activated RISC ${ }^{*}$ independent from the thermodynamic properties of the duplex [99]. The silencing activity of siRNA can be increased by the lengthening of the duplex and converting siRNA into Dicer substrate DsiRNA [160,161] or by the modifications of the overhands affecting duplex thermodynamic asymmetry [162]. Single stranded analogs of siRNA can be also used as inducers of RNAi, in this case the problem of the strand selection does not exist [40,77]. All mentioned approaches will be described in details below.

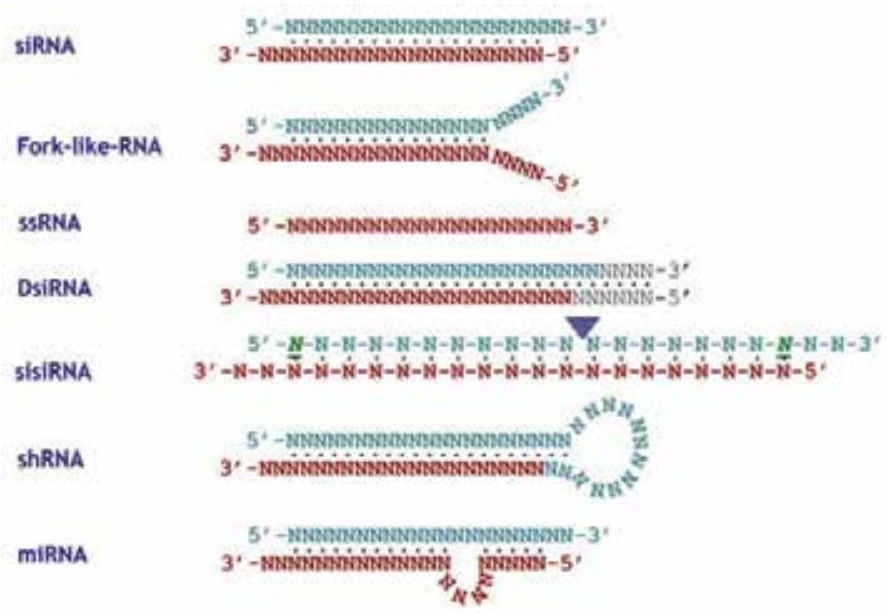

Figure 9. Structural repertoire of siRNA. The triangle indicates single stranded nick. 


\subsection{The influence of nucleotide substitutions on the silencing activity of siRNA}

Thermodynamic properties of siRNA play a key role on the stages of RISC activation and mRNA target cleavage, determining the efficiency of strand dissociation, strand selection and mRNA cleavage. Since that, nucleotide substitutions affecting thermodynamic stability of the duplex could change the silencing activity of siRNA. The nature of the changes depends on the type of substitutions and the location of the substitutions in siRNA structure. The data on the impact of nucleotide substitutions in the silencing activities of siRNAs reported in the literature are summarized in Table 4.

The kinetics of mRNA cleavage directed by anti-F.luciferase and anti-sod1 siRNA, containing nucleotide substitutions in one of the strands of the duplex were exanimated in details in [38]. It was shown, that the substitution of cytosine with uracil at the 5 '-end of the sense strand of anti-F.luciferase siRNA, resulting in U:G pair formation (stabilized by two hydrogen bonds)[163]), reduced the target mRNA cleavage rate. At the same time, the rate of the antisense RNA (complementary to the first target) cleavage increased substantially. The analysis of the binding of siRNA strands with RISC* revealed, that single nucleotide substitution at the position 1 of the sense strand reduce the incorporation of the antisense strand in the complex, whereas the efficiency of the sense strand incorporation into RISC* increased (fig. $10 \mathrm{~A})$.

Nucleotide substitution at the 3 '-end of the sense strand of anti-sod1 siRNA resulting in the formation of A:G mismatch (fig. $10 \mathrm{~B}$ ), caused the opposite effect: the rate of mRNA cleavage increased, while the rate of antisense mRNA cleavage decreased. This result indicates that the efficiency of the sense strand incorporation into RISC* decreases. The observed effect could be caused both by preferential incorporation of the antisense strand into RISC complex, and by non-perfect complementarity between the sense strand of siRNA and its target (antisense mRNA), but the results of the additional experiments with the substitution of $\mathrm{G}$ by $\mathrm{C}$ at the $5^{\prime}$-end of the antisense strand (fig. $10 \mathrm{~B}$ ) verified the key role of preferential RISC loading [38]. Thus, it was clearly demonstrated, that single nucleotide substitutions at the flank regions of siRNA could change the thermodynamic asymmetry and to determine the preferential incorporation of one strand into RISC*: destabilization of the duplex at the $3^{\prime}$-end of the sense strand increase the activity of siRNA, the destabilization of the opposite end of the duplex, reduce the silencing activity [38].

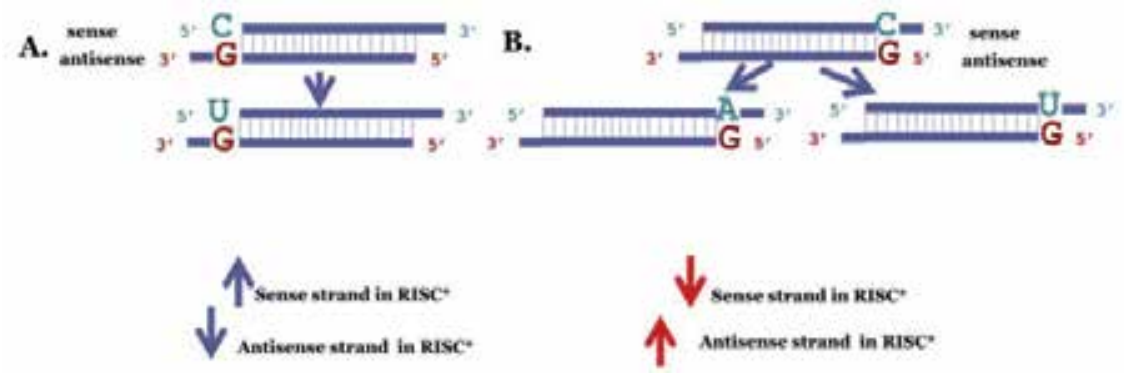

Figure 10. The scheme of the experiment described in ${ }^{38}$. A. Substitution at the $5^{\prime}$-end of the sense strand of anti-F.luciferase siPHK. B. Substitution at the $3^{\prime}$-end of the sense strand (left) or at the 5'-end of the antisense strand (right) of anti-sod1 siRNA. 


\begin{tabular}{|c|c|c|c|c|}
\hline Target, model system & $\begin{array}{l}\text { Type of } \\
\text { siRNA }^{1)}\end{array}$ & Substitutions ${ }^{2,3)}$ & $\begin{array}{c}\text { Silencing efficiency, } \\
\%\end{array}$ & Reference \\
\hline $\begin{array}{l}\text { P.luciferase, } \\
(+2 /+23), \\
\text { plasmid, transfected into } \\
\text { Hela cells }\end{array}$ & $\begin{array}{l}1 \\
2\end{array}$ & $\begin{array}{l}- \\
\mathrm{G}_{1} \mathrm{G} \rightarrow \mathrm{U}_{1} \mathrm{U} \\
\mathrm{A}_{19} \rightarrow \mathrm{U}_{19} \\
\mathrm{U}_{18} \mathrm{~A} \rightarrow \mathrm{A}_{18} \mathrm{U} \\
\mathrm{A}_{17} \mathrm{UA} \rightarrow \mathrm{U}_{17} \mathrm{AU} \\
\mathrm{C}_{16} \mathrm{AUA} \rightarrow \mathrm{U}_{16} \mathrm{UAU} \\
\mathrm{U}_{18} \mathrm{~A} \rightarrow \mathrm{A}_{18} \mathrm{U}, \mathrm{G}_{12} \rightarrow \mathrm{U}_{12}\end{array}$ & $\begin{array}{c}98 \\
94.5 \\
99.5 \\
99.8 \\
99.8 \\
99.6 \\
99 \\
\end{array}$ & 158 \\
\hline $\begin{array}{l}\text { P.luciferase, } \\
(+21 /+43), \\
\text { plasmid, transfected into } \\
\text { Hela cells }\end{array}$ & $\begin{array}{l}1 \\
2\end{array}$ & $\begin{array}{l}- \\
\mathrm{A}_{1} \mathrm{C} \rightarrow \mathrm{U}_{1} \mathrm{~A} \\
\mathrm{C}_{19} \rightarrow \mathrm{U}_{19} \\
\mathrm{G}_{18} \mathrm{C} \rightarrow \mathrm{U}_{18} \mathrm{U} \\
\mathrm{U}_{17} \mathrm{GC} \rightarrow \mathrm{A}_{17} \mathrm{UU} \\
\mathrm{C}_{16} \mathrm{UGC} \rightarrow \mathrm{U}_{16} \mathrm{AUU} \\
\mathrm{G}_{18} \mathrm{C} \rightarrow \mathrm{U}_{18} \mathrm{U}, \mathrm{G}_{12} \rightarrow \mathrm{U}_{12}\end{array}$ & $\begin{array}{c}50 \\
8 \\
76 \\
78 \\
75 \\
70 \\
78 \\
\end{array}$ & \\
\hline $\begin{array}{l}\operatorname{Lamin} A / C, \\
(+829 /+851) \\
\text { Hela cells }\end{array}$ & $\begin{array}{l}1 \\
2\end{array}$ & $\mathrm{~A}_{18} \mathrm{C} \rightarrow \mathrm{U}_{18} \mathrm{U}, \mathrm{A}_{12} \rightarrow \mathrm{U}_{12}$ & $\begin{array}{l}67.5 \\
79.5\end{array}$ & \\
\hline $\begin{array}{l}\operatorname{Lamin} A / C, \\
(+608 /+630) \\
\text { Hela cells }\end{array}$ & $\begin{array}{l}1 \\
2\end{array}$ & $\bar{C}_{18} \mathrm{~A} \rightarrow \mathrm{U}_{18} \mathrm{U}, \mathrm{U}_{12} \rightarrow \mathrm{A}_{12}$ & $\begin{array}{l}79 \\
92\end{array}$ & \\
\hline $\begin{array}{l}\text { Dnm T1, } \\
(+70 /+89) \\
\text { Hela cells }\end{array}$ & $\begin{array}{l}1 \\
2\end{array}$ & $\mathrm{G}_{18} \mathrm{~A} \rightarrow \mathrm{U}_{18} \mathrm{U}, \mathrm{G}_{12} \rightarrow \mathrm{U}_{12}$ & $\begin{array}{l}77.5 \\
84.5\end{array}$ & \\
\hline $\begin{array}{l}\text { Dnm T1, } \\
(+185 /+203) \\
\text { Hela cells }\end{array}$ & $\begin{array}{l}1 \\
2\end{array}$ & $\mathrm{U}_{18} \mathrm{U} \rightarrow \mathrm{A}_{18} \mathrm{~A}, \mathrm{G}_{12} \rightarrow \mathrm{U}_{12}$ & $\begin{array}{l}82.5 \\
89.5\end{array}$ & \\
\hline $\begin{array}{l}\text { R.luciferase/P.luciferase c }{ }^{4}{ }^{4)} \\
\text { plasmid, transfected into } \\
\text { Hela cells }\end{array}$ & $\begin{array}{l}1 \\
2\end{array}$ & $\begin{array}{l}\mathrm{A}_{1} \mathrm{C} \rightarrow \mathrm{U}_{1} \mathrm{~A} \\
\mathrm{G}_{18} \mathrm{C} \rightarrow \mathrm{U}_{18} \mathrm{U}\end{array}$ & $\begin{array}{l}86 \\
72 \\
95\end{array}$ & 159 \\
\hline $\begin{array}{l}\text { R.luciferase/ P.luciferase a } \\
\text { plasmid, transfected into } \\
\text { Hela cells }\end{array}$ & $\begin{array}{l}1 \\
2\end{array}$ & $\begin{array}{l}C_{18} A \rightarrow A_{18} U \\
C_{1} G \rightarrow U_{1} U\end{array}$ & $\begin{array}{l}92 \\
89 \\
20\end{array}$ & \\
\hline $\begin{array}{l}\text { F.luciferase, } \\
\text { plasmid, transfected into } \\
\text { NIH3T3 cells }\end{array}$ & 1 & $\begin{array}{l}G_{9} \rightarrow C_{9} \\
G_{6,9} \rightarrow C_{6,9} \\
G_{6,9,13} \rightarrow C_{6,9,13} \\
G_{3,6,9,13,17} \rightarrow C_{3,6,9,13,17}\end{array}$ & $\begin{array}{l}60 \\
57 \\
56 \\
30 \\
15\end{array}$ & 74 \\
\hline $\begin{array}{l}\text { MGC29643, } \\
(+576 /+596) \\
\text { Hela cells }\end{array}$ & 1 & $\begin{array}{l}C_{8} \rightarrow G_{8} \\
A_{12} \rightarrow \mathrm{U}_{12} \\
C_{8,} \mathrm{~A}_{12} \rightarrow \mathrm{G}_{8}, \mathrm{U}_{12} \\
\end{array}$ & $\begin{array}{c}50 \\
10 \\
55 \\
3 \\
\end{array}$ & 166 \\
\hline $\begin{array}{l}\text { GPR39, } \\
(M G C 29643 \\
\left.(+576 /+596)^{a}\right) \\
\text { Hela cells } \\
\end{array}$ & 1 & $\begin{array}{l}- \\
\mathrm{C}_{12} \rightarrow \mathrm{G}_{12} \\
A_{8} \rightarrow U_{8} \\
A_{8,} \mathrm{C}_{12} \rightarrow U_{8,} \mathrm{G}_{12} \\
\end{array}$ & $\begin{array}{c}50 \\
55 \\
5 \\
0 \\
\end{array}$ & \\
\hline $\begin{array}{l}\text { Jagged }-1, \\
(+3382 /+3402) \\
\text { plasmid, transfected into } \\
\text { HEK 293T cells }\end{array}$ & 1 & $\begin{array}{l}C_{18} \rightarrow U_{18} \\
C_{5} A_{12} C_{15} \rightarrow U_{5} G_{12} U_{15}\end{array}$ & $\begin{array}{c}0 \\
50 \\
0\end{array}$ & 165 \\
\hline
\end{tabular}




\begin{tabular}{|c|c|c|c|c|}
\hline Target, model system & $\begin{array}{l}\text { Type of } \\
\text { siRNA }{ }^{1)}\end{array}$ & Substitutions ${ }^{2,3)}$ & $\begin{array}{c}\text { Silencing efficiency, } \\
\%\end{array}$ & Reference \\
\hline $\begin{array}{l}\text { sod1/F.luciferase c, } \\
\text { plasmid, transfected into } \\
\text { HEK } 293 \text { cells }\end{array}$ & 1 & $\begin{array}{l}- \\
\mathrm{G}_{1} \rightarrow \mathrm{U}_{1} \\
\mathrm{C}_{19} \rightarrow \mathrm{A}_{19} \\
\mathrm{G}_{1} \rightarrow \mathrm{U}_{1} \\
\mathrm{C}_{19} \rightarrow A_{19}\end{array}$ & $\begin{array}{c}55 \\
0 \\
70 \\
70 \\
0\end{array}$ & \multirow[t]{2}{*}{164} \\
\hline $\begin{array}{l}\text { sod1/F.luciferase }{ }^{\text {a }} \\
\text { plasmid, transfected into } \\
\text { HEK } 293 \text { cells }\end{array}$ & 1 & $\begin{array}{l} \\
\mathrm{G}_{1} \rightarrow \mathrm{U}_{1} \\
\mathrm{C}_{19} \rightarrow \mathrm{A}_{19} \\
\mathrm{G}_{1} \rightarrow \mathrm{U}_{1} \\
\mathrm{C}_{19} \rightarrow A_{19} \\
\end{array}$ & $\begin{array}{l}75 \\
75 \\
45 \\
40 \\
80 \\
\end{array}$ & \\
\hline $\begin{array}{l}\text { APP }_{(\mathrm{L})} / \text { luciferase }^{5)} \\
\text { plasmid, transfected into } \\
\text { HEK } 293 \text { cells }\end{array}$ & 2 & $-\bar{C}{ }_{18} \mathrm{C} \rightarrow \mathrm{U}_{18} \mathrm{U}$ & $\begin{array}{c}0 \\
45\end{array}$ & 159 \\
\hline $\begin{array}{l}\text { APP }_{(\mathrm{S})} / \text { luciferase, } \\
\text { plasmid, transfected into } \\
\text { HEK } 293 \text { cells }\end{array}$ & 2 & $\overline{\mathrm{G}}_{18} \mathrm{C} \rightarrow \mathrm{U}_{18} \mathrm{~A}$ & $\begin{array}{c}0 \\
80\end{array}$ & 159 \\
\hline $\begin{array}{l}\text { EGFP, } \\
(+306 /+324) \\
\text { human lung carcinoma } \\
\text { H1299 }\end{array}$ & 1 & $\mathrm{G}_{19} \rightarrow \mathrm{C}_{19}$ & $\begin{array}{l}55 \\
50\end{array}$ & \multirow[t]{2}{*}{24} \\
\hline $\begin{array}{l}\text { EGFP, } \\
(+396 /+414) \\
\text { human lung carcinoma } \\
\text { H1299 }\end{array}$ & 1 & $\begin{array}{l}- \\
\mathrm{G}_{19} \rightarrow \mathrm{A}_{19} \\
\mathrm{G}_{19} \rightarrow \mathrm{C}_{19} \\
\mathrm{G}_{19} \rightarrow \mathrm{U}_{19} \\
\mathrm{C}_{19} \rightarrow A_{19} \\
\mathrm{C}_{19} \rightarrow \mathrm{U}_{19} \\
\mathrm{C}_{19} \rightarrow \mathrm{G}_{19} \\
\end{array}$ & $\begin{array}{l}65 \\
42 \\
40 \\
35 \\
52 \\
45 \\
45 \\
\end{array}$ & \\
\hline $\begin{array}{l}\text { F.luciferase, } \\
\text { plasmid, transfected into } \\
\text { Hela SS6 cells }\end{array}$ & 1 & $\begin{array}{l}- \\
\mathrm{C}_{1} \rightarrow \mathrm{A}_{1} \\
\mathrm{~A}_{19} \rightarrow \mathrm{C}_{19} \\
\mathrm{~A}_{19} \rightarrow \mathrm{G}_{19} \\
\mathrm{~A}_{19} \rightarrow \mathrm{U}_{19} \\
\mathrm{G}_{9} \rightarrow \mathrm{A}_{9} \\
\mathrm{G}_{9} \rightarrow \mathrm{C}_{9} \\
\mathrm{G}_{9} \rightarrow \mathrm{U}_{9} \\
\mathrm{~A}_{10} \rightarrow \mathrm{C}_{10} \\
\text { other substitutions in the sense } \\
\text { strand (2-8, } 12-18 \text { positions) }\end{array}$ & $\begin{array}{c}1^{\mathrm{b}} \\
0.3 \\
1.15 \\
1.2 \\
1.25 \\
4.5 \\
3.3 \\
2.15 \\
2.3 \\
0.2-1.3\end{array}$ & 36 \\
\hline
\end{tabular}

1)

\begin{tabular}{|c|c|c|c|}
\hline $\begin{array}{l}\text { siRNA } \\
\text { type }\end{array}$ & Structure of siRNA & Strand & $\begin{array}{l}\text { Length of the } \\
\text { strand, nt }\end{array}$ \\
\hline \multirow{2}{*}{1} & 5'-NNNNNNNNNNNNNNNNNNNNN-3' & sense & 21 \\
\hline & 3'-NNNNNNNNNNNNNNNNNNNNN-5' & antisense & 21 \\
\hline \multirow{2}{*}{2} & 5'-NNNNNNNNNNNNNNNNNNN-3' & sense & 19 \\
\hline & 3'-NNNNNNNNNNNNNNNNNNNNN-5' & antisense & 21 \\
\hline
\end{tabular}

2) Numeration of the nucleotides from the 5 '-end.

3) Substitutions in the sense strand are presented in normal letters; substitutions in the antisense strand are presented in italic.

4) P.luciferase s(a) - a gene or a fragment of gene encoded sense (s) or antisense (a) RNA.

5) APPL(S)/luciferase - recombinant gene, containing mutant London (L) or Swedish (s) type of APP allele.

b) Silencing activity of structurally modified siRNA is presented as the ration of IC50 (primary siRNA) / IC50 (modified siRNA), where IC50 is the concentration of siRNA, inducing -50\% silencing of the target gene.

Table 4. The influence of the nucleotide substitutions in siRNA on the efficiency of RNAi 
The elevation of the thermodynamic asymmetry by single nucleotide substitution at the 1th position of the antisense strand was reported to increase or have no influence on the silencing activity of different siRNAs, targeted to sod1/F.luciferase mRNA or the complementary sequence (antisense mRNA) [164] (Table 4). In turn, the equal efficiency of the cleavage of $h t t$ mRNA and $h t t$ antisense mRNA by thermodynamically symmetrical siRNA suggests the equal probability of sense or antisense strand incorporation into RISC* [38].

Introduction of the nucleotide substitutions at the $3^{\prime}$-end of the sense strand of siRNA duplex resulted in the formation of the mismatches, has led to the creation of the structurally new class of small interfering RNAs - fork-like siRNAs (fsiRNAs) [158]. More effective silencing of the expression of R.luciferase/P.luciferase gene by fsiRNAs then by classical siRNA targeted to the same sequences was demonstrated in Hela cells (Table 4). The most pronounced augmentation of the activity was observed for siRNA with initially moderate activity [158]. Fork-like siRNA with two nucleotide substitutions at the 3 '-end of the sense strand was found to be the most active [11, 158, 159] (Table 4). Introduction of from 2 to 4 nucleotide substitutions at the 3 '-end of the sense strand of siRNA targeted sod1 and $h t t$ mRNAa correspondingly increased the efficiency of silencing [38].

The increase of the thermodynamic asymmetry of the duplex by the introduction of the mismatches at the ends of the strands cannot guarantee the positive influence on the activity. It is known, that the region of the "guide" strand spanning from 2 to 6 position determines the efficiency of the interaction of siRNA with Ago2 [21, 30, 31]; this fact limits the introduction of substitutions in this region. Nevertheless, $C \rightarrow U$ substitution in $2^{\mathrm{d}}$ position of the antisense strand of siRNA targeted Jagged-1 mRNA increased the silencing effect from 0 to $50 \%$ in HEK $293 \mathrm{~T}$ cells transfected with vector, containing cDNA fragment of the target gene [165] (Table 4). This data suggests, that the substitutions in the antisense strand resulted in the formation of non-canonical base pairs is more tolerable than the substitutions resulted in the formation of mismatches. In the other study, single nucleotide substitutions at $19^{\text {th }}$ position of the sense strand or at the $1^{\text {st }}$ position of the antisense strand resulting in the formation of the mismatches as well as non-canonical base-pairs reduced the activity of anti-EGFP siRNA in H1299 cells. It was assumed that the reduction of the efficiency of the interaction of TRBP (but not the Dicer) with siRNA was the possible reason of the observed effect [24] (Table 4).

Introduction of nucleotide substitutions in the central part of siRNA duplex could be used for the destabilization the central region and the subsequent elevation of the silencing activity. Nevertheless, this type of structure modification could switch the mode of siRNA action to miRNA-like mechanism [167] (fig. 4). It was demonstrated in experiments on HeLa cells, that substitutions in $9^{\text {th }}$ and $10^{\text {th }}$ positions of the sense strand of siRNA leads to the 2 - 4.5-fold increase of IC50 as compared with IC50 for siRNA with classical duplex 36. Substitutions in the sense strand or in both strands of siRNA [167] reduced the efficiency of RNAi (Table 4).

Thus, the introduction of nucleotide substitutions could be successfully used for the design of siRNA characterized by favorable thermodynamic asymmetry and low thermostability of the central part of the duplex. This approach can be applied for the creation active siRNA 
targeted to any desired sequence for silencing of mutated or chimerical genes. However, there is not enough data publish up to date for the formulation of the universal rules for the design of fork-like siRNA and siRNA with destabilized center, the activity of resulted siRNA cannot be predicted and should be determined experimentally.

\subsection{The influence of the siRNA overhangs structure on the efficiency of gene silencing}

2 - 3 nt overhangs at the $3^{\prime}$-ends of siRNA are a distinguishing feature of both natural siRNA generated by Dicer and synthetic siRNA [40, 45, 162]. The overhangs are important for the interaction of siRNA with PAZ-domens of Dicer and Ago2 [21, 27, 168]. However, the data on the activity of siRNA with blunt ends (with no overhangs) are rather contradictory: in mammalian cells the activity of this type of siRNA did not vary significantly from the activity of siRNA with 2-nt overhangs targeted to the same sequence [59], controversially, in extracts from drosophila cells blunt ends siRNA were less active [40, 45]. The assumption that observed difference could be accounted for the difference in the mechanism of action of heterodimer Dicer-R2D2 in drosophila and its analogue Dicer-TRBP in humans [59], did not find the experimental support, since it was reported recently, that in HeLa S3 the activity of the blunt ended siRNA was similarly lower (60\% silencing) than the activity of classical siRNA (80 $\%$ silencing) [162]. Moreover, the presence of $2 \mathrm{nt}$ overhang at the $3^{\prime}$-end of only one of the strands leads to the selection of this strand as a guide strand due to the preferential loading into RISC ${ }^{*}[162]$. The presence of the mismatches at the $5^{\prime}$-end of the antisense strand resulting in the formation of thermodynamically asymmetrical duplex increases the efficiency of the incorporation of antisense strand, containing 2 nt overhang, into RISC*. Efficient silencing of the target gene expression by fork-like structurally asymmetrical siRNA was achieved in a number of studies conducted on HeLa cells (Table 4, 2nd type of siRNA).

It was found the $1 \mathrm{nt}$ shortening of the overhang ( $1 \mathrm{nt}$ overhang instead of 2 ) at the 3 ' end in some cases did not affect significantly the silencing activity [40]. The sequential lengthening of the 3'-end overhang in the sense or the antisense strand up to $7 \mathrm{nt}$ induced the substantial reduction of the silencing activity of siRNA [40], probably due to inefficient interaction of this siRNAs with PAZ-domen of Dicer [29]. siRNAs, containing overhangs at the 5 '-ends of the duplex were inactive in RNAi [40], and induced side effects related to the PKR-mediated activation of the innate immunity [169].

Therefore, exploitation of the structurally asymmetrical siRNAs, containing 2 nt overhang only at the $3^{\prime}$-end of the antisense strand together with the reduction of the thermodynamic stability of the duplex at the $5^{\prime}$-end region of the antisense strand could be successfully used in siRNA design for increasing the efficiency of RNAi.

\subsection{Single stranded analogous of siRNA (ss-siRNA)}

Before the discovery of RNAi, it was demonstrated that long (several hundred bases in length) single stranded RNA are capable of sequence specific silencing of the expression of 
homologous genes in C.elegans [170]. Later, it was assumed that the minor fraction of dsRNA in the preparations of ssRNA obtained by in vitro transcription was the agent that silence the expression of the target genes 1 . However, the ability of antisense, but not sense ssRNA (22 - $40 \mathrm{nt})$ to induce gene silencing was experimentally verified in C.elegans [171]. It was demonstrated that mRNA degradation induced by ssRNA proceeds via RISC* assembling (fig. 9) [131,122]. The evaluation of the silencing activity of anti-luciferase sssiRNA in HeLa cells demonstrated, that the required concentration of ss-siRNA was 8-fold higher, than the concentration of classical siRNA to reach the similar level of gene silencing. In the other report, it was shown, that the concentration of ss-siRNA essential for the RISC assembling was 10 - 100 higher than the concentration of double stranded siRNA analogous [122]. High sensitivity of single stranded RNA to ribonucleases [62], and less efficient interaction with the RNAi machinery proteins [131,122] were supposed to be the possible reasons of the lower efficiency of RNAi induction by ssRNA.

Thus, the application of non-modified ss-siRNA as inhibitors of gene expression is not beneficial in comparison with double stranded siRNA.

\section{4. dsRNA (27-30-mers) as Dicer substrates}

During the search of the effective inductors of RNAi, able to silence target gene at low (nanomolar and lower) concentrations, it was found that dsRNAs 25-30 bp in length which are substrates of Dicer (here and after DsiRNA - Dicer-substrate siPHK), are significantly more active in comparison with "classic" or "conventional" siRNAs [161, 172, 173, 174]. To compare interfering activity of DsiRNA and conventional siRNA the duplexes of different length (21-30 bp) and structure (presence or absence of $3^{\prime}$ or $5^{\prime}$ dinucleotide overhangs) targeting EGFP were used [174]. The interfering activity of dsRNA was evaluated at concentration ranging from $50 \mathrm{pMol}$ to $50 \mathrm{nMol}$ using HEK293 cells transfected with the plasmid encoded EGFP. It was shown that at subnanomolar concentrations (50 pM- 200 $p M)$ DsiRNAs were significantly more active than siRNA. Among duplexes tested the DsiRNA $27 \mathrm{bp}$ in length with blunt ends displays the highest interfering activity: this DsiRNA (1 $\mathrm{nM}$ ) inhibited expression of the target gene by $95 \%$ (Table 5) while corresponding siRNA was almost inactive [174]. It turned out, that this DsiRNA (27 bp, blunt ends) is processed by Dicer yielding pull of different siRNAs $21 \mathrm{bp}$ in length. Seven siRNAs (21 bp with 3'-dinucleotide overhangs) generated by single-nucleotide shift along the EGFP mRNA sequence homologous to DsiRNA were synthesized to answer the question can the synthetic siRNA be as active as this particular DsiRNA. It was shown that at the concentrations $50 \mathrm{pM}$ and $200 \mathrm{pM}$ neither each synthetic siRNA nor pull of synthetic siRNAs silence expression of EGFP as efficiently as corresponding DsiRNA. Interestingly, in the case if DsiRNA was processed by Dicer in vitro and then transfected into the cells the silencing efficiency of this processed DsiRNA was similar to the activity of synthetic siRNAs [174]. It has been suggested, than after DsiRNA cleavage Dicer did not dissociated from the complex with DsiRNA and thus governed the mode of interaction of R2D2 (D.melanogaster) or TRBP (H.sapiens) and, as a consequence, the unequivocal orientation of siRNA within the RISC. This provides for incorporation into the RISC the antisense strand of the duplex. 
However, if an equivocal binding of Dicer with dsRNA takes place which results in a formation of a set of siRNA cleavage products, the possibility of formation of incompetent RISC $^{*}$, containing sense strand of siRNA as a leading strand is increased. Thus, optimization of a structure of DsiRNA is a current task.

It is known, that one of the functions of Dicer within the cell is a processing of pre-miRNA via binding with their $3^{\prime}$-end, containing two hanging nucleotides followed by cleavage of the duplex at a distance of 21-22 nucleotides from the 3'-end [172]. Taking into account that Dicer has a low affinity to DNA, a set of asymmetric dsRNA was synthesized, containing at the 3 '-end of the sense or antisense strand two nucleotides overhang, while the opposite end was blocked for Dicer binding by the replacement of two ribonucleotides in the complementary strand by deoxyribonucleotides [161, 172] (Table 5, structures 25D/27 and $27 / 25 D$ ). It was anticipated that such a structure of DsiRNAs provide for an unequivocal binding of the duplex with Dicer.

According to mass-spectrometry the cleavage of these DsiRNA by Dicer (Table 5, structures 25D/27 and 27/25D) yields the same product in both cases [161]. However, the silencing activity of siRNAs derived from these DsiRNAs was different; which possibly is explained by the peculiarities of DsiRNA interactions with Dicer, determined the efficiency of sense or antisense incorporation into RISC*.

\begin{tabular}{|c|c|c|c|c|}
\hline Target gene / model system & $\begin{array}{l}\text { Optimal or the only } \\
\text { studied concentration } \\
\text { of DsiRNA, nM }\end{array}$ & $\begin{array}{l}\text { DsiRNA structure } \\
\text { peculiarities }\end{array}$ & $\begin{array}{l}\text { Level of target gene } \\
\text { silencing, } \%\end{array}$ & Ref \\
\hline $\begin{array}{c}\text { EGFP, } \\
\text { Plasmid DNA encoded } \\
\text { EGFP transfected in HEK } \\
293 \text { cells }\end{array}$ & 50 & $\left.\begin{array}{c}21+2 \text { 1) } \\
23-2 \\
23+2 \\
24-2 \\
24+2 \\
25-2 ; 25+2 ; 26+0 ; \\
27+0 ; 27+2 ; 27-2\end{array}\right\}$ & $\begin{array}{c}72 \\
80 \\
98 \\
82 \\
95 \\
\sim 100\end{array}$ & \multirow[t]{2}{*}{174} \\
\hline $\begin{array}{c}\text { EGFP, } \\
\text { Endogenous gene in } \\
\text { NIH3T3 cells }\end{array}$ & 1 & $\begin{array}{l}27+0 \\
30+0 \\
35+0 \\
40+0 \\
45+0\end{array}$ & $\begin{array}{c}98 \\
98 \\
97 \\
2 \\
<1\end{array}$ & \\
\hline $\begin{array}{c}\text { EGFP, } \\
\text { Plasmid DNA encoded } \\
\text { EGFP transfected in HEK } \\
293 \text { cells } \\
\end{array}$ & 1 & $\begin{array}{c}19+2 \\
27+0 \\
27 / 25 \mathrm{D}^{2)} \\
25 \mathrm{D} / 27 \\
\end{array}$ & $\begin{array}{l}<1 \\
68 \\
53 \\
95\end{array}$ & \multirow[t]{4}{*}{161} \\
\hline $\begin{array}{c}\text { F.luciferase } \mathbf{1}^{3)} \text {, } \\
\text { Plasmid DNA transfected in } \\
\text { HEK } 293 \text { cells }\end{array}$ & 10 & $\begin{array}{l}27 / 25 \mathrm{D} \\
25 \mathrm{D} / 27\end{array}$ & $\begin{array}{l}80 \\
97\end{array}$ & \\
\hline $\begin{array}{c}\text { F.luciferase } \mathbf{2}^{3)} \\
\text { Plasmid DNA transfected in } \\
\text { HEK } 293 \text { cells }\end{array}$ & & $\begin{array}{l}27 / 25 \mathrm{D} \\
25 \mathrm{D} / 27\end{array}$ & $\begin{array}{l}97 \\
96\end{array}$ & \\
\hline $\begin{array}{c}\text { F.luciferase } \mathbf{3}^{3} \text { ) } \\
\text { Plasmid DNA transfected in } \\
\text { HEK } 293 \text { cells }\end{array}$ & & $\begin{array}{l}27 / 25 \mathrm{D} \\
25 \mathrm{D} / 27\end{array}$ & $\begin{array}{l}23 \\
78\end{array}$ & \\
\hline
\end{tabular}




\begin{tabular}{|c|c|c|c|c|}
\hline Target gene / model system & $\begin{array}{c}\text { Optimal or the only } \\
\text { studied concentration } \\
\text { of DsiRNA, } \mathbf{n M}\end{array}$ & $\begin{array}{c}\text { DsiRNA structure } \\
\text { peculiarities }\end{array}$ & $\begin{array}{c}\text { Level of target gene } \\
\text { silencing, } \%\end{array}$ & Ref \\
\hline $\begin{array}{c}\text { F.luciferase } \mathbf{4}^{3)} \\
\text { Plasmid DNA transfected in }\end{array}$ & & $27 / 25 \mathrm{D}$ & 95 & 94 \\
HEK 293 cells & 10 & $25 \mathrm{D} / 27$ & 30 & 172 \\
\hline La antigene in & 1 & $27 / 25 \mathrm{D}$ & 82 & 173 \\
HEK 293 cells & & $25 \mathrm{D} / 27$ & 95 & \\
\hline STAT1, endogenous gene in & & & & \\
HeLa cells & & & & \\
\hline
\end{tabular}

1) The structures of dsRNA - substrates of Dicer: L+2 - duplex of L bp with two nucleotide overhangs at the 3'- ends, L-2 - duplex of L bp with two nucleotides overhangs at the 5 ' ends, L+0 - duplex of L bp with blunt ends where L varied from 19 to $45 \mathrm{bp}$.

\begin{tabular}{|c|c|c|}
\hline $\begin{array}{l}\text { The structure } \\
\text { of DsiRNA }\end{array}$ & Scheme of DsiRNA & Length of the strand (L), bp \\
\hline $\mathrm{L}+2$ & $5^{\prime}-$ NNNNNNNNNNNNNNNNNNNNNNN-3' & 23 \\
\hline L-2 & $\begin{array}{c}5^{\prime}-\text { NNNNNNNNNNNNNNNNNNNNNNNNN }-3 ' \\
3^{\prime}-\text {-NNNNNNNNNNNNNNNNNNNNNNNN-5' }\end{array}$ & 24 \\
\hline $\mathrm{L}+0$ & $\begin{array}{l}5^{\prime}-\text { NNNNNNNNNNNNNNNNNNNNNNNNNN }-3^{\prime} \\
3^{\prime}-\text { NNNNNNNNNNNNNNNNNNNNNNNNNN-5' }\end{array}$ & $\begin{array}{l}26 \\
26\end{array}$ \\
\hline
\end{tabular}

2) Asymmetric DsiRNA (25 bp), containing at the 3 '-end of the sense (antisense) strand 2 nt overhang, the opposite end was blocked by replacement of two ribonucleotides with deoxyribonucleotides $-\underline{\mathbf{N}}$.

\begin{tabular}{|c|c|c|c|}
\hline $\begin{array}{c}\text { The structure } \\
\text { of DsiRNA }\end{array}$ & Scheme of DsiRNA & Strand & $\begin{array}{c}\text { Length of the } \\
\text { strand (L), bp }\end{array}$ \\
\hline \multirow{2}{*}{$27 / 25 \mathrm{D}$} & 5'-NNNNNNNNNNNNNNNNNNNNNNNNNNN-3' $^{\prime}$ & sense & 27 \\
3'-NNNNNNNNNNNNNNNNNNNNNNNNN-5' & antisense & 25 \\
\hline \multirow{2}{*}{$25 \mathrm{D} / 27$} & 5'NNNNNNNNNNNNNNNNNNNNNNNNN-3' $^{\prime}$ sense & 25 \\
& 3'-NNNNNNNNNNNNNNNNNNNNNNNNNNN-5' $^{\prime}$ & antisense & 27 \\
\hline
\end{tabular}

3) The numbers correspond to different sequences within the target gene F.luciferase.

Table 5. The influence of DsiRNA structure in its silencing activity

It was shown, that in HEK293 and HeLa cells the DsiRNAs with antisense strand having dinucleotide overhang at the 3 '-end were more active than those with dinucleotide overhang at the $3^{\prime}$-end of the sense strand (Table 5) [161, 172]. Thus, it is possible to predict the structure of forming siRNAs upon processing of DsiRNA by Dicer [161, 172], however, the activity of these siRNAs will depend on the thermodynamic parameters of their duplexes. It worth mentioning that in the case of unfavorable context of the target sequence within mRNA DsiRNAs have a significant advantage over conventional siRNA [174].

The silencing activity of a longer dsRNAs with blunt ends ( $27-45 \mathrm{bp}$ ) decreases with the increase of dsRNA length. It turns out that this drop of silencing activity correlate well with the drop of efficiency of cleavage of longer dsRNAs by Dicer [174]. The silencing effect caused by DsiRNA was longer in comparison with conventional siRNA: DsiRNAs efficiently silence EGFP expression in NIH3T3 cells for 10 days while corresponding siRNA displays silence activity not longer than 4-7 days [174]. 
Despite the promising results on silence activity of Dicer substrates the optimization of the properties of DsiRNAs by chemical modification is relevant. The influence of 2'-F- and 2'-OMe modifications within DsiRNA type $25 \mathrm{D} / 27$ on the silencing activity and nuclease resistance was studied [173]. When choosing sites of modifications authors try to avoid the region needed for Dicer to execute dsRNA cleavage, despite presence there of nuclease sensitive motives (Fig. 11).

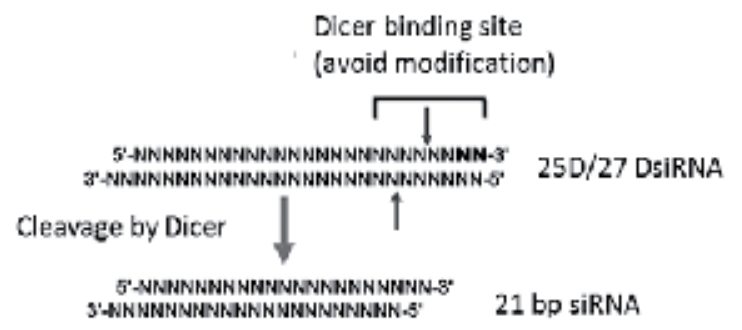

Figure 11. Design of chemically modified DsiRNA. By bold letter N deoxyribonucleotides are shown, short arrows - cleavage site of DsiRNA by Dicer.

It was shown, that chemical modification of DsiRNA affects its silencing activity in respect to STAT1 mRNA only at the lowest concentration used (100 pM), while at higher concentration of DsiRNA ( 1 and $10 \mathrm{nM}$ ) the drop of silencing activity was detected only for extensively modified molecules, containing combination of several 2'-F- and 2'-O-Me nucleotides in a row. DsiRNA containing $112^{\prime}$-O-Me nucleotides in the antisense strand was very nuclease resistant: the fraction of intact DsiRNA was found in reaction mixture after 24 $h$ of incubation in the presence of serum. However, such an extensive modification affects pattern of DsiRNA cleavage by Dicer and lead to the formation of two products of 21 and 22 $\mathrm{bp}$ instead of one product of $21 \mathrm{bp}$ formed with unmodified DsiRNA. Replacement of one or two 2'-O-Me nucleotides in the vicinity of Dicer cleavage sites by the natural ones facilitated formation of $21 \mathrm{bp}$ cleavage product. The introduction from 1 to 4 mismatches into central part of each strand of anti-EGFP DsiRNA significantly reduced its silencing activity [174].

On the other hand, chemical modification of DsiRNA can block induction of the unspecific immune response in mammalian cells, linked with the presence of triphosphate groups at the $5^{\prime}$ ends of DsiRNA or/and immunostimulating motives in the duplex [43,175, 176, 177, $178,179,180]$. So, DsiRNA, which contains in the antisense strand 10 -11 2'-O-Me nucleotides (including 2'-O-Me-cytidine and 2'-O-Me-uridine nucleotides known as the most effective blockers of interferon response activation via endosomal TLR [64]), blocked IFN- $\alpha$ secretion by human PBMC and did not induce immune response in RIG-I-dependent T98G cells opposite to unmodified DsiRNA and 2'-F-modified disPHK, respectively [173].

Full replacement of natural nucleotides by the analogs (2'-O-Me, 2'-F, etc.) is unacceptable as these DsiRNA will not be processed by Dicer [173]. Therefore, upon the introduction of chemical modifications in the structure of DsiRNA one should avoid modifying the nucleotides from 1 to 8 from $5^{\prime}$ end of the sense strand and complementary region of the sense strand (Fig. 11). Moreover, two overhanging nucleotides at the $3^{\prime}$-end of the antisense 
strand as well as two deoxyribonucleotides at the $3^{\prime}$-end of the sense strand facilitate the correct orientation of Dicer and selection of the antisense strand of DsiRNA as a leading strand.

\subsection{Small segmented RNA}

Recently another approach to enhance RNAi efficiency based on modification of siRNA structure was proposed [99]. It was shown that siRNA in which the sense strand is subdivided in two parts of 10-12 nucleotides long, are more active in comparison with conventional siRNA duplexes. The efficiency of this internally segmented siRNA or sisiRNA (small internally segmented interfering RNA) (Fig. 9) was provided by unequivocal incorporation of the antisense strand into RISC* ${ }^{*}$, since the nick in the central part of the sense strand blocked its usage as a guiding strand and facilitate the dissociation of the sense strand from the duplex [99]. Thus, sisiRNA have an advantage over conventional siRNA with unfavorable duplex thermoasymmetry. In addition unlike siRNAs with the classic structure the extensive chemical modification of antisense strand of sisiRNA has only little effect on its silencing activity. As an example, the silencing activity of anti-EGFP sisiRNA with antisense strand having 6 LNA-nucleotides or alternative 2'-F / 2'-O-Me-nucleotides and internally segmented sense strand with 6 LNA-nucleotides in each part was significantly higher than that of conventional siRNA bearing the same modifications [99]. Besides, both mentioned sisiRNA display high nuclease resistance. Thus, sisiRNAs as sequence specific inhibitors of gene expression are very promising, because they provide efficient RNAi even at the unfavorable thermodynamic parameters RNA duplex.

According to literary data the structural and thermodynamic peculiarities of interfering RNAs are a major factors providing for efficient gene silencing.

Prediction of silencing activity of any siRNA based on its thermodynamic profile is an efficient method of selection of active molecules. This approach is used in a number of software and algorithms for search and selection of such siRNA [37]. An alternative approach to the problem of creating efficient sequence-specific inhibitors of gene expression is the use of structure-modified siRNAs: fork-like siRNAs, DsiRNAs, or sisRNAs which silencing activity is less dependent on their thermodynamic parameters as compared with conventional siRNA diplex.

\section{Conclusion}

Small interfering RNAs are not optimal to be used as therapeutics in vivo, therefore, development of the approached aimed to improve or/and optimize the properties of siPHK is of significance nowadays. One of the approaches widely used is chemical modification of siRNAs. At the beginning this approach was proposed as a way to increase nuclease resistance of siRNA in the presence of serum, in the cells and bloodstream [61, 62]. The enhancement of nuclease resistance of siRNA by chemical modification not resulting in the drop of its silencing activity represent a complex task, since silencing activity of siRNA is 
strongly affected by the nature, number and locations within the duplex of chemical modifications [65, 76, 181]. Despite many works carried out in this area, a general algorithm which allows receiving active nuclease resistant siRNA is not developed yet.

Chemical modification of siRNA can be also used to develop delivery vehicles for siRNA. It is well known that naked or free siRNA is not able to penetrate through cellular membrane and accumulate in the cells. In the case of in vivo conditions, the small size of siRNA molecule promotes its rapid clearance [57]. In other words, the systems for in vivo delivery of siRNAs should provide for efficiency of cellular accumulation, specificity of delivery (if applicable), duration of silencing as well as safety and possibility of systemic administration. Today no one delivery approach encounters these criteria.

On the other hand, the low efficiency of RNAi can be a result of its unfavorable sequence determining thermodynamic properties and/or by stable secondary structure at the target site within mRNA $[37,38,51,52,54]$. There are several algorithms to select siRNAs of highperformance [182, 183, 184, 185, 186], however it is impossible to predict precisely silencing activity of any particular siRNA in vivo. In addition, the design of siRNA targeting chimeric or mutant genes represent of complex task since the limitations of target site selection does not allow to use these algorithms for choosing the sequence siRNA. This is why structure modifications of siRNA improving their thermodynamic properties are of interest. The forklike siRNA [158, 159], able to silence target gene more efficiently that conventional siRNA is a promising approach for creating high-performance siRNA targeted any sequence of the gene. Despite this, the number of works in this field is negligible so further research of properties of siRNA with modified structure are relevant.

To obtain high-performance siRNA often optimization of multiple parameters is required, so using modification of the siRNA structure together with its chemical modification is a promising approach. Thus, advanced research of chemically and/or structurally modified siRNA, as well as development and optimization of the algorithms for modification are important.

\section{Author details}

Natalya S. Petrova, Marina A. Zenkova and Elena L. Chernolovskaya

Institute of Chemical Biology and Fundamental Medicine SB RAS, Novosibirsk, Russia

\section{Acknowledgement}

This research was supported by the Russian Academy of Science under the programs Molecular and Cell Biology (grant no. 22-1) and Science to Medicine (grant no. 37), the Russian Foundation for Basic Research (grant nos. 11-04-01017-a and 11-04-12095-ofi-m2011) Ministry of Science and Education of the Russian Federation (grant no. 14.740.11.1058) and the Siberian Branch of Russian Academy of Sciences (grant no. 85). 


\section{References}

[1] Fire A., Xu S., Montgomery M. K., Kostas S. A., Driver S. E. and Mello C. C. (1998) Potent and specific genetic interference by double-stranded RNA in Caenorhabditis elegans. Nature. 391, 806-811.

[2] Bosher J. M. and Labouesse M. (2000) RNA interference: genetic wand and genetic watchdog. Nat Cell Biol. 2, E31-36.

[3] Sharp P. A. (1999) RNAi and double-strand RNA. Genes Dev. 13, 139-141.

[4] Vaucheret H. and Fagard M. (2001) Transcriptional gene silencing in plants: targets, inducers and regulators. Trends Genet. 17, 29-35.

[5] Hammond S. M., Bernstein E., Beach D. and Hannon G. J. (2000) An RNA-directed nuclease mediates post-transcriptional gene silencing in Drosophila cells. Nature. 404, 293-296.

[6] Aigner A. (2006) Gene silencing through RNA interference (RNAi) in vivo: strategies based on the direct application of siRNAs. J Biotechnol. 124, 12-25.

[7] Aronin N. (2006) Target selectivity in mRNA silencing. Gene Ther. 13, 509-516.

[8] Hannon G. J. (2002) RNA interference. Nature. 418, 244-251.

[9] Hutvagner G. (2005) Small RNA asymmetry in RNAi: function in RISC assembly and gene regulation. FEBS Lett. 579, 5850-5857.

[10] Rana T. M. (2007) Illuminating the silence: understanding the structure and function of small RNAs. Nat Rev Mol Cell Biol. 8, 23-36.

[11] Hohjoh H. (2002) RNA interference (RNA(i)) induction with various types of synthetic oligonucleotide duplexes in cultured human cells. FEBS Lett. 521, 195-199.

[12] Manche L., Green S. R., Schmedt C. and Mathews M. B. (1992) Interactions between double-stranded RNA regulators and the protein kinase DAI. Mol Cell Biol. 12, 52385248.

[13] Elbashir S. M., Harborth J., Lendeckel W., Yalcin A., Weber K. and Tuschl T. (2001) Duplexes of 21-nucleotide RNAs mediate RNA interference in cultured mammalian cells. Nature. 411, 494-498.

[14] Chiu Y. L., Ali A., Chu C. Y., Cao H. and Rana T. M. (2004) Visualizing a correlation between siRNA localization, cellular uptake, and RNAi in living cells. Chem Biol. 11, 1165-1175.

[15] Elbashir S. M., Harborth J., Weber K. and Tuschl T. (2002) Analysis of gene function in somatic mammalian cells using small interfering RNAs. Methods. 26, 199-213.

[16] Paddison P. J., Caudy A. A. and Hannon G. J. (2002) Stable suppression of gene expression by RNAi in mammalian cells. Proc Natl Acad Sci U S A. 99, 1443-1448.

[17] Preall J. B. and Sontheimer E. J. (2005) RNAi: RISC gets loaded. Cell. 123, 543-545.

[18] Zamore P. D., Tuschl T., Sharp P. A. and Bartel D. P. (2000) RNAi: double-stranded RNA directs the ATP-dependent cleavage of mRNA at 21 to 23 nucleotide intervals. Cell. 101, 25-33.

[19] Chendrimada T. P., Gregory R. I., Kumaraswamy E., Norman J., Cooch N., Nishikura K. and Shiekhattar R. (2005) TRBP recruits the Dicer complex to Ago2 for microRNA processing and gene silencing. Nature. 436, 740-744.

[20] Tomari Y., Matranga C., Haley B., Martinez N. and Zamore P. D. (2004) A protein sensor for siRNA asymmetry. Science. 306, 1377-1380. 
[21] Ma J. B., Ye K. and Patel D. J. (2004) Structural basis for overhang-specific small interfering RNA recognition by the PAZ domain. Nature. 429, 318-322.

[22] Zhang H., Kolb F. A., Brondani V., Billy E. and Filipowicz W. (2002) Human Dicer preferentially cleaves dsRNAs at their termini without a requirement for ATP. Embo J. 21, 5875-5885.

[23] Grunweller A. and Hartmann R. K. (2005) RNA interference as a gene-specific approach for molecular medicine. Curr Med Chem. 12, 3143-3161.

[24] Kini H. K. and Walton S. P. (2009) Effect of siRNA terminal mismatches on TRBP and Dicer binding and silencing efficacy. FEBS J. 276, 6576-6585.

[25] Liu X., Jiang F., Kalidas S., Smith D. and Liu Q. (2006) Dicer-2 and R2D2 coordinately bind siRNA to promote assembly of the siRISC complexes. Rna. 12, 1514-1520.

[26] Hutvagner G. and Simard M. J. (2008) Argonaute proteins: key players in RNA silencing. Nat Rev Mol Cell Biol. 9, 22-32.

[27] Song J. J., Smith S. K., Hannon G. J. and Joshua-Tor L. (2004) Crystal structure of Argonaute and its implications for RISC slicer activity. Science. 305, 1434-1437.

[28] Bernstein E., Caudy A. A., Hammond S. M. and Hannon G. J. (2001) Role for a bidentate ribonuclease in the initiation step of RNA interference. Nature. 409, 363-366.

[29] Ma J. B., Yuan Y. R., Meister G., Pei Y., Tuschl T. and Patel D. J. (2005) Structural basis for 5 '-end-specific recognition of guide RNA by the A. fulgidus Piwi protein. Nature. 434, 666-670.

[30] Parker J. S., Roe S. M. and Barford D. (2005) Structural insights into mRNA recognition from a PIWI domain-siRNA guide complex. Nature. 434, 663-666.

[31] Rivas F. V., Tolia N. H., Song J. J., Aragon J. P., Liu J., Hannon G. J. and Joshua-Tor L. (2005) Purified Argonaute2 and an siRNA form recombinant human RISC. Nat Struct Mol Biol. 12, 340-349.

[32] Meister G. and Tuschl T. (2004) Mechanisms of gene silencing by double-stranded RNA. Nature. 431, 343-349.

[33] Nykanen A., Haley B. and Zamore P. D. (2001) ATP requirements and small interfering RNA structure in the RNA interference pathway. Cell. 107, 309-321.

[34] Leuschner P. J., Ameres S. L., Kueng S. and Martinez J. (2006) Cleavage of the siRNA passenger strand during RISC assembly in human cells. EMBO Rep. 7, 314-320.

[35] Matranga C., Tomari Y., Shin C., Bartel D. P. and Zamore P. D. (2005) Passenger-strand cleavage facilitates assembly of siRNA into Ago2-containing RNAi enzyme complexes. Cell. 123, 607-620.

[36] Addepalli H., Meena, Peng C. G., Wang G., Fan Y., Charisse K., Jayaprakash K. N., Rajeev K. G., Pandey R. K., Lavine G., Zhang L., Jahn-Hofmann K., Hadwiger P., Manoharan M. and Maier M. A. (2010) Modulation of thermal stability can enhance the potency of siRNA. Nucleic Acids Res. 38, 7320-7331.

[37] Khvorova A., Reynolds A. and Jayasena S. D. (2003) Functional siRNAs and miRNAs exhibit strand bias. Cell. 115, 209-216.

[38] Schwarz D. S., Hutvagner G., Du T., Xu Z., Aronin N. and Zamore P. D. (2003) Asymmetry in the assembly of the RNAi enzyme complex. Cell. 115, 199-208.

[39] Haley B. and Zamore P. D. (2004) Kinetic analysis of the RNAi enzyme complex. Nat Struct Mol Biol. 11, 599-606. 
[40] Elbashir S. M., Martinez J., Patkaniowska A., Lendeckel W. and Tuschl T. (2001) Functional anatomy of siRNAs for mediating efficient RNAi in Drosophila melanogaster embryo lysate. Embo J. 20, 6877-6888.

[41] Liu J., Carmell M. A., Rivas F. V., Marsden C. G., Thomson J. M., Song J. J., Hammond S. M., Joshua-Tor L. and Hannon G. J. (2004) Argonaute2 is the catalytic engine of mammalian RNAi. Science. 305, 1437-1441.

[42] Braasch D. A. and Corey D. R. (2002) Novel antisense and peptide nucleic acid strategies for controlling gene expression. Biochemistry. 41, 4503-4510.

[43] Dorsett Y. and Tuschl T. (2004) siRNAs: applications in functional genomics and potential as therapeutics. Nat Rev Drug Discov. 3, 318-329.

[44] Bertrand J. R., Pottier M., Vekris A., Opolon P., Maksimenko A. and Malvy C. (2002) Comparison of antisense oligonucleotides and siRNAs in cell culture and in vivo. Biochem Biophys Res Commun. 296, 1000-1004.

[45] Elbashir S. M., Lendeckel W. and Tuschl T. (2001) RNA interference is mediated by 21and 22-nucleotide RNAs. Genes Dev. 15, 188-200.

[46] Prakash T. P., Allerson C. R., Dande P., Vickers T. A., Sioufi N., Jarres R., Baker B. F., Swayze E. E., Griffey R. H. and Bhat B. (2005) Positional effect of chemical modifications on short interference RNA activity in mammalian cells. J Med Chem. 48, 4247-4253.

[47] Chu C. Y. and Rana T. M. (2006) Translation repression in human cells by microRNAinduced gene silencing requires RCK/p54. PLoS Biol. 4, e210.

[48] Jakymiw A., Pauley K. M., Li S., Ikeda K., Lian S., Eystathioy T., Satoh M., Fritzler M. J. and Chan E. K. (2007) The role of GW/P-bodies in RNA processing and silencing. J Cell Sci. 120, 1317-1323.

[49] Chalk A. M., Wahlestedt C. and Sonnhammer E. L. (2004) Improved and automated prediction of effective siRNA. Biochem Biophys Res Commun. 319, 264-274.

[50] Reynolds A., Leake D., Boese Q., Scaringe S., Marshall W. S. and Khvorova A. (2004) Rational siRNA design for RNA interference. Nat Biotechnol. 22, 326-330.

[51] Brown K. M., Chu C. Y. and Rana T. M. (2005) Target accessibility dictates the potency of human RISC. Nat Struct Mol Biol. 12, 469-470.

[52] Gredell J. A., Berger A. K. and Walton S. P. (2008) Impact of target mRNA structure on siRNA silencing efficiency: A large-scale study. Biotechnol Bioeng. 100, 744-755.

[53] Holen T., Amarzguioui M., Wiiger M. T., Babaie E. and Prydz H. (2002) Positional effects of short interfering RNAs targeting the human coagulation trigger Tissue Factor. Nucleic Acids Res. 30, 1757-1766

[54] Westerhout E. M. and Berkhout B. (2007) A systematic analysis of the effect of target RNA structure on RNA interference. Nucleic Acids Res. 35, 4322-4330.

[55] Amarzguioui M., Brede G., Babaie E., Grotli M., Sproat B. and Prydz H. (2000) Secondary structure prediction and in vitro accessibility of mRNA as tools in the selection of target sites for ribozymes. Nucleic Acids Res. 28, 4113-4124.

[56] Braasch D. A., Paroo Z., Constantinescu A., Ren G., Oz O. K., Mason R. P. and Corey D. R. (2004) Biodistribution of phosphodiester and phosphorothioate siRNA. Bioorg Med Chem Lett. 14, 1139-1143.

[57] Soutschek J., Akinc A., Bramlage B., Charisse K., Constien R., Donoghue M., Elbashir S., Geick A., Hadwiger P., Harborth J., John M., Kesavan V., Lavine G., Pandey R. K., Racie T., Rajeev K. G., Rohl I., Toudjarska I., Wang G., Wuschko S., Bumcrot D., Koteliansky 
V., Limmer S., Manoharan M. and Vornlocher H. P. (2004) Therapeutic silencing of an endogenous gene by systemic administration of modified siRNAs. Nature. 432, 173-178.

[58] Chiu Y. L. and Rana T. M. (2003) siRNA function in RNAi: a chemical modification analysis. Rna. 9, 1034-1048.

[59] Czauderna F., Fechtner M., Dames S., Aygun H., Klippel A., Pronk G. J., Giese K. and Kaufmann J. (2003) Structural variations and stabilising modifications of synthetic siRNAs in mammalian cells. Nucleic Acids Res. 31, 2705-2716.

[60] Haupenthal J., Baehr C., Kiermayer S., Zeuzem S. and Piiper A. (2006) Inhibition of RNAse A family enzymes prevents degradation and loss of silencing activity of siRNAs in serum. Biochem Pharmacol. 71, 702-710.

[61] Morrissey D. V., Blanchard K., Shaw L., Jensen K., Lockridge J. A., Dickinson B., McSwiggen J. A., Vargeese C., Bowman K., Shaffer C. S., Polisky B. A. and Zinnen S. (2005) Activity of stabilized short interfering RNA in a mouse model of hepatitis B virus replication. Hepatology. 41, 1349-1356.

[62] Turner J. J., Jones S. W., Moschos S. A., Lindsay M. A. and Gait M. J. (2007) MALDI-TOF mass spectral analysis of siRNA degradation in serum confirms an RNAse A-like activity. Mol Biosyst. 3, 43-50.

[63] White P. J. (2008) Barriers to successful delivery of short interfering RNA after systemic administration. Clin Exp Pharmacol Physiol. 35, 1371-1376.

[64] Judge A. D., Bola G., Lee A. C. and MacLachlan I. (2006) Design of noninflammatory synthetic siRNA mediating potent gene silencing in vivo. Mol Ther. 13, 494-505.

[65] Manoharan M. (2004) RNA interference and chemically modified small interfering RNAs. Curr Opin Chem Biol. 8, 570-579.

[66] Robbins M., Judge A., Liang L., McClintock K., Yaworski E. and MacLachlan I. (2007) 2'O-methyl-modified RNAs act as TLR7 antagonists. Mol Ther. 15, 1663-1669.

[67] Judge A. and MacLachlan I. (2008) Overcoming the innate immune response to small interfering RNA. Hum Gene Ther. 19, 111-124.

[68] Takeda K. and Akira S. (2005) Toll-like receptors in innate immunity. Int Immunol. 17, $1-14$.

[69] Zamanian-Daryoush M., Marques J. T., Gantier M. P., Behlke M. A., John M., Rayman P., Finke J. and Williams B. R. (2008) Determinants of cytokine induction by small interfering RNA in human peripheral blood mononuclear cells. J Interferon Cytokine Res. 28, 221-233.

[70] Kleinman M. E., Yamada K., Takeda A., Chandrasekaran V., Nozaki M., Baffi J. Z., Albuquerque R. J., Yamasaki S., Itaya M., Pan Y., Appukuttan B., Gibbs D., Yang Z., Kariko K., Ambati B. K., Wilgus T. A., DiPietro L. A., Sakurai E., Zhang K., Smith J. R., Taylor E. W. and Ambati J. (2008) Sequence- and target-independent angiogenesis suppression by siRNA via TLR3. Nature. 452, 591-597.

[71] Braasch D. A., Jensen S., Liu Y., Kaur K., Arar K., White M. A. and Corey D. R. (2003) RNA interference in mammalian cells by chemically-modified RNA. Biochemistry. 42, 7967-7975.

[72] Choung S., Kim Y. J., Kim S., Park H. O. and Choi Y. C. (2006) Chemical modification of siRNAs to improve serum stability without loss of efficacy. Biochem Biophys Res Commun. 342, 919-927. 
[73] Kraynack B. A. and Baker B. F. (2006) Small interfering RNAs containing full 2'-Omethylribonucleotide-modified sense strands display Argonaute2/eIF2C2-dependent activity. RNA. 12, 163-176.

[74] Hamada M., Ohtsuka T., Kawaida R., Koizumi M., Morita K., Furukawa H., Imanishi T., Miyagishi M. and Taira K. (2002) Effects on RNA interference in gene expression (RNAi) in cultured mammalian cells of mismatches and the introduction of chemical modifications at the 3'-ends of siRNAs. Antisense Nucleic Acid Drug Dev. 12, 301-309.

[75] Parrish S., Fleenor J., Xu S., Mello C. and Fire A. (2000) Functional anatomy of a dsRNA trigger: differential requirement for the two trigger strands in RNA interference. Mol Cell. 6, 1077-1087.

[76] Watts J. K., Deleavey G. F. and Damha M. J. (2008) Chemically modified siRNA: tools and applications. Drug Discov Today. 13, 842-855.

[77] Allerson C. R., Sioufi N., Jarres R., Prakash T. P., Naik N., Berdeja A., Wanders L., Griffey R. H., Swayze E. E. and Bhat B. (2005) Fully 2'-modified oligonucleotide duplexes with improved in vitro potency and stability compared to unmodified small interfering RNA. J Med Chem. 48, 901-904.

[78] Layzer J. M., McCaffrey A. P., Tanner A. K., Huang Z., Kay M. A. and Sullenger B. A. (2004) In vivo activity of nuclease-resistant siRNAs. RNA. 10, 766-771.

[79] Muhonen P., Tennila T., Azhayeva E., Parthasarathy R. N., Janckila A. J., Vaananen H. K., Azhayev A. and Laitala-Leinonen T. (2007) RNA interference tolerates 2'-fluoro modifications at the Argonaute2 cleavage site. Chem Biodivers. 4, 858-873.

[80] Ikeda H., Fernandez R., Wilk A., Barchi J. J., Jr., Huang X. and Marquez V. E. (1998) The effect of two antipodal fluorine-induced sugar puckers on the conformation and stability of the Dickerson-Drew dodecamer duplex [d(CGCGAATTCGCG)]2. Nucleic Acids Res. 26, 2237-2244.

[81] Damha M. J., Noronha A. M., Wilds C. J., Trempe J. F., Denisov A., Pon R. T. and Gehring K. (2001) Properties of arabinonucleic acids (ANA \& 20'F-ANA): implications for the design of antisense therapeutics that invoke RNase $\mathrm{H}$ cleavage of RNA. Nucleosides Nucleotides Nucleic Acids. 20, 429-440.

[82] Ui-Tei K., Naito Y., Zenno S., Nishi K., Yamato K., Takahashi F., Juni A. and Saigo K. (2008) Functional dissection of siRNA sequence by systematic DNA substitution: modified siRNA with a DNA seed arm is a powerful tool for mammalian gene silencing with significantly reduced off-target effect. Nucleic Acids Res. 36, 2136-2151.

[83] Amarzguioui M., Holen T., Babaie E. and Prydz H. (2003) Tolerance for mutations and chemical modifications in a siRNA. Nucleic Acids Res. 31, 589-595.

[84] Dorn G., Patel S., Wotherspoon G., Hemmings-Mieszczak M., Barclay J., Natt F. J., Martin P., Bevan S., Fox A., Ganju P., Wishart W. and Hall J. (2004) siRNA relieves chronic neuropathic pain. Nucleic Acids Res. 32, e49.

[85] Chen X., Shen L. and Wang J. H. (2004) Poly-2'-DNP-RNAs with enhanced efficacy for inhibiting cancer cell growth. Oligonucleotides. 14, 90-99.

[86] Liao H. and Wang J. H. (2005) Biomembrane-permeable and Ribonuclease-resistant siRNA with enhanced activity. Oligonucleotides. 15, 196-205.

[87] Ashun M. A., Hu Y., Kang I., Li C. C. and Wang J. H. (1996) Inhibition of murine leukemia virus with poly-2'-O-(2,4-dinitrophenyl)poly[A]. Antimicrob Agents Chemother. 40, 2311-2317. 
[88] Bramsen J. B., Laursen M. B., Nielsen A. F., Hansen T. B., Bus C., Langkjaer N., Babu B. R., Hojland T., Abramov M., Van Aerschot A., Odadzic D., Smicius R., Haas J., Andree C., Barman J., Wenska M., Srivastava P., Zhou C., Honcharenko D., Hess S., Muller E., Bobkov G. V., Mikhailov S. N., Fava E., Meyer T. F., Chattopadhyaya J., Zerial M., Engels J. W., Herdewijn P., Wengel J. and Kjems J. (2009) A large-scale chemical modification screen identifies design rules to generate siRNAs with high activity, high stability and low toxicity. Nucleic Acids Res. 37, 2867-2881.

[89] De Mesmaeker A., Altmann K. H., Waldner A. and Wendeborn S. (1995) Backbone modifications in oligonucleotides and peptide nucleic acid systems. Curr Opin Struct Biol. 5, 343-355.

[90] Inoue H., Hayase Y., Imura A., Iwai S., Miura K. and Ohtsuka E. (1987) Synthesis and hybridization studies on two complementary nona(2'-O-methyl)ribonucleotides. Nucleic Acids Res. 15, 6131-6148.

[91] Dande P., Prakash T. P., Sioufi N., Gaus H., Jarres R., Berdeja A., Swayze E. E., Griffey R. H. and Bhat B. (2006) Improving RNA interference in mammalian cells by 4'-thiomodified small interfering RNA (siRNA): effect on siRNA activity and nuclease stability when used in combination with 2'-O-alkyl modifications. J Med Chem. 49, 1624-1634.

[92] Hoshika S., Minakawa N., Kamiya H., Harashima H. and Matsuda A. (2005) RNA interference induced by siRNAs modified with 4'-thioribonucleosides in cultured mammalian cells. FEBS Lett. 579, 3115-3118.

[93] Hoshika S., Minakawa N., Shionoya A., Imada K., Ogawa N. and Matsuda A. (2007) Study of modification pattern-RNAi activity relationships by using siRNAs modified with 4'-thioribonucleosides. Chembiochem. 8, 2133-2138.

[94] Braasch D. A. and Corey D. R. (2001) Locked nucleic acid (LNA): fine-tuning the recognition of DNA and RNA. Chem Biol. 8, 1-7.

[95] Grunweller A., Wyszko E., Bieber B., Jahnel R., Erdmann V. A. and Kurreck J. (2003) Comparison of different antisense strategies in mammalian cells using locked nucleic acids, 2'-O-methyl RNA, phosphorothioates and small interfering RNA. Nucleic Acids Res. 31, 3185-3193.

[96] Elmen J., Thonberg H., Ljungberg K., Frieden M., Westergaard M., Xu Y., Wahren B., Liang Z., Orum H., Koch T. and Wahlestedt C. (2005) Locked nucleic acid (LNA) mediated improvements in siRNA stability and functionality. Nucleic Acids Res. 33, 439-447.

[97] Hornung V., Guenthner-Biller M., Bourquin C., Ablasser A., Schlee M., Uematsu S., Noronha A., Manoharan M., Akira S., de Fougerolles A., Endres S. and Hartmann G. (2005) Sequence-specific potent induction of IFN-alpha by short interfering RNA in plasmacytoid dendritic cells through TLR7. Nat Med. 11, 263-270.

[98] Mook O. R., Baas F., de Wissel M. B. and Fluiter K. (2007) Evaluation of locked nucleic acid-modified small interfering RNA in vitro and in vivo. Mol Cancer Ther. 6, 833-843.

[99] Bramsen J. B., Laursen M. B., Damgaard C. K., Lena S. W., Babu B. R., Wengel J. and Kjems J. (2007) Improved silencing properties using small internally segmented interfering RNAs. Nucleic Acids Res. 35, 5886-5897.

[100] Herdewijn P., Allart B., De Bouvere B., De Winter H., Hendrix C., Hossain N., Schepers G., Verheggen I., Wroblowski B. and Van Aerschot A. (1999) Properties of oligonucleotides with six membered base moiety and the hydroxymethyl group mimics 
and a 1,4-relationship between the base moiety and the hydroxymethyl group. Nucleosides \& Nucleotides. 18, 1371-1376.

[101] Kozlov I. A., Zielinski M., Allart B., Kerremans L., Van Aerschot A., Busson R., Herdewijn P. and Orgel L. E. (2000) Nonenzymatic template-directed reactions on altritol oligomers, preorganized analogues of oligonucleotides. Chemistry. 6, 151-155.

[102] Herdewijn P., Allart B., De Bouvere B., De Winter H., Hendrix C., Hossain N., Schepers G., Verheggen I., Wroblowski B. and Van Aerschot A. (1999) Properties of oligonucleotides with six membered base moiety and the hydroxymethyl group mimics and a 1,4-relationship between the base moiety and the hydroxymethyl group. Nucleosides \& Nucleotides. 18, 1371-1376.

[103] Fisher M., Abramov M., Van Aerschot A., Xu D., Juliano R. L. and Herdewijn P. (2007) Inhibition of MDR1 expression with altritol-modified siRNAs. Nucleic Acids Res. 35, 1064-1074.

[104] Kenski D. M., Cooper A. J., Li J. J., Willingham A. T., Haringsma H. J., Young T. A., Kuklin N. A., Jones J. J., Cancilla M. T., McMasters D. R., Mathur M., Sachs A. B. and Flanagan W. M. (2010) Analysis of acyclic nucleoside modifications in siRNAs finds sensitivity at position 1 that is restored by 5 '-terminal phosphorylation both in vitro and in vivo. Nucleic Acids Res. 38, 660-671.

[105] Langkjaer N., Pasternak A. and Wengel J. (2009) UNA (unlocked nucleic acid): a flexible RNA mimic that allows engineering of nucleic acid duplex stability. Bioorg Med Chem. 17, 5420-5425.

[106] Kenski D. M., Cooper A. J., Li J. J., Willingham A. T., Haringsma H. J., Young T. A., Kuklin N. A., Jones J. J., Cancilla M. T., McMasters D. R., Mathur M., Sachs A. B. and Flanagan W. M. (2010) Analysis of acyclic nucleoside modifications in siRNAs finds sensitivity at position 1 that is restored by 5 '-terminal phosphorylation both in vitro and in vivo. Nucleic Acids Res. 38, 660-671.

[107] Werk D., Wengel J., Wengel S. L., Grunert H. P., Zeichhardt H. and Kurreck J. (2010) Application of small interfering RNAs modified by unlocked nucleic acid (UNA) to inhibit the heart-pathogenic coxsackievirus B3. FEBS Lett. 584, 591-598.

[108] Brown S. C., Thomson S. A., Veal J. M. and Davis D. G. (1994) NMR solution structure of a peptide nucleic acid complexed with RNA. Science. 265, 777-780.

[109] Demidov V. V., Potaman V. N., Frank-Kamenetskii M. D., Egholm M., Buchard O., Sonnichsen S. H. and Nielsen P. E. (1994) Stability of peptide nucleic acids in human serum and cellular extracts. Biochem Pharmacol. 48, 1310-1313.

[110] Potenza N., Moggio L., Milano G., Salvatore V., Di Blasio B., Russo A. and Messere A. (2008) RNA interference in mammalia cells by RNA-3'-PNA chimeras. Int J Mol Sci. 9, 299-315.

[111] Harborth J., Elbashir S. M., Vandenburgh K., Manninga H., Scaringe S. A., Weber K. and Tuschl T. (2003) Sequence, chemical, and structural variation of small interfering RNAs and short hairpin RNAs and the effect on mammalian gene silencing. Antisense Nucleic Acid Drug Dev. 13, 83-105.

[112] Hall A. H., Wan J., Shaughnessy E. E., Ramsay Shaw B. and Alexander K. A. (2004) RNA interference using boranophosphate siRNAs: structure-activity relationships. Nucleic Acids Res. 32, 5991-6000. 
[113] Schwarz D. S., Tomari Y. and Zamore P. D. (2004) The RNA-induced silencing complex is a Mg2+-dependent endonuclease. Curr Biol. 14, 787-791.

[114] Lee M., Simon A. D., Stein C. A. and Rabbani L. E. (1999) Antisense strategies to inhibit restenosis. Antisense Nucleic Acid Drug Dev. 9, 487-492.

[115] Prakash T. P., Kraynack B., Baker B. F., Swayze E. E. and Bhat B. (2006) RNA interference by 2',5'-linked nucleic acid duplexes in mammalian cells. Bioorg Med Chem Lett. 16, 3238-3240.

[116] Iwase R., Toyama T. and Nishimori K. (2007) Solid-phase synthesis of modified RNAs containing amide-linked oligoribonucleosides at their 3'-end and their application to siRNA. Nucleosides Nucleotides Nucleic Acids. 26, 1451-1454.

[117] Saenger W. (1984) Forces stabilizing association between bases: Hydrogen bonding and base stacking. In Principle of nucleic acids structure. Springer-Verlag, New York.

[118] Agris P. F., Sierzputowska-Gracz H., Smith W., Malkiewicz A., Sochacka E. and Nawrot B. (1992) Thiolation of uridine carbon-2 restricts the motional dynamics of the transfer RNA wobble position nucleoside. J. Am. Chem. Soc. 114, 2652-2656.

[119] Davis D. R., Veltri C. A. and Nielsen L. (1998) An RNA model system for investigation of pseudouridine stabilization of the codon-anticodon interaction in tRNALys, tRNAHis and tRNATyr. J Biomol Struct Dyn. 15, 1121-1132.

[120] Sipa K., Sochacka E., Kazmierczak-Baranska J., Maszewska M., Janicka M., Nowak G. and Nawrot B. (2007) Effect of base modifications on structure, thermodynamic stability, and gene silencing activity of short interfering RNA. RNA. 13, 1301-1316.

[121] Morrissey D. V., Lockridge J. A., Shaw L., Blanchard K., Jensen K., Breen W., Hartsough K., Machemer L., Radka S., Jadhav V., Vaish N., Zinnen S., Vargeese C., Bowman K., Shaffer C. S., Jeffs L. B., Judge A., MacLachlan I. and Polisky B. (2005) Potent and persistent in vivo anti-HBV activity of chemically modified siRNAs. Nat Biotechnol. 23, 1002-1007.

[122] Schwarz D. S., Hutvagner G., Haley B. and Zamore P. D. (2002) Evidence that siRNAs function as guides, not primers, in the Drosophila and human RNAi pathways. Mol Cell. 10, 537-548.

[123] Chen P. Y., Weinmann L., Gaidatzis D., Pei Y., Zavolan M., Tuschl T. and Meister G. (2008) Strand-specific 5'-O-methylation of siRNA duplexes controls guide strand selection and targeting specificity. RNA. 14, 263-274.

[124] Lorenz C., Hadwiger P., John M., Vornlocher H. P. and Unverzagt C. (2004) Steroid and lipid conjugates of siRNAs to enhance cellular uptake and gene silencing in liver cells. Bioorg Med Chem Lett. 14, 4975-4977.

[125] Wolfrum C., Shi S., Jayaprakash K. N., Jayaraman M., Wang G., Pandey R. K., Rajeev K. G., Nakayama T., Charrise K., Ndungo E. M., Zimmermann T., Koteliansky V., Manoharan M. and Stoffel M. (2007) Mechanisms and optimization of in vivo delivery of lipophilic siRNAs. Nat Biotechnol. 25, 1149-1157.

[126] Thomas M., Kularatne S. A., Qi L., Kleindl P., Leamon C. P., Hansen M. J. and Low P. S. (2009) Ligand-targeted delivery of small interfering RNAs to malignant cells and tissues. Ann N Y Acad Sci. 1175, 32-39.

[127] Moschos S. A., Jones S. W., Perry M. M., Williams A. E., Erjefalt J. S., Turner J. J., Barnes P. J., Sproat B. S., Gait M. J. and Lindsay M. A. (2007) Lung delivery studies 
using siRNA conjugated to TAT(48-60) and penetratin reveal peptide induced reduction in gene expression and induction of innate immunity. Bioconjug Chem. 18, 1450-1459.

[128] Muratovska A. and Eccles M. R. (2004) Conjugate for efficient delivery of short interfering RNA (siRNA) into mammalian cells. FEBS Lett. 558, 63-68.

[129] McNamara J. O., 2nd, Andrechek E. R., Wang Y., Viles K. D., Rempel R. E., Gilboa E., Sullenger B. A. and Giangrande P. H. (2006) Cell type-specific delivery of siRNAs with aptamer-siRNA chimeras. Nat Biotechnol. 24, 1005-1015.

[130] Chiu Y. L. and Rana T. M. (2002) RNAi in human cells: basic structural and functional features of small interfering RNA. Mol Cell. 10, 549-561.

[131] Martinez J., Patkaniowska A., Urlaub H., Luhrmann R. and Tuschl T. (2002) Singlestranded antisense siRNAs guide target RNA cleavage in RNAi. Cell. 110, 563-574.

[132] De Paula D., Bentley M. V. and Mahato R. I. (2007) Hydrophobization and bioconjugation for enhanced siRNA delivery and targeting. Rna. 13, 431-456.

[133] Gaglione M. and Messere A. (2010) Recent progress in chemically modified siRNAs. Mini Rev Med Chem. 10, 578-595.

[134] Burnett J. R. and Barrett P. H. (2002) Apolipoprotein B metabolism: tracer kinetics, models, and metabolic studies. Crit Rev Clin Lab Sci. 39, 89-137.

[135] Feinberg E. H. and Hunter C. P. (2003) Transport of dsRNA into cells by the transmembrane protein SID-1. Science. 301, 1545-1547.

[136] Potocky T. B., Menon A. K. and Gellman S. H. (2003) Cytoplasmic and nuclear delivery of a TAT-derived peptide and a beta-peptide after endocytic uptake into HeLa cells. J Biol Chem. 278, 50188-50194.

[137] Richard J. P., Melikov K., Vives E., Ramos C., Verbeure B., Gait M. J., Chernomordik L. V. and Lebleu B. (2003) Cell-penetrating peptides. A reevaluation of the mechanism of cellular uptake. J Biol Chem. 278, 585-590.

[138] Poehlmann T. G., Ruediger T., Schaefer H., Koehn S., Imhof D., Seyfarth L., Schubert U. S. and Markert U. R. (2009) Development of small interfering RNA selectively activated in target cells. In RNAi2009: ncRNA: Bridging Biology and Therapy (Media L. P., ed.)^eds.). pp. 16-17, Library Publishing Media, Oxford, UK.

[139] Hicke B. J. and Stephens A. W. (2000) Escort aptamers: a delivery service for diagnosis and therapy. J Clin Invest. 106, 923-928.

[140] Dassie J. P., Liu X. Y., Thomas G. S., Whitaker R. M., Thiel K. W., Stockdale K. R., Meyerholz D. K., McCaffrey A. P., McNamara J. O., 2nd and Giangrande P. H. (2009) Systemic administration of optimized aptamer-siRNA chimeras promotes regression of PSMA-expressing tumors. Nat Biotechnol. 27, 839-849.

[141] Xia C. F., Boado R. J. and Pardridge W. M. (2009) Antibody-mediated targeting of siRNA via the human insulin receptor using avidin-biotin technology. Mol Pharm. 6, 747-751.

[142] Xia C. F., Zhang Y., Boado R. J. and Pardridge W. M. (2007) Intravenous siRNA of brain cancer with receptor targeting and avidin-biotin technology. Pharm Res. 24, 23092316.

[143] Xia W. and Low P. S. Folate-targeted therapies for cancer. (2010) J Med Chem. 53, 6811-6824.

[144] Kim S. H., Mok H., Jeong J. H., Kim S. W. and Park T. G. (2006) Comparative evaluation of target-specific GFP gene silencing efficiencies for antisense ODN, 
synthetic siRNA, and siRNA plasmid complexed with PEI-PEG-FOL conjugate. Bioconjug Chem. 17, 241-244.

[145] Zhang K., Wang Q., Xie Y., Mor G., Sega E., Low P. S. and Huang Y. (2008) Receptormediated delivery of siRNAs by tethered nucleic acid base-paired interactions. RNA. 14, 577-583.

[146] Jeong J. H., Mok H., Oh Y. K. and Park T. G. (2009) siRNA conjugate delivery systems. Bioconjug Chem. 20, 5-14.

[147] Kawakami S. and Hashida M. (2007) Targeted delivery systems of small interfering RNA by systemic administration. Drug Metab Pharmacokinet. 22, 142-151.

[148] Leng Q., Woodle M. C., Lu P. Y. and Mixson A. J. (2009) Advances in Systemic siRNA Delivery. Drugs Future. 34, 721.

[149] Lv H., Zhang S., Wang B., Cui S. and Yan J. (2006) Toxicity of cationic lipids and cationic polymers in gene $\mathrm{d}$ Xia W. and Low P. S. Folate-targeted therapies for cancer. (2010) J Med Chem. 53, 6811-6824.elivery. J Control Release. 114, 100-109.

[150] de Fougerolles A., Vornlocher H. P., Maraganore J. and Lieberman J. (2007) Interfering with disease: a progress report on siRNA-based therapeutics. Nat Rev Drug Discov. 6, 443-453.

[151] Wu Y., Navarro F., Lal A., Basar E., Pandey R. K., Manoharan M., Feng Y., Lee S. J., Lieberman J. and Palliser D. (2009) Durable protection from Herpes Simplex Virus-2 transmission following intravaginal application of siRNAs targeting both a viral and host gene. Cell Host Microbe. 5, 84-94.

[152] Blidner R. A., Hammer R. P., Lopez M. J., Robinson S. O. and Monroe W. T. (2007) Fully 2'-deoxy-2'-fluoro substituted nucleic acids induce RNA interference in mammalian cell culture. Chem Biol Drug Des. 70, 113-122.

[153] Corey D. R. (2007) Chemical modification: the key to clinical application of RNA interference? J Clin Invest. 117, 3615-3622.

[154] Jeong J. H., Mok H., Oh Y. K. and Park T. G. (2009) siRNA conjugate delivery systems. Bioconjug Chem. 20, 5-14.

[155] Jackson A. L., Burchard J., Leake D., Reynolds A., Schelter J., Guo J., Johnson J. M., Lim L., Karpilow J., Nichols K., Marshall W., Khvorova A. and Linsley P. S. (2006) Positionspecific chemical modification of siRNAs reduces "off-target" transcript silencing. RNA. $12,1197-1205$.

[156] Macrae I. J., Zhou K., Li F., Repic A., Brooks A. N., Cande W. Z., Adams P. D. and Doudna J. A. (2006) Structural basis for double-stranded RNA processing by Dicer. Science. 311, 195-198.

[157] Myers J. W., Jones J. T., Meyer T. and Ferrell J. E., Jr. (2003) Recombinant Dicer efficiently converts large dsRNAs into siRNAs suitable for gene silencing. Nat Biotechnol. 21, 324-328.

[158] Hohjoh H. (2004) Enhancement of RNAi activity by improved siRNA duplexes. FEBS Lett. 557, 193-198.

[159] Ohnishi Y., Tokunaga K. and Hohjoh H. (2005) Influence of assembly of siRNA elements into RNA-induced silencing complex bу вилко-siRNA duplex carrying nucleotide mismatches at the 3 '- or 5'-end of the sense-stranded siRNA element. Biochem Biophys Res Commun. 329, 516-521. 
[160] Kubo T., Zhelev Z., Ohba H. and Bakalova R. (2007) Modified 27-nt dsRNAs with dramatically enhanced stability in serum and long-term RNAi activity. Oligonucleotides. 17, 445-464.

[161] Rose S. D., Kim D. H., Amarzguioui M., Heidel J. D., Collingwood M. A., Davis M. E., Rossi J. J. and Behlke M. A. (2005) Functional polarity is introduced by Dicer processing of short substrate RNAs. Nucleic Acids Res. 33, 4140-4156.

[162] Sano M., Sierant M., Miyagishi M., Nakanishi M., Takagi Y. and Sutou S. (2008) Effect of asymmetric terminal structures of short RNA duplexes on the RNA interference activity and strand selection. Nucleic Acids Res. 36, 5812-5821.

[163] Masquida B. and Westhof E. (2000) On the wobble GoU and related pairs. RNA. 6, 915.

[164] Ding H., Liao G., Wang H. and Zhou Y. (2007) Asymmetrically designed siRNAs and shRNAs enhance the strand specificity and efficacy in RNAi. J RNAi Gene Silencing. 4, 269-280.

[165] Patzel V., Rutz S., Dietrich I., Koberle C., Scheffold A. and Kaufmann S. H. (2005) Design of siRNAs producing unstructured guide-RNAs results in improved RNA interference efficiency. Nat Biotechnol. 23, 1440-1444.

[166] Hu X., Hipolito S., Lynn R., Abraham V., Ramos S. and Wong-Staal F. (2004) Relative gene-silencing efficiencies of small interfering RNAs targeting sense and antisense transcripts from the same genetic locus. Nucleic Acids Res. 32, 4609-4617.

[167] Patzel V. (2007) In silico selection of active siRNA. Drug Discov Today. 12, 139-148.

[168] Lingel A., Simon B., Izaurralde E. and Sattler M. (2004) Nucleic acid 3'-end recognition by the Argonaute2 PAZ domain. Nat Struct Mol Biol. 11, 576-577.

[169] Marques J. T., Devosse T., Wang D., Zamanian-Daryoush M., Serbinowski P., Hartmann R., Fujita T., Behlke M. A. and Williams B. R. (2006) A structural basis for discriminating between self and nonself double-stranded RNAs in mammalian cells. Nat Biotechnol. 24, 559-565.

[170] Guo S. and Kemphues K. J. (1995) par-1, a gene required for establishing polarity in C. elegans embryos, encodes a putative Ser/Thr kinase that is asymmetrically distributed. Cell. 81, 611-620.

[171] Tijsterman M., Ketting R. F., Okihara K. L., Sijen T. and Plasterk R. H. (2002) RNA helicase MUT-14-dependent gene silencing triggered in C. elegans by short antisense RNAs. Science. 295, 694-697.

[172] Amarzguioui M., Rossi J. J. and Kim D. (2005) Approaches for chemically synthesized siRNA and vector-mediated RNAi. FEBS Lett. 579, 5974-5981.

[173] Collingwood M. A., Rose S. D., Huang L., Hillier C., Amarzguioui M., Wiiger M. T., Soifer H. S., Rossi J. J. and Behlke M. A. (2008) Chemical modification patterns compatible with high potency dicer-substrate small interfering RNAs. Oligonucleotides. $18,187-200$.

[174] Kim D. H., Behlke M. A., Rose S. D., Chang M. S., Choi S. and Rossi J. J. (2005) Synthetic dsRNA Dicer substrates enhance RNAi potency and efficacy. Nat Biotechnol. 23, 222-226.

[175] Reynolds A., Anderson E. M., Vermeulen A., Fedorov Y., Robinson K., Leake D., Karpilow J., Marshall W. S. and Khvorova A. (2006) Induction of the interferon response by siRNA is cell type- and duplex length-dependent. RNA. 12, 988-993. 
[176] Bridge A. J., Pebernard S., Ducraux A., Nicoulaz A. L. and Iggo R. (2003) Induction of an interferon response by RNAi vectors in mammalian cells. Nat Genet. 34, 263-264.

[177] Persengiev S. P., Zhu X. and Green M. R. (2004) Nonspecific, concentration-dependent stimulation and repression of mammalian gene expression by small interfering RNAs (siRNAs). Rna. 10, 12-18.

[178] Sledz C. A., Holko M., de Veer M. J., Silverman R. H. and Williams B. R. (2003) Activation of the interferon system by short-interfering RNAs. Nat Cell Biol. 5, 834-839.

[179] Hornung V., Ellegast J., Kim S., Brzozka K., Jung A., Kato H., Poeck H., Akira S., Conzelmann K. K., Schlee M., Endres S. and Hartmann G. (2006) 5'-Triphosphate RNA is the ligand for RIG-I. Science. 314, 994-997.

[180] Judge A. D., Sood V., Shaw J. R., Fang D., McClintock K. and MacLachlan I. (2005) Sequence-dependent stimulation of the mammalian innate immune response by synthetic siRNA. Nat Biotechnol. 23, 457-462.

[181] Shukla S., Sumaria C. S. and Pradeepkumar P. I. (2010) Exploring chemical modifications for siRNA therapeutics: a structural and functional outlook. ChemMedChem. 5, 328-349.

[182] Amarzguioui M. and Prydz H. (2004) An algorithm for selection of functional siRNA sequences. Biochem Biophys Res Commun. 316, 1050-1058.

[183] Matveeva O., Nechipurenko Y., Rossi L., Moore B., Saetrom P., Ogurtsov A. Y., Atkins J. F. and Shabalina S. A. (2007) Comparison of approaches for rational siRNA design leading to a new efficient and transparent method. Nucleic Acids Res. 35, e63.

[184] Shabalina S. A., Spiridonov A. N. and Ogurtsov A. Y. (2006) Computational models with thermodynamic and composition features improve siRNA design. BMC Bioinformatics. 7, 65.

[185] Ui-Tei K., Naito Y., Takahashi F., Haraguchi T., Ohki-Hamazaki H., Juni A., Ueda R. and Saigo K. (2004) Guidelines for the selection of highly effective siRNA sequences for mammalian and chick RNA interference. Nucleic Acids Res. 32, 936-948.

[186] Walton S. P., Wu M., Gredell J. A. and Chan C. (2010) Designing highly active siRNAs for therapeutic applications. FEBS J. 277, 4806-4813. 


\title{
Microbiological Chitosan: Potential Application as Anticariogenic Agent
}

\author{
Thayza Christina Montenegro Stamford, Thatiana Montenegro Stamford- \\ Arnaud, Horacinna Maria de Medeiros Cavalcante, Rui Oliveira Macedo \\ and Galba Maria de Campos-Takaki
}

Additional information is available at the end of the chapter

http://dx.doi.org/10.5772/54453

\section{Introduction}

Dental caries is the most prevalent oral disease that affects a significant part of the world population, especially in less developed countries. It is universally accepted that the dental caries is a chronic and multifactorial disease [1-3]. The permanence of the bacterial plaque on the tooth surface will lead to loss of minerals constituents of the dental enamel promoting the installation of the carie disease. The carious lesion is characterized by the tooth structure (hydroxyapatite) demineralization by the production of organic acids, such as lactic acid, resulting from bacterial (dental biofilm) metabolism. This results in loss of calcium and phosphate ions which subsequently diffuse out of the tooth. In this complex process, the microorganisms, particularly Streptococcus species, have an important role in its etiology [3-5].

Many oral Streptococcus in the presence of carbohydrate produce organics acids and insoluble glucans which serve as binding sites for bacteria on the tooth surface, forming the biofilm. The sucrose plays an important role in carie development, influencing on biofilm acidogenicity and cariogenic microflora. The high cariogenicity of dental plaque formed in the presence of sucrose can be mainly explained by the high concentration of insoluble glucans on its matrix, the low inorganic concentration and its protein composition may have some contribution [6,7].

Specifics microorganisms are associated with dental plaque formation, development and maturation. The Streptococcus mutans is a member of the oral microbial community which plays a key role in formation of cariogenic biofilms. The key factors of $S$. mutans cariogenic are the production of a great variety of carbohydrates, which generate low $\mathrm{pH}$ and cause the consequent demineralization of the tooth enamel [3, 8-10]. 
Recent developments in the areas of biomaterials devices have resulted in a number of advances in the searches of natural substances which may inhibit the dental plaque formation and/or the hydroxyapatite demineralization [11, 12]. In particular, this research is focused on novel macromolecules and biocompatible materials for use in clinical applications. Chitosan is a non-toxic and natural polysaccharide, which have many biological applications, mainly as antimicrobial agent [12].

\section{Chitosan: General considerations}

Chitosan is a natural co-polymers of chitin, composed by units of 2-amino-2-desoxi-Dglycopyranose and of 2-acetamide-2-desoxi-D-glycopyranose interconnected by glycosidic bonds $\beta-1,4$ in variable proportions. The first type of units is frequently present in chitosan. This polymer is naturally found in the cell wall of fungi, mainly in the Mucorales order [5, $13,14]$. Chitosan is formed by the chitin deacetylation, and the group N-acetyl can be suffer several degrees of deacetylation (Figure 1). Chitosan is characterized according to its deacetylation level and molar mass, once such features may influence the degradability and in the polysaccharide hydrolysis $[15,16]$. According to the medium acetylation level (AL), chitosan may be obtained with physical-chemical properties differentiated regarding the solubility parameters, $\mathrm{pKa}$ and viscosity $[17,19]$. It is difficult to obtain chitosan with high deacetylation level as due long process of isolation, and the degradation of the polymer also increases $[5,15]$.

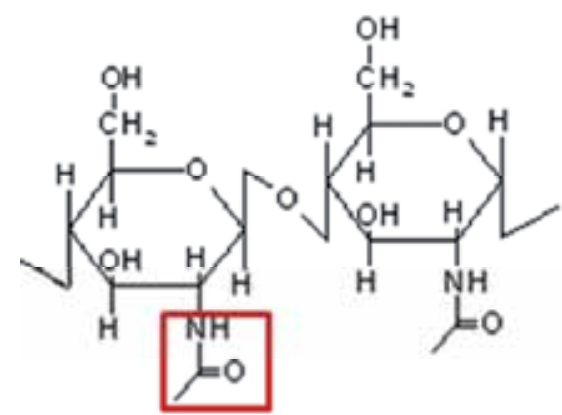

Chitin

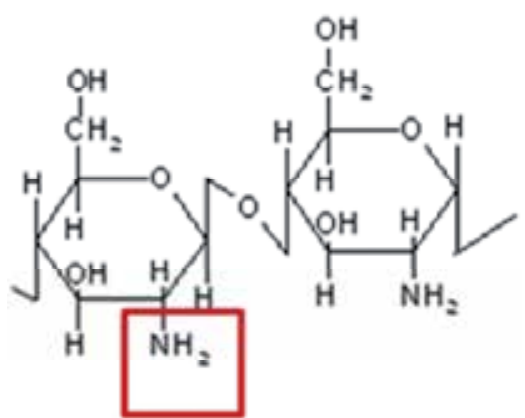

Chitosan

Figure 1. Chemical structure of Chitin and Chitosan (Source: authors)

The synthesis of chitin from fungi and crustaceans differs significantly; however, steps of enzymatic machinery for biosynthesis and catalytic regulation are similar. In crustaceans is initiated by conversion of glucose 6-phosphate $\mathrm{N}$-acetylglucosamine-1-phosphate, involving a series of stages that require several enzymatic reactions. $\mathrm{N}$-acetylglucosamine-1-phosphate reacts with UTP forming the UDP $\mathrm{N}$-acetylglucosamine, which finally transfers $\mathrm{N}$ acetylglucosamine for the polymerization of chitin chains in a process mediated by the 
enzyme chitin synthase and $\mathrm{Mg}^{+2}$ ion as catalyst, resulting in the formation of a long chain of subunits monosaccharide's linked by $\beta-1 \rightarrow 4$. Chitosan is thus obtained by deacetylation of chitin by chemical treatment with $\mathrm{NaOH}$ at high temperature [20].

Fungi the chitin and chitosan synthesis simultaneously were occurred. The synthesis of chitin is highly compartmentalized. The enzyme chitin synthase is the zymogen form and distributed in specific regions of the cell surface, vesicles in specialized, chitosome. The macromolecular assembly starts out of the cytoplasm, where the protease enzyme acts on the cell surface activating the zymogenes. In this way the UDP $\mathrm{N}$-acetylglucosamine is produced from glucose, and chitin synthase catalyzes the transfer of $\mathrm{N}$-acetylglucosamine for polymerization chain forming chitin. The chitosan present in cell walls of certain fungi (Mucorales) is formed from the deacetylation (chitin deacetylase) chain chitin source for the biosynthesis of chitin. The regulations of the synthesis of chitin and chitosan are determined by the spatial organization of the synthesis of chitin in the cell surface [20,21].

Crustacean chitosan is inconsistent in its physical--chemical properties due to the variability in raw materials, the harshness of the isolation and conversion processes, the caustic effects of the chemicals used in the isolation process, and variability in the levels of deacetylation and protein contamination [22-24].

The use of biomass from fungi have demonstrated a great advantages, such as: independence of seasonal factor, wide scale production, simultaneous extraction of chitin and chitosan, extraction process is simple and cheap resulting in reduction of the time and cost required for production, and also absence of proteins contamination, mainly the proteins that could cause allergy reactions in individuals with shellfish allergies. However, to optimize the production of chitin and chitosan from fungi, it's usually used complex or synthetics cultures media, which are expensive. It's becomes necessary to obtain economic culture media that promote the growth of fungi and stimulate the production of the polymers [25-32].

In order to obtain alternative sources of nutrients and low cost several research projects are being conducted. Table 1 shows the use of various synthetic media and low cost alternative media used for growth of fungi of the order Mucorales, and production of chitin and chitosan. The content of chitin and chitosan from fungal cell wall varies among different species and growth conditions. Many studies have been performed to verify the possibility of using the biomass of fungi, especially Mucorales, class Zygomycetes, as an alternative source of chitin and chitosan. Many of these studies test simple models to verify the production of chitin and chitosan, ie, the approach adopted experiments using only one variable at a time during the fermentation, eg after cultivation, agitation, $\mathrm{pH}$, temperature and concentration of nutrients. However, the literature report in recent years the scientific interest to reduce the numbers of tests and increase the accuracy of the results has been increased. Therefore, multivariate approach, using factorial design, allows the observation of the synergistic effect between the independent variables, since all variables are considered simultaneously, resulting in the final optimize conditions [30-34]. 


\begin{tabular}{|c|c|c|c|c|c|}
\hline Microorganism & Substrate & $\begin{array}{l}\text { Biomass } \\
\left(\mathrm{g} . \mathrm{L}^{-1}\right)\end{array}$ & $\begin{array}{l}\text { Chitin } \\
\left(\mathrm{mg} \cdot \mathrm{g}^{-1}\right)\end{array}$ & $\begin{array}{l}\text { Chitosan } \\
\left(\mathrm{mg} \cdot \mathrm{g}^{-1}\right)\end{array}$ & Reference \\
\hline Mucor circinelloides & Yam bean & 20.70 & 500 & 64 & 33 \\
\hline Cunninghamella elegans & Yam bean & 24.30 & 440 & 66 & 32 \\
\hline Cunninghamella elegans & $\begin{array}{l}\text { Hesseltine and } \\
\text { Anderson added } \\
\text { of } 5 \% \mathrm{NaCl} \text { and } \\
6 \% \text { glucose }\end{array}$ & 24.40 & 388 & 70 & 29 \\
\hline $\begin{array}{l}\text { Cunninghamella } \\
\text { bertholletiae }\end{array}$ & Sugar cane juice & 7.70 & - & 128 & 30 \\
\hline Aspergillus niger & $\begin{array}{l}\text { Potato Dextrose } \\
\text { Broth }\end{array}$ & 9.00 & - & 107 & 34 \\
\hline Lentinus edodes & $\begin{array}{l}\text { Potato Dextrose } \\
\text { Broth }\end{array}$ & 1.4 & - & 33 & 34 \\
\hline Zygosaccharomyces rouxii & $\begin{array}{l}\text { Yeast Malt } \\
\text { Extract Broth }\end{array}$ & 4.4 & - & 36 & 34 \\
\hline Candida albicans & $\begin{array}{l}\text { Yeast Malt } \\
\text { Extract Broth }\end{array}$ & 1.8 & - & 44 & 34 \\
\hline Rhizomucor miehei & $\begin{array}{l}\text { Sabouraod } \\
\text { dextrose (SDB) }\end{array}$ & 4.1 & - & 13.67 & 24 \\
\hline
\end{tabular}

- Data not shown

Table 1. Chitin and chitosan production by Mucorales strains compared with Cunninghamella cirnelloides using yam bean as substrate.

\section{Properties, antimicrobial and toxicity of fungi chitosan}

Chitosan has numerous applications in several areas, mainly biomedical and pharmaceutical fields, due to its specific properties Among their properties we highlight the excellent biocompatibility; almost any toxicity to human beings and animals; high bioactivity; biodegradability; reactivity of the group amino deacetylated; selective permeability; polyelectrolyte action; antimicrobial activity; ability to form gel and film; chelation ability and absorptive capacity [36-38]. These peculiar properties provide a variety of applications to the chitosan, such as: drug carrier of controlled release [36], anti-bacterial [37, 39] and anti-acid [38]; inhibition of the bacterial plaque formation and decalcification of dental enamel [2, 10]; promotes the osteogenesis [40]; and promotes the healing of ulcers and lesions [41, 42].

The applicability of chitosan is related to their physical-chemical properties considering the different sources (crustaceans, fungi, and mollusk's) and different processes for extraction and purification cause alterations in the degree of deacetylation, molecular weight, thermal stability and degree of crystallinity of the chitosan. Various derivatives of chitosan differ of the degree of deacetylation, molar weight, arrangement of residual $\mathrm{N}$-acetyl groups in the chain considering the reaction of - $\mathrm{NH} 2$ group at the carbon 2 (C2) or unspecific - $\mathrm{OH}$ group in position 3 carbons or 6 ( $\mathrm{C} 3$ or $\mathrm{C} 6)$ of the polymer with other functional groups are reported in the literature [15]. 
Chitosan is a weak base insoluble in water but soluble in dilute aqueous solutions of various acids, the most widely used is acetic acid [43]. The acid solubility is explained by the protonation of the free amino group, characteristic in the chitosan in natura, which change to $\mathrm{NH}_{2}$ to $\mathrm{NH}_{3}{ }^{+}$, whereas in alkaline condition, the hydro solubility is due to the formation of carboxylate, from the introduced carboxylic group [19,44]. The possibility to obtain a variety of polymer derivatives with differences solubility, thermal stability, reactivity with other substances and specificity regarding the binding site, providing several biological applications of the chitosan [15]. Some applications of the chitosan, it is highligh it's the use in the pharmaceutical industry, more specifically related to dental clinic [43].

Chitosan has a recognized antimicrobial activity, being this, one of the main properties of the polysaccharide. Several researchers demonstrated that this polysaccharide has antimicrobial action in a great variety of microorganisms, included gram-positive bacteria and various species of yeast [21,45]. In the literature is described that chitosan acts in the cellular wall of the microorganism modifying the electric potential of the cellular membrane [46]. This polysaccharide also acts potentiating other inhibition drugs, as the chlorhexidine gel, once it increases the drug permanence time action place [47, 48].

In reference [39] the authors report that chitosan has demonstrated low toxicity and the resistance development have not occurred. The antimicrobial action of the chitosan and its derivatives suffers influence from factors, which depending on the performed role may be classified in four main categories: 1 . Microbial factors as species, age of the cell); 2 . Intrinsic factors of the chitosan as: positive charge density, molecular weight, hydrophobic and hydrophilic characteristics, chelation capacity; 3. Physical state factors (soluble and solid state), and 4. Environmental factors ( $\mathrm{pH}$, ionic forces, temperature, and time).

The antimicrobial action mechanism of the chitosan is not yet fully elucidated, being several mechanisms are suggested by the literature. Some authors suggested the amino groups of the chitosan when in contact with physiological fluids are protonated and bind to anionic groups of the microorganisms, resulting in the agglutination of the microbial cells, and growth inhibition $[49,50]$. On the other hand, reference [49] report that when interacting with the bacterial cell, the chitosan, promotes displacement of $\mathrm{Ca}^{++}$of the anionic sites of the membrane, damaging them. Another postulate is the interaction between the positive load of the chitosan and the negative load of the microbial cell wall, because it causes the rupture and loss of important intracellular constituent of the microorganism life. Chitosan with low molecular weight penetrates in the cell and is linked to the microorganism DNA inhibiting the transcription and consequently the translation, whereas the chitosan of high molecular weight acts as a chelate agent, binding to the cell membrane [16].

Authors in reference [47] investigated the relation between antimicrobial activity of the chitosan and the characteristics of the cellular wall of bacteria. They verified that the chitosan is antibacterial agent more efficient to Gram-negative bacteria due the composition of phospholipids and carboxylic acids of the bacterial cellular wall. These results suggest that the effects of the chitosan are distinct in the two types of bacteria: in the case of the gram-positive, the hypothesis is that chitosan of high molecular mass may form films 
around the cell that inhibit the absorption of nutrients, while chitosan of low molecular mass penetrates more easily in gram-negative bacteria, causing riots in the metabolism of these microorganisms.

Chitosan from M. circineloides was obtained as described the literature [33]. The chitosan with different molecular weight was obtained by methods of extraction. Hight molecular weight of chitosan was obtained by methodology described by Stamford et al [32], and Fai et al.[ 33], and low molecular weight of chitosan by methodology described by $\mathrm{Hu}$ et al.[54]. The degree of deacetylation for chitosan was determined by infrared spectroscopy, the molecular weights by viscosity described by Stamford et al [32] and Fai et al.[33]. However, the chitosan with low molecular weight showed antimicrobial activity to Gram positive and negative bacteria than chitosan of high molecular weight (Table 2)

\begin{tabular}{lcccc}
\hline Bacteria & \multicolumn{2}{c}{$\begin{array}{c}\text { Minimum inhibitory concentration } \\
(\mathrm{mg} / \mathrm{ml})\end{array}$} & \multicolumn{2}{c}{$\begin{array}{c}\text { Minimum bactericidal } \\
\text { concentration }(\mathrm{mg} / \mathrm{mL})\end{array}$} \\
\cline { 2 - 5 } & \multicolumn{2}{c}{ Chitosan } & \multicolumn{2}{c}{ Chitosan } \\
& ChLMW & ChHMW & ChLMW & ChHMW \\
\hline S. aureus & 1.25 & 2.5 & 2.5 & 5.0 \\
E. coli & 1.25 & 2.5 & 2.5 & 5.0 \\
P. aeroginosa & 0.625 & 1.25 & 1.5 & 5.0 \\
S. mutans & 0.625 & 1.25 & 1.5 & 5.0 \\
\hline
\end{tabular}

Table 2. Minimum Inhibitory Concentration (mg. $\left.\mathrm{mL}^{-1}\right)$ of chitosan from Mucor circineloides with different molecular weight: Low molecular weight chitosan with $3.2 \times 10^{3} \mathrm{~g} / \mathrm{mol}$ (ChLMW) and higher molecular weight chitosan $2.72 \times 10^{5} \mathrm{~g} / \mathrm{mol}(\mathrm{ChHMW})$, against pathogenic bacteria

Studies in vitro were carried out in the Microbiology laboratory of UFPB to determine the Minimum Inhibitory Concentration (CIM) and the Minimum Bactericidal Concentration $(\mathrm{MBC})$ of chitosan from different origins, and varying the parameters deacetylation degree and molecular weight to oral Streptococcus species. The results showed greater influence of the molecular weight of the polymer on antibacterial activity. The low molecular weight of chitosan have shown higher antimicrobial activity to CIM and CBM, when compared with chitosan gel (soluble in acetic acid 1\%), and with higher molecular weight, as described in the Table 3.

In addition, chick embryo chorioallantoic membrane (CAM), is a rapid and inexpensive method for determination of tissue reactions to biomaterials, and was used to evaluate the chitosan toxicity and physiological compatibility. Positive control was used as sodium lauryl sulfate $1 \%$. The method allows prediction of the potential irritation by chitosan was studied.

Fertile hen's eggs at $10^{\circ}$ days of incubation at $37^{\circ} \mathrm{C}$, obtained from Guaraves Guarabira Aves Ltda, were used in the tests. Five eggs were used for each chitosan solution assayed. On day 10 of incubation, the egg shell above the air space was removed. The exposed membrane was moistened with a drop of $0.9 \%$ physiological saline and the saline was removed, uncovering the CAM. An aliquot of $200 \mu \mathrm{l}$ of chitosan solution was applied on the CAM. Signs of vasoconstriction, hemorrhage and coagulation for 5 minutes were observed to 
evaluate the potential for irritation according to the method of HET-CAM. Thus, biocompatibility of chitosan was evaluated including the search for signs of inflammation, edema or neovascularization [55].

\begin{tabular}{|c|c|c|c|c|c|c|c|c|}
\hline \multirow[t]{3}{*}{ Chitosan } & \multicolumn{8}{|c|}{ Streptococcus species } \\
\hline & \multicolumn{2}{|c|}{ S. mutans } & \multicolumn{2}{|c|}{ S. sanguis } & \multicolumn{2}{|c|}{ S. oralis } & \multicolumn{2}{|c|}{ S. mitis } \\
\hline & CIM & CBM & CIM & CBM & CIM & $\mathrm{CBM}$ & CIM & CBM \\
\hline $\begin{array}{l}\text { Crustaceans 65DA\% } \\
3,5 \times 10^{6}\end{array}$ & 1.25 & 5.0 & 1.25 & 7.5 & 1.25 & 5.0 & 1.25 & 5.0 \\
\hline $\begin{array}{l}\text { Crustaceaus } \\
\text { 90DA\% } 3,5 \times 10^{6}\end{array}$ & 1.25 & 2.5 & 1.25 & 5.0 & 1.25 & 2.5 & 1.25 & 2.5 \\
\hline $\begin{array}{l}\text { Crustaceans } \\
90 \mathrm{DA}^{2} 3,1 \times 10^{4}\end{array}$ & 0.625 & 1.25 & 0.625 & 2.5 & 0.625 & 1.25 & 0.625 & 1.25 \\
\hline $\begin{array}{l}\text { Fungi } \\
80 \% \text { DA } 2,72 \times 10^{6}\end{array}$ & 1.25 & 2.5 & 1.25 & 2.5 & 1.25 & 2.5 & 1.25 & 2.5 \\
\hline $\begin{array}{l}\text { Fungi } \\
90 \% \text { DA } 2,72 \times 10^{6}\end{array}$ & 1.25 & 2.5 & 1.25 & 2.5 & 1.25 & 2.5 & 1.25 & 2.5 \\
\hline $\begin{array}{l}\text { Fungi } \\
90 \% \text { DA } 2,3 \times 10^{4}\end{array}$ & 0.625 & 1.25 & 0.625 & 1.25 & 0.625 & 1.25 & 0.625 & 1.25 \\
\hline
\end{tabular}

Table 3. Studies in vitro performed by Microbiology laboratory, UFPB (João Pessoa-PB, Brasil) demonstrating the Minimum Inhibitory Concentration (CIM) and Minimum Bactericidal Concentration (CBM) of the chitosan (derivatives from different origins, 80 and $90 \%$ of deacetylation degree (DA), molecular weight (MW) $\mathrm{g} / \mathrm{mL}$ and solubility) to Streptococcus species

\begin{tabular}{c|c|c|c}
\hline Assays & Chmw & Clmw & SLS 1\% \\
\hline Vasoconstriction & $0.0 \pm 0.0$ & $0.0 \pm 0.0$ & $6.0 \pm 1.0$ \\
Hemorrhage & $0.0 \pm 0.0$ & $0.0 \pm 0.0$ & $48 \pm 3.0$ \\
Coagulation & $0.0 \pm 0.0$ & $0.0 \pm 0.0$ & $63 \pm 3.0$ \\
Irritation potential & $0.0 \pm 0.0$ & $0.0 \pm 0.0$ & $17,74 \pm 0,4$
\end{tabular}

Non-irritating: 0-0.9 ; slightly irritating: 1-4.9; Irritating: 5-8.9 and Severely irritating: 9-21

Table 4. Test of chitosan to high (Chmw) and low (Clmw) molecular weight against vasoconstriction, hemorrhage and coagulation. Positive control: sodium lauryl sulfate 1\% (SLS1\%).

\begin{tabular}{ccccccc}
\hline \multirow{2}{*}{ Assays } & \multicolumn{2}{c}{ Inflammation } & \multicolumn{2}{c}{ edema } & \multicolumn{2}{c}{ neovascularization } \\
\cline { 2 - 7 } & Chmw & Clmw & Chmw & Clmw & Chmw & Clmw \\
1 & 0.0 & 0.0 & 0.0 & 0.0 & 0.0 & 0.0 \\
2 & 0.0 & 0.0 & 0.0 & 0.0 & 0.0 & 0.0 \\
3 & 0.0 & 0.0 & 0.0 & 0.0 & 0.0 & 0.0 \\
4 & 0.0 & 0.0 & 0.0 & 0.0 & 0.0 & 0.0 \\
5 & 0.0 & 0.0 & 0.0 & 0.0 & 0.0 & 0.0 \\
\hline
\end{tabular}

Table 5. Test of chitosan to high (ChHMW) and low (ChLMW) molecular weight for inflammation, edema and neovascularization. 


\section{Anticariogenic effects of chitosan}

Experiments were performed in the Microbiology Laboratory-Nucleus of Research in Environmental Sciences- UNICAP (Recife, PE, Brazil) regarding the safety concentration for the dilution of fungi chitosan in acetic acid. The mechanisms of chitosan from crabs and fungi to inhibit the tooth colonization by S. mutans, S. sanguis, S. mitis and S. oralis were evaluated through the adherence test of chitosan to dental and bacteria surface, results showed in Figure 2. Chitosan from crabs and fungi, in all concentration tested, decreased the adsorption of Streptococcus strains to dental enamel, reduced the bacteria cell wall hydrophobicity and decreased the glucan production by bacteria. However, chitosan from fungi was more efficient than chitosan from crabs for the tree parameters studied.

Figure 2A shows the decrease of bacteria adsorption to dental enamel in the presence of chitosan from crabs and fungi, in all concentrations studied. Chitosan demonstrated best performance at the concentration of $2 \mathrm{mg} / \mathrm{mL}$ for $S$. mutans and of $3 \mathrm{mg} / \mathrm{mL}$ for $S$. sanguis, $S$. mitis and S. oralis. These results are in agreement with the one obtained by [8], which studied the effect of chitosan from crabs of low molecular weight in the adsorption of $S$. mutans, S. sanguis and S. oralis to the commercial hydroxyapatite.

Researchers $[9,10]$ investigated, "in vivo", the activity of a chitosan mouthrinse, respectively of $1 \%$ and $0.5 \%$, and verified significant reduction of dental plaque formation. The authors reported that chitosan might be altering of the electrostatic interaction between the bacterial cell surface in saliva and tooth pellicle surface. The electrostatic interaction is usually repulsive due to the fact that inature both bacteria and the pellicle surface are predominantly negatively charged. The chitosan chains attach themselves to the negatively charged bacterial cell surface by means of their positively charged groups. If these chains are of a sufficient length to bind more than one cell, bridges are formed between bacterial cells. As soon as the bridging becomes effectives flocs are formed, and the bacteria cannot colonize the tooth surface.

In literature [8-10] is reported that aggregating oral bacteria may reduce their adherence to tooth surface. The polycationic nature of chitosan might reduce the initial bacterial adherence onto the teeth surfaces, at least in part, by generating bacterial aggregation. There have also been suggestions that bacterial aggregates are removed more easily from the oral cavity than individual bacterial cells.

To verified the influence of chitosan, sublethal concentration, to modification in bacterial cell surface, it was evaluated the affinity of chitosan to xylene of bacteria grown in the presence or absence of chitosan sub-MICs, through hydrophobicity tests (Figure 2B), and for sucrose catabolization, through extracellular glucan production by bacteria strains (Figure 2C). The results in figure $2 \mathrm{~B}$ indicates that increasing concentration of chitosan in bacterial suspension caused a successive decrease of the bacteria cell wall hydrophobicity, being more evident for the $S$. mutans strain. These results are supported by findings of $[8,9]$. These authors suggested that chitosan induced a successive decrease in cell hydrophobicity, and that surface hydrophobicity is related to adherence ability of bacteria. Therefore, the 
inhibiting effects of chitosan may depend, at least, partially, on alterations of bacterial cell surface hydrophobicity expression.

In figure $2 \mathrm{C}$ is observed a decreasing of extracellular glucan production by bacteria strain in presence of sucrose, with increasing chitosan concentration. The ionic interaction between the cation, according to [61,62], from chitosan (amine group) and anionics parts of bacteria cell wall (phospholipids and carboxylic acids) can form a membrane polymer, which prevents nutrients from entering the cell. Since chitosan could adsorb the electronegative substance, this polymer can promote cell flocculate, and disturbs the physiological activities of the bacteria and kill them. Authors [63] reported that chitosan interact with the electronegative bacterial cell surface resulting in displacement of $\mathrm{Ca}^{++}$from anionic membrane sites, resulting in a changing the electric potential of the bacteria surface.

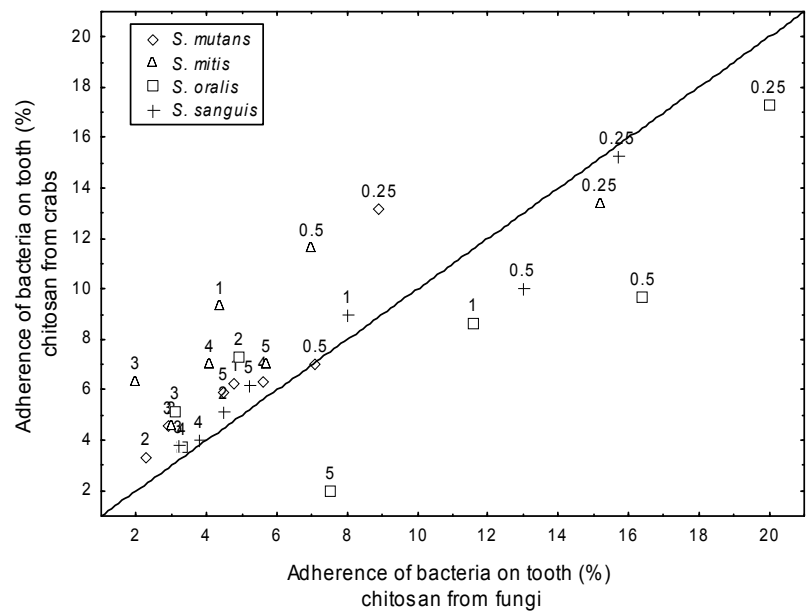

A

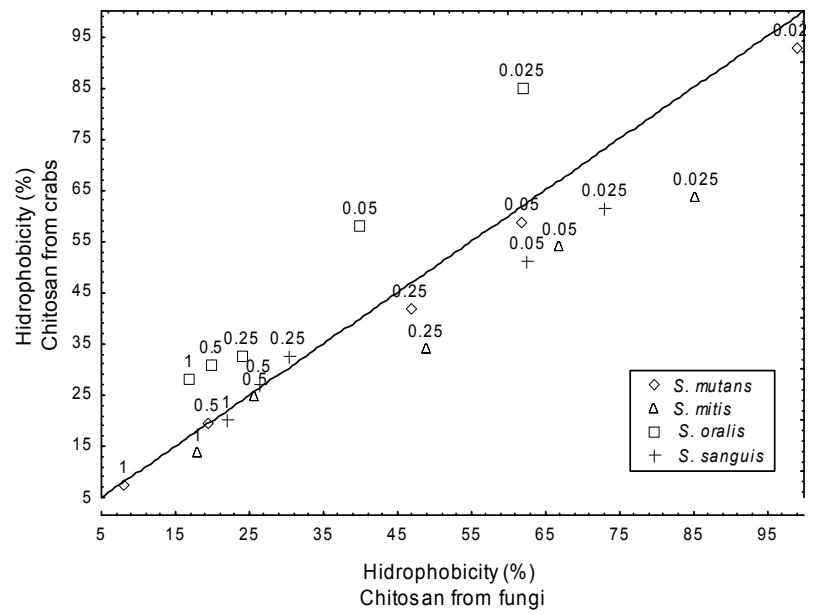

$\mathrm{B}$ 


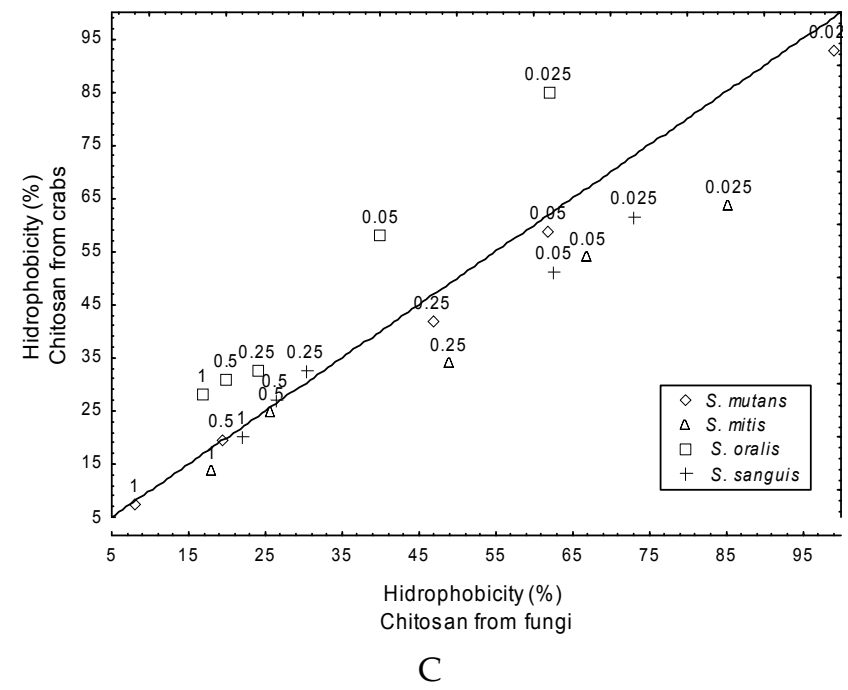

Figure 2. Diagram of stratification dispersion of chitosan from fungi and crabs activity: inhibition of bacteria adsorption on dental enamel (A); hydrophobicity of bacteria surface (B) and glucan production by bacteria $(\mathrm{C})$.

Experiments were conducted in the department of chemistry's fundamental UFPE verified the penetration depth of chitosan in teeth enamel for two specimens by Optical Coherence Tomography (OCT) images. Chitosan was applied only to half of the teeth surface. The image in the left was obtained for a chitosan concentration of $1.25 \mathrm{mg} / \mathrm{ml}$. it is clearly seen the penetration of chitosan as indicated by the brighter area (caused by light scattering) in the figure, only in the region where chitosan may act as a mechanical barrier for the acid penetration in the enamel, which would explain its effect in the demineralization inhibition [2].
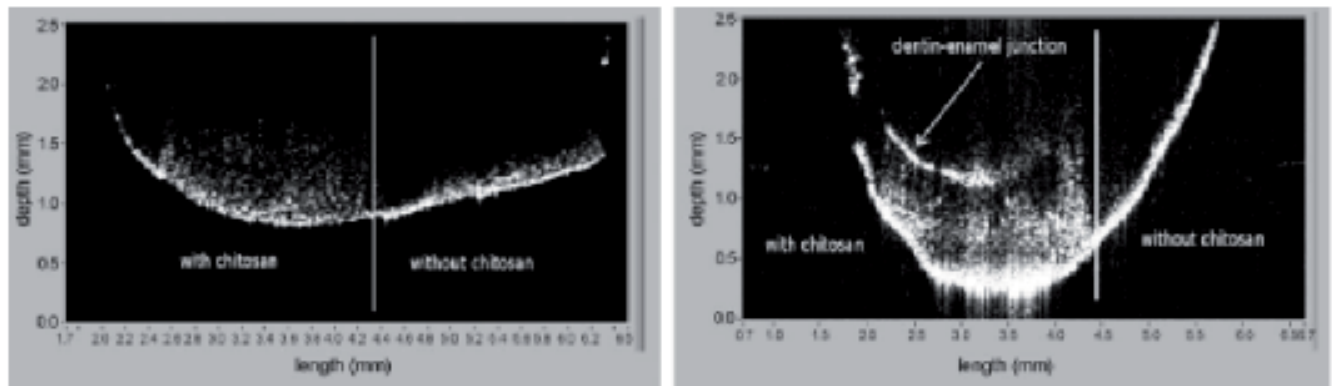

Sources: [2]

Figure 3. Optical Coherence Tomography (OCT) images of specimens treated with chitosan at $1.25 \mathrm{mg} / \mathrm{mL}$ (left) and $5.0 \mathrm{mg} / \mathrm{mL}$ (right)

\section{Conclusion}

The use of chitosan in different formulations, such as toothpaste (Chitodent $\left.{ }^{\circledR}\right)$, mouthwash solution and chewing gum, is mentioned in literature [8-10]. In all forms the chitosan has 
shown antibacterial action for bacteria belonging to the group Streptococcus, inhibits the growth and adherence of cariogenic bacteria and the desmineralization process of dental enamel in vitro, and stimulates salivation, in vivo. The results confirm a high biotechnological potential for chitosan from both fungi and crab sources as a cariostatic and anticariogenic agent, suggesting their application as dentistry biomaterial for prevention and therapeutic of dental carie.

\section{Author details}

Thayza Christina Montenegro Stamford

Department of Physiology and Pathology, Federal University of Paraíba, Paraíba-PB, Brazil

Thatiana Montenegro Stamford-Arnaud

Post-graduate in Materials Science, Federal University of Pernambuco, Recife-PE, Brazil

Horacinna Maria de Medeiros Cavalcante and Rui Oliveira Macedo

Department of Pharmaceutical Science, Federal University of Paraíba, Paraíba-PB, Brazil

Galba Maria de Campos-Takaki*

Nucleus of Research in Environmental Sciences, Catholic University of Pernambuco, Recife-PE, Brazil

\section{Acknowledgement}

The authors are gratefully to Conselho Nacional de Desenvolvimento Científico e Tecnológico (CNPq), Coordenação de Aperfeiçoamento de Pessoal de Nível Superior (CAPES), and Fundação de Amparo à Ciência e Tecnologia do Estado de Pernambuco (FACEPE).

\section{References}

[1] Tahmourespour A, Kermanshahi RK, Saleh R, Pero NG. Biofilm formation potential of oral streptococci in related to some carbohydrate substrates. African Journal of Microbiology Research. 2010; 4 (11) 1051-1056.

[2] Stamford-Arnaud TM, Barros Neto B, Diniz FB. Chitosan effect on dental enamel deremineralization: an in vitro evaluation. Journal of Dentistry. 2010; 38 (11) 848-852.

[3] Keegan GM, Smart JD, Ingram MJ, Barnes LM, Burnett GR, Rees GD. Journal of Dentistry. 2012; 40, 229-240.

[4] Fitzgerald RJ, Keyes PH. Demonstration of the etiologic role of streptococci in experimental caries in the hamster. Journal of the American Dental Association. 1960; 61, 9-19.

[5] Carvalho MMSG, Stamford TCM, Santos EP, Tenorio P, Sampaio F. Chitosan as n oral antimicrobial agent. In: Mendez A (ed). Science against microbial pathogens:

" Corresponding Author 
Communicating current research and technological advances. Badajoz, Formatex. 2012. 542-550.

[6] Steinberg D; Poran S; Shapira L. The effect of extracellular polysaccharides from Streptococcus mutans on the bactericidal activity of human neutrophils. Archives of Oral Biology. 1999; 44, 437-444.

[7] Cury LA; Rebelo MAS; Del Bel Cury AA; Derbyshire MTVC. Biochemical composition and cariogenicity of dental plaque formed in the presence of sucrose, glucose and fructose. Caries Research. 2000; 34, 491-497.

[8] Tarsi R; Corbin B; Pruzzo C; Muzzarelli RAA. Effect of low-molecular-weight chitosans on the adhesive properties of oral streptococci. Oral Microbiology and Immunology. 1999;13, 217-224

[9] Sano H; Shibasaki K; Matsukubo T; Takaesu Y. Comparison of the activity of four chitosan derivatives in reducing initial adherence of oral bacteria onto tooth surfaces. Bulletin of Tokyo Dental College. 2001; 42, 243-9.

[10] Sano H; Shibasaki K; Matsukubo T; Taraesu Y. Effect of chitosan rinsing on reduction of dental plaque formation. Bulletin of Tokyo Dental College. 2003; 44(1), 9-16.

[11] Alexander C, Marsh L. Creating the optimum environment for pressure area care. Brazilian Journal of Nursery. 1992;1(15),751-757.

[12] Ji QX, Zhong DY, Lu R; Zhang WQ; Jing D; Chen XG. In vitro evaluation of biomedical propesties of chitosan and quaternized chitosan for dental applications. Carbohydrate Research. 2009; 344, 1297-1302.

[13] Canella KMNC, Garcia RB. Caracterização de quitosana por cromatografia de permeação em gel . influência do método de preparação e do solvente. Quimica Nova 2001,;24(1),13-17.

[14] Silva MCF, Moraes-Filho M, Jara AMT, Silva AC, Stamford TCM, Campos-Takaki GM. Effect of phenanthrene on germination, radial growth and chitin and chitosan production by Cunninghamella elegans Lendner. p. 219-222. In: Current Research Topics in Applied Microbiology and Microbial Biotechnology:proceedings id the II International Conference on Environmental, Industrial and apllied microbiology (BioMicroWorld 2007). Editor Antonio Mendez-Vilas. World Scientific, 2009

[15] Costa Silva HSR, Santos KSCR, Ferreira EI. Quitosana: derivados hidrossolúveis, aplicações farmacêuticas e avanços. Quimica Nova; 2006, 29(4), 776-785.

[16] Pedro AS, Cabral-Albuquerque E, Ferreira D, Sarmento B. Chtosan: na option for development of essencial oil delivery systerms for oral cavity care? Carbohydrate Polymers, 2009, 76, 501-508

[17] Franco LO, Stamford TCM, Stamford NP, Campos-Takaki GM. Cunninghamella elegans (IFM 46109) como fonte de quitina e quitosana. Revista Analytica, 2005; 3(10),4044.

[18] Stamford-Arnaud TM, Stamford TCM. Caracterização da quitosana e sua aplicação na nanotecnologia. Cap 6 p.733-761. In: Biotecnologia aplicada à agricultura: Textos de apoio $e$ protocolos experimentais. Edtores FIGUEIREDO MVB; BURITY HA OLIVEIRA JP; 
SANTOS CERS; STAMFORD NP. Embrapa Informação Tecnológica; Instituto Agronômico de Pernambuco (IPA), p.761, 2010

[19] Garcia RB, Silva DLP, Costa M, Raffin FN, Ruiz NMS. Avaliação de géis obtidos a partir da acetilação da quitosana em meio heterogêneo Quím. Nova (2008), 31(3).

[20] Campos-Takaki GM The fungal versatility on the copolymers chitin and chitosan production. In: Dutta PK (ed.). Chitin and chitosan opportunities and challenges. India, SSM: International Publication. 2005, 69-94.

[21] Stamford TCM, Stamford TLM, Franco LO. Produção, propriedades e aplicações da quitosana na agricultura e no ambiente. Cap. 21 p 487-506. In: Microrganismos e agrobiodiversidade: o novo desafio para a agricultura. Ed Figueiredo MVB; Burity HA; Stamford NP; Santos CERS. Agro livros, 568p. 2008

[22] Nadarajah K; Kader J; Mohd M; Paul DC. Production of chitosan by fungi, Journal of Biolical Science. 2001, 4 (3), 263-265.

[23] Nwe N; Chandrkrachang S; Stevens WF; Maw T; Tan TK; Khor E; Xong SM. Production of fungal chitosan by solid state and submerged fermentation. Carbohydrate Polymer, 2002,49 (2), 235-237

[24] Tajdinia F; Ali Aminia M; Nafissi-Varchehc N; Faramarzi MA. Production, physiochemical and antimicrobial properties of fungal chitosan from Rhizomucor miehei and Mucor racemosus. International Journal of Biological Macromolecules, 2010, 47, 180-183

[25] Andrade VS; Barros Neto B; Souza W; Campos-Takaki GM. A factorial designs analysis of chitin production by Cunninghamella elegans. Canadian Journal of Microbiology, 2000, 46, (11), 1042-1045.

[26] Amorim RVS; Souza W; Fukushima K; Campos-Takaki GM. Faster chitosan production by mucoralean strains in submerged culture. Brazilian Journal of Microbiology, 2001, 32 (1), 20-23.

[27] Andrade VS; Barros Neto B; Fukushima K; Campos-Takaki GM. Effect of medium components and time of cultivation on chitin production by Mucor circinelloides (Mucor javanicus IFO 4570)-A factorial study. Revista Iberoamericana de Micologia, 2003, 20, 149-153.

[28] Amorim RVS; Ledingham WM; Fukushima EK; Campos-Takaki GM. Screening of chitin deacetylase from Mucoralean strains (Zygomycetes) and its relationship to cell growth rate. Journal of Industrial Microbiology and Biotechnology, 2005, 31, 19-23.

[29] Silva MCF; Stamford TCM; Franco LO; Campos-Takaki GM. Effects of salinity and glucose on chitin and chitosan production by Cunninghamella elegans. Asian Chitin Journal, 2006, 2, 29-38.

[30] Amorim RVS; Pedrosa RP; Kazutaka F; Martínez CR; Ledingham WM; Campos-Takaki GM. Alternative carbon sources from sugar cane process for submerged cultivation of Cunninghamella bertholletiae to produce chitosan. Food Technology and Biotechnology, 2006, 44 (4), 519-523. 
[31] Silva MCF; Barros Neto B; Stamford TCM; Campos-Takaki GM. Effect of environmental conditions on chitin and chitosan production by Cunninghamella elegans UCP 542 using factorial design. Asian Chitin Journal, 2007, 3, 15-22.

[32] Stamford TCM; Stamford TLM; Stamford NP; Barros Neto B; Campos-Takaki GM. Growth of Cunninghamella elegans UCP 542 and production of chitin and chitosan using yam bean medium. Electronic Journal of Biotechnology 2007, 10, 1. Available at: < http://www.ejbiotechnology.info/content/vol10/issue1/full/1/>.Accessed on: 04/16/2011.

[33] Fai AEC; Stamford TCM; Stamford-Arnaud TM; Santa-Cruz PDA; Silva MCF; CamposTakaki GM; Stamford TLM. Physico-chemical characteristics and functional properties of chitin and chitosan produced by Mucor circinelloides using yam bean as substrate. molecules Molecules 2011, 16, 7143-7154; doi:10.3390/molecules16087143

[34] Pochanavanich, P.; Suntornsuk, W. Fungal chitosan production and its characterization. Lettes Applied Microbiology. 2002, 35, 17-21.

[35] Winterowd JG, Sandford PA Chitin and Chitosan. In: Stephen AM. Food Polysaccharides and Their Applications. New York: Marcel Dekker, 1995.

[36] Hamman, JH. Chitosan Based Polyelectrolyte Complexes as Potential Carrier Materials in Drug Delivery Systems. Marine Drugs; 2010, 8, 1305-1322

[37] Feng Y, Xia W. Preparation, characterization and antibacterial activity of water-soluble O-fumaryl-chitosan .Carbohydrate Polymers, 2011, 83, 1169-1173

[38] Shibasaki K, Sano H, Matsukubo T, Takaesu Y. The influence of the buffer capacity of various substances on $\mathrm{pH}$ chenges in dental plaque. Bullet Tokyo Dental College (1994), 35(1):27-32.

[39] Kong M, Chen XG, Xing K, Park HJ. Antimicrobial properties of chitosan and mode of action: A state of the art review. International Journal of Food Microbiology, 2010, 144: 51-63

[40] Murugan R, Ramakrishna S. Bioresorbable composite bone paste using polysaccharide based nano hydroxyapatite. Biomaerial, 2004, 25:3829-3835

[41] Tanodekaew S, Prasitsilp M, Swasdison S, Thavornyutikarn B, Pothsree, Pateepasen, R. Preparation of acrylic grafted chitin for wound dressing application. Biomaterial, 2004, 25:1453-1460.

[42] Maia RCC, Franco LO, Stamford TCM, Fukushima K, Porto ALF, Campos-Takaki GM. Chitin producted by Cunninghamella elegans (IFM 46109) and applied to wound healing. Asian Chitin Journal, 2006, 2:11-20

[43] Laranjeira MCM, Fávere VT. Quitosana: biopolímero funcional com potencial industrial biomédico. Quimica Nova 2009, 32(3): 672-678.

[44] Pillai CKS, Paul W, Sharma CP. Chitin and chitosan polymers: Chemistry, solubility and fiber formation. Progress Polymer Science, 2009, 34, 641-678

[45] Xia W, Liu P, Zhang J, Chen J. Biological activities of chitosan and chitooligosaccharides. Food Hydrocolloids,2011, 25,170-179

[46] Yadav AV, Bhise .B. Chitosan: a potencial biomaterial effective against typhoid. Currient Science, 2004, 87(9), 1176-1178. 
[47] Chung YC, Su YP, Chen CC, Jia G, Wang HL, WU JCG, LIN JG. Relationship between antibacterial activity of chitosan and surface characteristics of cell wall. Acta Pharmacologica Sinica.2004, 25(7),932-936.

[48] Muzzarelli R, Tarsi R, Filippini O, Giovanetti E, Biagini G, Varaldo PE. Antimicrobial properties of N-carboxybutyl chitosan. Antimicrob. Agents Chemotherapy, 1990, 34,2019-2023.

[49] Senel S, Ikinci G, Kas S, Yousefirad A, Sargon M,; Hincal AA. Chitosan films and hydrogels of chlorhexidine gluconate for oral mucosal delivery. Internatinal Journal of Pharmeutical, 2000,5(2),197-203.

[50] Avadi, MR., Sadeghi, AMM., Tahzibi, A., Bayati, KH., Pouladzadeh, M.,ZohuriaanMehr, MJ.,et al. Diethylmethyl chitosan as na antimicrobial agent: synthesis, characterization and antibacterial effects. European Polymer Journal, 2004, 40:13551362.

[51] Tsai GJ, Hwang SP. In vitro and in vivo antibacterial activity of shrimp chitosan against some intestinal bacteria. Fisher. Scientific. 2004, 70: 675-681.

[52] Ikinci G, Senel S, Akincibay H, Kas S, Ercis S, Wilson CG, Hincal A.A.: Effect of chitosan on a periodontal pathogen Porphyromonas gingivalis. International Journal of Pharmeutical, 2002,235:121-7

[53] Zheng LY, Zhu JF. Study on antimicrobial activity of chitosan with different molecular weights. Carbohydrate Polymer, 2003, 45: 527-530

[54] Hu KJ; Yeung KW; Ho KP; Hu JL. Rapid extraction of high-quality chitosan from mycelia of Absidia glauca. Journal of Food Biochemistry, 1999, 23(2), 187-196.

[55] Bagley DM; Waters D; Kong BM. Development of a 10-day chorioallantoic membrane vascular assay as an alternative to the Draize rabbit eye irritation test. Food and Chemical Toxicology, 1994, 32, 1155-1160.

[56] Vargas A; Zeisser-Labouèbe M; Lange N; Gurny R; Delie F. The chick embryo and its chorioallantoic membrane (CAM) for the in vivo evaluation of drug delivery systems. Advanced Drug Deliver Review, 2007, 59, 1162-1176.

[57] Alany, R. G.; Rades, T.; Nicoll, J.; Tucker, I. G.; Davies, N. M. W/O microemulsions for ocular delivery: Evaluation of ocular irritation and precorneal retention. Journal of Controlled Release. 2006, 111, 145-152.

[58] Lagarto, A.; Vega, R.; Guerra, I.; González, R. In vitro quantitative determination of ophthalmic irritancy by the chorioallantoic membrane test with trypan blue staining as alternative to eye irritation test. Toxicology In Vitro. 2006, 20, 699-702.

[59] Valdes, T. I.; Kreutzer, D.; Moussy, F. The chick chorioallantoic membrane as a novel in vivo model for the testing of biomaterials .Journal of Biomedical Materials Research. 2001, 62(2) 273-282.

[60] Zwadlo-Klarwasser, G.; Gorlitz, K.; Hafemann, B.; Klee, D.; Klosterhalfen, B. The chorioallantoic membrane of the chick embryo as a simple model for thestudy of the angiogenic and inflammatory response to biomaterials. Journal of Materials Science Materials in Medicine. 2001,12, 195-199. 
[61] Ikinci G, Senel S, Akincibay H, Kas S, Ercis S, Wilson CG, Hincal A.A.: Effect of chitosan on a periodontal pathogen Porphyromonas gingivalis. Intrnational Journal of Pharmaceutical, 2002,235,121-7

[62] Zheng LY, Zhu JF. Study on antimicrobial activity of chitosan with different molecular weights. Carbohydrate Polymer, 2003, 45, 527-530

[63] Yadav AV, Bhise .B. Chitosan: a potencial biomaterial effective against typhoid. Currient. Science, 2004, 87(9), 1176-1178. 


\title{
Antimicrobial and Anti-Adhesive Potential of a Biosurfactants Produced by Candida Species
}

\author{
Raquel Diniz Rufino, Juliana Moura de Luna, Leonie Asfora Sarubbo, \\ Lígia Raquel Marona Rodrigues, José Antônio C. Teixeira \\ and Galba Maria de Campos-Takaki
}

Additional information is available at the end of the chapter

http://dx.doi.org/10.5772/52578

\section{Introduction}

Several compounds with tensioactive properties are synthesized by living organisms, from plants (e.g. saponins) to microorganisms (e.g. glycolipids) and humans (e.g. surface-active lipoprotein complex), being considered natural surfactants [1]. Additionally, these compounds have been produced through biotechnological processes broadening their diversity and potential applications [2]. Surfactants are usually organic compounds that are amphiphilic, meaning they contain both hydrophobic groups (tails) and hydrophilic groups (heads), and that act preferably in the interface of fluid phases with different levels of polarity and bridges of hydrogen, such as oil/water or air/water interfaces. Many microbes appear to produce a complex mixture of biosurfactants, particularly during their stationary growth on water-immiscible substrates. Generally, biosurfactants are secondary metabolites with the typical amphiphilic structure of a surfactant, where the hydrophobic moiety is either a long-chain fatty-acid, hydroxyl fatty acid, or $\alpha$-alkyl- $\beta$-hydroxy fatty acid and the hydrophilic moiety can be a carbohydrate, an amino acid, a cyclic peptide, a phosphate, a carboxylic acid, or alcohol, among others [1].

Physical and chemical properties, surface tension reduction, and stability of the emulsion formed are important characteristics in biosurfactant that make possible its use in countless biological applications. Most work on biosurfactant applications has been focused on their use in environmental applications owing to their diversity, environmentally friendly nature, suitability for large-scale production and selectivity [3]. Biosurfactants have several advantages over chemical surfactants, such as lower toxicity, higher biodegradability and effectiveness at extreme temperatures or $\mathrm{pH}$ values [4]. Many of the potential applications that have been considered for biosurfactants depend on whether they can be produced 
economically; however, much effort in process optimization and at the engineering and biological levels have been carried out [5]. Despite their potential and biological origin only a few studies have been carried out on applications related to the biomedical field [6]. Some biosurfactants are suitable alternatives to synthetic medicines and antimicrobial agents and may be used as safe and effective therapeutic agents [6].

Furthermore, biosurfactants have been found to inhibit the adhesion of pathogenic organisms to solid surfaces or to infection sites hampering biofilm formation that is the cause of many diseases, as for example cystic fibrosis [7]. Therefore, prior adhesion of biosurfactants to solid surfaces might constitute a new and effective means of combating colonization by pathogenic microorganisms and subsequent biofilm formation $[8,9]$.

Pre-coating vinyl urethral catheters by running a surfactin solution through them before inoculation with media resulted in a decrease in the amount of biofilm formed by Salmonella typhimurium, S. enterica, Escherichia coli and Proteus mirabilis [10]. Given the importance of opportunistic infections with Salmonella species, including urinary tract infections of AIDS patients, these results have great potential for practical applications. In addition, the use of lactobacilli as a probiotic for the prevention of urogenital infections has been widely studied [11].

Microbial surfactants are not yet competitive with those produced by the chemical industry, but efforts should be made on the different production aspects to find suitable and economic substrates and to develop new strategies to increase the volumetric productivity. We have shown that the co-utilization of ground-nut oil refinery residue and corn steep liquor is an attractive choice for biosurfactant production. The biosurfactant adhesive mechanism is based in the inhibition of microorganisms to different surfaces can interact with interfaces of the molecule. In this sense, they are an alternative to synthetic surface-active agents because of their low toxicity and biodegradability $[7,12]$.

Considering the lack of studies with yeasts biosurfactants for medical purposes, and is an attractive characteristics showed by produced by the Candida lipolytica (Rufisan) and Candida sphaerica (Lunasan). In this sense the revision shown the role and applications of rufisan and lunasan biosurfactant as a antimicrobial and antiadhesive activities were investigated against pathogenic and nonpathogenic microorganisms, and indicated the therapeutic perspectives.. Results gathered in the current work showed the potential of those molecules in this field of application; however, its use still remains limited, possibly as due to high production costs, as well as on their toxicity towards human systems.

\section{Adhesion and microbial biofilms}

The interest of biosurfactants as alternative medicines and antimicrobial agents increased considering the safe use as effective therapeutic agent on human and animal cells [12]. In consequence, the microbial adhesion is mediated by specific interactions between cells surface structures and molecular mass on the substratum surface, or by non-specific interaction forces, including electrostatic forces, acid-base interactions and Vander Waals forces [13]. 
during exponential growth, presumably as a result of increased cell wall hydrophobicity during this growth phase

The conditioning film on the biomaterial surface (and on the bacterial cell surface) plays an important role, as it changes the physicochemical properties of the interacting surfaces. Albumin is a strong adhesion inhibitor, for unknown reasons, although changes in hydrophobiciy and sterical hindrance are proposed as mechanisms [14].

The adhesion of microorganisms to a surface is one of the first stages in the development of a biofilm and is believed to be influenced by a number of factors. As the substrate is essential in the development of a biofilm, an understanding of how substrate properties affected the adherence of bacterial cells my assist in designing or modifying substrates inhibitory to bacterial adhesion. Many of these molecules are proteinaceous constitution, such as serum albumin, fibrogen and collagen, and some have been shown to affect subsequent bacterial adhesion [13].

The formation of infectious biofilm on biomaterial appeared to involve several sequential steps. Immediately after exposure of a device to body fluids, such as blood, saliva, or urine, macromolecular components adsorb to form a conditioning film [15]. The most microbial surfactants are complex molecules, comprising different structures that include peptides, glycolipids, glycopeptides, fatty acids and phospolipids, as reviewed recently. Among the many classes of biosurfactants, lipopeptides are particularly interesting because their high surface activities and antibiotic potential. Lipopeptides are molecules act as antibiotics, antiviral and antitumoral agents, and enzyme inhibitors. Those molecules enhance or decrease the bacterial surface hydrophobicity following that the surface is less or more hydrophobic[16]. Morikawa et al. [17] identified and characterized a biosurfactant, arthrofactin, produced by Arthrobacter.

Glycolipids are the most common class of biosurfactans of which the most effective from the point of view of surface active properties arethe trehalose lipids of Mycobacterium and related bacteria, the rhamnolipids of Pseudomonas sp and the sophorolipids of yeasts [18]. Otto et al. [19] described the production of sophorose lipids from deproteinized whey concentrate by a two-stage process. Several antimicrobial, immunological and neurological properties have been attributed to mannosylerythritol lipid (MEL), a yeast glycolipid biosurfactant, produced from vegetable oils by Candida strains.

\section{Antimicrobial activity of biosurfactant}

Several biosurfactants which exhibit antimicrobial activity against various microorganisms have been previously described. They include surfactin and iturin produced by Bacillus subtilis strains [20], rhamnolipids from Pseudomonas species [21], mannosylerythritol lipids from C. antarctica [22] and biosurfactants produced by some fungi [23].

Among the genus Bacillus, B. subtilis produces a broad spectrum of bioactive lipopeptides which have a great potential for biotechnological and biopharmaceutical applications. The 
characteristic structure of lipopeptides is a fatty acid combined with an amino-acid moiety. Several lipopeptides have potent antibiotic activity and have been the subject of several studies on the discovery of new antibiotics. The list includes surfactin, produced by $B$. subtilis, the most powerful of biosurfactant known to date. These compounds have many pharmacological activities: antibacterial, antifungal, antiviral, and antimycoplasma properties; inhibition of the fibrin clot formation and hemolysis; formation of ion channels in lipid bilayer membranes [24]; antitumour activity against Ehrlich's ascites carcinoma cells; and inhibition of the cyclic adenosine 3,5-monophosphate phosphodiesterase [25].

The evaluation of the antimicrobial activity of these compounds was carried out against 29 bacteria. Enterococcus faecalis (11 strains), Staphylococcus aureus (6 strains) and Pseudomonas aeruginosa (7 strains) and Escherichia coli CI 18 (1 strain) displayed a profile of well defined drug resistance. All strains were sensitive to the surfactants, in particular Enterococcus faecalis. The results demonstrated that lipopeptides have a broad spectrum ofaction, including antimicrobial activity against microorganisms with multidrug-resistant profiles [26].

The antimicrobial activity of the crude biosurfactant isolated from Candida lipolytica UCP 0988 was determined by measuring the growth inhibition percentages obtained for several microorganisms (Table 1).

\begin{tabular}{lccccc}
\hline \multirow{2}{*}{\multicolumn{1}{c}{ Microorganism }} & \multicolumn{5}{c}{ Growth inhibition (\%) } \\
\cline { 2 - 6 } & 0.75 & 1.5 & 3.0 & 6.0 & 12.0 \\
\hline Lactobacillus casej & $4.5 \pm 0.02$ & $9.1 \pm 0.07$ & $15 \pm 0.02$ & $27.2 \pm 0.08$ & $28.4 \pm 0.05$ \\
Lactobacillus casej72 & $4.5 \pm 0.03$ & $14.2 \pm 0.08$ & $18.1 \pm 0.06$ & $32.9 \pm 0.06$ & $33.7 \pm 0.03$ \\
Lactobacillus reuteri 104R & $5.9 \pm 0.07$ & $9.7 \pm 0.01$ & $15.5 \pm 0.01$ & $24.4 \pm 0.05$ & $25.4 \pm 0.08$ \\
Lactobacillus reuteri ML1 & $8.2 \pm 0.06$ & $11.3 \pm 0.02$ & $16.3 \pm 0.02$ & $31.1 \pm 0.02$ & $32.1 \pm 0.02$ \\
Escherichia coli & 0 & 0 & $3.0 \pm 0.01$ & $5.0 \pm 0.01$ & $5.0 \pm 0.01$ \\
Streptococcus agalactiae & 0 & $10.9 \pm 0.03$ & $35.3 \pm 0.03$ & $35.8 \pm 0.02$ & $35.5 \pm 0.02$ \\
Streptococcus mutans N S & $15.6 \pm 0.07$ & $20.1 \pm 0.04$ & $23.8 \pm 0.13$ & $46.0 \pm 0.1$ & $46.4 \pm 0.01$ \\
Streptococcus sanguis 12 & $13.7 \pm 0.07$ & $21.5 \pm 0.04$ & $31.7 \pm 0.05$ & $48.1 \pm 0.08$ & $48.0 \pm 0.01$ \\
Streptococcus mutans & $22.5 \pm 0.02$ & $34.8 \pm 0.01$ & $44.6 \pm 0.01$ & $58.3 \pm 0.01$ & $58.0 \pm 0.06$ \\
Streptococcus oralis J22 & $12.8 \pm 0.04$ & $13.2 \pm 0.03$ & $14.2 \pm 0.03$ & $18.7 \pm 0.08$ & $62.8 \pm 0.06$ \\
Streptococcus mutans HG985 & $41.8 \pm 0.02$ & $43.1 \pm 0.01$ & 55.60 .04 & $64.6 \pm 0.03$ & $64.9 \pm 0.01$ \\
Pseudomonas aeruginosa & 0 & $7.9 \pm 0.02$ & $10 \pm 0.01$ & $11.6 \pm 0.01$ & $16.5 \pm 0.04$ \\
Staphylococcus aureus & 0 & 0 & $1.57 \pm 0.06$ & $3.15 \pm 0.06$ & $15.1 \pm 0.03$ \\
Staphylococcus epidermidis & $10.1 \pm 0.01$ & $12.9 \pm 0.07$ & $14.9 \pm 0.07$ & $18.1 \pm 0.01$ & $18.0 \pm 0.06$ \\
Candida albicans & 0 & 0 & $3.1 \pm 0.03$ & $5.95 \pm 0.01$ & $6.0 \pm 0.02$ \\
\hline
\end{tabular}

Table 1. Percentages of growth inhibition obtained with the biosurfactant Rufisan isolated from Candida lipolytica at different concentrations ( $\mathrm{mg} / \mathrm{l})$. Results are expressed as means \pm standard deviations of values obtained from triplicate experiments

The biosurfactant was effective against the microorganisms tested, albeit to different degrees. The highest anti-adhesive percentages were obtained for a biosurfactant 
concentration of $12 \mathrm{mg} / \mathrm{l}$ or $4 \times \mathrm{CMC}$. Non-pathogenic species associated with the oral cavity of Streptococcus were used (S. mutans HG $-64.9 \%$; S. oralis J22 $-62.8 \%$; S. mutans $-58 \%$; S. sanguis $12-48 \%$; S. mutans NS - 46\%). On the other hand, the biosurfactant did not show an effective antimicrobial activity against the Lactobacillus strains studied. It inhibited only $32.1 \%$ of the growth of L. reuteri ML1 at the maximum concentration tested (12 mg/l).

The growth of the other microorganisms tested was poorly inhibited. Percentages of $5 \%, 5 \%$, $15 \%, 16 \%$ and $18 \%$ were observed for C. albicans, E. coli, S. aureus, P. aeruginosa and S. epidermidis, respectively.

The antimicrobial activity of the biosurfactant isolated from Candida sphaerica was determined by measuring the growth inhibition percentages obtained for several microorganisms (Table 2).

\begin{tabular}{|c|c|c|c|c|c|}
\hline \multirow{3}{*}{ Microorganism } & \multicolumn{5}{|c|}{ Growth inhibition (\%) } \\
\hline & \multicolumn{5}{|c|}{ Biosurfactant mg/L } \\
\hline & 0.625 & 1.25 & 2.5 & 5.0 & 10.0 \\
\hline Lactobacillus casej & $5.5 \pm 0.2$ & $12.0 \pm 0.3$ & $15.0 \pm 0.1$ & $30.0 \pm 0.4$ & $40.4 \pm 0.2$ \\
\hline Lactobacillus casej72 & $5.5 \pm 0.3$ & $11.0 \pm 0.2$ & $13.0 \pm 0.4$ & $25.0 \pm 0.3$ & $43.3 \pm 0.1$ \\
\hline Lactobacillus reuteri 104R & $10.0 \pm 0.1$ & $17.3 \pm 0.3$ & $22.0 \pm 0.5$ & $4.40 \pm 0.3$ & $46.5 \pm 0.1$ \\
\hline Lactobacillus reuteri ML1 & $8.5 \pm 0.3$ & $11.0 \pm 0.2$ & $16.0 \pm 0.2$ & $27.0 \pm 0.2$ & $49.0 \pm 0.2$ \\
\hline Escherichia coli & $7.3 \pm 0.2$ & $10.0 \pm 0.3$ & $11.0 \pm 0.3$ & $35.0 \pm 0.2$ & $46.0 \pm 0.2$ \\
\hline Streptococcus agalactiae. & $14.0 \pm 0.1$ & $17.8 \pm 0.1$ & $22.6 \pm 0.1$ & $38.3 \pm 0.1$ & $40.2 \pm 0.6$ \\
\hline Streptococcus mutans NS & $14.2 \pm 0.3$ & $15.6 \pm 0.4$ & $20.0 \pm 0.3$ & $23.8 \pm 0.1$ & $36.0 \pm 0.1$ \\
\hline Streptococcus sanguis 12 & $22 \pm 0.2$ & $33.1 \pm 0.1$ & $45.6 \pm 0.3$ & $46.0 \pm 0.4$ & $48.0 \pm 0.1$ \\
\hline Streptococcus mutans. & $10.3 \pm 0.6$ & $15.4 \pm 0.1$ & $28.0 \pm 0.1$ & $32.2 \pm 0.5$ & $42.5 \pm 0.2$ \\
\hline Streptococcus oralis J22 & $13.6 \pm 0.3$ & $15.0 \pm 0.4$ & $15.5 \pm 0.5$ & $28.0 \pm 0.8$ & $39.0 \pm 0.1$ \\
\hline Streptococcus mutans HG985 & $11.0 \pm 0.4$ & $13.2 \pm 0.3$ & $15.2 \pm 0.3$ & $30.7 \pm 0.4$ & $68.0 \pm 0.2$ \\
\hline Pseudomonas aeruginosa & $8.3 \pm 0.1$ & $13.5 \pm 0.2$ & $25.0 \pm 0.2$ & $42.0 \pm 0.1$ & $57.6 \pm 0.3$ \\
\hline Staphylococcus aureus & $10.6 \pm 0.1$ & $20.0 \pm 0.2$ & $27.3 \pm 0.3$ & $32.2 \pm 0.2$ & $43.9 \pm 0.1$ \\
\hline Staphylococcus epidermidis & $7.7 \pm 0.1$ & $8.0 \pm 0.1$ & $12.5 \pm 0.1$ & $13.6 \pm 0.4$ & $47.0 \pm 0.2$ \\
\hline Candida albicans & $12.5 \pm 0.2$ & $17.3 \pm 0.3$ & $32.0 \pm 0.3$ & $44.2 \pm 0.1$ & $57.0 \pm 0.2$ \\
\hline
\end{tabular}

Table 2. Percentages of growth inhibition obtained with the crude biosurfactant Lunasan isolated from Candida sphaerica at different concentrations ( $\mathrm{mg} / \mathrm{l})$. Results are expressed as means \pm standard deviations of values obtained from triplicate experiments

The tested biosurfactant presented antimicrobial activity against all microorganisms used, although, depending on the microorganism, the biosurfactant presents different effective concentrations. The highest concentration of biosurfactant tested $\left(10 \mathrm{mg} \mathrm{ml}^{-1}\right)$ showed high percentages of inhibition for Streptococcus oralis J22 (68\%), C. albicans (57\%) and Staphylococcus epidermidis (57.6\%). The antimicrobial activity of the crude biosurfactant isolated from Candida sphaerica with concentrations between 5 and $10 \mathrm{mg} \mathrm{ml}^{1}$ against $C$. albicans, Staph. aureus and Staph. epidermidis was less to that obtained with the biosurfactants 
isolated from Lact. paracasei ssp A20, which completely inhibited the growth of those microrganisms with concentrations between 25 and $50 \mathrm{mg} \mathrm{ml}^{-1}$ ) [27] .

The crude biosurfactant showed antimicrobial activity against a broad range of microorganisms, including Gram-positive and Gram-negative bacteria and yeasts.

Biosurfactants antimicrobial activity has been described, as for example surfactin, a cyclic lipopeptide produced by Bacillus subtilis [28]. The antimicrobial activity of surfactin was tested against several microbes. All tested bacteria, except for B. subtilis, showed susceptibility to surfactin. P. aeruginosa was the most sensitive Gram-negative bacteria, while E. coli, Salmonella choterasius and Serratia marcescens were inhibited in a lower level. Also, the lipopeptide affected the growth of Gram-positive bacteria, especially Micrococcus luteus and Bacillus cereus [18]. Other examples have been reported by Rodrigues and coworkers [8, 27]. Crude biosurfactants isolated from Lactococcus lactis 53 and Streptococcus thermophilus A showed antimicrobial activity against C. troplicalis GB in low concentrations.

Some biosurfactants are able, even in low concentrations, to destabilize the microorganism's membranes, killing them or disabling their growth $[29,30]$. The interest in biosurfactants was first expressed due to its potential antimicrobial properties, being the first reported and actually the most studied biosurfactants, rhamnolipid and surfactin [31]. Gram-positive bacteria are more sensitive to biosurfactants than Gram-negative bacteria, which are weakly inhibited or not inhibited at all [32]. C. bombicola and C. apicola were reported to produce a glycolipid-type biosurfactant (sophorolipid) that inhibit the growth of B. subtilis, $S$. epidermidis and Streptococcus faecium in concentrations between 6 and $29 \mathrm{mg} / \mathrm{l}$ [33]. Other glycolipids inhibit not only the growth of Gram-positive bacteria, but also Gram-negative ones, such as E. coli and S. marcescens [31]. Kitamoto et al. [34] reported in their work an antimicrobial activity against $S$. aureus, E. coli, P. aeruginosa and C. albicans for a mannosylerythritol roduced by $C$. antarctica, a sophorolipid produced by $C$. apicola and a rhamnolipid produced by $P$. aeruginosa.

Several biosurfactants that exhibit antimicrobial activity have been previously described. However, there are few reports about the antimicrobial activity of biosurfactants isolated from Candida; only biosurfactants obtained from S. thermophilus A and L. lactis 53 showed significant antimicrobial activity against several bacterial and yeast strains isolated from explanted voice prostheses [35].

\section{Anti-adhesive activity of biosurfactant}

Involvement of biosurfactants in microbial adhesion and desorption has been widely described, and adsorption of biosurfactants to solid surfaces might constitute an effective strategy to reduce microbial adhesion and combating colonization by pathogenic microorganisms, not only in the biomedical field, but also in other areas, such as the food industry [36, 37]. 
Biosurfactants have been found to inhibit the adhesion of pathogenic organisms to solid surfacesor to infection sites, thus prior adhesion of biosurfactant to solid surfaces might constitute a new and effective means of combating colonization by pathogenic microorganisms [12]. Precoating vinyl urethral catheters by running a surfactin solution through them before inoculation with media resulted in a decrease of the amount of biofilm formed by Salmonella typhimurium, Salmonella enteric, Escherichia coli and Proteus mirabilis [38]. Given the importance of opportunistic infections with Salmonella species, including urinary tract infections of AIDS patients, these results have great potential for practical applications.

In addition to the antimicrobial properties, the anti-adhesive activity of the biosurfactant was evaluated against a variety of bacterial and fungal strains. The biosurfactant showed anti-adhesive activity against most of the microorganisms tested, but the anti-adhesive effect depends on the concentration and the micro-organism tested (Table 3).

\begin{tabular}{|c|c|c|c|c|c|c|}
\hline \multirow{3}{*}{ Microorganism } & \multicolumn{6}{|c|}{ Anti-adhesive activity (\%) } \\
\hline & \multicolumn{5}{|c|}{ Biosurfactant mg/L } & \multirow[b]{2}{*}{$\begin{array}{c}\text { PBS } \\
\text { (control) }\end{array}$} \\
\hline & 0.75 & 1.5 & 3.0 & 6.0 & 12.0 & \\
\hline Lactobacillus casei & $91 \pm 0.1$ & $91 \pm 0.1$ & $99 \pm 0.1$ & $99 \pm 0.1$ & $99 \pm 0.1$ & 0 \\
\hline Lactobacillus casei72 & $81 \pm 0.0$ & $87 \pm 0.0$ & $89 \pm 0.0$ & $91 \pm 0.0$ & $95 \pm 0.0$ & Q \\
\hline Lactobacillus reuteri 104R & $84 \pm 0.2$ & $93 \pm 0.2$ & $94 \pm 0.2$ & $95 \pm 0.2$ & $97 \pm 0.2$ & Q \\
\hline Lactobacillus reuteri ML1 & $81 \pm 0.0$ & $82 \pm 0.0$ & $84 \pm 0.0$ & $87 \pm 0.0$ & $89 \pm 0.0$ & Q \\
\hline Escherichia coli & $8 \pm 0.1$ & $17 \pm 0.1$ & $18 \pm 0.1$ & $25 \pm 0.1$ & $27 \pm 0.1$ & Q \\
\hline Streptococcus agalactiae & $80 \pm 0.0$ & $81 \pm 0.0$ & $81 \pm 0.0$ & $84 \pm 0.0$ & $96 \pm 0.0$ & Q \\
\hline Streptococcus mutans NS & $91 \pm 0.1$ & $95 \pm 0.1$ & $96 \pm 0.1$ & $98 \pm 0.1$ & $99 \pm 0.1$ & Q \\
\hline Streptococcus sanguis 12 & $61 \pm 0.0$ & $62 \pm 0.0$ & $68 \pm 0.0$ & $70 \pm 0.0$ & $77 \pm 0.0$ & Q \\
\hline Streptococcus mutans. & $76 \pm 0.1$ & $84 \pm 0.1$ & $85 \pm 0.1$ & $88 \pm 0.1$ & $97 \pm 0.1$ & 0 \\
\hline Streptococcus oralis J22 & $73 \pm 0.0$ & $85 \pm 0.0$ & $87 \pm 0.0$ & $89 \pm 0.0$ & $90 \pm 0.0$ & Q \\
\hline Streptococcus mutans HG985 & $76 \pm 0.1$ & $76 \pm 0.1$ & $81 \pm 0.1$ & $83 \pm 0.1$ & $85 \pm 0.1$ & Q \\
\hline Pseudomonas aeruginosa & $13 \pm 0.0$ & $26 \pm 0.0$ & $33 \pm 0.0$ & $41 \pm 0.0$ & $49 \pm 0.0$ & 0 \\
\hline Staphylococcus aureus & $88 \pm 0.0$ & $91 \pm 0.0$ & $92 \pm 0.0$ & $97 \pm 0.0$ & $98 \pm 0.0$ & Q \\
\hline Staphylococcus epidermidis. & $2 \pm 0.0$ & $5 \pm 0.0$ & $6 \pm 0.0$ & $16 \pm 0.0$ & $21 \pm 0.0$ & Q \\
\hline Candida albicans & $8 \pm 0.0$ & $8 \pm 0.0$ & $16 \pm 0.0$ & $36 \pm 0.0$ & $51 \pm 0.0$ & Q \\
\hline
\end{tabular}

Table 3. Anti-adhesive properties of crude biosurfactant isolated from Candida lipolytica. Negative controls were set at $0 \%$ to indicate the absence of biosurfactant. Positive percentages indicate the reductions in microbial adhesion when compared to the control. Results are expressed as means \pm standard deviation of results from triplicate experiments

The crude biosurfactant showed anti-adhesive activity against most of the microorganisms tested from the minimum concentration used $(0.75 \mathrm{mg} / \mathrm{l})$. The anti-adhesive property was proportional to the concentration of the biosurfactant. For the microorganisms of the Lactobacillus anti-adhesive values around $81 \%$ were observed at the minor concentration tested $(0.75 \mathrm{mg} / \mathrm{l})$. The major anti-adhesive specificity was observed against $L$. casei with 
values of $91 \%$ and $99 \%$ with the minimum concentration used. Low inhibitions were observed for S. epidermidis and E. coli, with values of $27 \%$ and $21 \%$, respectively, at the maximum biosurfactant concentration. For the other microorganisms, the anti-adhesive activity was above $45 \%$.

Gudina et al. [24] observed an anti-adhesive activity for the biosurfactant from Lactobacillus paracasei against several pathogenic microorganisms such as $S$. aureus, S. epidermidis and $S$. agalactiae. However, this biosurfactant showed low anti-adhesive activity against E. coli, C. albicans and $P$. aeruginosa, in contrast with the antimicrobial activity exhibited against these strains at the same biosurfactant concentrations.

The use and potential commercial applications of biosurfactants in the medical field has increased considerably in the last years. Their antimicrobial and anti-adhesive properties make them relevant molecules for use in combating many diseases and infections and as therapeutic agents [18].

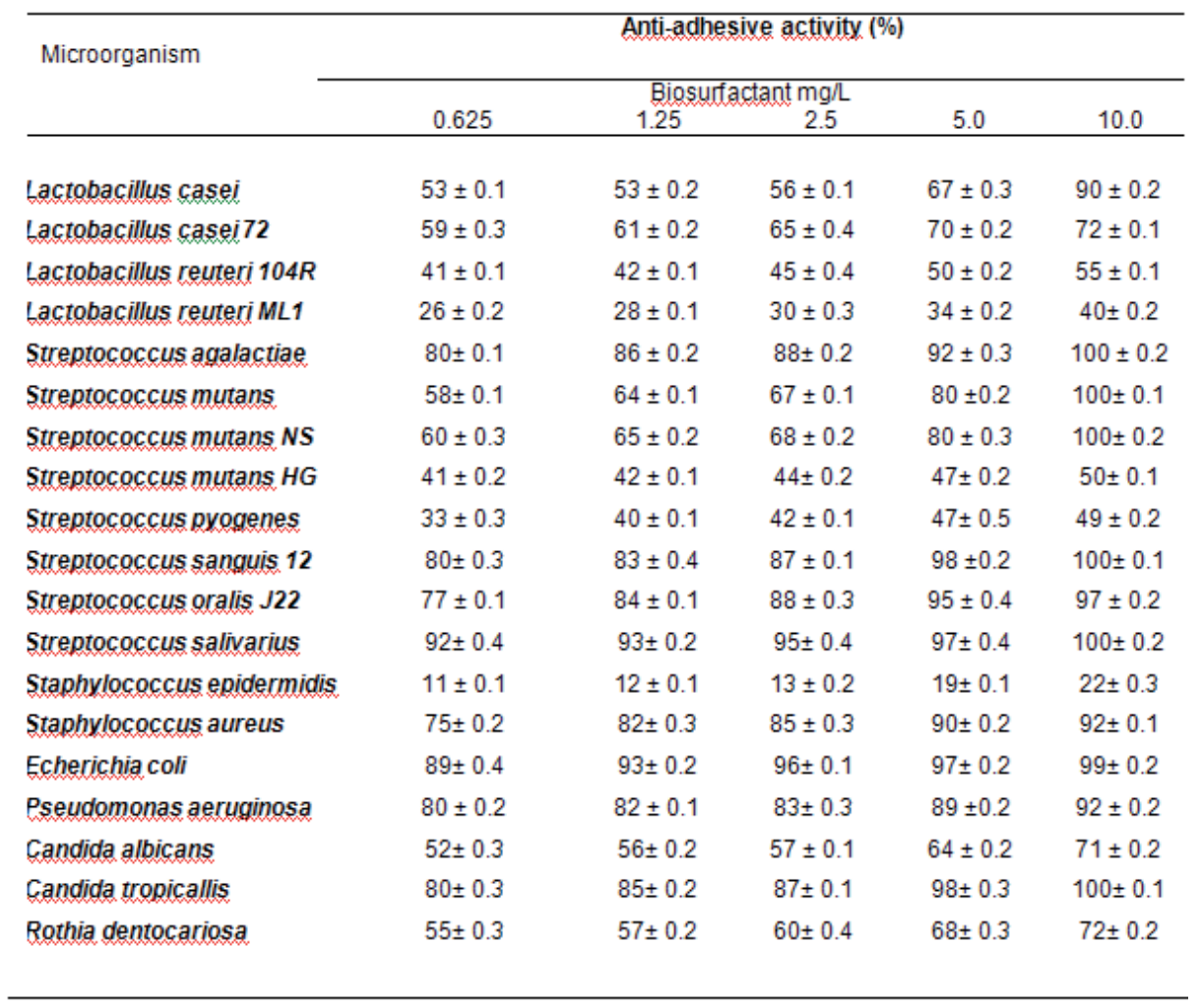

Table 4. Anti-adhesive properties of crude biosurfactant isolated from Candida sphaerica. Negative controls were set at $0 \%$ to indicate the absence of biosurfactant. Positive percentages indicate the reductions in microbial adhesion when compared to the control

Adhesion to surfaces and subsequent biofilm formation consist in a surviving strategy used by microorganisms in several hostile environments, protecting them from dehydration, 
predators, biocides and extreme conditions [22]. The antiadhesive activity of this biosurfactant was evaluated against a variety of bacterial and fungal strains. The biosurfactant showed antiadhesive activity against most of the microrganisms tested, but the antiadhesive effect depends on the concentration and the microrganism tested (Table 4).

This biosurfactant was effective against all the microorganisms tested, albeit to different degree. With regard to the Lactobacillus strains, the antiadhesive activity was higher against Lact. casei (90\%), Lact. casei 72 (72\%), Lact. reuteri 104R (55\%) and Lact. reuteri ML1 (40\%). For the pathogenic bacteria studied (Streptococcus agalactiae, Streptococcus mutans, Streptococcus mutans NS, Streptococcus sanguis 12, Streptococcus salivarius GB and Echerichia coli), a complete inhibition of adhesion was also achieved with biosurfactant concentrations of $10 \mathrm{mg} \mathrm{ml}^{-1}$.

Regarding the yeast, a total inhibition of adhesion was also observed for $C$. tropicalis GB at a biosurfactant concentration of $10 \mathrm{mg} \mathrm{ml}^{-1}$. The highest percentages of adhesion inhibition were obtained for $P$. aeruginosa (92\%), Staphylococcus aureus $(92 \%)$, Streptococcus oralis J22 $(97 \%)$ and Rothia dentocariosa GBJ (72\%), while low activity was obtained for Streptococcus mutans HG 985(50\%) and Staphylococcus epidermidis GB(22\%).

The antiadhesive activity of the crude biosurfactant isolated from C. sphaerica completely inhibited the adesion with a concentration of $10 \mathrm{mg} \mathrm{ml}^{-1}$ against Streptococcus agalactiae, Streptococcus mutans, Streptococcus mutans NS, Streptococcus sanguis 12, Streptococcus salivarius GB and Echerichia coli. These results were higher to that obtained with the biosurfactants isolated from Lact. paracasei ssp A20 [24]. A role of biosurfactants as defense weapons in competition with post-adhesion has been suggested for biosurfactants produced by Streptococcus mitis and S. mutans [39].

Besides possessing antifungal, antibacterial and antiviral activities, biosurfactants have also proved to be great inhibitors of microbial adhesion and of biofilm formation. For example, the biosurfactant released by S. mitis was found to reduce the adhesion of Streptococcus. mutans [40]. Similarly, Lactobacillus fermentum RC-14 releases surfactant compounds that can inhibit the adhesion of uropathogenic bacteria, including Enterococcus faecalis.

The adsorption of a biosurfactant on surface was found to change its hydrophobicity, which might caused interference in the adhesion and desorption processes [41]. Furthermore, Velraeds et al. [42] reported the inhibition of adhesion of pathogenic enteric bacteria by a biosurfactant produced by Lactobacillus fermetum RC-14. The authors suggested the use of this anti-adhesive agent in catheters aiming at decreasing biofilm formation. Falagas and Makris [37] have proposed the application of biosurfactants isolated from probiotic bacteria to patient care equipments (such as catheters and other medical insertional devices) in hospitals, with the aim of decreasing colonization by microorganisms responsible for nosocomial infections.

\section{Conclusion}

In conclusion, in this work we have demonstrated the antimicrobial and anti-adhesive properties of the crude biosurfactant isolated from C. lipolytica UCP0988 against several pathogenic and nonpathogenic microorganisms, including bacteria, yeasts and filamentous fungi. The results obtained suggest the possible use of this biosurfactant as an alternative 
antimicrobial agent in the medical field for applications against microorganisms responsible for diseases and infections, making it a suitable alternative to conventional antibiotics.

\section{Author details}

Raquel Diniz Rufino, Juliana Moura de Luna and Galba Maria de Campos-Takaki

Nucleus for Research in Environmental Sciences and Center for Sciences and Technology, Catholic University of Pernambuco, Rua do Príncipe, Boa Vista, Recife- PE, Brazil

Leonie Asfora Sarubbo

Centre of Science and Technology, Catholic University of Pernambuco, Boa Vista, Recife-Pernambuco, Brazil

Lígia Raquel Marona Rodrigues and José Antônio C. Teixeira

IBB, Institute for Biotechnology and Bioengineering, Centre of Biological Engineering,

University of Minho, Campus de Gualtar, Braga, Portugal

\section{Acknowledgement}

This work was financially supported by Coordenação de Aperfeiçoamento de Pessoal de Nível Superior (CAPES), Fundação de Amparo à Ciência e Tecnologia do Estado de Pernambuco (FACEPE), Institute for Biotechnology and Bioengineering, Centre of Biological Engineering, Universidade do Minho, Braga, Portugal. We are grateful to Núcleo de Pesquisas em Ciências Ambientais (NPCIAMB) laboratories, Universidade Católica de Pernambuco, Brazil.

\section{References}

[1] Rufino RD, Sarubbo LA, Barros-Neto B, Campos-takaki GM. Experimental design for the production of tensio-active agent by Candida lipolytica. Journal Industrial Microbiology and Biotechnology 2008; 35: 907-914.

[2] Luna JM, Rufino RD, Sarubbo LA, Rodrigues LRM, Teixeira JAC, Campos-Takaki GM. Evaluation Antimicrobial and Antiadhesive Properties of the Biosurfactant Lunasan Produced by Candida sphaerica UCP 0995. Current Microbiology 2011; 62: 1527-1534.

[3] Sarubbo LA, Farias CBB, Campos-Takaki GM. Co-utilization of canola oil and glucose on the production of a surfactant by Candida lipolytica. Current Microbiology 2007; 54: 68-73.

[4] Sobrinho HBS, Rufino RD, Luna JM, Salgueiro AA, Campos-takaki GM, Leite LFC, Sarubbo LA. Utilization of two agroindustrial by-products for the production of a surfactant by Candida sphaerica UCP0995. Process Biochemistry 2008; 912-917.

[5] Rahman KSM, Gakpe E. Production, characterization and applications of biosurfactants- Review. Biotechnology 2008; 7: 360-370.

[6] Benincasa M. Rhamnolipid produced from agroindustrial wastes enhances hydrocarbon biodegradation in soil. Curr Microbial 2007; 56: 445-449.

[7] Das P, Mukherjee S, Sen R. Antiadhesive action of a marine microbial surfactant. Colloids and Surfaces B: Biointerfaces 2009; 71: 183-186. 
[8] Rodrigues LR, Moldes A, Teixeira JA, Oliveira R. Kinetic study of fermentative biosurfactant production by Lactobacillus strains. Biochemical Engineering Journal 2006e; 28: 109-116.

[9] Rodrigues LR, Van Der Mei HC, Teixeira JA, Oliveira R. Influence of biosurfactants from probiotic bacteria on formation of biofilms on voice prostheses. Applied and Environmental Microbiology 2004b; 70: 4408 - 4410.

[10] Mireles JR, Toguchi A, Harshey RM. Salmonella enterica serovar Typhimurium swarming mutants with altered biofilm-forming abilities: surfactin inhibits biofilm formation. J Bacteriol 2001; 183: 5848-54.

[11] Boris S, Barbe's C. Role played by lactobacilli in controlling the population of vaginal pathogens. Microbes Infect. 2000; 2: 543-546.

[12] Singh P, Cameotra S Potential applications of microbial surfactants in biomedical sciences. Trends Biotechnol 2004; 22: 142-6.

[13] Rodrigues LR. Biosurfactants production by probiotic bacteria and inhibition of voice prostheses microbial colonization. PhD Thesis. Universidade do Minho; 2005.

[14] Kulakovskaya T, Kulakovskaya E, Golubev W. ATP leakage from yeast cells treated by extracellular glycolipids of Pseudozyma fusiformata. FEMS Yeast Res 2003; 3: 401-404.

[15] Reid G. In vitro testing of Lactobacillus acidophilus NCFM as a possible probiotic for the urogenital tract. Int Dairy 2000; 10: 415-419.

[16] Rufino RD, Luna JM, Sarubbo LA, Rodrigues LRM, Teixeira JAC, Campos-Takaki GM. Antimicrobial and anti-adhesive potential of a biosurfactant Rufisan produced by Candida lipolytica UCP 0988. Colloids and surfaces B: Biointerfaces 2011;84: 1-5.

[17] Golubev WI, Kulakovskaya TV, Golubeva W. The yeast Pseudozyma fusiformata VKM Y2821 producing an antifungal glycolipid. Microbiol 2001; 70: 553-556.

[18] Rodrigues LR, Teixeira JA, Van Der Mei HC, Oliveira R. Isolation and partial characterization of a biosurfactant produced by Streptococcus thermophilus A. Colloids and Surfaces B: Biointerfaces 2006a; 53: 105 -112.

[19] Dunne Jr WM. Bacterial adhesion: seen any good biofilms lately. Clin. Microbiol Rev 2002 15: 155-166.

[20] Ahimou F, Jacques P, Deleu M. Surfactin and iturin A effects on Bacillus subtilis surface hydrophobicity. Enzyme and Microbial Technology 2001; 27: 749-754.

[21] Abalos A, Pinazo A, Infante MR. Physicochemical and anti-microbial properties of new rhamnolipds produced by Pseudomonas aeruginosa AT10 from soybean oil refinery wastes. Langmuir 2001; 17: 1367-1371.

[22] Wright JR. Pulmonary surfactant: a front line of lung host defense. J Clin Invest 2003; 111: 1453-1455.

[23] Bai G, Brusseau ML, Miller RM. Influence of a rhamnolipid biosurfactant on the transport of bacteria through a sandy soil. Appl Environ Microbiol 1997; 63: 1866-1873.

[24] Gudiña EJ, Rocha V, Teixeira JA, Rodrigues LR. Antimicrobial and antiadhesive properties of a biosurfactant isolated from Lactobacillus paracasei SSP. Paracasei A20. Applied Microbiology 2010a; 50: 419-424.

[25] Fernandes A, Arruda IR, Santos AFAB, Araújo A, Maior AM, Ximenes EA. Antimicrobial activity of surfactants produced by Bacillus subtilis r14 against multidrugresistant bacteria Brazilian Journal of Microbiology 2007; 38: 704-709. 
[26] Gudiña EJ, Teixeira JA, Rodrigues LR. Isolation and functional characterization of a biosurfactant produced by Lactobacillus paracasei. Colloids and Surfaces B: Biointerfaces 2010; 76: 298-304.

[27] Rodrigues LR, Banat IM, Van Der Mei HC, Teixeira JA, Oliveira R Interference in adhesion of bacteria and yeasts isolated from explanted voice prostheses to silicone rubber by rhamnolipid biosurfactants. Journal of Applied Microbiology 2006 d; 100: 470-480.

[28] Elving GJ, Van Der Mei HC, Busscher HJ, Van Weissenbruch R, Albers FW. Comparision of the microbial composition of voice prosthesis biofilms from patients requiring frequent versus infrequent replacement. Annals of Otology, Rihinol Laryngol 2002; 111: 200-203.

[29] Calvo C, Manzanera M, Silva-Castro GA, González-Lopéz J. Application of bioemulsifiers in soil oil bioremediation processes. Future Prospects. Science of the Total Envrironment 2009; 407: 3634-3640.

[30] Carrilo C, Teruel JA, Aranda FJ, Ortiz A Molecular mechanism of membrane permeabilization by the peptide antibiotic surfactin. Biochimica et Biophysica Acta 2003; 1611: 91-97.

[31] Singh A, Van Hamme JD, Ward OP. Surfactants in microbiology and biotechnology: Part 2. Application aspects Biotechnol 2007; 25: 99-121.

[32] Elving GJ, Van Der Mei HC, Busscher HJ, Amerogen EC, Van Weissenbruch R, Albers FW. Antimicrobial activity of synthetic salivary peptides against voice prosthetic microorganisms Laryngoscope 2000; 110: 321-324.

[33] Lang S, Katsiwela E, Wagner F. Antimicrobial effects of biosurfactants. Fat Science Technology 1989; 9: 363-366.

[34] Kitamoto D, Isoda H, Nakahara T. Functions and potential applications of glycolipid biosurfactants - from energy-saving materials to gene delivery carriers. Journal of Bioscience and Bioengineering 2002; 94: 187-201.

[35] Busscher HJ, Van Hoogmoed CG, Geertsema-Doornbusch GI, Van Der Kuijl-Booij M, Van Der Mei HC Streptococcus thermophilus and its biosurfactants inhibit adhesion by Candida spp. On silicone rubber. Applied Environ Microbiol 1997; 63: 3810-3817.

[36] Nitschke M, Ferraz C, Pastore GM. Selection of microorganisms for biosurfactant production using agroindustrial wastes. Brazilian Journal of Microbiology 2004; 35: 336-341.

[37] Falagas MF, Makris GC. Probiotic bacteria and biosurfactants for nosocomial infection control: a hypothesis. Journal Hosp Infect 2009; 71: 301-306.

[38] Mireles JR, Toguchi A, Harshey RM. Salmonella enterica serovar Typhimurium swarming mutants with altered biofilm-forming abilities: surfactin inhibits biofilm formation. J Bacteriol 2001; 183: 5848-54.

[39] Busscher HJ, Van Hoogmoed CG, Geertsema-Doornbusch GI, Van Der Kuijl-Booij M, Van Der Mei HC. Streptococcus thermophilus and its biosurfactants inhibit adhesion by Candida spp. On silicone rubber. Applied Environ Microbiol 1997; 63: 3810-3817.

[40] Pratt- Terpstra IH, Busscher HJ. Microbial factors in a thermodynamic approach of oral streptococcal adhesion to solid substrata. Journal Colloid Interface Science 1989; 129: 568-574.

[41] Fischer W. Molecular analysis of lipid macroamphiphiles by hydrophobic interaction chromatography. Journal of Microbiological Methods 1996; 25: 129 -144.

[42] Velraeds M, Van Der Mei HC, Reid G. Interference in initial adhesion of uropathogenic bacteria and yeasts to silicone rubber by a Lactobacillus acidophilus biosurfactant. J Med Microbiol 1998; 47: 1081-1085. 


\title{
Biocompatibility and Immunocompatibility Assessment of Poly(2-Oxazolines)
}

\author{
Juraj Kronek, Ema Paulovičová, Lucia Paulovičová, \\ Zuzana Kroneková and Jozef Lustoň
}

Additional information is available at the end of the chapter

http://dx.doi.org/10.5772/51501

\section{Introduction}

Synthetic polymers are considered to be the promising materials for biomedical applications. Various polymer formulations have been employed to achieve the desired chemical, physical and biological properties. Recently, there has been much interest in the development of environmentally responsive polymers for use as biomaterials [1]. Such behavior is significant for the controlled release of drugs upon the application of a stimulus, such as $\mathrm{pH}$, temperature, light or ionic strength. These mentioned properties are necessary for the utilization of polymeric materials for biomedical applications, such as drug and gene delivery, biomembrane technology and biocatalysis [2,3]. Polymer materials can be used in medicine as a part of implant, dialysis membranes, bone scaffolds or components of artificial organs. It means that polymers covers very broad range of biomedical applications. A critical point of the usage of synthetic polymers in living bodies is their utilization, accompanied with the interactions of the foreign material, with the living matter (cells, tissues etc.). The implantation of polymeric materials to a body is usually associated with the inflammation and biofouling. The inflammation is the first defense mechanism of the immune system followed by unspecific cell and protein adhesion and the formation of fibrotic tissue which leads to implant's dysfunctions. The fundamental role in the implantation of these materials is to increase the tolerance of body to implanted material and to avoid the foreign body reaction $[4,5]$.

Assessments of polymeric material biocompatibility and immunotoxicity are key issues to consider material to be suitable for biomedical applications. Biocompatibility assessment of a polymeric material includes adequate testing for undesired responses. To evaluate biocompatibility, examinations of acute and system toxicity, tissue cultures, cell growth inhibition, mutagenicity, carcinogenicity, teratogenicity and allergenic potential should be 
conducted [6]. In vitro cytotoxicity can be tested by a number of methods that are mostly based on the colorimetric assay of dyes that are sensitive to viable or dead cells. Such assays include 3-(4,5-dimethylthiazol-2-yl)-2,5-diphenyltetrazolium bromide (MTT), 2,3-bis-(2methoxy-4-nitro-5-sulfophenyl)-2H-tetrazolium-5-carboxanilide (XTT), water soluble tetrazolium salts (WST), trypan blue or sulforhodamine B as dyes.

The immunocompatibility of polymers is obviously compulsory for their potential medical application. Several aspects of targeted immune responses have to be taken into account. The immune system is an integrated system composed of two major sub-systems, the innate, non-specific immune system and the adaptive, specific immune system. The innate immune system represents a primary defense mechanism against invading microorganisms and foreign substances and the adaptive immune system operates as a second part of defense. Systemic host immune responses involve complex and multisided interactions between numerous mechanisms of innate and adaptive immunities and their cellular and humoral components. Apart from systemic immunity, common mucosal immune system is effective in all mucosal surfaces i.e. mucosa-associated lymphoid tissue (MALT), including aggregates and diffuse population of immune cells within mucosae; gut-associated lymphoid tissue (GALT), including isolated lymphoid follicles, Peyer's patches, the appendix, and cells in the lamina propria; bronchus-associated lymphoid tissue (BALT), including defined aggregates and diffuse populations of lymphoid cells around the airways [7].

Several specifically programmed cells are involved in innate and adaptive immunity. These include neutrophiles and macrophages, which are involved in phagocytosis, basophils and mast cells, which are involved in inflammation and B and T lymphocytes which account for antibody mediated immunity and cell mediated immunity, respectively. Most of immune system's immunocompetent effector cells are derived from specific precursors in the bone marrow. In addition to red blood cells and platelets, pluripotent stem cells give rise to the lymphocytes, the monocytes and macrophages, and to the group of cells with collective term "the granulocytes", covering neutrophils, basophils and eosinophils.

Dendritic cells (DCs) comprise a subset of cells with different characteristics, derived from bone marrow precursor cells. Dendritic cells are professional antigen presenting cells that play a central role in the running and regulation of immune responses, as well as control the innate (NK cells, NKT cells, $\gamma \delta$ T cells) and adaptive (T and B cells) arm of immunity DCs have a crucial role in initiating T-cell mediated immunity. DCs'can control a substantial part of the adaptive immune response by internalizing and processing antigen through MHCclass I and class II pathways and, finally, presenting antigenic peptides to $\mathrm{CD}^{+}$and CD8+ T lymphocytes [8-10].

The specific adaptive immune system requires some time period to act in a response to an invader (microorganism or foreign substance), whereas the non-specific innate immune system comprises fundamental defenses mostly constitutively present and mobilized immediately upon the invasion and recognition of foreign invader. Macrophages and the progeny of granulocyte differentiation, tissue-based mast cells and NK cells are decisive cellular components of the innate immune response. Monocyte-derived macrophages represent the first line of host defence after the epithelial barrier against invader that reaches 
mucosal surfaces. Hence they are one of the most crucial cells acting in innate immune responses to exogenous challenge. They are equipped with restricted number of pattern recognition receptors (PRRs) to recognize foreign invader via their pathogen-associated molecular patterns (PAMPs). Pattern recognition receptors (PRRs) are membrane-bound or secreted microbial sensory receptors that include Toll-like receptors (TLRs), Nod-like receptors (NLRs), and C-type lectin receptor (CLRs). The main functions of PRRs' include opsonization, activation of complement and coagulation cascades, phagocytosis, induction of inflammatory cytokines, and induction of apoptosis. Innate immune responses are triggered upon PAMPs recognition by the Toll-like receptors (TLR), type I transmembrane signalling cell surface molecules. Activation of TLRs induces, via the transcriptional activator nuclear factor $\mathrm{kB}(\mathrm{NF}-\mathrm{kB})$, the triggering of a wide variety of inflammatory and immune-response genes and expression of a variety of molecules in macrophages including pro-inflammatory cytokines e.g. tumor necrosis factor - $\alpha$ (TNF- $\alpha)$, interleukin-1 (IL-1), interleukin 6 (IL-6), interferons (IFN- $\gamma$, IFN $\beta$ ), granulocyte-colony stimulating factor (G$\mathrm{CSF})$, granulocyte-macrophage colony-stimulating factor (GM-CSF), transforming growth factor (TGF- $\beta$ ), macrophage inflammatory protein 1 (MIP-1) [11-13].

Host immune responses towards biomaterials and biocompatibility and immunocompatibility of polymeric materials are essentially important for their biomedical application. Recently, strategies of triggering appropriate immune responses by functional biomaterials and approaches of biomaterials that mimic the physiological extracellular matrix and modify cellular immune responses have been highlighted [14]. The targeting of dendritic cells with biopolymers [15] and targeting antigen to immune cells via PAMPmodified biomaterials [16] have received considerable attention and represents the novel strategy to control the subsequent immune responsiveness. Immunological inflammation comprising the temporal variation in the acute inflammatory response, chronic inflammatory response, granulation tissue development, and foreign-body reaction represents one of the most important reactions to biomaterials observed in a host $[17,18]$. Immunogenicity of synthetic biopolymers, their immunomodulative character and induction of cell and antibody immunities are crucial aspects of in vivo applications. The involvement of the immune system in incompatibility reactions towards biomaterials is not desirable in special cases, thus, various possibilities to shield the biomaterial and cell surfaces from recognition by the innate immune system are studied [19].

The aim of this chapter is to summarize and evaluate the available methods for the biocompatibility and immunocompatibility assesment of 2-oxazoline-based polymers. 2Oxazoline-based polymers serve a significant role in the field of polymers suitable for biological and medical use. Examples of their applications in field of biology and medicine were also given.

\section{Poly(2-oxazolines) as materials for biomedical applications}

From a variety of synthetic polymers suitable for different biomedical applications, polymers based on 2-oxazoline chemistry represent very versatile and promising materials. 
Poly(2-oxazolines) can be prepared by the living cationic polymerization of 2-oxazolines containing aliphatic or aromatic substituent [20] (Fig. 1). This means that polymers with defined structure, size and narrow dispersity can be prepared. Living character of polymerization of 2-oxazolines can be employed for the preparation of polymers with complex architecture, such as star-shaped polymers [21,22] or polymer combs and brushes [23], and also for the synthesis of block copolymers.<smiles>[R]C1=NCCO1</smiles>
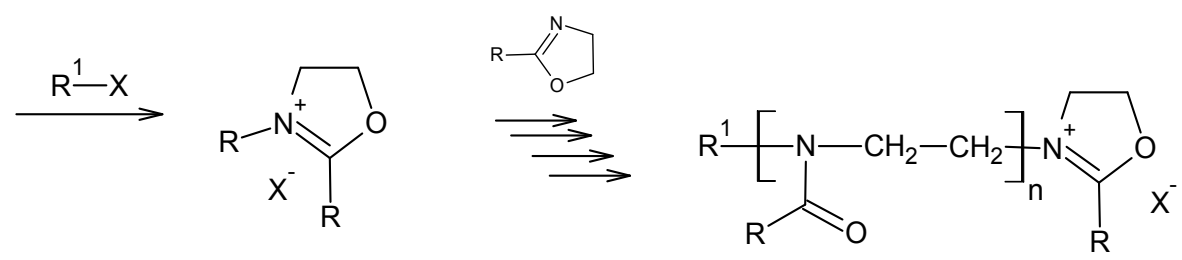

Figure 1. Scheme of living cationic polymerization of 2-substituted 2-oxazolines.

Polymers containing functional groups represent an important part of polymer therapeutics, especially in the drug delivery systems. Polymers containing functional groups in the side chain can be prepared directly from the 2-oxazoline monomer containing the required group, or by polymer analogous reaction of polymer precursors. Preparation of polymers containing amino, carboxyl, mercapto, and aldehyde groups in the side chain was reported [24-26]. In all cases, monomers containing a protected functional group were used for the polymer synthesis. The protecting group was removed after the polymerization process was completed. Copolymers containing a free amino group were prepared directly by the cationic copolymerization of 2-ethyl-2-oxazoline and 2-(4-aminophenyl)-2-oxazoline [27].

Poly(2-alkyl-2-oxazolines) with shorter alkyl chain are water soluble and exhibit thermosensitive behaviour (Fig. 2). Poly(2-ethyl-2-oxazoline) (PETOX) represents a thermoresponsive polymer with the lower critical solution temperature (LCST) equal to 69 ${ }^{\circ} \mathrm{C}$ [28]. Poly(2-isopropyl-2-oxazoline) (PIPOX) also exhibits thermosensitive behavior, and its LCST is near $37^{\circ} \mathrm{C}$, which is necessary for its utilization in drug and gene delivery [29]. Poly(2-propyl-2-oxazoline) (PPOX) has LCST equal to $25^{\circ} \mathrm{C}$ [30]. These results show that the length of alkyl chain has a great impact on solution properties of poly(2-alkyl-2-oxazolines) and phase transition can be adjusted also by copolymerization of monomers with different alkyl chain.<smiles>CCC(=O)N1CCC1(C)C</smiles>

PETOX

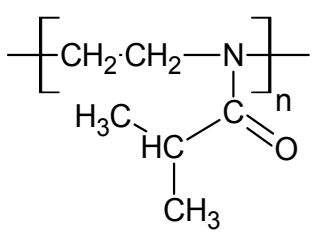

PIPOX<smiles>CCCC(=O)N1CCC1(C)C</smiles>

PPOX

Figure 2. Structure of thermosensitive poly(2-oxazolines). 
Due to their versatility and ability to form functional materials and nanostructures, 2oxazoline based polymers have a promising use in biomedical applications. One of the principal application areas for polymers in biological and medicinal contexts is drug and gene delivery. The concept of drug delivery is based on either encapsulation of a drug in polymer and its delivery through diffusion or covalent attachment onto functional polymer matrix through labile bond [31]. The majority of polymers that have been investigated for use in drug and gene delivery applications have focused on linear polymers such as poly(ethylene oxide) (PEO), poly(N-2-hydroxypropyl methacrylamide) (HPMA) and others. One possibility is based on the preparation of liposome-polyoxazoline conjugates $[32,33]$. Prepared poly(2-methyl-2-oxazoline) (PMEOX) and poly(2-ethyl-2-oxazoline) (PETOX) containing the carboxylic group positioned at either the initiation or termination ends of the polymer chains. Distearoylphosphatidylethanolamine was covalently attached to the polymers through the carboxyl end groups, resulting in conjugates which incorporate readily into liposomes. Both polymers exhibit long plasma lifetimes and low hepatosplenic uptake.

Another possibility is the preparation of amphiphilic block copolymers consisted of either blocks of hydrophilic and hydrophobic 2-oxazoline monomers or blocks of hydrophilic poly(2-oxazoline) and another hydrophobic polymer. In such manner, amphiphilic block copolymers containing poly(2-ethyl-2-oxazoline) as hydrophilic block and polycaprolactone as hydrophobic block have been prepared (Fig. 3b). Such block copolymers are able to form micelles in aqueous solution and can be exploited for delivery of paclitaxel (Fig. 3a) [34]. Similarly, sustained release of the antibody bevacizumab (Avastin) from a biocompatible material based on triblock copolymer of poly(2-ethyl-2-oxazoline)-block- poly(epsiloncaprolactone)-block- poly(2-ethyl-2-oxazoline) has been reported by Wang et al. [35]. Bevacizumac has been used clinically to treat intraocular neovascular diseases based on its antivascular endothelial growth factor (VEGF) character.

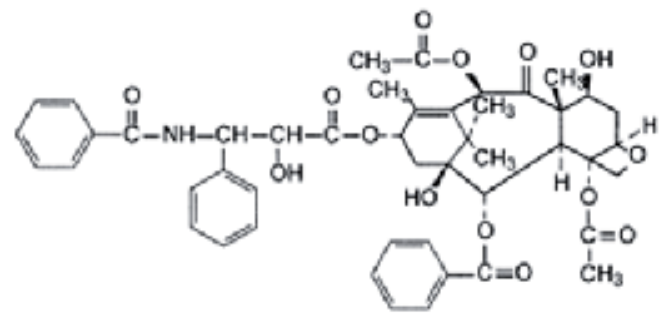

(a)

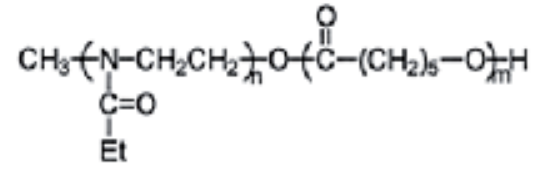

(b)

Figure 3. Structure of paclitaxel (a) and poly(2-ethyl-2-oxazoline)-block- poly(epsilon-caprolactone) (b).

Amphiphilic graft copolymers with a thermosensitive poly(N-isopropylacrylamide) (PNiPAAM) backbone and pH-sensitive hydrophilic poly(2-carboxyethyl-2-oxazoline) graft chains were prepared and used for the formation of stable micelle-like aggregates with reversible thermosensitive and $\mathrm{pH}$ dependent swelling behaviour [36]. The temperatureswitchable hydrophilicity or swelling of the core as well as the pHdependent stretching of 
the side chains in these core-shell nanogels offers a great potential for applications such as drug delivery systems or nanoreactors/carriers in biotechnology. Nanoparticles based on block copolymers of 2-ethyl-2-oxazoline and 2-phenyl-2-oxazolines were studied as promising tools for targeted delivery of drugs in the treatment of neuropsychiatric disorders [37].

Polymers based on 2-oxazoline chemistry were also used in gene delivery. Polyethylene imine (PEI) is one of widely used vectors for nucleic acid delivery. It was found that linear PEI is more effective than branched PEI prepared by conventional polymerization of aziridine. Therefore, DNA complex with fully deacylated PEI can be used in gene therapy for lung diseases [38]. On the other hand, partially hydrolyzed poly(2-ethyl-2-oxazoline) showed lower cytotoxicity comparing to PEI and hasstill high transfection efficiency in the gene expression [39]. Linear PEI can be prepared by basic or acidic hydrolysis of poly(2ethyl-2-oxazoline). Recently, microwave synthesizer induced hydrolysis of poly(2-ethyl-2oxazoline) was described as powerful method for the preparation of "pharmagrade" PEI [40]. A block copolymer of a hyperbranched poly(ethylene glycol)-like core and linear polyethylenimine (HBP) was prepared by cationic polymerization of 2-ethyl-2-oxazoline initiated by hyperbranched initiator bearing p-chloromethylbenzoyl initiating groups and subsequent hydrolysis [41]. Linear PEI-bearing hyperbranched polycations (HBP) is able to form a complex with DNA with an average diameter of $50 \mathrm{~nm}$ that is stable for several weeks and showed resistance to DNAse I-mediated degradation. The 'inverted' block copolymers showed several orders higher transfection efficiency than block copolymers of PEG-b-LPEI in vitro. Comb-like copolymers suitable for DNA transfection consisted of linear poly(ethyleneimine)-graft-poly(2-ethyl-2-oxazoline) (LPEI-comb-PETOX) and (ethyleneimine)-graft-poly(ethyleneimine) (LPEI-comb-LPEI) [42]. Successful gene delivery systems with high transfection efficiency was prepared also by condensation of therapeutic DNA with different copolymers of poly(L-lysine)-graft-poly(2-methyl-2-oxazoline) of variable grafting densities [43]. Amphiphilic poly[(propylene glycol)-block-(2-methyl-2oxazoine)] block copolymers were studied for gene transfer in skeletal muscle [44].

Poly(2-oxazolines) have found utilization also in vaccine technology. Recently, a novel glycoconjugates consisting of detoxified lipopolysaccharide of Vibrio cholerae O135, linear poly(2-oxazoline) copolymer containing free amino groups as a carrier, and an immunogenic protein, BSA, has been prepared. Multiple attachments of small effector antigen molecules to a suitable matrix is considered a novel technique for creating efficient glycoconjugate vaccines $[45,46]$.

Antimicrobial polymers are becoming increasingly important materials in the face of spreading microbial infections and increasing microbial resistance to antibiotics. Waschinski et al. recently reported the synthesis of poly(2-methyl-2-oxazolines) and poly(2-ethyl-2oxazolines) with quarternary ammonium terminating groups [47]. The polymers were prepared via standard cationic ring-opening polymerisation and terminated using a series of $\mathrm{N}$-alkyl-N,N-dimethyl amines as well as pyridine (Fig. 4). The study of antimicrobial properties toward Staphylococcus aurea showed that only poly(2-methyl-2-oxazoline)-based 
polymers containing alkyl ammonia functions with alkyl chains of twelve carbon atoms or longer exhibit antimicrobial activity.

Polymers based on 2-oxazoline chemistry were used in biotechnology for different applications. Biofouling release properties are important for the development of coatings exhibiting marine biofouling resistance. It was found that copolymers prepared from perfluoroalkyl acrylates and poly(2-isopropenyl-2-oxazoline) exhibited unprecedented resistance to marine biofouling [48]. Another example is the development of an optical biosensor using an electrically controlled-release system for measurement of peroxide concentration. The response is based on the hydrogen bond of carboxylic group of currentsystem and 2-oxazoline group of a polymer complex [49]. Activating enzymes in organic solvents is of great interest in current biocatalysis. Amphiphilic and liposome-like polymers have exploitation also in membrane technology. Amphiphilic polymer tether consisted of linear hydrophilic poly(2-methyl-2-oxazoline) chains with defined length with surfacecoupling silane groups on one chain-end and hydrophobic n-alkyl chains on second end [50]. These polymers represent linear polymer spacers between supported lipid membranes and solid substrates. Lipid-tehtered poly(2-methyl-2-oxazoline) lipopolymer chains can be used as Langmuir monolayers at the air-water interface [51]. Recently, novel alpha,omegafunctionalized amphiphilic lipopolymers based on a proximal lipid moiety and hydrophilic poly(2-oxazoline) were used in asymmetric functionalized model lipid membranes for transmembrane transport and cell adhesion/recognition [52]. Amphiphilic co-networks composed of more hydrophilic poly(2-hydroxyethyl acrylate) and more hydrophobic, telechelic poly(2-ethyl-2-oxazoline) segments were prepared as free-standing membranes for entrapment of an enzyme molecule [53]. Amphiphilic miktoarm star copolymers containing cyclodextrin core can be used as nanocarriers in drug delivery but also in biocatalysis. Amphiphilic copolymers were consisted of poly(2-ethyl-2-oxazoline) and polylactide arms covalently linked to cyclodextrine core [54].

All reported applications of poly(2-oxazolines) confirm a great potential of 2-oxazoline based polymers as biomaterials. Detailed study of their biocompatibility and immunocompatibility is essential and necessary to understand their interactions with the living systems (biomolecules, cells, tissues etc.).

\section{Methods for the assessment of cytotoxicity of poly(2-oxazolines)}

Generally, biocompatibility is the ability of a material to perform with an appropriate host response in a specific application [55]. It was already mentioned that biocompatibility assessment includes different assays of acute and system toxicity, tissue cultures, cell growth inhibition, mutagenicity, carcinogenicity, teratogenicity and allergenic potential. In vitro cytotoxicity can be measured by different laboratory assays, such as the 3-(4,5dimethylthiazol-2-yl)-2,5-diphenyltetrazolium bromide (MTT) assay, 2,3-bis-(2-methoxy-4nitro-5-sulfophenyl)-2H-tetrazolium-5-carboxanilide (XTT) assay, Trypan blue (TB) assay, Sulforhodamine B (SRB) assay, WST assay and clonogenic assay. 
The MTT assay is a laboratory test and a standard colorimetric assay for measuring cellular growth. It can also be used to determine cytotoxicity of potential medicinal agents and other toxic materials [56]. Yellow MTT is reduced to purple formazan in the mitochondria of living cells (Fig. 4). A solubilization solution (usually dimethyl sulfoxide, an acidified ethanol solution or a solution of the detergent sodium dodecyl sulfate in dilute hydrochloric acid) is added to dissolve the insoluble purple formazan product into a colored solution. The absorbance of this colored solution can be quantified by measuring at a certain wavelength (usually between 500 and $600 \mathrm{~nm}$ ) by a UV-Vis spectrophotometer. The absorption maximum is dependent on the solvent employed. This reduction takes place only when mitochondrial reductase enzymes are active, and therefore conversion can be directly related to the number of viable (living) cells. When the amount of purple formazan produced by cells treated with an agent is compared with the amount of formazan produced by untreated control cells, the effectiveness of the agent in causing death of cells can be deduced (Fig. 4.).
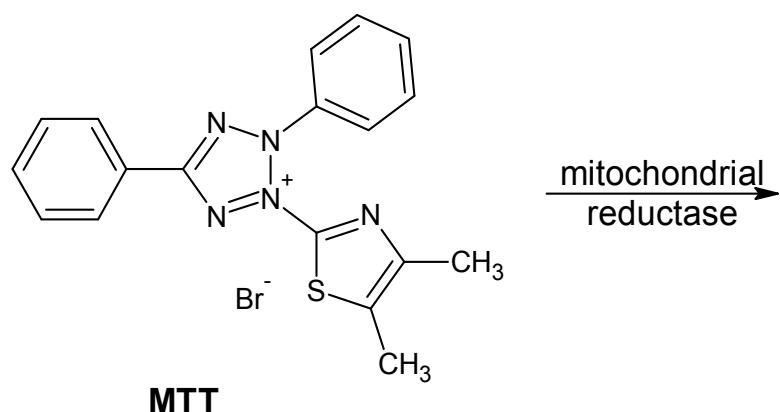

Figure 4. Scheme of reduction of the MTT to fozmazan.

The principle of the WST assay is the same as in the case of MTT assay. In contrast to the MTT assay, no extraction step was necessary due to the water solubility of the reduced form of 2-(4-iodophenyl)-3-(4-nitrophenyl)-5-(2,4-disulfophenyl)-2H-tetrazolium (WST-1) so that absorbance could be determined directly at $450 \mathrm{~nm}$ after conversion [57]. The same principle is used in the XTT assay which employs 2,3-bis-[2-methoxy-4-nitro-5-sulphophenyl]-2Htetrazolium-5-carboxanilide (XTT) as reagent.

Trypan blue is a vital stain used to selectively color dead tissues or cells blue (Fig. 5). Live cells or tissues with intact cell membranes are impermeable for Trypan bluesince cells are very selective in the compounds that pass through the membrane. Trypan blue is not absorbed. In dead cells, the dye traverses the membrane and dead cells are stained blue under a microscope.

Sulforhodamine B or Kiton Red is a fluorescent dye with uses spanning from L.I.F.(Laser Induced Fluorescense) to the quantification of cellular proteins of cultured cells. The red, solid, water soluble dye is primarily used as a polar tracer. The dye absorbs $565 \mathrm{~nm}$ light and emits $586 \mathrm{~nm}$ light. It does not exhibit $\mathrm{pH}$ dependent absorption or fluorescence over the range of 3 to 10 [58]. 


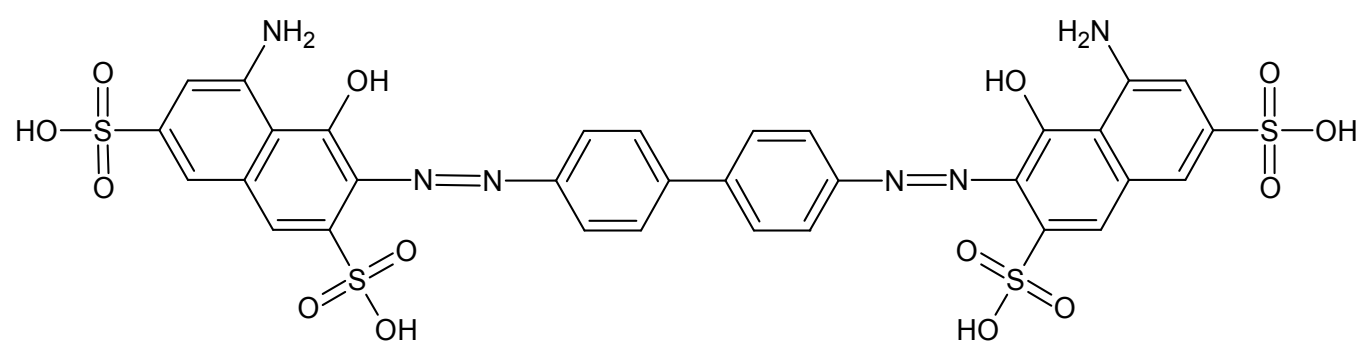

trypan blue

Figure 5. Structure of Trypan blue

A clonogenic assay is a microbiology technique for studying the effectiveness of specific agents on survival and proliferation of cells. It is frequently used in cancer research laboratories to determine the effect of drugs or radiation on proliferating tumor cells [59]. Although this technique can provide accurate results, the assay is time-consuming to set up and analyse and can only provide data on tumor cells that can grow in culture. The word "clonogenic" refers to the fact that these cells are clones of one another.

The experiment involves three major steps:

1. The treatment is applied to a sample of cells.

2. The cells are "plated" in a tissue culture vessel and allowed to grow.

3. The colonies produced are fixed, stained, and counted.

At the conclusion of the experiment, the percentage of cells that survived the treatment is measured. A graphical representation of survival versus drug concentration or dose of ionizing radiation is called a cell survival curve.

Although a number of assays can be employed for the estimation of in vitro cytotoxicity of different materials, the MTT assay was used almost exclusively for the assessment of in vitro cytotoxicity of poly(2-oxazoline)s and 2-oxazoline related polymers. The cytotoxicity of poly(2-ethyl-2-oxazoline) (PETOX) as a function of hydrolysis to linear poly(ethylene imine) (PEI) was studied by conducting an MTT assay [60]. It was found that cell viability decreased with an increasing degree of hydrolysis, and above $50 \%$ hydrolysis the cell viability decreased to $20 \%$. Another example of this polymer as a suitable material for medical use is employing polymeric micelles of poly(2-ethyl-2-oxazoline)-block-poly( $\varepsilon$ caprolactone) copolymer (PETOX-PCLs) as a carrier for paclitaxel as mentioned above. It was showed that the viability of KB human epidermoid carcinoma cells in the presence of PETOX-PCL copolymers were in the range 80 to $100 \%$ depending on the concentration of the copolymer solution and also on the ratio of both building blocks [34]. Similarly, polymeric micelles prepared from poly(2-ethyl-2-oxazoline)-b-poly(1-lactide) diblock copolymers (PETOX-PLLA) have been considered biomaterials for drug delivery. PETOXPLLA polymeric micelles had low cytotoxicity in human normal fibroblast HFW cells for 72 $h$ by using MTT assay. It was found that cell viability was higher than $80 \%$ for the concentration range below $1 \mathrm{mg} / \mathrm{ml}$ [61]. Structure-uptake relationships of a series of 
amphiphilic poly(2-oxazolines) block copolymers that have different architectures, molar mass and chain termini were reported [62]. The relative cytotoxicity of poly(2-oxazolines) was tested on MCF7-ADR cells derived from human breast carcinoma cell line, MCF7 (ATCC HT-B22). All tests confirmed that these polymers are typically not cytotoxic even at high concentrations. The rate of endocytosis can be fine-tuned over a broad range by changing the polymer structure. The cellular uptake of the polymers increased with their hydrophobic character.

Low cytotoxicity of aliphatic poly(2-alkyl-2-oxazoline)s was confirmed also in in vitro studies with Rat-2 fibroblasts using the MTT test [63] (Figs. 6 and 7). The dependence of cell viability on molar mass confirmed the expected trend; the viability increased with the higher molar mass of poly(2-ethyl-2-oxazoline), up to $15000 \mathrm{~g} / \mathrm{mol}$. The effect of incubation time and concentration was also studied. It was shown that cell viability is in the range of untreated control even after 48 hours and the polymer concentration up to 5 $\mathrm{mg} / \mathrm{ml}$ (Fig. 7b).<smiles>CC(=O)N(C)CCCC(C)(C)O</smiles>

PMEOX<smiles>CCCCN(CCCC(C)(C)O)C(=O)CC</smiles><smiles>CCC(=O)N(CCC(=O)O)CCC(=O)C1=CC=C2CC=C3CC=CC4=CC=C1C2C43</smiles>

Figure 6. Structures of measured poly(2-alkyl-2-oxazolines)
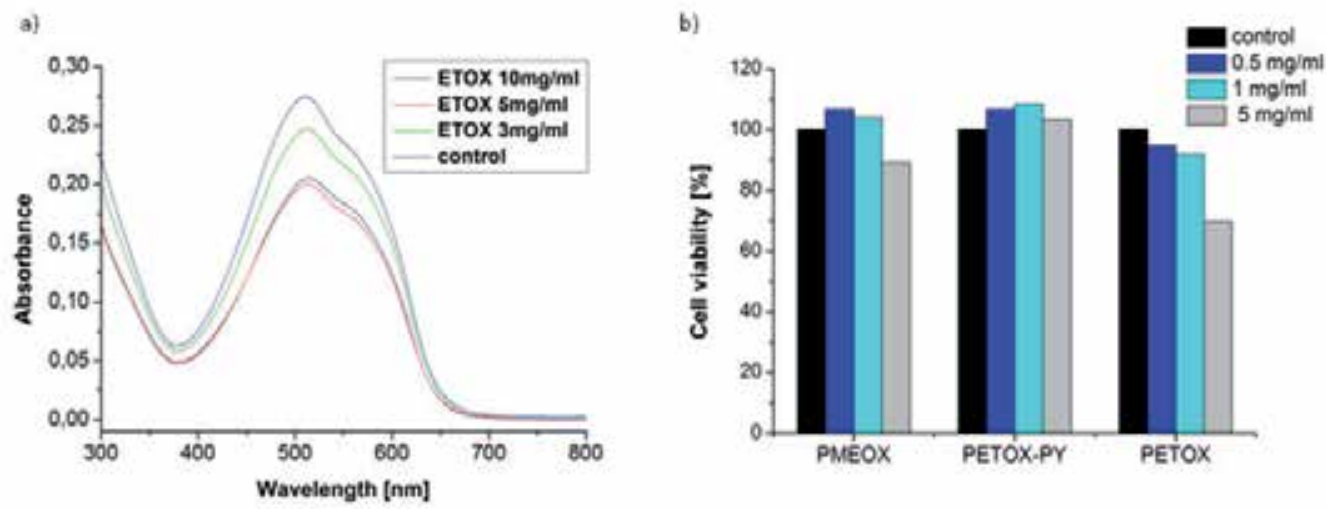

Figure 7. a) UV spectra of the reduced formazan dissolved in DMSO after incubation of rat fibroblasts with PETOX, b) relative cell viability of selected 2-oxazolines. PMEOX - poly(2-methyl-2-oxazoline), PETOX - poly(2-ethyl-2-oxazoline), PETOX-py - pyrene labelled poly(2-ethyl-2-oxazoline).

The results obtained for the polymers with aliphatic side chains were compared with the analogues that possessed an aromatic moiety. It is known from the literature that the presence of benzene ring in many cases increases their toxicity as was showed for 
different aromatic drugs [64,65]. In spite of these results, our measurements indicated that the presence of a benzene ring in poly[2-ethyl-2-oxazoline-co-2-(4-aminophenyl)-2oxazoline] with 10 mol. \% aromatic moiety (AEOX10) (synthesis see Fig. 8) has no affect on the growth and proliferation of cells and that the values of cytotoxicity measured in the MTT test were on the same level as in the case of PETOX and PMEOX [27,63]. All results confirmed low cytotoxicity of the polymers prepared by cationic polymerization of 2-alkyl- and 2-aryl-2-oxazolines, which supports their utilization in biomedical applications.

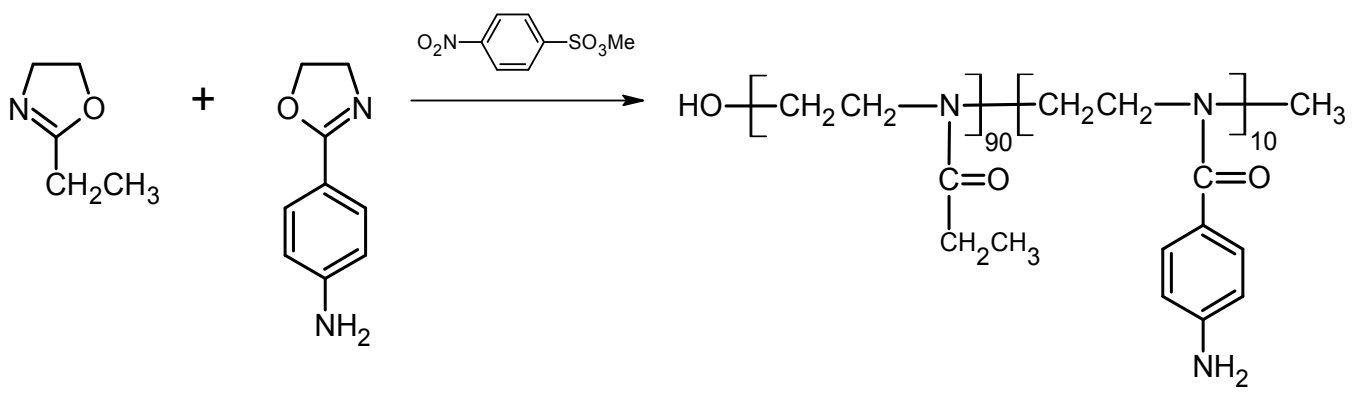

AEOX10

Figure 8. Synthesis of poly[2-ethyl-2-oxazoline-co-2-(4-aminophenyl)-2-oxazoline] (9:1).

\section{Methods for the assessment of immunocompatibility of poly(2-oxazolines)}

Selected techniques as flow cytometric simultaneous evaluation of phagocytosis accompanied by oxidative burst, immunocytometric determination of $\mathrm{T}_{\mathrm{H}} 1 / \mathrm{T}_{\mathrm{H}} 2 / \mathrm{T}_{\mathrm{H}} 17$ cytokines using bead application and enzyme-linked spot evaluation of cytokine producing cells comprise novel approaches to characterise and consider immunomodulation of basic functions of immunocompetent cells targeted by biopolymers.

\subsection{Assessment of phagocyte functions by immunocytometry}

Macrophages are tissue-based cells belonging to the reticuloendothelial system, capable of phagocytosis and production of an array of immunomodulatory mediators. Foreign substance or invader triggered macrophage activation result in a release of proinflammatory interleukins, growth factors and chemokines and also in up regulation of induction of inducible oxide synthase and other potent reactive oxidants inflicting cell damage. Phagocytosis represents a complex process comprising several subsequent steps i.e. chemotaxis, attachment/adherence onto a phagocytotic membrane, internalization and fusion of the cell membrane, intra- and extracellular digestion of targets, and oxidative burst (Fig. 9). The rate of endocytosis can be fine-tuned over a broad range by changing the polymer structure. The cellular uptake increases with the hydrophobic character of the polymers and is observed even at nanomolar concentrations [62]. 


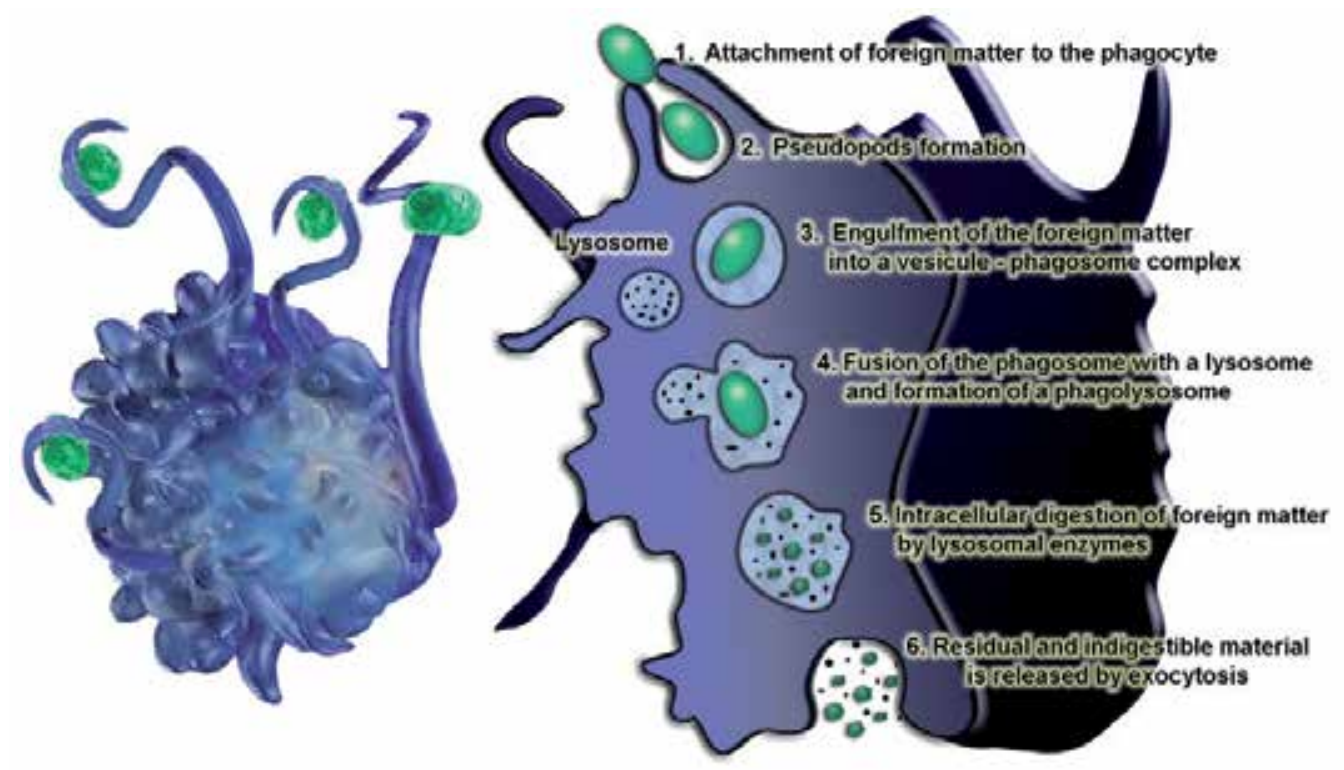

Figure 9. The sequential mechanism of phagocytosis.

Phagocytosing cells undergo a burst of oxygen consumption that is caused by an NADPH oxidase complex that assembles at the phagosomal membrane. Neutrophils and the other phagocytes create $\mathrm{O}_{2}{ }^{-}$(superoxide) by the one-electron reduction of oxygen at the expense of NADPH. A variety of soluble and particular stimuli induce extracellular superoxide production. Most of the oxygen consumed can be accounted for as hydrogen peroxide, which is formed from dismutation of superoxide radical. From these agents a large number of highly reactive microbicidal oxidants are formed, including $\mathrm{HOCl}$ (hypochlorous acid), which is produced by the myeloperoxidase-catalyzed oxidation of $\mathrm{Cl}^{-}$by $\mathrm{H}_{2} \mathrm{O}_{2}$; $\mathrm{OH}^{*}$ (hydroxyl radical), produced by the reduction of $\mathrm{H}_{2} \mathrm{O}_{2}$ by $\mathrm{Fe}^{2+}$ or $\mathrm{Cu}^{+}$; $\mathrm{ONOO}^{*}$ (peroxynitrite), formed by the reaction between $\mathrm{O}_{2}^{-}$and $\mathrm{NO}^{\circ}$, and many others [66]. Reactive oxygen species (ROS) comprises not only free radicals, such as superoxide radical, hydroperoxyl radical, hydroxyl radical, peroxyl radical etc., but also non-radicals, namely hydrogen peroxide, singlet oxygen, and hypochlorous acid. These reactive oxidants are manufactured not only as microbicidal agents, but they also impose cell damage.

Hydroethidine (dihydroethidium; HE) is frequently used as fluorescence probe for the evaluation of cell oxidative burst by detection of ROS (Fig. 10). HE has the ability to cross-cellular membranes becoming therefore efficient in cell oxidative burst studies. When inside the cell the $\mathrm{HE}$ is oxidized by $\mathrm{O}_{2}{ }^{-}$, it originates ethidium $\left(\mathrm{E}^{+}\right)$, which becomes locked in the cell by virtue of its cationic nature. The ethidium intercalates into DNA and is red fluorescent when excited with either visible or UV light. HE can also be oxidized by different reactive species, being the relative reactivity ranked by ONOO$>\mathrm{HO}^{\circ}>\mathrm{O}_{2}{ }^{\circ}>\mathrm{H}_{2} \mathrm{O}_{2}$. Thus, HE provides an index of reactive oxygen and nitrogen species production [67]. 


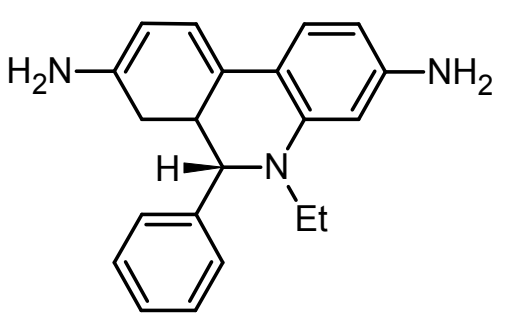

HE

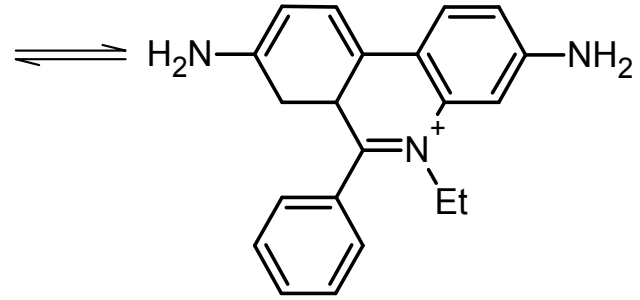

$\mathrm{E}^{+}$

$$
\begin{aligned}
& \lambda_{\text {exc }}=520 \mathrm{~nm} \\
& \lambda_{\text {em }}=610 \mathrm{~nm}
\end{aligned}
$$

Figure 10. Chemical structure of hydroethidine (HE) and ethidium $\left(\mathrm{E}^{+}\right)$.

To follow up the production of ROS throughout the oxidative burst of phagocyte, the fluorescence methodology, associated with the use of suitable fluorescence probes, is an excellent research approach applicable both for fluorescence microscopy and flow cytometry (Fig. 11).

Generally, flow cytometry measures optical and fluorescence characteristics of single cells or any other particles flowing in a single file in a stream of fluid. Light scattering at different angles can distinguish differences in size and internal complexity. Intensity of light scattered in the forward direction i.e. forward angle light scatter (FSC) roughly equates to the particle's size and can be used to distinguish between cellular debris and living cells. Light measured approximately at a $90^{\circ}$ angle to the excitation line is called side scatter. The side scatter channel (SSC) provides information about the granular content within a particle. Fluorescence measurements at different wavelengths $\left(\lambda_{\mathrm{em}}\right)$ can provide qualitative and quantitative data about fluorochrome-labelled cell surface receptors or intracellular molecules such as DNA and cytokines [68,69].

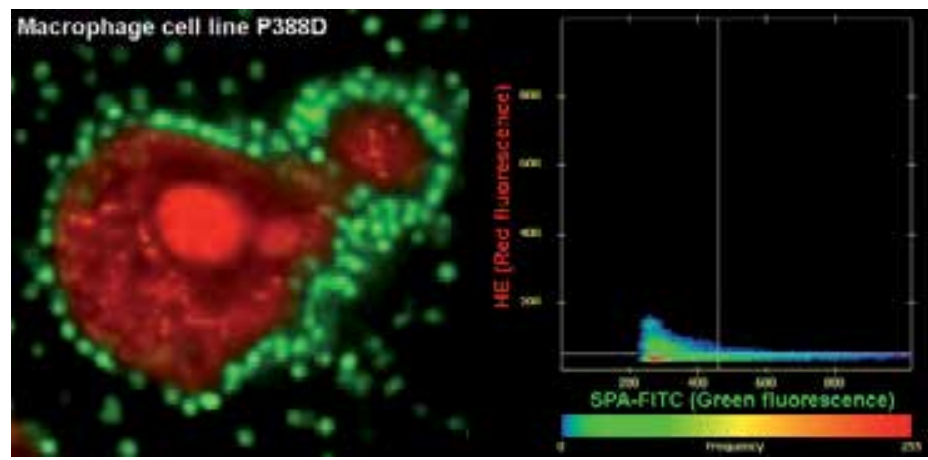

Figure 11. Simultaneous phagocytosis and oxidative burst in mouse macrophage line P388D, by S.aureus BioParticles ${ }^{\circledast}$ FITC labeled (Molecular Probes) in conjunction with dihydroethidium. Visualisation: Confocal Laser Scanning Microscopy LSM 710, Cell Observer (Zeiss). 
Assessment of phagocyte functions i.e phagocytosis and oxidative burst using S. aureusFITC and fluorescent probe HE by flow cytometry is an approach based on the complex imunocytometric analysis of multiple characteristic of individual cells i.e neutrophils, monocyte-derived macrophages (Fig. 12). Analyses were performed with a BeckmanCoulter FC 500 flow cytometer with 488-nm excitation and filters for the detection of green fluorescence $(525 \mathrm{~nm})$ of $S$. aureus BioParticles ${ }^{\circledR}$ FITC labelled and red fluorescence of ethidium (610 nm). Two parameters, MFI (mean fluorescence intensity) and percentage of fluorescence-positive cells were determined separately from approx. 5000 macrophages with a flow rate of 200-300 events/s by gating the cell population according to the forward scatter (FS)/side scatter (SS) histogram. To exclude cell debris and non-ingested S. aureus BioParticles from FS/SS histogram, discriminator was set into the FS channel.
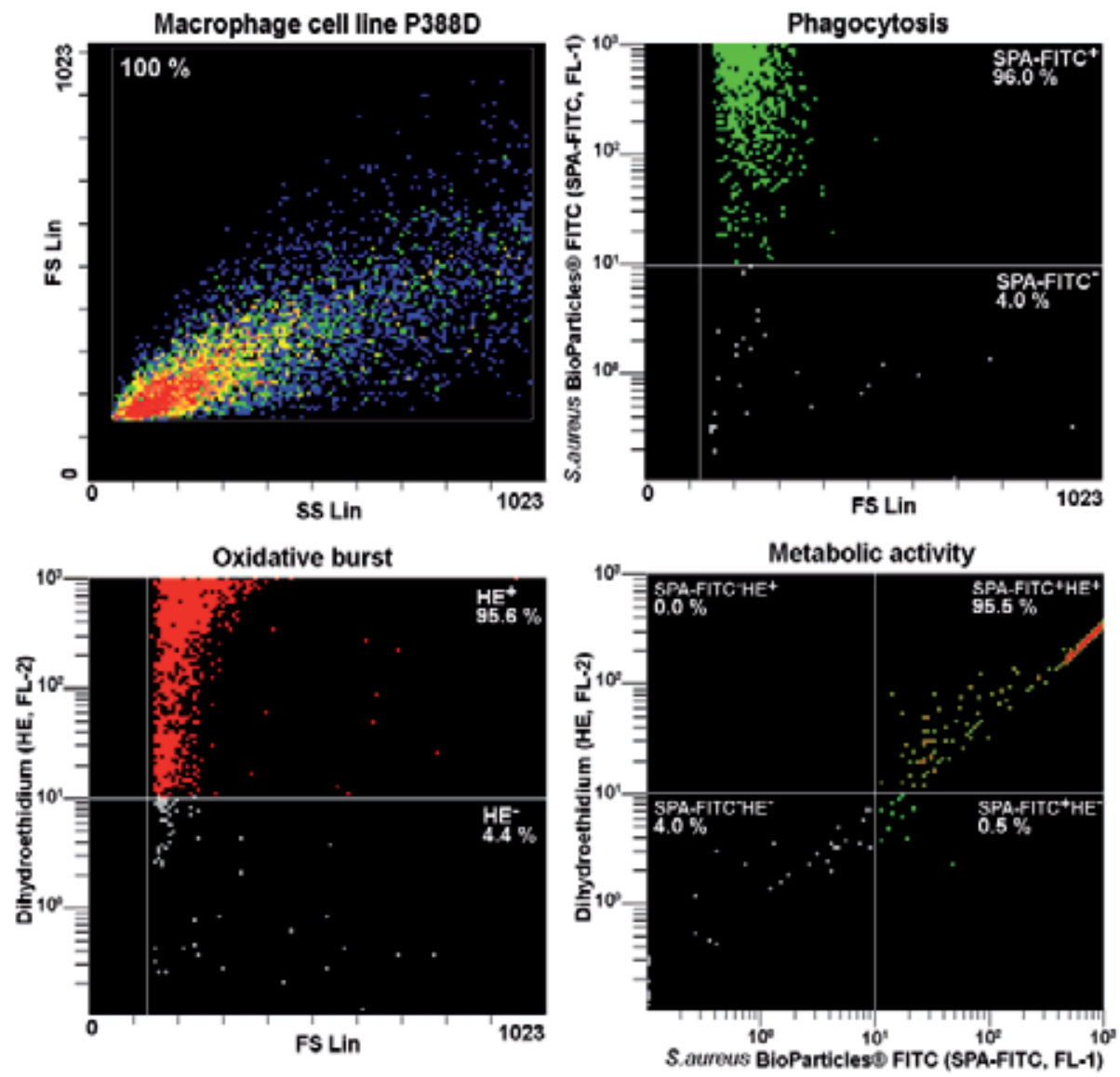

Figure 12. Simultaneous flow cytometric evaluation (Beckman Coulter FC 500) of phagocytosis and oxidative burst in mouse macrophage line P388D, by S.aureus BioParticles ${ }^{\circledR}$ FITC labeled (Molecular Probes), (SPA-FITC) in the conjunction with dihydroethidium (HE). Mean \% of phagocytic cells represents the percentage of granulocytes ingesting at least one SPA-FITC particle; mean \% of oxidative burst represents the percentage of granulocytes tagged by ethidium; mean metabolic activity $\%$ represents the percentage of granulocytes ingested at least one SPA-FITC and were tagged by ethidium. 


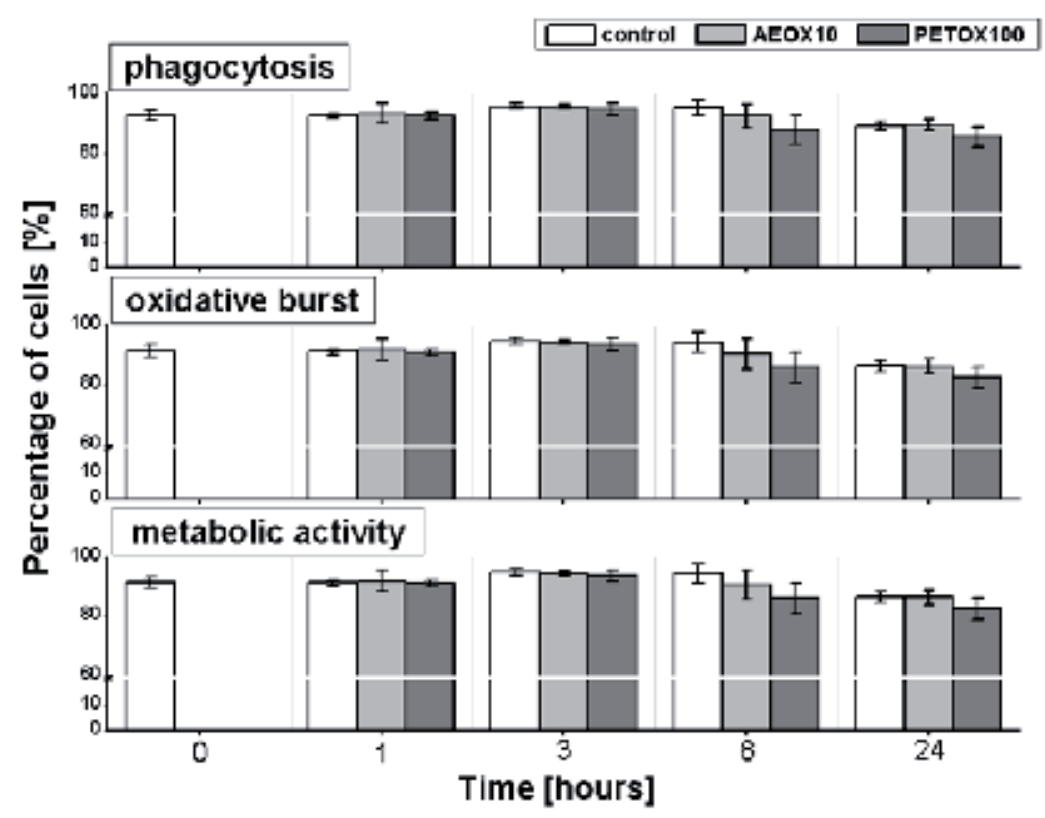

Figure 13. The influence of AEOX10 and PETOX100 ( $5 \mathrm{mg} / \mathrm{ml})$ on phagocytic effectiveness, oxidative burst and mtabolic activity (phagocytosis and oxidative burst) of macrophages.

In our experiments we analyzed the time and concentration dependent influence of poly(2oxazoline)s: AEOX10 and PETOX100 (PETOX with theoretical degree of polymerization equal to 100) on phagocytosis and oxidative burst of macrophages as representative professional phagocytic cells belonging to innate immune system compartments [63]. The treatment was performed for 1, 3, 6 and 24 hours with selected concentrations $0.5 \mathrm{mg} / \mathrm{ml}$ and $5 \mathrm{mg} / \mathrm{ml}$, respectively. In all experiments the metabolic activity, including phagocytosis and oxidative burs, treated macrophage cell line P388D remained comparable with untreated control cells without any significant immunosuppressive effect of poly(2-oxazoline)s (Fig. 13).

\subsection{Assessment of triggered cytokine release and $\mathrm{T}_{\mathrm{H}}$ polarisation using ELISpot and FlowCytomix ${ }^{\mathrm{TM}}$ assays}

Cytokines are proteins secreted by different cells of the immune system and serve as molecular messengers between cells. Cytokines interact with cells of the immune system in order to regulate the host response to pathological disorders; apart from this they also mediate physiological cellular processes. Different types of cytokines as colony stimulating factors, growth and differentiation factors, immunoregulatory and proinflammatory cytokines are involved in direct cell stimulation, differentiation, development and polarisation of immune response (Fig. 14). $\mathrm{CD}^{+} \mathrm{T}$ lymphocytes are crucial mediators of the cellular immune response. The early response of naive $\mathrm{CD} 4^{+} \mathrm{T}$ cells to antigenic stimulation (via pattern-recognition receptors) is characterized by induced proliferation. Further differentiation give arise of cells with a significant potential for cytokine expression. Depending upon the balance of local cytokines, co-stimulatory molecules, antigen levels, 
and genetic factors, $\mathrm{T}_{\mathrm{H}} 1, \mathrm{~T}_{\mathrm{H}} 2, \mathrm{~T}_{\mathrm{H}} 17$ effector and/or memory cells and Tregs' are generated by immune responses. Functionally-polarized $\mathrm{CD}^{+} \mathrm{T}$ cell subsets have been identified based on their exclusive patterns of various cytokine secretions. As a signature cytokine, $\mathrm{T}_{\mathrm{H}} 1$ cells selectively produce large amounts of interferon-gamma (IFN- $\gamma$ ). TH2 cells selectively produce IL-4, next characteristic $\mathrm{T}_{\mathrm{H}} 2$ cytokines are IL-5 and IL-13. The population of $\mathrm{T}_{\mathrm{H}} 17$ cells expresses high levels of IL-17A, followed by IL-17AF , IL-17F, IL-21 and IL-22. Unique cytokine expression for Tregs' represents TGF- $\beta$ and IL-10. The $\mathrm{T}_{\mathrm{H}} 1 / \mathrm{T}_{\mathrm{H}} 2 / \mathrm{T}_{\mathrm{H}} 17 / \mathrm{Treg}$ paradigm provides a useful model system for investigating the cellular and molecular mechanisms that mediate protective as well as harmful immune responses.

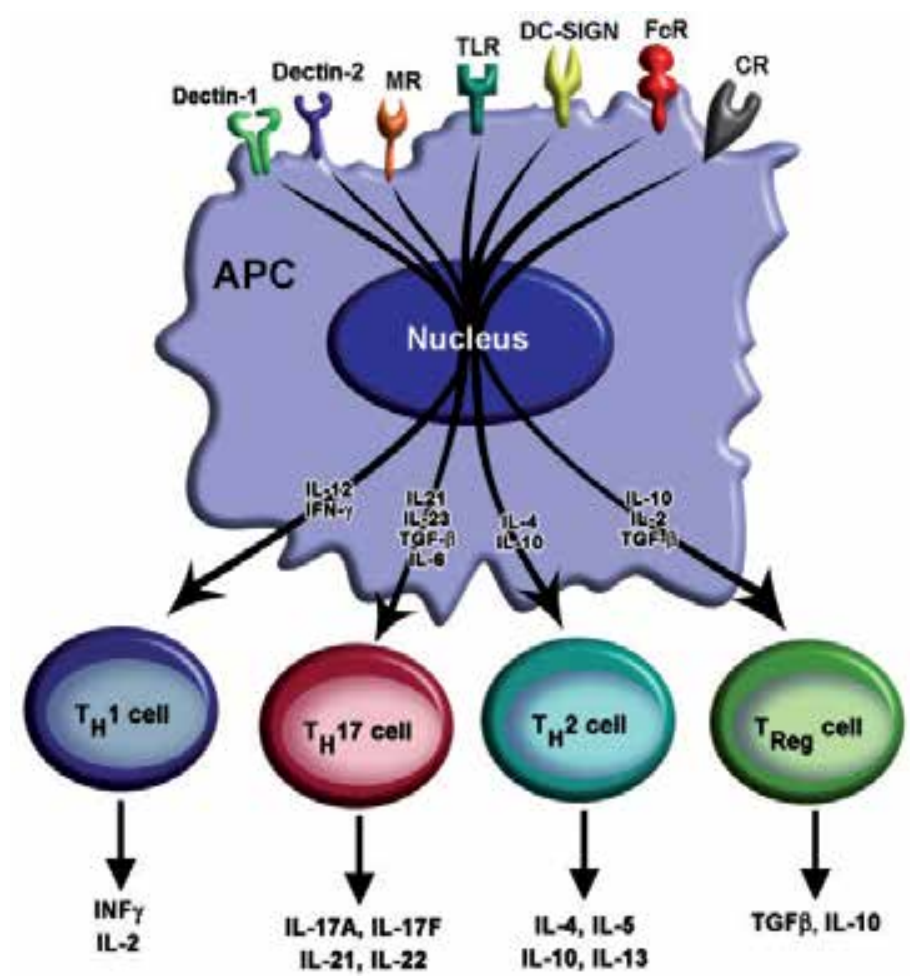

Figure 14. APC-derived cytokines and $\mathrm{T}_{\mathrm{H}}$ cell polarization. Pattern-Recognition Receptors (PRR): Dectin-1, Dectin-2, mannose receptor (MR), Toll-like receptor, DC-SIGN (Dendritic Cell-Specific Intercellular adhesion molecule-3-Grabbing Non-integrin), Fc receptor ( $\left.F_{C} R\right)$ - binding specificity for a Fc region of immunoglobulins, complement receptor (CR) - receptor of the complement system

It has been documented that the surface with neutral hydrophilic surface properties was found to show the greatest inhibition of cell adhesion and IL-1, IL- 6 and TNF- $\alpha$ release [72]. Recently, Brodbeck et al. have demonstrated that the surface chemistry (hydrophilicity and surface charge) of the biomaterial modulates the expression of anti- and pro-inflammatory $\mathrm{T}_{\mathrm{H}} 1$ and $\mathrm{T}_{\mathrm{H}} 2$ cytokines [71]. The influence of the PETOX100 and AEOX10 treatments of the mouse lymphoid line P388.D1 [clone 3124] on the changes of the IL-1 $\alpha$, IL-6 and TNF- $\alpha$ was evaluated. The exposition of P388.D1 macrophages with both AEOX10 and PETOX100 resulted in significant enhancement of IL-1 $\alpha$ IL-6 and TNF- $\alpha$ compared to the baseline 
results at immediately after the PETOX100 treatment. When compared to the time-kinetics of untreated cell control, the significance was confirmed only for TNF- $\alpha$ after 24 hours of exposure. The observed secretion levels of cytokines were significantly (10-100 times, depending on cytokine) lower than the cytokine levels observed with classical bacterial inducer, such as LPS. Based on these results, it could be assumed that both polymers, AEOX10 and PETOX100, represent bioavailable materials with immunobiological properties without strong undesirable inflammatory effectiveness with respect to their capacity to induce pro-inflammatory cytokines [70].

Apart from enzyme linked immunosorbent assay (ELISA) the more sensitive bead-based multiplex immunoassay for the flow cytometer and the enzyme linked immunosorbent spot assay (ELISpot) are frequently used for quantitative follow up of cytokine secretion by APC cells (Fig. 15).
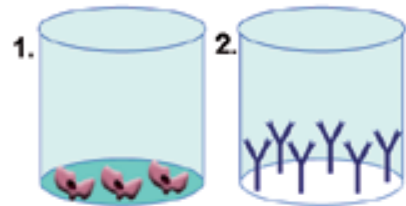

3.

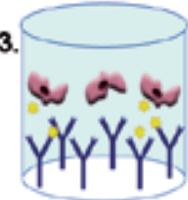

4.
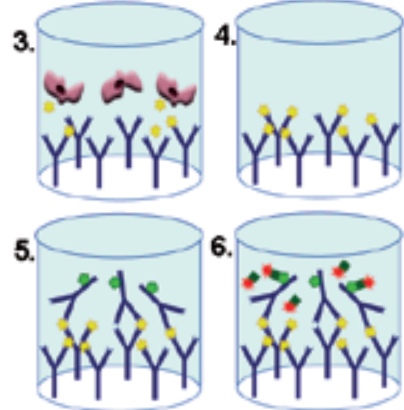

6.

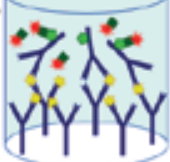

7.

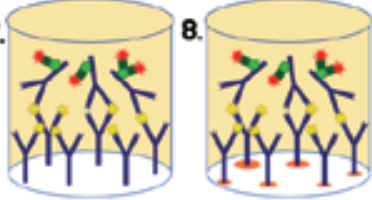

9.

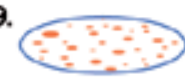

1. antigen activation of cells in cell - culture plate

2. 96 - membrane bottomed well plate is activated with cthanol and cytokine-specific antibodies are immobilized on membrane

3. pre - activated cells are transferred to coated wells and secreted cytokine is captured by primary antibody

4. removing of cells by washing, cytokines remine bound to the primary antibodies

5. detection antibody either directly conjugated with enzyme or biotinylated is added, detection antibody bounds to cytokine - antibody complex

6. if biotinylated detection antibody is used, a streptavidin-enzyme conjugate is added

7. incubation with detection substrate

8. substrate is converted into colored product and form a colored spot around the secreting cells

9. evaluation of resulting spots by spots counting in stimulated cultures and controls without stimulus, the frequency of responding cells could be determined

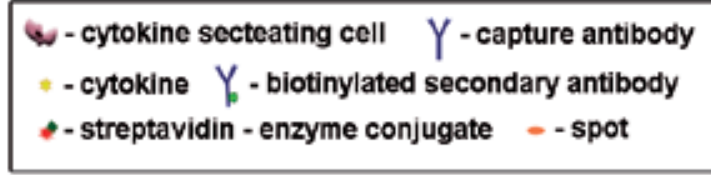

Figure 15. Scheme of ELISpot assay for quantification of cytokine one cell-based production.

Ultra-sensitive ELISPOT assays (limit of detection less than 1 in 100,000) can provide accurate measurements of antigen-specific cell immune responses and cytokine release. ELISPOT assays can measure both the magnitude and the quality of the response, thus providing more information about the samples in comparison with other methods for determination of cytokines e.g. ELISA. The methodology of ELISpot (developed by Czerkinsky et al. [73]) is rational up-grade of previously used plaque forming cells (PFC) technique with combination of enzyme immunoassay. ELISPOT assay was originally 
developed to enumerate B lymphocytes secreting antigen-specific antibodies. Nowadays, this technique has been adapted also for the identification and enumeration of cytokineproducing cells at the single cell level. ELISPOT assay allows visualization of the secretory product of individual activated or stimulated cells. Each spot represents an antigen-induced response of single reactive cell. Thus, the ELISPOT assay provides both qualitative (type of cytokine, antibody etc.) and quantitative (number of responding cells) information [74].

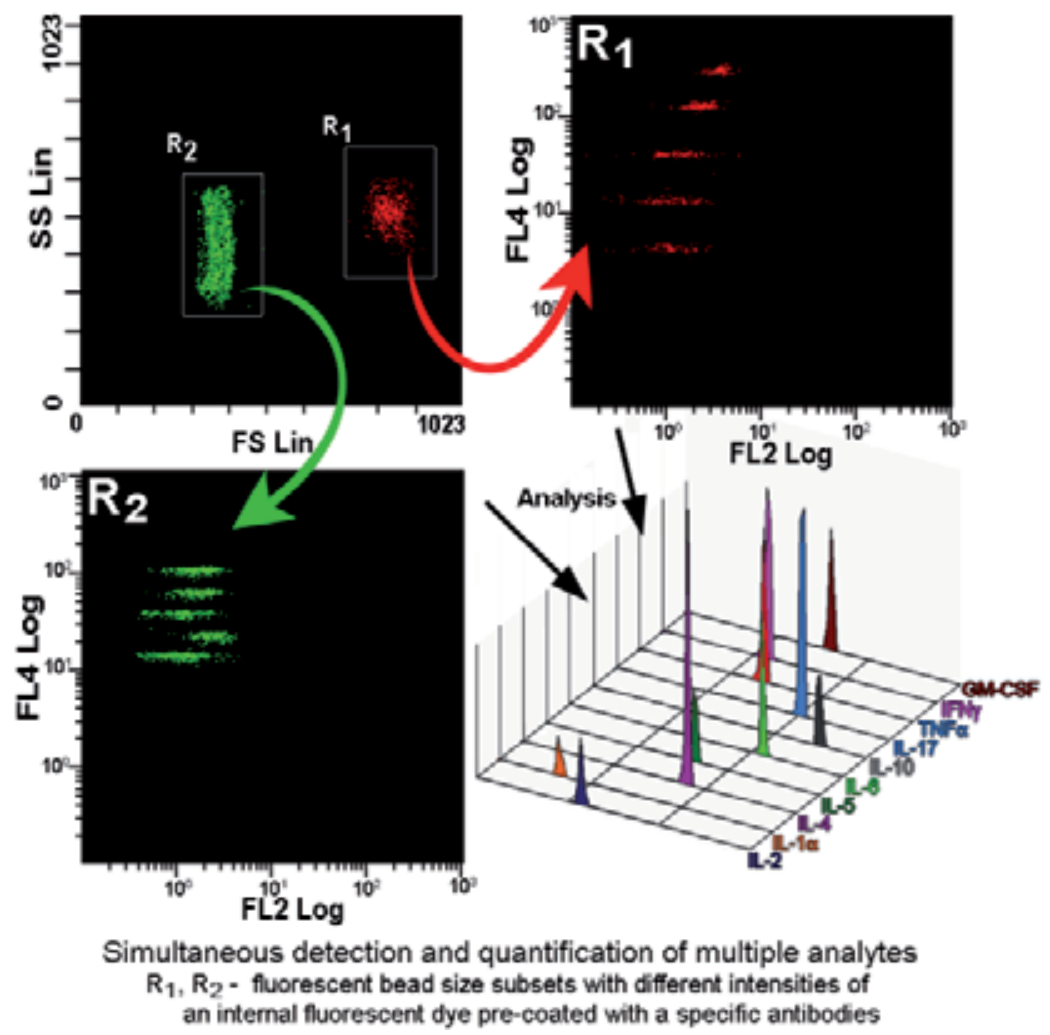

Figure 16. Example of FlowCytomix ${ }^{\mathrm{TM}}$ simultaneous quantification of Th1, Th2, Th17 and Treg cytokines in mice splenocytes (unpublished data)

Bead-based multiplex immunoassays for the immunocytometry are applied for the multiple detection of cytokines, chemokines, growth factors and related proteins. The FlowCytomix ${ }^{\mathrm{TM}}$ Multiple Analyte Detection System is designed to simultaneously quantify up to 20 protein targets. The methodology significantly reduces sample volumes and time compared to traditional ELISA and Western blot techniques. The FlowCytomix technology is based on the sandwich immunoassay technology where microspheres are used as a solid phase instead of a 96-well plate (Fig. 16). Within two bead size populations $4 \& 5 \mu \mathrm{m}$ latex beads coated with specific antibodies are multiple bead subsets, differentiated by varying intensities of an internal fluorescent dye Starfire Red. This dye can be excited at $488 \mathrm{~nm}$ by an Argon, He-Ne, or UV laser, and emits at $690 \mathrm{~nm}$ (the far red spectrum), which is detected in the FL-3/FL-4 channel. The combination of the two different bead sizes and different internal dye intensities 
makes it possible to distinguish up to 20 bead sets in one fluorescent channel. Reporter fluorochrome PE bound to strepavidin, binds to the biotin conjugate, emits at $578 \mathrm{~nm}$ and is detected in the FL-2 channel, allowing the quantification of the cytokine.

\section{In vitro and in vivo visualization methods}

The in vivo applications of polymers require investigating the intracellular metabolic pathways of distribution of polymer in cells and tissues. This can be examined by employing labeled systems. One way is the utilization of fluorescent probes for the visualization through labelling or staining. Labelled polymeric materials can be used for monitoring of cell uptake of desired materials using fluorescence microscopy or confocal laser scanning microscopy (CLSM). CLSM is a technique for obtaining high-resolution optical images with high depth resolution. This technique is widely used in cell biology, genetics, microbiology or developmental biology. Clinically, CLSM is an important method for the evaluation of different diseases, in endoscopic procedures and also in pharmaceutics for imaging the drug distribution. In the field of biocompatible polymers, this technique can be employed for the imaging of polymer uptake and distribution in cell, tissues or whole organs. For example, MCF-7 cancer cell uptake of coumarin-6-loaded poly(lactic-co-glycolic acid) (PLGA) nanoparticles was visualized using CLSM technique by overlaying the images obtained through the FITC filter and the PI filter [75]. The described system was used for sustainable release of Taxol in the treatment of cancer. CLSM was used for the examination of cell uptake and gene release from the ternary polyplexes of the plasmid DNA with B-PEI and $\mathrm{pH}$ responsive diblock copolymer poly(2-ethyl-2-oxazoline)-block-poly(methacrylic acid) (PETOX-b-PMAA) [76]. Time-dependent observations in three channels (green fluorescence of LysoTracker, blue fluorescence of $4^{\prime}, 6$-diamidino-2-phenylindole -DAPI andred channel of Cyanin 5.5) revealed the cell uptake process of the polyplex started after 3 hours and accelerated after 6 hours of incubation. Nanoparticles composed of statistical copolymers prepared by cationic copolymerization 2-ethyl-2-oxazoline and 2-decenyl-2oxazoline were prepared for encapsulation of various biomolecules [77]. Their cellular uptake of the prepared nanoparticles labeled with fluorescein was monitored with CLSM. Confocal micrographs revealed a high loading of mouse connective tissue fibroblasts with nanoparticles although no transfection agents were used to promote particle uptake. The overlay of the transmission and the fluorescence image shows the uniform distribution of the particles in intracellular compartments after endocytosis, which is the common way for the nanoparticle uptake. The results obtained by confocal microscopy were confirmed by flow cytometry. It was showed that the particles did not show toxic effects during the time of incubation.

Biodistribution of PETOX labeled with pyrene moiety in cells has been monitored by the Carl-Zeiss confocal laser-scanning microscopy [63]. The cuts of the treated cells were used for constructing 3D model (Fig. 17). The confocal micrographs supported the hypothesis of the polymer accumulation in specific organelles as observed also in the case of fluorescence microscopy measurements. 


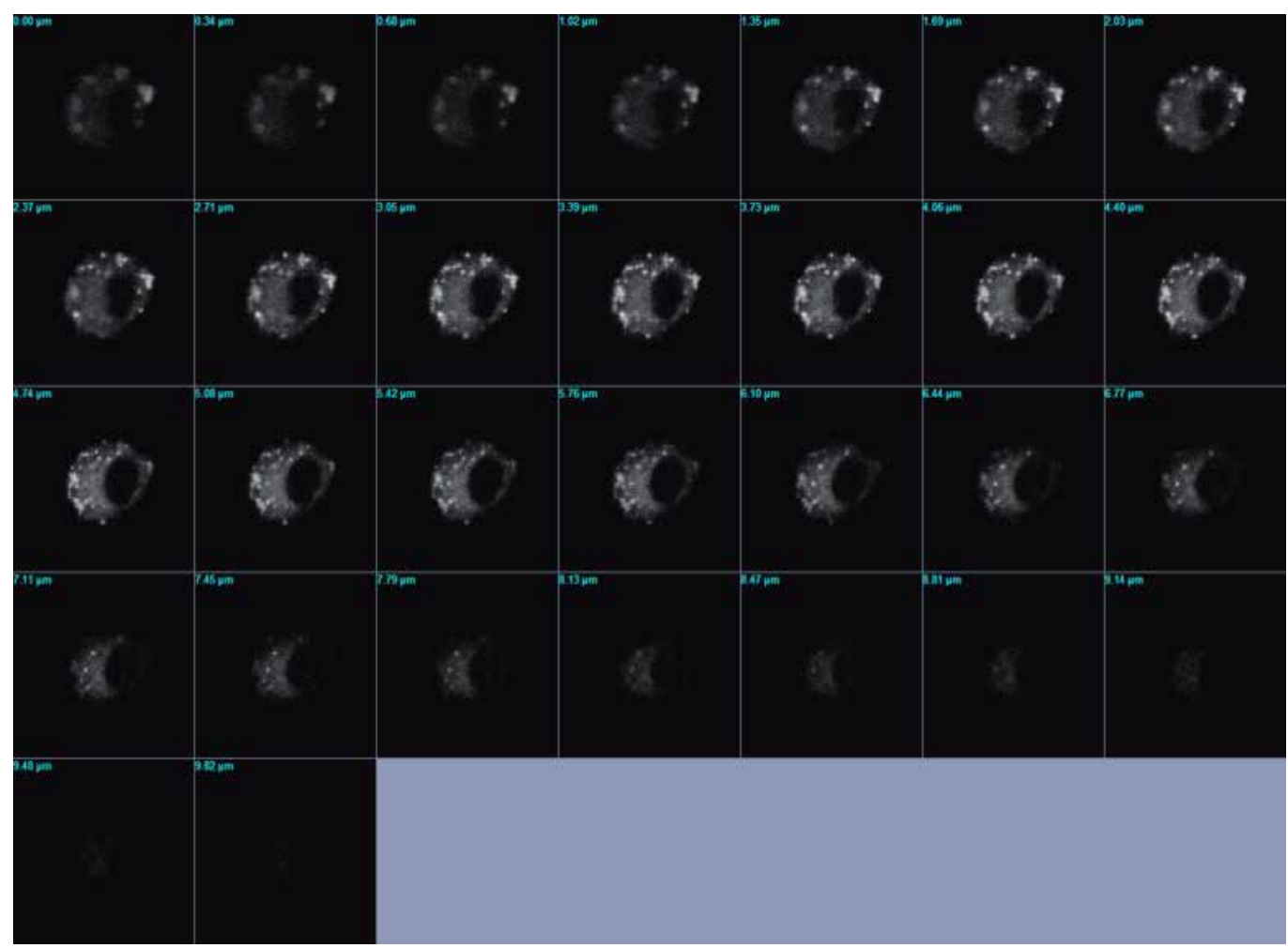

Figure 17. The cuts of the mouse macrophages treated loaded with pyrene-labeled PETOX conducted on the Carl-Zeiss confocal laser-scanning microscopy.

Similarly, cell uptake of polymers can be examined by fluorescence microscopy. A fluorescence microscope is an optical microscope used to study properties of organic or inorganic substances using the phenomena of fluorescence using two filters. An illumination (or excitation) filter ensures almost monochromatic excitation of a sample and a second emission (or detection) filter which ensures none of the excitation light source reaches the detector. The technique of fluorescence microscopy has become an essential tool in biology and the biomedical sciences, as well as in materials science due to attributes that are not readily available in other contrast modes with traditional optical microscopy. The application of an array of fluorochromes has made it possible to identify cells and submicroscopic cellular components with a high degree of specificity amid non-fluorescing material. In fact, the fluorescence microscope is capable of revealing the presence of a single molecule. Different probes can simultaneously identify several target molecules through the use of multiple fluorescence labeling. Although the fluorescence microscope cannot provide spatial resolution below the diffraction limit of specific specimen features, the detection of fluorescing molecules below such limits is readily achieved. Recently, infrared microscopy became very powerful method for optical visualization in the fields of biology, biochemistry, microbiology or genetics. Fluorescence microscopy was used for the visualization of cellular uptake of 2-oxazoline polymers. The principle was related to 
experiments on CLSM. Studied polymers were labeled with fluorescent probe. For these purposes, a pyrene moiety was introduced into the macromolecule of poly(2-ethyl-2oxazoline) (PETOX-py) using 1-(bromoacetyl)pyrene as initiator of cationic polymerization of 2-ethyl-2-oxazoline (Fig. 18).
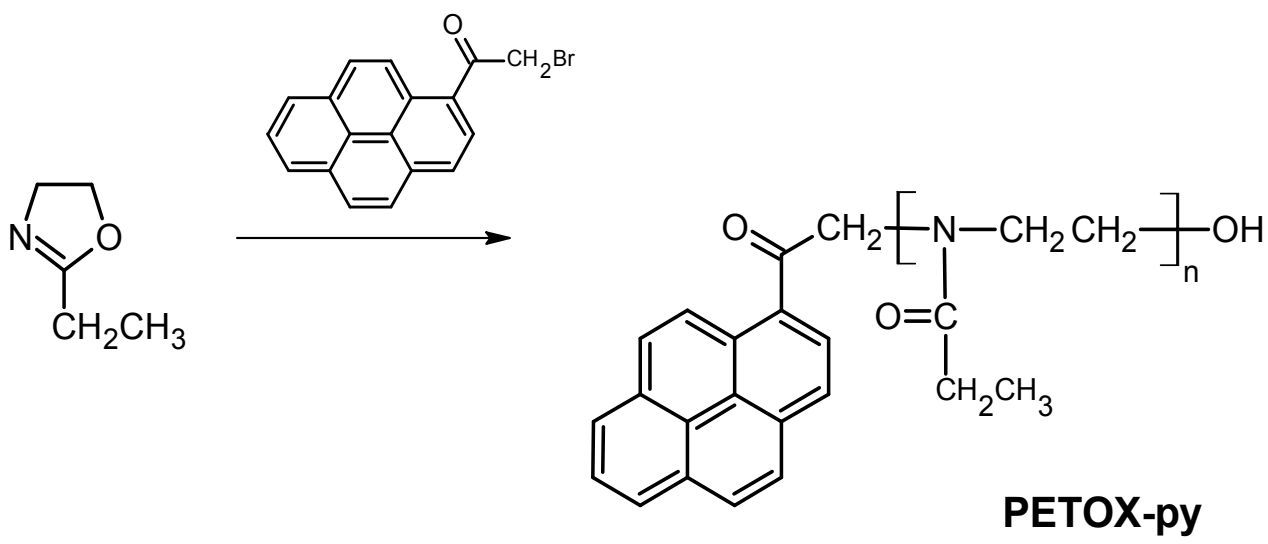

Figure 18. Synhesis of pyrene-labeled poly(2-ethyl-2-oxazoline) (PETOX-py).

Pyrene-based probes are in a class of hydrophobic dyes and can be used not only for the visualization and analysis of polymer concentrations within cells but also for the investigation of the environment polarity and the formation of agglomerates. Fig.19 shows the fluorescence microscopy of Rat-2 fibroblasts incubated in the presence of PETOX-py in PBS with the concentration of $5 \mathrm{mg} / \mathrm{ml}$. It can be seen that macromolecules are accumulated in the cells in specific organelles, most probably in peroxisomes [63].

(a)

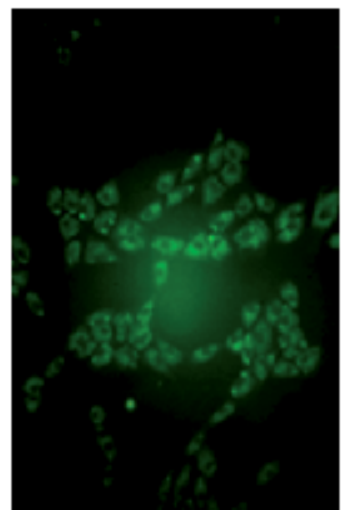

(b)

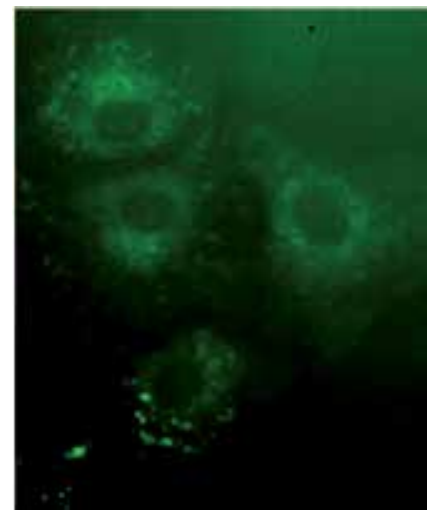

Figure 19. Fluorescence micrographs of rat fibroblasts loaded with pyrene-labeled PETOX for 24 hours in DMEM medium in the concentration equal to $5 \mathrm{~mol} / \mathrm{l}$ (a) and a detail of polymer uptake in cells (b). 
Peroxisomes are small membrane-enclosed organelles that contain enzymes involved in a variety of metabolic reactions, including several aspects of energy metabolism. Peroxisomes contain at least 50 different enzymes, which are involved in a variety of biochemical pathways in different types of cells. Peroxisomes were defined as organelles that carry out oxidation reactions leading to the production of hydrogen peroxide. A variety of substrates are broken down by such oxidative reactions in peroxisomes, including uric acid, amino acids, and fatty acids [78].

Biodistribution, targeting and excretion can also be investigated by radiolabeled poly(2alkyl-2-oxazolines). Authors used ${ }^{111}$ In-labeled polymers to study the distribution and excretion of such polymers in mice [79]. Similarly, ${ }^{125}$ I-labeled 2-oxazoline-based copolymers were examined after their intravenous administration to mice, and their biodistribution was assessed [80]. Beside the described visualization techniques, other modern methods such as scanning electron microscopy (SEM), transmission electron microscopy (TEM), atomic force microscopy (AFM) or tomography are available for the analysis of biodistribution.

\section{Conclusion}

The aim of this chapter was to survey the currently used experimental methods for evaluation of polymeric materials in biological systems concerning various methods for biocompatibility, immunological, bioimmunological and immunotoxicological assessment. This covers practical aspects throughout assay development and specific methodology adjustment. Decisive stages in development and application of these methods and practical suggestions are discussed. Such kind of research is essential due to enormous progress in the development of polymeric materials for different biomedical applications.

Among a number of polymers suitable for the use in medicine, poly(2-oxazolines) belong to polymeric materials with a great potential for different biomedical application. Their possible application areas in biological and medicinal contexts were reported. Especially the drug delivery, gene therapy and tissue engineering belong to the principal areas of their applications. Nowadays, several immunobiological and immunotoxicological methods were employed for the assessment of their biocompatibility and bioavailability. In vitro cytotoxicity of poly(2-oxazolines) was evaluated mainly by colorimetric MTT assay. However, other laboratory methods, like XTT, TB, SRB or WST assays, can be also used. All published results proved high biocompatibility of poly(2-oxazolines). Additionally, polymers prepared by cationic polymerization of 2-alkyl-2-oxazolines behave as "stealth" materials. This behaviour was confirmed by several independent immunological methods. Selected techniques as flow cytometric evaluation of phagocyte functions, immunocytometric determination of $\mathrm{T}_{\mathrm{H}} 1 / \mathrm{T}_{\mathrm{H}} 2 / \mathrm{T}_{\mathrm{H}} 17$ cytokines and ELISpot evaluation of cytokine producing cells comprise novel approaches to characterise immunomodulation of basic functions of immune competent cells targeted with poly(2-oxazolines). Last part of the chapter summarized in vitro and in vivo visualisation methods. 


\section{Author details}

Juraj Kronek, Zuzana Kroneková and Jozef Lustoň

Polymer Institute, Centre of Excellence GLYCOMED, Slovak Academy of Sciences, Bratislava, Slovakia

Ema Paulovičová and Lucia Paulovičová

Institute of Chemistry, Centre of Excellence GLYCOMED, Slovak Academy of Sciences, Bratislava, Slovakia

\section{Acknowledgement}

The authors appreciate the financial support of the Slovak Scientifical Agency VEGA in the Project nr. 2/0151/12 and the Centre of Excellence of the Slovak Academy of Sciences with the acronym GLYCOMED.

\section{References}

[1] He C, Kim SW, Lee DS (2008) In situ gelling stimuli-sensitive block copolymer hydrogels for drug delivery. J. control. release.127: 189-207.

[2] Roy I, Gupta MN (2003) Smart Polymeric Materials: Emerging Biochemical Applications. Chem. biol.10: 1161-1171.

[3] Jeong B, Gutowska A (2002) Lessons from nature: stimuli responsive polymers and their biomedical applications. TRENDS in biotechnol. 20: 305-311.

[4] Ř́hová B (2000). Immunocompatibility and biocompatibility of cell delivery systems. Adv. drug deliv. rev. 42: 65-80.

[5] Ř́hová B (1996) Biocompatibility of biomaterials: haemocompatibility, immunocompatibility and biocompatibility of solid polymeric materials and soluble targetable polymeric carriers. Adv. drug deliv. rev. 21: 157-176.

[6] Shalaby SW (1994) Biomedical Polymers: Designed-to-Degrade Systems. Cincinnati, USA: Hansen/Gardner Publ. ISBN 3-446-16531-2.

[7] Brauer H, Bienenstock J, Stead RH (2003) Mucosal Immunology. Munich: Sankyo Pharma GmbH. pp.1-63.

[8] Caminshi I, Shortman K (2012) Boosting antibody responses by targeting antigens to dendritic cells. Trends in immunol. 33: 71-77.

[9] McKenna AS, Beignon N, Bhardwaj A (2005): Plasmacytoid Dendritic Cells: Linking Innate and Adaptive Immunity. J. virol. 79: 17-27.

[10] Pulendran B, Palucka K, Banchereau J (2001) Sensing Pathogens and Tuning Immune Responses. Science. 293: 253-256.

[11] Kawai T, Akira S (2010) The role of pattern-recognition receptors in innate immunity: update on Toll-like receptors. Nat. immun. 11: 275-384.

[12] van Duin D, Medzhitov R, Shaw AC (2006) Triggering TLR signaling in vaccination. Trends in immunol. 27: 49-55. 
[13] Ishii KJ, Koyama S, Nakagawa A, Coban C, Akira S (2008) Host Innate Immune Receptors and Beyond: Making Sense of Microbial Infections, Cell host \& microbe, 2008: 352-363.

[14] Franz S, Rammelt S, Scharnweber D, Simon JC (2011) Immune responses to implants. A review of the implications for the design of immunomodulatory biomaterials. Biomaterials, 32: 6692-6709.

[15] Reddy ST, Swartz MA, Hubbell JA (2006) Targeting dendritic cells with biomaterials: developing the next generation of vaccines. Trends in immunol. 27: 573-579.

[16] Demento SL, Siefert AL, Bandyopadhyay A, Sharp FA, Fahmy TM (2011) Pathogenassociated molecular patterns on biomaterials: a paradigm for engineering new vaccines. Trends in biotechnol. 29: 294-306.

[17] Anderson JM, Cook G, Costerton B, Hanson SR, Hensten- Pettersen A. Jacobsen N, Johnson RJ, Mitchell RN, Pasmore M, Schoen FJ, Shirtliff M, Stoodley P (2004) Host Reactions to Biomaterials and Their Evaluation. In: Biomaterials Science, Elsevier Inc.2nd Edition, chapter 4: 293-338.

[18] Ř́hová B (2002) Immunomodulating activities of soluble synthetic polymer-bound drugs. Adv. drug deliv. Rev. 54: 653-674.

[19] Nilsson B, Korsgren O, Lambris JD, Nilsson Ekdahl K (2009) Can cells and biomaterials in therapeutic medicine be shielded from innate immune recognition? Trends in immunol. 31: 32-38.

[20] Kobayashi S (1990) Ethylenimine polymers. Prog. polym. sci. 15: 751-823.

[21] Weberskich R, Hettich R, Nuyken O, Schmaljohann D, Voit B (1999) Synthesis of new amphiphilic star polymers derived from a hyperbranched macroinitiator by the cationic 'grafting from' method. Macrom. Chem. Phys. 200: 863-73.

[22] Kowalczuk A, Kronek J, Bosowska K, Trzebicka B, Dworak A (2011) Star poly(2-ethyl-2oxazoline)s - synthesis and thermosensitivity. Polym. Int. 60: 1001-1009.

[23] Puts RD, Sogah DY (1997) Universal multifunctional initiator containing orthogonal reactive sites. Synthesis of macromonomers and comb polymers using consecutive controlled free radical and cationic ring-opening polymerizations. Macromolecules, 30: 7050-7055.

[24] Cesana S, Auernheimer J, Jordan R, Kessler H, Nuyken O (2006) First Poly(2-oxazoline)s with Pendant Amino Groups. Macrom. chem. phys. 207: 183-192.

[25] Cesana S, Kurek A, Baur MA, Auernheimer J, Nuyken O (2007) Polymer-Bound Thiol Groups on Poly(2-oxazoline)s. Macromol. rapid commun. 28: 608-615.

[26] Taubmann C, Luxenhofer R, Cesana S and Jordan R (2005) First AldehydeFunctionalized Poly(2-oxazoline)s for Chemiselective ligation. Macromol. Biosci. 5: 603612.

[27] Kronek J, Lustoň J, Kroneková Z, Paulovičová E, Farkaš P, Petrenčíková N, Paulovičová L, Janigová I (2010) Synthesis and bioimmunological efficiency of poly(2-oxazolines) containing a free amino group. J. mater. sci. mater. med. 21: 879-886.

[28] Christova D, Velichkova R. Loos W, Goethals EJ, Du Pre, F (2003) New thermoresponsive polymer materials based on poly(2-ethyl-2-oxazoline) segments. Polymer, 44: 2255-2261. 
[29] Park JS, Akiyama Y, Winnik FM, Kataoka K (2004) Versatile synthesis of endfunctionalized thermosensitive poly(2-isopropyl-2-oxazolines). Macromoleules, 37: 6786-6792.

[30] Bloksma MM, Paulus RM, van Kuringen HPC, van der Wert F, Lamberhort-Thijs HML, Schubert US, Hoogenboom R (2012) Thermoresponsive Poly(2-oxazine)s. Macrom. rapid commun. 33: 92-96.

[31] Charrois GJR, Allen TM (2004) Drug release rate influences the pharmacokinetics, biodistribution, therapeutic activity, and toxicity of pegylated liposomal doxorubicin formulations in murine breast cancer, Biochim. Biophys. acta, 1663: 167-177.

[32] Zalipsky S, Hansen CB, Oak JM, Allen TM (1996) Evaluation of blood clearance rates and biodistribution of poly(2-oxazoline)-grafted liposomes. J. pharm. sci. 85: 133.

[33] Luedtke K, Jordan R, Hommes P, Nuyken O, Naumann CA (2005) Lipopolymers from new 2-substituted-2-oxazolines for artificial cellmembrane constructs, Macromol. biosci. 5: 384-393.

[34] Lee SC, Kim C, Kwon IC, Chung H, Jeong SY (2003) Polymeric micelles of poly(2-ethyl2-oxazoline)-block-poly(caprolactone) copolymer as a carrier for paclitaxel. J. control. rel. 89: 437-446.

[35] Wang CH, Hwang YS, Chiang PR, Shen CR, Hong WH, Hsiue GH (2012) Extended Release of Bevacizumab by Thermosensitive Biodegradable and Biocompatible Hydrogel. Biomacromolecules, 13: 40-48.

[36] Zschoche S, Rueda JC, Binner M, Komber H, Janke A, Arndt KF, Lehmann S, Voit B (2012) Reversibly Switchable $\mathrm{pH} /$ and Thermoresponsive Core-Shell Nanogels Based on Poly(NiPAAm)-graft-poly(2-carboxyethyl-2-oxazoline)s. Macromol. chem. phys. 213: 215-226.

[37] Donev R, Koseva N, Petrov P, Kowalczuk, Thome J (2011) Characterization of different nanoparticles with potential use for drug delivery in neuropsychiatric disorders. World j. biol. psych. 12: 44-51.

[38] Thomas M, Lu JJ, Ge Q, Zhang CC, Cheng JZ, Klibanov AM (2005) Full deacylation of polyethylenimine dramatically boots its gene delivery efficiency and specifity to mouse lung. Proc. nat. acad. sci. USA, 102: 5679-5684.

[39] Hsiue GH, Chiang HZ, Wang CH, Juang TM (2006a) Nonviral gene carriers based on diblock copolymers of poly(2-ethyl-2-oxazoline) and linear polyethylenimine. Bioconjug. chem. 17: 781-786.

[40] Tauhardt L, Kempe K, Knop K, Altuntas E, Jager M, Schubert S, Fischer D, Schubert US (2011) Linear Polyethyleneimine: Optimized Synthesis and Characterization - On the Way to "Pharmagrade" Batches. Macromol. chem. phys. 212: 1918-1924.

[41] Banerjee P, Weissleder R, Bogdanov A (2006) Linear polyethyleneimine- grafted to hyperbranched poly(ethylene glycol)-like core: a co-polymer for gene delivery. Bioconjug. chem. 17: 125-131.

[42] Halacheva S, Price GJ, Garamus VM (2011) Effect of Temperature and Polymer Composition upon the Aqueous Solution Properties of Comblike Linear Poly(ethylene imine)/Poly(2-ethyl-2-oxazoline)-Based Polymers. Macromolecules, 44: 7394-7404. 
[43] Von Erlach T, Zwicker S, Pidhatika B, Konradi R, Textor M, Hall H, Luhmann T (2011) Formation and characterization of DNA-polymer-condensates based on poly(2-methyl2-oxazoline) grafted poly(L-lysine) for non-viral delivery of therapeutic DNA. Biomaterials, 32: 5291-5303.

[44] Brissault B, Kichler A, Leborgne C, Jarroux N, Cheradame H, Guis C (2007) Amphiphilic poly[(propylene glycol)-block-(2-methyl-2-oxazoine)] copolymers for gene transfer in skeletal muscle. ChemMedChem 2: 1202-1207.

[45] Farkas P, Korcova J, Kronek J, Bystricky S (2010) Preparation of synthetic polyoxazoline based carrier and Vibrio cholerae O-specific polysaccharide conjugate vaccine. Eur. j. med. chem. 45: 795-799.

[46] Korcova J, Machova E, Farkas P, Bystricky S (2010) Immunomodulative properties of conjugates composed of detoxified lipopolysaccharide and capsular polysaccharide of Vibrio cholerae 0135 bound to BSA-protein carrier. Biologia, 65: 768-775.

[47] Waschinski CJ, Tiller JC (2005) Poly(oxazoline)s with telechelic antimicrobial functions, Biomacromolecules, 6: 235-243.

[48] Schmidt DL, Brady RF, Lam K, Schmidt DC \& Chaudhury MK (2004) Contact angle hysteresis, adhesion and marine biofouling. Acta biomater. 20: 2830-2836.

[49] Choi JW, Lim IH, Kim HH, Min JH, Lee WH (2001) Optical perxide biosensor using the electrically controlled-release technique. Biosensors\&Bioelectronic, 16: 141-146.

[50] Purrucker O, Goennenwein S, Foertig A, Jordan R, Rusp M, Baermann M, Moroder L, Sackmann E, Tanaka M (2007) Polymer-tethered membranes as quantitative models for the study of integrin-mediated cell adhesion. Soft matter, 3:333-336.

[51] Luedtke K, Jordan R, Furr N, Garg S, Forsythe K, Naumann CA (2008) Twodimensional center-of-mass diffution of lipid-tethered poly(2-methyl-2-oxazoline) at the air-water interface studied at the single molecule. Langmuir, 24: 5580-5584.

[52] Reif M, Jordan R (2011) Alpha,omega-Functionalized Poly(2-Oxazoline)s Bearing Hydroxyl and Amino Functions. Macrom. chem. phys. 212: 1815-1824.

[53] Dech S, Wruk V, Fik CP, Tiller JC (2012) Amphiphilic polymer conetworks derived from aqueous solutions for biocatalysis in organic solvents. Polymer, 53: 701-707.

[54] Adeli M, Zarnegar Z, Kabiri R (2008) Amphiphilic star copolymers containing cyclodextrin core and their application as nanocarrier. Eur. polym. j. 44: 1921-1930.

[55] Williams DF (1999) In William's dictionary of Biomaterials. Liverpool University Press. ISBN 0-85323-921-5.

[56] Mosmann T (1983) Rapid Colorimetric Assay for Cellular Growth and Survival: Application to Proliferation and Cytotoxicity Assays, J. immunol. methods, 65: 55-63.

[57] Ishiyama M, Shiga M, Sasamoto K, Mizoguchi M, He PG (1993) A new sulfonated tetrazolium salt that produces a highly water-soluble formazan dye, Chem. pharm. bull. 41: 1118-1122.

[58] Coppeta R (1998) Dual Emission Laser Induced Fluorescence for Direct Planar Scalar Behavior Measurements. Experiments in Fluids, 1998: 1-15.

[59] Hoffman RM (1991) In vitro sensitivity assays in cancer: a review, analysis, and prognosis. J. clin. lab. anal. 5: 133-143. 
[60] Jeong JH, Song SH, Lim DW, Lee H, Park TG (2001) DNA transfection using linear poly(ethylenimine) prepared by controlled acid hydrolysis of poly(2-ethyl-2-oxazoline). J. control. rel. 73: 391-399.

[61] Hsiue GH, Wang CH, Lo CL, Wang CH, Li JP, Yang JL (2006) Environmental-sensitive micelles based on poly(2-ethyl-2-oxazoline)-b-poly(L-lactide) diblock copolymer for application in drug delivery. Int. j. pharm. 317: 69-75.

[62] Luxenhofer R, Sahay G, Schulz A, Alakhova D, Bronich TK, Jordan R, Kabanov AV Structure-property relationship in cytotoxicity and cell uptake of poly(2-oxazoline) amphiphiles. J. control. rel. 153: 73-82.

[63] Kronek J, Kroneková Z, Lustoň J, Paulovičová E, Paulovičová L, Mendrek B (2011) In vitro bio-immunological and cytotoxicity studies of poly(2-oxazolines). J. mater. sci. mater. med. 22: 1725-1734.

[64] Ross D (1996) Metabolic basis of benzene toxicity. Eur. j. hemat. 57: 111-118.

[65] Klopman G, Shi LM, Ramu A (1997) Quantitative Structure-Activity Relationship of Multidrug Resistance Reversal Agents. Mol. pharm. 52: 323-334.

[66] Babior BM (2000) Phagocytes and oxidative stress. Am. j. med. 109: 33-44.

[67] Gomes A, Fernandes E, Lima JLFC (2005) Fluorescence probes used for detection of reactive oxygen species. J. biochem. biophys. Methods, 65: 45-80.

[68] Brown M, Wittwer C (2000) Flow cytometry: Principles and Clinical Applications in Hematology. Clinical chem. 46: 1221-1229.

[69] Shapiro HM (2003) In: Practical Flow cytometry. J.Wiley \& Sons, Inc. 4th. Edition: 1-562.

[70] Kronek J, Paulovičová E, Paulovičová L, Kroneková Z, Lustoň J (2012). Immunomodulatory efficiency of poly(2-oxazolines). J. mater. sci. mater. med. 23: 14571464.

[71] Brodbeck WG, Nakayama Y, Matsuda T, Colton E, Ziats NP, Anderson JM (2002). Biomaterial surface chemistry dictates adherent monocyte/macrophage cytokine expression in vitro. Cytokine, 18: 311-319.

[72] Bernatchez SF, Merkli A, Minh TL, Tabatabay C, Anderson JM, Gurny R (1994). Biocompatibility of a new semisolid bioerodible poly(orthoester) intended for ocular delivery of 5-fluorouracil. J. biomed. mater. res. 28: 1037-1046.

[73] Czerkinsky C, Nilsson L, Nygren H, Ouchterlony O, Tarkowski A (1983). A solid-phase enzyme-linked immunospot (ELISPOT) assay for enumeration of specific antibodysecreting cells. J. immunol. methods, 65: 109-121.

[74] Kalyuzhny AE (2005). Handbook of ELISPOT. In Methods in Molecular Biology, Vol. 302, pp.1-323, Humana Press Inc.

[75] Feng SS, Zhao L, Zhang Z, Bhakta G, Win KY, Dong Y, Chien S (2007). Chemotherapeutic engineering: Vitamin E TPGS-emulsified nanoparticles of biodegradable polymers realized sustainable paclitaxel chemotherapy for $168 \mathrm{~h}$ in vivo. Chem. eng. sci. 62: 6641-6648.

[76] Lin CP, Sung YC, Hsiue GH (2011). A pH Responsive Ternary Gene Carrier based on Branched-Poly(ethylenimine) and Poly(2-ethyl-2-oxazoline)-block-Poly(methacrylic acid). Biotech. biomater. 1: 1-8. 
[77] Kempe K, Vollrath A, Schaefer HW, Poehlmann TG, Biskup C, Hoogenboom R, Hornig S, Schubert US (2010). Multifunctional Poly(2-Oxazoline) Nanoparticles for Biological Applications. Macrom. rapid commun. 31: 1869-1873.

[78] Cooper GM (2000) In: The cell: Molecular Approach. 2nd edition, Sunderland (MA): Sinauer Associates. ISBN-10: 0-87893-106-6.

[79] Gaentner FC, Luxenhofer R, Blechert B, Jordan R, Essler M (2007). Synthesis, biodistribution and excretion of radiolabeled poly(2-alkyl-2-oxazoline)s. J. control. rel. 119: 291-300.

[80] Goddard P, Hutchinson LE, Brown J, Brookman LJ (1989). Soluble polymeric carriers for drug delivery. Part 2. Preparation and in vivo behavior of $\mathrm{N}$-acylethyleneimine copolymer. J. control. rel. 10: 5-16. 


\title{
Bio-Medical Applications of the Electron-Beam Plasma
}

\author{
T. Vasilieva
}

Additional information is available at the end of the chapter

http://dx.doi.org/10.5772/52576

\section{Introduction}

The Electron-Beam Plasma (EBP) is generated by injecting an electron beam (EB) into a gaseous medium. The EBP composition is complex: generally it contains molecules, atoms, radicals and ions in stable and excited states, plasma electrons and injected beam electrons as well. At moderate pressures $\left(P_{m}<10 \mathrm{kPa}\right)$ the EBP is strongly nonequilibrium. It means that the function of the electron energy distribution in the EBP is non-Maxwellian and heavy plasma particles mentioned above are produced in super-equilibrium concentrations, i. e. very high densities of ionized and excited particles can be reached. As a result, the EBP appears to be chemically active even at low temperature. With respect to non-equilibrium plasmas generated in conventional ways (for instance, the plasma of gas discharges) the EBP has the following advantages:

- $\quad$ the EB can be injected into any gases, vapors and gas-vapor mixtures;

- $\quad$ the EBP bulk does not contract even at very high gas pressures ( $P_{m} \sim 10 \mathrm{kPa}$ and higher);

- the solid powders and liquid droplets injected into the gas do not prevent the EBP generation; large-size bodies can be inserted into the plasma bulk;

- $\quad$ both solid powders and thing films can be treated in the EBP;

- $\quad$ very high concentrations of chemically active particles can be obtained even at low (up to room) temperatures;

- the process of the EBP-treatment is absolutely controllable and the treatment results are replicable.

Due to its properties the EBP seems to be very promising for biomaterial treatment and especially for the modification of biopolymers.

The main objective of the study is to experimentally prove the applicability and advantages of the electron-beam plasma for actual biological, pharmacological, and medical problems. 
The production of substances with novel biological and pharmacological properties on the base of EBP-modified proteins and polysaccharides is considered as example.

The products of the fibrinogen proteolytic degradation are known to inhibit the platelet aggregation [1]. Being the product of the intermediate stage of the fibrinogen-fibrin polymer conversion, fibrin-monomer strongly affects the platelet activity due to two very active sites in its molecule. These sites are formed by the proteolytic cleavage in sequence the N-temini of the fibrinogen $\mathrm{A} \alpha$ and $\mathrm{B} \beta$ chains and release of the fibrinopeptides $\mathrm{A}$ and $\mathrm{B}$ [2]. Low molecular weight products of the fibrin-monomer proteolytic degradation are considered to be promising compounds for the platelets inhibition. Unfortunately the industrial fibrinmonomer can not be degradated by proteolytic enzymes (such as trypsin, plasmin, thrombin, and etc.) due to its high polymerization tendency. Therefore, the alternative techniques for controllable modification of the fibrin-monomer structure should be found to produce peptides with the high antiaggregating activity and without polymerization tendency.

The natural renewable biopolymers chitin and, especially, chitosan are very promising for technological and industrial applications such as agriculture, food processing, cosmetics production and others [3, 4]. Chitosan, linear heterocopolymers of $\beta$-1,4-linked 2-amino-2deoxy-D-glucopyranose and 2-acet-amido-2-deoxy-D-glucopyranose units, has many unique biological properties namely high biocompatibility with living tissues, biodegrability, ability to the complexation, and low toxicity. In medicine and pharmaceutics the water-soluble low molecular weight chitosans (less than $10 \mathrm{kDa}$ ) are usually required. These substances can be used as immune response-modulating or antibacterial agents, sorbents, radioprotectors, and for the production of microcapsules, thing films, and substrates for cell cultures [3,4]. To produce the low molecular weight chitosans (LMWC) several techniques, including chemical, enzymatic, and radical treatment have been suggested [5]. Simple and rather low-cost chemical treatment is a conventional method, however toxic wastes and environment contamination are inherent in the chemical chitin and chitosan processing as well as in all techniques mentioned above. Besides, the chemical treatment is very time consuming and usually takes several hours. Thus, the development of the effective techniques for quick and environment friendly chitosan degradation is the burning issue of the day.

The aims of the present study were as follows:

1. to experimentally prove the possibility of the EBP-stimulated hydrolysis of proteins and polysaccharides as a result of the chitosans plasmachemical processing;

2. to prove the controlablity of the EBP-treament and to develop the methods for the EBPtreament control;

3. to obtain the high yield of the low molecular weight products by optimizing the treatment conditions;

4. to characterize both the structure products of the plasmachemical treatment and their bioactivity;

5. to obtain low molecular products of fibrin-monomer and chitosan with antiaggregating and antimicrobial activity, respectively. 


\section{The Electron-Beam Plasmachemical reactor and the treatment procedure}

For the controllable biopolymers modification and low molecular mass substances production the special Electron Beam Plasmachemical Reactor (EBPR) was designed.

Figure 1 illustrates the design and operation of the EBPR. The focused continuous EB 3 generated by the electron-beam gun 1 which is located in the high vacuum chamber 2 is injected into the working chamber 5 filled with the plasma-generating gas through the specially designed double-stage gas-dynamic injection window 4 [6]. Oxygen, nitrogen, noble gases, gaseous hydrocarbons and other atomic and molecular gases, water vapor, and vapors of some organic substances can be used for the EBP generation. The electrically heated evaporator 11 is placed inside the reaction chamber, as shown in figure 1, to add the vapor to the plasma-generating gas. Evidently, the pure vapor can be used for the EBP generation and, in this case, the reaction chamber is kept at given constant pressure by adjusting the heater power.

In passing through the gas the EB is scattered in elastic collisions and the energy of fast electrons gradually diminishes during various inelastic interactions with the medium (ionization, excitation, dissociation). As a result, the cloud 10 of the EBP is generated, all plasma parameters being functions of $x, y$, and $z$ coordinates $(z$ is the axis of the EB injection).

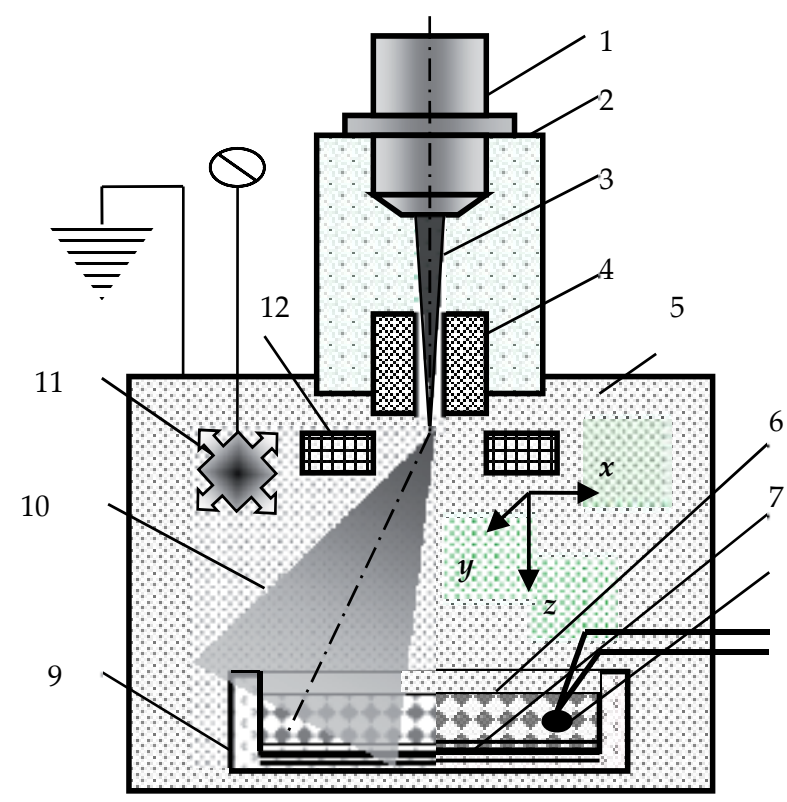

1 - electron beam; 2 - high vacuum chamber; 3 - EB; 4 - injection window; 5 - working chamber; 6 - mixing layer of the powder to be treated; 7 - piezoceramic plate; 8 - temperature sensor; 9 - glass container; 10 - EBP cloud; 11 water evaporator; 12 - scanning system.

Figure 1. The design of the plasmachemical reactor and the treatment procedure 
The electromagnetic scanning system 12, which is placed inside the working chamber near the injection window is able to deflect the injected EB axis in $x$ and $y$ directions and, therefore, to control the spatial distribution of the plasma particles over the plasma bulk. The working chamber is preliminary evacuated to pressure $\sim 10^{-2}$ Torr and then filled with plasma generating media. The samples to be treated were inserted into the EBPR reaction zone as solid powders with characteristic particle size $\sim 100 \mathrm{mcm}$ and as thing films. The powder of the substance to be treated partially fills the glass container 9 . Thin plate 7 made of piezoelectric ceramics is placed on the container bottom. Being fed with AC-voltage the plate vibrates, throws up the powder particles and forms the mixing layer 6 of the treated material. The miniature thermo-sensor 8 is inserted into the container to monitor the material temperature $T_{s}$ during the treatment. To prevent the thermal distraction of the biological material all samples were processed at $T_{s}<50{ }^{\circ} \mathrm{C}$. In the case of proteins $T_{s}$ was $\sim 37{ }^{\circ} \mathrm{C}$. The temperature control was carried out by selecting the EB current $I_{b}\left(1<I_{b}<100 \mathrm{~mA}\right)$.

Special software was developed to calculate irradiation doses as functions of the electron beam characteristics, gas pressure and chemical composition, treatment duration, and beam scanning parameters [7], see section "The control of EBP-modification process".

\section{The operation modes of the EBPR}

The reaction zone (item 6 in figure 1) of the EBPR is the plasma of an aerosol, as it is sometimes called "dusty plasma". In general, the operation parameters of the reactor responsible for the properties of the plasma of this kind are as follows.

1. The beam parameters: the initial electron energy $(E b)$, that is equal to the accelerating voltage of the electron gun, and the EB power $(N)$ injected into the reaction chamber.

2. The power $N$ is less than the original power of the EB $\left(N_{b}=E_{b} I_{b}\right.$, where $I_{b}$ is the current of the beam generated by the gun) since the EB is partially absorbed by the injection window. The transparency coefficient of the injection window was specially measured under various conditions of the plasma generation and the data of the measurements were used to characterize the parameters of the material treatment. The values of both $E_{b}$ and $I_{b}$ are measured inside the high voltage power source, supplying the gun, and are displayed on the control panel of the EBP generator.

3. The plasma-generating gas parameters: the pressure $\left(P_{m}\right)$ and temperature $\left(T_{m}\right)$ of the gas; its chemical composition.

4. The parameters of the dust: dimensions and shape of the dust particles; the dust density, i.e. the number of the dust particles per unit volume $\left(n_{d}\right)$; physical properties of the dust material (chemical composition, density, coefficients of the electron emission and some others);

5. The geometry of the plasma bulk, especially the distance $\left(z_{0}\right)$ between the injection window outlet and mixing layer.

In comparison with the plasma treatment of conventional powder materials (e.g. metals, ceramics, carbon, etc.) the biomaterials processing has at least three important peculiarities. 
1. The biomaterials should be processed at low temperatures $\left(\mathrm{T}_{\mathrm{s}}<50{ }^{\circ} \mathrm{C}\right)$ to prevent the material destruction caused by overheating.

2. The effect of the biomaterials modification was found to crucially depend on the dose of the biomaterial irradiation by the EBP particles. Usually, the material properties, especially its bioactivity, change abruptly when the certain irradiation dose has been accumulated, but if the dose exceeds this threshold level the bioactivity doesn't increase any more [8].

3. The direct bombardment by high energy electrons ( $E_{b} \sim 100 \mathrm{keV}$ and higher) is known to damage or destruct bio-molecules $[9,10]$.

It means that biomaterials should be processed by the EBP generated by the beams of the moderate $E_{b}$ and that the plasma particles densities and the local power input must be uniform over the reaction bulk, predictable and controllable.

The controlling system of the EBPR is able to vary $E_{b}\left(25<E_{b}<40 \mathrm{keV}\right)$ and $I_{b}\left(1<I_{b}<100\right.$ $\mathrm{mA})$. The system supports various scanning modes of the EB injected into the reaction chamber (linear in $x$ or $y$ directions, circle or ellipse, rectangular raster, multi-triangle or saw-tooth), and both the amplitudes and frequencies of the scanning can be varied. The controlling system also supports the intermittent modes of the EB generation that enables to the biomaterials treatment in the decaying EBP. The pulse frequency can be tuned within the range 1-1000 Hz; the shortest and longest pauses between the EB pulses are $10^{-3} \mathrm{~s}$ and 1.0 s respectively.

The EBPR controlling system also includes the gas-feeding unit which has a feedback with the valves of the vacuum system. The gas-feeding system supplies one or two plasmagenerating gases to the reaction chamber from separate external vessels, thereby keeping the total pressure of the gas mixture (within the range $0,1<P_{m}<100$ Torr) and partial pressures of the mixture components constant.

\section{The tests of the EBPR and optimization of the biomaterial treatment procedure}

The computer simulation of the dusty EBP was carried out to preliminary estimate optimal parameters of the biomaterial treatment. The following processes were taken into account:

- $\quad$ The scattering of high-energy electrons by the gas molecules and dust particles;

- The gas ionization by high-energy electrons;

- $\quad$ The capture of high-energy electrons by dust particles;

- The gas and dust heating;

- The heat transfer between the gas and dust particles;

- The plasmachemical reactions in the gas;

- The electron-ion recombination on the dust surface;

- The electric charging of the dust.

The local EB power input $Q(x, y, z)$, densities of charged particles of the EBP in various zones of the plasma bulk (including the mixing layer), and the temperature of the dust particles 
$\left(T_{s}\right)$ were calculated for various modes of the EB scanning modes and various distances $z 0$. The results of the computer simulations were verified experimentally for some modeling plasma-generating gases at pressures $1,0<P_{m}<50$ Torr and dust materials $\left(10^{3}<n_{d}<10^{6} \mathrm{~cm}^{-}\right.$ $\left.{ }^{3}\right)$. The diagnostic complex containing optical, microwave, calorimetric and some other devices was developed to measure values of $Q(x, y, z), T_{s}$, and densities of the plasma particles.

Figure 2 illustrates the obtained experimental data in comparison with the calculations. It presents the radial distributions of the local power input (curves 1-3) and steady-state temperature of the dust particles (curves 4,5 ) in the air-EBP at pressure $P_{m}=4$ Torr; $z_{0}=200$ $\mathrm{mm}$. All quantities plotted along the axis of ordinates are normalized by their values at the point $x=0, y=0$, i.e. at the center of the given cross-section $z_{0}$ of the plasma cloud.

The best combinations of the EBPR operating parameters were found for various plasmagenerating gases $\left(1,0<P_{m}<50\right.$ Torr $)$ and dust densities. For the experiments described they were: plasma generating gases - water vapor, oxygen, and helium, $E_{b}=30 \mathrm{keV}, I_{b}=2-5 \mathrm{~mA}$, $P_{m}=9$ Torr (water vapor), 4 Torr (oxygen) and 40 Torr (helium). The axis of the injected EB was scanned in $x$ - and $y$-directions to form the square raster $13 \times 13 \mathrm{~cm}^{2}$ in the plane of the mixing layer. Both continuous and intermittent modes of the EBP generation were applied. The container with the powder to be treated was located at the distance $z_{0}=25 \mathrm{~cm}$ from the injection window.

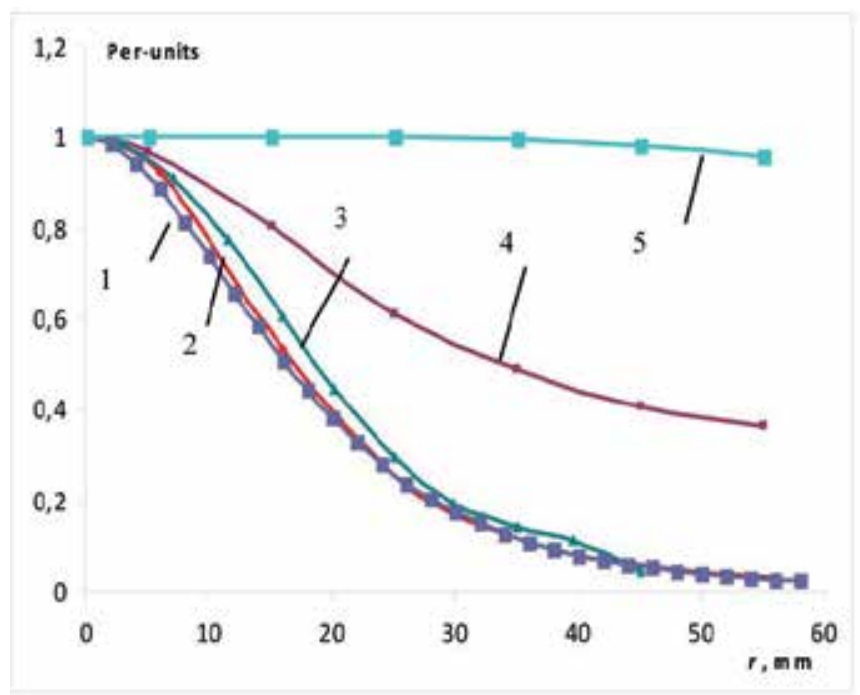

Figure 2. Radial distributions of the local power input (curves 1-3) and steady-state temperature of the dust particles (curves 4, 5): 1 - simulations, 2 - calorimetric measurements, 3 - optical measurements, 4 - thermo-sensor measurements in the non-scanning plasma cloud, 5 - thermo-sensor measurements in the plasma generated by the EB scanning along the $x$-axis.

Both the experiments and simulation showed that the powder is uniformly heated to the temperature $T_{s}<50{ }^{\circ} \mathrm{C}$ and the concentrations of the chemically active plasma particles can reach $10^{9}-10^{11} \mathrm{~cm}^{-3}$ in the vicinity of the material to be treated. The concentrations of the 
charged plasma particles (secondary electrons and ions) were measured by means of an open barrel-shaped microwave cavity [11] and data of the computer simulation can be used to estimate the concentrations of the neutral ones (molecules, atoms, radicals) [6]. The appropriate duration of the treatment procedure was experimentally found to achieve the required dose of the material irradiation.

\section{The control of EBP-modification process}

In general the following parameters are responsible for the processes (and eventually for the results) of the materials modification:

- $\quad$ The parameters of the EB being injected: electron energy $E_{b}$, integral current of the beam $I_{b}$ and current density $j_{b}$. For the EBPR under consideration these parameters are completely predetermined by the reactor design: the designs of the electron gun and injection window, the gun accelerating voltage $U$.

- The medium properties: chemical composition, pressure $P_{\mathrm{m}}$ and temperature $T_{\mathrm{m}}$ of the gas.

- The geometry of the reaction bulk: location of the reaction zone inside the EBP cloud, amplitude and frequency of the EB scanning.

- Characteristic size of the powder particles and density of the aerosol in the reaction volume.

- The total dozes of the powder particle irradiation by plasma particles of various kinds; the dozes being, obviously, determined by the fluxes of these particles on the powder surface and by the treatment duration.

Special software was developed to simulate the processes of the EBP generation and plasma-powder interaction, predict the effect of the modification under various experimental conditions, and optimize the treatment procedure. The description of the numerical algorithms and physical and plasmachemical models used for the software is beyond the paper involved. Here we demonstrate only the application of the software. Figure 3 presents the incident fluxes of the plasma particles on one $\mathrm{cm}^{2}$ of the container bottom (item 9 in Figure 1) calculated as a function of $x$ - and $y$-distances from the container center. The EB axis scans in $x$ - and $y$-directions, the amplitudes of scanning being equal to $\pm 13 \mathrm{~cm}$. Figure 3(a) illustrates the bio-material treatment in helium at pressure 40 Torr and Figure 3(b) - in water vapor at pressure 10 Torr. In the first case, four rigid peaks of the incident power appear at the raster corners and a sufficiently flat valley $\approx 5 \times 5 \mathrm{~cm}^{-2}$ occurs between the peaks. The intensity of irradiation in the valley is about half of that on the peaks. In the EBP of water vapor a flat plateau $\approx 7 \times 7 \mathrm{~cm}^{-2}$ with a uniform flux of plasma particles is formed. In real experiments the container with the material to be treated was placed in the zones of uniform incident fluxes.

If the container location and scanning parameters are known the values of plasma particles fluxes can be found in terms of local concentrations $n\left(\mathrm{~cm}^{-3}\right)$ of these particles inside the container. The functions $n=n\left(P_{m}, I_{b}, U\right)$ were calculated for every plasmagenerating gas used in our experiments. The variations $n=n\left(P_{m}\right)$ of the ion concentration 
in the EBP of argon excited by the EB at two different values of the beam current $I_{b}=15$ and $I_{b}=7,5 \mathrm{~mA}$ (curves 1 and 2 respectively) are presented in Figure 4 as the example of numerical simulations. In this figure all concentration values $n$ are divided by the maximum value of the particles concentration $n_{\max }$ for $I_{b}=15 \mathrm{~mA}$. The Figure 3 shows that there are optimal values of $P_{m}$ for given $I_{b}$ and $U$, these optimal values being individual for each gas.
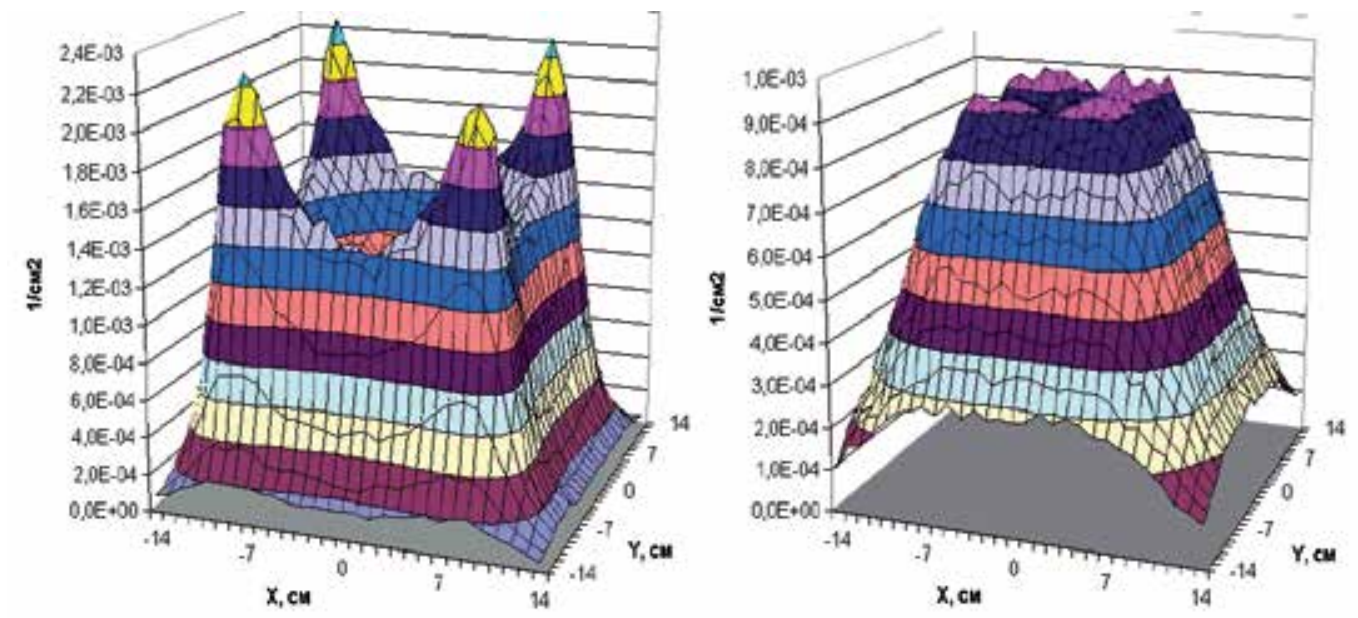

Figure 3. a.The incident radiation power of fast electrons on $1 \mathrm{~cm}^{2}$ of the sample surface calculated as a function of $x$ - and $y$-distances from the sample center: the treatment in the helium EBP at pressure 40 Torr. b.The incident radiation power of fast electrons on $1 \mathrm{~cm}^{2}$ of the sample surface calculated as a function of $x$ - and $y$-distances.

If optimal values of $P_{m}$ are found the total dose of the powder particles irradiation can be calculated. In particular, the treatment durations $\tau$ required to irradiate the powder by equal dozes at variable gas pressures were calculated and relevant experiments in various gases were planned and carried out. Experiments of this kind showed the modification effect in the organic materials under consideration to appear when the accumulated doze exceeded some threshold value.

The material temperature can be controlled by varying the gas pressure value during the treatment. The lower the gas pressure - the higher the sample temperature since the scattering and absorption of the EB in gaseous media are more intensive at higher pressures than at lower ones. If higher sample temperature is required to obtain the desirable modification effect the gas pressure should be reduced. For this reason the polysaccharides were treated at lower pressures than the proteins because the higher temperature (about 400 $\mathrm{K})$ was optimal for effective polysaccharides modification. On the contrary, when the proteins were treated in helium the higher pressures $\left(P_{m} \approx 40\right.$ Torr) were required to prevent the sample from overheating. It was because of the restrictions on the material temperature that the working pressures in the reaction chamber of the EBPR sometimes differed from their optimal values found as the maximum of the functions $n=n\left(P_{m}, I_{b}\right)$. 


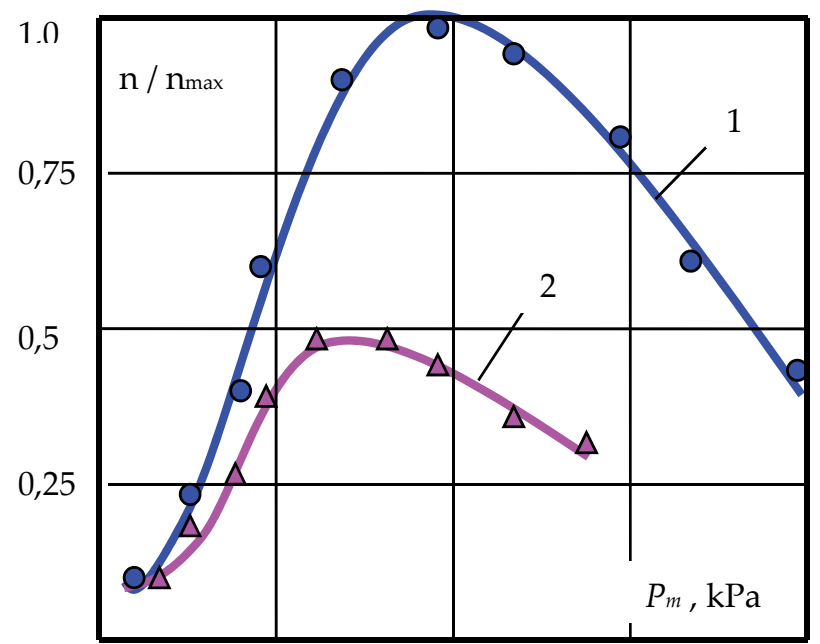

Figure 4. Concentrations of excited particles in the EBP of argon filling the reaction chamber as a function of the gas pressure, $U=25 \mathrm{kV}, z=130 \mathrm{~mm}: I_{b}=15 \mathrm{~mA}$ (curve 1), $I_{b}=7,5 \mathrm{~mA}$ (curve 2).

\section{Original substances and methods used to investigate products of their EBP-modification}

The plasmachemical modification of the following natural materials was studied in detail:

- $\quad$ synthetic derivative of 2-aminopropanoic acid (alanine) - natural alpha-amino acid with artificially inserted pyrozolidine cycles;

- $\quad$ bovine serum albumin (BSA, $M_{r}=66 \mathrm{kDa}$ )

- fibrin-monomer (FM, $M_{r} \sim 340 \mathrm{kDa}$ ) - the natural protein which is contained in the blood of mammalians.

- cellulose materials (pure cellulose, wooden sawdust, and crushed paper);

- chitosan from crab or shrimp shells with the degree of deacetylation and molecular weight of $95 \%$ and $500 \mathrm{kDa}$, respectively;

- peat of some peat deposits in Russia.

The original powders of polysaccharides were additionally ground in the laboratory mill before treatment. Average final sizes of the powder particles were within the range 10-40 $\mu \mathrm{m}$. The proteins were treated both in forms of solid powders (BSA and FM) and thing films (BSA). To form a film BSA was dissolved in distilled water and than water evaporation under vacuum was performed. The thickness of the formed film was $\sim 1 \mu \mathrm{m}$.

The following properties of EBP-modified products were of particular interest:

- $\quad$ water solubility of the materials after plasma treatment;

- molecular mass;

- $\quad$ spectral characteristics in IR- and UV-regions; 
- $\quad$ electrophoretic mobility;

- $\quad$ specific antigenic properties;

- biological activities.

Solubility measurements. $100 \pm 0,1 \mathrm{mg}$ of the preliminary dried sample $\left(m_{s}\right)$ were placed into a tube and $1,5 \mathrm{ml}$ of distilled water were added to the sample. The resulting mixture was incubated for $24 \mathrm{~h}$ at room temperature under periodic mixing. After the incubation the mixture was centrifuged for $5 \mathrm{~min}$ and $1 \mathrm{ml}$ of centrifugate was taken and dried. The mass of the dry residue $(m d r)$ was measured with an accuracy $\pm 0,1 \mathrm{mg}$. The sample solubility was calculated as the $\left(m_{d r} / m_{s}\right) \times 100 \%$ ratio.

Molecular mass characterization. To characterize the molecular masses of the EBP-treatment products the exclusion chromatography was applied. The chromatograph Staier (Russia) and the chromatographic column Phenomenex BioSep-Sec-S-3000 (USA) with the efficiency of 30000 theoretical plates were used. The analysis conditions were as follows: the elutriating agent $-0,1 \mathrm{M}$ phosphate buffer $\left(\mathrm{pH} 6,86\right.$ ) containing $0,05 \% \mathrm{NaN}_{3}$; the elution rate $-1 \mathrm{ml} / \mathrm{min}$; temperature $-30^{\circ} \mathrm{C}$; UV-detector with the wavelength $280 \mathrm{~nm}$.

The effects of the EBP-treatment on proteins molecular mass and structure were detected also by means of the UV- and IR-spectroscopy, ion-exchange chromatography, immunoelectrophoresis and PAGE-electrophoresis as well [12].

$U V$-spectroscopy. The measurements were performed with the spectrometer Shimadzu UV3600 (Japan). The IR-spectra were registered within wavelength $\lambda=226-418 \mathrm{~nm}$.

IR-spectroscopy. The measurements were performed with the IR-spectrometer Portmann Instruments $A G$ (Switzerland) equipped with the ZnSe crystal. The IR-spectra were registered within wave numbers $v=500-3600 \mathrm{~cm}^{-1}$. To improve the spectral resolution Fourier analysis was performed.

Ion-exachange chromatography. The ion-exchange chromatography (with the preliminary acid hydrolysis of the protein) was performed to reveal the changes in the amino acid composition of proteins due to the EBP-modification. The analyzer AAA-339 $M$ (Hungary) was used. To quantitatively analyze the sulfur-containing amino acids (cystine and methionine) the biomaterial was treated with the performic acid before the hydrolysis procedure. Totally 17 basic amino acids contents were measured. The accuracy of the mesuarment was $10 \%$.

Immunoelectrophoresis. The electrophoresis was performed in the 1,4\% agar gel [13]. The commercial specific antiserum to human fibrinogen was used to characterize and compare antigenic structure of the FM before and after plasmachemical treatment.

PAGE-electrophoresis was performed according to Laemmli U.K. [14].

Biological activity of the EBP-produced low molecular weight chitosans (LMCW). The inhibition of the bacteria growth in vitro was measured to quantitatively characterize the bioactivity of LMCW obtained by the plasma treatment, gram-positive (S. aureus), gram-negative (E. coli, Ps. aeruginosa) microorganisms and yeast-like fungi (C. albicans) being used in these experiments. 
Biological activity of the EBP-treated alanine derivative and fibrin-monomer. The EBP-modification products of fibrin-monomer were tested as the platelet aggregation inhibitors [15]. The platelet aggregation $A(\%)$ was measured by the turbodimetric method [16] and $A$ was defined as the ratio of the light transparency of the platelet suspension after ceasing the aggregation process to the initial value of the light transparency. The aggregation was monitored by the aggregometer Chronolog Corporation (USA), adenosine diphosphoric acid (ADP, final concentration $1 \times 10^{-5} \mathrm{M}$; Boechringer Mannheim, Germany) being used as an aggregation agent.

\section{The amino-acids treatment in the Electron-Beam Plasma}

Some natural amino acids with artificially inserted pirozolidine cycles into their structures were used as original substances and the products of their modification in the EBP of helium and water vapor were tested as inhibitors of the human platelet aggregation. Preliminary analysis showed the substances of this class to be promising as active agents for medical therapy of acute coronary events, and cardiovascular diseases that remain the leading cause of mortality. Their advantages are due to selectivity of the pharmacology action and limited side effects.

The powder samples ( $\approx 50 \mathrm{mg}$ in mass) of the original derivative of alanine were treated in the EBP of water vapor at pressure $P_{m} \approx 9$ Torr for variable time duration $\tau=45-300 \mathrm{~s}$. The typical EB power was $N_{b} \approx 0,1 \mathrm{~kW}$, the sample temperature $T_{s}$ under the treatment could be varied within the range $30-110{ }^{\circ} \mathrm{C}$.

The untreated compound was not dissolvable in distilled water at room temperature and the water heating up to $90{ }^{\circ} \mathrm{C}$ followed by cooling to $25{ }^{\circ} \mathrm{C}$ was required to carry out the control experiments and to study its effect on human platelet aggregation in vitro. The treated substance became partially water-soluble at room temperature and the solution at maximum concentration was added to the platelet suspension to measure the aggregation degree.

The untreated derivative decreased human platelet aggregation only to $46 \pm 2 \%$ with respect to control (56 $\pm 2 \%)$. The water-soluble products of plasma treatment reduced the aggregation degree up to $\approx 30 \%$, i.e. being treated by the EBP for $5 \mathrm{~min}$ the studied substance reduced the platelet aggregation activity by approximately $45 \%$ (Table 1 ).

\begin{tabular}{ccccccc}
\hline \multirow{2}{*}{ ADP } & ADP + & \multicolumn{5}{c}{ ADP + treated amino acid } \\
\cline { 3 - 7 } & \begin{tabular}{c} 
untreated \\
\cline { 3 - 7 }
\end{tabular} & $\tau=45 \mathrm{~s}$, & $\tau=90 \mathrm{~s}$, & $\tau=180 \mathrm{~s}$, & $\tau=180 \mathrm{~s}$, & $\tau=300 \mathrm{~s}$, \\
\hline $56 \pm 2 \%$ & $46 \pm 2 \%$ & $41 \pm 3 \%$ & $41 \pm 3 \%$ & $34 \pm 3 \%$ & $32 \pm 3 \%$ & $31 \pm 3 \%$ \\
\hline
\end{tabular}

Table 1. The effect of the plasma modification in the EBP of water vapor on the anti-aggregation activity of the tested alanine derivative (in vitro): the aggregation degree as a function of the treatment duration $\tau$ and temperature of the substance $T_{\mathrm{s}}$ under the treatment procedure

The effect of the treatment duration on their anti-aggregation activity increased as the treatment prolonged, the anti-aggregation activity rising sharply at $90<\tau<180 \mathrm{~s}$. At shorter durations $\tau<\tau_{0}$ the plasma did not modify the original substance and the longer treatment $\tau$ 
$>\tau_{0}$ resulted in insignificant additional effect. Moderate sample heating amplified the treatment effect slightly, i.e. plasmachemical processes are responsible for the modification.

\section{The proteins treatment in the Electron-Beam Plasma}

Originally water-indissoluble native FM was found to become soluble at room temperature without bunching. Figure 5 presents the UV-spectra of absorption for original and treated fibrin-monomer. The spectral curves of the modified products differ radically from the curve representing the original substance. This supports the hypothesis of the changes in the physical-chemical properties due to the plasmachemical treatment in the EBP.

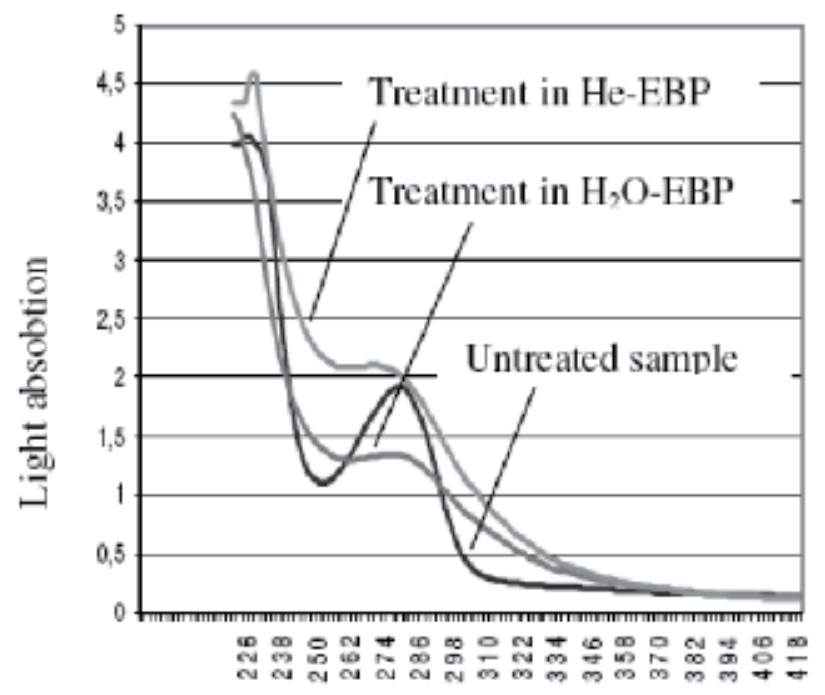

\section{Wavelength, nm}

Figure 5. UV-spectra of the light absorption of fibrin-monomer solutions

Radical changes in the FM structure after the EBP-treatment were detected by means of immunoelectrophoresis. The EBP-modified products did not exhibit the specific antigenic properties of original FM and did not react with specific antibodies, while the native substance gave specific precipitation line (Figure 6).

To reveal the changes in the primary and secondary structure of the EBP-treated FM the IRspectroscopy was used. The IR-spectra analysis showed (Figure $7 \mathrm{a}, \mathrm{b}$ ):

1. The maximum of the absorbance in the band complex $v=1711-1714 \mathrm{~cm}^{-1}$ (complex of amide II bands) displaced from $1711 \mathrm{~cm}^{-1}$ (native FM) to $1712 \mathrm{~cm}^{-1}$ and $1712 \mathrm{~cm}^{-1}$ (FM treated in the EBP of helium and water vapor, respectively). This indicates the partial destruction of peptide -CO-NH-bonds in the primary FM structure.

2. The spectral bands $v=3354 \mathrm{~cm}^{-1}$ and $v=3475 \mathrm{~cm}^{-1}$ characterize the valence oscillations of $-\mathrm{N}-\mathrm{H}$-bonds. The expansion of this band in the spectra of the EBP-modified FM 
confirms the partial destruction of the native protein molecule and low molecular weight peptides formation.

3. The spectral bands $v=1163 \mathrm{~cm}^{-1}$ and $v=1471 \mathrm{~cm}^{-1}$ which characterize the oxidation of disulfide bounds are most intensive in the IR-spectra of EPB-treated FM.

These facts confirm that the EBR-treatment of the FM for $\tau=5$ min has caused the partial destruction of the peptide - $\mathrm{CO}-\mathrm{NH}$-bonds in the primary FM structure and the oxidation of the disulfide bonds responsible for the tertiary peptides structure.

All changes were more significant in the case of the water vapor EBP, which could result from the higher chemical activity of the water plasmolysis products (e.g. $\left.\mathrm{OH}^{\bullet}\right)$.

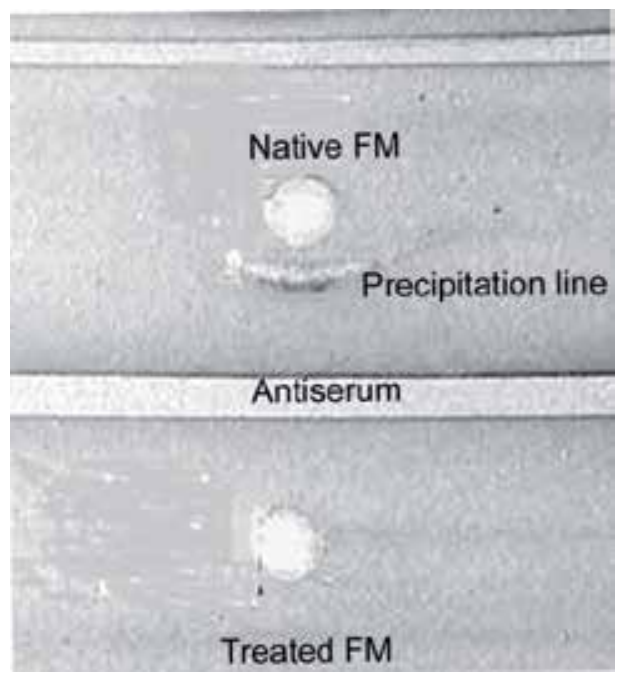

Figure 6. The immunoelectrophoresis of FM before and after the EBP-treatment

The plasma treatment reduced the amount of amino acids forming the primary protein structure (the content of 17 amino acids was studied). The percentages of lysine, threonine, glutamic acid, cystine, tyrosine and phenylalanine were found to be reduced significantly (down to 2 times with respect to the native FM, Table 2). The reduction of aspartic acid, serine, glycine, valine, methionine, leucine and isoleucine was not so sharp (only 1,3-1,5 times with respect to the native FM). The intensive peak of free $\mathrm{NH}_{3}$ was detected in the chromatogram of the modified products whereas it was not found in the chromatogram of the original peptide.

To characterize the molecular masses of the EBP-treatment products the exclusion chromatography was applied. The peaks corresponding to 6 individual peptides with the elution times 12,$30 ; 12,55 ; 13,17 ; 13,70$; and 13,94 min were observed in the exclusion chromatograms of the FM modified by the EBP (Figure 8).

The water-soluble products of the FM appropriately treated by the EBP of both helium and water vapor were found to decrease the platelet aggregation down to $\approx 33-35 \%$ in vitro at final concentrations $1 \times 10^{-5}-1 \mathrm{mg} / \mathrm{ml}$, treatment in the water vapor EBP being more effective 
than that in helium (Figure 9). The effect of the treatment on the FM anti-aggregation activity increased sharply with the time within the range $90<\tau<180 \mathrm{~s}$. The longer treatment resulted in a negligible additional effect. The peak corresponding to the elution time 12,3 min (molecular weight $\approx 650 \mathrm{Da}$ ) was observed at the exclusion chromatograms of the FM modified in the EBP of helium and EBP of water vapor at a moderate irradiation dose whereas the FM treated EBP of water vapor at lower or higher irradiation doses did not produce the peak (Figure 8). This peptide is likely to be responsible for the inhibiting of the platelet aggregation.
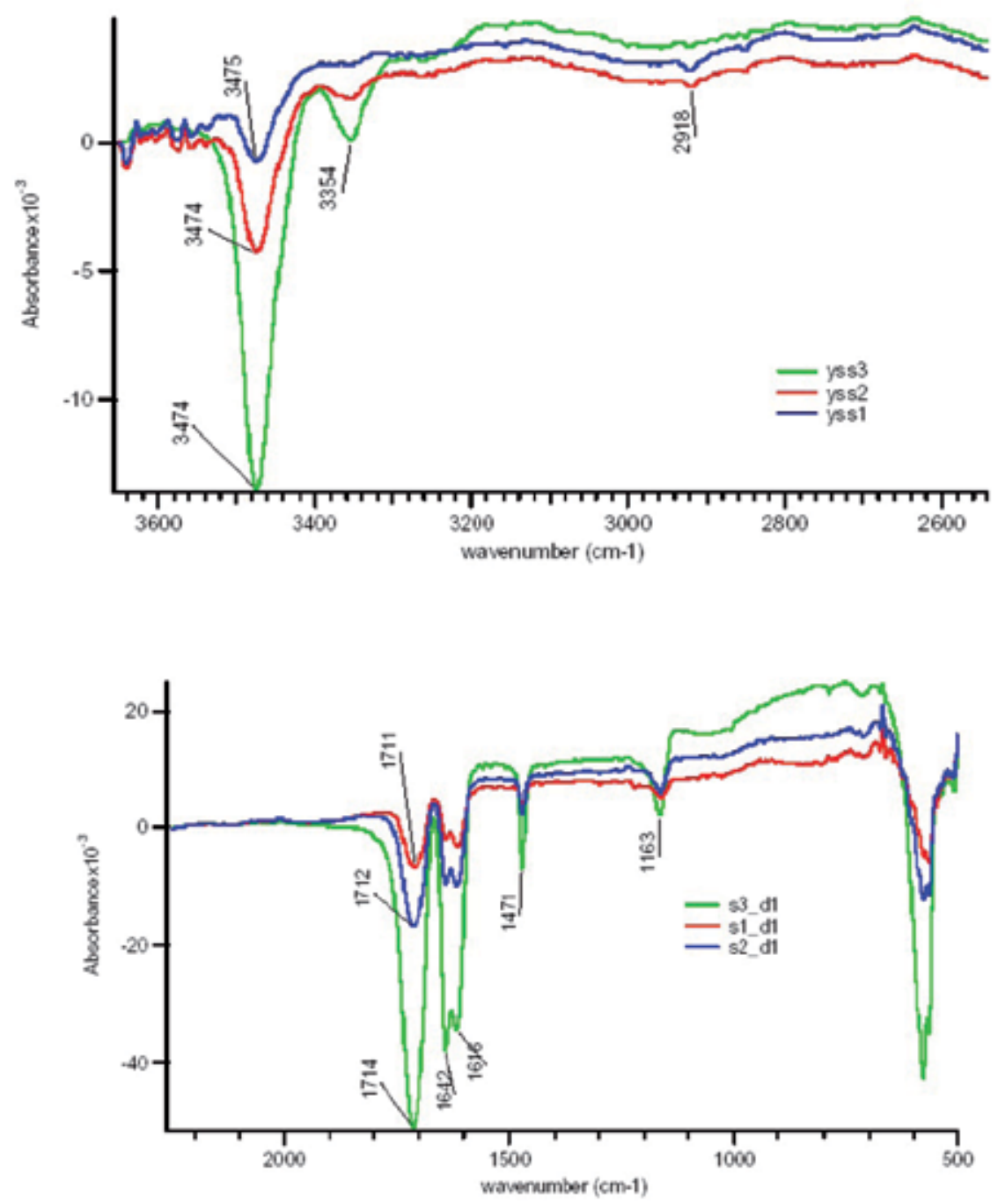

Figure 7. a. IR-spectra of the native and EBP-treated fibrin-monomer ( $\tau=5 \mathrm{~min}$ ): native FM - blue line, FM treated in the EBP of helium - red line, FM treated in the EBP of water vapor - green line; b.IRspectra of the native and EBP-treated fibrin-monomer $(\tau=5 \mathrm{~min})$ : native FM - red line, FM treated in the EBP of helium - blue line, FM treated in the EBP of water vapor - green line 


\begin{tabular}{llll}
\hline \multicolumn{1}{c}{ Amino acid } & \multicolumn{3}{c}{ Amino acid content, $\%$} \\
\cline { 2 - 4 } & & Fative FM & Fapor EPB \\
Lysine & 0,26 & 0,13 & 0,13 \\
\hline Threonine & 0,24 & 0,15 & 0,18 \\
\hline Glutamic acid & 0,53 & 0,36 & 0,40 \\
\hline Cystine & 0,068 & 0,039 & 0,051 \\
\hline Tyrosine & 0,1 & 0,09 & 0,09 \\
\hline Phenylalanine & 0,16 & 0,09 & 0,10 \\
\hline Aspartic acid & 0,64 & 0,49 & 0,53 \\
\hline Serine & 0,29 & 0,22 & 0,21 \\
\hline Glycine & 0,24 & 0,22 & 0,22 \\
\hline Valine & 0,15 & 0,12 & 0,13 \\
\hline Methionine & 0,15 & 0,11 & 0,12 \\
\hline Leucine & 0,24 & 0,17 & 0,19 \\
\hline Isoleucine & 0,14 & 0,10 & 0,12 \\
\hline
\end{tabular}

Table 2. The amino acid content of FM before and after EBP-treatment

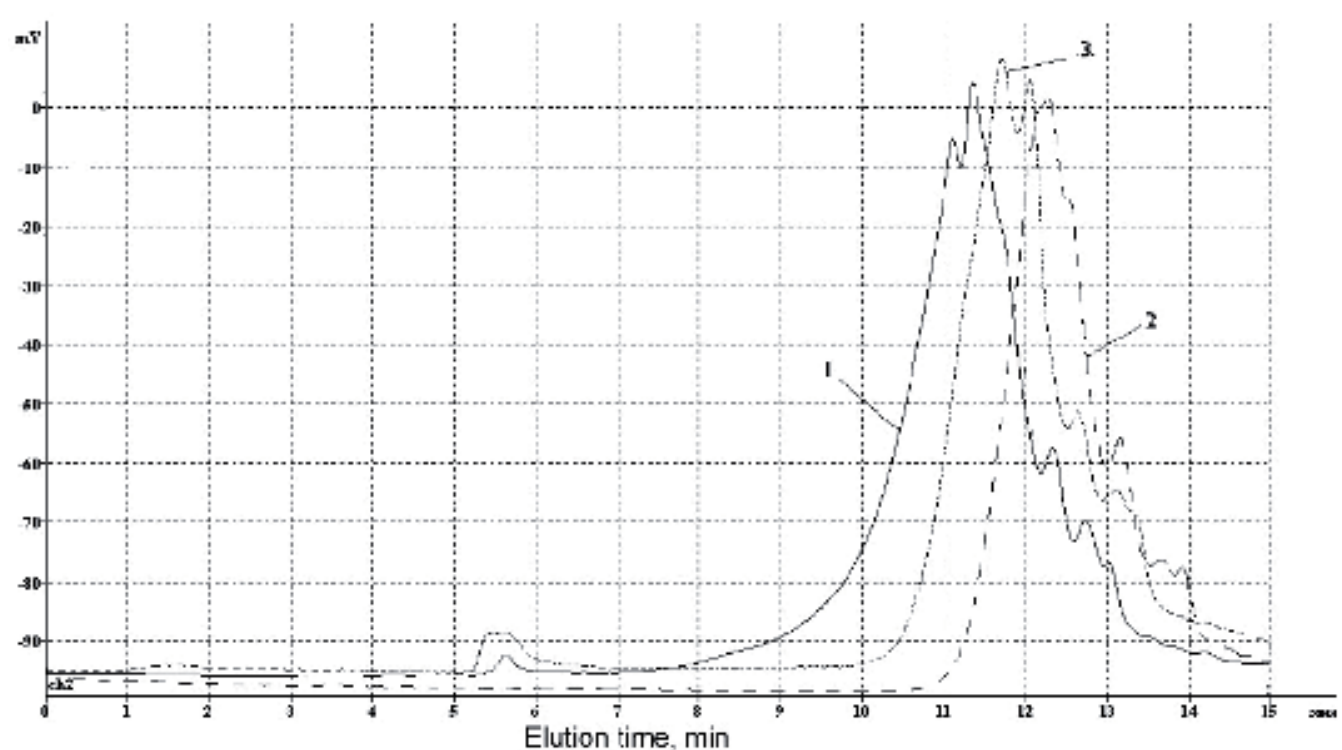

Figure 8. The chromatograms of the FM treated in the EBP of helium (curve 1), water vapor at moderate irradiation dose ( curve 2) and at low irradiation dose (curve 3) 


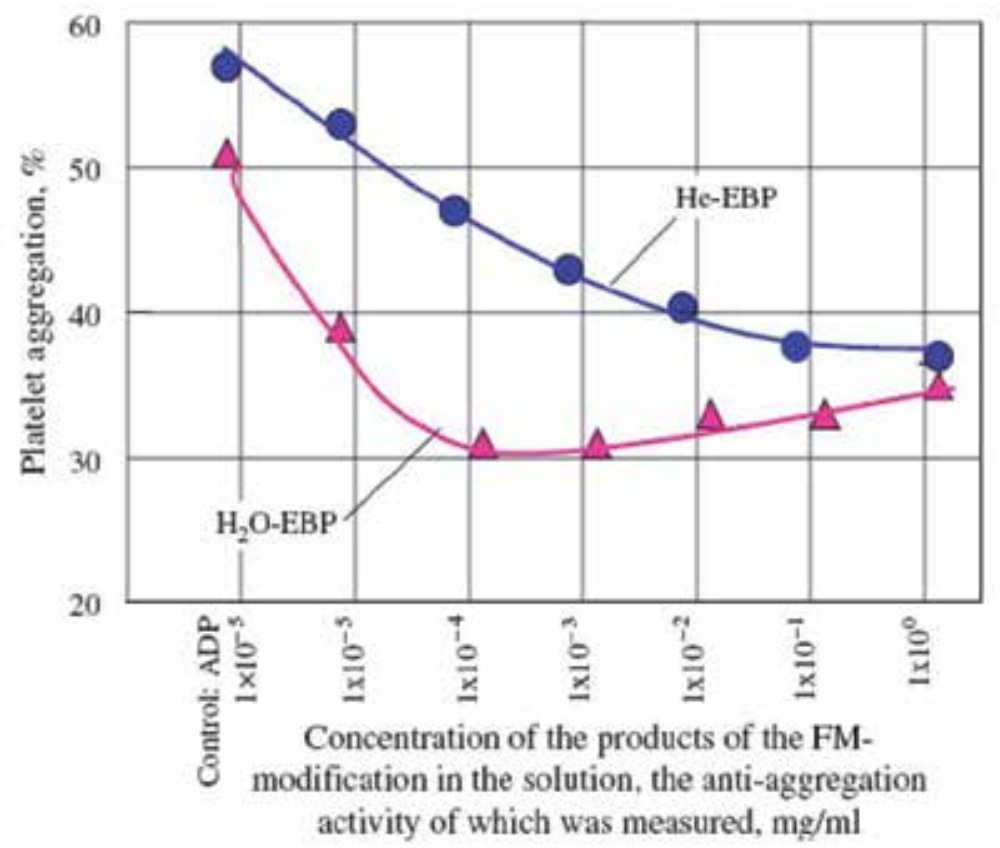

Figure 9. The reduction of the ADP-induced platelet aggregation by products of the FM modification in the EBP of water vapor and helium

The powder of globular protein BSA was treated in the EBP as well. The solubility of the native BSA was $100 \%$. Contrary to the EPB-treated FM the products of the EBP-modification became partially indissoluble in distilled water and the solubility decrease could be observed by the unaided eye. The water solution of the treated BSA contained the of undissolved protein particles, the solution of native BSA was absolutely clear. The latter indicates that the BSA polymerization has occurred due to the EBP-treatment.

No differences in either BSA structure were found in the IR-spectrograms, PAGEs (Figure $10)$, changes in its amino acid composition were not observed also. Since the molecular mass of the BSA is significantly less than that of the fibrin-monomer the BSA may be more resistant to the EBP treatment and no changes in the amino acids content and their structure in the EBP-modified BSA occur. On the other hand, minor quantities of the low molecular products which could be formed due to the EBP-treatment of the BSA are undetectable by the relatively low-sensitive PAGE technique.

The exclusion chromatography, that has higher sensitivity, was used to detect the products of the BSA treatment. The peaks corresponding to the individual peptides with molecular weights $>800 \mathrm{kDa}, 350 \mathrm{kDa}, 150 \mathrm{kDa}, 66 \mathrm{kDa}$ and less (elution times 5,6; 7,2; 8,0; 8,7 min and more than $8,7 \mathrm{~min}$, respectively) were observed in the exclusion chromatograms of the BSA modified by the EBP of both oxygen and water vapor (Figure 11).The treatment of the BSA for longer time (up to $20 \mathrm{~min}$ ) did not result in the additional changes of its properties and composition. 
Whereas the products of the EBP-induced polymerization and very small concentration of low molecular peptides are detected the most significant peak $(>2400 \mathrm{mV})$ corresponds to protein with molecular mass $66 \mathrm{kDa}$ i.e. to the unmodified BSA. This confirms that though the EBP-treatment of the BSA causes some changes in its structure they are not as pronounced as in the case of the fibrin-monomer treatment. Therefore the modification effect of the plasma treatment depends on the size and structure of the original protein molecule.

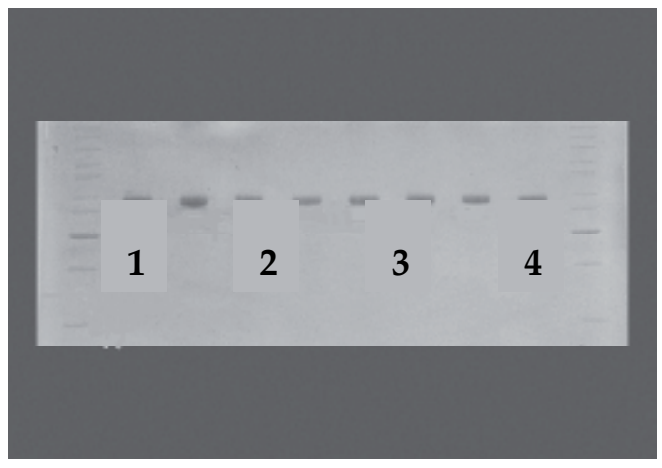

Figure 10. PAGE-electrophoresis of four BSA samples: 1 - control BSA; 2,3 and 4 - BSA treated in the EBP of water vapor for 2,5, 5 and $10 \mathrm{~min}$, respectively

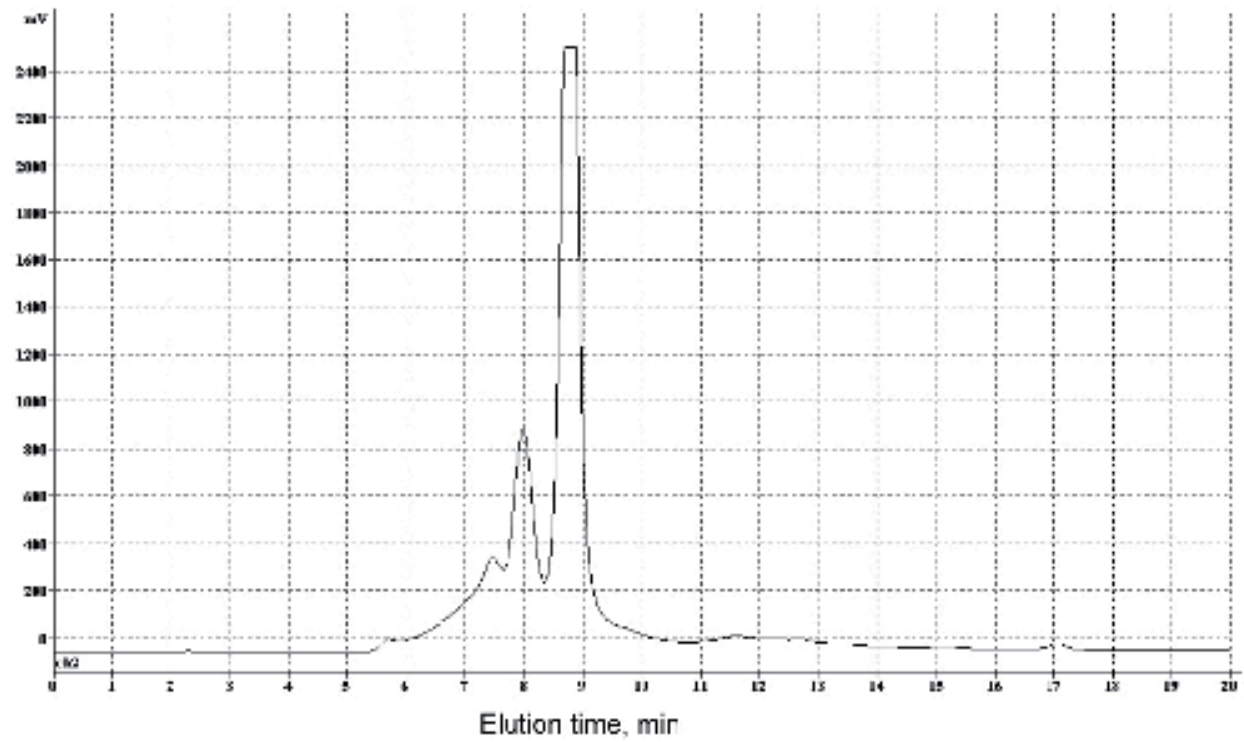

Figure 11. The exclusion chromatogram of BSA powder treated in EBP of oxygen $(\tau=10 \mathrm{~min})$

On the other hand the small concentration of low molecular weight peptides is probably due to a small depth (a few micrometers) of a powder particle involved in the plasmachemical treatment. To enhance the homogeneity of the EBP-modification, the samples of globular protein were treated in the form of thin films. Such treatment arrangement can considerably 
increase the yield of low molecular weight products with the comparison to the powdered samples.

The PAGE of the BSA samples modified as films in EBP revealed a dramatic decrease in the intensity of the albumin band and the formation of a number of low molecular peptides, of which most have a molecular mass below $14 \mathrm{kDa}$. The exclusion chromatograms also displayed numerous peaks corresponding to low molecular mass products with elution times of 11,8; 15,1; and 17 min (Figure 12). The concentration of the low molecular mass compounds depended on the plasma treatment time and the nature of the plasma gas: as the treatment time increased, their concentration grew, and BSA degradation being more significant in the water vapor EBP. A decrease in the content of almost all amino acids was observed. The most significant decrease (by 2-3,5 times with respect to untreated BSA) was in the case of lysine, aspartic and glutamic acids, tyrosine, and cystine (Table 3).

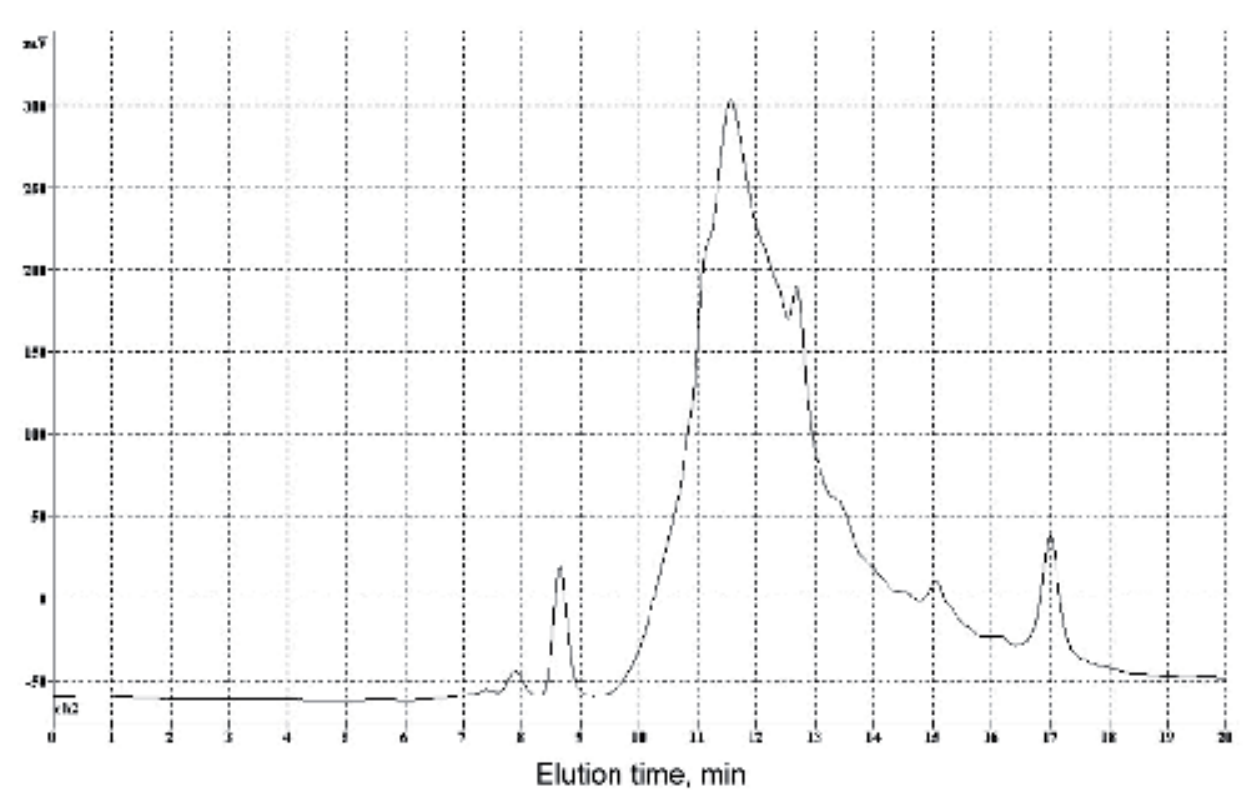

Figure 12. The exclusion chromatogram of BSA thing film treated in EBP of oxygen $(\tau=10 \mathrm{~min})$ 


\begin{tabular}{lllcl}
\hline \multicolumn{1}{c}{ Amino acid } & \multicolumn{4}{c}{ Amino acid content, $\%$} \\
\cline { 2 - 5 } & Native BSA & $\begin{array}{c}\text { BSA, treated in } \\
\text { water vapor EPB } \\
\tau=5 \text { min }\end{array}$ & $\begin{array}{c}\text { BSA, treated in } \\
\text { oxygen EPB } \\
\tau=5 \text { min }\end{array}$ & $\begin{array}{c}\text { BSA, treated in } \\
\text { oxygen EPB } \\
\tau=10 \text { min }\end{array}$ \\
\hline Lysine & 10,25 & 3,68 & 2,73 & 2,45 \\
\hline Threonine & 5,02 & 2,65 & 2,63 & 2,27 \\
\hline Glutamic acid & 16,78 & 5,78 & 6,64 & 5,90 \\
\hline Cystine & 4,73 & 1,78 & 2,07 & 1,75 \\
\hline Tyrosine & 4,30 & 1,70 & 1,55 & 1,79 \\
\hline Phenylalanine & 5,01 & 3,43 & 3,37 & 2,90 \\
\hline Aspartic acid & 9,17 & 3,86 & 5,12 & 5,14 \\
\hline Serine & 3,75 & 2,03 & 2,11 & 1,89 \\
\hline Glycine & 1,45 & 2,13 & 2,48 & 2,54 \\
\hline Valine & 4,45 & 3,70 & 3,25 & 3,51 \\
\hline Methionine & 2,02 & 0,76 & 0,89 & 0,63 \\
\hline Leucine & 9,61 & 6,55 & 6,05 & 5,47 \\
\hline Isoleucine & 1,85 & 1,34 & 3,37 & 2,90 \\
\hline
\end{tabular}

Table 3. The amino acid content of BSA before and after EBP-treatment (BSA was treared as thing film)

\section{Discussions and conclusions}

1. Our experiments show that the powders of various proteins can be effectively modified in the EBP and that the plasmachemical processes are responsible for the modification. With the use of special procedures of sample preparation for the EBPtreatment, it is possible not only to increase the yield of plasma modification products but also to effectively control their composition. It was found that unlike powdered samples, the materials in the form of a freeze dried film of $\sim 1 \mu \mathrm{m}$ thickness can be homogeneously treated to an extent sufficient for the practical use of the compounds produced.

2. Plasmachemical reactions in thin films occur throughout the entire volume of the sample, leading to the formation of final low molecular mass products. The powdered samples are characterized by a thin surface layer (of at most $10 \mu \mathrm{m}$ in thickness) in which the effective modification of the protein takes place. The protein remains intact in deep layers of such samples, and polymerization of protein molecules is likely to occur in intermediate layers. Our results are consisted with the data obtained in some other studies [17, 18]. The destruction of BSA thing films was also observed in the experiments using the atmospheric pressure glow discharge. Nevertheless the effective BSA degradation in the atmospheric pressure glow discharge was observed when the very small amounts of the protein sample $(\sim 20 \mu \mathrm{l})$ were treated [18]. Thus, the EBPtreatment seems to be more powerful technique for the protein degradation and low molecular peptides formation with respect to the gas-discharge plasmas.

The following factors influence the biomaterial placed into the plasma cloud: 
- the fast electrons of the partially degraded EB that bombard the sample;

- the secondary electrons of moderate energy produced in the EBP can also act on the powder;

- $\quad$ the EBP-radiation, especially UV one and X-ray (bremsstrahlung);

- chemically active heavy particles of the EBP (excited molecules and atoms, ions, radicals). This factor is likely plays the pivotal role for the low molecular peptides formation and for the production of bioactive products [19]. Due to the action of the reactive oxygen species (ROS) the destruction of the peptide bonds occures. The Figure 13 illustrates the possible mechanism of peptide bonds cleavage [20]. The formation of intermolecular cross-links can also occure under the ROS influence and the production of some high molecular weight substances was detected by the exclusion chromatography after the EBP-treament of fibrin-monomer powder (see Figure 8).

Some new chemical groups might be formed in the molecule structure during the treatment in the EBP. For instance, the ROS generated in the EBP of the water vapor or oxygen can react with the amino acid residues resulting in their oxidation and the formation of various keto-products. The formation of such oxidized substances (e.g. pyruvic acid, kynurenine, glutamic semialdehyde, disulfides, cysteic acid [21]) is possible reason for the changes in the UV-spectra and amino acid composition (see Figure 5 and Tables 2 and 3).

3. the EBPR with the aerosol reaction zone was successfully used to produce compounds with new pharmacological activity and therapeutic effect, e.g. for the production of effective platelet aggregation antagonists. Also the destruction of peptides in the EBP can be used for the effective sterelization of medical instruments, inactivation of pathogenic bateria and prion proteins.

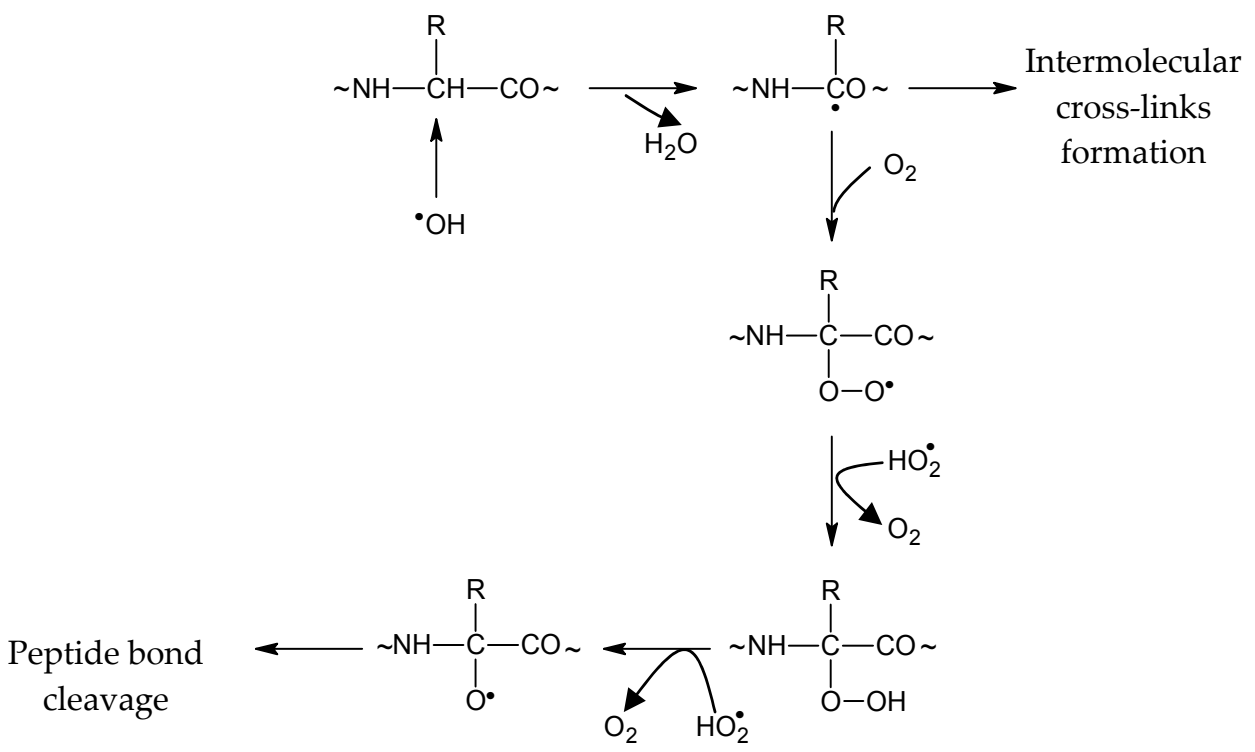

Figure 13. The scheme of peptide bound degradation and cross-links formation under ROS action in the EBP of the water vapor or oxygen 


\section{The polysaccharides treatment in the Electron-Beam Plasma}

The plasmachemical modifications of cellulose materials, peat, and chitosan in EBP of $\mathrm{O}_{2}$, $\mathrm{He}$, and $\mathrm{H}_{2} \mathrm{O}$, are studied. The yield $(S, \%)$ and the average molecular mass of water-soluble products extracted from the solution were taken as the quantitative criteria for the modification. Note, typical content of water-soluble substances in untreated cellulose materials does not exceed $1-2 \%$.The molecular mass of the cellulose may reach several million. The water-soluble products yield depends upon the processing conditions and has a maximum value $S_{\max }$ for each plasma-generating gas: for oxygen plasma $S_{\max } \approx 45 \%$, for plasma of water vapor $S_{\max } \approx 56 \%$, which is reached during certain time periods of the treatment $\tau$. At first the dependence $S(\tau)$ increases smoothly, then - steeply in the vicinity of $\tau_{0}$ after which the yield of the water-soluble products does not change whatever the time of the treatment could be (Figure 14). The NMR- and IR-spectroscopy analyses showed the final water-soluble products of the cellulose modification to be $\beta-(\mathrm{C} 1-4)$-tetrasaccharide, the molecule structure and chemical bonds of the products being identified. In particular, low-molecular products $(M \approx 250-800 \mathrm{Da}$ ) have attached carbonyl and/or carboxyl groups on their ends if the original materials have been treated in $\mathrm{O}_{2}$ or $\mathrm{H}_{2} \mathrm{O}$ plasmas.

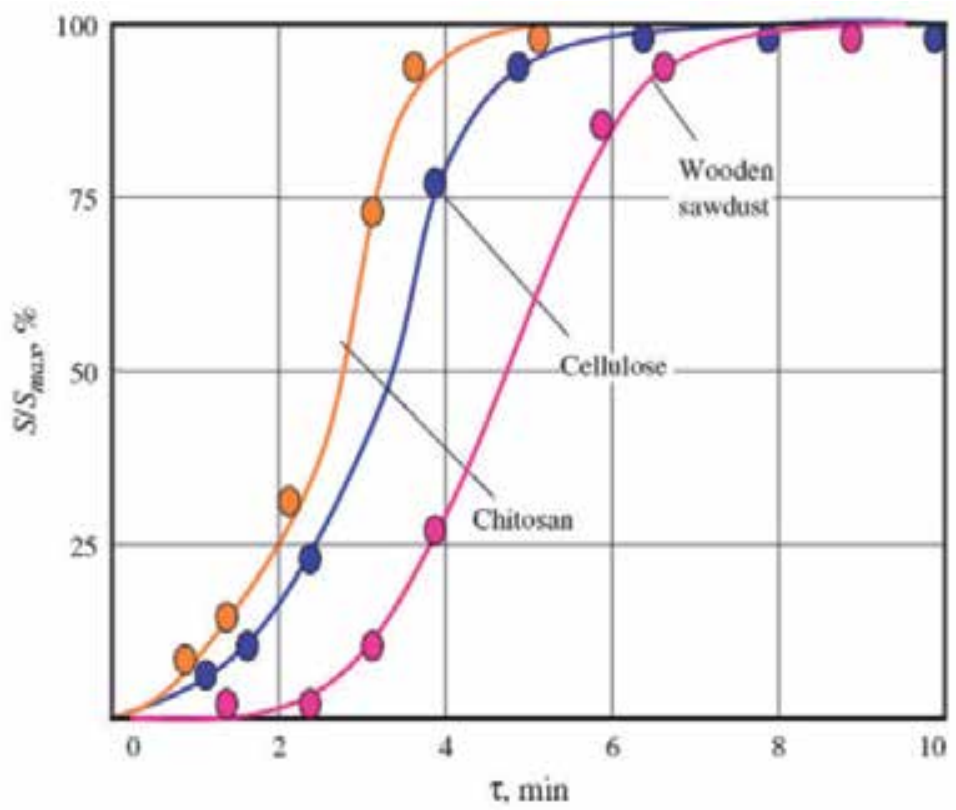

Figure 14. The modification effect in studied polysaccharides as a function of the treatment duration in the EBP of water vapor

The products of the EBP modification of all the substances mentioned above turned out to be bioactive. For instance, modified cellulose and peat are effective substratesfor microorganisms and fungi: the yield of the yeastrel on the plasmachemically treated peat reached $0,14 \mathrm{~kg}$ per $1 \mathrm{~kg}$ of the peat, whereas the productivity of the untreated substrate was only $0,053 \mathrm{~kg}$. 
The original chitosan was not water-soluble while its EBP-treament products became soluble and the effect increased with the prolongation of $\tau$. The maximum yield of watersoluble substances was obtained after $10 \mathrm{~min}$ and the solubility of these products was up to 95\% up at room temperature.

The exclusion chromatography of the EBP-treated chitosan revealed the formation of a number of LMWC with molecular weight varied from $18 \mathrm{kDa}$ to monomeric fragments (Figure 15). The majority of products formed with the EBP-treatment were oligosaccharides with the molecular mass $1 \mathrm{kDa}$ (the elution time 11,3 min).

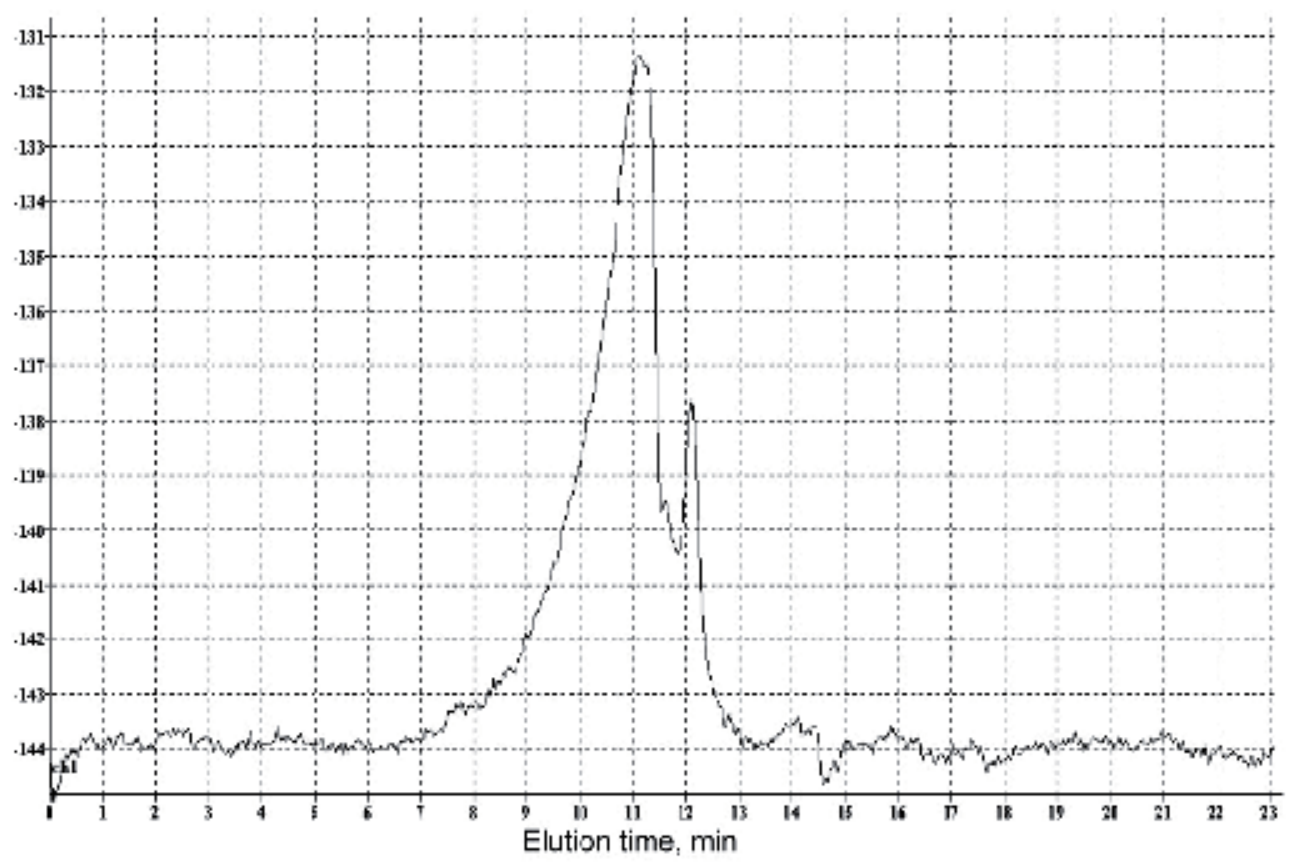

Figure 15. The exclusion chromatogram of chitosan treated in the EBP of water vapor $(\tau=10 \mathrm{~min})$

The degradation of the original polymer is due to the action of free radicals formed in the EBP. Active oxygen particles $\left(\mathrm{O}, \mathrm{O}^{*}\right.$, singlet oxygen) that are produced in plasmachemical processes and the products of the water plasmolisys (e.g. $\left.\mathrm{OH}^{*}\right)$ are likely to be of the most importance. These chemically active particles break the $\beta-1,4$ glycosidic bound and decrease the chitosan molecular weight Figure 16 illustrates the possible degradation mechanism [22].

To quantitatively characterize the bioactivity of the EBPproduced LMCW the bacteria growth in vitro was studied. The LMCW produced by the EBP-treatment in water vapor at concentration $1000 \mu \mathrm{g} / \mathrm{ml}$ were found to completely suppress the multiplication of colon bacillus, aurococcus and yeast-like fungi. At lower doses the EBP-treatment products were also active and strongly inhibited the microorganism multiplication (Table 4). 


\begin{tabular}{lc|c|c|c|c|}
\hline \multirow{2}{*}{ Test } & \multicolumn{4}{c|}{ EBP-treated chitosan concentration, $\mu \mathrm{g} / \mathrm{ml}$} & \multirow{2}{*}{ Control } \\
\cline { 2 - 5 } microorganism & 1000 & 500 & 250 & 125 & \\
\hline E. coli & --- & \pm & \pm & + & + \\
\cline { 2 - 6 } Ps. aeruginosa & + & + & + & + & + \\
\cline { 2 - 6 } S. aureus & --- & \pm & + & + & + \\
C. albicans & ---- & \pm & + & + & + \\
\hline
\end{tabular}

--- the absence of microorganism growth; \pm weak microorganism growth; + microorganism growth comparable with reference sample

Table 4. The microorganism growth under EBP-treated chitosan
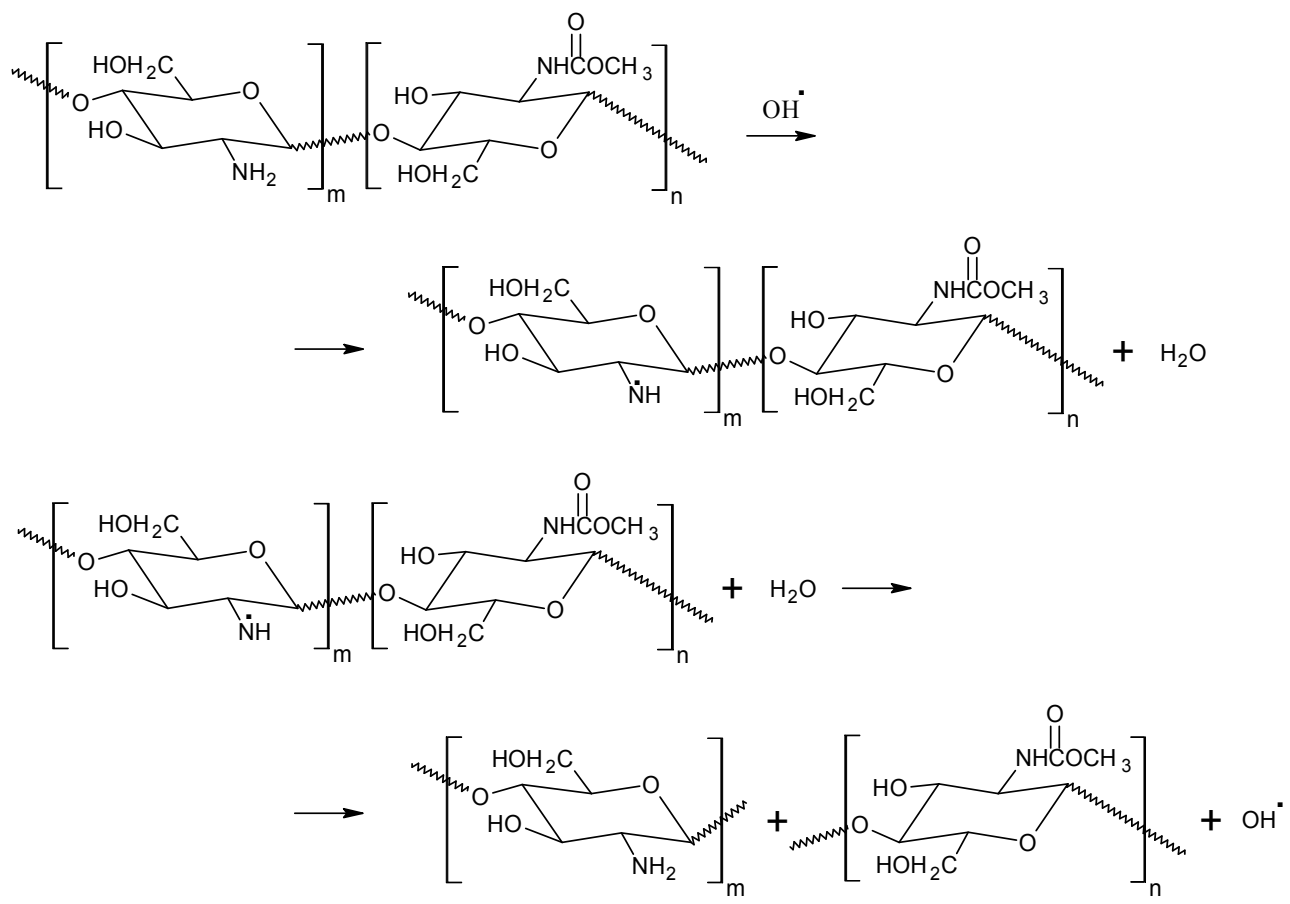

Figure 16. The scheme of chitosan degradation under hydroxyl radical action in the EBP of the water vapor [22]

\section{Discussions and conclusions}

- The possibility of the EBP-stimulated hydrolysis of native polysaccharides and formation of water-soluble low molecular weight bioactive products was proved experimentally. The yield of the water-soluble products depends on the doze of the irradiation by the plasma particles. Other things being equal, the yield of the bioactive products began to increase abruptly at particular duration of treatment $\tau_{0}$. At shorter duration $\tau<\tau_{0}$ the plasma didn't modify the original substance and the longer treatment $\tau>\tau_{0}$ resulted in an insignificant additional effect. 
- The 95\% yield of low molecular weight EBP-treatment products was attained by optimizing the treatment procedure. The high yields of low molecular weight water soluble products are obtained at treatment time $\sim 10 \mathrm{~min}$ whereas the traditional chitosan hydrolysis usually takes several days. The hazardous by-products and toxic wastes are not generated during the EBP-treatment.

- The low molecular water-soluble forms of the chitosan obtained by its treatment in the EBP of oxygen and water vapor were found to inhibit the multiplication of colon bacillus, aurococcus and yeast-like fungi. The LMWC with molecular weight 30-180 $\mathrm{kDa}$ and chitooligosaccharide mixtures are known to possess antimicrobial properties $[22,23]$. We suppose that the antibacterial activity of the EBP-produced LMCW results from the LMCW interaction with the cell walls of microorganisms. This mechanism was considered in detail in [24].

- The EBP-stimulated degradation mechanisms occurring both in polysaccharides and high molecular weight proteins are similar and occur due to the plasmachemical processes, whereas the attendant of fast electrons and X-ray irradiations are minor factors only.The active oxygen species produced in plasmachemical reactions and the products of water plasmolisys are responsible for the LMWC and chitooligosaccharides formation. Parameters of the treatment process can be adjusted by the control system of the EBPR and the results of the modification are predictable.

\section{Future developments}

1. Our experiments have demonstrated that the EBP can be used for the effective and controllable modification of some natural biopolymers. Using various plasmagenerating gases the functional groups containing sulfur, nitrogen, phosphorus can be introduced into the biopolymer structure to obtain substances with unique pharmacological and biological activities (anticoagulant, hemostatic, antibacterial, etc.);

2. On the basis of present study the novel strategies of plasma-stimulated synthesis and plasma-assisted bioactive coating deposition can be developed. Two different ways of the plasma-assisted formation of bioactive coatings by means of the deposition of organic and inorganic vapors activated by the electron beam are possible: 1) on a preliminary prepared plane substrate; 2 ) on a surface of powder particles levitating in the plasma trap. The following materials can be synthesized:

- supramolecular complexes "protein-drug agent" or "polysaccharide- drug agent" for the addressed drug delivery;

- medical hybrid materials and coatings with high bio- and hemo-compatibility and/or combined pharmacological action (e.g. haemostatics with antimicrobial or regenerative properties);

3. The technique is potentionally interesting for:

- increasing the wettability of the original organic and inorganic materials to obtain effective sorbents, membranes for hemodialysis, and microbiological substrates;

- improving membranes selectivity and stability and production more sensitive biosensors. 


\section{Author details}

T. Vasilieva

Department of General Chemistry, Moscow Institute of Physics and Technology, Dolgoprudny, Moscow region, Russia

\section{References}

[1] Solum N.O., Rigollot C., Budzynski A.Z., Marder V.J. A quantitative evaluation of the inhibition of platelet aggregation by low molecular weight degradation products of fibrinogen. Brit. J. Haematol. 1973; 24(4) 419-434.

[2] Lord S.T. Fibrinogen and fibrin: scaffold proteins in hemostasis. Curr. Opin. Hematol. 2007; 14(3) 236-241.

[3] Ray S.D. Potential aspects of chitosan as pharmaceutical excipient. Acta Pol. Pharm. 2011; 68(5) 619-622.

[4] Laurienzo P. Marine polysaccharides in pharmaceutical applications: an overview. Mar. Drugs 2010; 8(9) 2435-2465.

[5] Aam B.B., Heggset E.B., Norberg A.L., Sorlie M., Varum K.M., Eijsink V.G.H. Production of chitooligosaccharides and their potential applications in medicine. Mar. Drugs 2010; 8(5) 1482-1517.

[6] Vasiliev M.N. In: Fortov V. E. (ed.) Encyclopedia of the Low-Temperature Plasma. Nauka: Moscow; 2001. V. XI p436-44.

[7] Vasilieva T., Lysenko S. Factors responsible for biomaterials modification in the electron-beam plasma. J. Phys.: Conf. Ser. 2007; 63(1) 012033.

[8] Vasiliev M., Vasilieva T. Electron-beam plasma in the production of bioactive agents and drugs. J. Phys.: Conf. Ser. 2006; 44(140) 140-145.

[9] Henderson R. The potential and limitations of neutrons, electrons and X-rays for atomic resolution microscopy of unstained biological molecules. Q. Rev. Biophys. 1995; 28(171) 171-193.

[10] Glaeser R.M. Review: electron crystallography: present excitement, a nod to the past, anticipating the future. J. Struct. Biol. 1999; 128(3) 3-14.

[11] Aleksandrov N. L., Vasil'ev M. N., Lysenko S. L., Makhir A. Kh. Experimental and Theoretical Study of a Quasi-Steady Electron-Beam Plasma in Hot Argon. Plasma Physics Reports 2005; 31(5) 425-435.

[12] Vasilieva T. A beam-plasma source for protein modification technology. IEEE Transac. Plasma Sci. 2010; 38(8) 1903-1907

[13] Axelsen M., Kroll B., Weeke B. A manual of quantitative immuno-electrophoresis. Mehtods and applications; 1977.

[14] Laemmli U.K. Cleavage of structural proteins during the assembly of the head of bacteriophage T4. Nature 1970; 227(5259) 680-685

[15] Vasilieva T. The controllable production of peptides inhibiting the platelet aggregation by the electron-beam plasma technologies. In: Aimoto S. and Ono S. (ed.) Peptide Science 2007. The Japanese Peptide Society; 2008. p.35-38. 
[16] Born G. Aggregation of blood platelets by adenosine diphosphate and its reversal. Nature 1962; 194(12) 927-930.

[17] Deng X.T., Shi J.J., Chen H.L., Kong M.G. Protein destruction by atmospheric pressure glow discharges. Appl. Phys. Lett. 2007; 90(1) 013903.

[18] Deng X.T., Shi J.J., Kong M.G. Protein destruction by a helium atmospheric pressure glow discharge: capability and mechanisms. J. Appl. Phys. 2007; 101(7) 074701.

[19] Lushchak V.I. Free radical oxidation of proteins and its relationship with functional state of organisms. Biochemistry. 2007; 72(8) 809-827.

[20] Vasilieva T. The properties of peptides modified by the electron-beam plasma nanotechnologies. In: Aimoto S. and Ono S. (ed.) Peptide Science 2006. The Japanese Peptide Society; 2006. p.386-387.

[21] Cabiscol E., Tamarit J., Ros J. Oxidative stress in bacteria and protein damage by reactive oxygen species. Internatl. Microbiol. 2000; 3(1) 3-8.

[22] Chang K.L.B., Tai M.C., Cheng F.H.J. Kinetics and products of the degradation of chitosan by hydrogen. Peroxide. Agric. Food Chem. 2001; 49(10) 4845-4851.

[23] Fernandes J. C., Tavaria F. K., Fonseca S. C., Ramos O. S., Pintado M. E., Malcata F. X. Antimicrobial effects of chitosans and chitooligosaccharides, upon Staphylococcus aureus and Escherichia coli, in food model systems. J. Microbiol. Biotechnol. 2010; $20311-318$.

[24] Wang Y., Zhou P., Yu J., Pan X., Wang P., Lan W., Tao S. Antimicrobial effect of chitooligosaccharides produced by chitosanase from Pseudomonas CUY8.Asia Pac. J. Clin. Nutr. 2007; 16(Suppl. 1) 174-177. 


\title{
Functional Inorganic Nanohybrids for Biomedical Diagnosis
}

\author{
Thai-Hoa Tran and Thanh-Dinh Nguyen \\ Additional information is available at the end of the chapter
}

http://dx.doi.org/10.5772/55266

\section{Introduction}

Functional hybrid inorganic nanomaterials have received substantial attention for their promising performance in nanotechnological applications.[1] A combination of more than one nanocomponent into a hybrid structure gives rise to new collective properties different from the constituents.[1] The hybrid nanostructures not only have multifunctional properties, but also may induce synergistic properties, arising from interfacial particleparticle interactions.[2] Coupling of two or more components produces a hybrid nanostructure that allows electronic transfer across the junction to change local electronic structure. The engineering chemical reactivity on the particle surface is thus dependent on the internal and external interfacing capacilities and the particle size distribution of the deposited particles onto the nanosupports. $[1,3]$ These behaviours make them to generally have potential applications in solar energy conversion, catalysis, and potential biomedical methods for drug delivery, bioimaging, and cancer theragnosis.[4-6]

The morphology of the hybrid nanostructures impacts a critical factor affecting their active performance.[7] The shape-controlled synthesis of these materials have been made in recent years. This objective could be performed by conducting heterogeneous nucleation-growth kinetics during the synthesis. Wet-chemistry methods, such as seed-mediated growth, ionexchange deposition, thermal decomposition, hydro-solvothermal process used to synthesize single nanoparticles have been extended to the hybrid nanostructures. The nanohybrids with specific shapes (e.g., dumbbell and core-shell) formed by sequential growth of second components onto the preformed seeds through directed attachment. The hybrid structures formed during heterogeneous growth are dependent on organic linkers used and lattice parameters of each components, which is relative to the electron transferred capacity at the particle-particle interfaces. A combination of synthetic control along with 
understanding of the interfacial interactions is therefore a significant basis for the fabrication of the multifunctional hybrid nanomaterials.

The functional inorganic hybrid nanomaterials with combined plasmonic-magnetic, plasmonic-fluorescent, or magnetic-fluorescent structures have unique optoelectronic properties for biomedical applications.[5, 8-10] The optical, electronic, magnetic properties could be controlled by adjusting their sizes, shapes, compositions, and surface chemistry. Several types of the hybrid nanostructures are potentially useful for biomedical applications.[5, 8] For example, the plasmonic-fluorescent materials would be interesting as dual-use biological tags, giving the ability to visualize labeled cells using both magnetic resonance and fluorescence imaging techniques, while external magnetic fields could be employed for the directed assembly of such materials.[11] In order to be compatible with biomedical environment, the surface of the nanohybrids is functionalized with appropriate amphiphilic polymer, silica, targeting ligand through chemisorption, covalent linkage, and ligand exchange.[12] After surface modification, the coated hybrid nanoparticles have the possibility of highly colloidal stabilization in aqueous media compatible with bioconjugation.[13] The delivery of the hybrid nanoparticles to targeted cells is an important and challenging approach in medical diagnosis and therapy. Development of biomoleculeconjugated strategies allows the hybrid nanoparticles delivering to targeted tissues through either antibody-antigen or ligand-receptor interactions.[12] Biomolecules, such as small molecules (vitamin, peptide, lipid, sugar) and larger ones (protein, DNA, antibody, enzyme, carbohydrate), are often used to conjugate onto the hybrid particles. Biomolecule-conjugated nanohybrid colloids have the possibility of highly selective binding with alive organ made them to be "biocompatible" behaviour and superior bioactivity at the supermolecular level.[5, 14, 15] These features have potentially applied in smart drug delivery vehicles, contrast agents, and theranostic biomedical applications.

This Chapter reviews the design, fabrication, and theranostic biomedical applications of the inorganic hybrid nanomaterials with combined plasmonic-magnetic, plasmonic-fluorescent, or magnetic-fluorescent structures. New collected properties of the hybrid nanostructures arising from the particle-particle interactions and the geometries are discussed from specific results of recent publications. The functionalizations of the hybrid nanostructures with amphiphilic polymer and silica coatings yielded water-soluble nanohybrid colloids are described. The subsequent conjugation of biomolecules with the coated nanohybrids afforded cellular targeting agents and the use of them as multimodal bioprobes for multimodal imaging and therapy are highlighted.

\section{Seed-mediated growth toward hybrid nanostructures}

Wet-chemically seed-mediated growth provides an effective method for the synthesis of the hybrid nanoparticles with well-controlled structures, where the secondary species attach and sequentially grow on the preformed seeds. To ensure the deposited species welldispersed on the supports, heterogeneous nucleation and growth through atomic addition must be achieved and homogeneous nucleation should be avoided. Significant progress has 
(a)

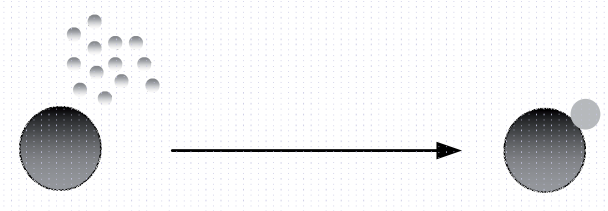

(b)

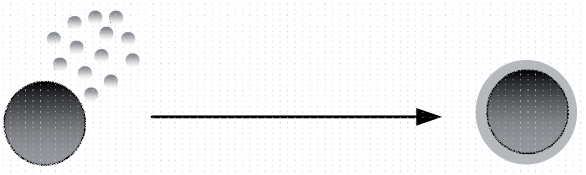

(c)

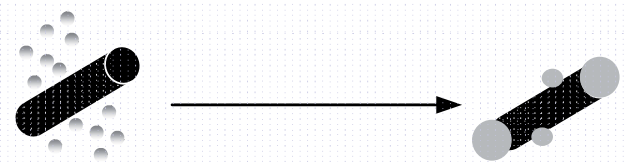

(d)

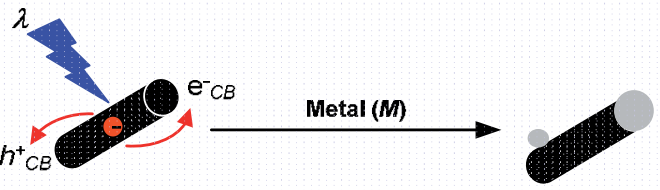

(e)

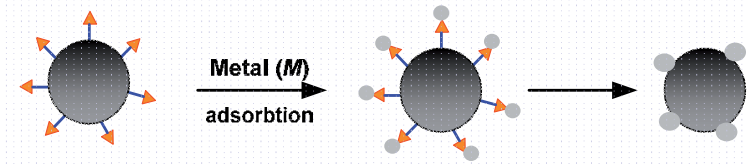

(f)

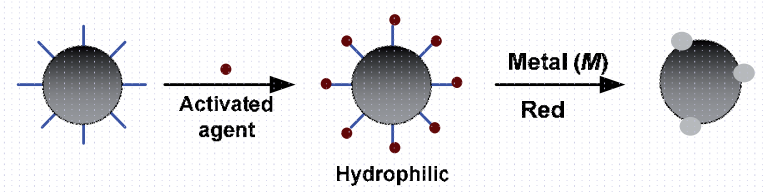

(g)

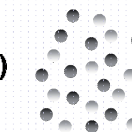

Figure 1. A general sketch of reaction mechanisms for the formation of the hybrid nanostructures: (a-c) Heterogeneous nucleation and growth of secondary precursors on the preformed seeds; (d) Secondary precursors adsorbed on the opposite charged surfaced supports by photo-irradiation; (e) Reduced precipitation of secondary precursors on hydrophilic-surfaced supports; (f) Secondary precursor growth on the activated-surfaced supports; (g) One-pot self-controlled nucleation-growth.

been achieved in the synthesis of the core-shell and dumbbell nanoparticles combining two different components. When the individual components involved have similar crystal structures and lattice parameters, each component fuses together giving the dumbbell shape. In a dumbbell structure consisted of one particle-bounded another, charged transfer across nanoscale junction could significantly change local electronic configuration that give the remarkable properties. While large lattice space difference of the individual components 
results in the core shell-shaped structure obtained by growing a uniform layer of a shell material around colloidal particles. An isolation of core from surroundings could create the specific materials with emerged properties to those of the bare nanoparticles.[16, 17]

The colloidal nanohybrids are generated upon reaction of molecular precursors in solution in the presence of surfactants. Once the synthesis is activated at a suitable temperature, monomers are generated, and then induced nucleation of nanoparticles and sustain their subsequent enlargement. The organic surfactants play key roles along the courses of the hybrid formation. Figure 1 shows general growth models for the fabrication of dumbbelland core shell-shaped hybrid nanostructures, that can be classified into the synthetic routes: (i) the direct heterogeneous growth of secondary precursors on the sites or tips of the preformed seeds (Figure 1 a-c); (ii) metal clusters adsorbed on the opposite-charged surface of the supports by photo-irradiation (Figure 1d); (iii) reduced precipitation of secondary precursors adsorbed on the hydrophilic-surfaced nanohybrids through ion-exchange deposition (Figure 1e); (iv) secondary precursor growth on the support surface after the chemical activation of their surface (Figure 1f); (v) one-pot growth of secondary precursors on the supports through self-assembled control (Figure 1g). Following these pathways, a large variety of the nanohybrids including metal-metal, metal-oxide, oxide-oxide, metalsemiconductor structures has been synthesized successively. In the following sections, we present types of the plasmonic-magnetic, plasmonic-fluorescent, and magnetic-fluorescent hybrid nanomaterials synthesized using seed-mediated growth methods along functionalization and subsequent bioconjugation of the hybrid nanostructures for biomedical applications.

\subsection{Synthesis of plasmonic-magnetic hybrid nanostructures}

Plasmonic-magnetic nanohybrids provide a promising platform for developing optical and magnetic multifunctional probes for cell imaging applications. Materials researchers have recently been efforted to synthesize the hybrid nanostructures. Alivisatos et al.[18] synthesized gold-iron oxide core-shells via thermolysis of $\mathrm{Fe}(\mathrm{CO})_{5}$ at the surface of gold nanoparticles in octadecene/oleylamine/oleic acid. These hybrid nanostructures were formed by deposition of an iron shell around the gold core and subsequent oxidation of the metallic iron shell to form an iron oxide hollow through Kirkendall effect. The heterogeneous growth of iron precursors on the gold seeds was dependent on the molar ratio of oleylamine/oleic acid capping agents. The thinnest oxide shell $(\sim 2 \mathrm{~nm})$ surrounded the gold nanoparticles was formed at oleylamine/oleic acid ratio of 1:1. Irregular polycrystalline iron oxide shells connected with the gold core were formed in the presence of oleylamine without oleic acid. With increasing the oleic acid concentration led to the iron nucleation and growth to be slowed because of the formation of stable iron oleate complex in high-temperature reaction. The gold-iron oxide core-shells exhibited the surface plasmon resonance shift of the gold particles and the magnetic hysteresis loop shift of the iron oxide particles, originating from the core-shells with particle-particle interactions. Sun et al.[19] synthesized $\mathrm{Au}-\mathrm{Fe}_{3} \mathrm{O}_{4}$ dumbbells via thermolysis of $\mathrm{Fe}(\mathrm{CO})_{5}$ on $\mathrm{Au}$ particles and subsequent 
oxidation in 1-octadecene. The sizes $(\sim 2-8 \mathrm{~nm})$ of $\mathrm{Au}$ particles were controlled either by tailoring $\mathrm{HAuCl}_{4} /$ oleylamine molar ratio or by controlling temperature at which $\mathrm{HAuCl}_{4}$ precursors was injected. The sizes $(\sim 4-20 \mathrm{~nm})$ of $\mathrm{Fe}_{3} \mathrm{O}_{4}$ particles were tuned by adjusting the $\mathrm{Fe}(\mathrm{CO})_{5} / \mathrm{Au}$ ratio. Hyeon el al.[20] synthesized metal/oxide core-shells $\left(\mathrm{Au}-\mathrm{Fe}_{3} \mathrm{O}_{4}, \mathrm{Ni}-\mathrm{Fe}_{3} \mathrm{O}_{4}\right.$, $\left.\mathrm{Au}-\mathrm{MnO}, \mathrm{Pt}_{-} \mathrm{Fe}_{3} \mathrm{O}_{4}\right)$ through thermolysis of the mixtures of transition metal-oleate (Fe, $\mathrm{Mn}$ ) complexes and metal-oleylamine $(\mathrm{Au}, \mathrm{Ag}, \mathrm{Pt}, \mathrm{Ni})$ complexes in oleylamine/octadecene. These complexes were prepared from transfer-phase reaction of the inexpensive metal salts and surfactants. Due to the high solubility of the prepared complex precursors in 1octadecene solvent, this method was able to large-scale synthesis of the well-shaped nanohybrids per a singe preparation. The $\mathrm{Au}-\mathrm{Fe}_{3} \mathrm{O}_{4}$ particles became soluble in water by encapsulating their surface with a PEG-phospholipid shell. The amino-functionalized Au$\mathrm{Fe}_{3} \mathrm{O}_{4}$ nanohybrids conjugated with thiolated oligonucleotide sequences exhibited the red shift of the surface plasmon resonance of the gold particles and the enhanced signal intensity of the $\mathrm{Fe}_{3} \mathrm{O}_{4}$ particles in $\mathrm{T}_{2}$-weighted magnetic resonance imaging. These conjugated hybrid nanostructures have potential in multimodal biomedical probes.

Figure 2 shows plasmonic-magnetic Au-Co core-shell and Au-Ni spindly nanostructures synthesized by $\mathrm{Li}$ et al.[21] through one-pot solvothermal reaction of $\mathrm{HAuCl}_{4}$ and metal
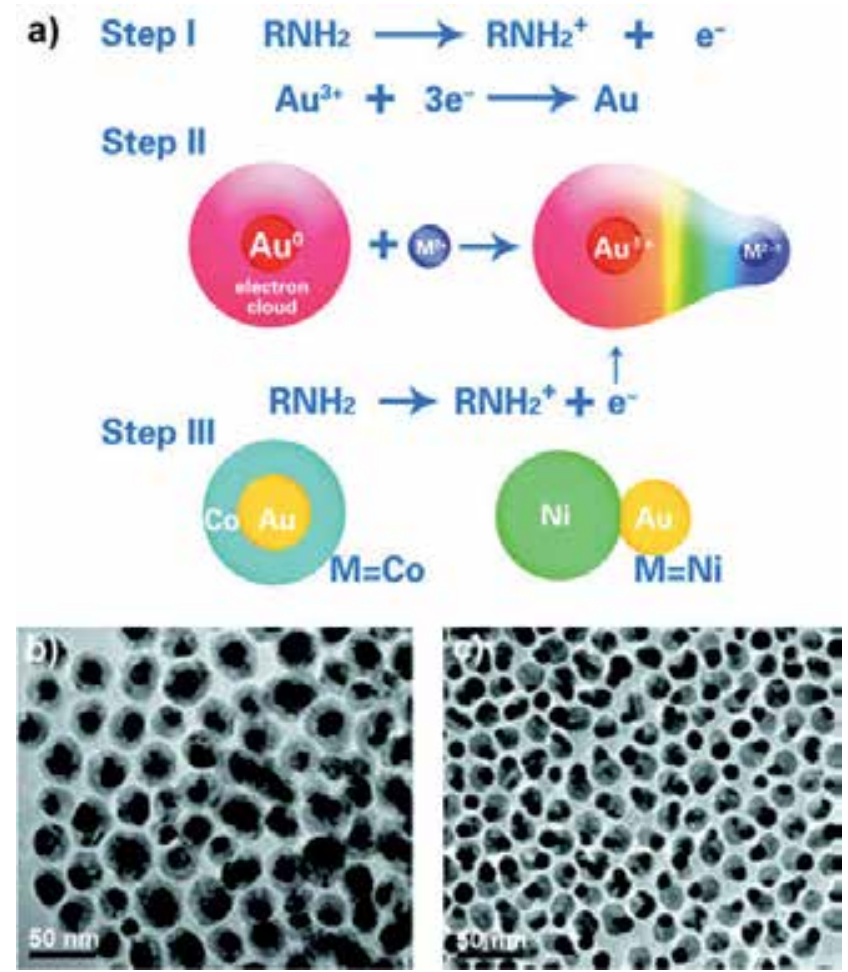

Figure 2. One-pot protocol for plasmonic-magnetic hybrid nanostructures via noble-metal-induced reduction process. (a) Schematic illustration of the noble-metal-induced reduction process. TEM images of Au-Co core-shell (b) and Au-Ni spindly nanostructures (c). Reproduced with permission from ref. [21]. Copyright 2010, American Chemical Society. 
nitrate $\left(\mathrm{Co}\left(\mathrm{NO}_{3}\right)_{2} \cdot 6 \mathrm{H}_{2} \mathrm{O}\right.$ or $\left.\mathrm{Ni}\left(\mathrm{NO}_{3}\right)_{2} \cdot 6 \mathrm{H}_{2} \mathrm{O}\right)$ in octadecylamine used as both solvent and surfactant. The formation of the hybrid structures was illustrated by metal-induced reduction process namely, octadecylamine-supplied electron cloud surrounded $\mathrm{Au}$ atoms reduced transition metal ions to form the nanohybrids. The strong magnetism of the plasmonic-magnetic nanohybrids and the $\mathrm{CO}$ superior catalytic activity of the Au-Co coreshells were showed as a result of incorporating magnetic heterometals into gold particles. The authors [22] also synthesized bifunctional $\mathrm{Au}-\mathrm{Fe}_{3} \mathrm{O}_{4}$ nanohybrids through the conjugation of $\mathrm{Au}$ particles to thiol-modified $\mathrm{Fe}_{3} \mathrm{O}_{4}$ nanoparticles. Lysine contained both amino and carboxylic groups played dual roles as both linker and capping agent in attaching metals on $\mathrm{Fe}_{2} \mathrm{O}_{3}$ particles. The hybrid nanostructures can be used for magnetic separation of biosubstances and for protein separation. The authors [27] also synthesized hexagonal pyramid-like shaped $\mathrm{Au}-\mathrm{ZnO}$ nanohybrids based on the growth of $\mathrm{ZnO}$ particles derived from a mixture of zinc acetate/oleylamine/dodecanol on $\mathrm{Au}$ seeds. The nanopyramids composed of Au particles as the tip and hexagonal $\mathrm{ZnO}$ pyramid as the tail. The Au-ZnO hybrid nanopyramids with homogeneous composition and controlled morphology showed better photocatalytic efficiency than $\mathrm{ZnO}$ nanocrystals.

\subsection{Synthesis of plasmonic-fluorescent and fluorescent-magnetic hybrid nanostructures}

Plasmonic-fluorescent hybrid nanostructures have seen a renewed interest in photocatalytic degradation of organic contaminants and photocatalytic water splitting.[4] Banin et al.[1, 3] synthesized various classes of these materials via photodeposition of metals on semiconductors. Au/CdSe/CdS hybrid nanorods were synthesized through growing $\mathrm{Au}$ precursors on the reactive facets exposed tips of the seeded rods.[23] Under UV excitation, large $\mathrm{Au}$ domains were exclusively deposited at one end of the seeded $\mathrm{CdSe} / \mathrm{CdS}$ rod because of electron migration to one of the reactive facets of the tips. The strong Au-Se coordination allowed Au precursors at high concentration to grow further on the side facets of the seeds. Au-CdSe pyramids were obtained by growth and reductive transformation of a gold shell around a CdSe pyramid under electron beam irradiation. The deposition of the $\mathrm{Au}$ clusters along the anisotropic semiconductors was strongly influenced by reaction temperature and ligand-mediated defect sites. Decreasing the amount of surfactant allowed the $\mathrm{Au}$ tips to interact and coalesce, forming disordered networks of the fused hybrid nanostructures. The selective growth of the Au precursors on the tips of the CdSe rod, tetrapod, pyramid exhibited a significant modification of the optical property compared to the corresponding singe components.

Figure 3 shows Au-CdSe rod- and pyramid-shaped hybrids synthesized by Klinke et al.[24] via growth and reductive transformation of the $\mathrm{Au}$ shell around the pyramidal CdSe nanocrystals in the presence of chlorine under either electron beam irradiation or addition of reducing agent. The size of the deposited Au dots can be tuned from 1.4 to $3.9 \mathrm{~nm}$ by varying the synthesis conditions, such as ligands and shape of the CdSe. The shape rod and pyramid were thermodynamically favored for the size-controlled growth of Au precursors 

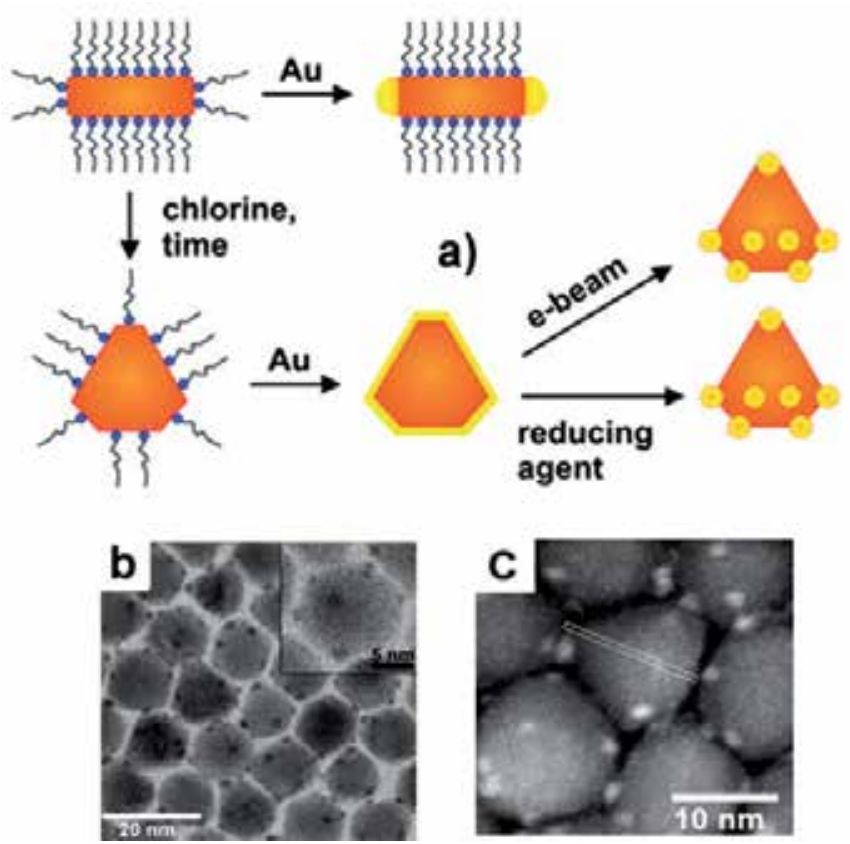

Figure 3. Growth of Au shell around pyramidal CdSe nanocrystals. (a) Scheme of the Au growth process onto CdSe nanocrystals. (b) Incubation of CdSe with Au-tetra- $n$-butylammonium borohydride/dodecyltrimethylammonium bromide led to Au clusters positioned at apexes of the CdSe pyramids. (c) STEM image of Au/CdSe hybrids with sharp angles as reactive sites for the nucleation of Au particles. Reproduced with permission from ref. [24]. Copyright 2010, The Royal Society of Chemistry.

on the corners and tips of the CdSe nanocrystals through the suitable choice of the metal precursor and the surface ligand concentration. The pyramid structure provided relatively sharp angles which were highly reactive sites for the nucleation of the gold particles. Banin et al. ${ }^{[25]}$ also synthesized cage-shaped $\mathrm{Ru} / \mathrm{Cu}_{2} \mathrm{~S}$ nanohybrids by adding $\mathrm{Ru}(\mathrm{acac})_{3}$ to $\mathrm{Cu} 2 \mathrm{~S}$ seed suspension in octadecylamine. Metallic Ru selectively grew on the crystal edges of the $\mathrm{Cu}_{2} \mathrm{~S}$ nanocages to form a symmetrical cage around a $\mathrm{Cu}_{2} \mathrm{~S}$ core. The cage formation could be due to the capping of thiol ligands on the $\mathrm{Cu}_{2} \mathrm{~S}$ facets to block the growth of these crystal facets. These $\mathrm{Ru} / \mathrm{Cu}_{2} \mathrm{~S}$ nanocages were used as an excellent electrocatalyst for $\mathrm{H}_{2} \mathrm{O}_{2}$ sensing with catalytic activity higher than $\mathrm{Cu}_{2} \mathrm{~S}$ electrode.

The recent progress made in the synthesis of magnetic-fluorescent nanohybrids with combined magneto-transport properties has been reported. Cozzoli et al.[26] reported the two-step seeded-growth for the selective synthesis of Co-tipped CdSe/CdS core-shell nanorods. A two-step procedure consisted of injection of tri-n-octyl phosphine sulphide/CdSe seeds to Cd-surfactant complexes and heated at $350-380{ }^{\circ} \mathrm{C}$ to form $\mathrm{CdSe} / \mathrm{CdS}$ core-shells. Co clusters were then attached with the $\mathrm{CdSe} / \mathrm{CdS}$ seeds by injecting $\mathrm{CO}_{2}(\mathrm{CO})_{8}$ solution into octadecene solvent containing $\mathrm{CdSe} / \mathrm{CdS}$ seeds at $200-240{ }^{\circ} \mathrm{C}$ under inert atmosphere. An excess volume of oleic acid was added to the growing mixture to stabilize the hybrid nanostructures formed after reaction completion. The formation of the 
nanohybrids with matchstick-like topology was predicted by crystal-oriented-attachment occurred the directional fusion of the generated Co nanocrystals to the nanorod tips. These Co-tipped CdSe/CdS heterostructures exhibited unusual room-temperature ferromagnetism and fluorescent emission despite photoexcited charge transfer from the semiconductor to the metal domain. Talapin et al.[27] synthesized FePt/PbS and FePt/PbSe nanohybrids with magneto-transport properties by coupling ferromagnetic FePt particles with either $\mathrm{PbS}$ or $\mathrm{PbSe}$ in form of core-shells or dumbbells. The formation of the hybrid products by injecting bis(trimethylsilyl) sulfide to reaction mixture containing FePt nanoparticles, oleic acid, $\mathrm{Pb}$ oleate complex dissolved in octadecene at $120-150{ }^{\circ} \mathrm{C}$. The nanohybrid shape was controlled by capping of the ligand on the surface of the FePt seeds and the reaction temperature. These magnet-in-the-semiconductor hybrid nanostructures showed semiconductor-type transport properties with magnetoresistance characteristic of combining the advantages of both functional components. The maghemite-metal sulphide (ZnS, CdS, HgS) nanohybrids were synthesized by adding sulphur and appropriate metallorganic precursors to the $\mathrm{Fe}_{2} \mathrm{O}_{3}$ particles followed by heating treatment.[28] In other reports, the large lattice mismatch between $\mathrm{Fe}_{2} \mathrm{O}_{3}$ and metal sulphide resulted in the formation of non-centrosymmetric nanostructures. The formations of trimers and oligomers were observed for $\mathrm{ZnS}$ and dimers for $\mathrm{CdS}$ and $\mathrm{HgS}$ nanocomposites. Alloy-cadmium selenide core-shells including FePt/CdSe, $\mathrm{NiPt} / \mathrm{CdSe}, \mathrm{FePt} / \mathrm{CdSe}, \mathrm{NiPt} / \mathrm{CdSe}$ were synthesized by hydrolysis of cadmium stearate in oleylamine, hexadecylamine/octyl ether, 1,2-hexadecandiol in the presence of alloy seeds.[29]

\section{Surface modification of hybrid nanostructures}

Functionalization of the hybrid nanostructures has received a great deal of interest because of their biomedical applications in targeted drug delivery, diagnosis and therapy. The synthesis of the hybrid nanomaterials has mostly been achieved in organic and aqueous media containing either hydrophobic or hydrophilic organic linkers. The hybrid nanostructures synthesized usually involves organic linkers binding to the surface of two or more components to stabilize the nuclei and larger nanoparticles against aggregation by repulsive force for controlled growth of the sized and shaped nanohybrids. As capped by the linkers, the resulting hybrid nanostructures become hydrophobic character and is insoluble in water, resulting in incompatible with biological systems. A surface modification of hydrophobic-surfaced nanohybrids to produce water-soluble functional nanohybrids are therefore a indispensable step prior to biomedical applications. These interactions with the environment ultimately affect the colloidal stability of the particles and may yield to the delivery of the appropriate functional nanoparticles to targeted species. There are common functionalization strategies of silica and amphiphilic polymer coatings. Since the coated hybrid nanostructures retained their original physical properties, the resulting nanocomposites showed the multifunctional properties. These routes can serve as a powerful paradigm for the further fabrication of antibody-conjugated nanohybrids for multifunctional theranostic applications. 


\subsection{Polymer adsorption}

The nanohybrid colloids could be soluble in water by adsorbing amphiphilic polymers onto hydrophobic molecules-capped nanohybrids. The amphiphilic polymer adsorption are carried out through hydrophobic interaction of hydrocarbon chains and van der Waals force between the molecules. The desorption of polymer molecules from the nanoparticles is usually prevented because numerous contact points formed from the interactions of organic linkers and polymer chains. Advantage of this approach is mainly not dependent on the types of the inorganic hybrid cores and the organic linkers, and the physiochemical surface properties of the coated particles are significantly unchanged. A popular example is the gold particles in aqueous media prepared by citrate reduction. Citrate ions adsorbed on the gold surface resulted in their negative charge and colloidal stability within several years by electrostatic repulsion. The citrate layer was replaced by stronger-binding ligands of mercaptocarboxylic acids. The surface modification of the particles with mercaptocarboxylic acids allowed for achieving concentrated particle solutions, that can precipitate out of particles by salt-induced aggregation and redissolved in low-salt buffers.[30] The surface functionalization of TOP/TOPO-capped CdSe/ZnS quantum dots was substituted phosphine-based hydrophobic ligands with hydrophilic mercaptocarboxylic acid molecules.[31] The quantum dots in aqueous solution stabilized with mercaptoacetic acid were modified by co-adsorption of polyethylene glycol and peptides.

Polyethylene glycol (PEG) is a linear polymer consisted of ethylene oxide units and well soluble in water. PEG is high biocompatibility due to its inertness and non-toxic properties.[32] Apart from the post-modification approach by covalent chemistry, PEGmodified nanoparticles can be obtained by PEG-contained ligand molecules with functional group that can bind to the particle surface.[33] Owing to the solubility of PEG itself, the PEG-coated particles can also be dispersed in polar organic solvents.[34] Figure 4 shows the modular design toward The synthesis of the amphiphilic polymer of poly(maleic anhydridealt-1-octadecene) (PMAO)-polyethylene glycol was carried out through reaction between maleic anhydride and primary amine-terminated polyethylene glycol methyl ethers and subsequent coating of PMAO-polyethylene glycol amphiphilic polymer with hydrophobic ligand-capped quantum dots.[35] The functionalized materials dissolved in water had the same optical spectra and quantum yield as those pre-synthesized quantum dots. The watersoluble encapsulated nanoparticles contain free carboxylic acid groups for conjugating antiHer2 antibody to the polyethylene glycol-coated nanoparticles. The antibody-conjugated quantum dots were used as a probe to recognize human breast cancer cells with Her2 receptor with nonspecific binding of polyethylene glycol with cell receptors on the particle surface. Mattoussi et al.[33] achieved the capping of water-soluble ligand of mixed dihydrolipoic acid (DHLA) and poly(ethylene glycol) (PEG) on quantum dots. These ligands are consist of a poly(ethylene glycol) (PEG) segment attached anchoring DHLA group on one end to drive binding to the quantum dot. Cap exchange with these hydrophilic ligands made quantum dots to be stabilized in water over extended periods of time and over a broad $\mathrm{pH}$ range. The quantum dots capped with DHLA-PEG-biotin interacted with streptavidin coupled to proteins which were subsequently taken up by live cells. Yu et al. 


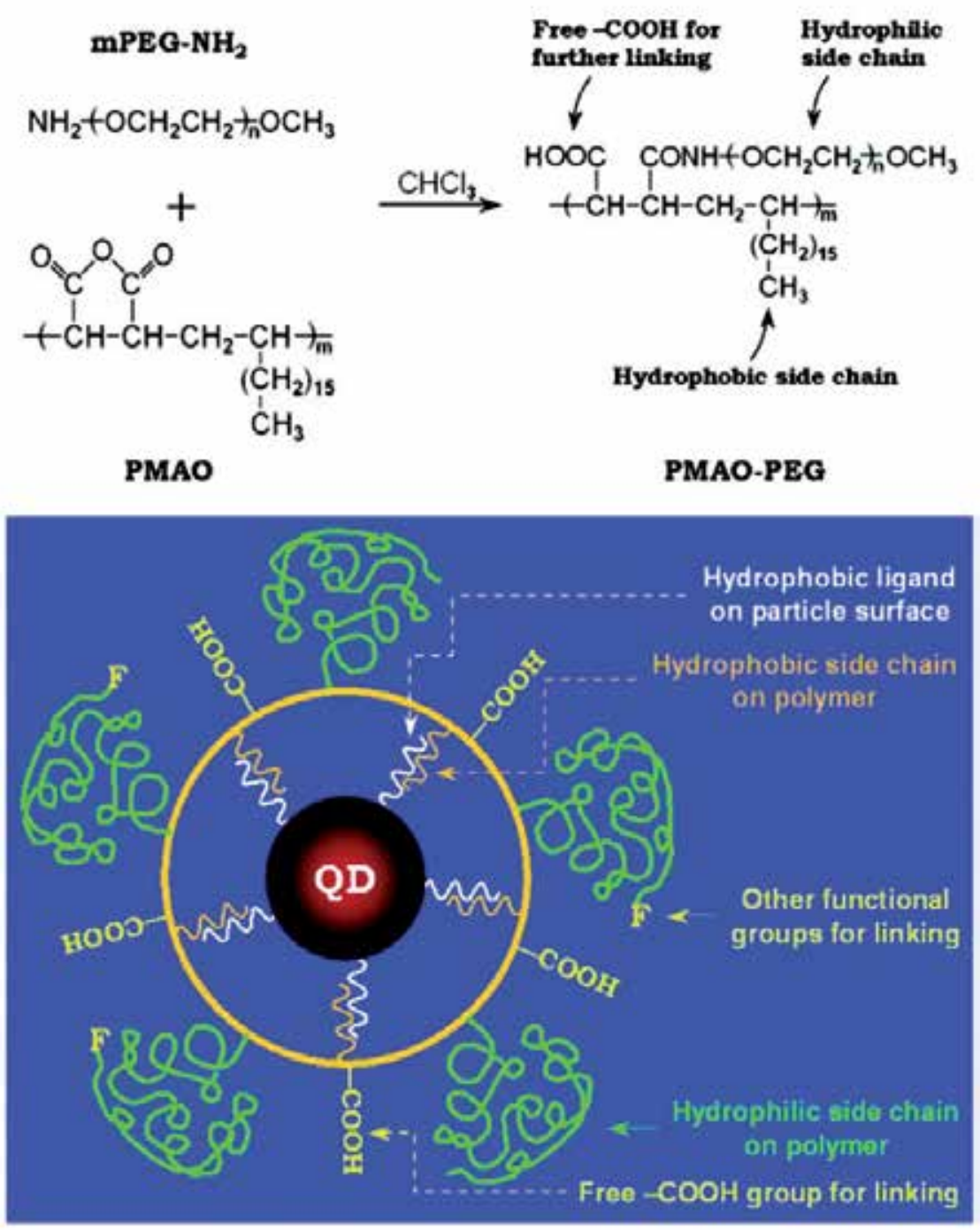

Figure 4. Forming biocompatible and nonaggregated nanocrystals in water using amphiphilic polymers. Top: one-step formation of poly(maleic anhydride-alt-1-octadecene) (PMAO)-polyethylene glycol (PEG) amphiphilic polymers through reaction between maleic anhydride and amino groups. Bottom: schematic structure of water-soluble quantum dots (F stands for a functional group instead of $\mathrm{OCH}_{3}$, such as $-\mathrm{OH},-\mathrm{COOH},-\mathrm{NH}_{2}$ ). Quantum dots were encapsulated by PMAO-PEG amphiphilic polymer hydrophobic interaction. Reproduced with permission from ref. [35]. Copyright 2007, American Chemical Society.

[35] prepared amphiphilic polymer of PMAO-PEG through reaction between poly(maleic anhydride-alt-1-octadecene) (PMAO) and primary amine-terminated polyethylene glycol methyl ethers (PEG). The quantum dots were mixed with PMAO-PEG in chloroform. These PMAO-PEG-coated quantum dots were found to have the optical properties and recognized the cancer cells with Her2 receptor. 
Poly(acrylic acid)-based polymers with hydrophobic side chains were usually used for surface modification with aliphatic amine- or thiol-capped particles.[36] These polymers are soluble in organic solvent and can bind to the hydrophobic particle surface. After solvent evaporation, the particle solids can be dissolved in aqueous buffer, providing stable watersoluble particles. Poly(acrylic acid) was coated onto hydrophobic dodecylamine-capped $\mathrm{CdTe} / \mathrm{CdSe}$ quantum dots to form amphiphilic double-layered nanohybrids soluble in either water or organic media.[37] The coating of these polymers can carry out in ethanol solvent, resulting of poly(acrylic acid) backbone linked with mixed octylamine and isopropylamine, giving numerous hydroxyl groups on the particle surface.[38, 39] The hydrophobic side chains of the polymers commonly cover or intercalate the hydrophobic ligand molecules, and the exposed hydrophilic backbone outwards to aqueous media. In addition, poly(maleic anhydride) copolymers prepared from copolymerization of maleic anhydride with olefin are used as alternating copolymers. In aqueous media, the maleic anhydride rings hydrolyze and open giving two carboxylic groups, which gives access to further functionalization. Each maleic anhydride ring yields a free carboxylic group, indicating that the surface of the polymer-coated particles could be covalently grafted to amino acids for biomolecule conjugation.

Poly(vinyl pyrilidone) was also used to graft directly on the surface of the particles through one-pot process.[37] The further surface functionalization of the grafted products can be achieved by adding a next layer or exchanging original capping agents. Other polymers contained a mixture of aliphatic side chains and others with primary amines at their ends can bind to the nanoparticle surface through the amino groups.[40] Additionally, poly(acrylic acid) modified with free thiol and amino groups at the ends of the side chains was demonstrated as coating for quantum dots to form a thin shell with little effect on the quantum yield of the coated particles. The hydrophobic-hydrophilic block-copolymers formed in micellar structure dispersible in solvent were also used for the coating of the nanoparticles. The coating by block-copolymer micelles yields the particle aggregates instead of the monodisperse particles that could be suitable for further generation of the multifunctional porous materials.

\subsection{Silica coating}

Coating of a cross-linked silica shell to protect the organic agent-capped hybrid cores from external environment is carried out to produce the silica-coated hydrophobic nanohybrids. Coating with silica layer is one of the most widely used methods for surface modification of the inorganic nanoparticles, because the unique properties of the nanoparticles can be preserved by silica shells. After silica coating, the colloids stabilized in aqueous media and have low nonspecific interaction with biosystems and inert silica layer against degradation of optical properties. Silica can also be easily surface modified to link bioconjugators with interesting biofunctionalities. To this goal, water-to-oil microemulsion and Stober sol-gel have generally been achieved for silica coating. 
Reverse microemulsion is a promising method for the synthesis of the monodisperse silicacoated nanoparticles.[41] Ying et al.[42] developed the reverse microemulsion-mediated route to encapsulate the hydrophobic trioctylphosphine oxide (TOPO)-capped quantum dots and magnetic particles within silica shells to form the silica-coated quantum dotmagnetic hybrid structures. Figure 5 describes the water-in-oil (W/O) reverse microemulsion system for silica coating of the hydrophobic particles, where water droplets are stabilized by nonionic Igepal surfactant in a continuous oil phase (e.g., cyclohexane). After the addition of silane (TEOS), hydrolysis and condensation occur at W/O interface or in water phase to encapsulate the inorganic particles within a silica shell. The magnetic particles and quantum dots were confined in the silica layer to afford the hybrid structure. The silica-coated magnetic-quantum dot nanohybrids preserved the magnetic property of $\gamma$ - $\mathrm{Fe}_{2} \mathrm{O}_{3}$ and optical property of CdSe quantum dots. The authors also used this route to synthesize silica-coated oleylamine-coated $\mathrm{Au}$ and Ag core-shells and subsequent conjugation with activated polymeric dextran.[43] The resulting materials were potentially used as glycobiological

a)

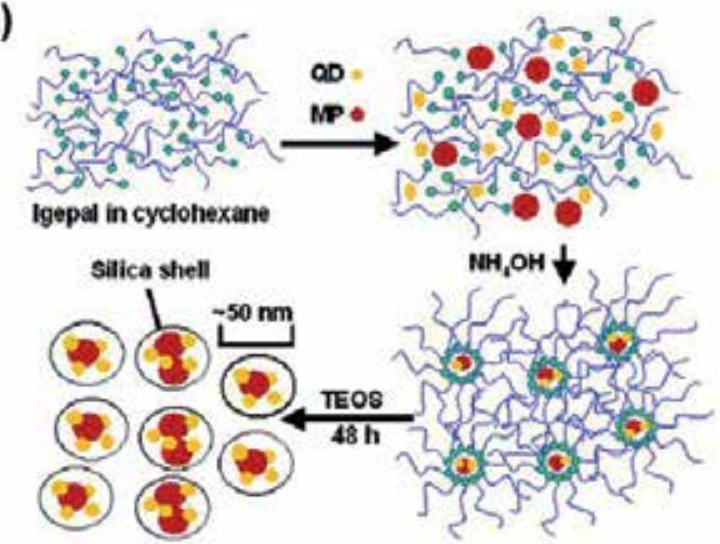

b)

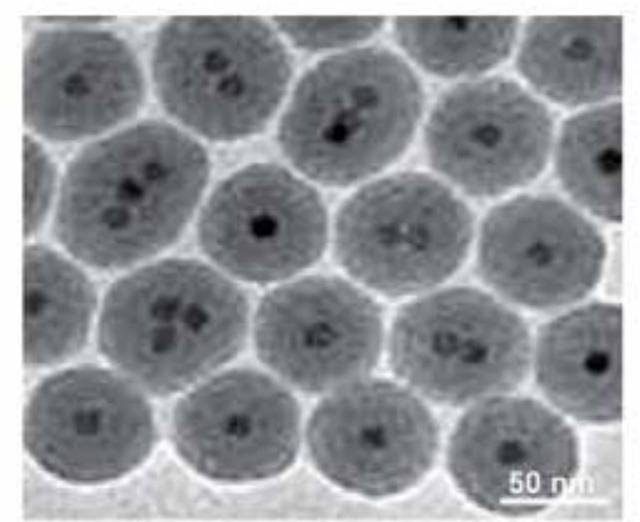

Figure 5. Silica-coated magnetic-quantum dot hybrid nanoparticles. (a) A scheme of the reverse microemulsion system for the synthesis of silica-coated magnetic-quantum dot nanohybrids; (b) TEM image of the silica-coated magnetic-quantum dot nanohybrids. Reproduced with permission from ref. [42]. Copyright 2005, American Chemical Society. 
probes. Similarly, the $\mathrm{SiO}_{2}$-coated $\mathrm{Fe}_{2} \mathrm{O}_{3}$ rattle-type nanoball structures were also synthesized and used as support for decorating the Pd nanoclusters onto the support surface by mercapto- or amino-functionalized silica.[44]

The reverse microemulsion process was also used by other research groups. Meijerink et al. [45] elucidated the mechanism of incorporating hydrophobic quantum dots into monodisperse silica spheres. In water-in-oil reverse microemulsion system, the hydrolyzed TEOS had a high affinity for the quantum dot surface for replacement of hydrophobic amine ligands, which enabled the transfer of the quantum dots to the hydrophilic interior of the micelles where silica growth occurred. Serna et al.[46] achieved in-situ synthesis and further silica coating of the Fe nanoparticles in microemulsion system. The lamellar-like coated nanostructures were formed through the subtle interplay controlling the formation of nanospherical silica particles by the ammonia base-catalyzed hydrolysis of tetraethoxysilane (TEOS) in water-in-oil. Kang et al.[47] designed the reverse microemulsion based on the Igepal CO-520 surfactant to produce the silica-coated NiPt nanohybrids prepared from the reduction of nickel acetylacetionate and platinum acetylacetonate in oleic acid/oleylamine. Tsang et al.[48] reported that the templated sol-gel encapsulation of CTAB-stabilized micelles containing metal precursors with ultra-thin porous silica coating allows solvent extraction of organic based stabilizer from silica-coated Ag-Pt alloys. The water-in-oil microemulsion for silica coating on $\mathrm{Y}_{3} \mathrm{Al}_{5} \mathrm{O}_{12}$ :Ce nanoparticles was presented by Chen et al. [49] through hydrolysis of tetraethyl orthosilicate. The silica shell thickness can be turned from $8-16 \mathrm{~nm}$ by varying the ratio of NiPt particles to TEOS precursor.

The Stober method of base-catalyzed hydrolysis and condensation of tetraethyl orthosilicate (TEOS) to produce silica used to coat on the hybrid cores. [50] This reaction has several advantages such as mild conditions, low cost, without surfactant used. Earlier, Kotov et al.[51] coated hydrophilic CdTe quantum dots within 40-80 nm silica spheres using modified Stober method, which resulted in reduced emission intensity with broadening of the spectrum. The quantum dots acted as seeds for the silica growth in ethanol/water. This method yielded single or multiple quantum dot per silica sphere, but the size and dispersion of the silica-coated quantum dots were hard to control. Alivisatos et al.[52] achieved Stober silanization approach for the functionalization of mercapto-silane/(3-mercaptopropyl-trimethoxysilane) siloxane with thiol and/or amine groups to produce silica shell-coated hydrophobic CdSe/ZnS coreshells. Mercaptopropyltris(methyloxy)silane (MPS) was replaced TOPO molecules on the surface. The methoxysilane groups ( $\left.\mathrm{Si}-\mathrm{OCH}_{3}\right)$ of (MPS) hydrolyzed into silanol groups (Si$\mathrm{OH})$, and formed a primary polymerization layer. The silane precursors containing functional groups $\left(\mathrm{F}=-\mathrm{SH},-\mathrm{NH}_{2}\right)$ were then incorporated into the shell and may tailor the nanoparticle surface functionality. Adopting the Stober method, Figure 6a shows the seminal silica coating of citrate-reduced Au particles by Mulvaney et al. [53] involved the weak surface attachment with bifunctional (3-aminopropyl) trimethoxysilane in aqueous media. The $-\mathrm{NH}_{2}$ groups were bound to the gold surface and $-\mathrm{Si}(\mathrm{OEt})_{3}$ groups and facilitated for hydrolysis and condensation with sodium silicate to deposit a surface-coated silica layer. Later, the thicker silica shells can be grown on the surface-stabilized $\mathrm{Au}$ particles by further hydrolysis/condensation of tetraethyl orthosilicate (TEOS). 
In some cases prior to silica coating, the particle surface should be attached with hydrophilic molecules to create the surface-protected nanoparticles stabilized in aqueous media. This could facilitate hydrolysis/condensation of tetraethyl orthosilicate. For example, Han et al. [54] synthesized the monodisperse silica-coated gold particles derived from the citratestabilized gold particles. The prepared citrate-reduced gold particles are low stable for silica coating in alcoholic media. The colloidal stability needs to be increased by introducing a certain amount of sodium citrate into the synthetic solution to replace the surface charge of the gold particles. Chang et al.[55] presented the synthesis of the silica nanohybrids composed of the CuInS $2 / \mathrm{ZnS}$ quantum dots and magnetite nanocrystals. The outside silica shell grafted with poly(ethyleneglycol) and amine groups to provide better biocompatibility and to allow further bioconjugation. These materials exhibited the exert excellent properties for drug delivery vehicles and magnetic resonance imaging. The conjugation of $\mathrm{Pt}(\mathrm{IV})$ anticancer drug onto the nanohybrids resulted in higher cytotoxicity than the free $\mathrm{Pt}(\mathrm{IV})$ anticancer drug, indicative of the multifunctional feature of the synthesized nanohybrids.

(A)
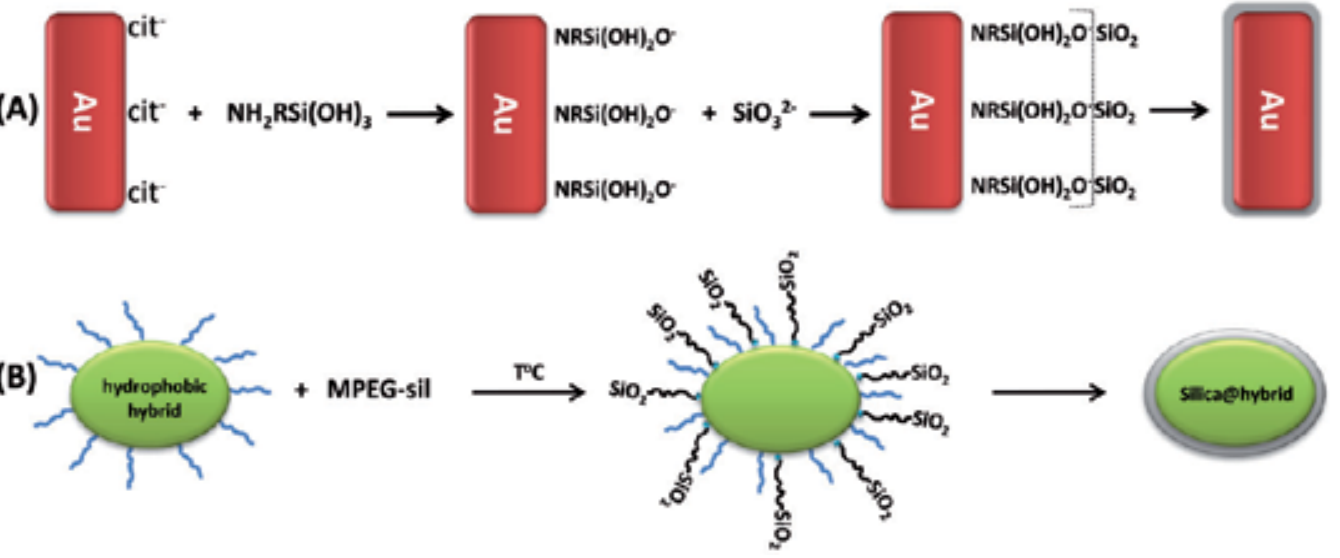

Figure 6. Schemes of silica coatings of hydrophilic (a) and hydrophobic (b) nanoparticles.

Figure $\mathbf{6 b}$ shows a successful example involved the surface adsorption of methoxypoly(ethylene glycol) silane to replace oleylamine capped on silver nanoparticles by Yang et al.[56] The functionalized silver nanoparticles were then hydrolyzed and condensed further to form thin silica layer-stabilized silver nanoparticles followed by thick silica coating with the Stober process. Bifunctional $\mathrm{Gd}_{2} \mathrm{O}\left(\mathrm{CO}_{3}\right)_{2} \cdot \mathrm{H}_{2} \mathrm{O} /$ silica/Au hybrid nanoparticles prepared by condensation of TEOS followed by conjugation with the gold shells were demonstrated potential as a MRI and therapeutic agent.[57] The hybrid particles showed the capability of absorbing NIR radiation for photothermal destruction of cancer tumors, in which the Au shell thickness strongly influenced the NIR optical absorption and photothermal effect. Pratsinis et al.[58] achieved coating of a thin silica shell on $\mathrm{Ag} / \mathrm{Fe}_{2} \mathrm{O}_{3}$ Janus-shaped hybrids via one-step flame aerosol method. The silica coating still intacted their shape and plasmonic-magnetic properties but minimizes the release of toxic $\mathrm{Ag}^{+}$ions from the Ag particle surface and their direct contact with live cells. Well-defined $\mathrm{Au} / \mathrm{SiO}_{2} / \mathrm{CdSe}$ hybrid nanostructures constituted a gold core overcoated with a silica shell 
followed by a dense monolayer of CdSe quantum dots were formed via multistep procedure.[59] The formed products involved the synthesis of the gold particles, gold surface activation, silica-shell deposition, modification of the silica surfaces with $-\mathrm{NH}_{2}$ groups, and final self-assembly of the CdSe quantum dots onto the particle surfaces. In order to the surface activation of the gold particles, (3-mercaptopropyl)-trimethoxysilane was found to be better than (3-aminopropyl)-trimethoxysilane because of stronger binding of $-\mathrm{SH}$ groups to the gold surfaces. These hybrid structures were used to perform the accurate quantitative analysis of the effect of the metal on quantum dot photoluminescence intensity. Khlebtsov et al.[60] preformed the silica coating on Au-Ag nanocages through adding water-ammonia solution and TEOS to the reaction solution containing Au-Ag particles. Silica-coated $\mathrm{Au}-\mathrm{Ag}$ nanocages were then functionalized with photodynamic sensitizer $\mathrm{Yb}$-2,4-dimethoxyhematoporphyrin to form the nanocomposites potential in multifunctional capability of IR-luminescence detection, photosensitization, and photothermolysis.

\section{Nanotechnology in cancer treatments}

Cancer is the greatest challenge in human healthcare today. It is a result of unregulated cell division leading to the uncontrolled growth and spread of abnormal cells. This behaviour causes the formation of malignant tumors consisted of cancer cells plus some healthy cells (normal tissue) invade nearby parts of the body. At early growth stages, the cancer cells mostly do not look or act like the normal cells because they are readily disguised by the healthy cells on their surface. This mainly behaves an extremely danger of the cancer cells. Tumor cells have a strong tendency to displace healthy cells until the tumor reaches a diffusion-limited maximal size, frequently resulting of changes to the DNA (mutations), leading to deaths.

Traditional cancer diagnosis and treatment modalities basically include post-surgical chemotherapy, radiotherapy, hormone therapy, and immunotherapy.[61] Each of these modalities has constantly limitations in treatment and also contribute to the rising costs of healthcare. Because of most human cancers relevant to solid tumors, so that the current cancer therapies are usually achieved some surgeries for removal of tumors, followed by chemotherapy and radiotherapy to kill the remaining tumor cells. However, the efficacy of the chemotherapy, serious side-effects on different healthy organs, the increased costs are a great obstruction by the fact that cancer stem cells be still survive and could continue to spread back. It is reasonably why cancer symptoms come back within relative short duration in patients who has passed through the post-surgical chemotherapy.

Thermal therapies (hyperthermia) have often employed a variety of heat sources including laser light, focused ultrasound, microwaves to destroy the solid tumors.[62] The benefits of hyperthermia are minimally or non-invasive, relatively simple to perform in the absence of surgical resection. However, simple heating techniques have trouble discriminating between tumors and surrounding healthy tissues, and often heat intervening tissue between source and target site. To irradiating beams reached underlying tumors or dispersed into large 
tumors, high activating energy source must achieve at long duration of time, leading to sufficiently penetrate and damage healthy tissues.

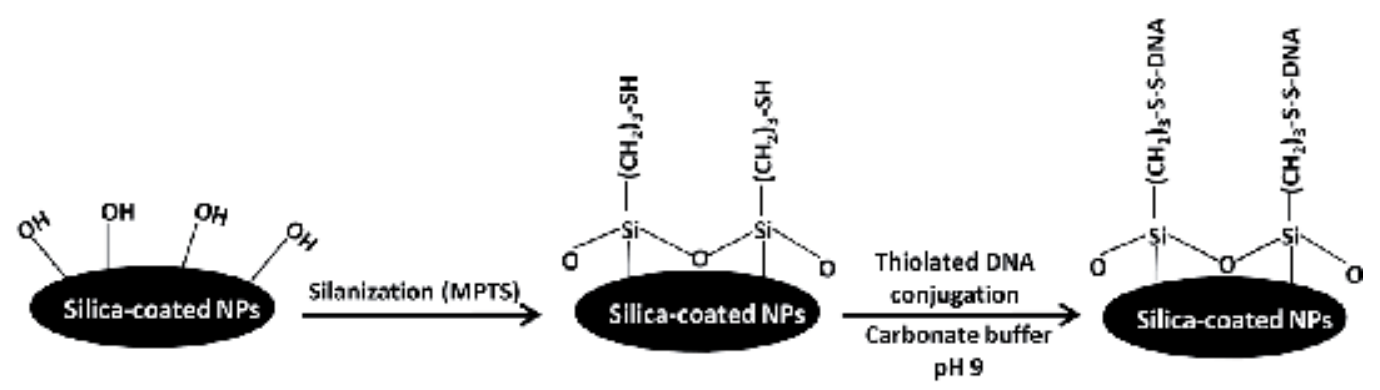

Figure 7. A scheme of thiolated DNA conjugation onto the silica-coated nanoparticles.

To the goal of the cost and performance, the development of new efficient approaches based on an advanced combination between "smart drug delivery" chemotherapy and photoradiation companied by "near-infrared (NIR) laser-adsorbing nanomaterials" to create the most effective results have been interested in medicine technology.[63] Lack of target specificity is one of the major disadvantages of many drugs. When drugs administered into human body are distributed to all organs through bloodstream, rather than to specific target organ that needs the pharmacological treatment. Biochemical and physiological barriers of certain organs also limit drug delivery to the desired organ. Chemotherapeutic drugs may destroy the cancer cells along with destroy the healthy tissue and cytotoxic effect of the drugs. To overcome these disadvantages, newer and effective methods should be developed to safely shepherd a pharmacological agent to avoid specific organs, where healthy tissue might be adversely affected.

The nanoparticles with the size smaller 100-10,000 times than the cells can be conjugated with various complementary biomolecules including DNA strands and antigens, as shown in Figure 7. The conjugated nanoparticles can easily pass through the cell membrane and accumulate into target sites by manipulation, which is advantageous in targeted imaging, diagnosis, and delivery.[64] The functionalization of the silica-coated nanohybrids is usually achieved by adsorption or chemical conjugation of the biomolecules to the particle surface. The silica-coated gold-based nanohybrid colloids can be surface-functionalized with mercapto-, amino-, carboxy-terminated silanes for biomolecule conjugation. Homogeneously water-dissolved biomolecules-conjugated silica-coated nanoparticles could bind to the surface of the cancer cells with greater affinity than to the noncancerous cells.

\section{Gold nanorods-activated NIR laser plasmonic photothermal therapy}

The targeted delivery of gold-based nanohybrids to solid tumors is one of the most important and challenging problems in cancer medicine. The strongly plasmonic absorption and photothermal conversion of the gold nanoparticles have been exploited in cancer 
therapy through the selective localized photothermal heating of the cancer tumors.[65] To treat a tumor, the gold particles conjugated with biomolecules can be selectively targeted to cancer cells without significant binding to healthy cells. The nanoparticles in the bloodstream generally have to firstly move across the tumor blood vessels. The tumors are then exposed to an excitation source, such as NIR laser light, radiowave, or an alternating magnetic field. When the gold nanoparticles are exposed to the light radiation at their resonance wavelength, the electric field of light causes the collective oscillation of the conduction-band electrons at the particle surface. The coherent oscillation of the metal free electrons in resonance with the electro-magnetic field is called the surface plasmon resonance (SPR). The excitation of the maximum SPR absorption results in enhancement of the photophysical properties of gold particles.[66] The gold nanoparticles absorb the incident energy and convert them to heat, which raises the temperature $\left(\sim 42^{\circ} \mathrm{C}\right)$ of the tissue and ablates the cancerous cells by disrupting the cell membrane. The photoradiations do not often kill healthy cells because the laser power requires to heat/destroy the cancer cells much low than the healthy cells to which nanoparticles do not bind specifically. The physical heating mechanism of ablative therapies would provide an advantage against chemotherapy-resistant cancers, as well as improved tumor response when combined with chemotherapy and photoradiation.

Key features to consider when selecting a compatible particle for hyperthermia are the wavelength of maximal absorption, absorption cross-section, and shape/size of the particle. NIR laser light is ideal for in-vivo hyperthermia applications because of its low absorption by tissue chromophores (hemoglobin and water), which prevents them from damaging healthy tissue. The absorption coefficient of these tissue chromophores is as much as two orders of magnitude greater in the visible region $(400-600 \mathrm{~nm})$ as compared to the NIR region (650-900 nm).[66] Gold-nanoparticle-mediated photothermal therapy is predominantly designed to operate in this window of wavelengths ("NIR window") to minimize energy interaction of light-tissue, preventing damaging heating of healthy tissue. Upon tumor laser irradiation, NIR light is absorbed by the nanoparticles and heat dissipation is generated as a consequence of electron-phonon interactions. For successful cancer ablation, the tissue must be heated to a minimum temperature for a minimum duration of time to induce tumor cell death.

The plasmon absorbance of the gold particles can be easily tuned from the visible region into the NIR by simple manipulation of their aspect ratio (from sphere to rod).[66] For the gold nanospheres, this resonance occurs in the visible spectral region at about $520 \mathrm{~nm}$, originating from the brilliant colour of the gold particle solution. Owing to their distinctive rod shape, the gold nanorods have two absorption peaks attributed to the free electron oscillation along the longitudinal and transverse axis, resulting in a stronger resonance band in the NIR region and a weaker band in the visible region ( $\sim 520 \mathrm{~nm}$ for gold nanospheres). The synthesis of the colloidal gold nanorods would therefore prove effectively for photothermal therapy because they can absorb low-energy NIR light and convert it to heat in the usual way. 
The gold species conjugated to antibodies can be selectively targeted to cancer cells without significant binding to healthy cells. In general, the routes to nanoparticle delivery are mainly based on an "active" mechanism and a "passive" mechanism.[67] In the active mode, the molecule ligands of antibodies, DNA, and peptides are used to recognize specific receptors on the tumor cell surface. In the passive mode, the nanoparticles without targeting ligands are accumulated and retained in the tumor interstitial space mainly. In both mechanisms, the nanoparticles in the bloodstream must first move across the tumor blood vessels. Some reports were used PEG ligand to attach the lysine-capped Au nanoparticles through lysineterminated PEG link.[68] The targeted delivery of the gold nanoparticles to solid tumors is one of the most important and challenging problems in cancer nanomedicine. It was recently observed that the colloidal gold nanoparticles were found in dispersed and aggregated forms within the cell cytoplasm and provided anatomic labeling information. The anti-EGFR antibody-conjugated nanoparticles homogeneously bind to the surface of the cancer type cells with greater affinity than to the noncancerous cells. These results were detected by using SPR scattering imaging and SPR absorption spectroscopy, in which a relatively sharper SPR absorption band with a red shifted maximum compared to that observed on noncancerous cells.[69] We recently reviewed the aqueous-based synthetic pathways of the metal nanocrystals potential in biomedical applications.[67]

\section{Hybrid nanostructures for biomedical diagnosis and therapy}

\subsection{Plasmonic-magnetic hybrid nanostructures}

Magnetic nanoparticles with unique magnetic properties are a direct consequence of the behaviour of electrons within the particle. The electrons are similar to tiny bar magnets, with a surrounding magnetic field that corresponds to the electron spin in an applied field. When electrons move between different energy levels, they absorb energy and can generate light or heat.[13] Because of their low toxicity and good sensitivity, the magnetic particles conjugated with biomolecules are widely studied and applied in biomedicine, where these particles are used as magnetic carriers that could travel to targeted cancer tumors under orientation of an external magnetic field. The magnetic carriers then absorb externally magnetical energy and convert them to heat to kill the cancer cells. Magnetic resonance imaging (MRI) is presently one of the most powerful diagnosis tools for imaging the central nervous system and for detecting tumors and cancer cells. The paramagnetic gadolinium chelate complexes (e.g., Gd-DTPA) were widely used for MRI contrast agents.[70] The porous/hollow iron oxide nanocapsules made by wrap-bake-peel process from $\mathrm{FeOOH}$ nanorods via silica coating were used for this goal.[14]

The plasmonic-magnetic nanohybrids retain plasmonic properties and strong response to magnetic fields, where scattering of the plasmon alongside with magnetic contrast could be envisioned. Many researchers have recently developed the plasmonic-magnetic nanohybrids to improve the contrasting abilities with extra functions. As a typical material for cancer diagnostics and therapeutics, the $\mathrm{Au} / \mathrm{Fe}_{3} \mathrm{O}_{4}$ nanohybrids as a bifunctional probe offer two functional surfaces for attachment of the plasmonic and magnetic particles.[71] 
The $\mathrm{Au}$ and $\mathrm{Fe}_{3} \mathrm{O}_{4}$ particles both in a hybrid system are known to be highly compatible with biomedicine and a consequence of extending for diagnostics and therapeutics. The $\mathrm{Au}$ and $\mathrm{Fe}_{3} \mathrm{O}_{4}$ interfaces result in drastically change the local electronic structure, leading to an enhancement of their synergistic properties. The plasmonic and magnetic properties of the nanohybrids could be optimized by adjusting the size of the two particles. In comparison with the single $\mathrm{Au}$ and $\mathrm{Fe}_{3} \mathrm{O}_{4}$ particles, the $\mathrm{Au} / \mathrm{Fe}_{3} \mathrm{O}_{4}$ hybrids possessing simultaneous plasmonic and magnetic detection facilitate for cancer diagnosis and therapy. When the biomolecules-conjugated $\mathrm{Au} / \mathrm{Fe}_{3} \mathrm{O}_{4}$ hybrids are dispersed in body, they could travel to the targeted cancer tumors by influence of external magnetic field. The $\mathrm{Au}$ and $\mathrm{Fe}_{3} \mathrm{O}_{4}$ particles in the hybrid system adsorb energy from irradiating laser light and from exposing external magnetic field, respectively, and convert them into heat to kill the cancer cells. By using these bifunctional materials, an abundant amount of synergistic heating within the cancer tumors is created by a simultaneous adsorption-conversion of $\mathrm{Au}$ and $\mathrm{Fe}_{3} \mathrm{O}_{4}$ species facilitating the heating of the cancer cells.

Figure 8 shows the use of $\mathrm{S} 6$ aptamer-conjugated plasmonic-magnetic $\mathrm{Au}-\mathrm{Fe}_{3} \mathrm{O}_{4}$ nanohybrids for the targeted diagnosis, isolation, and photothermal destruction of human breast cancer cells.[72] Cy3-modified S6 aptamers were attached to magnetic/plasmonic nanoparticles through -SH linkage. In the multifunctional nanoparticles, gold plasmonic shells were used as both a photothermal agent and a nanoplatform; the magnetic core was used for cell isolation. The S6 aptamer-conjugated magnetic/plasmonic nanoparticles attached to the cancer cells due to the S6 aptamer-cancer cell interaction. The bioconjugated magnetic/plasmonic nanoparticles highly selectively bind to the cells, which can be used for targeted imaging and magnetic separation of a specific kind of cell from a mixture of different cancer cells. The photothermal destruction results showed a selective irreparable cellular damage to most of the cancer cells, due to the absorption of $670 \mathrm{~nm}$ continuous NIR irradiation of the hybrid nanostructures.

Sun et al.[10] was achieved the thermolysis of $\mathrm{Fe}(\mathrm{CO})_{5}$ on the surface of the Au nanoparticles in octadecene to produce $\mathrm{Au}-\mathrm{Fe}_{3} \mathrm{O}_{4}$ dumbbells followed by coupling with Herceptinand $\mathrm{Pt}$ complex. The cisplatin complex was linked to $\mathrm{Au}$ surface by reacting Au-S$\mathrm{CH}_{2} \mathrm{CH}_{2} \mathrm{~N}\left(\mathrm{CH}_{2} \mathrm{COOH}\right)_{2}$ with cisplatin. The coating of Herceptin antibody on $\mathrm{Fe}_{3} \mathrm{O}_{4}$ particles was performed by PEG3000-CONH-Herceptin. Cisplatin-Au-Fe3 $\mathrm{O}_{4}$-Herceptin nanohybrids were tested to HER2-positive breast cancer cells and HER2-negative breast cancer cells. The

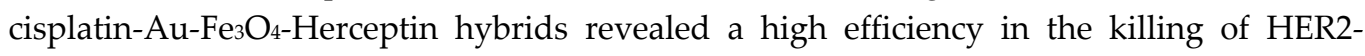
positive cancer cells. The presence of the hollow inside the nanohybrids was witnessed as an efficient carrier for targeted delivery and controlled release of cisplatin. These materials illustrated the possibility of acting as a multifunctional platform for target-specific platin delivery. Besides, other plasmon-magnetic materials of $\mathrm{FePt} / \mathrm{Fe}_{2} \mathrm{O}_{3}$ yolk-shells[6] and mesoporous silica-coated $\mathrm{Au}-\mathrm{Fe}_{3} \mathrm{O}_{4}$ core shells[73] were also synthesized and extensively studied for strong MRI contrast agent enhancement and carriers for anticancer drug delivery. 


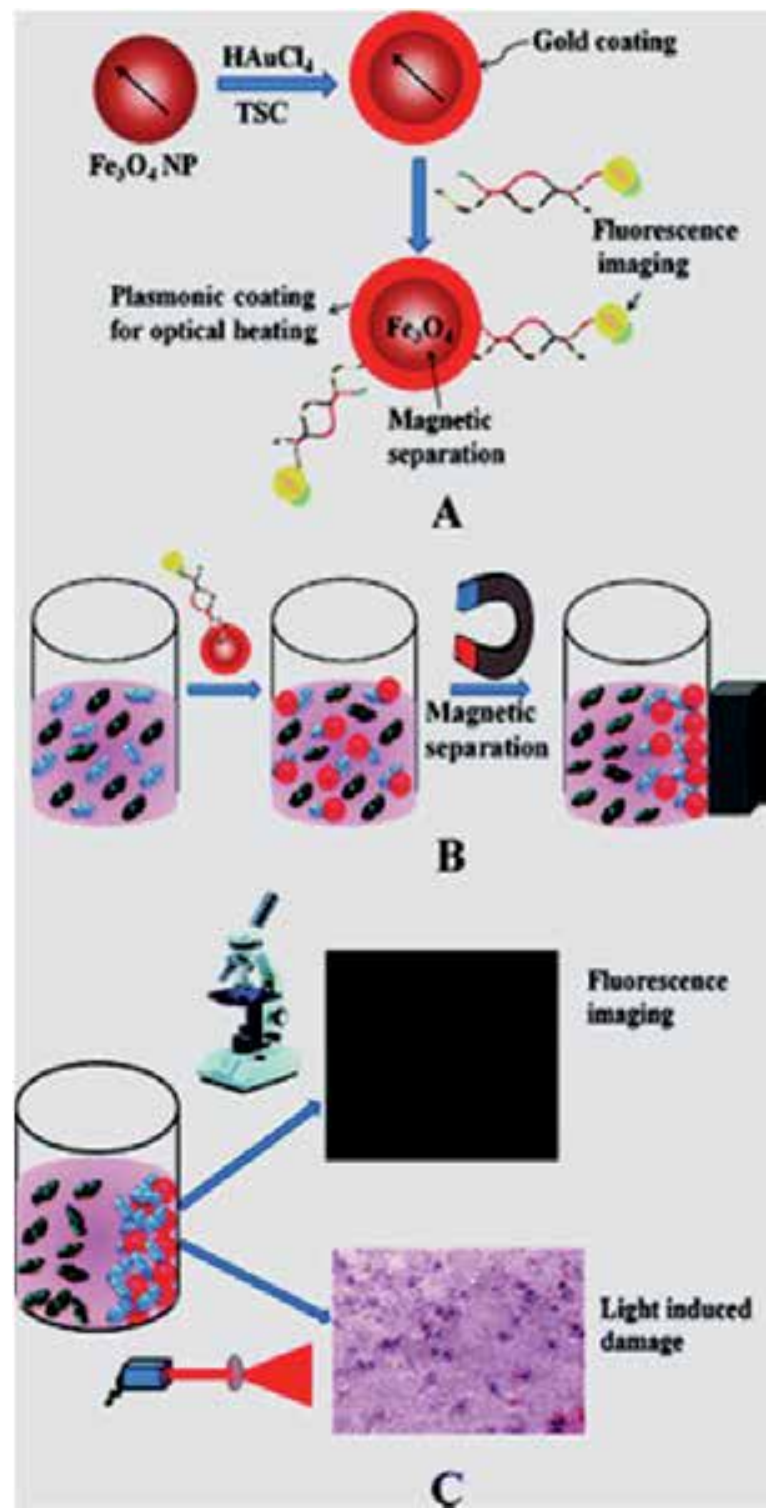

Figure 8. Plasmonic-magnetic $\mathrm{Au}-\mathrm{Fe}_{3} \mathrm{O}_{4}$ shell cores for targeted diagnostics, isolation, and photothermal destruction of tumor cells. (a) Scheme showing the synthesis of S6 aptamer-conjugated core shells. (b) Scheme showing the separation of breast cancer cells using S6 aptamer-conjugated plasmonic-magnetic nanoparticles. (c) Scheme showing the selective fluorescence imaging and targeted photothermal destruction of breast cancer cells. Reproduced with permission from ref. [72]. Copyright 2012, American Chemical Society.

\subsection{Fluorescent-magnetic hybrid nanostructures}

In comparison with conventional organic dyes and fluorescent proteins, the quantum dots have unique optoelectronic properties with size-tunable light emission, superior signal 
brightness, resistance to photobleaching, and broad absorption spectra for simultaneous excitation of multiple fluorescence colours. In order to establish the utility of the quantum dots for biological sensing application, the quantum dots are injected into desired cells of animals (e.g., mouse), emitted green and red labels are spectrally resolved to the eye clearly under the excitation of a single light source by a laser scanning microscope. For example, Liu et al.[74] used the synthesized triantennary dendritic galactoside-capped $\mathrm{ZnS} / \mathrm{CdSe}$ nanohybrids as a hydrophilic, fluorescent, multivalent probe for detecting metastatic lung cancer cells. The water-soluble nanohybrids were selectively uptaken by lung cancer cells enriched with membrane-bound asialoprotein receptors. The results suggested the stronger interaction between polyhydroxylended nanohybrids in the membrane composition and cancer cells.

Nevertheless, the use of the quantum dots for biosensor application still has some limitations. Namely, the quantum dots are frequently emitted at UV and visible regions, meanwhile biological samples (water and tissues) also induce autofluorescence by the absorption of ultraviolet and visible light, possibly leading to an inexact diagnosis. Moreover, if the biological samples are prolonged exposure to UV radiation, it would cause the photo-damage and mutation. Therefore, the use of the quantum dots emitted in the near-infrared spectrum is an alternative approach for the imaging of tumour structures in vivo. The fluorescent emission peaks of these desired nanoparticles are in the low-energy NIR (800-1000 nm), distant from the typical UV-vis spectrum $(400-600 \mathrm{~nm})$ of tissue autofluorescence. This unique properties of the near-infrared quantum dots makes probes easily recognisable under near-infrared light, even in the tissues with high fluorescent background.

To overcome this barrier, one novel approach is based on the conjugation of two components in a fluorescent particle. The fluorescent emission peaks of the quantum dots could be shifted from ultraviolet to near-infrared region, arising from particle-particle interface. Because the quantum dots did not show the magnetism, consequently, the quantum dots coupled with superparamagnetic particles could provide the two-in-one multimodal fluorescent-magnetic nanohybrids, which could act as multi-targeting, multifunctional and multi-treating tools. The bifunctional magnetic-fluorescent nanoprobe allows for a preoperative diagnosis via MRI owing to the magnetic-fluorescent properties. They ally the high sensitivity and resolution of the fluorescence phenomenon to the high spatial resolution and noninvasiveness of MRI. Furthermore, the interaction between quantum dots and magnetic particles could result in NIR fluorescent emission of the nanohybrids.

Visual sorting and manipulation of tumor cells through using fluorescent-magneticbiotargeting multifunctional nanobioprobes were reported by Pang et al.[75] Avidinconjugated fluorescent $\mathrm{CdSe} / \mathrm{ZnS}$-magnetic $\gamma$ - $\mathrm{Fe}_{2} \mathrm{O}_{3}$ nanohybrids synthesized were used to perform detection and extraction of multiple types of cancer cells via high affinity between antigens and antibodies. Figure 9 presents the synthetic procedure for these materials. $\mathrm{CdSe} / \mathrm{ZnS}$ and $\gamma-\mathrm{Fe}_{2} \mathrm{O}_{3}$ were coupled together in the chloroform/butanol suspension containing hydrazine-treated poly-(styrene/acrylamide) copolymer. Avidin-coupled fluorescent-magnetic nanohybrids were then obtained by incubating aldehyde-containing 

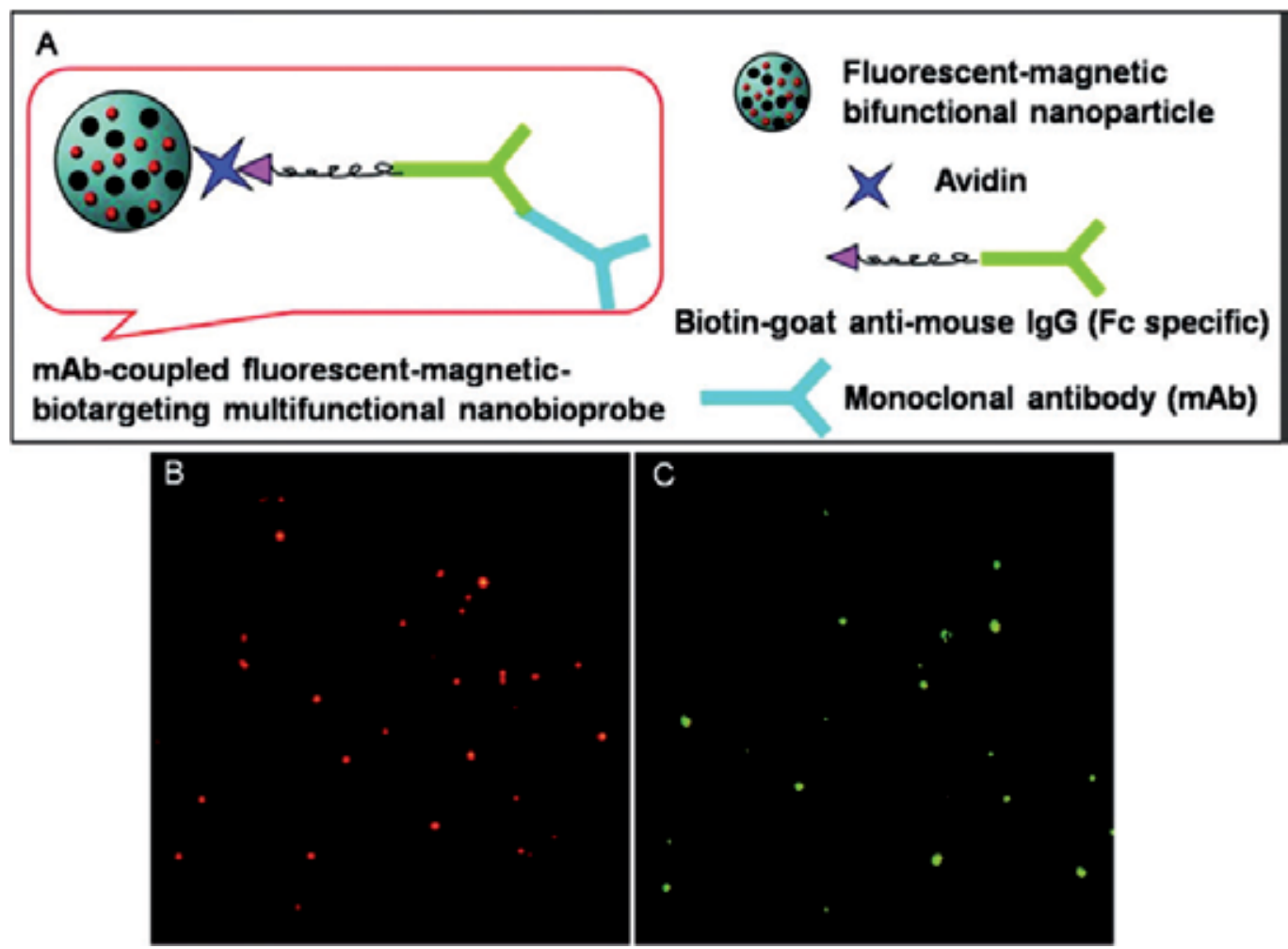

Figure 9. Fluorescent-magnetic biotargeting nanobioprobes for detecting and isolating tumor cells. (a) Scheme of avidin-coupled fluorescent-magnetic nanohybrids. Fluorescent-magnetic nanohybrids were covalently coupled with avidin and then coated with biotinylated goat anti-mouse IgG via biotin-avidin interaction. Mouse monoclonal antibody $(\mathrm{mAb})$ was then attached to the nanohybrids via binding to goat antibody; (b) Fluorescence microscopic images of anti-CD3 mAb-coupled red nanobioprobes; (c) Anti-prostate-specific membrane antigen mAb-coupled yellow nanobioprobes. Reproduced with permission from ref. [75]. Copyright 2011, American Chemical Society.

avidin with fluorescent-magnetic nanohybrids. The authors demonstrated that the avidincoupled fluorescent-magnetic nanobioprobes detected and extracted two different types of tumor cells from complex samples containing both normal cells and the target cancer cells and the capture efficiencies were of about $96 \%$ and $97 \%$, respectively. Upon exposing magnet and fluorescence microscopes, these multifunctional materials were sensitively detect and isolate target tumor cells at low concentration of $0.01 \%$ in the mixed cells. Shi et al. [76] synthesized fluorescent-magnetic nanospheres by co-embedding quantum dots and magnetite nanoparticles into hydrazide-functionalized copolymer nanospheres followed by coupled on the surface with IgG, avidin and biotin to form the fluorescent-magnetic bio-targeting trifunctional nanospheres. The nanoscale biocomposites can selectively link to apoptotic cells, allowing their visualisation and isolation. The fluorescent-magnetic particles were inert with respect to cell proliferation and tumour formation and served as both a negative contrast agent for in vivo MRI, as well as a fluorescent tumour marker for optical imaging in vivo and in vitro. The multifunctional capability of the nanocomposite nanoparticles as MRI and 
fluorescence imaging probes, along with their potential as drug delivery vehicles made them novel candidates for simultaneous cancer diagnosis and therapy.

The surface functionalization of the nanohybrids consisted of a polymer-coated maghemite superparamagnetic core and a CdSe/ZnS quantum dot shell, with anticycline E antibodies was permitted the separation of MCF-7 breast cancer cells from serum solution. The surface immobilised anticycline $\mathrm{E}$ antibodies bound specifically to cyclin, a protein which is expressed on the surface of breast cancer cells. The separated cells were monitored by fluorescence imaging microscopy, due to the strong luminescence of these nanohybrids.[42] An interesting external magnetic motor effect on floating cells, treated with fluorescentmagnetic nanocomposites, was reported by Lee et al.[77] In an another report, CdS:Mn/ZnS fluorescent-magnetic core-shells were prepared by using water-in-oil microemulsion. The peptide-conjugated fluorescent-magnetic core-shells possessing fluorescent, radio-opacity, and paramagnetic properties were used to label and visualise brain tissue without manipulating the blood-brain-barrier. The fluorescent visualisation of the whole rat brain was achieved using a simple low power handheld UV lamp, indicating that these materials are potentially applicable for advanced multimodal detection.[78]
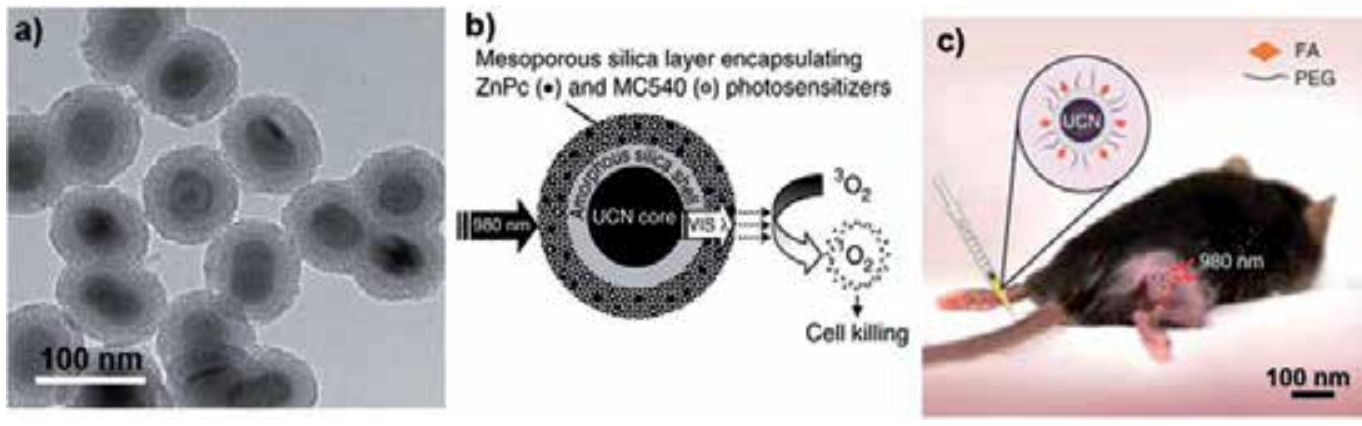

Figure 10. In vivo photodynamic therapy using mesoporous silica-coated $\mathrm{NaYF}_{4} \mathrm{Yb}$, Er upconversion fluorescent nanoparticles (UCNs) as remote-controlled nanotransducers. (a) TEM image of the mesoporous silica-coated UCNs, scale bar $=100 \mathrm{~nm}$. (b) Change in tumor size as a function of time after treatment to assess the effectiveness of UCN-based mediated targeted PDT in tumor-bearing mice intravenously injected with folic acid (FA)-PEG-UCNs. (c) Photograph showing showing UCN-based targeted conventional photodynamic therapy (PDT) in a mouse model of melanoma injected with UCNs surface modified with FA and PEG moieties. Reproduced with permission from ref. [82]. Copyright 2012, Nature.

One of the main problems in the preparation of the fluorescent-magnetic nanohybrids is the risk of quenching of the fluorophore on the surface of the particle by the magnetic core. This quenching process could be occurred because of the fluorophore contact with the particle surface, resulting in an energy transfer process. The problem of quenching can be partially resolved by coating of a stable shell (e.g., coating of a thin silica layer) on the magnetic nanoparticles prior to the introduction of the fluorescent molecules. As a proof-of-concept, Ying et al.[79] employed the silanization in a reverse microemulsion to produce a thin silica coating on the bare quantum dots or magnetic particles with surface $\mathrm{NH}_{2}$ groups. The 
silanized particles were conjugated to oleyl-O-poly(ethylene glycol)succinyl-Nhydroxysuccinimidyl ester through binding of the surface amine groups with heterofunctional polyethylene glycol. The biocompatible silica-coated can effectively target the cell membranes of HepG2 human liver cancer cells, NIH-3T3 mouse fibroblast cells, and 4T1 mouse breast cancer cells. These results demonstrated that these materials have potential for drug loading and delivery into cancer cells to induce cell death.

The biological applications of the down-conversion luminescent materials are currently restricted because they frequently emit at UV and visible regions, given the autofluorescence from biological tissues. These limitations would be breakthrough if we discover an alternate materials emitted in near-infrared (NIR)-to-vis upconversion.[80] This expectation was proved by Stucky et al.[81] who recently developed the new mesoporous multifunctional materials based on the combination of both up-converting luminescent and magnetic properties. Nanorattle hollow spheres consisted of the rare-earth-doped $\mathrm{NaYF}_{4}$ shells with a $\mathrm{SiO}_{2}$-coated $\mathrm{Fe}_{3} \mathrm{O}_{4}$ inner particle fabricated through ion-exchange process. The silica coating of the $\mathrm{Fe}_{3} \mathrm{O}_{4}$ nanoparticles were carried out by hydrolysis of TEOS in reverse-microemulsion system. The $\mathrm{Fe}_{3} \mathrm{O}_{4} @ \mathrm{SiO}_{2} @ \mathrm{Y}_{2} \mathrm{O}_{3} / \mathrm{Yb}, \mathrm{Er}$ magnetic upconversion oxide nanospheres were prepared by coating with the layer of $\mathrm{Y} / \mathrm{Yb}, \mathrm{Er}(\mathrm{OH}) \mathrm{CO}_{3} . \mathrm{H}_{2} \mathrm{O}$ via homogeneous precipitation in the aqueous solution of yttrium nitrate and urea and subsequent calcination at $550{ }^{\circ} \mathrm{C}$ for 2 h. The $\mathrm{Fe}_{3} \mathrm{O}_{4} @ \mathrm{SiO}_{2} @ \alpha-\mathrm{NaYF}_{4} / \mathrm{Yb}, \mathrm{Er}$ magnetic upconversion fluoride nanorattles were formed via ion-exchange of the $\mathrm{Fe}_{3} \mathrm{O}_{4} @ \mathrm{SiO}_{2} @ \mathrm{Y}_{2} \mathrm{O}_{3} / \mathrm{Yb}$, Er particles in $\mathrm{HF}$ and NaF solution. To demonstrate the material's potential use as a drug delivery system, the magnetic upconversion fluoride nanorattles were conjugated with antitumor drug doxorubicin. Through in vitro experiments in mice cells, the authors demonstrated that the material emits visible luminescence upon NIR excitation and can be directed by external magnetic field to specific target, making it an attractive system for targeted chemotherapy. Figure 10 shows the use of mesoporous silica-coated $\mathrm{NaYF}_{4}: \mathrm{Yb}$,Er upconversion fluorescent nanoparticles (UCNs) as a remote-controlled nanotransducer for photodynamic therapy reported by Zhang et al. ${ }^{[82]}$ The nanoparticle matrix can efficiently upconvert the energy of penetrating near-infrared light to visible light and transfer it to the encapsulated photosensitizers. The UCN materials exhibited a spectral overlap between the emitted visible light and the maximum absorption wavelengths of the photosensitizers to generate cytotoxic singlet oxygen in water. The authors discovered that the inhibition of tumor-cell growth in mice as a result of singlet oxygen generation from the UCNs, even at a very low 980-nm laser powers. This enabled selective fluorescent labelling, imaging and potentially sorting of the cells opening new prospects in cancer diagnostics and therapy.

\section{Conclusions and outlooks}

Most strategies of conjugating functional surfaced nanoparticles with biomolecule imaging agents produce antibody-conjugated particles that are considered as efficient biomedical diagnosis agents and universal platforms for engineering of multifunctional nanodevices. Inorganic nanohybrids containing more than one component have drawn considerable interest 
in materials science. In the multicomponent system, one can expect unique properties originated from collective interactions between the constituents. The outstanding understanding of how growth joining of two individual materials could allow for controlling the nanohybrid geometries with unexpected properties. In this Chapter, we review theranostic biomedical applications of diverse types of the colloidal inorganic nanohybrids synthesized using seed-mediated growth. The structure and shape of the nanohybrids are predominantly dependent on the crystal structure and lattice parameter of each component and seed-toprecursor ratio in heterogeneous growth. A major goal in nanomedicine is the coherent implementation of multifunctional platforms within a single targeted nanodelivery system, which would simultaneously perform diagnosis, targeted delivery and efficient therapy. With the goal of creating synergistic properties, multifunctional hybrid materials with combined plasmonic-magnetic, plasmonic-fluorescent, fluorescent-magnetic structures are reviewed. These hybrid structures were then functionalized with either silica or amphiphilic polymer layers to convert water-soluble particles compatible with biomedical applications, while retaining their individual functional characteristics. For theranostic biomedical applications, the water-soluble nanomaterials were conjugated with biomolecules (antibody, DNA, peptide). The resulting bioconjugated hybrid nanoparticles show to be viable not only as dualfunctional probes for imaging but also as an anticancer drug delivery vehicle even orientation of NIR excitation and external magnetic field. Through in vitro experiments demonstrated that these conjugated hybrid nanoparticles were actively delivered and targeted to the tumor sites in alive organs.

Since recent efforts on the synthesis of the multifunctional hybrid materials and studies them on animal species for theranostic biomedical applications, however it still presents major challenges for transferring into the clinical practices. Integrated collaborations of materials scientists with biologists and clinicians to be interdisciplinary teams should be established to systematic demonstrate and evaluate specific properties of the preformed hybrid nanomaterials, such as long-term toxicity, pharmacokinectics, and disinfection byproduct effects. In the future, the unexpected properties of these materials effective to human cancer cells are conducted by medical doctors, the multifunctional hybrid nanomaterials will provide powerful tools for simultaneous diagnosis and therapy of cancer diseases.

\section{Author details}

Thai-Hoa Tran

Department of Chemistry, Faculty of Sciences, Hue University, Hue 84054, Vietnam

Thanh-Dinh Nguyen*

Department of Chemical Engineering, Laval University, Quebec G1K 7P4, Canada

\footnotetext{
" Corresponding Author
} 


\section{Acknowledgement}

This work was supported by the National Foundation for Science and Technology development of Vietnam-NAFOSTED (No. 104.03-2012.54).

\section{References}

[1] Costi, R., A.E. Saunders, and U. Banin, Colloidal Hybrid Nanostructures: A New Type of Functional Materials. Angewandte Chemie International Edition, 2010. 49(29): p. 48784897.

[2] Banin, U., Nanocrystals: Tiny seeds make a big difference. Nat Mater, 2007. 6(9): p. 625-626.

[3] Mokari, T., et al., Formation of asymmetric one-sided metal-tipped semiconductor nanocrystal dots and rods. Nat Mater, 2005. 4(11): p. 855-863.

[4] Linic, S., P. Christopher, and D.B. Ingram, Plasmonic-metal nanostructures for efficient conversion of solar to chemical energy. Nat Mater, 2011. 10(12): p. 911-921.

[5] Yiu, H.H.P., et al., Designed Multifunctional Nanocomposites for Biomedical Applications. Advanced Functional Materials, 2010. 20(10): p. 1599-1609.

[6] Gao, J., et al., Multifunctional Yolk-Shell Nanoparticles: A Potential MRI Contrast and Anticancer Agent. Journal of the American Chemical Society, 2008. 130(35): p. 1182811833.

[7] Glotzer, S.C., Nanotechnology: Shape matters. Nature, 2012. 481(7382): p. 450-452.

[8] Yang, J., et al., Fluorescent magnetic nanohybrids as multimodal imaging agents for human epithelial cancer detection. Biomaterials, 2008. 29(16): p. 2548-2555.

[9] Service, R.F., Nanotechnology Takes Aim at Cancer. Science, 2005. 310(5751): p. 1132-1134.

[10] Xu, C., B. Wang, and S. Sun, Dumbbell-like Au-Fes $\mathrm{O}_{4}$ Nanoparticles for Target-Specific Platin Delivery. Journal of the American Chemical Society, 2009. 131(12): p. 4216-4217.

[11] Sailor, M.J. and J.-H. Park, Hybrid Nanoparticles for Detection and Treatment of Cancer. Advanced Materials, 2012. 24(28): p. 3779-3802.

[12] Katz, E. and I. Willner, Integrated Nanoparticle-Biomolecule Hybrid Systems: Synthesis, Properties, and Applications. Angewandte Chemie International Edition, 2004. 43(45): p. 6042-6108.

[13] Kim, B.Y.S., J.T. Rutka, and W.C.W. Chan, Nanomedicine. New England Journal of Medicine, 2010. 363(25): p. 2434-2443.

[14] Piao, Y., et al., Wrap-bake-peel process for nanostructural transformation from [beta]-FeOOH nanorods to biocompatible iron oxide nanocapsules. Nat Mater, 2008. 7(3): p. 242-247.

[15] Lee, J.E., et al., Multifunctional Mesoporous Silica Nanocomposite Nanoparticles for Theranostic Applications. Accounts of Chemical Research, 2011. 44(10): p. 893-902.

[16] Reiss, P., M. Protière, and L. Li, Core/Shell Semiconductor Nanocrystals. Small, 2009. 5(2): p. 154-168.

[17] Zhang, J., et al., Nonepitaxial Growth of Hybrid Core-Shell Nanostructures with Large Lattice Mismatches. Science, 2010. 327(5973): p. 1634-1638.

[18] Shevchenko, E.V., et al., Gold/Iron Oxide Core/Hollow-Shell Nanoparticles. Advanced Materials, 2008. 20(22): p. 4323-4329. 
[19] Yu, H., et al., Dumbbell-like Bifunctional Au-Fes $\mathrm{O}_{4}$ Nanoparticles. Nano Letters, 2005. 5(2): p. 379-382.

[20] Choi, S.-H., et al., Simple and Generalized Synthesis of Oxide-Metal Heterostructured Nanoparticles and their Applications in Multimodal Biomedical Probes. Journal of the American Chemical Society, 2008. 130(46): p. 15573-15580.

[21] Wang, D. and Y. Li, One-Pot Protocol for Au-Based Hybrid Magnetic Nanostructures via a Noble-Metal-Induced Reduction Process. Journal of the American Chemical Society, 2010. 132(18): p. 6280-6281.

[22] Bao, J., et al., Bifunctional $\mathrm{Au}-\mathrm{Fe}_{3} \mathrm{O}_{4}$ Nanoparticles for Protein Separation. ACS Nano, 2007. 1(4): p. 293-298.

[23] Mokari, T., et al., Selective Growth of Metal Tips onto Semiconductor Quantum Rods and Tetrapods. Science, 2004. 304(5678): p. 1787-1790.

[24] Meyns, M., et al., Growth and reductive transformation of a gold shell around pyramidal cadmium selenide nanocrystals. Journal of Materials Chemistry, 2010. 20(47): p. 1060210605.

[25] Macdonald, J.E., et al., Hybrid nanoscale inorganic cages. Nat Mater, 2010. 9(10): p. 810815.

[26] Deka, S., et al., Fluorescent Asymmetrically Cobalt-Tipped CdSe@CdS Core@Shell Nanorod Heterostructures Exhibiting Room-Temperature Ferromagnetic Behavior. Journal of the American Chemical Society, 2009. 131(35): p. 12817-12828.

[27] Lee, J.-S., et al., "Magnet-in-the-Semiconductor" FePt-PbS and FePt-PbSe Nanostructures: Magnetic Properties, Charge Transport, and Magnetoresistance. Journal of the American Chemical Society, 2010. 132(18): p. 6382-6391.

[28] Kwon, K.-W. and M. Shim, $\gamma$-Fe2O3/II-VI Sulfide Nanocrystal Heterojunctions. Journal of the American Chemical Society, 2005. 127(29): p. 10269-10275.

[29] Tian, Z.-Q., et al., Core/Shell Structured Noble Metal (Alloy)/Cadmium Selenide Nanocrystals. Chemistry of Materials, 2009. 21(14): p. 3039-3041.

[30] Lin, S.-Y., et al., Two-Step Functionalization of Neutral and Positively Charged Thiols onto Citrate-Stabilized Au Nanoparticles. The Journal of Physical Chemistry B, 2004. 108(7): p. 2134-2139.

[31] Clarke, S.J., et al., Photophysics of dopamine-modified quantum dots and effects on biological systems. Nat Mater, 2006. 5(5): p. 409-417.

[32] Daou, T.J., et al., Effect of Poly(ethylene glycol) Length on the in Vivo Behavior of Coated Quantum Dots. Langmuir, 2009. 25(5): p. 3040-3044.

[33] Susumu, K., et al., Enhancing the Stability and Biological Functionalities of Quantum Dots via Compact Multifunctional Ligands. Journal of the American Chemical Society, 2007. 129(45): p. 13987-13996.

[34] Kim, M., et al., Super-Stable, High-Quality Feз $\mathrm{O}_{4}$ Dendron-Nanocrystals Dispersible in Both Organic and Aqueous Solutions. Advanced Materials, 2005. 17(11): p. 1429-1432.

[35] Yu, W.W., et al., Forming Biocompatible and Nonaggregated Nanocrystals in Water Using Amphiphilic Polymers. Journal of the American Chemical Society, 2007. 129(10): p. 28712879. 
[36] Wang, K.T., I. Iliopoulos, and R. Audebert, Viscometric behaviour of hydrophobically modified poly(sodium acrylate). Polymer Bulletin, 1988. 20(6): p. 577-582.

[37] Kairdolf, B.A., A.M. Smith, and S. Nie, One-Pot Synthesis, Encapsulation, and Solubilization of Size-Tuned Quantum Dots with Amphiphilic Multidentate Ligands. Journal of the American Chemical Society, 2008. 130(39): p. 12866-12867.

[38] Petruska, M.A., A.P. Bartko, and V.I. Klimov, An Amphiphilic Approach to Nanocrystal Quantum Dot-Titania Nanocomposites. Journal of the American Chemical Society, 2003. 126(3): p. 714-715.

[39] Luccardini, C., et al., Size, Charge, and Interactions with Giant Lipid Vesicles of Quantum Dots Coated with an Amphiphilic Macromolecule. Langmuir, 2006. 22(5): p. 2304-2310.

[40] Potapova, I., et al., CdSe/ZnS Nanocrystals with Dye-Functionalized Polymer Ligands Containing Many Anchor Groups. Angewandte Chemie International Edition, 2005. 44(16): p. 2437-2440.

[41] Arriagada, F.J. and K. Osseo-Asare, Synthesis of Nanosize Silica in a Nonionic Water-in-Oil Microemulsion: Effects of the Water/Surfactant Molar Ratio and Ammonia Concentration. Journal of Colloid and Interface Science, 1999. 211(2): p. 210-220.

[42] Yi, D.K., et al., Silica-Coated Nanocomposites of Magnetic Nanoparticles and Quantum Dots. Journal of the American Chemical Society, 2005. 127(14): p. 4990-4991.

[43] Han, Y., et al., Reverse Microemulsion-Mediated Synthesis of Silica-Coated Gold and Silver Nanoparticles. Langmuir, 2008. 24(11): p. 5842-5848.

[44] Yi, D.K., et al., Nanoparticle Architectures Templated by $\mathrm{SiO}_{2} / \mathrm{Fe}_{2} \mathrm{O}_{3}$ Nanocomposites. Chemistry of Materials, 2006. 18(3): p. 614-619.

[45] Koole, R., et al., On the Incorporation Mechanism of Hydrophobic Quantum Dots in Silica Spheres by a Reverse Microemulsion Method. Chemistry of Materials, 2008. 20(7): p. 25032512.

[46] Tartaj, P. and C.J. Serna, Synthesis of Monodisperse Superparamagnetic Fe/Silica Nanospherical Composites. Journal of the American Chemical Society, 2003. 125(51): p. 15754-15755.

[47] Li, Y., et al., Chemical Synthesis and Silica Encapsulation of NiPt Nanoparticles. The Journal of Physical Chemistry C, 2007. 111(29): p. 10747-10750.

[48] Yu, K.M.K., D. Thompsett, and S.C. Tsang, Ultra-thin porous silica coated silver-platinum alloy nano-particle as a new catalyst precursor. Chemical Communications, 2003(13).

[49] Nien, Y.-T., K.-M. Chen, and I.-G. Chen, Improved Photoluminescence of $\mathrm{Y}_{3} \mathrm{Al}_{5} \mathrm{O}_{12}: \mathrm{Ce}$ Nanoparticles by Silica Coating. Journal of the American Ceramic Society, 2010. 93(6): p. 1688-1691.

[50] Stöber, W., A. Fink, and E. Bohn, Controlled growth of monodisperse silica spheres in the micron size range. Journal of Colloid and Interface Science, 1968. 26(1): p. 62-69.

[51] Rogach, A.L., et al., "Raisin Bun"-Type Composite Spheres of Silica and Semiconductor Nanocrystals. Chemistry of Materials, 2000. 12(9): p. 2676-2685.

[52] Gerion, D., et al., Synthesis and Properties of Biocompatible Water-Soluble Silica-Coated CdSe/ZnS Semiconductor Quantum Dotst. The Journal of Physical Chemistry B, 2001. 105(37): p. 8861-8871. 
[53] Liz-Marzán, L.M., M. Giersig, and P. Mulvaney, Synthesis of Nanosized Gold-Silica Core-Shell Particles. Langmuir, 1996. 12(18): p. 4329-4335.

[54] Liu, S.H. and M.Y. Han, Synthesis, Functionalization, and Bioconjugation of Monodisperse, Silica-Coated Gold Nanoparticles: Robust Bioprobes. Advanced Functional Materials, 2005. 15(6): p. 961-967.

[55] Hsu, J.-C., et al., Silica nanohybrids integrated with CuInS $2 / \mathrm{ZnS}$ quantum dots and magnetite nanocrystals: multifunctional agents for dual-modality imaging and drug delivery. Journal of Materials Chemistry, 2011. 21(48).

[56] Shen, R., et al., Silane-Based Poly(ethylene glycol) as a Primer for Surface Modification of Nonhydrolytically Synthesized Nanoparticles Using the Stober Method. Langmuir, 2008. 24(19): p. 11189-11195.

[57] $\mathrm{Hu}, \mathrm{K} .-W$. ., et al., Fabrication of $\mathrm{Gd}_{2} \mathrm{O}\left(\mathrm{CO}_{3}\right)_{2} . \mathrm{H}_{2} \mathrm{O} /$ silica/gold hybrid particles as a bifunctional agent for MR imaging and photothermal destruction of cancer cells. Journal of Materials Chemistry, 2009. 19(15).

[58] Sotiriou, G.A., et al., Hybrid, Silica-Coated, Janus-Like Plasmonic-Magnetic Nanoparticles. Chemistry of Materials, 2011. 23(7): p. 1985-1992.

[59] Ge, J., et al., Hierarchical Magnetite/Silica Nanoassemblies as Magnetically Recoverable Catalyst-Supports. Nano Letters, 2008. 8(3): p. 931-934.

[60] Khlebtsov, B., et al., Nanocomposites Containing Silica-Coated Gold-Silver Nanocages and Yb-2,4-Dimethoxyhematoporphyrin: Multifunctional Capability of IR-Luminescence Detection, Photosensitization, and Photothermolysis. ACS Nano, 2011. 5(9): p. 7077-7089.

[61] Petros, R.A. and J.M. DeSimone, Strategies in the design of nanoparticles for therapeutic applications. Nat Rev Drug Discov, 2010. 9(8): p. 615-627.

[62] Lal, S., S.E. Clare, and N.J. Halas, Nanoshell-Enabled Photothermal Cancer Therapy: Impending Clinical Impact. Accounts of Chemical Research, 2008. 41(12): p. 1842-1851.

[63] Kennedy, L.C., et al., A New Era for Cancer Treatment: Gold-Nanoparticle-Mediated Thermal Therapies. Small, 2011. 7(2): p. 169-183.

[64] Erathodiyil, N. and J.Y. Ying, Functionalization of Inorganic Nanoparticles for Bioimaging Applications. Accounts of Chemical Research, 2011. 44(10): p. 925-935.

[65] Lim, Z.-Z.J., et al., Gold nanoparticles in cancer therapy. Acta Pharmacol Sin, 2011. 32(8): p. 983-990.

[66] Huang, X., S. Neretina, and M.A. El-Sayed, Gold Nanorods: From Synthesis and Properties to Biological and Biomedical Applications. Advanced Materials, 2009. 21(48): p. 4880-4910.

[67] Tran, T.-H. and T.-D. Nguyen, Controlled growth of uniform noble metal nanocrystals: Aqueous-based synthesis and some applications in biomedicine. Colloids and Surfaces B: Biointerfaces, 2011. 88(1): p. 1-22.

[68] Dixit, V., et al., Synthesis and Grafting of Thioctic Acid-PEG-Folate Conjugates onto Au Nanoparticles for Selective Targeting of Folate Receptor-Positive Tumor Cells. Bioconjugate Chemistry, 2006. 17(3): p. 603-609.

[69] Jain, P.K., et al., Noble Metals on the Nanoscale: Optical and Photothermal Properties and Some Applications in Imaging, Sensing, Biology, and Medicine. Accounts of Chemical Research, 2008. 41(12): p. 1578-1586. 
[70] Caravan, P., et al., Gadolinium(III) Chelates as MRI Contrast Agents: Structure, Dynamics, and Applications. Chemical Reviews, 1999. 99(9): p. 2293-2352.

[71] $\mathrm{Xu}, \mathrm{C}$. , et al., $\mathrm{Au}-\mathrm{Fe}_{3} \mathrm{O}_{4}$ Dumbbell Nanoparticles as Dual-Functional Probes. Angewandte Chemie, 2008. 120(1): p. 179-182.

[72] Fan, Z., et al., Multifunctional Plasmonic Shell-Magnetic Core Nanoparticles for Targeted Diagnostics, Isolation, and Photothermal Destruction of Tumor Cells. ACS Nano, 2012. 6(2): p. 1065-1073.

[73] Chen, Y., et al., Core/Shell Structured Hollow Mesoporous Nanocapsules: A Potential Platform for Simultaneous Cell Imaging and Anticancer Drug Delivery. ACS Nano, 2010. 4(10): p. 6001-6013.

[74] Chen, C.-T., et al., A Triantennary Dendritic Galactoside-Capped Nanohybrid with a $\mathrm{ZnS} / \mathrm{CdSe}$ Nanoparticle Core as a Hydrophilic, Fluorescent, Multivalent Probe for Metastatic Lung Cancer Cells. Advanced Functional Materials, 2008. 18(4): p. 527-540.

[75] Song, E.-Q., et al., Fluorescent-Magnetic-Biotargeting Multifunctional Nanobioprobes for Detecting and Isolating Multiple Types of Tumor Cells. ACS Nano, 2011. 5(2): p. 761-770.

[76] Wang, G.-P., et al., Biofunctionalization of fluorescent-magnetic-bifunctional nanospheres and their applications. Chemical Communications, 2005(34).

[77] Yoon, T.-J., et al., Multifunctional Nanoparticles Possessing A "Magnetic Motor Effect" for Drug or Gene Delivery. Angewandte Chemie International Edition, 2005. 44(7): p. 10681071.

[78] Santra, S., et al., Synthesis of Water-Dispersible Fluorescent, Radio-Opaque, and Paramagnetic CdS:Mn/ZnS Quantum Dots: A Multifunctional Probe for Bioimaging. Journal of the American Chemical Society, 2005. 127(6): p. 1656-1657.

[79] Selvan, S.T., et al., Synthesis of Silica-Coated Semiconductor and Magnetic Quantum Dots and Their Use in the Imaging of Live Cells. Angewandte Chemie International Edition, 2007. 46(14): p. 2448-2452.

[80] Melancon, M.P., M. Zhou, and C. Li, Cancer Theranostics with Near-Infrared LightActivatable Multimodal Nanoparticles. Accounts of Chemical Research, 2011. 44(10): p. 947-956.

[81] Zhang, F., et al., Mesoporous Multifunctional Upconversion Luminescent and Magnetic "Nanorattle" Materials for Targeted Chemotherapy. Nano Letters, 2011. 12(1): p. 61-67.

[82] Idris, N. M., Gnanasammandhan, M. K., Zhang, J., Ho, P. C., Mahendran, R., Zhang, Y., In vivo photodynamic therapy using upconversion nanoparticles as remote-controlled nanotransducers, Nature Medicine, 2012, 12, p. 1580-1585. 
Section 3

\section{Biomedical Image Processing}





\title{
Study of the Murine Cardiac Mechanical Function Using Magnetic Resonance Imaging: The Current Status, Challenges, and Future Perspectives
}

\author{
Christakis Constantinides \\ Additional information is available at the end of the chapter
}

http://dx.doi.org/10.5772/51364

\section{Introduction}

While cardiac mechanical function studies initially focused on large mammals and the human, the mouse emerged as the preferred animal species for such research in recent years [Collins 2003]. Despite the fact that evidence supports that bio-energetically and hemodynamically the mouse scales in a linear fashion with larger mammals and humans [Dobson 1995, Nielsen 1958], nevertheless, important physiological questions still remain [Kass 1998, Balaban 2001] on whether such a model is the most appropriate for extrapolation of conclusions to man [Schaper 1998, Balaban 2012]. With the complete characterization of the mouse and human genomes (a National Institutes of Health initiative) in 2002 and 2003 respectively [Collins 2003, Gregory 2002], a plethora of mouse studies emerged targeting the cardiovascular system in animals with genetic modifications [James 1998, Hoit 2001, Gehmann 2000, Ehmke 2003], marking the onset of the molecular physiology, proteomics, and (structural and functional) genomics era. Collectively, these studies [Milano 1994, Barbee 1994, MacGowan 2001] initially targeted six important areas of cardiac function including the: (a) excitation-contraction cascade; (b) the beta-adrenergic system; (c) the cytosolic/structural system and the cytoskeleton; (d) the extracellular matrix and its coupling to important cytosolic elements that assist the mechanical force generation or propagation; (e) molecules that determine spatial-temporal mechanical changes (due to differential gene expression, phosphorylation, or recruitment of fetal development gene programs); and ( $f$ ) the energetic-metabolic status of the muscle. Equally important in most of these studies was the non-invasive imaging of such animals for phenotypic and genotypic screening, often conducted under inhalational anesthesia [Erhart 1984, Hart 2001, Price 1980, Kober 2005, Constantinides_ILAR 2011]. 
Despite the existence of a plethora of cardiac functional techniques for characterization of mechanical structure, function and dysfunction, a parallel need exists for development of invasive and non-invasive tools and techniques to describe the left ventricular (LV) tissue material properties as these relate to the: (a) mechanical pumping function of the LV; (b) myocardial oxygen demand defining myocyte metabolic status; (c) coronary blood flow and its auto-regulation; (d) arhythmogenic risk; (e) cell-signaling pathways responsible for growth and remodeling during development and disease. Reinforcing basic physiology work, invasive catheterization experiments [Georgakopoulos 1998] have also allowed determination of inotropic and lusitropic cardiac status, while Magnetic Resonance Imaging (MRI) experimentation, methodologies and technology advances have facilitated migration of such work to a non-invasive imaging platform, with tremendous potential for future basic science and translational research.

Specifically, advances in MRI techniques (myocardial spin tagging [Zerhouni 1988, Axel 1989], DENSE [Aletras 1999], and harmonic phase imaging [Osman 1999]) have been introduced over the last decades to quantify cardiac function, allowing myocardial tracking, motion and strain quantification in normal and genetically engineered mice [Rockman 1991, Franco 1999, Brede 2001, Yang 2002, Engel 2004, Wilding 2005]. Critical to such work has been the validation of the underlying hypothesis of morphological and functional scaling from mouse to human (through consideration of global cardiac function, circulatory control, blood flow distribution, $\mathrm{Ca}^{2+}$ storage and cycling, myosin light chain distribution, and force frequency reserve), for comparative studies.

This chapter provides an overview of the major physiological issues and challenges for mouse MR imaging and discusses the most recent and major advances in conventional and new cardiac Magnetic Resonance imaging strategies, that ultimately allow quantification of motion, global, and regional cardiac function, strain, and elasticity, characterizing inotropic and lusitropic contractile function and dysfunction in humans and transgenic mice for image-based phenotyping.

Specifically, this chapter attempts a detailed reference to the mouse as a research model, focusing on its genetic background and homology with the human genome and to the developmental and morphological differences between mouse and man, thus addressing cellular and global organ similarities and differences. As a basic determinant of structure, cardiac functional differences are associated, justified by carefully-controlled indices that determine integrative physiological control and functional activities, including metabolism, perfusion, angiogenetic, collateral flow, and coronary reserve. The importance and impact of anesthesia for image-based phenotyping in patho-physiological status is addressed with brief references to the possible mechanisms and cellular and sub-cellular target sites of anesthesia action. The section is complemented with recent findings on heart rate variability (as a result of the widely used inhalational anesthesia use) under optimal anesthesia conditions using isoflurane and long term physiological stability elicited from the use of the balancing anesthetic, Nitrous Oxide.

A historical overview of the evolution of mouse cardiac MRI is also attempted (in direct correlation with the evolution and progress of human clinical cardiac MRI, radiofrequency 
(RF), and gradient technologies) based on recent work and advances in miniature RF coils for imaging, state-of-the-art imaging techniques and pulse sequences based on rectilinear and non-cartesian sampling schemes, including functional MRI, atlas-based approaches for morphology assessment and four-dimensional (4D) motional variability, as well as regional cardiac functional characterization and quantification methods, Displacement Encoding with Stimulated Echoes (DENSE) [Aletras 1999], Harmonic Phase (HARP) [Osman 1999, Kuijer 2001], tagging and advanced imaging techniques. MRI-based, accurate threedimensional (3D) and 4D surface and finite element mesh model extractions, in association with advanced segmentation - seed-based or semi-automatic - and registration techniques diffeomorphic or landmark-based, are shown to facilitate efficient mouse inter-strain cardiac hemodynamical comparisons of both right and left-ventricular chambers. Based on recently published DENSE human-mouse comparative studies, and findings from image-based regional functional quantifications, similar transmural motional patterns are observed in both species, lending additional support to long-standing hypotheses for the existence of allometric scaling in metabolism, energetics [Dobson 1995, Nielsen 1958, Phillips 2012], and mechanical function in mouse and man.

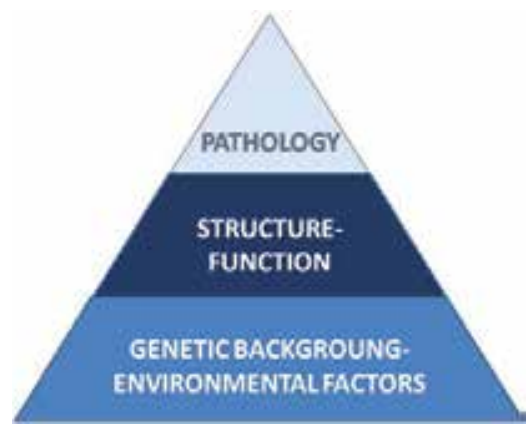

Figure 1. Hierarchical structure for studying human-animal pathology. Fundamental to the elucidation of pathological mechanisms is the detailed understanding of the structure and physiological function of tissues-organs as determined by their genetic background, or as influenced by the local environment.

\section{MRI of the mouse: Challenges for cardiac image-based mouse phenotyping}

\subsection{The mouse as a research model}

The mouse emerged as an attractive animal research model following the rapid advances in experimental molecular biology techniques that allowed targeted mutagenesis in single genes [Capecchi 1989], in addition to the tremendous success for extraction, manipulation, and use of embryonic stem cells [Koller 1989]. Practical and ethical advantages were also associated with mice, such as their stable genetic lines, immune system, short gestation periods, low cost, and ease of use. Initial research strides were supported by US National initiatives including the Human and Mouse Genome projects administered through the National Institutes of Health (NIH) for cloning and mapping the entire human and mouse genomes [Collins 2003, Gregory 2002], efforts that were successfully completed in 2003. 
Todate, multiple thousands of different knockout mice have been constructed (by individual Institutions, Laboratories, or National or International Consortia), most of which affect cardiovascular function. As attempts to match genotype to phenotype continue, an increasing number of knockout or loss-of-function mice is expected to be generated.

However, despite the usefulness, practicality, and low costs associated with the study of the mouse, important genetic, developmental, morphological, and physiological differences exist between mouse and man [Doevendans 1998, Schaper 1998]. The section that follows addresses succinctly common and distinctly differing features and functional differences in mouse and man and discusses the mechanisms of anesthesia effects on the mouse physiology and cardiac contractile machinery.

\subsubsection{Genetic background and gene homology}

Through various structural genomics attempts to map and compare human and mouse genomes [Schaper 1998, Gregory 2002], an overall homology of more than $85 \%$ and an identity of more than $80 \%$ were recorded [Schaper 1998], suggestive of the increased conservation during differentiation of the two species. Based on such findings, it is shown that the homeobox (hox) genes (transcription factors responsible for development), other transcription factors that bind to promoters encoding acute phase genes, and heat shock proteins, exhibit increased identity with humans. Based on Schaper et al. [Schaper 1998], however, structural genomics shows that (except primates) all other species (including mouse, rat, horse, bovine, etc) are equidistant from man, and evidence is thus inconclusive for the choice of the best species (based only on genetic homology and protein identity) for assessment of cardiovascular function and dysfunction.

\subsubsection{Developmental and morphological differences between mouse and human}

\section{Cardiac dimensions}

Indicatively, the human heart weighs around 250-300 $\mathrm{g}$ (left and right ventricular [RV, LV] and atrial chambers), and has an intrinsic rhythm of around 60-70 beats per minute (bpm), while the murine heart has a weight of $0.2-0.3 \mathrm{~g}$ and beats at a rate of $600-700 \mathrm{bpm}$. Additionally, the most evident external morphological difference between the adult mouse heart and the human heart is its shape and size (Figure 2). Developmental differences also exist; human development starts during the $3^{\text {rd }}$ week of gestation and lasts approximately 7 months to complete, while mouse cardiac development spans a little more than 2 weeks. At human birth, most of the cardiac organ development has been completed while in the infant mouse cardiac development may still be in progress.

\section{Cardiac anatomy}

No major differences exist in the outer morphology, ventricular structure, or valves. Noted differences exist, however, in atrial and venous parts. Specifically, the left superior caval vein drains directly into the right atrium in the mouse, whereas the pulmonary vein has an opening in the left atrium [Doevendans 1998], and a secondary atrial septum is lacking 
[Webb 1996]. While availability of data on the cardiomyofiber structure is scarce, a prior publication [McLean 1992] indicates some fiber orientation differences, primarily on the middle layer of the myocardium.

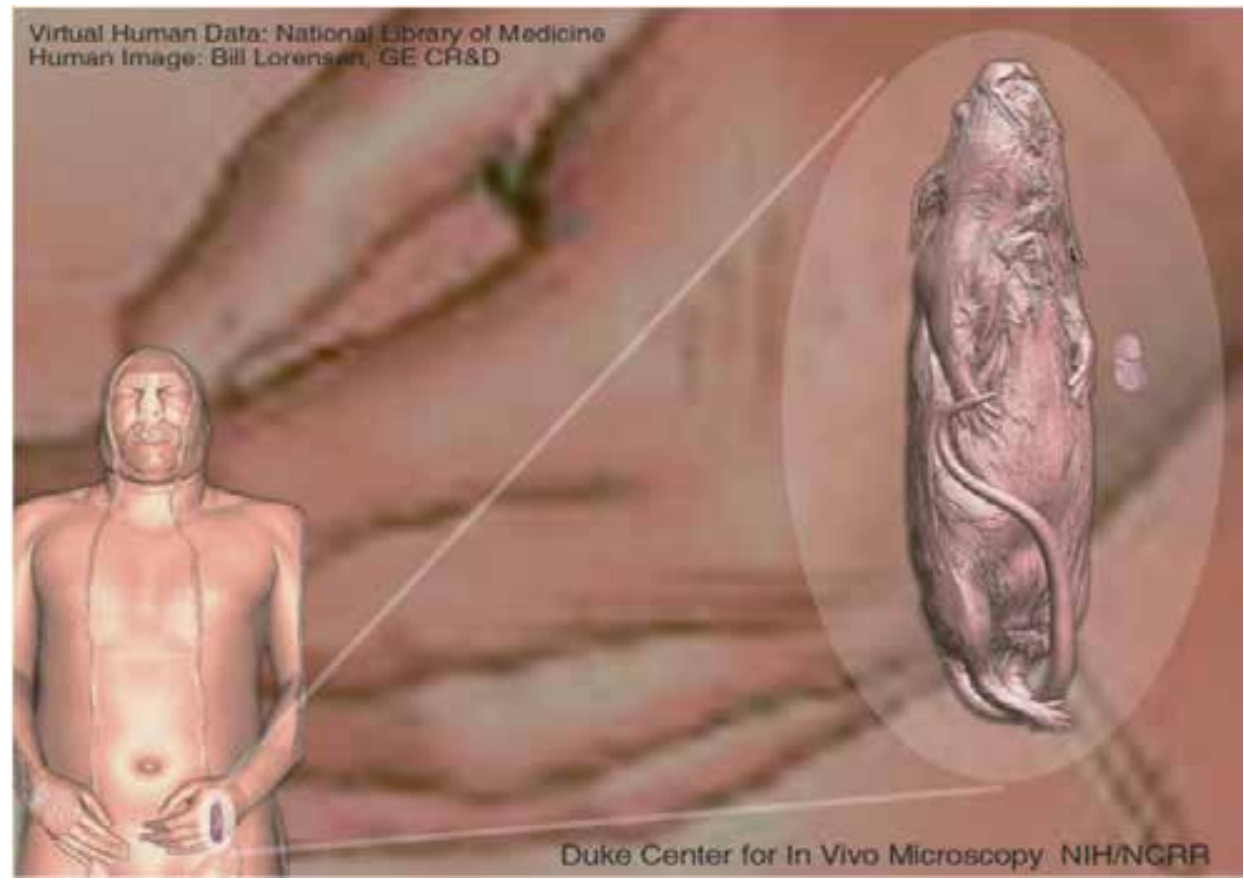

Figure 2. Relative size scales of the human, adult, and mouse embryo [Courtesy of the Duke Center for In Vivo Microscopy, an NIH/NIBIB National Biomedical Technology Resource Center (P41 EB015897)].

Prominent differences also exist for the conduction system and coronary anatomy. In contrast to the human where the sino-atrial (SA) node is distinctly located on the right atrium, in the mouse it lies on the superior vena cava (SVC), at the juncture of the SVC with the right atrium. Coronary anatomy exhibits branching of a septal coronary artery from the left coronary system supplying the left anterior ventricular wall, while the right coronary artery branches into a right coronary and a circumflex vessel supplying the posterior ventricular wall [Doevendans 1998].

\section{Cellular size and content}

Cardiomyocytes are reported to have lengths of approximately 80-100 $\mu \mathrm{m}$ along their major axis, and lengths of 10-20 $\mu \mathrm{m}$ along their minor axis [Doevendans 1998, Smaill 1991). Eightyfive percent $(85 \%)$ of the total number of cardiac cells (amounting to 7-10 million approximately) are interstitial while the remaining 15\% that represent cardiomyocytes occupy $90 \%$ of the available total ventricular tissue volume [Doevendans 1998]. Also important is an increased capillary to fiber ratio (of the order of 1.4) in the mouse, a direct necessity for the higher energetic and metabolic demands compared to rats, canine, or humans, (with a fiber ratio of the order of 1-1.1) [Sabbah 1995, Przylenk 1983, Olivetti 1989, 
Doevendans 1998]. The duration of the cell cycle (once G1 is entered) is reported to be almost the same in mouse and man [Schaper 1998].

\subsubsection{Functional physiological differences}

\section{Cardiovascular metabolism}

Energetic requirements in biological organisms are often assessed by their oxidative capacity, myosin ATPase and SERCA activities [Blank 1989] with a commensurate increased demand in small animals, such as rodents. In compensation of such increased energetic demand, an increased volume density of mitochondria is reported by Doevendans et al. [Doevendans 1998] in mice (37.9\%) compared to humans (25.3\%), with similar myofibril density (approximately 50\%). However, recent findings by Phillips et al. [Phillips 2012] argue in favor of a constant density of mitochondria across species (approximately 21\%), yet an increased enzymatic activity of mitochondrial enzymes in mice and a smaller dynamic range of metabolism is reported. Also of interest are the relative patterns of cardiac myosin chain isoforms $(\alpha \alpha, \beta \beta, \alpha \beta)$, a mouse $\alpha \alpha$-isoform compared to a human- $\beta \beta$ isoform dominance [Sheuer 1979], and a developmental switch in mice compared to humans, resulting in higher myosin ATPase activity. Noteworthy and more important is the increased basal activity of metabolic enzymes (Complex V) [Phillips 2012] and the smaller energetic reserve in mice compared to humans [Blank 1989, Phillips 2012], raising concerns for the appropriateness of the mouse as a proper model for comparative pharmacologic (doputamine, dipyridamole) stress studies in ischemia-infarction-reperfusion models, compared to human disease [van Rugge 1992, Wiesmann_Circ_Res 2001, Williams 2001].

Perfusion, angiogenesis-collateral flow, coronary reserve

Based on the extensive number of literature publications that focused on the elucidation of the basic principles of cardiovascular physiology (during the early and latter half of the $20^{\text {th }}$ century), including the autoregulation of blood flow in rats and canine [Feng 2001, Sandgaard 2002], important differences exist in mouse and man with regard to perfusion, capillary density [Stoker 1982, Rakusan 1994], total blood volume (2.5 $\mathrm{ml}$ in the mouse compared to $400-500 \mathrm{ml}$ in humans), relative distribution of blood flow in the capillary bed and redistribution capability subject to stimuli (temperature, anesthesia) [Barbee 1992, Rosenblum 1997, Sarin 1990, Constantinides 2011], angiogenetic capacity and formation of collateral vessels, as well as the resting coronary reserve, as factors that primarily project to ischemia-reperfusion studies (or other cardiovascular pathology models) and comparative analyses between pigs, canine, and man.

\section{In vivo cardiac function}

While direct comparative studies between mouse and man are still few, nevertheless published results favor similar hemodynamic and global cardiac functional indices, including blood pressure [Janssen 2002, Constantinides, 2011], inotropic (ejection fraction $[\mathrm{EF}]$, maximum developed ventricular pressure rates - $\mathrm{dP} / \mathrm{dtmax}$, stroke work $(\mathrm{SW})$, preload adjusted maximum power (PAMP), Preload recruitable stroke work (PRSW), end-systolic 
elastance (ES), and the end-systolic pressure volume relationship) and lusitropic (minimum developed ventricular pressure rates - $\mathrm{dP} / \mathrm{dtmin}$, Weiss and Glantz relaxation constants, and the end-diastolic pressure-volume relationship), left ventricular contractile indices [Constantinides_ABME 2011, Doevendans 1998]. Recent MRI findings also support similar torsional patterns, as reported by normalized (to left ventricular lengths) twist and torsional angles [Henson 2000, Zhou 2003, Liu 2006, Zhong 2010], however, limited and less steep responses in the force-frequency relationship-response of the mouse compared to humans [Stull 2002] refers to altered calcium kinetics [Stuyvers 1997] and handling under stress.

Integrative physiological control

In consideration of the various morphological and anatomical comparisons listed, the murine cardiovascular system (under normal physiological conditions) resembles relatively closely evoked responses in larger mammals. As mentioned, numerous cardiovascular indices in mice match corresponding values in rats and humans, predicted by allometric scaling laws.

At the integrative physiological level, the major blood pressure regulating systems, namely the baroreceptor reflex [Ma 2002] and the renin-angiotensin system (regulating electrolyte balance) [Cholewa 2001] seem to resemble closely those in larger mammals.

However differences do exist, including the increased sensitivity of blood pressure to anesthesia and temperature, especially under conditions of stress [Barbee 1992, Rosenblum 1997, Sarin 1990, Janssen 2004, Constantinides_ILAR 2011], higher resting cardiac sympathetic tone [Janssen 2000, Janssen 2002], neurotransmitter release [Ehmke 2003], and others.

\section{Allometric scaling of function, energetics, and metabolism}

Overall, under physiological conditions, evidence supports that bioenergetically and hemodynamically the mouse scales linearly with larger mammals and humans [Dobson 1995, Nielsen 1958, Phillips 2012], exhibiting a similar maximal aerobic capacity across species [Phillips 2012]. Preliminary evidence for allometric scaling to heart size in mechanical kinematic performance has also been presented [Popovic 2005, Zhong 2010] supported by preliminary comparative mouse-human results in this present work.

Given all considerations, extrapolations of inferences from mice to man is appropriate under physiological conditions, however, similar attempts in pathological models or states may be indeed risky.

\subsection{The impact of anesthesia for mouse studies}

Even if advances in telemetric techniques have allowed the pursuit of mouse studies under conscious conditions to a large extent, most of the research (terminal, invasive, or noninvasive imaging) studies utilize anesthetics. Anesthetics, however, are known to cause severe cardio-depression [Hart 2001] with adverse physiological effects on hormonal release, centrally to the heart and peripherally to the vasculature [Price 1980, Ohnishi 1974] at the cellular level, affecting calcium entry through L-type $\mathrm{Ca}^{2+}$ channels, the calcium 
binding sensitivity of the contractile proteins to calcium, on conduction and excitability, and possibly on other sarcoplasmic reticular sites [Price 1980]. Also prominent are effects on the central and peripheral nervous system, but most notable are effects on the metabolism (through mitochondrial vasomotor changes in coronary circulation and perfusion, vasodilation, and blood flow changes in the microvasculature [Kober 2005], possibly synergistic to Nitric Oxide), and the decoupling of oxidative phosphorylation manifested through myocardial oxygen consumption changes.

\subsubsection{Mechanisms of anesthesia action}

Linus Pauling [Pauling 1952] was one of the first scientists to attempt to explain the molecular and cellular mechanisms of action of anesthetics. Nevertheless, his proposed theory on clathrate formation post-anesthesia administration, proved inaccurate. With recent advances in molecular biology, new published evidence justifies the prominent role of the cellular chlorine channel as a mediator for anesthesia induction and maintenance [Brunson 2008, Maze 2008]. Specifically, hyperpolarizing chlorine channel currents lead to inhibition of cellular excitability and hypnotic action. It appears that one of the major hypnotic action centers is the locus coeruleus in the central nervous system, with mediatory action referred to the $\alpha_{2}$-adrenergic receptors via cyclic-AMP-mediated transduction pathways.

At the integrative physiological level, the effects of anesthesia target multiple cellular sites (Figure 3) and thereby often lead to cardio-depression. Major effects include their potency in inducing vasodilation of both the cerebral and coronary vasculature [Toyama 2004] leading to increased perfusion. A rapid hyperglycemic effect is often expressed (primarily via the sympathetic nervous system innervating the liver) causing immediate hormonal (catecholamine) release from the adrenal medulla [Durand 2009]. Glucose metabolic rates are also down-regulated (via inhibition of ATP synthesis in mitochondria), eventually leading to impairment of glucose tolerance (mediated via enzymatic protein activity in the liver). Also observed is an eventual oxidative phosphorylation decoupling.

\subsubsection{Types of anesthesia}

Widely used anesthetics for animal research are categorized into injectable (such as Ketamine/Xylazine, propofol, lidocaine, nembutal) and inhalational (such as isoflurane, halothane, and sevoflurane). Hedlund et al. [Hedlund 2008] and Brunson et al. [Brunson 2008] have published excellent summaries of the different types of anesthetics used for rodents to which the reader is referred.

\subsubsection{Basal physiology and maintenance}

While anesthesia usage causes physiological changes [Hildebrandt 2008], proper protocols and administration methodologies (type, dose) can achieve optimal conditions of animal study, maintain animal stability and homeostasis, and minimize time-induced accumulated 


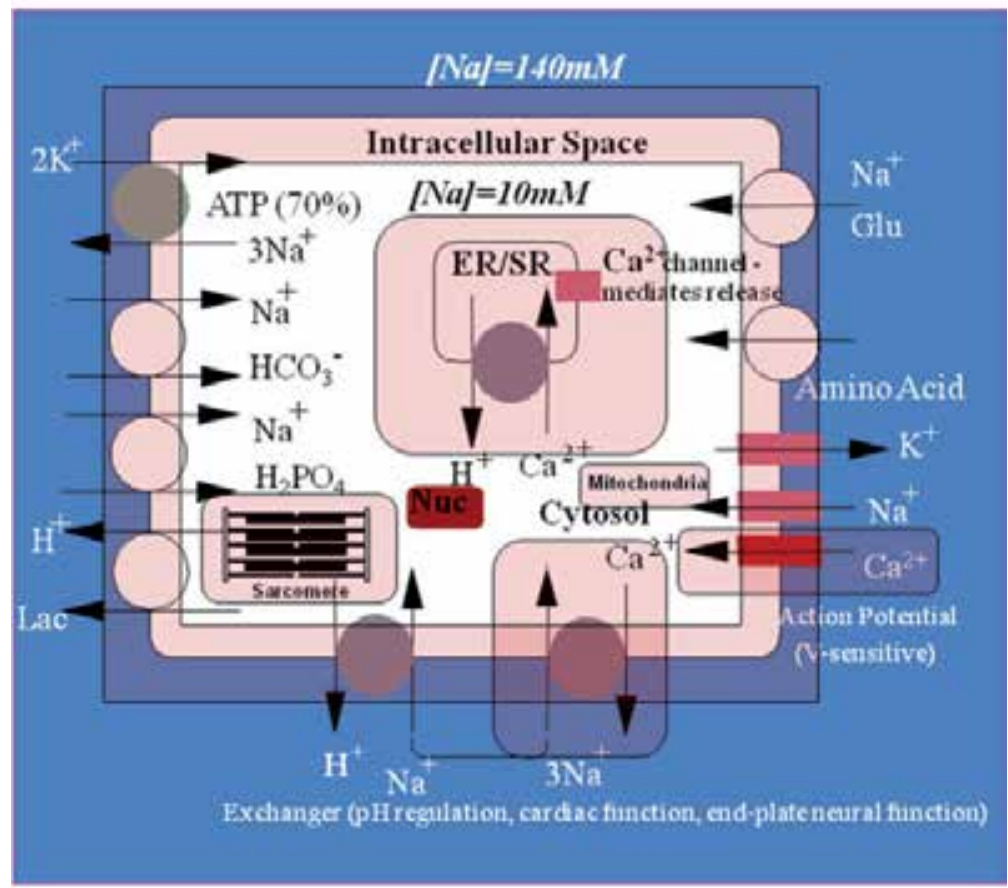

Figure 3. Schematic representation of a typical myocyte with indicative (highlighted) target sites of inhalational anesthesia.

effects, especially for prolonged imaging studies often exceeding 45 minutes in duration. Apart from physiological indices of hormonal release, respiration and metabolism, carefully controlled cardiac indices (such as heart rate, ejection fraction, arterial pressure, and heart variability) ensure proper conditions of study of the cardiovascular system avoiding detrimental hypotension-induced blood volume changes, metabolic and contractile downregulation, and arrhythmogenicity. Heart variability (HRV) analyses have also been applied for phenotypic screening of transgenic mice, study of heart rhythm mediators through signaling pathways as well as the effects of pharmacologic intervention on intrinsic heart rhythm and arrythmogenesis [Thireau 1997, Bernston 1997, Gehrmann 2000]. Standardization of HRV analyses tools for mice have, however, been limited due to the numerous data acquisition types and analysis techniques employed. Most importantly, variability of HRV is dependent on the type of anesthesia being used, that may also be further influenced by anesthetic balancing agents such as nitrous oxide $\left(\mathrm{N}_{2} \mathrm{O}\right)$, medical air, and oxygen. Interpretation of HRV results has also been difficult [Hoit 2004], due to their dependence on a number of factors including aging, posture, circadian variability, and the duration of the sampling periods used, with recent evidence supporting the fact that the primary contribution of autonomic activity in the mouse is due to the sympathetic tone, in contrast to the parasympathetic vagus contribution [Janssen 2002, Janssen 2000, Constantinides_IEEE 2010]. 

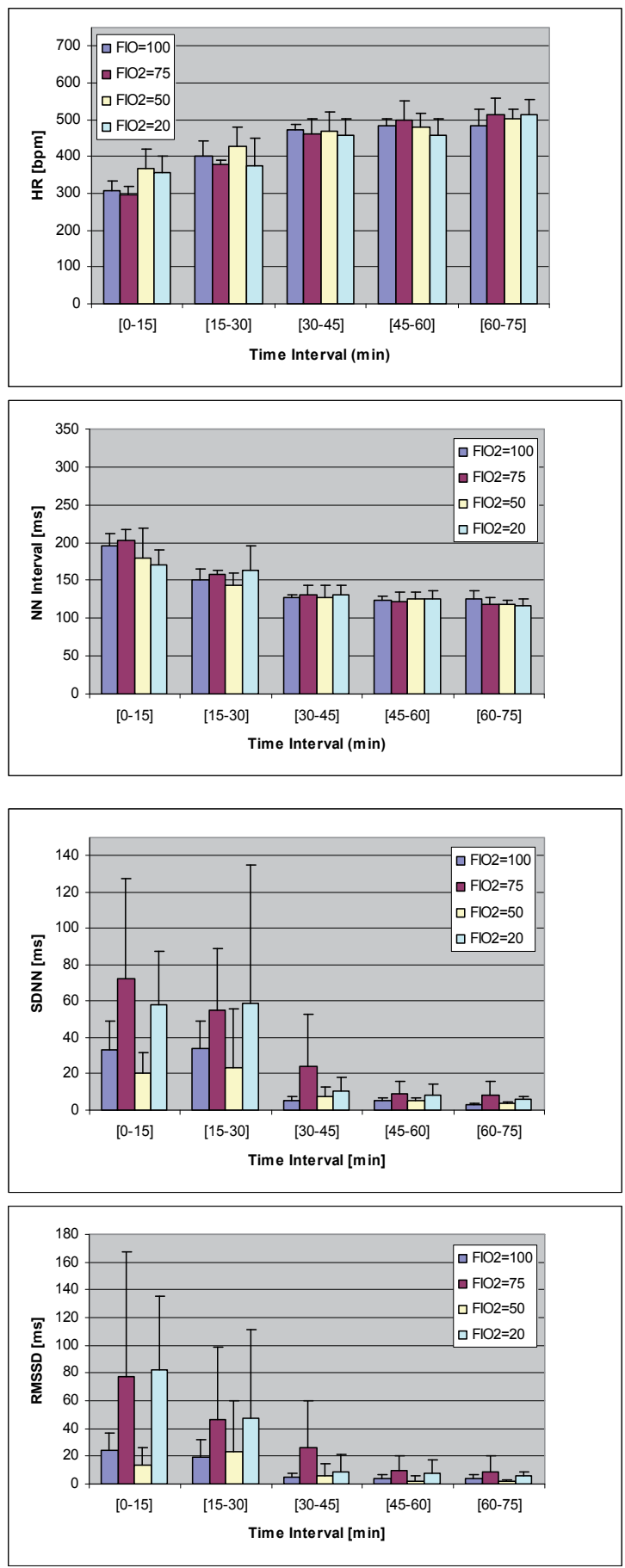

Figure 4. Heart rate variability indices at varying $\mathrm{FiO}_{2}$ values. (Top) $\mathrm{HR}$, R-R intervals (NN), (bottom) SDNN, and RMSSD variation. [Reproduced from Constantinides C et al. [Constantinides_IEEE 2010] with IEEE permission]. 
Despite such previous work, the extent of the contribution of sympathetic and parasympathetic tone to heart rate (HR) and HRV in the mouse is still a matter of debate. Recent, carefully controlled studies based on the level of anesthesia and additional factors that relate to animal preparation indicate that overall, HRV is expected to be generally lower during anesthesia in comparison to the conscious state. Indicative in HRV is an SDNN reduction under $\mathrm{FiO}_{2}$ conditions at $50 \%$ but also a noted increase in the standard deviation of averages of all normal R-R intervals (SDNN) and the square root of mean squared differences between adjacent normal R-R intervals (RMSSD) as a result of $\mathrm{N}_{2} \mathrm{O}$ administration. Variation of $\mathrm{FiO}_{2}$ seems to result in prominent effects only at specific levels (50,100\%). Since HRV indices are determined by an inter-play between both mechanical and neural factors, the exact mechanisms responsible for this effect are still unknown.
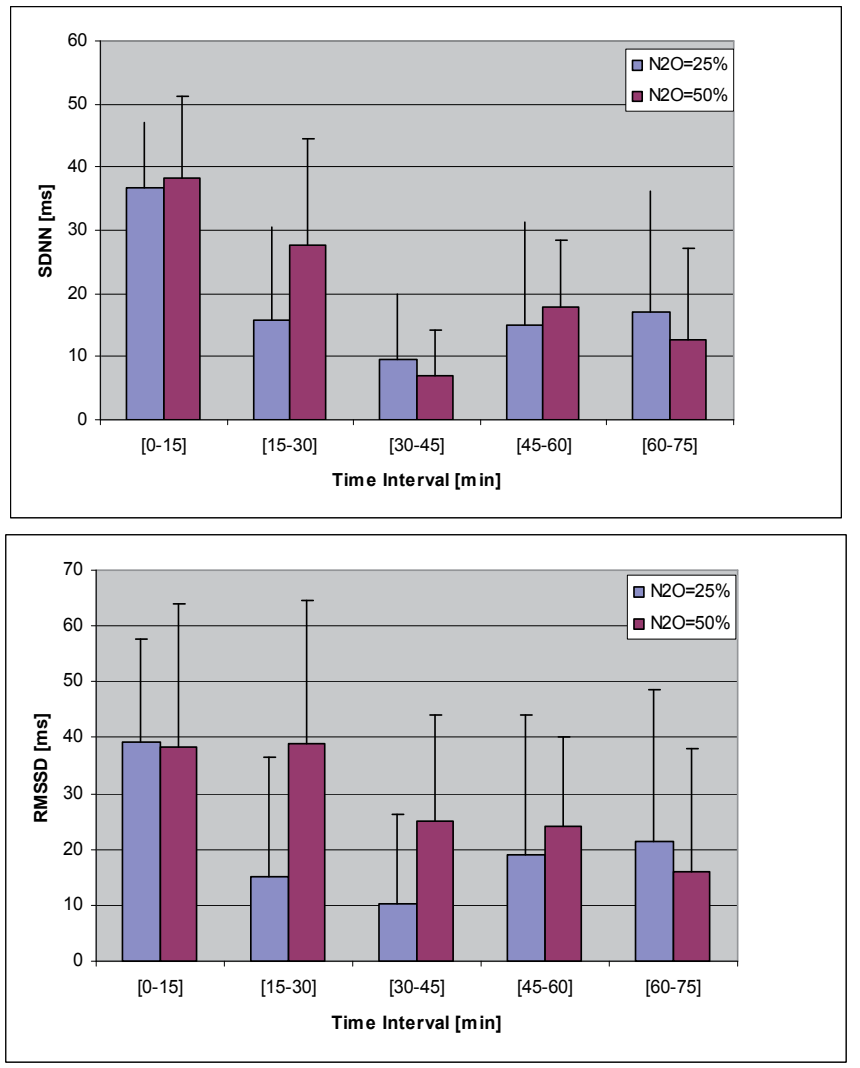

Figure 5. Heart rate variability indices with varying $\mathrm{N}_{2} \mathrm{O} / \mathrm{O}_{2}$ values $(25-75 \%$ and $50-50 \%)$ at different time intervals post-anesthesia induction. (Top) SDNN and (bottom) RMSSD variation ( $\mathrm{n}=3-6$ per group). [Reproduced from Constantinides et al. [Constantinides_IEEE 2010] with IEEE permission].

Overall, the major practical benefit is that the protocol described for physiological studies of mice under anesthesia has the potential for high reproducibility in diagnostic modalities including MRI, microCT, ultrasound, and microPET. Elicited results show that the optimal ISO anesthetic regimen for mice is a dose of approximately $1.5 \% \mathrm{v} / \mathrm{v}$ mixed with $25-50 \% \mathrm{O}_{2}$ 
and $75-50 \% \mathrm{~N}_{2} \mathrm{O}$. However, despite the optimization of murine physiological conditions under anesthesia, it is yet not possible from this study to determine whether the mechanism of action involves transient sympathetic activation, steroid release, a direct effect of ISO, or a combination of such effects. The overall effects of ISO may be the result of opposing vasodilatory and vasoconstrictive effects either directly (vasodilation) or secondary to an anesthesia-induced decrease of the heart's metabolic demand. Surely, the major limitation of all studies under anesthesia is that a noted cardio-depressive effect on basic cardiovascular function exists, compared to the conscious state.

\section{MRI cardiac imaging: The current status and future perspectives}

Mouse cardiac MRI emerged as a logical consequence to the human and mouse genome mapping initiatives and parallel developments and advances in human cardiac MRI in the late 1980's and early 1990's, focusing on establishing MRI as the 'one-stop-shop' in clinical practice. Correspondingly, technological advances and developments of novel hardware and pulse sequences were fairly limited, merely revolving on the scalability of existing technology to match smaller field-of-view acquisitions for mouse and rat imaging. Manning et al. [Manning 1990] and Shapiro [Shapiro 1994] first reported cardiac MRI-based LV mass estimations, and Siri et al. [Siri 1997] the first mouse cardiac imaging results from a $9.4 \mathrm{~T}$ system. Subsequent to such early studies were the first quantitative studies of function [Ruff 1998] and myocardial mass in diseased mice post-hypertrophy induction using the $\beta$ adrenergic agonist isoproterenol (ISO) [Slawson 1998]. Despite attempts early on to utilize conventional 1.5T clinical systems to conduct mouse cardiac MRI [Franco 1998], it was soon realized that dedicated, high field, high resolution mouse systems were necessary for imaging, a realization that drove technological evolution and migration to field strengths of 4.7-11.7 T systems [Wiesmann 1998].

Collectively, efforts over the last 14 years targeted the study of global [Ruff 1998, Wiesmann 1998, Wiesmann 2002, Schneider 2003, Zhou 2003] and regional cardiac function [Epstein 2002, Zhou 2003, Heijman 2004, Zhong 2010] in transgenic and wildtype-littermate control mice as a basis for image-based phenotyping [Wiesmann 2000, Wiesmann_Card_Magn_Reson 2001, Chuang 2010]. They also addressed models of disease (ischemia-infarction-reperfusion [Nahrendorf 2000, Ross 2002] and heart failure [Wiesmann 2002, Schneider 2003, Schneider 2004]). During the evolution of such prior attempts, noted advances in the mouse physiological status and its maintenance in high-field horizontal or vertical systems [Schneider 2003, Schneider 2004, Frydrychowicz 2007], and electrocardiographic (ECG) and respiratory gating strategies have been reported [Cassidy 2004, Bishop 2006, Hiba 2007, Heijman 2008, Bovens 2011] over the years. Other prominent work on mouse MRI also includes cardiac studies in imaging-based embryogenesis and development [Johnson 2002, Wiesmann 2000], perfusion [Kober 2005, Streif 2005], metabolism [Chacko 2000, Weiss 2002], coronary artery imaging [Ruff 2000], and stress testing [Williams 2001].

Only recently have efforts been completed to quantify the murine motional patterns and regional cardiac mechanical function [Zhou 2003, Streif 2005, Zhong 2010] towards the study 
of function and dysfunction using advanced techniques, including black-blood CINE [Berr 2005], spin-tagging [Epstein 2002, Liu 2006], DENSE [Zhong 2010], and HARP [Osman 1999, Kuijer 2001]. This section discusses some of the fundamental aspects of mouse preparation, positioning, physiology and its maintenance during MRI, advances in hardware and pulse sequence acquisitions, image processing techniques, and global and regional cardiac phenotyping, as these are complemented with recent findings from our group.

\subsection{Mouse physiology - Maintenance and stability during MRI}

Even if physiological protocols are easier to optimize on the bench, murine cardiac MRI imposes additional stringent challenges that relate to the animal's heart rate, pressure monitoring, and thermoregulation [Schneider 2004, Hedlund 2008, Constantinides_ILAR 2011]. While most research sites are equipped with commercial mouse imaging systems, a number of adjustments often need to be implemented to ensure proper physiological maintenance (including, but not limited to, computer controlled ECG, breathing, temperature monitoring systems and MR-compatible [fiber-optic and other] devices) [Figure 6]. Often specially designed negative-feedback air-flow systems are interfaced with closedbore scanner systems to facilitate fast and efficient bore air-heating, compensating for the lower bore temperatures due to the cryogenic environment of the magnet and gradient coils.

Proper mouse positioning almost always requires specially designed cradles to fit imaging probes (often constructed on specially designed casings that fit in fast-switching, high-slew rate gradient inserts) and scanner high-performance RF coil and gradient inserts.

Mouse cradle designs have evolved in complexity over the past 14 years and include those custom-made and commercially available types. Most of them are customized to allow mouse placement in prone and supine positions, placement of non-magnetic metallic or carbon-fiber ECG electrodes on the front paws and limbs, a rectal probe and an inflated air-bellow, for temperature and respiration monitoring [Figure 6]. Also of importance is the administration of anesthesia gases, often accomplished via a nose cone (with mice being obligatory nostril breathers), with tubing that connects to a vaporized-flowmeter device system (freely breathing [Schwartz 2000]), or directly to a ventilator device (achieving time-synchronous ventilation) as reported previously [Gilson 2005, Constantinides_ILAR 2011]. Special arterial or venous lines (tail vein, carotid vein or artery) are often adapted to allow pharmacologic infusions or arterial/ventricular blood pressure monitoring [Constantinides_ABME 2011]. Intraperitoneal injections are also preferred for anesthesia or contrast agent infusions.

Maintaining the mouse under optimal physiological conditions for a 60-90 minute MR imaging study, is a formidable and challenging task. Often, the HR value may be misleading as an indicative biomarker of proper physiological status (masking an underlying hypotension or cardiac contractile down-regulation [Constantinides_ILAR 2011]), especially after prolonged anesthesia exposure, excessive infusion of electrolytes (for blood volume maintenance), or following pharmacologic challenges. Therefore, care must be exercised in the initial induction, proper inhalational anesthesia administration, proper acclimatization of the animal to its imaging environment, and careful control and monitoring of its temperature and 
other critical physiological parameters (including, but not limited to HR, coefficient of variation $[\mathrm{CV}]$, mean arterial pressure $[\mathrm{MAP}]$, blood $\mathrm{pH}$, glucose, and insulin) maintaining homeostasis throughout the study as shown in Table 1 [Constantinides_ILAR 2011].

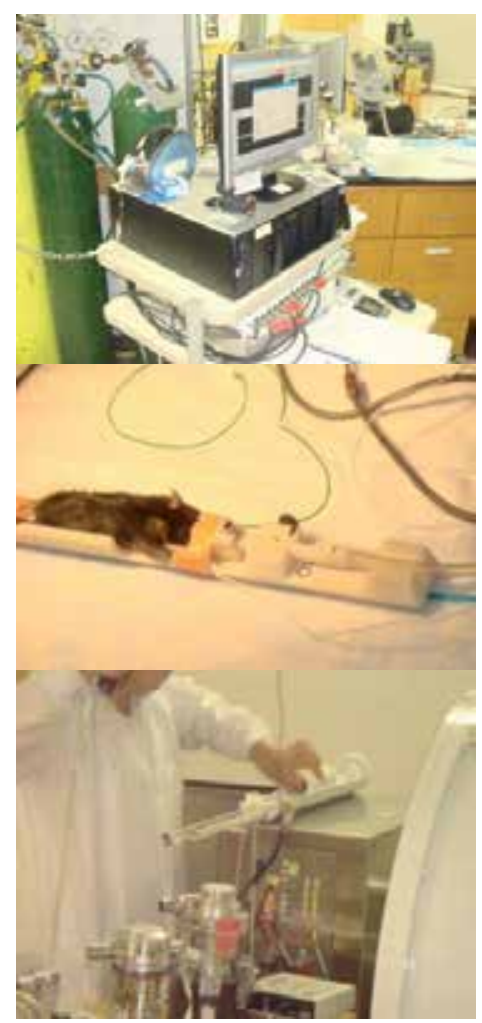

Figure 6. Physiological monitoring of mice during MRI using a dedicated recording system and the Labview software; (b) specially designed mouse positioning cradle, and (c) RF coil-mouse cradle assembly for a small-bore, high field animal scanner.[Photographs acquired from the Center for In Vivo Microscopy at Duke University Medical Center, an NIH/NIBIB National Biomedical Technology Resource Center].

\subsection{Advances in specialized hardware for high field animal MRI}

\subsubsection{High field magnets}

While gradient and RF coil technology spans numerous decades of history (as early as Paul Lauterbur's ground-breaking inception of zeugmatography [Lauterbur 1973]), the stringent technical requirements imposed by the mouse physiology for cardiac imaging have led to optimization of prior technology or to recent introductions of new technologies, as reviewed and discussed briefly in this section. Interestingly, large-bore high-field systems emerged during the past decade at field strengths spanning 4.7, 7.1, 9.4, and 11.7T maintaining, however, the same design technology as originally developed for human superconducting systems in the late 1980's and 1990's. 


\begin{tabular}{|c|c|c|c|c|c|c|c|c|c|}
\hline \multicolumn{2}{|c|}{$\begin{array}{l}\text { Anesthesia/Inhalati } \\
\text { on Mixture }\end{array}$} & \multirow[t]{2}{*}{$\begin{array}{c}\text { HR } \\
{[\mathrm{bpm}]}\end{array}$} & \multirow{2}{*}{$\begin{array}{c}\mathrm{CV}_{\mathrm{HR}} \\
\begin{array}{c}14+2 \\
(\mathrm{~N}=7, \mathrm{M})\end{array}\end{array}$} & \multirow[t]{2}{*}{$\begin{array}{c}\text { MAP } \\
{[\mathrm{mmHg}]} \\
\end{array}$} & \multirow{2}{*}{$\begin{array}{c}\mathrm{CV}_{\mathrm{MAP}} \\
13 \pm 3 \\
(\mathrm{~N}=7, \mathrm{M})\end{array}$} & \multirow[t]{2}{*}{$\begin{array}{c}\mathrm{t} R \mathrm{R} \\
{[\mathrm{ms}]}\end{array}$} & \multirow{2}{*}{$\begin{array}{c}\mathrm{pH} \\
7.3 \pm 0.1- \\
7.4 \pm 0.1 \\
(\mathrm{~N}=9 / 5, \\
\mathrm{M})\end{array}$} & \multirow{2}{*}{$\begin{array}{c}\begin{array}{c}\text { Glucose } \\
{[\mathrm{mg} / \mathrm{ml}]}\end{array} \\
176.3 \pm 26.1 \\
- \\
188.8 \pm 11.1 \\
(\mathrm{~N}=4 / 5 \\
\mathrm{M})\end{array}$} & \multirow{2}{*}{$\begin{array}{c}\begin{array}{c}\text { Insulin } \\
\text { [ng/ml] }\end{array} \\
\\
3.4 \pm 0.5- \\
4.2 \pm 0.9 \\
(\mathrm{~N}=4 / 5, \mathrm{M})\end{array}$} \\
\hline $\begin{array}{c}\mathrm{ISO}=1.0 \\
\%\end{array}$ & $\begin{array}{c}\mathrm{O}_{2}=100 \\
\%\end{array}$ & & & & & & & & \\
\hline $\begin{array}{c}\mathrm{ISO}=1.5 \\
\%\end{array}$ & $\begin{array}{c}\mathrm{O}_{2}=100 \\
\%\end{array}$ & $\begin{array}{l}478 \pm 11.4 \\
(\mathrm{~N}=7, \mathrm{M})\end{array}$ & $\begin{array}{c}11 \pm 1 \\
(\mathrm{~N}=7, \mathrm{M})\end{array}$ & $\begin{array}{c}92+2 \\
(\mathrm{~N}=7, \mathrm{M})\end{array}$ & $\begin{array}{c}8 \pm 2 \\
(\mathrm{~N}=7, \mathrm{M})\end{array}$ & $\begin{array}{c}114- \\
134 \mathrm{~ms} \\
(\mathrm{~N}=4, \mathrm{M})\end{array}$ & & & \\
\hline $\begin{array}{c}\mathrm{ISO}=2.0 \\
\%\end{array}$ & $\begin{array}{c}\mathrm{O}_{2}=100 \\
\%\end{array}$ & & $\begin{array}{c}14 \pm 3 \\
(\mathrm{~N}=7, \mathrm{M})\end{array}$ & & $\begin{array}{c}33+2 \\
(\mathrm{~N}=7, \mathrm{M})\end{array}$ & & $\begin{array}{c}7.3 \pm 0.1- \\
7.3 \pm 0.2 \\
(\mathrm{~N}=9 / 6, \\
\mathrm{M})\end{array}$ & $\begin{array}{c}139.4 \pm 30.1 \\
-192 \pm 11.9 \\
(\mathrm{~N}=4, \mathrm{M})\end{array}$ & $\begin{array}{c}3.54 \pm 0.3- \\
4.24 \pm 0.6 \\
(\mathrm{~N}=4, \mathrm{M})\end{array}$ \\
\hline $\begin{array}{c}\mathrm{ISO}=1.5 \\
\%\end{array}$ & $\mathrm{O}_{2}=75 \%$ & & $\begin{array}{c}10 \pm 2 \\
(\mathrm{~N}=7, \mathrm{M})\end{array}$ & & $\begin{array}{c}25 \pm 6 \\
(\mathrm{~N}=7, \mathrm{M}) \\
\end{array}$ & & & & \\
\hline $\begin{array}{c}\mathrm{ISO}=1.5 \\
\%\end{array}$ & $\mathrm{O}_{2}=50 \%$ & $\begin{array}{c}475+21.6 \\
(\mathrm{~N}=6 \\
\mathrm{M})\end{array}$ & $\begin{array}{c}9 \pm 1 \\
(\mathrm{~N}=6, \mathrm{M})\end{array}$ & $\begin{array}{c}90 \pm 13 \\
(\mathrm{~N}=6, \mathrm{M})\end{array}$ & $\begin{array}{c}6 \pm 2 \\
(\mathrm{~N}=6, \mathrm{M})\end{array}$ & $\begin{array}{c}108- \\
150 \mathrm{~ms} \\
(\mathrm{~N}=3-6, \\
\mathrm{M})\end{array}$ & & & \\
\hline $\begin{array}{c}\mathrm{ISO}=1.5 \\
\%\end{array}$ & $\mathrm{O}_{2}=20 \%$ & & $\begin{array}{c}8 \pm 1 \\
(N=5, M)\end{array}$ & & $\begin{array}{c}10 \pm 4 \\
(\mathrm{~N}=5, \mathrm{M})\end{array}$ & & & & \\
\hline $\begin{array}{c}\mathrm{ISO}=1.5 \\
\%\end{array}$ & $\begin{array}{c}\mathrm{O}_{2}=50 \% \\
\mathrm{~N}_{2} \mathrm{O}=50 \\
\%\end{array}$ & $\begin{array}{c}490 \pm 15.0 \\
(\mathrm{~N}=5 \\
\mathrm{M})\end{array}$ & $\begin{array}{c}10 \pm 2 \\
(\mathrm{~N}=5, \mathrm{M})\end{array}$ & $\begin{array}{c}93 \pm 3 \\
(\mathrm{~N}=5, \mathrm{M})\end{array}$ & $\begin{array}{c}6 \pm 3 \\
(\mathrm{~N}=5, \mathrm{M})\end{array}$ & $\begin{array}{c}114- \\
124 \mathrm{~ms} \\
(\mathrm{~N}=3-4, \\
\mathrm{M})\end{array}$ & & & \\
\hline $\begin{array}{c}\mathrm{ISO}=1.5 \\
\%\end{array}$ & $\begin{array}{c}\mathrm{O}_{2}=75 \% \\
\mathrm{~N}_{2} \mathrm{O}=25 \\
\%\end{array}$ & $\begin{array}{c}516 \pm 8.7 \\
(\mathrm{~N}=5 \\
\mathrm{M})\end{array}$ & $\begin{array}{c}7 \pm 2 \\
(\mathrm{~N}=5, \mathrm{M})\end{array}$ & $\begin{array}{c}94+3 \\
(\mathrm{~N}=5, \mathrm{M})\end{array}$ & $\begin{array}{c}10 \pm 3 \\
(\mathrm{~N}=5, \mathrm{M})\end{array}$ & & & & \\
\hline
\end{tabular}

Table 1. Summary of various cardiovascular and biochemical physiological indices of anesthetized mice using Isoflurane from bench studies. Stable mouse responses were obtained under both bench and scanner experimental conditions for periods spanning 1-1.5 hrs post-induction. [This table originally appeared in Constantinides C et al., Effects of Isoflurane Anesthesia on the Cardiovascular Function of the C57BL/6 Mouse, published in ILAR e-Journal 52(2), available online at $h$ ttp://delsold.nas.edu/ilar_n/ilarjournal/52_2/PDFs/v5202e-Constantinides.pdf].

\subsubsection{Gradient coil technology}

The requirements for increased spatial resolution acquisitions (of orders matching cellular size of approximately 100-200 $\mu^{3}$ ) led to fast switching, high-amplitude (up to $1000 \mathrm{mT} / \mathrm{m}$ ), high-slew rate (up to $11250 \mathrm{mT} / \mathrm{s}$ ) gradients (exhibiting linearity of better than $\pm 3-5 \% / \mathrm{mm}$ ) for ex-vivo constructions of atlases [Johnson 2002] or in-vivo high-resolution isotropic cardiac imaging [Bucholz 2008, Perperidis 2011]. Often associated with extra inserts (of approximately 6-20 cm in inner diameter), such gradients impose further spatial restrictions in animal placement and monitoring during cardiac imaging. However modern, commercially available systems are often equipped with actuator-controlled manual or computerized cradle positioning systems. 


\subsubsection{RF coils}

In a similar fashion to the evolution and migration of magnet and gradient technology from human to mouse applications, advances were noted in RF coils. Of all three hardware components (magnets, gradients, transmit/receive coils), RF coils received most of the attention of the research community for mouse cardiac MRI [Doty 2007]. Despite the use of simple resonant coil loops for cardiac studies early on, image-based phenotyping attempts [Wiesmann 2000, Wiesmann_Card_Magn_Reson 2001] redirected attention to highthroughput imaging and to the deployment and construction of multiple-mouse phased arrays [Bock 2003, Ramirez 2010]. Availability of multiple receivers, in combination with parallel imaging techniques (SENSE, GRAPPA), have reinforced attempts for highthroughput mouse acquisitions at high fields [Sosnovick 2007, Schneider 2010]. More recent developments of scaled-down birdcage coils have provided optimal imaging solutions for single mouse MRI as exemplified by direct comparative studies with simple surface [Markiewicz 2006], planar, and cylindrical spiral multi-turn coils (Figure 7) [Constantinides_Concepts 2011].

Traditionally, RF surface coils reduce the spatial coverage (and hence the field-of-view) compared to volume coils, while maintaining higher local signal-to-ratio (SNR). The limitation for expansion of the spatial region of surface coil coverage (while maintaining or improving SNR), yielded to phased array designs, often in combination with circularlypolarized volume transmit coils. Introduction of alternative coils to phased array designs, such as the one shown presents one of the first attempts to ameliorate such a surface coil limitation, in consideration of the small spatial scales, and the complexity and cost of phased arrays. Additionally, the use of such a surface coil is not prohibitive in terms of its concurrent use with dedicated and specially designed transmit (or receive coil arrays).

Specifically, imaging results showed improved performance of the cylindrical spiral coil in comparison to the flat counterpart. The cylindrical coil has increased field of penetration that allows visualization of the entire lateral and inferior myocardial walls with adequate relative SNR (rSNR). Its performance compares well and outperforms its flat equivalent in septal, inferior, anterior, and lateral myocardial areas (rSNR improvement between 27 and $167 \%$ ), despite its non-optimal placement and positioning on the mouse (anteriorly and laterally), in this particular case. Its design and response, that clearly exploits the additive effect of the transverse $\mathrm{B}_{1}$-field component, also compares favorably with a commercially available birdcage coil within the region where the mouse heart resides. However, the birdcage coil exhibits the best performance associated with three to five times higher rSNR values over the entire left ventricular myocardial regions. Promising are anticipated uses and applications of new hyperpolarized cryostat volume (birdcage) coils that have recently become commercially available [Kovacs 2005].

The fact that the constructed coils are transceiver surface resonators, certainly presents a conceptual and practical limitation for their wide-spread use, in the context of inhomogeneous excitation and their ability to allow quantitative cardiac mouse imaging. Direct use of such coils for high field mouse cardiac imaging is likely to result in increased 
loading effects between the RF and gradient coil inserts, imposing the need for use of specially constructed isolation shields. The inhomogeneous response of such coils, obviously translates to issues associated with artifacts, imperfect application of magnetization preparation pulses, or biased quantitative measures in cardiac functional and perfusion imaging. Furthermore, flip-angle sensitive techniques, such as fat saturation in cardiac MRI cannot be applied, due to the inherent variations in the flip angle spatial distribution, thereby limiting their potential applications.

\subsection{Conventional and new imaging methodologies for mouse cardiac imaging}

Similar to hardware developments numerous pulse acquisition schemes migrated from human cardiac applications to the mouse. Nevertheless, a number of new techniques emerged over the years that include cartesian (rectilinear) and radial (spiral, twisted projection, other) data acquisition schemes for fast mouse cardiac imaging. A brief review and analysis of the most-successful imaging and reconstruction practices (fast Fourier and non-uniform data regridding-Fourier transformations) is attempted below.

\subsubsection{Cartesian $k$-space pulse sequence acquisitions}

Two were the major types of Cartesian imaging acquisition techniques that migrated from prior human MRI efforts, extensively used for mouse cardiac imaging. They are both gradient echo sequences and adhere to lexicographic k-space or rectilinear sampling and are based on spin-warp imaging. The first, a steady state incoherent pulse sequence, was developed by Haase [Haase 1986] and became known as Fast Low Angle Shot and was dubbed with the acronym FLASH (equivalent to Spoiled Gradient Recalled Imaging, later developed by General Electric). The second type, a steady state coherent sequence known as Steady State Free Precession (SSFP) was developed early on by Carr [Carr 1958], employing ultra-short repetition times (TR) for imaging later adopted in an equivalent sequence with gradients by Hinshaw [Hinshaw 1976] and Frahm et al. [Frahm 1987], a sequence known as Fast Imaging with Steady Precession (FISP) [with the equivalent acronyms of Gradient Refocussed Acquisition at Steady State (GRASS) and balanced Fast Field Echo (B-FFE), as the GE's and Phillip's equivalent versions]. While SSFP was neglected for many years it regained tremendous interest over the last decade following advances in gradient technology in clinical scanners that eventually allowed ultra-short TR imaging.

For mouse cardiac imaging, segmented k-space acquisitions in an interleaved fashion are executed (to allow reconstruction of multi-frame images at spatial resolutions reaching 87 $\mu \mathrm{m}$ isotropic) based on a trigger pulse (prospective [Cassidy 2004, Bovens 2011], retrospective [Bishop 2006, Bovens 2011], or self-gated [Hiba 2007]), or a combination of the ECG and the respiratory [of order of 50-120 breaths/minute approximately] signal (thereby minimizing cardiac motion and respiratory artifacts). Navigator echoes may also be used prior to the gating pulse with a number of preparation pulses (pre-pulses) often executed immediately after the trigger pulse (Figure 8). Such optional pulses may include fat suppression and spectral-spatial pulses for black-blood imaging and/or fat suppression, as well as other prep-pulses including inversion, DENSE, spin-tagging and others. 


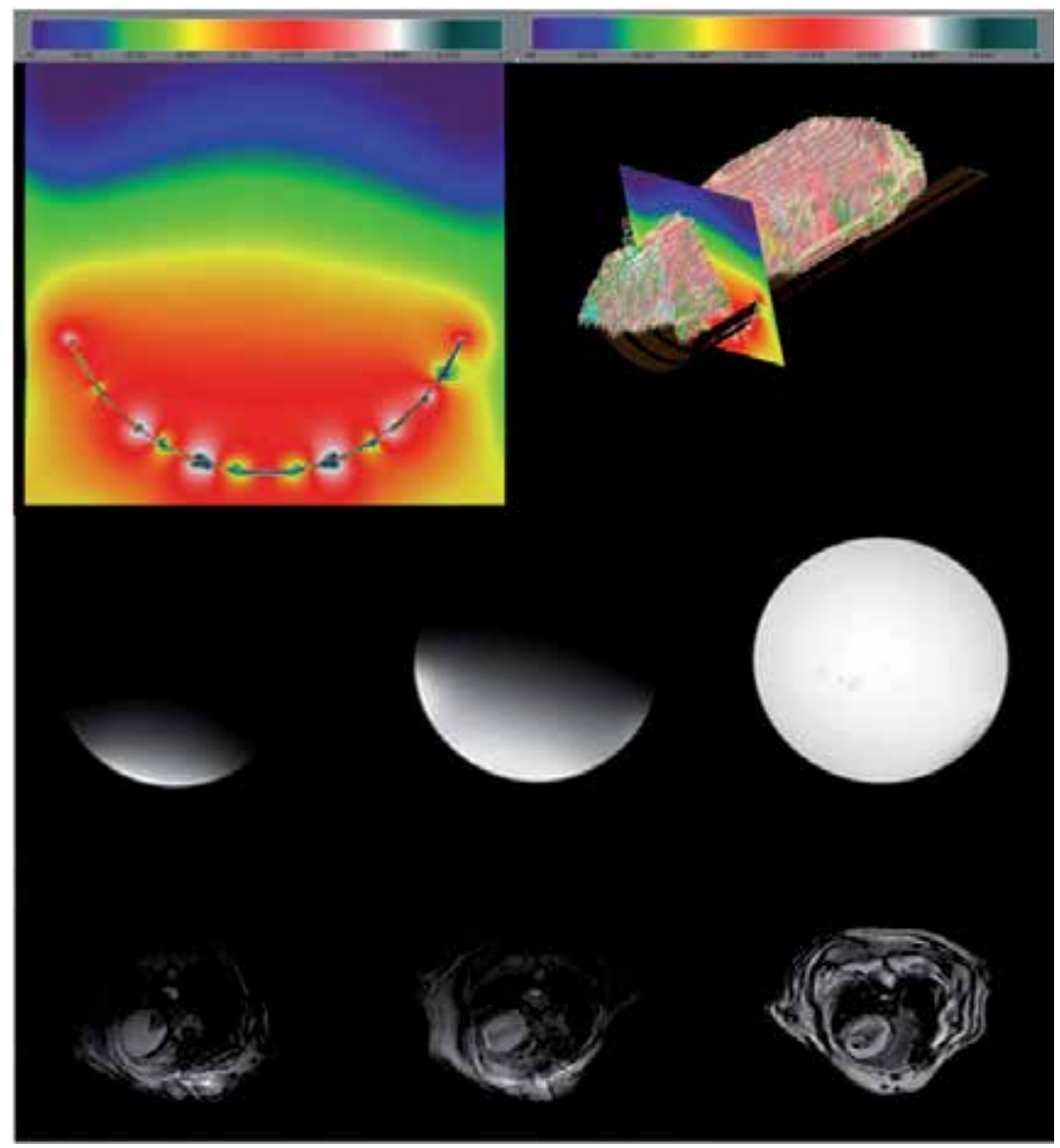

Figure 7. (Top, Left) Mouse XFdtd B1-field simulation of a four-spiral cylindrical coil under loaded conditions with a mouse computational model (top, right) [color bar varies between 0 - -40 $\mathrm{dB}]$;(Bottom) Flat, cylindrical spiral, and birdcage axial phantom response using conventional SPGR pulse sequence acquisitions and corresponding cardiac MRI from each of the three coils in mice postmortem showing improved performance by the birdcage coil. [This figure originally appeared in Constantinides $C$ et al., Intercomparison of performance of RF Coil Geometries for High Field Mouse Cardiac MRI. Concepts of Magnetic Resonance Part A, 38A(5):236-252, 2011, DOI 10.1002/cmr.a.20225, reproduced with permission from Wiley Publications].

\subsubsection{Spiral or non-cartesian $k$-space pulse sequences}

The ultrafast mouse heart rates, even under anesthesia, necessitate fast imaging acquisitions (within trR=100-120 ms). While Cartesian sampling schemes cover k-space adequately nevertheless, non-rectilinear and spiral k-space acquisitions have gained tremendous interest recently as efficient and fast sampling schemes. Radial acquisition variants by Bucholz et al. [Bucholz 2008], and STEAM-based DENSE encoding followed by interleaved spiral acquisitions proposed by Zhong [Zhong 2010] (Figure 9) are also adopted for mouse cardiac functional imaging. 


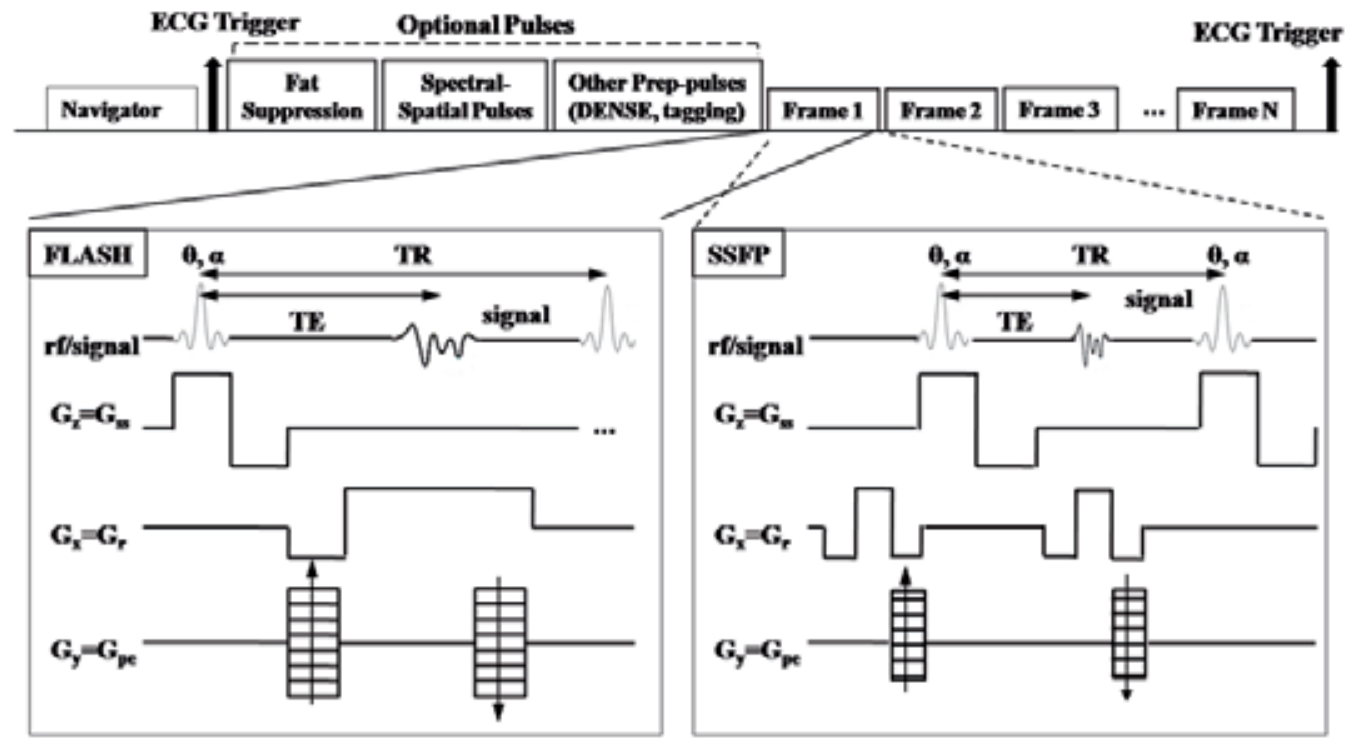

Figure 8. Rectilinear k-space sampling schemes employed for mouse cardiac imaging. (Left) FLASH imaging, and (right) SSFP. Shown on top are optional preparatory pulses and the inherent capability to perform multi-phase CINE acquisition based on the ECG (or a combined cardiac-respiratory) trigger pulse. $\left[G_{x}, G_{r}=\right.$ readout gradient, $G_{y}, G_{p e}=$ phase encoding gradient, $G_{z}, G_{s s}=$ slice selection gradient, $\theta=$ flip angle, $\alpha=$ RF pulse angle].

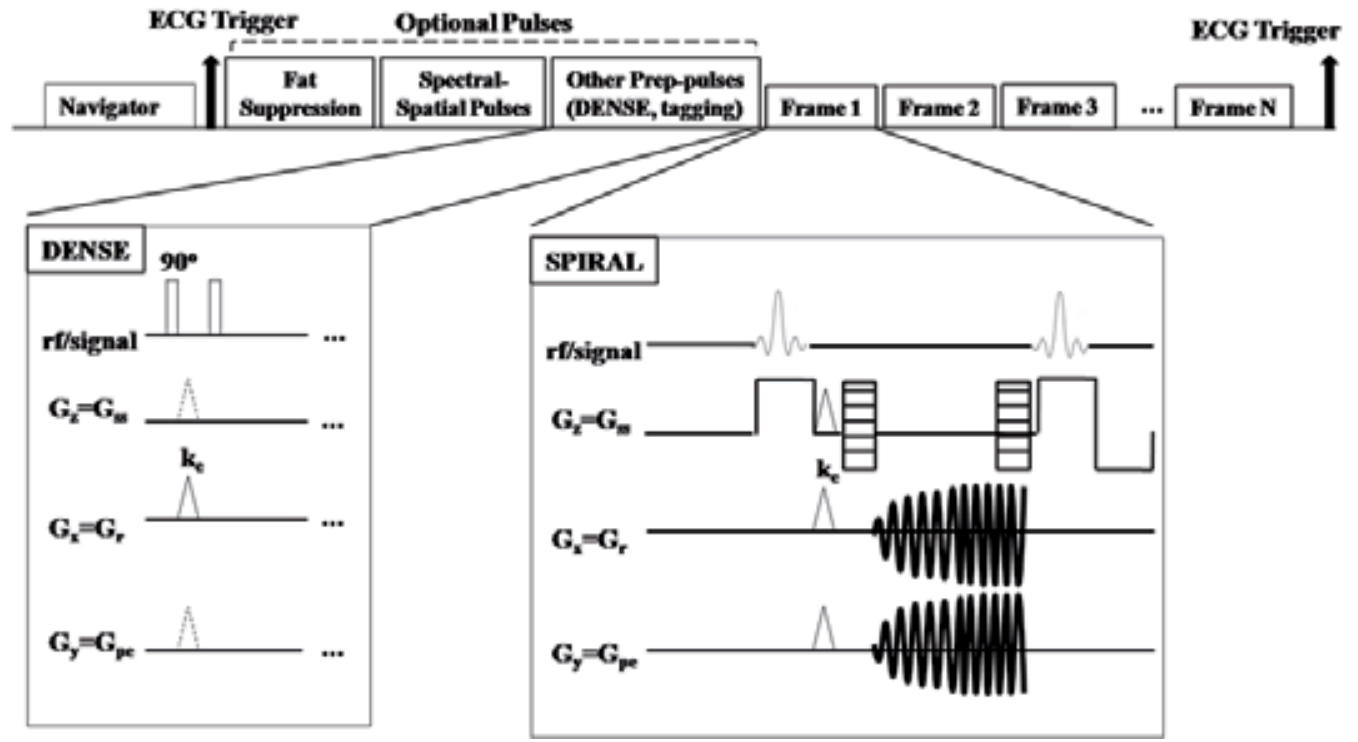

Figure 9. Non-rectilinear k-space sampling for cardiac imaging. Shown is a typical spiral readout scheme used in association with the DENSE (STEAM-based) preparatory pulses. (Left) ke represents the encoding pulse. The same pulse (un-encoding) is executed during the spiral readout (right). [Gx, $\mathrm{Gr}=$ readout gradient, $\mathrm{G}_{\mathrm{y}}, \mathrm{G}_{\mathrm{pe}}=$ phase encoding gradient, $\mathrm{G}_{\mathrm{z}}, \mathrm{G}_{\mathrm{ss}}=$ slice selection gradient, $\theta=$ flip angle, $\alpha=$ RF pulse angle]. 
In summary, numerous practical benefits are associated with mouse cardiac MR imaging, including the non-invasive nature of the technique, the inherent capability to map cardiac morphology and function, for both LV and RV chambers, and their motional patterns. High spatial and temporal resolution imaging can thus be achieved, through execution of highthroughput protocols, yielding direct, accurate estimates of global and regional indices of cardiac function, avoiding any assumptions whatsoever or model-based derivation approaches endorsed by other imaging techniques such as ultrasound.

Despite the extensive use of cartesian imaging with adequate SNR performance and spatial resolution (using FLASH, SSFP, or FISP), 3D acquisition studies maybe more efficiently completed using radial or spiral imaging sequences, especially for dynamic cardiac imaging (with pharmacologic interventions or contrast agent infusions). Nevertheless important and critical drawbacks are associated with such sequences, including the necessity to maintain data density as sampling extends to outer k-space regions, the convoluted and complex reconstructions (often associated with data re-gridding, kernel de-convolution, filtering, and inverse fourier transformation), and inherently lower SNR performance than rectilinear imaging. Thus, the choice between cartesian and radial imaging reduces to a tradeoff between SNR, spatial resolution, and efficiency of data sampling for 3D coverage.

\subsection{Image-based phenotyping}

Quantitative characterization of ventricular function has become important for the assessment of murine cardiac performance in heart disease [Wiesmann 2000, Wiesmann_Circ_Res 2001, Dawson 2004]. As the manipulation of the mammalian genome becomes routine, it is now possible to generate animal models to study cardiovascular function and dysfunction [Wiesmann_Card_Magn_Reson 2001, Epstein 2002, Epstein 2007, Bucholz 2008, Stuckey 2012]. Critical to successful phenotypic screening of mouse models of the cardiovascular system using MRI are highly efficient four-dimensional (4D) acquisition ex-vivo and [Zamyadi 2010] in-vivo protocols [Bucholz 2010]. Such protocols ought to span the scales of the embryo to the adult, fully-developed mouse, and ought to lead to the reduction of the computational image processing complexity for accurate quantification of motion, global and regional cardiac function, strain, elasticity, and others.

\subsubsection{Quantification of global mechanical function and hemodynamic indices}

Traditionally, quantification of global hemodynamic indices has been associated with calculation of hemodynamic indices of function. MRI and other diagnostic techniques, (including microCT and ultrasound) are not the only existing techniques to accomplish functional characterization. While ultrasonic techniques are associated with inherent limitations for such quantification [Dawson 2004], microCT has been gaining interest and popularity [Badea 2005]. Miniature catheterization techniques introduced in the late 1990s [Georgakopoulos 1998] have revolutionized cardiac-based phenotypic in transgenic mice. However, their major drawbacks include their invasive nature and other technical limitations [Porterfield 2009, Constantinides_IEEE 2011, Constantinides 2012]. As newly 
emerging techniques and applications develop to investigate in-vivo sarcomeric force generation with contrast agents [Constantinides_ABME 2011], the current scientific focus is still on image-based calculations of global indices of function.
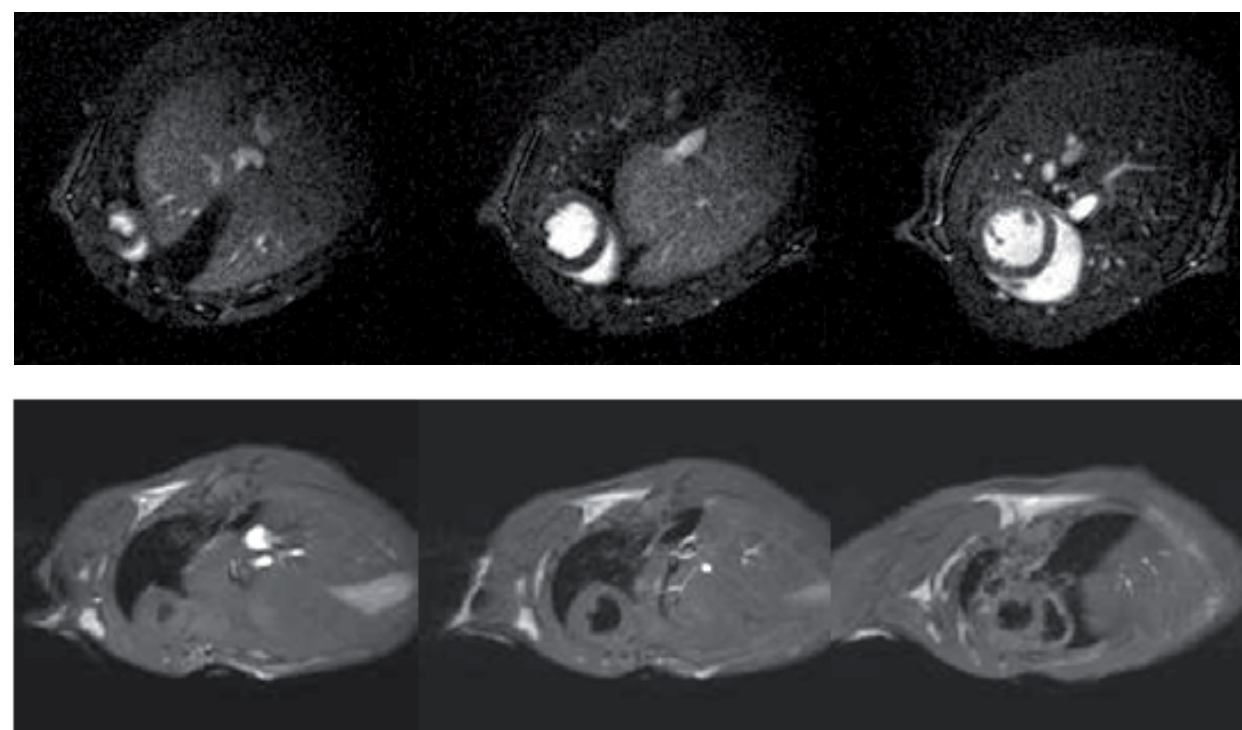

Figure 10. (Top) Typical bright blood true-short axis cardiac imaging at an apical, middle, and basal levels of the C57BL/6 murine heart using a liposomal contrast agent; (Bot) Black-blood spin-echo images of the murine heart at basal, mid, and apical levels.

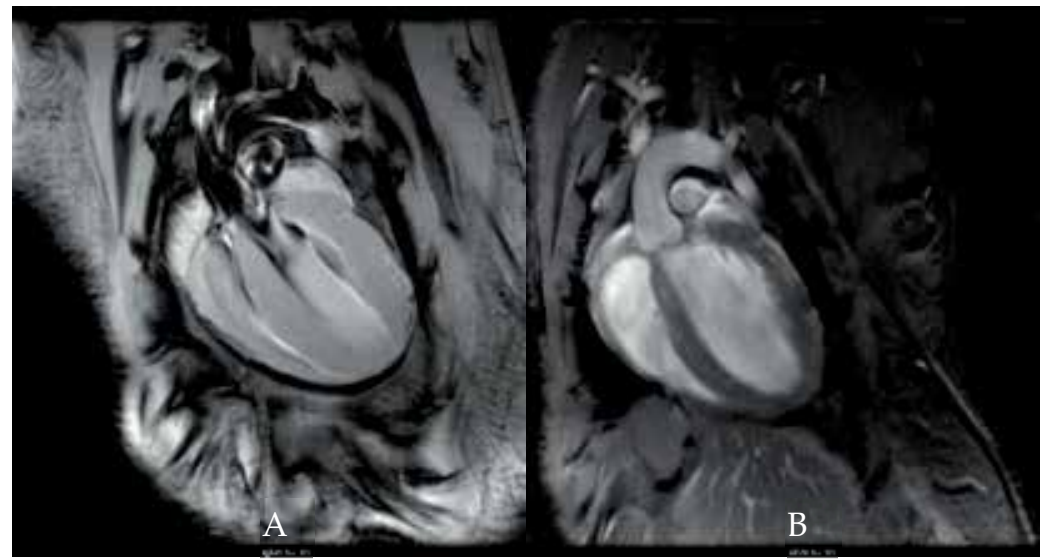

Figure 11. (A) ECG-gated four chamber view of a healthy mouse heart al $78 \times 78 \mu \mathrm{m}^{2}$ in-plane resolution, and (B) corresponding self-gated (retrospective) view of a separate mouse using a navigator echo at the same spatial resolution, at 11.7 T using FLASH-MRI [Images courtesy of Bruker Biospin MRI, Ettlingen, Germany].

Correspondingly, estimated cardiac volumes can easily be computed using standard image processing tools and converted to absolute volume units using the voxel dimension and the myocardial tissue density. Hemodynamic indices such as end-diastolic (EDV) and end- 
systolic volumes (ESV), stroke volume (SV), cardiac output (CO), and ejection fractions (EF) can now be routinely calculated according to standard cardiac mechanical functional relations [Constantinides_SBI 2009], based on CINE-MRI (Figure 11). CINE based local contractile function, including estimation of wall thickness, motion and fractional shortening, indicators of systolic function and long-term prognostic biomarkers in dysfunction remain the gold-standard for assessment of motional patterns and cardiac function in small animals due to the excellent soft tissue contrast of MRI and its high spatial and temporal resolution (Figure 12).

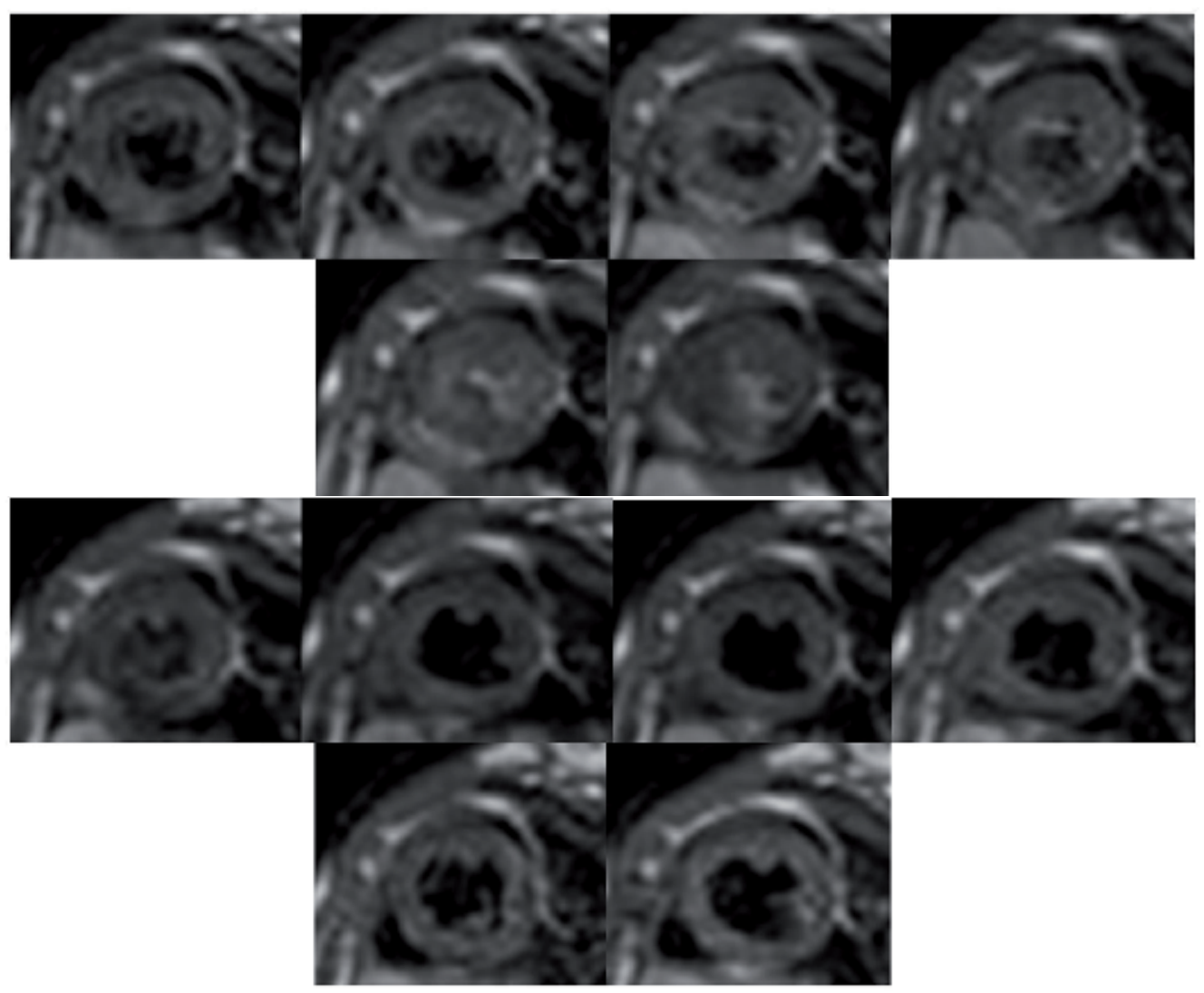

Figure 12. Single-slice, multiphase CINE functional cardiac imaging of the C57BL/6 mouse heart over 12 cardiac phases.

\section{Interstrain morphological and 4D motional variability - Statistical altases}

The advances in high-field, high-resolution cardiac imaging techniques, have also allowed the development of atlas-based approaches for the description of anatomical structures and their function [Ali 2005, Sharief 2008] in normal [Ruff 1998, Bucholz 1998, Badea 2005, Zamyadi 2010] and transgenic mice [Chien 2000, Epstein 2007]. Completion of the sequencing of the mouse genome has led to increased requirements for identifying the specific phenotype 
elicited post-transgenic modifications and the genetic basis of pathology [Henkelman 2010]. Therefore mouse imaging, combined with probabilistic, statistical atlas constructions and morphometric methods [Zamayadi 2010], is envisaged to play an increasing role in imagebased phenotyping and gene expression mapping of genetically altered mice [ $\mathrm{Ng} \mathrm{2010]}$ in the future. Construction of probabilistic and statistical atlases [Perperidis 2011] can potentially enable the study of murine, global cardiac structure and function with increased quantitative accuracy, identifying modal components of shape variability (from embryogenesis to adulthood), and disseminating components of global mechanical motion. Similar to existing cardiac atlases [Perperidis 1995, Hoogendoorn 2007], these can be population-based instead of single-subject. Prior efforts have focused on modeling cardiac anatomy in humans [Helm 2006] but only limited attempts have been made to construct accurate, high-spatial and hightemporal resolution computerized atlases for mice [Perperidis 2011]. Despite multiple prior efforts with construction of human brain atlases [Young 2009], Frangi et al. [Frangi 2002], Mitchell et al. [Mitchell 2002], and Lotjonen et al. [Lotjonen 2004] were the first to develop human ventricular statistical shape models. More recently Ordas et al. [Ordas 2007] developed a computational atlas of the entire heart using registration-based techniques. Perperidis et al. [Perperidis 1995, Perperidis 2005] and Hoogendoorn et al. [Hoogendoorn 2007] proposed 4D spatio-temporal human cardiac probability atlases from (MRI), while Beg et al. [Beg 2004] developed a large deformation diffeomorphic mapping (LDDM) for construction of cardiac statistical atlases. As an extension to Beg's work, Helm et al. [Helm 2006] employed LDMM to achieve inter-subject registration to a reference anatomical template to compare cardiac geometric variability using Principle Component Analysis (PCA) from diffusion tensor MRI in normal and failing human hearts.

\section{Manual and semi-automated segmentation and registration approaches}

Critical to successful constructions of atlases and to the transformation of constructed surface models to the finite element models (for subsequent computational work of mechanical function), are efficient and accurate segmentation and registration techniques (Figures 13, 14). Using recently developed techniques in our group [Perperidis 2011], a segmented (template) reference MRI sequence is selected from the mouse database consisting of imaged anatomic structures (left and right ventricular myocardium, left and right ventricular blood pools, and papillary muscles) as representative sets of typical anatomic objects of interest. This template is subsequently used to register population images via global and local non-rigid transformations. Although such high-dimensional transformation differs from established one-to-one (invertible) diffeomorphic transformations [Helm 2006], its performance is comparable in accuracy, precision, simplicity, and computational intensity, as recently reported by a multi-center quantitative ranking of 14 different non-linear deformation algorithms [Klein 2009].

Figure 13 show examples of manual, user-based spline segmentation and subsequent correction for left and right ventricular feature extraction, as discussed previously [Perperidis 2011]. Implementation of a global and local registration scheme is also diagrammatically summarized in Figure 14 [Perperidis 2011]. 


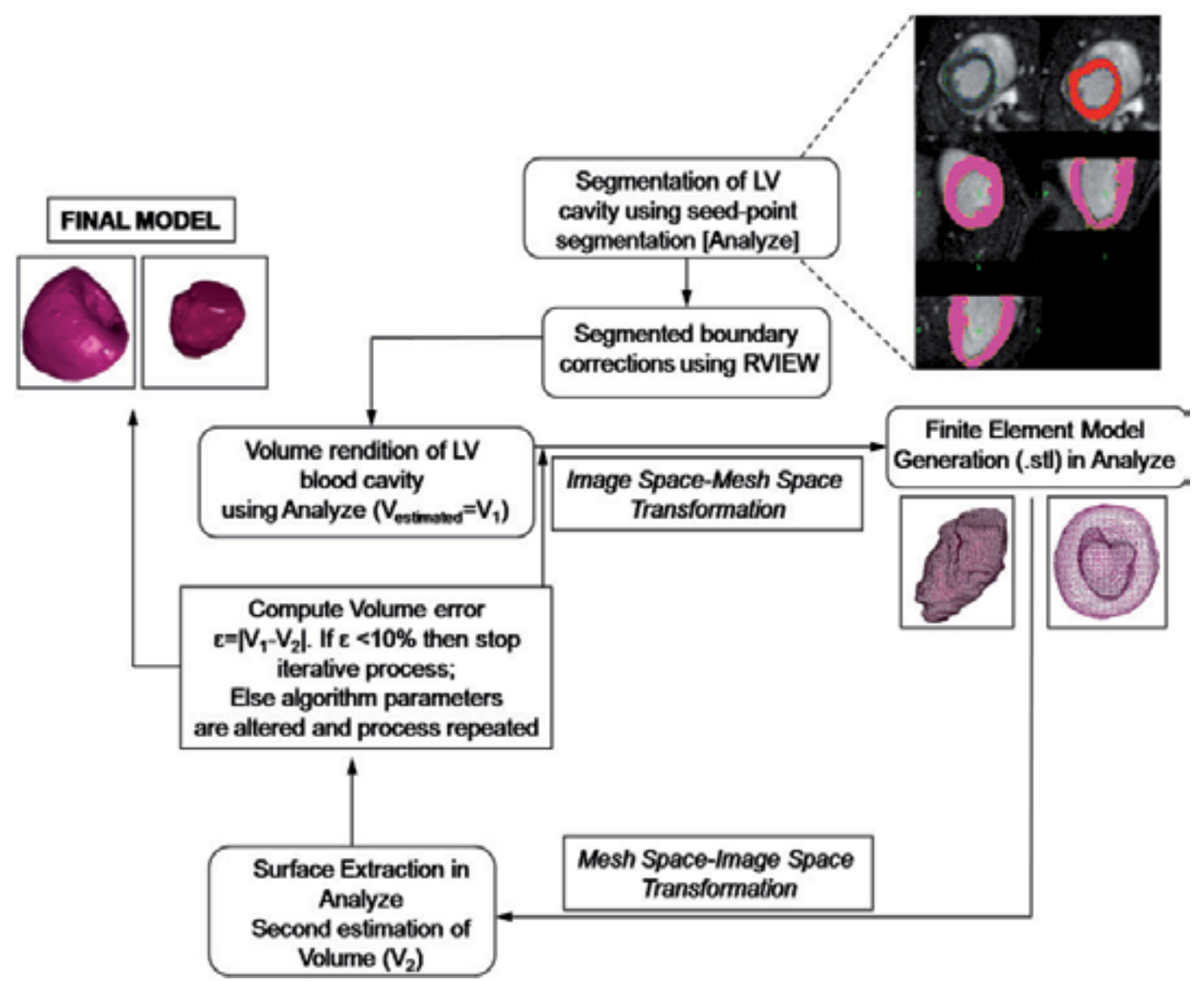

Figure 13. Flow diagrammatical representation of the empirical optimization process for generation of quantitatively accurate finite element models of the murine myocardium. [Reproduced from Constantinides et al. [Constantinides_SBI_2010] with permission from IEEE]. Insert (top right): (top row) Seed-point segmentation and binary mask construction in short axis MRI; (middle and bottom rows) RView 3D binary mask correction. [This work originally appeared in Constantinides et al. [Constantinides 2010] and Perperidis et al. [Perperidis et al. 2011], and was published in Computerized Medical Graphics and the IEEE Proceedings of the International Society of Biomedical Imaging; Reproduced with the permission of Elsevier and IEEE].

Manual, image-based techniques seem to provide the best avenue for segmentation (despite their inherent intra- and inter-observer variability inaccuracies).

Certainly, atlas-based approaches are envisaged to be of tremendous benefit and value for cardiac phenotyping characterization in the upcoming years, aiding spatial mapping of gene expression using novel cellular and sub-cellular probes and markers, understanding embryogenesis and development, accurately mapping strain-, age-, and sex-based morphological dependencies, quantifying patterns of motional variability, and efficiently screening cardiac functional changes based on semi-automatic template segmentation techniques for efficient estimation of hemodynamic indices of function, and high throughput phenotypic screening. 


\begin{tabular}{|c|c|c|c|c|}
\hline Reference Landmaxked & $\begin{array}{l}\text { Non rigid } \\
\text { registration }\end{array}$ & Mouse Heart & Tranformation & $\begin{array}{l}\text { Registered Landnarkoc } \\
\text { Mouse Heart }\end{array}$ \\
\hline
\end{tabular}
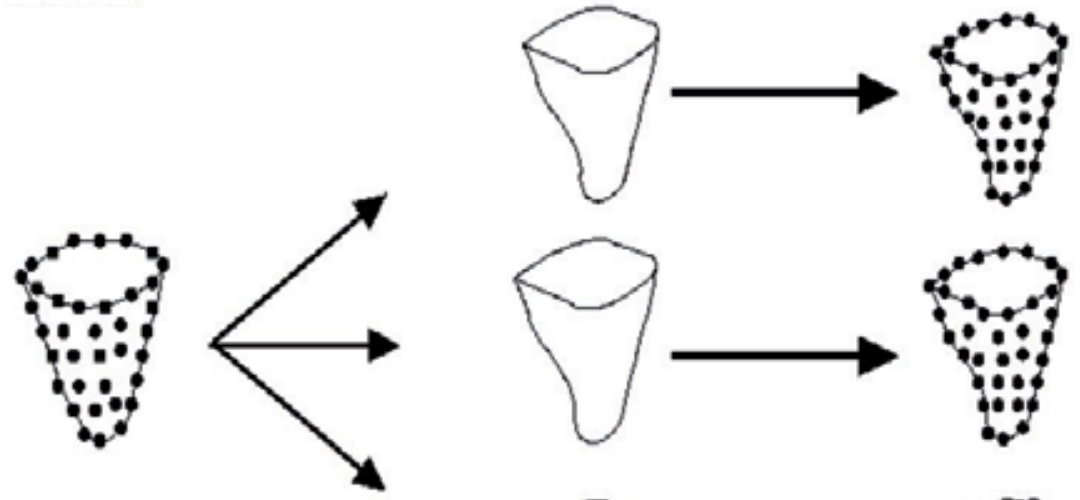

(A)
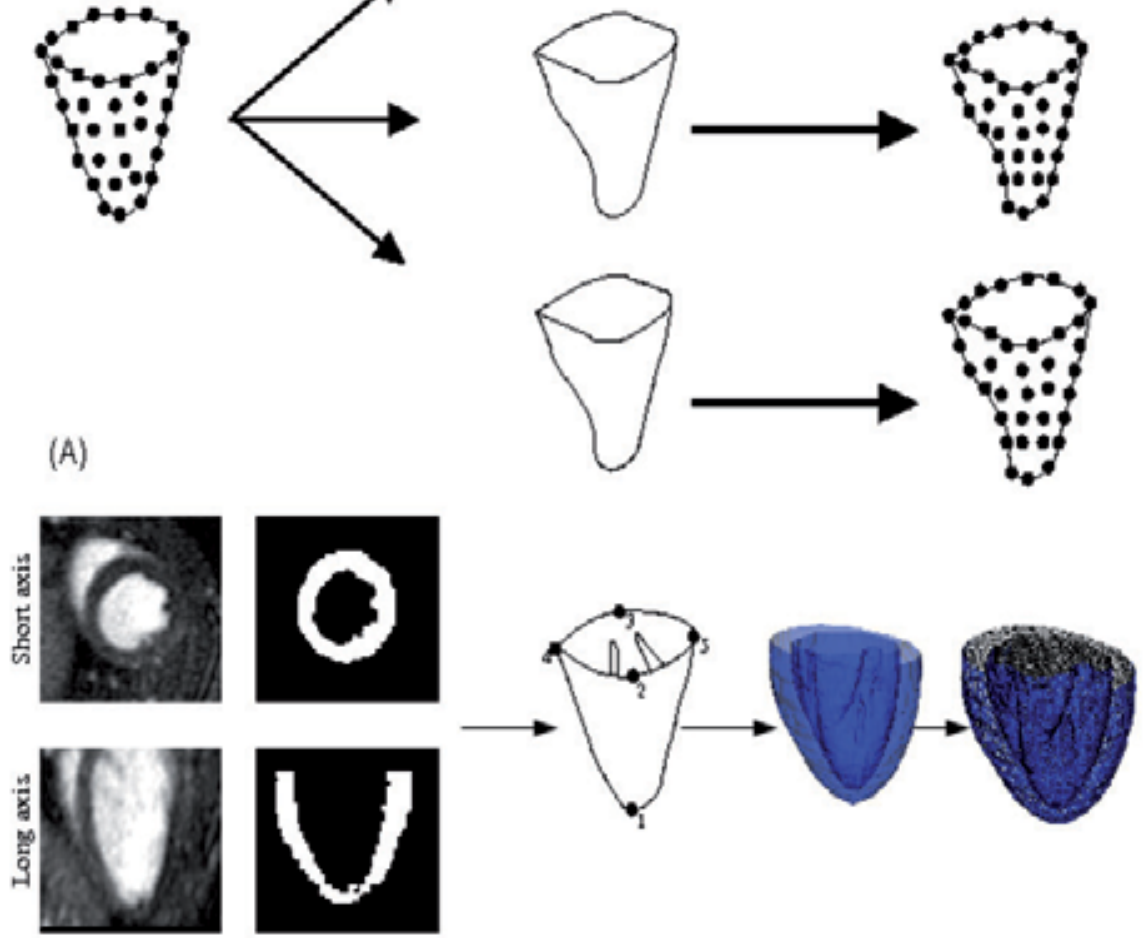

(B)

(C)

(D)

(E)

(F)

Figure 14. Graphical representation of land-marking as an initial step to image registration. The landmarks are propagated from a reference landmarked template heart model to each remaining mouse heart of each cohort. (B) Typical mid-short and long-axis images from a mouse heart; (C) RView corrected binary masks; (D) Example of five-point landmarked mesh for global registration; (E) 3D epicardial/endocardial reference surface model rendition used for local registration, and (F) construction of the three-dimensional (3D) mesh volumetric dataset using the marching cubes algorithm. [This work originally appeared in Perperidis et al. (Perperidis et al. 2011), published in Computerized Medical Graphics; Reproduced with the permission of Elsevier].

Despite its potential usefulness, the major limitations of such a technique is associated with the significant image post-processing, the need for accurate registration methods (often a challenging task for cardiac datasets), the inherent assumptions associated with the normality of distribution of independent datasets, and confounding factors on attempts to analyze motion and variability (often as a direct modulation of anesthesia effects). 


\subsubsection{Interstrain comparisons of global cardiac function}

Apart from early attempts [Wiesmann 2000], little work has been published on interstrain comparisons of global cardiac functional differences in various mouse strains [Constantinides_SBI 2010, Bucholz 2010]. Instead, studies of cardiac dysfunction have often included quantitative comparisons with normal control or sham mice [Zhang 2008]. Interstrain variability on cardiac function exists in different mouse strains, which may be dependent or independent of strain, but there are certainly developmental factors and other factors that ought to be investigated. Reported results must thus be carefully considered for age, weight, sex, and genetic background. In recent findings from our group (Figure 15) no significant variations in cardiac function were observed from age-, sex-matched (male), normal C57BL/6J and DBA/2J mice. Image-derived hemodynamic indices of function reported in Figure 16 exemplify similar cardiac functional responses from both the right and left ventricular cavities, in agreement with prior reports [Zhang 2008].

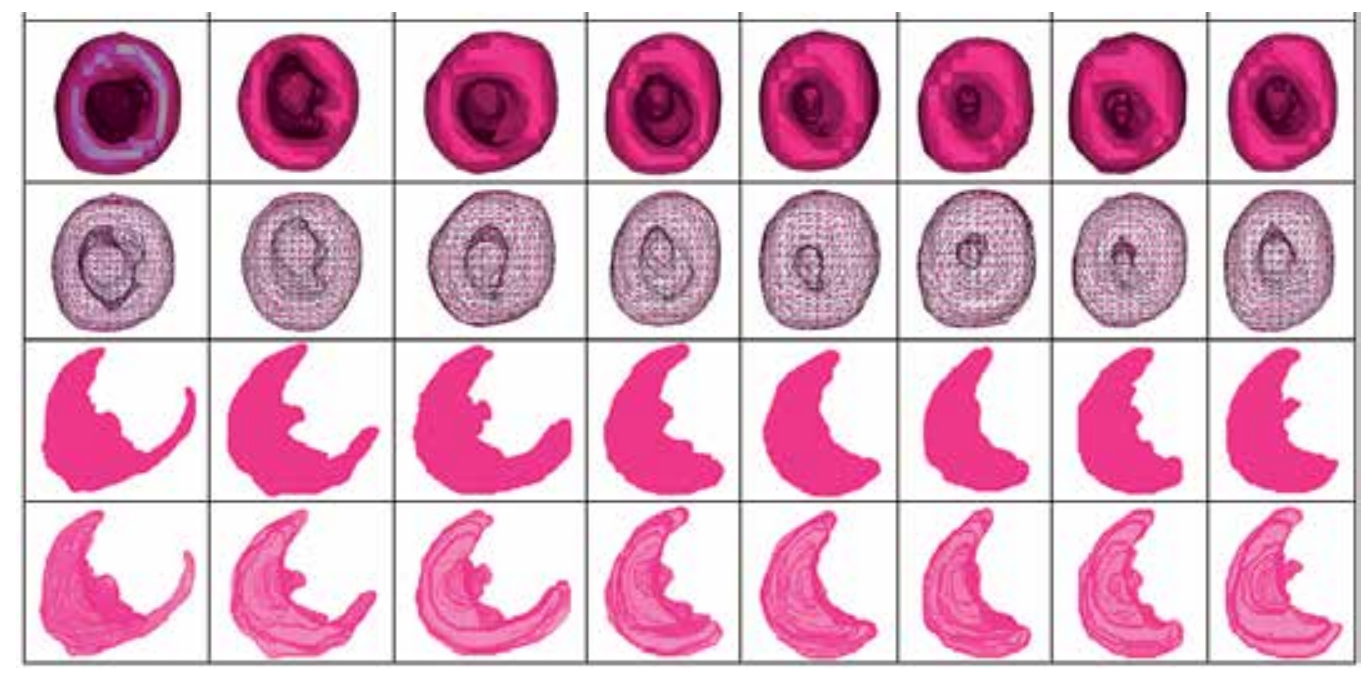

Figure 15. Typical (top two rows) LV surface and finite element, and (bottom two rows) corresponding $\mathrm{RV}$ representation of the $4 \mathrm{D}$ dynamic surface and finite element model of DBA/2J mice through eight cardiac phases over the entire cardiac cycle.

\subsubsection{Quantification of regional cardiac function - Comparison of mouse and human}

Despite the usefulness of global cardiac index comparisons, the value and importance of regional cardiac functional analyses in disease is paramount. Further to the use of tagging [Liu 2006] and DENSE [Gilson 2005, Zhong 2010] as non-invasive techniques for assessment of regional cardiac displacement and strain in mice, similar patterns of motional responses were observed in mice and humans with noted finite, but distinct, differences. Particularly, Gilson [Gilson 2005] reports basal displacement but almost no apical displacements in the mouse during the cardiac cycle, in comparison to both apical and basal motion in humans [Moore 2000]. Furthermore, circumferential and radial displacements seem to scale proportionally with values previously reported in humans [Constantinides_Phantom 2012]. 
Finite differences may exist but careful consideration of other factors (such as age, sex, or anesthesia effects) must also be considered in such analyses.

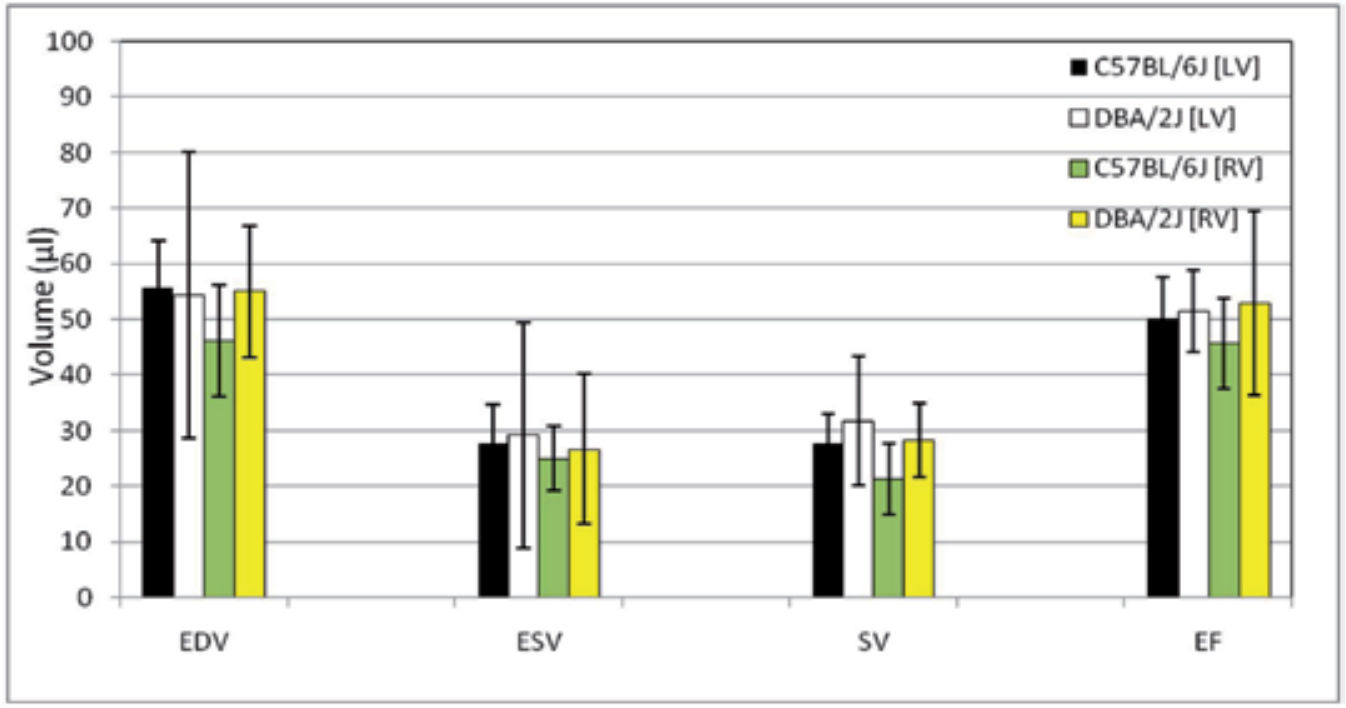

Figure 16. Interstrain hemodynamic (EDV, ESV, SV, EF) index comparisons for left (LV) and right (RV) ventricular performance in male C57BL/6J $(n=5)$ and DBA/2J mice $(n=5)$ based on MRI.

Apart from tissue displacement encoding techniques other motion tracking [Osman 1999], velocity [Streif 2003], and acceleration techniques [Staehle 2011] have been reported.

Despite the importance of all such techniques they nevertheless necessitate access to dedicated high-field MRI scanners and invariably require development, or use, of complex algorithms and reconstruction software. An easier methodology to assess regional cardiac function for comparison with humans was recently reported by Constantinides et al. [Constantinides_ISMRM 2011] with the use of dedicated software (Figure 17) based on segmented epicardial and endocardial boundaries, according to:

$$
\begin{aligned}
& \text { Wall Motion }=E D_{\text {epicardial_wall_diameter }}-E S_{\text {epicardial_wall_diameter }} \\
& \text { WallThickening }=100 \cdot \frac{\left(E S_{\text {wall_thickness }}-E D_{\text {wall_thickness }}\right)}{E D_{\text {wall_thickness }}} \\
& r E F=100 \cdot \frac{\left(E D_{\text {endocardial_diameter }}{ }^{2}-E S_{\text {endocardial_diameter }}{ }^{2}\right)}{E D_{\text {endocardial_diameter }}{ }^{2}}
\end{aligned}
$$


Based on such analyses, bullseye-plots of regional cardiac function were generated in 17sector representations of the murine and human hearts (from independent studies in two separate Institutions) showing similar patterns in transmural variations in wall motion and thickness, and regional ejection fraction (Figure 18) in mouse and man. Such spatial patterns observed for mouse and human (in agreement with prior tagging work [Moore 2000]) are supported by two-tailed paired t-tests indicating absence of statistical significant differences in the mean values of wall thickness $(\mathrm{p}=0.07)$, wall motion $(\mathrm{p}=0.051)$, or regional $\mathrm{EF}(\mathrm{p}=0.065)$ at the $1 \%$ significance. Repeated measures ANOVA indicated significant differences in regional mouse and human for wall thickness $(p=0.002)$ and regional $E F(p<0.0001)$ and insignificant differences for wall motion $(\mathrm{p}=0.016)$ at the $1 \%$ significance. However, despite the similarity in such patterns, quantification of global and regional functional indices (Table 2) shows distinct, finite differences, in agreement with prior reports. Unknown at this stage is whether such differences can be attributed to species variability or endogenous or exogenous parameter dependencies, as they relate to the conduct of such studies and data analyses, or the modus operandi of the human and murine hearts.

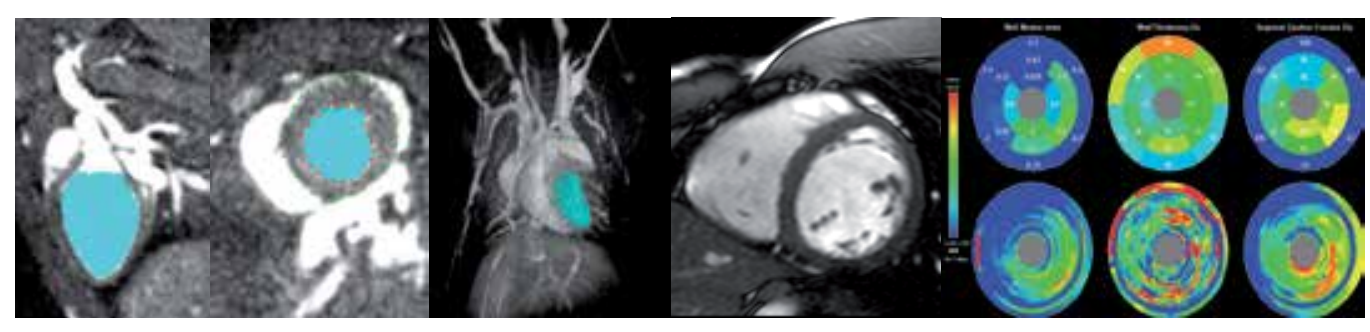

Figure 17. (Left to right) Epicardial and endocardial LV contour definition in mouse long and short axis MRI, and 3D LV blood cavity segmentation; 3D ventriculogram rendition using Vitrea from murine MRI; short axis human MRI, and Vitrea reconstruction of wall motion, wall thickening, and regional ejection fraction from a typical mouse dataset.

Therefore, numerous practical benefits are associated with dedicated, state-of-the-art mouse cardiac MR imaging, including the non-invasive nature of the techniques, the inherent capability to map cardiac morphology and function, for both LV and RV chambers, and their motional patterns. High spatial and temporal resolution imaging can thus be achieved, through execution of high-throughput protocols, yielding direct, accurate estimates of global and regional indices of cardiac function, avoiding any assumptions whatsoever or model-based derivation approaches endorsed by other imaging techniques such as ultrasound. 


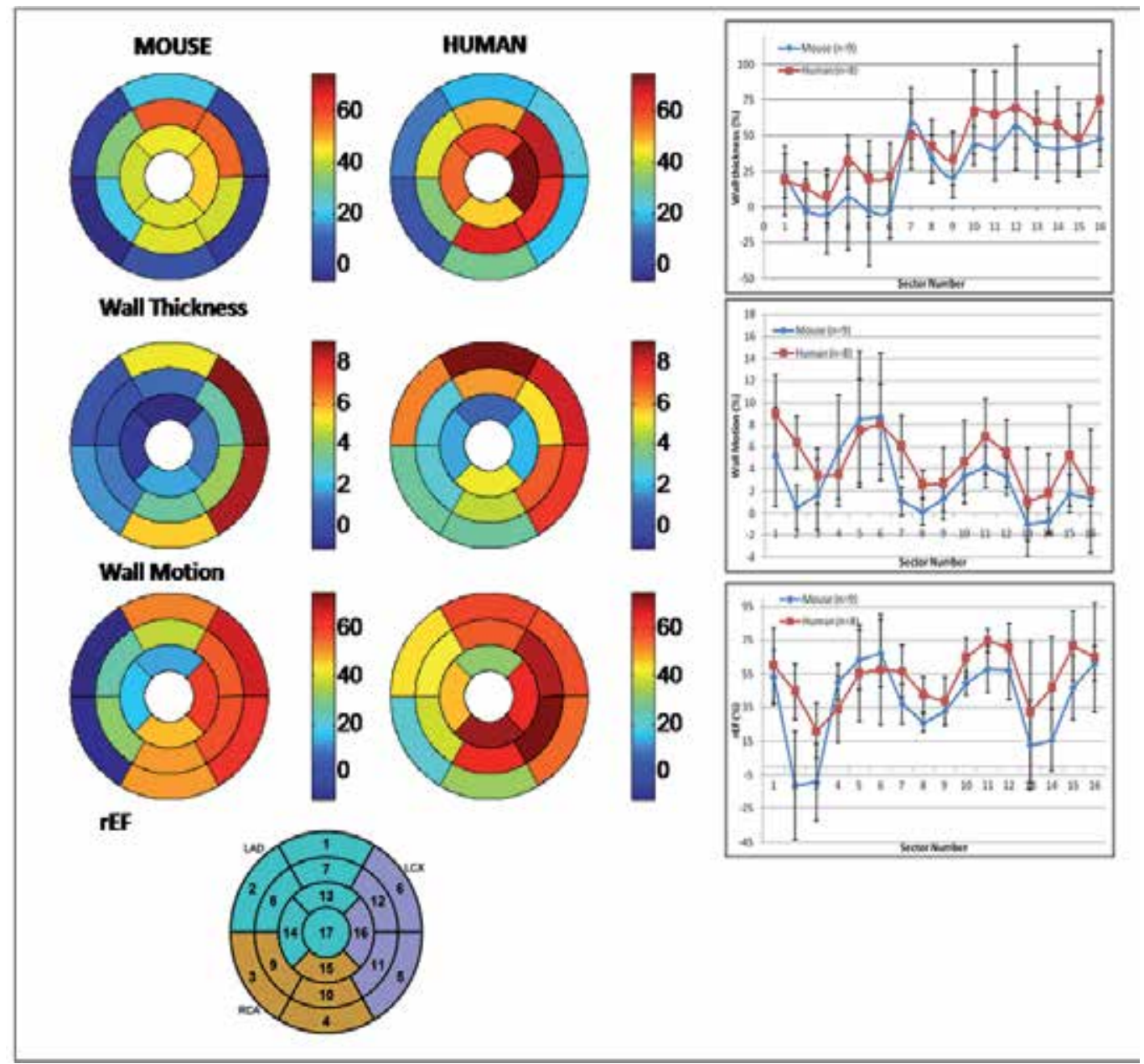

Figure 18. (Left, middle) Regional parameter quantification and comparisons in the mouse and the human using VITREA. Mean bullseye plots for wall motion, wall thickening, and regional ejection fraction for C57BL/6J mice $(n=9)$ and human $(n=8)$ datasets over the entire cardiac cycle. Schematic diagram of sectoral representation of the heart according to AHA guidelines; (right) Regional cardiac performance inter-comparison of mouse and human, including wall thickness, wall motion, and rEF variation in the various sectors of the murine and human hearts (sector 17 is excluded from presented results). 
As an extension of the development and use of such techniques (DENSE, tagging, HARP) has been the tremendous value for regional cardiac functional quantification and direct applicability to transgenic mice and in pathological states (myocardial infarction, heart failure).

Such approaches are, however, associated with a number of limitations and drawbacks, including the necessity for use of complex algorithms and laborious data post-processing (tagging, HARP, and DENSE), the inherently low spatial resolution for strain quantification (tagging vs. DENSE), the T1-tag dependence, the low SNR performance of DENSE (often with a temporal decreasing dependence following ECG-triggering), and the necessity to eliminate the anti-echo and free-induction decay signals in DENSE through proper acquisition adjustments and/or image subtractions.

\begin{tabular}{|c|c|c|}
\hline Cardiac Index & $\begin{array}{c}\text { Mouse } \\
(\mathbf{n}=9)\end{array}$ & $\begin{array}{c}\text { Human } \\
(\mathbf{n}=8)\end{array}$ \\
\hline EF & $50.7 \pm 3.7 \%$ & $64.4 \pm 2.4 \%$ \\
\hline EDV & $45.4 \pm 11.0 \mu \mathrm{l}$ & $117 \pm 32.7 \mathrm{ml}$ \\
\hline ESV & $22.5 \pm 6.2 \mu \mathrm{l}$ & $46.5 \pm 10.3 \mathrm{ml}$ \\
\hline SV & $22.9 \pm 5.4 \mu \mathrm{l}$ & $83 \pm 15.4 \mathrm{ml}$ \\
\hline CO & $1.4 \pm 0.3 \mathrm{ml} / \mathrm{min}$ & $5.0 \pm 0.91 / \mathrm{min}$ \\
\hline Myocardial Mass & - & $153.1 \pm 37.2 \mathrm{~g}$ \\
\hline rEF & $38.0 \pm 25.0 \%$ & $52.2 \pm 15.8 \%$ \\
\hline Wall Thickness & $27.9 \pm 22.4 \%$ & $42.6 \pm 21.8 \%$ \\
\hline Wall Motion & $2.8 \pm 3.0 \%$ & $4.8 \pm 2.4 \%$ \\
\hline
\end{tabular}

Table 2. Summary of mean global and regional cardiac mechanical functional indices ( \pm sd) of murine and human myocardium.

\section{Future perspectives}

In the new era of molecular imaging, MRI faces major challenges in accomplishing detection of molecular probes with increased sensitivity and specificity, comparable to other diagnostic techniques (such as PET/SPECT). Currently, 10-100 $\mu$ molar sensitivity is attained by MRI in contrast to the established nano- and picomolar sensitivity of PET and SPECT. While a new generation of contrast agents is anticipated to extend current limits shedding new light into cellular and molecular mechanisms, current efforts focus primarily on stem cell technology, cellular tracking, and construction of hybrid PET/MRI systems, as discussed below.

\subsection{Molecular imaging}

\section{Regenerative techniques - Stem cell technology and cellular tracking}

Advances in the biology of stem cells have evoked great interest in cell replacement therapies for the regeneration of heart tissue after myocardial infarction. Despite the initial 
controversial results from human trials [Rosenzweig 2006] due to the uncertain and unclear long-term fate, target-destination of the injected cells, their engraftment and viability, scientific interest and excitement remains high.

Employing high-resolution MRI in association with metabolism, iron-oxide labeled cells [Stuckey 2006] can be tracked and visualized thereby monitoring their migration patterns and ultimate engraftment fate in tissues of interest.

\subsection{Hybrid system imaging}

The superb ability of PET to detect ligand-receptor binding at the nano- to picomolar concentrations and the excellent spatial resolution of MR imaging, have stimulated efforts for the construction of hybrid PET/MRI systems. While the first prototype systems have already been completed [Herzog 2010, Pichler 2008], it will be of interest to see if corporate interest will aid establish such hybrid imaging techniques as tools of the arsenal of other diagnostic tools for clinical practice and for basic science research.

\section{Conclusion}

In the short-lived period of mouse cardiac MRI of the past 15 years, tremendous strides have been made for image-based phenotyping of the cardiovascular system. Such were realized in terms of the scalability of equipment, ease of handling and maintaining animals under proper physiological homeostatic conditions, adaptation of conventional imaging techniques, and inception of new, fast imaging acquisition schemes that have revolutionized cardiac, image-based phenotyping with efficient, high-throughput imaging protocols.

In conclusion, despite the usefulness, practicality, and low costs associated with the study of the mouse, important genetic, developmental, morphological, and cardiovascular system differences exist between mouse and man especially as such are unmasked in pathological conditions or under stress. Physiological results indicate that the optimal ISO anesthetic regimen for mouse studies under anesthesia is approximately $1.5 \% \mathrm{v} / \mathrm{v}$, yielding stable MAP and HR values comparable to those observed in the animal's conscious state, with a minute-tominute variability in MAP and $\mathrm{HR}$ of $\leq 11 \%$. Based on such recordings, the optimal $\mathrm{FiO}_{2}$ appears to be $50 \%$. The additional use of $\mathrm{N}_{2} \mathrm{O}$ was associated with higher and more stable values of MAP and HR (at a mixture of $25-50 \% \mathrm{O}_{2}$ and $75-50 \% \mathrm{~N}_{2} \mathrm{O}$ ). Arterial $\mathrm{pH}$ values are within the physiological range and varied between 7.20 and 7.43 . ISO anesthesia at $1.5 \% \mathrm{v} / \mathrm{v}$ is also associated with mild hyperglycemia $(+47 \%)$ whereas insulin levels remain unaltered. The protocol described for physiological studies of mice under anesthesia has the potential for high reproducibility in diagnostic modalities, including MRI, microCT, ultrasound, and microPET. This regimen can be useful in phenotypic screening and pharmacological studies of cardiac function in mice and can facilitate the transfer of such work to noninvasive imaging platforms, with tremendous potential for both basic science and translational research.

Basic MRI studies of murine global cardiac structure and function under optimal physiological conditions, in combination with PCA and other image processing techniques, 
can identify modal components of shape variability and disseminate components of global mechanical motion. Such atlases can be population-based instead of single-subject based and can serve as a powerful reference tool for morphological and functional inter-strain mouse studies, complementary to current ongoing efforts for image-based phenotyping that target the cardiovascular system. Based on constructed morphometric maps and atlases using principal component analysis in C57BL/6J, it is found that in probabilistic atlases, a gradient of probability exists for both strains in longitudinal locations from base to apex. Based on the statistical atlases, differences in size (49.8\%), apical direction (15.6\%), basal ventricular blood pool size $(13.2 \%)$, and papillary muscle shape and position $(17.2 \%)$ account for the most significant modes of shape variability for the left ventricle of the C57BL/6J mice. Correspondingly, for DBA mice, differences in left ventricular size and direction $(67.4 \%)$, basal size $(15.7 \%)$, and position of papillary muscles $(16.8 \%)$ account for significant variability. These data reason in favor of existing variability in the apical location in both strains, a direct consequence of the heart's effort to re-establish the position of the apex in a consistent manner at end-diastole. Additionally, higher variability exists in DBA mice in the location of the papillary muscles, as well as in the epicardial areas of the left ventricle.

On the forefront of direct, high-field acquisitions using RF technologies with mouse cardiac MRI, the commercially available birdcage outperforms cylindrical spiral multi-turn surface coils in relative signal-to-ratio (SNR) by a factor of 3-5 times as assessed by experimental measurements, simulations, and experiments in free space, and under phantom and animal loading conditions. Nevertheless, quantitative comparison of the performance of the two spiral coil geometries in anterior, lateral, inferior, and septal regions of the murine heart yield maximum mean percentage rSNR increases to the order of $27-167 \%$ post-mortem (cylindrical compared to flat coil), values that are by far higher than previous designs of surface coils and comparable to receiver phased array performance.

Such hardware improvements, in association with fast radial pulse sequence acquisitions, may also lead to quantification of global and regional functional parameters in various mouse strains. While morphological differences appear only to relate to increased papillary muscle variability in the $\mathrm{DBA} / 2 \mathrm{~J}$ mice, nevertheless interstrain cardiac hemodynamics, based on dynamic cardiac MRI acquisitions, do not exhibit significant differences for neither the LV nor RV in C57BL/6J or DBA/2J mice. Comparative to previous reports of global functional indices [Bucholz 2010], similar mouse and human results were also observed. The work supports the validity of the hypothesis of functional scaling in mice and humans.

\section{Author details}

Christakis Constantinides

Laboratory of Physiology and Biomedical Imaging (LBI),

Department of Mechanical and Manufacturing Engineering, School of Engineering, University of Cyprus, Nicosia, Cyprus 


\section{Acknowledgement}

I would like to thank Professors G. A. Johnson, G. Truskey, L. Hedlund, and Dr. E. Bucholz, Mr. G. Cofer, and Mr. J. Cook at Duke University, Drs. R. Balaban and A. Koretsky, and Mr. D. Despres and Dr. M. Lizak at the National Institutes of Health, Prof. D. Rueckert at Imperial College, London, Professor BA Janssen at Maastricht Cardiovascular Institute, and my fellows and students Dr. D. Perperidis, Mr. N. Aristocleous, Mr. R. Mean, Mr. S. Gkagkarelis, and Mr. S. Angeli at the University of Cyprus. I am indebted to the help and support from Remcom Inc. I am also grateful for the availability of the Image Registration Toolkit used under License from IXICO Ltd and to Dr. E. Treiber from Bruker Biospin for the provision of the cardiac MR images.

I would also like to acknowledge grant support from the Research Promotion Foundation (RPF) under grants RPF/TEXNOLOGY/MHXAN/0609(BE)/05, RPF/STOXOS/0302/02, and RPF/PROSVASI/0302/01.

\section{References}

[1] Aletras A, Ding S, Balaban RS, Wen H. DENSE: Displacement encoding with stimulated echoes in cardiac functional MRI. J. Magnetic Resonance 1999; 137: 247-252.

[2] Ali A, Dale AM, Badea A, Johnson GA. Automated segmentation of neuroanatomical structures in multispectral MR microscopy of the mouse brain. Neuroimage 2005; 27:425-35.

[3] Axel L, Dougherty L. MR Imaging of motion with spatial modulation of magnetization. Radiology 1989; 171(3):841-5.

[4] Badea C, Fubara B, Hedlund L, Johnson G. 4D micro-CT of the mouse heart. Molecular Imaging 2005; 4(2):110-116.

[5] Balaban RS, Hampshire VA. Challenges in Small Animal Noninvasive Imaging. ILAR 2001; 42(3):248-262.

[6] Barbee RW, Perry BD, Re RN, Murgo TP. Microsphere and dilution techniques for the determination of blood flows and volumes in conscious mice. Am J Physiol Regulatory Integr Comp Physiol 1992; 263:R728-R733.

[7] Barbee RW, Perry BD, Re RN, Murgo JP, Field LJ. Hemodynamics in Transgenic Mice with Overexpression of Atrial Natriuretic Factor. Circ. Res. 1994; 74:747-751.

[8] Beg MF, Helm PA, McVeigh E, Miller MI, Winslow RL. Computational cardiac anatomy using MRI. Magn Reson Med 2004; 52:1167-74.

[9] Bernston GC, Bigger JT Jr, Eckberg DL, Grossman P, Kaufmann PG, Malik M, Nagaraja HN, Parges SW, Paul JP, Stone PH, Van Der Molen MW. Heart rate variability: origins, methods, and interpretive caveats. Psychophysiol. , 1997; 34:623- 648.

[10] Berr SS, Roy RJ, French BA, Yang Z, Gilson W, Kramer CM, Epstein FH. Black blood gradient echo Cine magnetic resonance imaging of the mouse heart. Magn Reson in Med 2005; 53:1074-1079. 
[11] Bishop J, Feintuch A, Bock NA, Nieman B, Dazai J, Davidson L, Henkelman RM. Retrospective gating for mouse cardiac MRI. Magn Reson in Med 2006; 55(3):472477.

[12] Blank S, Chen V, Hamilton N, Salerno T, Ianuzzo C. Biochemical characteristics of mammalian myocardial. J Mol Cell Cardiol 1989; 21:367-373.

[13] Bock NA, Konyer NB, Henkelman RM. Multiple-mouse MRI. Magn Reson in Med 2003; 49(1):158-167.

[14] Bovens SM, te Boekhorst BC, den Ouden K, van de Kolk KW, Nauerth A, Nederhoff MG, Pasterkamp G, ten Hove M, van Echteld CJ. Evaluation of infracted murine cardiac function: comparison of prospectively triggered with self-gated MRI. NMR in Biomedicine 2011; 24(3):307-315.

[15] Brede M, Hadamek K, Meinel L, Wiesmann F, Peters J, Engelhardt S, Simm A, Haase A, Lohse MJ, Hein L. Vascular hypertrophy and increased P70S6 kinase in mice lacking the angiotensin II AT(2) receptor. Circulation 2001; 104(31):2602:2607.

[16] Brunson DB. Pharmacology of inhalation anesthetics (Chapter 2). In: Fish RE, Brown MJ, Danneman PJ, Karas AZ (eds.) Anesthesia and Analgesia in Laboratory Animals. 2nd ed. London: Academic Press. 2008; p29-41.

[17] Bucholz E, Ghaghada K, Qi Y, Mukundan S, Johnson GA. Four-dimensional MR microscopy of the mouse heart using radial acquisition and liposomal gadolinium contrast agent. Magn Reson Med 2008; 60:111-8.

[18] Bucholz E, Ghaghada K, Qi Y, Mukundan S, Rockman H, Johnson GA. Cardiovascular phenotyping of the mouse heart using a $4 \mathrm{D}$ radial acquisition and liposomal Gd-DTPABMA. Magn Reson in Med 2010; 63(4):979-97.

[19] Carr HY. Steady-State Free Precession in Nuclear Magnetic Resonance. Physical Review 1958; 112:1693-1701.

[20] Cassidy PJ, Schneider JE, Grieve SM. Lygate C, Neubauer S, Clarke K. Assessment of Motion Gating Strategies for Mouse Magnetic Resonance at High Magnetic Fields. Journal of Magnetic Resonance Imaging 2004; 19:229-237.

[21] Chacko VP, Aresta F, Chacko SM, Weiss RG. MRI/MRS assessment of in vivo murinecardiac metabolism, morphology, and function at physiological heart rates. Am J Physiol Heart Circ Physiol 2000; 279(5):H2218-H2224.

[22] Chien KR. To Cre or not to Cre: the next generation of mouse models of human cardiac diseases. Circ Res 2000; 88:546-9.

[23] Cholewa BC, Mattson DL. Role of the renin-angiotensin system during alterations of sodium intake in conscious mice. Am J Physiol Regul Integr Comp Physiol 2001; 281:R987-R933.

[24] Chuang JS, Zemlijic-Harpf A, Ross RS, Frank LR, McCulloch AD, Omens JH. Determination of three-dimensional ventricular strain distributions in gener-targeted mice using tagged MRI. Magnetic Reson Med 2010; 64(5):1281-8.

[25] Collins FS, Green ED, Guttmacher AE, Guyer MS. A vision for the future of genomics research: A blueprint for the genomic era. Nature 2003; 422(6934):835-847. 
[26] Constantinides C, Mean R, Janssen BJ. Effects of Isoflurane Anesthesia on the Cardiovascular Function of the C57BL/6 Mouse. ILAR 2011; 52:e21-e31.

[27] Constantinides C, Angeli S, Mean R. Changes in Murine Cardiac Ionotropy and Hemodynamics following Manganese administration under isoflurane anesthesia. Annals in Biomedical Engineering 2011; 39(11):2706-2720; DOI: 10.1007/s10439-0110367-5 (accessed August 5, 2011).

[28] Constantinides C, Angeli S, Gkagarelis S, Cofer G. Intercomparison of performance of RF Coil Geometries for High Field Mouse Cardiac MRI. Concepts of Magnetic Resonance Part A 2011; 38A(5):236-252. DOI 10.1002/cmr.a.20225 (accessed 4 August 2011).

[29] Constantinides C, Mean R, Janssen BA. Heart rate and blood pressure variability effects as a result of oxygen and nitrous oxide administration in the anesthetized Mouse 2010: $32^{\text {nd }}$ Annual International Conference of the IEEE Engineering in Medicine and Biology Society (IEEE-EMBS) 'Merging Medical Humanism and Technology', August 2010, 2010, Buenos Aires, Argentina.

[30] Constantinides C, Aristocleous A, Johnson A, Perperidis D. Static and Dynamic Cardiac modeling: initial strides and results towards a quantitatively accurate mechanical heart model 2009: Proceedings of the IEEE Society on Biomedical Imaging (SBI), Rotterdam, The Netherlands, February 2009.

[31] Constantinides C, Angeli A, Mean R. Murine cardiac catheterizations and hemodynamics: On the issue of parallel conductance, IEEE Transactions of Biomedical Engineering 2011; 58(11), 3260-3268.

[32] Constantinides C, Angeli S, Kossivas F, Ktorides P. Underestimation of cardiac hemodynamics using invasive catheters: errors, limitations, and remedies. Cardiovascular Engineering Technology 2012; 3(2):179-193; DOI: 10.1007/s13239-0120084-8 (accessed June 2012).

[33] Constantinides C, Nearchou D, Constantinou C, Ktorides P, Gravett R, Tzagarakis V. A Novel Cardiac Phantom to Study Murine and Human Cardiac Motion and Function using MRI 2011: Proceedings of the International Society of Magnetic Resonance in Medicine, Montreal, Canada 2011.

[34] Constantinides C, Zhong X, Tzangarakis G, Cofer G, Gravett R. A Computer-Controlled Cardiac Phantom to Emulate Human and Murine Motion using DENSE MRI. Submitted, June 2012.

[35] Dobson GP, Headrick JP. Bioenergetic scaling: Metabolic design and body-size constraints in mammals. Proc. Natl. Acad. Sci. USA 1995; 92:7317-7321.

[36] Doevendans PA, Daemen MJ, de Muinck ED, Smits JF. Cardiovascular phenotyping in mice. Cardiovascular Research 1998; 39:34-49.

[37] Dawson D, Lygate C, Saunders J, Schneider JE, Ye X, Hulbert K, Noble JA, Neubauer S. Quantitative 3-Dimensional Echocardiography for accurate and rapid cardiac phenotype characterization in mice. Circulation 2004; 110:1632-1637.

[38] Ehmke H. Mouse gene targeting in cardiovascular physiology. Am J Physiol Regul Integr Comp Physiol 2003; 284: R28-R30. 
[39] Engel D, Peshock R, Armstong RC, Sivasubramanian N, Mann DL. Cardiac myocyte apoptosis provokes adverse cardiac remodeling in transgenic mice with targeted TNF overexpression. Am J Physiol Heart Circ Physiol 2004; 287(3):H1303H1311.

[40] Epstein FH, Yang Z, Gilson WD, Berr SS, Kramer CM, French BA. MR tagging early after myocardial infarction in mice demonstrates contractile dysfunction in adjacent and remote regions. Magn Reson in Med 2002; 48(2):399-403.

[41] Epstein FH. MR in mouse models of cardiac disease. NMR Biomed 2007; 20(3):23855.

[42] Feng MG, Dukaez SAW, Kline RL. Selective effect of tempol on renal medullary hemodynamics in spontaneously hypertensive rats. Am J Physiol Regul Integr Comp Physiol 2001; 281:R1420-R1425.

[43] Frahm J, Hanicke W, Merboldt KD. Transverse coherence in rapid FLASH NMR Imaging. Journal of Magnetic Resonance 1987;72:307-314.

[44] Frangi A, Rueckert D, Schnabel J, Niessen W. Automatic construction of multiple-object three-dimensional statistical shape models: applications to cardiac modelling. IEEE Trans Med Imag 2002; 21(9):1151-66.

[45] Franco F, Dubois SK, Peshock RM, Shohet RV. Magnetic resonance imaging accurately estimates LV mass in a transgenic mouse model of cardiac hypertrophy. Am J Physiol 1998; 274:H679-H683.

[46] Franco F, Thomas GD, Giroir B, Bryant D, Bullock MC, Chwialkowski MC, Victor RF, Peshock RM. Magnetic resonance imaging and invasive evaluation of heart failure in transgenic mice with myocardial expression of tumor necrosis factor-alpha. Circulation 1999; 99(3):448-454.

[47] Frydrychowicz A, Spindler M, Rommel E, Erti G, Haase A, Neubauer S, Wiesmann F. Functional assessment of isolated right heart failure by high resolution in-vivo cardiovascular magnetic resonance in mice. Journal of Cardiovascular Magnetic Resonance 2007; 9:623-627.

[48] Gehrmann, Hammer PE, Maguire CT, Wakimoto H, Triedman JK, Berul CI. Phenotypic screening for heart rate variability in the mouse. Am. J. Physiol. Heart Circ Physiol 2000; 279:H733-H740.

[49] Georgakopoulos D, Mitzner W, Chen CH, Byrne BJ, Millar HD, Hare JM, and Kass DA, "In vivo murine left ventricular pressure-volume relations by miniaturized conductance micromanometry," Amer. J. Physiol. Heart Circ. Physiol 1998; 274(43): H1416-H1422.

[50] Gilson WD, Yang Z, French BA, Epstein FH. Measurement of myocardial mechanics in mice before and after infarction using multislice displacement-encoded MRI with 3D motion encoding. Am J Physiol Heart Circ Physiol 2005; 288:H1491H1497.

[51] Gregory SG, Sekhon M, Schein J, Zhao S, Osoegawak K, Scott CE, Evans RS, Burridge PW, Cox TV, Fox CA, Hutton RD, Mullenger IR, Phillips KJ, Smith J, Stalker J, Threadgold GJ, Birney E, Wylie K, Chinwalla A, Wallis J, Hillier L, Carter J, Gaige T, Jaeger S, Kremitzki C, Layman D, Maas J, McGrane R, Mead K, Walker R, 
Jones S, Smith M, Asano J, Bosdet I, Chan S, Chittaranjan S, Chiu R, Fjell C, Fuhrmann D, Girn N, Gray C, Guin R, Hsiao L, Krzywinski M, Kutsche R, Sen Lee S, Mathewson C, McLeavy C, Messervier S, Ness S, Pandoh P, Prabhu A-L, Saeedi P, Smailus D, Spence L, Stott J, Taylor S, Terpstra W, Tsai M, Vardy J, Wye N, Yang G, Shatsman S, Ayodeji B, Geer K, Tsegaye G, Shvartsbeyn A, Gebregeorgis E, Krol M, Russell D, Overton L, Malek JA, Holmes M, Heaney M, Shetty J, Feldblyum T, Nierman WC, Catanesek JJ, Hubbard T, Waterston RH, Rogers J, de Jongk PJ, Fraser CM, Marra M, McPherson JD, Bentley DR. A Physical Map of the Mouse Genome. Nature 2002; 418:743-750.

[52] Haase A, Frahm J, Matthaei D, Hanicke W, Merboldt KD. FLASH imaging rapid NMR imaging using low flip-angle pulses. Journal of Magnetic Resonance 1986; 67(2):258-266.

[53] Hart CYT, Burnett JC, Redfield MM. Effects of avertin versus xylazine-ketamine anesthesia on cardiac function in normal mice. Am. J. Physiol. Heart Circ Physiol. 2001; 281:H1938-H1945.

[54] Hedlund LW, Gluckman TL. Basics of Small Animal Handling for In Vivo Imaging (Chapter 24). In: Pomper M, Gelovani J, (eds.) Molecular Imaging in Oncology. First Edition, INFRMAN-HC; 2008; p377-390.

[55] Heijman E, Strijkers GJ, Habets J, Janssen B, Nicolay K. Magnetic resonance imaging of regional cardiac function in the mouse. MAGMA 2004; 17: 170-178.

[56] Heijman E. Mouse Cardiac MRI. Doctoral Thesis. University of Eindhoven, The Netherlands; 2008.

[57] Helm PA, Younes L, Beg MF, Ennis DB, Leclercq C, Faris OP, et al. Evidence of structural remodelling in the dyssynchronous failing heart. Circ Res 2006; 98:12532.

[58] Henkelman RM. Systems biology through mouse imaging centers: experience and new directions. Annu Rev Biomed Eng 2010; 12:143-66.

[59] Henson RE, Song SK, Pastorek JS, Ackerman JJ, Lorenz CH. Left ventricular torsion is equal in mice and humans. Am J Physiolo Heart Circ Physiol 2000; 278(4):H1117H1123.

[60] Herzog H,Pietrzyk U, Shah NJ, Ziemons K. The current state, challenges and perspectives of MR-PET. NeuroImage 2010; 49(3):2072-82.

[61] Hildebrandt IJ, Su H, Weber WA. Anesthesia and other considerations for in vivo imaging of small animals. ILAR , 2008; 49(1): 17-26.

[62] Hiba B, Richard N, Thibault H, Janier M. Cardiac and respiratory self-gated Cine MRI in the mouse: comparison between radial and rectilinear techniques at 7T. Magn Reson in Med 2007; 58:745-753.

[63] Hinshaw WS. Image formation by nuclear magnetic resonance: The sensitive point method. Journal of Applied Physics 1976; 8:3709-3721.

[64] Hoit BD. New Approaches to Phenotypic Analysis in Adult Mice. J. Mol. Cell Cardiol. $2001 ; 33: 27-35$. 
[65] Hoit BD. Murine physiology: measuring the phenotype. J. Mol. Cell. Cardiol. 2004; 37:377-387.

[66] Hoogendoorn C, Sukno FM, Ordas S, Frangi AF. Bilinear models for spatiotemporal point distribution analysis: application to extrapolation of whole cardiac dynamics 2007: Proceedings of mathematical methods in biomedical image analysis. Rio de Janeiro, Brazil: IEEE Computer Society Press, p 1-8, 2007.

[67] James JF, Hewett TE, Robbins J. Cardiac Physiology in Transgenic Mice. Circulation Research 1998; 82:407-415.

[68] Janssen BJ, Leenders PJ, Smits JF. Short-term and long-term blood pressure and heart rate variability in the mouse. Am. J. Physiol. Reg. Integr. Comp. Physiol. 2000; 278(1): R215R225.

[69] Janssen B, Smits JFM. Autonomic control of blood pressure in mice: basic physiology and effects of genetic modification. Am. J. Physiol. Reg. Integr. Comp. Physiol. 2002; 282:R1545-R1564.

[70] Janssen BJA, Celle TD, Debets J, Brouns A, Callahan M, Smith T. Effects of anesthetics on systemic hemodynamics in mice. Am J Physiol Heart Circ Physiol 2004; 287:H1618H1624.

[71] Johnson GA, Cofer GP, Gewalt SL, Hedlund LW. Morphologic Phenotyping with MR Microscopy: The Visible Mouse. Radiology 2002; 222:789-793.

[72] Kass DA, Hare JM, Georgakopoulos D. Murine Cardiac Function: A Cautionary Tail. Circulation Research 1998; 82:519-522.

[73] Klein A, Andersson J, Ardekani BA, Ashburner J, Avants B, Chiang MC, et al. Evaluation of 14 nonlinear deformation algorithms applied to human brain MRI registration. Neuroimage 2009; 46:786-802.

[74] Kober F, Iltis I, Cozzone PJ, Bernard M. Myocardial Blood Flow Mapping in Mice Using High Resolution Spin Labeling Magnetic Resonance Imaging: Influence of Ketamine/Xylazine and Isoflurane Anesthesia. Magn. Reson. in Med. 2005; 553:601606.

[75] Kovacs H, Moskau D, Sraul M. Cryogenically cooled probes: a leap in NMR technology. Magnetic Resonance Spectroscopy 2005; 46:131-255.

[76] Kuijer JPA, Jansen E, Marcus JT, van Rossum AC, Heethaar RM. Improved Harmonic Phase Myocardial Strain Maps. Magnetic Resonance in Medicine 2001; 46:993999.

[77] Lauterbur PC. Image formation by induced local interactions: examples employing nuclear magnetic resonance. Nature 1973; 242:190-191.

[78] Liu W, Ashford MW, Chen J, Watkins MP, Williams TA, Wickline SA, Yu X. MR tagging demonstrates quantitative differences in regional ventricular wall motion in mice, rats, and men. Am J Physiol Heart Circ Physiol 2006; 291: H2515-H2521.

[79] Lotjonen J, Kivisto S, Koikkalainen J, Smutek D, Lauerma K. Statistical shape model of atria, ventricles and epicardium from short- and long-axis MR images. Med Image Anal 2004; 8:371-86. 
[80] Ma X, Abboud FM, Chapleau MW. Analysis of afferent, central, and efferent components of the baroreceptor reflex in mice. Am J Physiol Regul Integr Comp Physiol 2002; 283:R1033-R1040.

[81] Manning WJ, Wei JY, Fossel ET, Burnstein D. Measurement of left ventricular mass in rats using electrocardiogram-gated magnetic resonance imaging. Am J Physiol 1990; 258:H1181-H1186.

[82] Markiewicz FX, Zamora M, Karczmar GS, Roman BB. Comparison and evaluation of mouse cardiac MRI acquired with open birdcage, single loop surface, and volume birdcage coils. Phys Med Biol 2006; 51(24):N451-9.

[83] Maze M, Daunt DA, Salonen M. Current Research in Anesthesia and Trends in Clinical Applications (Chapter 17): In: Fish RE, Brown MJ, Danneman PJ, Karas AZ (eds.) Anesthesia and Analgesia in Laboratory Animals. 2nd ed. London: Academic Press 2008; p387-408.

[84] MacGowan GA, Du C, Cowan DB, Stamm C, McGowan FX, Solaro RJ, Koretsky AP, Del Nido PJ. Ischemic Dysfunction in transgenic mice expressing troponin I lacking protein kinase C phosphorylation sites. Am. J. Physiol. Heart Circ. Physiol 2001; 280:H835H843.

[85] McLean M, Prothero J. Determination of relative fiber orientation in heart muscle: methodological problems. Anat Rec 1992; 232:459-465.

[86] Milano CA, Allen LF, Rockman HA, Dolber PC, McMinn TR, Chien KR, Johnson TD, Bond RA, Lefkowitz RJ. Enhanced Myocardial Function in Transgenic Mice Overexpressing the beta-Adrenergic Receptor. Science 1994; 264:5158:582-586.

[87] Moore CC, Lugo-Olivieri CH, McVeigh ER, Zerhouni EA. Three-dimensional systolic strain patterns in the normal human left ventricle: characterization with tagged MR Imaging. Radiology 2000; 214(2):453-466.

[88] Mitchell S, Bosche J, Lelieveldt B, van der Geest R, Reiber J, Sonka M. 3-D active appearance models: segmentation of cardiac MR and ultrasound images. IEEE Trans Med Imag 2002; 21(9):1167-78.

[89] Nahrendorf M, Wiesmann F, Hiller KH, Han H, Hu K, Waller C, Ruff J, Haase A, Ertl G, Bauer WR. In vivo assessment of cardiac remodeling after myocardial infarction in rats by cine-magnetic resonance imaging. J. Cardiovascular Magn Reson 2000; 2(3):171-180.

[90] Ng L, Lau C, Sunkin SM, Bernard A, Chakravarty MM, Lein ES, Jones AR, Hawrylycz M. Surface-based mapping of gene expression and probabilistic expression maps in the mouse cortex. Methods 2010; 50:55-62.

[91] Nielsen KS, Larimer JL. Oxygen Dissociation Curves of Mammalian Blood in Relation to Body Size. Am. J. Physiol. 1958; 195(2):424-428.

[92] Ohnishi TS, Pressman GS, Price HL. A possible mechanism of anesthetic-induced myocardial depression. Biochemical and Biophysical Research Communications 1974; 57(1):316-322.

[93] Olivetti G, Lagstra C, Quanini F et al. Capillary growth in anemia-induced ventricular wall remodeling in the rat heart. Circ Res 1989; 65:1182-1192. 
[94] Ordas S, Oubel E, Sebastian R, Frangi AF. Computational anatomy atlas of the heart. In: International symposium on image and signal processing and analysis (ISPA), 2007; 338-42.

[95] Osman NF, Kerwin WS, McVeigh ER, Jerry L. Prince JL. Cardiac Motion Tracking Using CINE Harmonic Phase (HARP) Magnetic Resonance Imaging. Magn Reson Med. 1999; 42(6): 1048-1060.

[96] Pauling L, Marsh RE. The structure of chlorine hydrate. Proceedings of the National Academy of Sciences 1952; 38:112-118.

[97] Perperidis D, Mohiaddin RH, Rueckert D. Construction of probabilistic and statistical atlases of the cardiac anatomy and function using MR image sequences. Lect Notes Comput Sci, LNCS 1995; 3750:402-10.

[98] Perperidis D, Mohiaddin R, Rueckert D. Spatio-temporal free-form registration of cardiac MR image sequences. Med Image Anal 2005; 9(5):441-56.

[99] Perperidis D, Bucholz E, Johnson GA, Constantinides C. Morphological Studies of the Murine Heart Based on Probabilistic and Statistical Atlases. Computerized Graphics and Medical Imaging 2012; 36(2):119-29. DOI 10.1016/j.compmedimag 2011.07.001 (accessed August 2011).

[100] Phillips D, Covian R, Aponte A, Glancy B, Taylor JF, Chess D, Balaban RS. Regulation of oxidative phosphorylation complex activity: effects of tissue specific metabolic stress within an allometric series and acute changes in workload. Am J Physiol Regul Integr Comp Physiol. 2012; 302(9):R1034-48.

[101] Popovic ZB, Sun JP, Yamada H, Drinko J, Mauer K, Greenberg NL, Cheng Y, Moravec CS, Penn MS, Mazgalev TN, Thomas JD. Differences in left ventricular long-axis function from mice to humans follow allometric scaling to ventricular size. J Physiol 2005; 568.1: 255-265.

[102] Pichler BJ, Judenhofer MS, Wherl HF. PET/MRI hybrid imaging: devices and initial results. European Radiology 2008; 18(6):1077-86.

[103] Price HL, Ohnishi T. Effects of anesthetics on the heart. Federation Proceedings 1980; 39:1575-1579.

[104] Porterfield JE, Kottam ATG, Raghavan K, Escobedo D, Jenkins JT, Larson ER, Trevino RJ, Valvano JW, Pearce JA, Feldman MD. Dynamic correction for parallel conductance, $\mathrm{G}_{\mathrm{p}}$, and gain factor, $\alpha$, in invasive murine left ventricular volume measurements. J Appl Physiol 2009; 107:1693-1703.

[105] Przylenk K, Groom AC. Microvascular evidence for a transition zone around a chronic myocardial infarct in the rat. Can J Physiol Pharmacol 1983; 61:1516-1522.

[106] Rakusan K, Nagal J. Morphometry of arterioles and capillaries in hearts of senescent mice. Cardiovascular Res 1994; 28(7):969-972.

[107] Ramirez MS, Esparza-Coss E, Bankson JA. Multiple-Mouse MRI with Multiple Arrays of Receive Coils. Magn Reson Med. 2010; 63(3): 803-810.

[108] Rockman HA, Ross RS, Harris AN, Knowlton KU, Steinhelper ME, Field LJ, Ross J, Chien KR. Segregation of atrial-specific and inducible expression of an atrial natriuretic factor transgene in an in vivo murine model of cardiac hypertrophy. Proc Natl Acad Sci USA 1991; 88(18):8277-8281. 
[109] Rosenblum W. Regional cerebral blood flow in the anesthetized mouse as measured by local hydrogen clearance. Stroke 1977; 8:103-106.

[110] Rosenzweig A. Cardiac cell therapy - mixed results from mixed cells. The New England Journal of Medicine 2006; 355(12):1274-1277.

[111] Ross AJ, Yang Z, Berr SS, Gilson WD, Petersen WC, Oshinski JN, French BA. Serial MRI evaluation of cardiac structure and function in mice after reperfused myocardial infarction. Magn Reson in Med 2002; 47:1158-1168.

[112] Ruff J, Wiesmann F, Hiller KH, Voll S, von Kienlin M, Bauer WR, Rommel E, Neubauer $S$, Haase A. Magnetic resonance microimaging for noninvasive quantification of myocardial function and mass in the mouse. Magn Reson Med 1998; 40:43-48.

[113] Ruff J, Wiesmann F, Lanz T, Haase A. Magnetic resonance imaging of coronary arteries and heart valves in a living mouse: techniques and preliminary results. J. Magn Reson 2000; 146(2):290-296.

[114] Sabbah HN, Sharov VG, Lesch M, Goldstein S. Progression of heart failure: a role for interstitial fibrosis. Mol Cell Biochem 1995; 147:29-34.

[115] Sandgaard NCF, Andersen JL, Holstein-Rathlou NN, Bie P. Aortic blood flow subtraction: an alternative method for measuring total renal blood flow in conscious dogs. Am J Physiol Regul Integr Comp Physiol 2002; 282:R1528-R1535.

[116] Sarin SK, Sabba C, Groszmann RJ. Splanchnic and systemic hemodynamics in mice using a radioactive microsphere technique. Am J Physiol Gastrointest Liver Physiol 1990; 258:G365-G369.

[117] Schaper W, Winkler B. Of mice and men - the future of cardiovascular research in the molecular era. Cardiovascular Research 1998; 39:3-7.

[118] Schneider JE, Cassidy PJ, Lygate C, Tyler DJ, Wiesmann F, Grieve SM, Hulbert K, Clarke K, Neubauer S. Fast, High- Resolution In Vivo Cine Magnetic Resonance Imaging in Normal and Failing Mouse Hearts on a Vertical 11.7 T System. Journal of Magnetic Resonance Imaging 2003; 18:691-701.

[119] Schneider JE, Hulbert KJ, Lygate CA, Hove MT, Cassidy PJ, Clarke K, Neubauer S. Long-term stability of cardiac function in normal and chronically failing mouse hearts in a vertical-bore MR system. MAGMA 2004; 17: 162-169.

[120] Schneider JE, Lanz T, Barnes H, Lee-Anne Stork, Bohl S, Lygate CA, Ordidge RJ, Neubauer S. Accelerated cardiac magnetic resonance imaging in the mouse using an eight-channel array at 9.4 Tesla. Magn Reson in Med 2010; 65(1):60-70.

[121] Schwartz LA, Zuurbier CJ, Ince C. Mechanical ventilation of mice. Basic Res Cardiol 2000; 95:510-520.

[122] Shapiro EP. Evaluation of left ventricular hypertrophy by magnetic resonance imaging. Am J Card Imaging 1994; 8:310-315.

[123] Sharief A, Badea A, Dale AM, Johnson GA. Automated segmentation of the actively stained mouse brain using multi-spectral MR microscopy. Neuroimage 2008; 39:136-45.

[124] Sheuer J, Bahn AK. Cardiac contractile proteins: adenosine triphosphatase activity and physiological function. Circ Res 1979; 45:1-12. 
[125] Siri FM, Jelicks LA, Leinwand LA, Gardin JM. Gated magnetic resonance imaging of normal and hypertrophied murine hearts. Am J Physiology Am. J. Physiol. Heart Circ. Physiol. 1997; 272(41): H2394-H2402.

[126] Slawson SE, Roman BB, Williams DS, Koretsky AP. Cardiac MRI of the normal and hypertrophied mouse heart. Magnetic Reson in Med 1998; 39:980-987.

[127] Smaill B, Hunter P. Structure and function of the diastolic heart: Material properties of passive myocardium. In Glass L, Hunter P, McCulloch A, Theory of heart: biomechanics, biophysics, and nonlinear dynamics of cardiac function. Chapter 1: Springer-Verlag; 1991. p 1-29.

[128] Sosnovick DE, Dai G, Nahrendorf M, Rosen BR, Seethamraju R. Cardiac MRI in mice at 9.4 Tesla with a transmit-Receive surface coil and a cardiac-tailored intensitycorrection algorithm. J. Magn Reson Imaging 2007; 26:27-287.

[129] Staehle F, Jung BA, Bauer S, Leupold J, Bock J, Lorenz R, Foll D, Markl M. ThreeDirectional acceleration phase mapping of myocardial function. Magn Reson in Med 2011; 65:1335-1345.

[130] Streif JUG, Herold V, Szimtenings M, Lanz TE, Nahrendorf M, Wiesmann F, Rommel E, Haase A. In Vivo Time-Resolved Quantitative Motion Mapping of the Murine Myocardium With Phase Contrast MRI. Magnetic Resonance in Medicine 2003; 49:315321.

[131] Streif JU, Nahrendorf M, Hiller KH, Waller C, Wiesmann F, Rommel E, Haase A, Bauer WR. In vivo assessment of absolute perfusion and intracapillary blood volume in the murine myocardium by spin labeling magnetic resonance imaging. Magn Reson in Med 2005; 53(3):584-592.

[132] Stoker ME, Gerdes AM, May JF. Regional differences in capillary density and myocyte size in the normal human heart. Anato Rec 1982; 202(2):187-191.

[133] Stuckey DJ, Carr CA, Martin-Rendon E, Tyler DJ, Wilmott C, Cassidy PJ, Hale SMJ, Schneider JE, Tatton L, Harding SE, Radda GK, Clarke K. Iron particles for non-invasive monitoring of bone marrow stromal cell engraftment and isolation of viable engrafted donor cells from the heart. Stem Cells 2006; 24: 1986-75.

[134] Stuckey DJ, Carr CA, Camelliti P, Tyler DJ, Davies KE, Clarke K. In vivo MRI characterization of progressive cardiac dysfunction in the $\mathrm{mdx}$ mouse model of muscular dystrophy. PLoS ONE 2012; 7(1):e28569.

[135] Stull LB, Leppo MK, Marban E, Janssen PM. Physiological determinants of contractile force generation and calcium handling in mouse myocardium. J Mol Cel Cardiol 2002; 34(10):1367-76.

[136] Stuyvers BDMY, Miura M, Ter Keuers HEDJ. Dynamics of viscoelastic properties of rat cardiac sarcomeres during the diastolic interval: involvement of $\mathrm{Ca}^{2+}$. J. Physiol Lond 1997; 502:661-677.

[137] Thireau J, Zhang BL, Poisson D, Babuty D. Heart rate variability in mice: a theoretical and practical guide. Exp. Physiol 2008; 93(1): 83-94.

[138] van Rugge P, van der Wall EE, Bruschke AVG. New Developments in Pharmacologic Stress Imaging. Progress in Cardiology 1992; 124(2):468-484. 
[139] Webb S, Brown NA, Anderson RH. The structure of the mouse heart in late fetal stages. Anat Embryol Berl 1996; 194:37-47.

[140] Wiesmann F, Ruff J, Haase A. High-resolution MR imaging in mice. MAGMA 1998; 6:186-188.

[141] Wiesmann F, Ruff J, Dienesch C, Leupold A, Rommel E, Haase A, Neubauer S. Cardiovascular phenotype characterization in mice by high resolution magnetic resonance imaging. MAGMA 2000; 11(1-2):10-5.

[142] Wiesmann F, Neubauer S, Haase A, Hein L. Can we use vertical bore magnetic resonance scanners for murine cardiovascular phenotype characterization? Influence of upright body position on left ventricular hemodynamics in mice. J. Cardiovascular Magn Reson 2001; 3(4):311-315.

[143] Wiesmann F, Frydrychowicz A, Rautenberg J, Illinger R, Rommel E, Haase A, Neubauer S. Analysis of right ventricular function in healthy mice and a murine model of heart failure by in vivo MRI. Am J Physiol Heart Circ Physiol 2002; 283: H1065H1071.

[144] Wiesmann F, Ruff J, Engelhardt S, Hein L, Dienesch C, Leupold A, Illinger R, Frydrychowicz A, Hiller KH, Rommel E, Haase A, Lohse MJ, Neubauer S. DobutamineStress Magnetic Resonance Microimaging in mice: acute changes of cardiac geometry and function in normal and failing murine hearts. Circulation Research 2001; 88:563569.

[145] Wilding JR, Schneider JE, Sang AE, Davies KE Neubauer S, Clarke K. Dystrophin- and MLP-deficient mouse hearts: marked differences in morphology and function, but similar accumulation of cytoskeletal proteins. FASEB J 2005; 19(1):79-81.

[146] Williams SP, Gerber HP, Giordano FJ, Peale FV, Bernstein LJ, Bunting S, Chien KR, Ferrara N, van Bruggen N. Dobutamine Stress Cine-MRI of Cardiac Function in the Hearts of Adult Cardiomyocyte-Specific VEGF Knockout Mice. J. Magnetic Resonance Imaging 2001; 14:374-382.

[147] Young A, Frangi AF. Computational cardiac atlases: from patient to population and back. Exp Physiol 2009; 94(5):578-96.

[148] Zamyadi M, Baghdadi L, Lerch JP, Bhattacharya S, Schneider JE, Henkelman RM, Sled JG. Mouse embryonic phenotyping by morphometric analysis of MR images. Physiol Genomics 2010; 42A:89-95.

[149] Zerhouni EA, Parish DM, Rogers WJ, Yang A, Shapiro EP. Human heart: tagging with MR Imaging - a method for noninvasive assessment of myocardial motion. Radiology 1988; 169(1):59-63.

[150] Zhang W, ten Hove M, Schneider JE, Stuckey DH, Sebag-Montefiore L, Bia BL, Radda GK, Davies KE, Neubauer S, Clarke K. Abnormal cardiac morphology, function and energy metabolism in the dystrophic mdx mouse: an MRI and MRS study. J. Mol Cell Cardiol 2008; 45(6):754-60.

[151] Zhou R, Pickup S, Glickson JD, Scott CH, Ferrari VA. Assessment of global and regional myocardial function in the mouse using cine and tagged MRI. Magn. Reson in Med 2003; 49(4):760-764. 
[152] Zhong X, Spottinswoode BS, Meyer CH, Kramer CM, Epstein FH. Imaging threedimensional myocardial mechanics using navigator-gated volumetric spiral cine DENSE MRI. Magn Reson in Med 2010; 64(4):1089-1097. 


\title{
Biomedical Engineering in Epidural Anaesthesia Research
}

\author{
Venketesh N. Dubey, Neil Vaughan, \\ Michael Y. K. Wee and Richard Isaacs \\ Additional information is available at the end of the chapter
}

http://dx.doi.org/10.5772/50764

\section{Introduction}

The application of engineering techniques into biomedical procedures has proved extremely beneficial in many areas of medicine. A developing area is in epidural analgesia and anaesthesia, a technique employed for the relief of pain in both acute and chronic, and for anaesthesia to enable pain-free surgery. The aim of this chapter is to demonstrate several specific areas of research and how biomedical engineering techniques are used to improve and enhance the experience and training in the epidural procedure. The overall goal is to reduce the risks and subsequent morbidity in patients using advanced technologies to recreate the epidural procedure replicating as far as possible the in-vivo procedure. This would allow anaesthetists to practice the procedure in a safe and controlled environment without risk to patients. This could be achieved by recreating the sensation of the needle passing through the tissues and ligaments and by the generation of forces that match exactly those felt in-vivo. Epidural simulators are currently used as a training aid for anaesthetists, however existing simulators lack realism to various degrees and their operation is not based on measured invivo data that can accurately simulate the procedure. The techniques of advanced simulation and biomedical engineering detailed in this chapter can provide a solution.

Haptic devices have been used previously to reproduce needle forces but the forces are often not based on measured data. Needle insertion forces in-vivo are largely unknown as there are few studies in this specific area. Without accurate measurement of resultant pressure on the syringe plunger of the epidural needle, as the needle passes through the various ligaments and tissues of the spine, it is difficult to create accurate simulation of the epidural procedure. The ideal model would require other features such as a palpable spine, ability to accommodate for patient variation, 3D graphics visualisation and an adjustable needle insertion point. Techniques in biomedical engineering can provide solutions through 
the design of devices capable of making precise measurements and utilising them in a novel high fidelity epidural simulator. Adequate training on an advanced simulator will help alleviate the risks of epidural failures from inaccurate placement and also reduce potential morbidity to patients thereby improving the safety of the procedure.

This chapter is laid out in various sections to illustrate different aspects of current epidural anaesthesia research. Section 2 describes the actual epidural procedure and its challenges. Section 3 discusses the needle insertion forces in epidurals. Section 4 describes an interspinous pressure measurement device for wireless data collection during needle insertion leading to a porcine trial discussed in Section 5. Section 6 describes an image processing technique for non-contact needle depth measurement that could be used in conjunction with pressure measurement for fully characterising the needle insertion. In Section 7, 3D-modelling of spine with bending and flexing is discussed for flexibility of patient's positions together with heterogeneous volumetric modelling of spinal ligaments in Section 8. Stereo 3D visualisation for depth perception of epidural procedure has been discussed in Section 9. Section 10 applies a haptic force feedback device configured with the measured force data to create an electronic human-computer interface which is described in Section 11. Finally, section 12 brings all these technologies together and demonstrates the complete system that makes up our current epidural simulator prototype with conclusions provided in section 13 .

\section{Epidural procedure and challenges of clinical simulation}

Epidural analgesia and anaesthesia is commonly used as a form of pain relief during childbirth, for the treatment of chronic back pain or as a means to provide anaesthesia or analgesia during specific operations. Monitoring the depth of the needle during an epidural insertion is crucial because once the needle tip enters the epidural space, an epidural catheter is usually sited to a specific length. This enables the intermittent or continuous use of the epidural for anaesthesia or pain relief. If the needle is advanced too far it will puncture the dural sac and cause leakage of cerebrospinal fluid. Post dural puncture headaches may result, which can be extremely disabling for the patient. Other potential risks include nerve damage or bleeding which may very rarely lead to paralysis. If the needle is not within the epidural space, the analgesia or anaesthesia may be ineffective or absent due to incorrect placement of the catheter.

During an epidural insertion, the operator tries to perceive which tissue layer the needle tip is passing through by feeling the resistances on the needle. This is a process known as "haptic" feedback. A simulator can assist the development of this visuospatial awareness of spinal anatomy and 'feel' of the procedure to allow practice prior to attempts on patients. Not only will this serve to enhance patient safety but it also creates a safe and controlled environment in which to learn.

The procedure of inserting an epidural needle into the lumbar spine requires the operator to visualise in their mind a three-dimensional (3D) anatomical image of the bony alignments and the various tissue layers from skin, through to subcutaneous fat, supraspinous and 
interspinous ligaments, ligamentum flavum and then to the epidural space. Epidural needle insertion is essentially a blind procedure, but utilises a well-known technique referred to as "loss of resistance" (LOR). LOR essentially involves identification of the epidural space by compression of either fluid or air as the epidural needle encounters the various ligaments of the lumbar vertebral column [1]. Initially, the back of the patient is palpated, and using surface landmarks such as the iliac crests, an assessment is made of suitable intervertebral spaces and of midline. For lumbar epidurals, this may be between lumbar vertebra 3 (L3) and lumbar vertebra 4 (L4) for instance. The epidural or Tuohy needle, as it is commonly called, is inserted into the interspinous ligament and a syringe filled with saline is attached to the end of the needle. These LOR syringes are specially manufactured so that there is less friction between the plunger and the inner wall of the LOR syringe. A constant or intermittent force is then applied to the plunger by the operator's thumb as the needle is slowly advanced forward. As the tougher and more fibrous ligamentum flavum is encountered, a higher resistive force to injection is encountered. Once the needle tip traverses the ligamentum flavum, the epidural space is then entered into and saline can be quite easily injected, hence the phenomenon of LOR. It is this haptic perception that informs the operator of needle location within the various tissue layers, obstruction from bone and loss of resistance from potential spaces such as those between the ligaments. Combining this with the creation in one's mind of a three-dimensional image of lumbar spinal anatomy enables successful placement of an epidural catheter.

The ideal epidural simulator should be capable of replicating the above procedure and aim to recreate as far as possible the in-vivo procedure. A real Tuohy needle could be inserted at any intervertebral space in the lumbar or thoracic region using the midline or paramedian approach [2]. It would contain a force feedback haptic device, with force data originating from measured Tuohy needle insertions from patients. Using measured in-vivo data from patients and integrating this into the epidural simulator software, the resistance would automatically adjust to give patient variation on weight, height and body shape. This could simulate random patients or match measurements from a specific patient. The $3 \mathrm{D}$ virtual patient and virtual vertebrae can also be adjusted in size and shape to match measurements from actual patients. As the needle advances, the resultant force should represent each tissue layer and a LOR on reaching the epidural space. Once the epidural space is reached, saline would be released. During the entire insertion, a 3D virtual spine could be displayed on the monitor showing the trajectory of the needle in real time. The manikin could bend forwards to mimic spinal flexion to increase spacing between the vertebrae or alternatively bend backwards (extension) to simulate increased difficulty in locating the interspinous space for training purposes.

Variation in patient size, height, weight and other characteristics should be possible based upon actual patient measurements. Currently, most simulators have only two or three options such as obese, elderly and normal [3-6] which is perhaps not enough to encapsulate reality and could therefore be improved. Simulators could have unlimited patient variation by including parameters such as height, weight, body shape, age, obesity which could be adjustable. Ideally, the settings should match measurements from real patient data. The 
adjustments can be programmed to occur automatically based on basic patient data, so that the user does not have to manually configure all the settings. The simulator could then recreate a virtual model of a particular patient. Clinicians planning on performing the epidural can practice beforehand on a virtual model of the patient thereby reducing the learning curve during the procedure on the patient. The four common patient positions adopted during epidural insertion are sitting, sitting with lumbar flexion, lateral decubitus and lateral decubitus with lumbar flexion. These four common positions at least should be modelled in an epidural simulator to give a greater level of realism than static epidural simulators. Ideally, variable spine flexibility could be achieved by modelling 3D flexible spine vertebrae and extended to other positions to simulate difficult spinal anatomy. This may allow simulation of spinal conditions such as curvatures and rotations caused by kyphosis and scoliosis. These conditions cause difficulties in placing the needle due to unusually positioned landmarks. Also the accuracy of the forces in epidural simulators is a topic of recent discussion [7-9], so it is important that the forces required to insert a needle during simulation match those achieved in reality. Skills learned during this simulation can then be transferred to the actual clinical environment.

\section{Modelling the needle insertion forces}

Epidural insertion consists of a complicated interaction of many forces, needle position and intrinsic properties of the epidural equipment: a) Each tissue has various viscosity, elasticity, density and frictional properties. b) Bubbles of air in saline can compress. c) The method of insertion can vary depending upon needle inclination angle, paramedian angle, speed of insertion and twisting of the needle. d) Properties of the needle can vary, including the angle of the tip, tip type - side tipped or two-plane symmetric, needle gauge from 15-20 and width of the metallic walls in hollow needles vary. e) Plunger resistance is caused by friction on the inner syringe walls. f) The flow of saline is restricted by the funnel narrow opening of the syringe at LOR. g) The needle orifice can plug with tissue obstructing saline release.

Theoretically, a model can partition reaction force down into its individual constituents. The thumb applies force onto the plunger of the syringe and this force interacting with the frictional and resistive tissue forces contributes to the 'resultant pressure', see Figure 1. This pressure cannot escape so it causes the needle to push forwards. This causes the 'reaction force' which is equal and opposite to the applied force and comprised of several factors: a) The cutting force required for the needle tip to pierce the tissue. b) Friction caused by needle shaft resistance on the tissue. c) Static friction to get the stationary needle moving. d) Side compression force is caused by the surrounding tissues. e) Torque is caused by twisting of the needle. f) All of these forces, resistances and torque vary according to depth and tissue stiffness.

It is not feasible to measure all of these forces individually in-vivo and it would not make sense to measure the exact proportions of each force that make up the reaction force. In practice, it may be sufficient to measure the resultant pressure of the saline instead. Measuring resultant pressure provides a combination of all reaction forces, which is felt by 
the anaesthetist during insertion, and this combination of all forces is what simulators need to re-create to simulate the feeling on insertion.

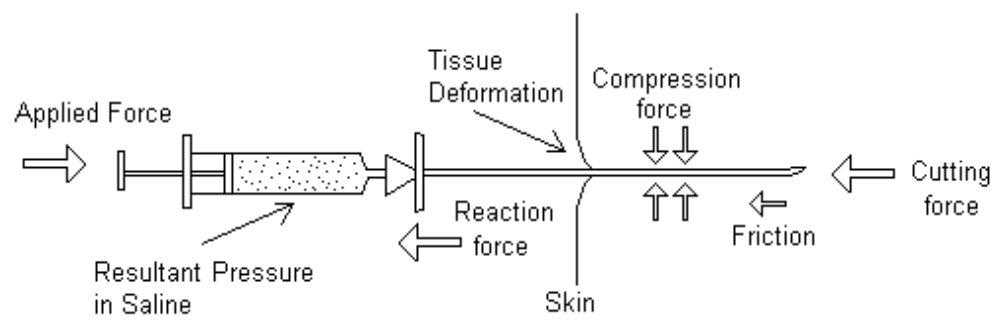

Figure 1. Several forces involved with needle insertion

\section{Pressure measurement for realistic epidural simulation}

A sterile wireless measurement device was developed to record the resultant pressure of the saline inside the syringe during an epidural needle insertion. This measurement device is used to enable data collection to quantify the pressure during the epidural procedure. Quantifying the pressure will enable accurate configuration of an epidural simulator.

Our novel pressure measurement device has wireless functionality and by using entirely sterile components allows in-vivo trials to be conducted with patients. A wireless data transmitter is utilized to minimize the equipment and disruption in the hospital room (Figure 2).
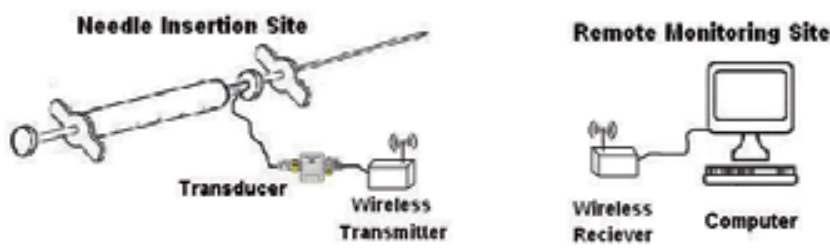

Figure 2. Remotely monitored wireless epidural pressure measurement system.

The design aims to minimise changes to the standard epidural set up. A small sterile threeway tap (BD Connecta ${ }^{\mathrm{TM}}$ ) is connected between the Tuohy needle and syringe (Figure 3). The tap is connected to the pressure transducer via a one metre length of saline-filled sterile manometer tubing. The transducer's electrical plug is connected by a short electrical cable to our wireless transmitter. At the remote site, a wireless receiver is connected via Universal Serial Bus (USB) to the computer.

The UTAH Medical Deltran disposable transducer is used for the pressure measurement sensor. These transducers are commonly used in hospitals to monitor systemic blood pressure and central venous pressure. Transducers produce a small electrical signal based on the pressure of the liquid inside the manometer tubing. Disposable transducers are designed to have accuracy of $+/-3 \%$ with the average output of $100.03+/-0.55 \mathrm{~mm} \mathrm{Hg}$ and the worst cases being 98.53 and 101.36 when $100 \mathrm{~mm} \mathrm{Hg}$ was applied [10]. 


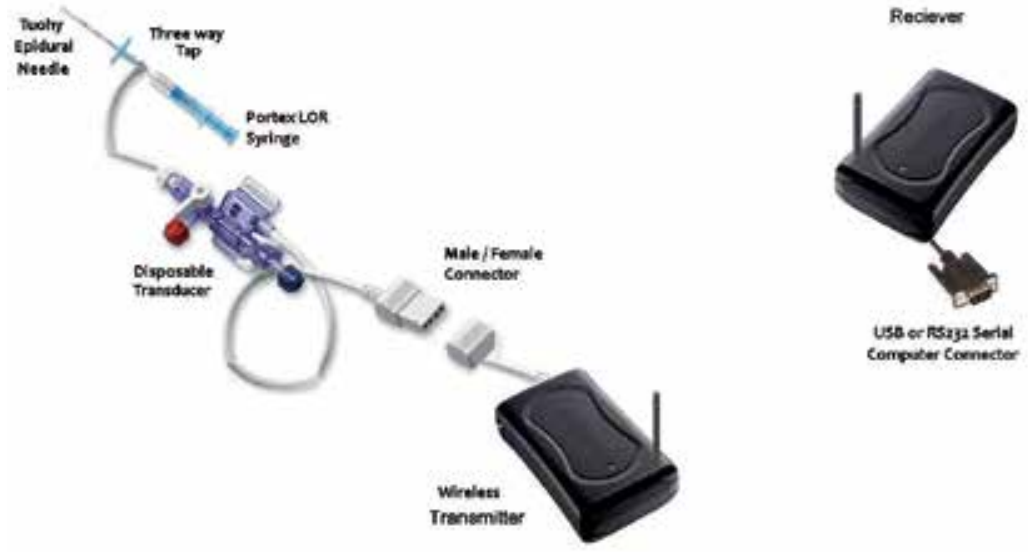

Figure 3. Wireless Device for recording measured pressure of saline during insertion.

The computer can process pressure data, display a real-time graph on screen and simultaneously record the data to a file. When the anaesthetist presses on the syringe plunger, the saline inside the syringe is pressurised and the device quantifies this pressure. The computer runs our custom built software (Figure 4) which monitors pressure data as it arrives [11]. The software displays the live data on screen in the form of a real-time graph, can save graphs as images to file and writes data to a text file. The data files can be used for further analysis using statistical software. Before each insertion, the graph and start-time are reset and a new data file is created. Pressure can be converted into various units. In the current implementation the pressure is measured in $\mathrm{mmHg}$ or $\mathrm{kPa}$ and also a provision is given to determine force on the plunger in Newtons. This directly provides actual pressure measurement of saline inside the needle as applied to a continuum. To test this device a pilot trial was conducted on a porcine cadaver.

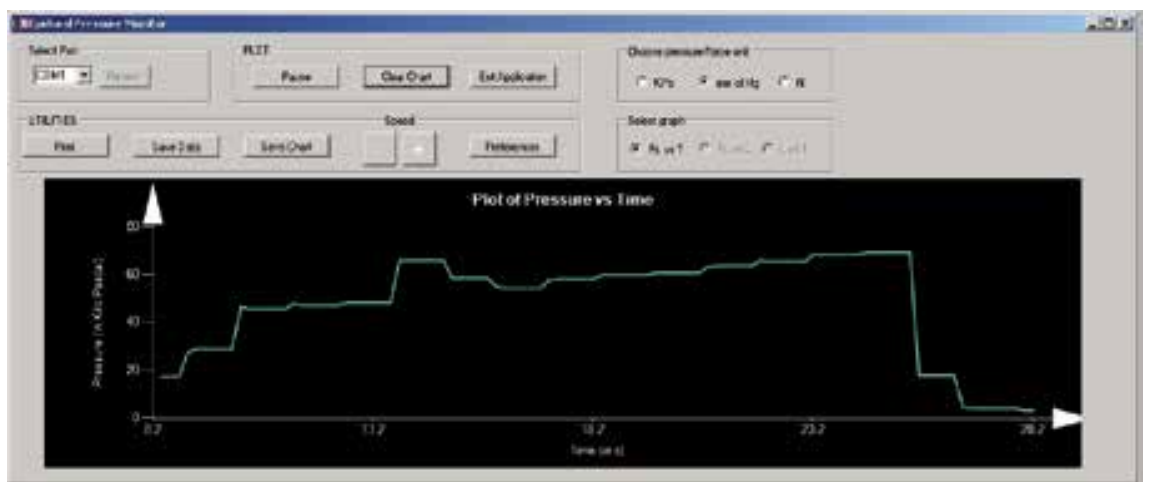

Figure 4. Screen print of the software to monitor and record pressure of saline during insertion

\section{Trial on porcine cadaver}

A trial using a section of a porcine cadaver was conducted to test the pressure measurement device during epidural insertions. The pig is claimed to be the closest animal model for 
human spinal research and can be a representative anatomical model for the human spine and tissues [12]. The porcine tissue specimen was a double loin saddle cut. The cadaver was obtained from a livestock farm within 24 hours of slaughter without being frozen or modified in any way to avoid desiccation and deterioration of the spinal tissues which would affect the pressure measurements. The pig was a standard hybrid Large White cross Saddleback. The specimen contained the entire back in one piece, with the whole spine, and all tissue layers from external skin, through to the thoracic cavity. The porcine tissue was mounted vertically against a wooden support to mimic sitting position, resting upon, but not attached onto, a platform beneath (Figure 5).

Epidural insertions were performed by two experienced anaesthetists. The epidural space was located using a Portex 16-gauge Tuohy needle (Smiths Medical International Ltd, Kent, $\mathrm{UK}$ ) at L2/3 or L3/4 intervertebral levels using a midline approach. Subsequently a number of different vertebral levels ranging from T12-L5 were targeted. The porcine spine was palpated to locate anatomical landmarks prior to insertion. The Tuohy needle with its introducer stylet penetrates the skin as is standard procedure. The recordings of pressure were then started and continuously recorded throughout needle insertion until after the loss of resistance had been experienced.
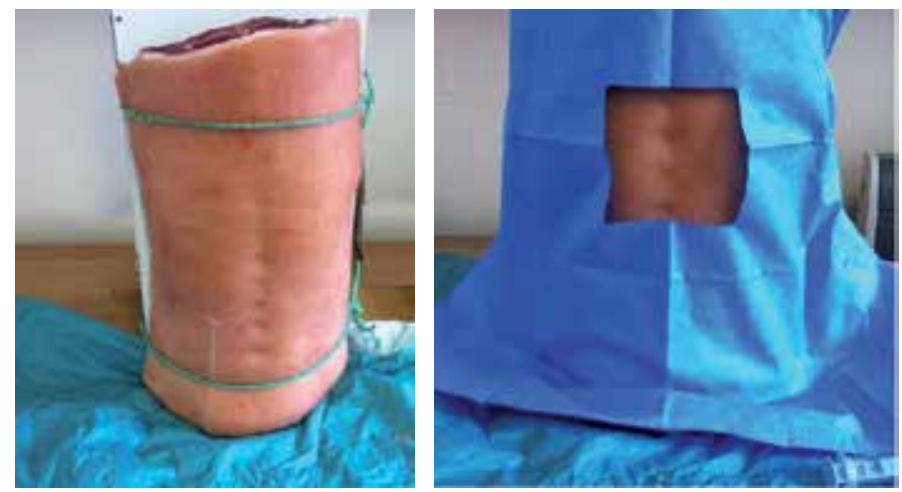

Figure 5. Porcine cadaver set up for Tuohy needle insertions

The majority of insertions located the epidural space during the first attempt. Data from hitting bone was also recorded to analyse the effect on pressure. In some cases, the number of attempts to find the space was greater than three so those recordings were abandoned. The maximum pressure during ligamentum flavum was $500 \mathrm{mmHg}$. The highest pressures were obtained when the Tuohy needle hit bone.

The results demonstrated that during needle insertion the saline pressure started low and gradually built up, although the increase was not entirely steady due to the various tissues encountered. A similar pressure trend was found; a depression occurred on insertion 2 during 3-6 seconds and insertion 3 during 12-15 seconds (Figure 6, circular area). This may have been caused by the interspinous ligament and the pressure required to traverse this was $350 \mathrm{mmHg}$ on insertion 2 and $470 \mathrm{mmHg}$ on insertion 3. The final peak pressure was $500 \mathrm{mmHg}$ which was caused by the ligamentum flavum (Figure 6, rectangular area). It was 
also noted that after the final drop of pressure there was often a 'step' before the bottom pressure was reached (square area). One explanation is that the initial pressure is the effect of opening up the epidural space which is a potential space and also saline pushing the dura away.
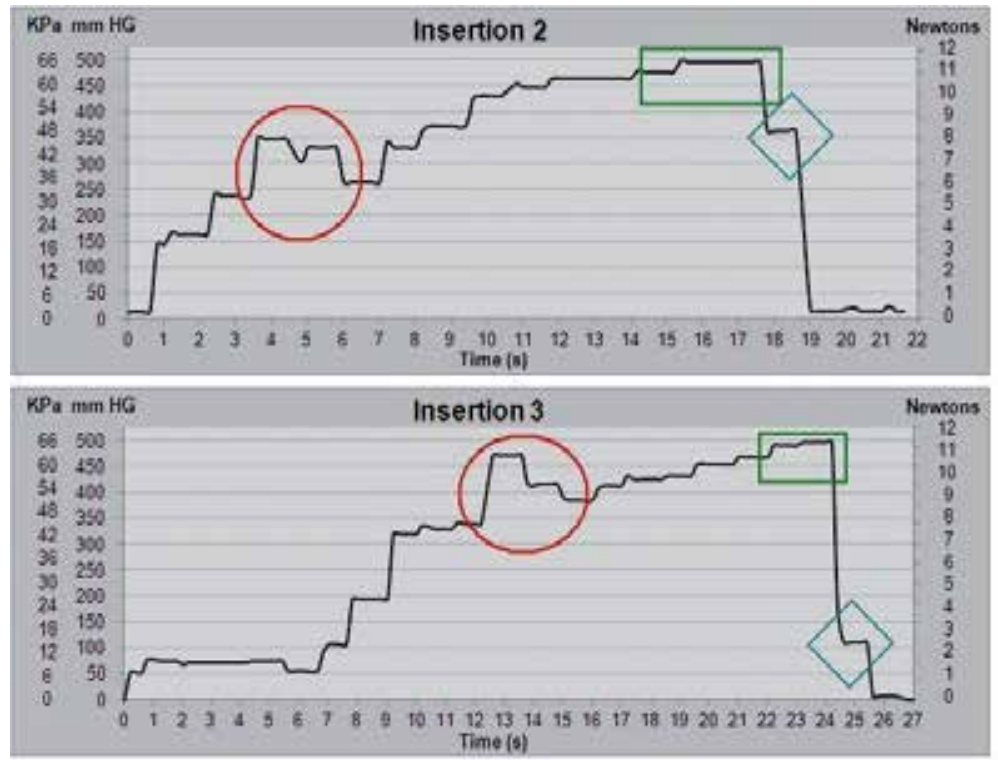

Ligamentum Flavum

Step caused by saline release

Figure 6. Pressure recordings durings two successful insertions to the epidural space.

The opinion of the trial anaesthetists was that porcine tissue did feel like a close approximation to human tissue and the shape of the graphs were similar to graphs previously reported from human insertions [8]. In most cases the resulting pressure-time graphs clearly show a drop when the loss of resistance occurred as the needle entered the epidural space (Figure 6). The maximum pressure peak during successful insertions ranged from 470 to $500 \mathrm{mmHg}(62.7-66.7 \mathrm{kPa}$ ) caused by ligamentum flavum. After this the needle tip enters the epidural space causing a sudden loss of pressure back to the starting pressure. The shapes of each graph in successive trials were similar but also different to reflect individual variations.

The results of this pilot trial demonstrate that the wireless pressure measuring system is accurate and responsive in the porcine model. Such measurements from patients could be used to create realistic epidural simulators.

\section{Image processing for non-contact needle depth measurement}

The reason why needle depth is important is that it relates the depths at which each resultant pressure occurred during the epidural procedure. This can also provide 
information about the depth of ligaments. We have developed image processing algorithms to measure the needle depth by a wireless camera during insertion [13].

During the epidural insertion procedure, the needle is slowly advanced through layers of tissue into the epidural space which is on average somewhere between $40-80 \mathrm{~mm}$ deep. It is possible to record the depth of the needle tip by viewing the $10 \mathrm{~mm}$ markings printed on the metallic needle; however, it is important to measure the needle depth precisely so that the needle travel can be guided with available measurements from techniques such as ultrasound scanning or magnetic resonance imaging for precise needle placement in the actual procedure. We have developed a novel image processing technique which aims to measure insertion depth of an epidural Tuohy needle in real-time. The implemented technique uses a single wireless camera to transmit depth data remotely to a host computer. Combining length and pressure data enables more accurate interpretation of the data in that the various changes in pressure can be linked to the actual depth at which the changes occurred.

The 16 gauge Portex Tuohy needle of $80 \mathrm{~mm}$ length (Figure 7 ) is the most common epidural needle used in hospitals. The needle has grey and silver markings on the metallic shaft at $10 \mathrm{~mm}$ intervals which are used by the software as a reference length. The blue handle is the plastic part at the base which is held by the operator and connected to a LOR syringe. This is used for colour detection.

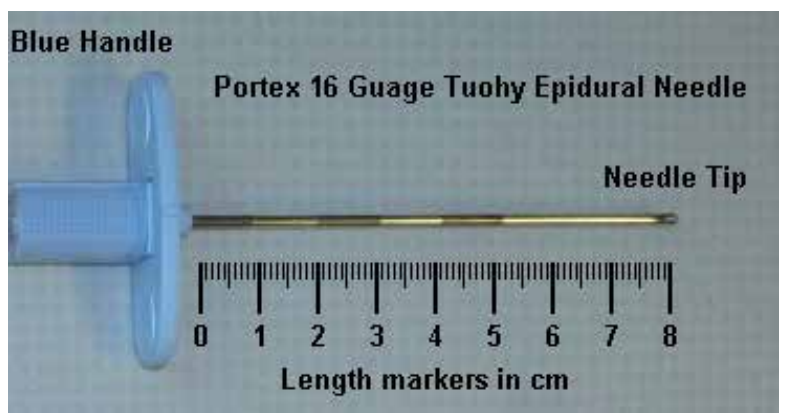

Figure 7. Properties of the $80 \mathrm{~mm}$ Tuohy needle used for image processing

The actual technique of length and size measurement by digital image processing is well established, however, in this specific circumstance, image processing is much more complex and challenging due to many reasons; (i) the needle is a thin, narrow object, (ii) the needle is composed of reflective stainless steel, (iii) the needle is circular in cross-section causing colour changes around the shaft of the needle, (iv) wireless camera introduces transmission noises, (v) as the needle is tilted it reflects in different directions, (vi) the needle will not be the only object in the foreground due to the operator's hands and patient's back, (vii) lighting conditions vary from room to room. We have overcome these problems by advanced analysing techniques focusing on a small area of the dynamic environment.

The actual technique involves placing a wireless camera in the procedure room, one metre away from the needle insertion, which will transmit data to a remotely located computer. 
The camera transmits a $640 \times 480$ pixel image in full colour over a $20 \mathrm{MHz}$ wireless link. The computer contains the image processing algorithm to detect the visible needle in the image and measure its length. The first step in the algorithm is to automatically calibrate the background model. For ten seconds, with no objects in the foreground, the colour values (HSV) for each pixel are analyzed. Maximum and minimum values are stored in an array and used later as a background model. HSV values from each frame are compared to the background model. Foreground objects are identified by HSV values outside of the expected range. The pixels from foreground objects are scanned for HSV values which match the blue handle. The centre point of the blue handle is found by taking an average and is stored for object tracking in subsequent frames, and is assumed to be approximately at the level of the needle shaft. The rightmost edge is stored and assumed to be the start point of the metal shaft. The blue handle is removed from further processing. The algorithm scans horizontally from the position of the blue handle to find the metal needle shaft by matching HSV values. The leftmost and rightmost pixels in the metal shaft are identified. These are stored for tracking in subsequent frames. At this point a strip of image remains over the needle. For each column, an average HSV value is taken. This average is used to create four separate histograms for $\mathrm{H}, \mathrm{S}, \mathrm{V}$ and the total along the length of the metallic shaft. The histograms identify sudden changes in colour, caused by the boundaries between $10 \mathrm{~mm}$ markings (Figure 8). Histograms make the markings more detectable under reflective conditions. The number of visible $10 \mathrm{~mm}$ markings is counted. The number of pixels in each division is counted to find how many pixels equate to $10 \mathrm{~mm}$. If the final marking is only partially visible the length is calculated by comparing it to a full division.

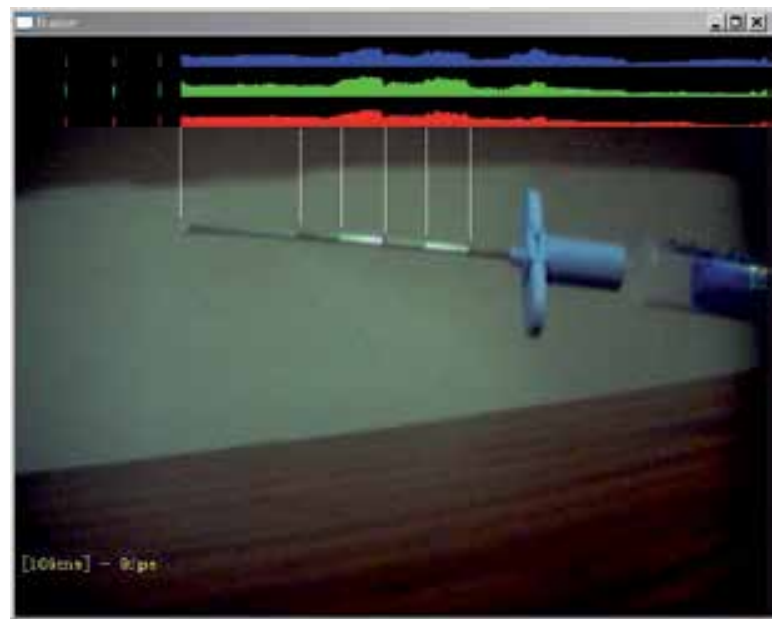

Figure 8. Output of the algorithm to measure needle length with histograms

The image processing algorithm was tested during insertions. The needle was successfully detected and measured accurately in most frames. The developed software was used to draw a graph of the length in real time and write the length data into a data file. Figure 9 shows the graph during an insertion in which the needle was slowly advanced and then rapidly withdrawn. We found that length measurement was accurate to within $+/-3 \mathrm{~mm}$, 
when the needle was $500 \mathrm{~mm}$ from the camera. The graph shows two erroneous readings at about 4 and 8 seconds, which was due to camera noise and in these frames the needle shaft was not detected properly, but all other frames were successfully measured and verified by the actual measurement. The total insertion took about fifteen seconds with 10 frames per second. The failure rate was 3 frames out of 150 which gave an overall $97.8 \%$ reliability during this insertion [13]. Errors like this could potentially be removed by ignoring sudden jumps in the data. The graph currently displays length but this can be converted from length to needle depth by simply subtracting the value from $80 \mathrm{~mm}$, which is the total length of the needle.

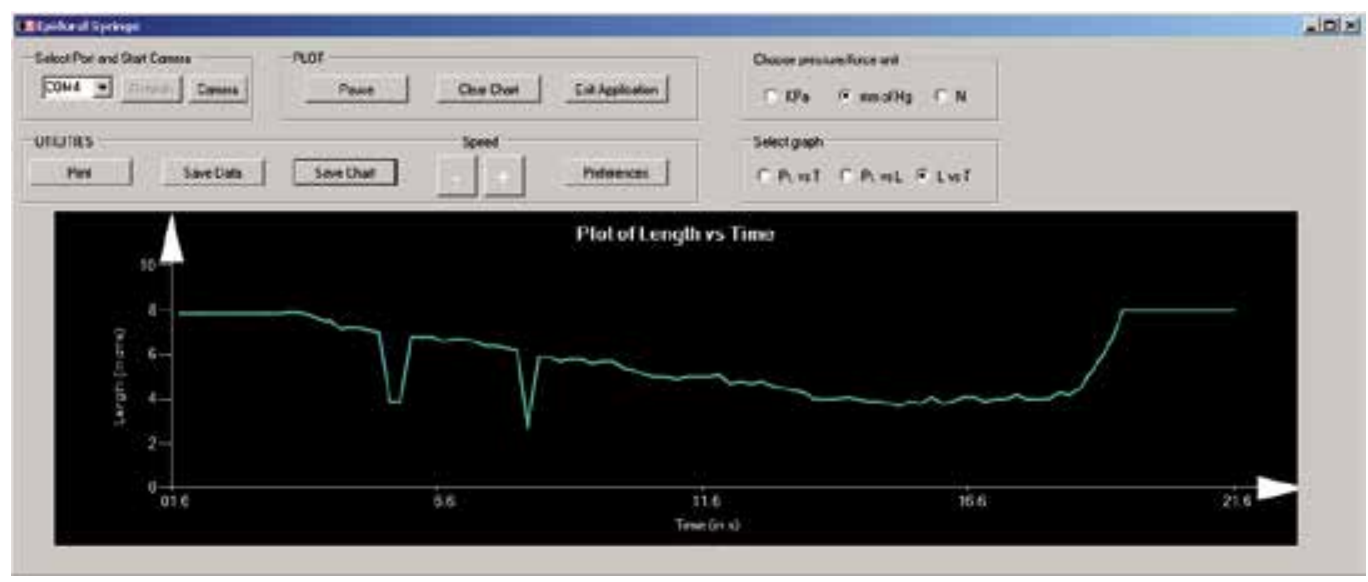

Figure 9. Software showing plot of needle length during insertion

The distance between needle and camera can be varied because length is measured using the $10 \mathrm{~mm}$ markings as a reference length. At distances over $150 \mathrm{~cm}$ the reliability dropped but this could be improved with a higher resolution camera. The needle can be tilted up or down to $+/-30$ without any adverse effect to measurements. Tilting towards or away from the camera does not affect measurement as long as the divisions are clearly visible because division length differentiates between length reductions caused by tilt and caused by insertion. Failures occurred on some frames, due to blur in the image, or at certain angles where silver and grey areas became merged. The background model successfully removed the majority of background, even with cluttered multi-colour backgrounds.

\section{3D spine modelling for epidural training}

In order to simulate the whole epidural procedure a realistic user interface must be provided together with the flexibility of 3D visualization and haptic interaction. The 3D models for the epidural simulator were generated with an object modelling software. Each vertebra is an individual wireframe model, constructed from 514 vertices. The vertices are positioned and then wrapped by a texture. Shadows and light sources are applied through OpenGL interfaces. The spine in the simulator contains 26 separate objects for the thoracic, cervical and lumbar spinal vertebrae, sacrum and coccyx. Layers of tissue, fat, muscle and 
skin were appended as layers above the bones. The different parts of the model were exported into separate format files. The format is text based with each vertex on a separate line. A custom C++ OpenGL graphics application then parses the text file to re-create each vertex. The epidural Tuohy needle was created as separate 3D models allowing it to be moved around independently. This is important to allow the operator to place the needle anywhere along the spine for training purposes.

The 3D objects can be viewed as stereograms (Figure 10) by displaying two images of the same object side by side with slight rotation around the $\mathrm{Y}$ axis [14]. The epidural simulator also supports this method of stereo in addition to page-flip stereo.

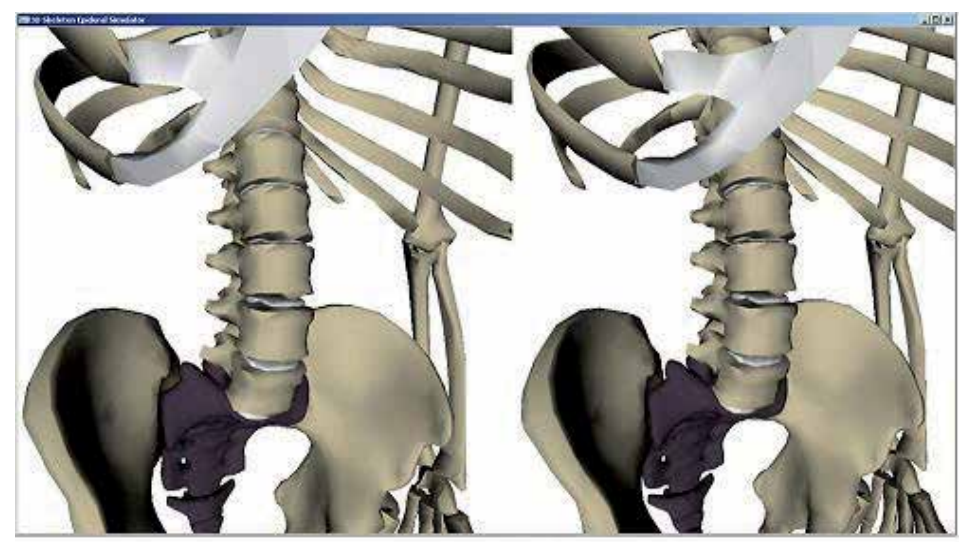

Figure 10. Stereogram view of the spine model with two perspectives and binocular parallax

Transparency is applied to skin, subcutaneous fat, supraspinous ligament, interspinous ligament and ligamentum flavum. This allows the user to see the position of the needle tip in the tissue layers. Transparency can be adjusted during the simulation by a control on the keyboard. Rotation is enabled allowing the camera angle to rotate around the scene. This is applied by OpenGL translation and rotation which gives an effect of camera movement whilst the other objects all remain stationary. During rotation, the tip of the needle remains at the central focus point of the screen. Zoom can be applied to move closer or further away from the site of insertion in the working epidural simulator. Pan can also be applied which is a translation of the camera which allows the user to view other areas or to move up and down the spine when selecting the insertion site.

Another issue equally important is the flexibility built into the spine model. There are four common patient positions adopted during the administration of spinal or epidural anaesthesia [15]. Lateral decubitus (Figure 11) involves lying down sideways on the patients left or right, usually the right side is used for caesarean patients, because it is the opposite side from which the patient will lie on during surgery in the left lateral tilt position, which helps to increase the spread of anaesthetic. When the patient lies in lateral positions their back should be close and parallel to the edge of the bed, with their spine in a straight line. However, a variation to this position, maximal lumbar flexion in the lateral decubitus position can be used. The sitting position is preferred and often required in obese patients to 
enable the palpation of spinal processes and identification of the midline. Finally, the sitting position combined with maximal lumbar flexion is also used, and having the patient bend forward is advantageous to the anaesthetists because it increases the space between the vertebrae, which increases the target space for the needle to pass through.

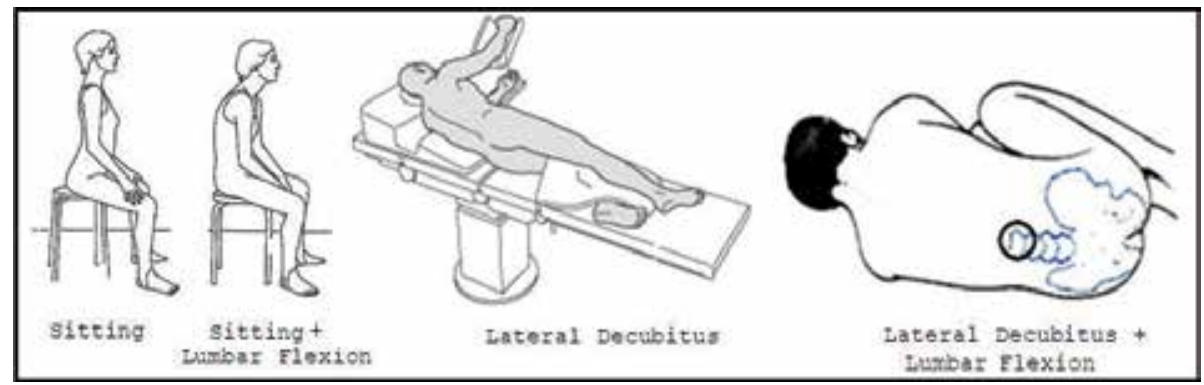

Figure 11. Four common patient positions used for epidural insertion

Based on this, the patient could bend the spine to various positions, so the epidural simulator is required to use computer graphics models of the human spine which can bend, flex and twist. The model can realistically duplicate the shape of the spine during various sitting positions adopted by patients during surgery and epidural anaesthesia. The extent of bending and flexing is kept within the limits of human spine flexibility. Also the model vertebrate adapt in size to match weight and height of specific patient bodies based on parametric modelling [16]. Our spine model is flexible for epidural simulation which offers accurate models of spinal vertebrae.

The human spine consists of twenty six vertebrae. Each of the vertebrae connects with numerous ligaments. Internally, there is a protective space running through the centre of the spine, housing the spinal cord. The column of vertebrae also provides connection points with the ribs and back muscles. The twenty six vertebrae are segmented into five regions, each with varying characteristics. From cranial to caudal there are cervical vertebrae (C1C7), thoracic vertebrae (T1 - T12), lumbar vertebrae (L1 - L5), sacrum and coccyx. The human spine is able to bend, flex and rotate in various directions. Lumbar flexion occurs when the patient bends forwards and lumbar extension occurs when bending backwards. The spine was modelled using 3D design software, formed from 26 individual vertebrae, shown in Figure 12. The 26 vertebrae were each loaded as 3D models into a custom made software graphics application. The software renders 3D objects using vertices with the OpenGL graphics library and its utility toolkit (GLUT). The colours of each region of vertebrae bone, flesh and the spinal discs were set using materials.

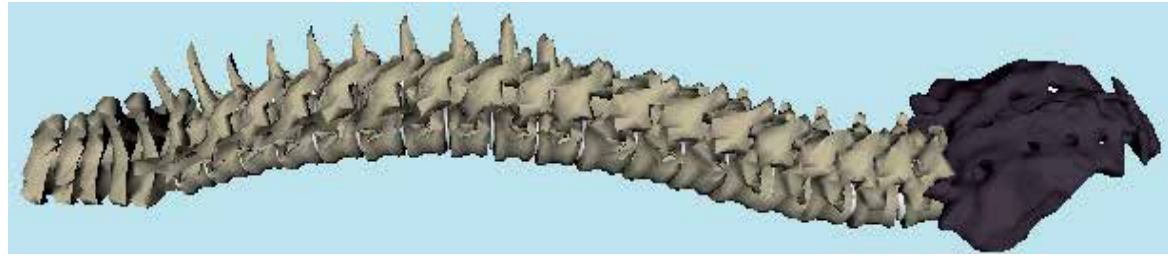

Figure 12. The model spine consisting of 26 individually rendered 3D vertebrae 
Initially the vertebrae are positioned in the standing position and are then adjusted by mathematical equations to match the current patient position. The curvature of the spine for four common patient positions was calculated using the equations. The shape of the spine was based on the four common patient positions used for epidural insertion. Our model's prediction for the spine shape for each of the positions is shown in Figure 13 [14].

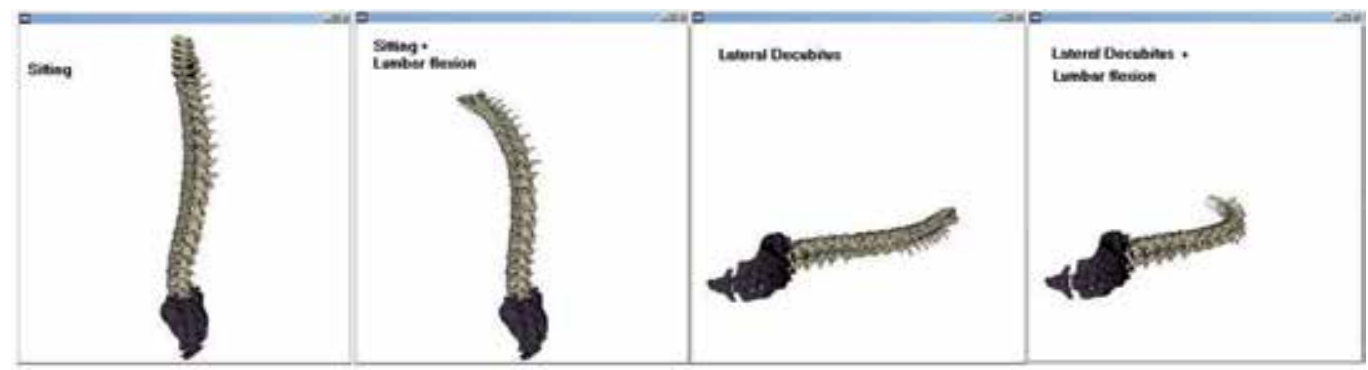

Figure 13. The spine model with flexion for four common patient positions

The ability to flex and rotate the spine has provided the opportunity to simulate epidural insertions on patients in various positions. This is important because the feeling of insertion is different for each patient position. This novel aspect has not been attempted in epidural simulation before and will increase versatility of the simulation.

\section{Heterogeneous ligament modelling}

Since the introduction of traditional computer graphics and modelling techniques, the primary focus has been to display and modelling of homogenous objects which have uniform interior and consist of one material throughout. This was acceptable for many situations, however, such surface-based approaches were aimed to represent the visual appearance of the external layer of objects, leaving the interior untouched. Recently, with the availability of increased computing power, the focus has shifted from surface-based to volume-based graphics, whereby volume-based architecture attempt to describe the material structure of internal regions by the use of voxels [17]. This can allow manipulation and experimentation on the physical properties of the materials, such as density, friction, elasticity, tensile strength and in so doing opens up new possibilities for experimentation. Heterogeneous objects are a step further, being solid physical objects, which consist of two or more material primitives but offering the advantage of materials that may be distributed continuously blending with each other.

For epidural needle insertion, the needle passes through several ligaments along its path to the epidural space, with each of the ligaments having different properties such as density, resistance to insertion and friction (see Section 3). A model is required to encompass these aspects of each ligament if the graphics are to be capable of displaying a true likeness of the materials in-vivo. Ligamentum flavum (LF) is heterogeneous in nature, containing both elastic tissue and fibrous tissue. Certain data describing the ligamentum flavum has been recorded in the literature and can be used to set up a heterogeneous model of the ligament. 
As LF thickness increases, fibrosis increases and elastic tissue decreases. The dorsal side of LF contains more fibrous tissue and less elastic tissue than the dural and middle sides, as indicated by a fibrosis Score of 1.58, 1.63, and 2.63 for dural, middle, and dorsal sides respectively [18]. The loss of elastic fibres caused by increased thickness is more pronounced along the dorsal side. A single patient has several ligamentum flava, one at each spinal level between the lamina and their thicknesses vary according to the spinal level. A study of 77 patients measured LF at spinal level L2/3, L3/4, L4/5, and L5/S1, the mean LF thickness is $2.41,3.25,4.08$, and $2.68 \mathrm{~mm}$ [18]. It was shown that the thickest part of ligamentum flavum is consistently at L4/5, which is the level that endures the greatest mechanical stress. LF is crescent shaped in cross section on the horizontal plane with the thickest part in the middle. It wraps around the circular epidural space and dura. It connects to lamina above and below. The elastic fibres are yellow in colour, hence 'flava' being Latin for yellow. Each flava is a separate ligament which is clearly seen from the side of the lamina.

Object modelling software was used to create a model of the vertebrae. At the location of L2/L3 a ligamentum flavum was modelled with the thickness $2.41 \mathrm{~mm}$ which was internally comprised of bundles of fibres (Figure 14).

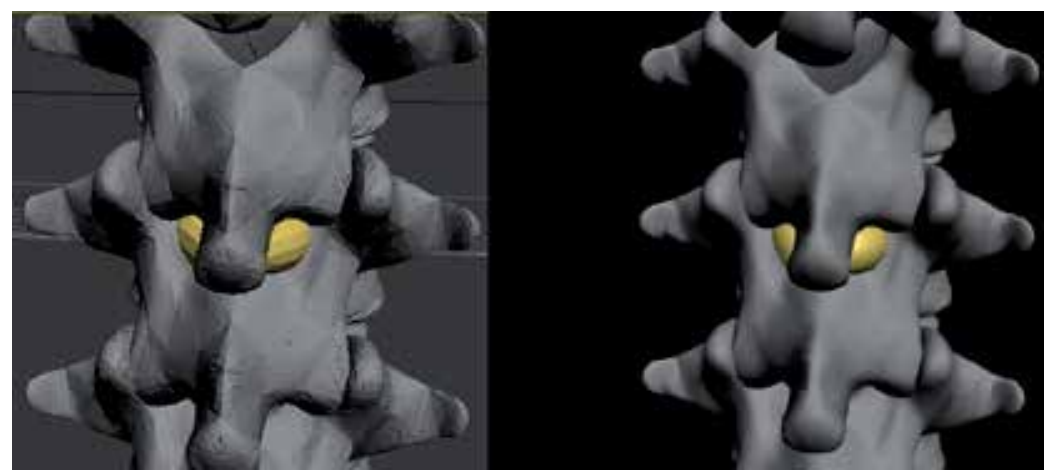

Figure 14. The modelled ligamentum flavum between L2/L3 vertebrae.

The interior structure of the ligamentum flavum has been modelled by numerous bundles of fibres extending vertically and parallel to one another, as do the elastic and fibrous tissues in-vivo. By creating this heterogeneous model of the internal structure of ligamentum flavum, the model will describe more accurately how the material responds to a needle being inserted through it. Similar models may be created for interspinous ligament and supraspinous ligament which are also both heterogeneous in nature, consisting of over three types of elastic fibres that can used to provide realistic haptic feedback.

\section{3D visualisation of epidural procedure}

We have applied stereoscopic 3D computer graphics for visualization of epidural insertions. The stereoscopic images are viewed through a head mounted visor containing two OLED micro-displays in stereo using the page-flipped method. The 3D graphics are built from several vertex models of the anatomical structures as described in section 7 . The stereo 
simulation allows depth to be perceived so that the operator can judge depth of the needle tip in relation to tissue layers and bones, which aids to the location of the epidural space. Applying stereoscopic vision to epidural simulators helps the operator to visualize the depths required for correct needle placement in the epidural space [14].

Depth judgement is crucial to the technique and since stereographics allows the perception of depth in 3D graphics, epidural simulators can benefit greatly from stereo-technology. Here the aim is to apply stereo vision technology to simulate epidural needle insertion. Without stereo graphics the depths of objects in simulations are not perceived accurately. By viewing 3D graphics on a flat computer screen there is no way of knowing the actual distance between objects other than by estimating their size. Estimation is not always accurate and some medical applications may require far more precision in depth perception. Epidural simulators require the needle tip to penetrate several layers of tissue between 42 $47 \mathrm{~mm}$ thick and must stop within the $6 \mathrm{~mm}$ epidural space [19], which is difficult to achieve without depth perception. With stereo vision, distance can be perceived natively allowing the user to intuitively view the depth and distance between objects by perceiving differences between the two images, if images are appropriately scaled.

Stereo glasses contain two small OLED screens, one for each eye. Alternatively, glasses can be polarized, which allows viewing of a polarized screen, which has both images superimposed, one of which arrives at each eye. Shutter glasses can be used which contain moving mechanisms to consecutively close each eye similar to a camera shutter. The screen then displays images for left and right eye consecutively at the same shutter speed. Alternatively, a glasses free approach, vertically dispersive holographic screen (VDHS) can be used by directing two beams of light containing the images into each eye separately [20]. Mirror screens contain two monitors mounted at 110 degrees with a plane of silver-coated glass combining the two images and cross-polarized glasses are worn to separate the images. For all stereo systems, once the two images arrive separately at each eye, the brain combines them to generate $3 \mathrm{D}$ with depth perception based on some calibrated data.

For this epidural simulator, we have used stereo glasses containing two OLED microdisplays, one for each eye, with magnifying lenses. Figure 15 shows how the epidural simulator is being used with the stereo glasses displaying the 3D spine model. The glasses have advantages that the user can see the image whichever direction they look in and as they turn their head motion detectors can rotate the image to follow. The glasses produce a 40-degree diagonal field of view for each eye. The image appears the same size as a 105 inch projection screen viewed from 12 feet. Magnifying lenses allow the eye to focus further away avoiding eye strain. The graphic resolution must be fixed at $800 \times 600$ pixels which display sufficient details. Two separate images are displayed on each eye display. Stereo is achieved by using the page-flip method. A signal is generated by the graphics card at $60 \mathrm{~Hz}$, with the images consecutively swapped between left eye and right eye. The swapping is done by the graphics card drivers. The hardware inside the 3D glasses splits this into two separate $30 \mathrm{~Hz}$ signals and delivers one to each eye, this results in stereoscopic images. 


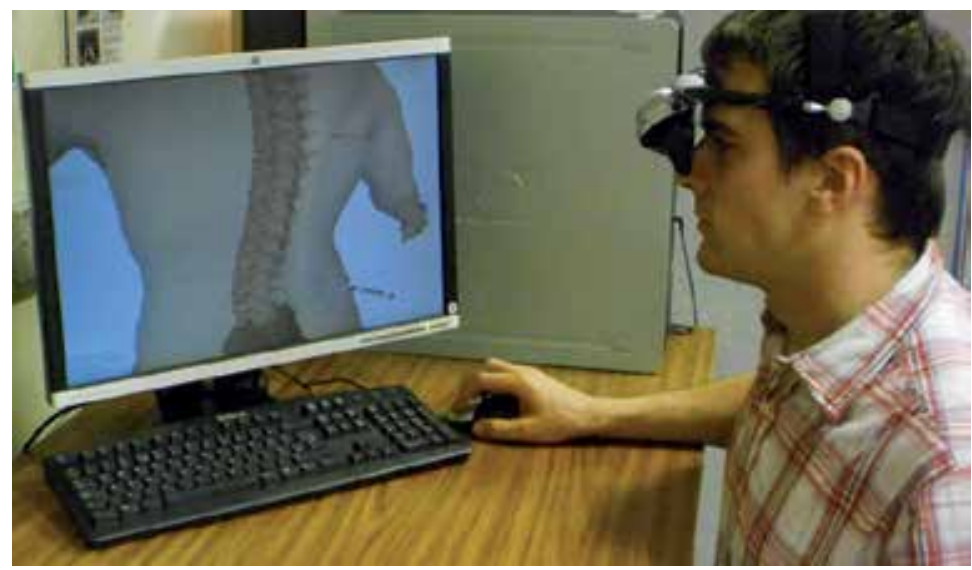

Figure 15. Stereo glasses used for epidural insertion visualization

The epidural simulator software interfaces with head motion detectors. When the user turns their head, the 3D objects rotate by the same degree in the opposite direction to create an illusion of camera rotation. This interface allows the user to change the view point to different directions by turning their head, so that the mouse and keyboard are no longer required. The feedback from experienced anaesthetists suggested that the flexible spine model will be useful for modelling patient position. The options for adjustable body shape and size was seen as a positive step to encapsulate the variety of patients which has not previously been accomplished.

\section{Haptic interface for epidural insertion}

Haptic devices have become a more popular and accepted tool for medical simulation and provide an accurate way of re-creating the feel of surgery [21, 22]. The insertion of an epidural is a procedure which relies almost entirely upon feeling the forces on the needle. Epidural simulators are therefore ideally suited to haptic technology. This section describes methods for configuration of a haptic device to interact with 3D computer graphics as part of a high fidelity epidural simulator development program.

Haptic devices have been used in epidural simulators previously, although they are not based on measured patient data from needle insertions. Instead, they are configured by 'experts' trialling and adjusting the system. It is therefore hard to assess the accuracy of the forces generated and so creates a real potential for improvement. The haptic device has currently been set up to reconstruct the force data found during the porcine trial. The force data from the graphs were divided into sections to represent each of the tissue layers separately [23].

A haptic device has been connected and used as an input to move the needle in 3D, and also to generate force feedback to the user during insertion (Figure 16). A needle insertion trial was conducted on a porcine cadaver to obtain resultant pressure data (Section 5). The data generated from this trial was used to recreate the feeling of epidural insertion in the simulator. The interaction forces have been approximated to the resultant force obtained during the trial 
representing the force generated by the haptic device. The haptic device is interfaced with the 3D graphics (see Sections 7-9) for visualization. As the haptic stylus is moved, the needle moves on the screen and the depth of the needle tip indicates which tissue layer is being penetrated. Different forces are generated by the haptic device for each tissue layer as the epidural needle is inserted. As the needle enters the epidural space, the force drops to indicate loss of resistance. An advantage to the use of haptic devices for epidural simulators is that they can accept various adjustable settings, so that patient variation including weight, height, age and sex can be accounted for, which helps to train for a range of patients. Patient variety is becoming an even more important aspect than ever since the current obesity epidemic poses great challenges for the anaesthetist. In obese patients, the depth to the epidural space is increased, anatomical landmarks are harder to feel and the midline is more difficult to locate. The resultant effect is that the risk of injury is increased.

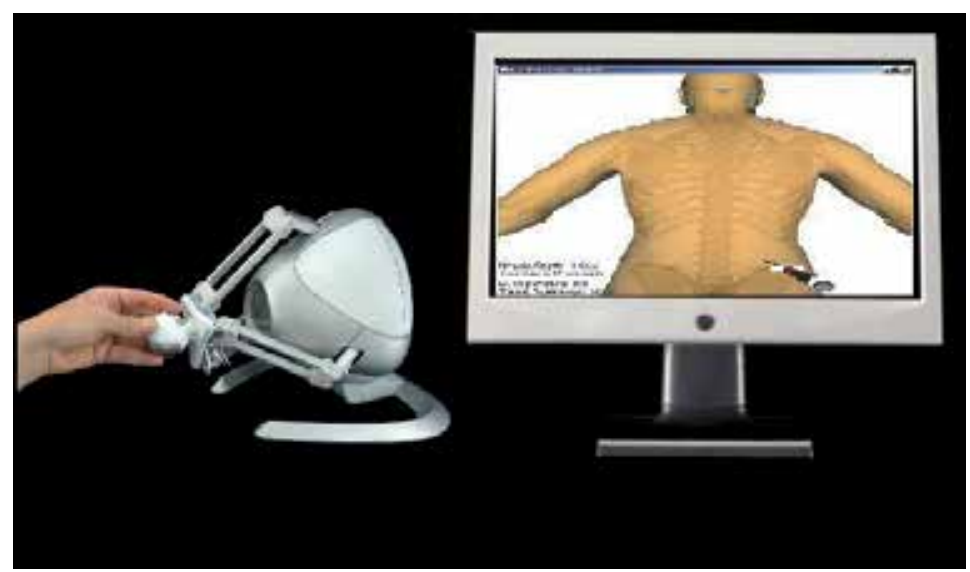

Figure 16. The haptic device interfaced with the graphics

To apply different forces to each layer, 3D vector regions were defined within the graphics model. As the needle tip enters these regions, the software identifies which tissue layer the needle is in, based on the depth data from the trial (Table 1). The software then uses a lookup table to find the appropriate force for each layer, and instructs the haptic device to generate that force. The forces generated represent the resultant pressure on the syringe which is a sum of all resistances to insertion, which are the equal and opposite to the force applied by the user. For example, if a particular layer has insertion force of $4.3 \mathrm{~N}$, and the user is pressing with only $3.2 \mathrm{~N}$, then the haptic device exerts $3.2 \mathrm{~N}$, so the stylus remains stationary. Only if the user increases the force to over $4.3 \mathrm{~N}$ the stylus will move forward. Table 1 is based on measurements taken from our porcine trial in line with [24].

The haptic device is also able to simulate palpation of the lumbar region. Palpation is the process for choosing which location to insert the needle. The haptic device was configured for palpation by creating a surface hardness profile of the lumbar region, with a hardness value for each point in the region (see Section 8). The haptic device can be used to press at any point and the user can feel the hardness at that point. This allows the user to locate landmarks and choose a point to commence needle insertion. Our advanced haptic interface is based on the 
measured data and the aim is to develop a generic simulator based on measured data to offer a realistic in-vitro experience before attempting the procedure on actual patients.

\begin{tabular}{|l|c|c|c|}
\hline Porcine Tissue Layer & $\begin{array}{c}\text { Tissue thickness } \\
(\mathrm{mm})\end{array}$ & $\begin{array}{c}\text { Needle depth } \\
(\mathrm{mm})\end{array}$ & $\begin{array}{c}\text { Insertion force } \\
(\mathrm{N})\end{array}$ \\
\hline Skin & 3 & 0 & 12.9 \\
Subcutaneous fat & 6 & 3 & 6 \\
Supraspinous ligament & 4 & 9 & 9 \\
Interspinous ligament & 26 & 13 & 8 \\
Ligamentum flavum & 3 & 39 & 11.1 \\
Epidural space & 6 & 42 & 0 \\
Dura & 15 & 48 & 2.0 \\
\hline
\end{tabular}

Table 1. Insertion forces in porcine $[23,24]$

\section{Human-computer interface for loss of resistance syringe}

With the above developed components, a hardware device has been created consisting of a regular Portex LOR syringe connected to the computer via a serial data transfer device. This allows a regular clinical syringe to be used as part of an interactive system for the epidural simulator development. The syringe was also combined with the haptic device to create a comprehensive human-computer interface. The simulator can measure force applied to the plunger and the resultant pressure of the saline inside the syringe barrel. This interface enables a real clinical syringe to interface with a 3D graphical visualization showing the simulated insertion of the Tuohy epidural needle through the spinal ligaments.

The developed hardware interface makes use of the equipment as developed in Sections 4 \& 6 by incorporating custom made hardware with the developed software and the graphical visualization of the needle insertion procedure. The hardware device takes measurements of the forces applied onto the needle and the resultant pressure of the saline inside the barrel of the syringe caused by the pressure from the operators thumb on the plunger. The measurements are sent to the computer by a custom-made hardware interface device (see Sections 4 \& 6). The graphical simulation uses these measurements to update the needle in the simulation and calculates the needle position. The graphical software calculates if any collisions have occurred between the needle and any bone structures, plus the resistance of insertion to saline, and the force required for the needle to move forwards through the current ligament.

The developed human-computer interface uses an actual syringe and an epidural Tuohy needle as shown in Figure 17. During insertions, the LOR syringe is normally connected directly onto the Tuohy needle. We have introduced a three-way tap between the needle and syringe. This connects onto a one metre length of saline manometer tubing which runs to a disposable pressure transducer. The transducer converts the pressure of the saline into an electrical signal. The electrical signal is connected into a hardware device which amplifies and sends the pressure reading to the computer. This allows the graphics visualization to 
update according the pressure applied by the operator's thumb on the plunger of the syringe. This has the advantage that the user can control the visualization with the same equipment that would be used in-vivo, which is a more natural interface than simply using keyboard or mouse. Additionally, since the saline line separates the hardware device from the needle, the user can move the needle around since it is attached only by the saline line.

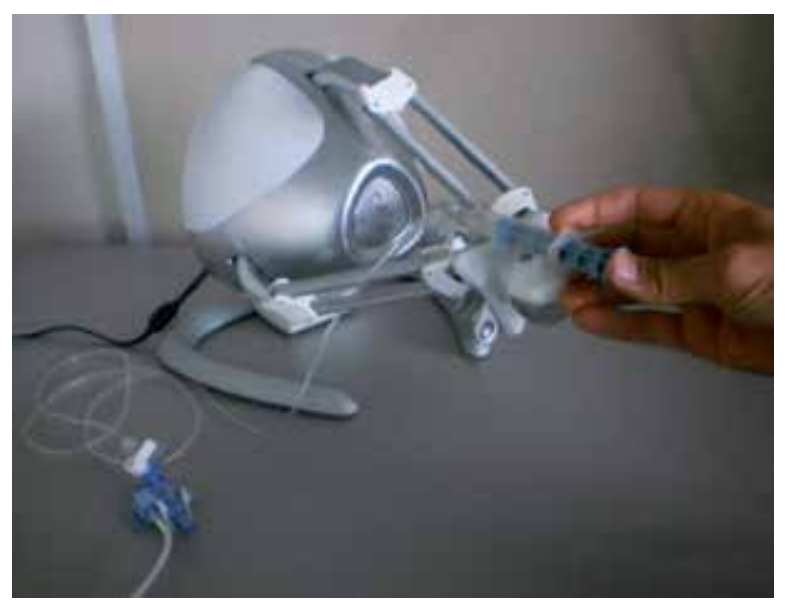

Figure 17. The syringe connected to the computer as an input device.

The hardware device runs at $8 \mathrm{MHz}$. Data is transmitted from the hardware device to the computer using the serial RS232 port. The serial bit rate is running at 22000 bits per second. The serial data transfer protocol uses $-12 \mathrm{~V}$ DC as a positive bit and $+12 \mathrm{~V}$ DC as a negative bit. The serial transfer cycle starts with a negative start bit, followed by 8 data bits sent consecutively and finished with a positive stop bit. As shown in Figure 18, the following start bit can then occur either immediately or after a pause of arbitrary length.

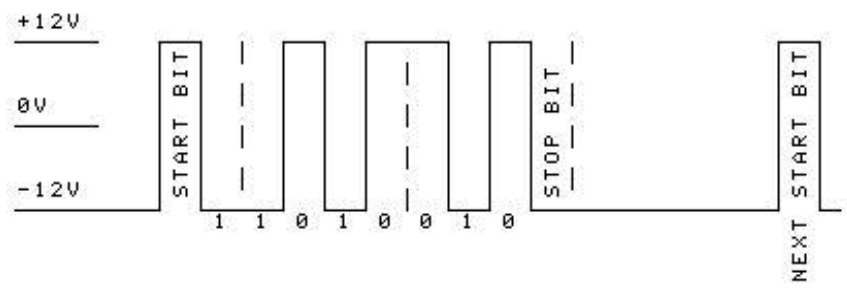

Figure 18. Binary serial data transfer protocol.

The 8 data bits are received and interpreted as binary and converted into a decimal number from 0 to 255 for use in the software. The decimal value represents the pressure of the saline between 0 to $70 \mathrm{kPa}$, which is 0 to $550 \mathrm{mmHg}$. The 256 possible values give an accuracy resolution to within $+/-0.14 \mathrm{kPa}$. This can be easily increased to 1024 with 10 bits data transfer which will then provide accuracy of within $+/-0.03 \mathrm{kPa}$. The speed could also increase beyond the current 22000 bits per second but it does not seem necessary since no delay is noticed between pressing the plunger and seeing the results on screen. Currently at 22000 bits per second the time delay between bits is $45 \mu \mathrm{S}$ so the start bit is identified by 
testing the pin for $+12 \mathrm{~V}$, and then checking again after $22 \mu \mathrm{S}$ for the same high value. The computer runs the custom designed software which monitors the data as it arrives. Also the values are received by the graphics application which updates the visualisation to match the pressure applied on the physical syringe.

This study has demonstrated the development of a human-computer interface based around a clinical Portex LOR syringe connected via a custom made hardware interface device to a computer for use in an epidural simulator. The results show that the device is both fast and accurate enough to be used seamlessly in the simulation. The addition of the Portex LOR syringe with a pressure monitoring device has undoubtedly improved the human-computer interaction. Using the actual medical components in the implementation is beneficial because epiduralists will be familiar with the syringe and use it to interact with the 3D graphics visualization intuitively. The interface could be modified to be bi-directional i.e. the graphics software could send back data to the device which could control a motor to cause forces which affect the physical needle so that the user can feel the forces through the needle as in-vivo.

\section{Creation of a novel epidural simulator}

The presented biomedical engineering ideas have enabled us to develop a simulator with a combination of engineering, computing and clinical technologies as discussed in previous sections above. Data from the developed measurement devices have been used to configure a realistic force feedback epidural simulator [25]. Numerous improvements have been identified that could enhance existing epidural simulators. Manikin models are generally static and only able to represent one or two patient variations, such as normal and obese. An advanced simulator would be able to simulate insertions on a variety of body mass indices because excess fat deposition has the potential to generate very different changes in patient characteristics.

The developed system offers a virtual reality based epidural simulator (Figure 19) incorporating a 3D graphically modelled spine complete with skin, fat and tissue layers, supraspinous, interspinous ligaments and ligamentum flavum. In the current prototype, a Novint Falcon haptic device is used in combination with a Portex LOR syringe connected as a human-computer interface via a custom made electronic serial interface. As the haptic stylus is moved, the needle follows on the screen in 3D in real time. When pressure is applied to the plunger by the operator's thumb, this is displayed in the graphic model. As the needle is advanced through the tissues, the forces are generated by the haptic device to reconstruct the feelings of needle insertion through each tissue layer. The forces of the needle insertion are based on the recorded forces measured during the clinical trial, and this data based approach is more accurate than previous simulators which have used a user evaluation approach to configure the forces.

Novel aspects of our epidural simulator include stereo graphics, modelled vertebrae, spine flexibility, patient variation, haptic force feedback based on measured needle insertion data, custom made syringe interface. The simulated needle can be inserted at any spinal position 


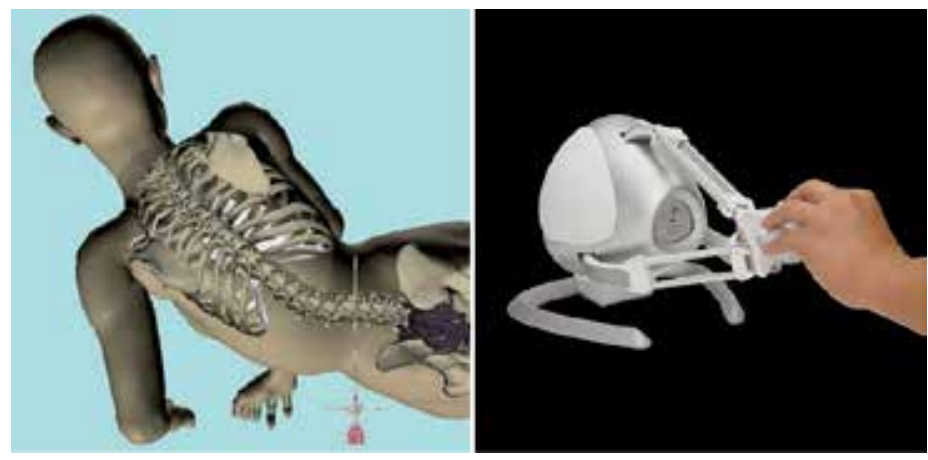

Figure 19. Prototype 3D graphics epidural simulator with haptic device interface

from T2 - L5 and needle direction from midline to paramedian. The 3D graphics allow a close-up real time view of the needle internally during insertion. The virtual patient can adjust to various body shapes, weights and heights since body size considerably affects insertion force. These all have roots in biomedical engineering that can potentially enhance many clinical procedures.

\section{Conclusions}

The application of biomedical engineering approaches can help simplify many clinical problems as demonstrated for the epidural procedure.

We have described in this chapter, the developed measuring devices which have successfully recorded the data on resultant pressure and depth of epidural Tuohy needles during insertions in a porcine model. These data are very useful in developing a realistic high fidelity epidural simulator. We aim to measure pressures in-vivo with obstetric patients in labour of differing body mass indices and integrating this data with ultrasound and MRI scan imaging data. It is our belief that the resulting epidural simulator based on such data will replicate the in-vivo procedure more accurately since it is going to be based on patient specific information. No such simulator exists at the present time.

The overall benefits of applying biomedical engineering techniques to this research are that we are able to achieve a high degree of accuracy and improved technology for replicating the epidural procedure. By achieving higher realism and accuracy of simulation, epiduralists will be better trained with the procedure and this in turn will improve patient safety by minimizing the risk of failure and harm to patients.

\section{Author details}

Venketesh N. Dubey and Neil Vaughan

School of Design, Engineering and Computing, Bournemouth University, Bournemouth, UK

Michael Y. K. Wee and Richard Isaacs

Department of Anaesthesia, Poole Hospital NHS Foundation Trust, Poole, UK 


\section{References}

[1] Dogliotti AM. Research and Clinical Observations on Spinal Anesthesia: With Special Reference to the Peridural Technique. Anesthesia and Analgesia 1933;12:59-65.

[2] Carvalho JCA. Ultrasound-Facilitated Epidurals and Spinals in Obstetrics. Anesthesiology Clinics 2008;26:145-158.

[3] Wilson JG, Pallotta OJ, Reynolds KJ, Owen H. An epidural injection simulator. World Congress on Medical Physics and Biomedical Engineering, Sydney. 2003.

[4] Mayooran Z, Watterson L, Withers P, Line J, Arnett W, Horley R. Mediseus epidural: full-procedure training simulator for epidural analgesia in labour. Proc. SimTecT Healthcare Simulation Conference 2006.

[5] Magill JC, Byl MF, Hinds MF, Agassounon W, Pratt SD, Hess PE. A novel actuator for simulation of epidural anesthesia and other needle insertion procedures. Simul Healthc 2010;5(3):179-84.

[6] Dang T, Annaswamy T, Srinivasan M. Development and evaluation of an epidural injection simulator with force feedback for medical training. Stud Health Technol Inform 2001;81:97-102.

[7] Vidal FP, John NW, Healey AE, Gould DA Simulation of ultrasound guided needle puncture using patient specific data with 3D texture and volume haptics. Comp Anim Virtual Worlds 2008;19:111-127.

[8] Tran, D, Hor, K, Kamani, A, Lessoway V and Rohling, R. Instrumentation of the loss-ofresistance technique for epidural needle insertion, IEEE Transactions on Bio-Medical Engineering, 56(3), 2009, pp.820-7.

[9] Abolhassani, N Patel, R Moallem, M. Needle insertion into soft tissue: A survey. Medical Engineering \& Physics 2007;29(4):413-431.

[10] Gardner, R. Accuracy and reliability of disposable pressure transducers coupled with modern pressure monitors, Critical Care Medicine, 24(5), 1996, pp.879-82.

[11] Vaughan N, Dubey VN, Wee MYK, Isaacs R. Measuring Tuohy Needle Insertion Force on a Porcine Spine, IEEE 6th International Conference on Bioinformatics and Biomedical Engineering (iCBBE 2012), 17-20 May, Shanghai, China.

[12] Busscher, I, Ploegmakers, JJ, Verkerke GJ. and Veldhuizen, AG. Comparative anatomical dimensions of the complete human and porcine spine, European Spine Journal, 19, 2010, pp.1104-1114.

[13] Vaughan N, Dubey VN, Wee MYK, Isaacs R. Epidural Needle Length Measurement by Video Processing, The IET Image Processing Conference 2012, 3-4 July, London.

[14] Vaughan N, Dubey V, Wee M, Isaacs R. Virtual Reality based Enhanced Visualisation of Epidural Insertion, ASME 2012 International Design Engineering Technical Conferences \& Computers and Information in Engineering Conference, DETC2012-70951, August 12-15, 2012, Chicago, IL, USA.

[15] Chestnut DH. Obstetric anesthesia: principles and practice: 2009.

[16] Kasap M, Magnenat-Thalmann N. Skeleton-aware size variations in digital mannequins. The Visual Computer 2011;27:263-274. 
[17] Pasko, A., Adzhiev, V. and Comninos, P., Heterogeneous Objects Modelling and Applications, 2008, Springer, Germany.

[18] Sairyo, K, Biyani, A, Goel, V, Leaman, D, Booth, R, Thomas, J, Gehling, D, Vishnubhotla, L, Long, R, Ebraheim, N. Pathomechanism of Ligamentum Flavum Hypertrophy: A Multidisciplinary Investigation Based on Clinical, Biomechanical, Histologic, and Biologic Assessments, Spine. Issue: Volume 30(23), 1 December 2005, pp 2649-2656.

[19] Cheng, PA. 1963, The anatomical and clinical aspects of epidural anesthesia, Anesthesia and Analgesia; 42(1), pp. 398-406.

[20] Magalhães, DSF, Serra, RL, Vannucci, AL, Moreno, AB, and Li, LM. 2012, Glasses-Free 3D Viewing Systems for Medical Imaging, Optics and Laser Technology, 44(3), pp. 650655.

[21] Coles, T. R, Meglan, D, and John, N. W. 2011, The Role of Haptics in Medical Training Simulators: A Survey of the State of the Art, IEEE Transactions on haptics, 4(1), pp. 5166.

[22] Halvorsen, FH, Elle, OJ, and Fosse, E. 2005, Simulators in Surgery, Minimally Invasive Therapy and Allied Technologies, 14(4), pp. 214-223.

[23] Vaughan N, Dubey VN, Wee MYK, Isaacs R. Haptic interface on measured data for epidural simulation, ASME 2012 International Design Engineering Technical Conferences \& Computers and Information in Engineering Conference DETC201270891, August 12-15, 2012, Chicago, IL, USA.

[24] Hiemenz, L, 2001, Force models for needle insertion created from measured needle puncture data, Medicine meets virtual reality, pp. 180-6.

[25] Vaughan N, Dubey VN, Wee MYK, Isaacs R. Advanced Epidural Simulator with 3D Flexible Spine and Haptic Interface, DMD2012-6837, ASME Design of Medical Devices Conference (DMD 2012), 10-12 April, Minneapolis (USA). 

